\title{
ETSUTM霖
}

\author{
Doctoral Thesis
}

\section{In $\mathbf{G a}_{1-\mathrm{x}} \mathbf{N}$ layers, nanowires, and nanodots on Silicon for clean energy applications}

Presented by:

D. Pavel Aseev

M.Sc. in Nanoelectronics and Nanotechnology
Supervisor:

D. Enrique Calleja Pardo

Prof. Dr.

Catedrático de Universidad

Co-supervisor:

D. Žarko Gačević

Prof. Dr. 


\section{Table of Contents}

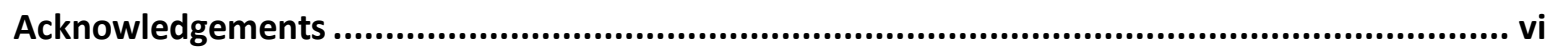

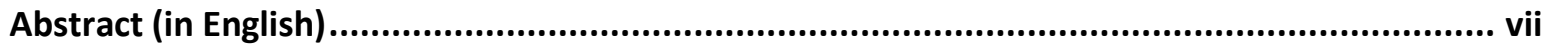

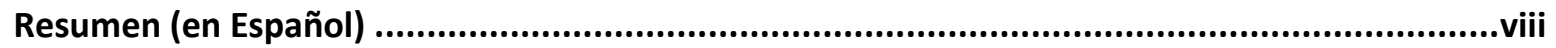

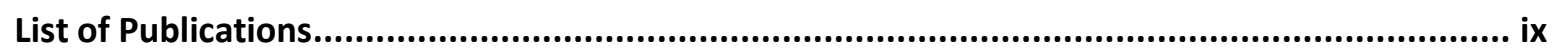

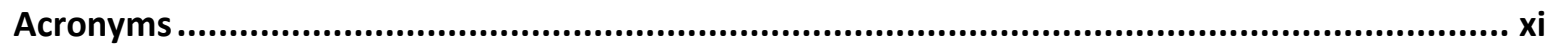

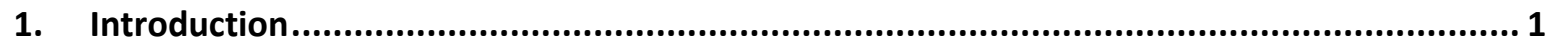

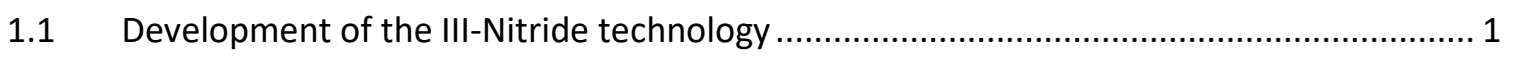

$1.2 \quad$ Further development of III-Nitride epitaxy ................................................................... 2

$1.3 \quad$ Novel potential application: solar-assisted water splitting ............................................. 2

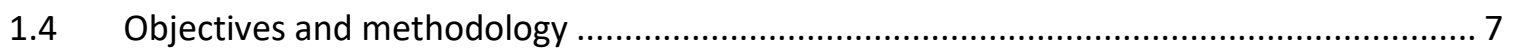

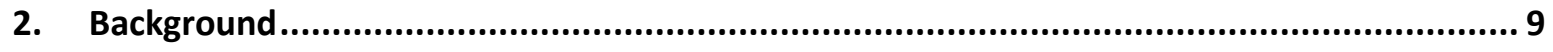

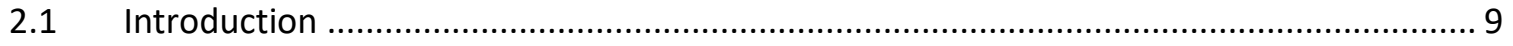

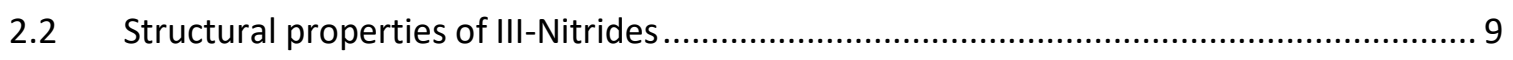

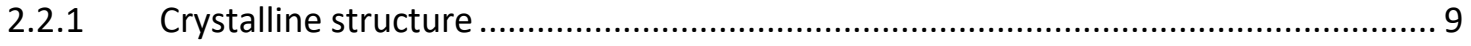

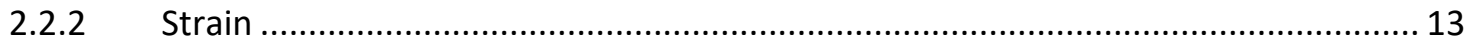

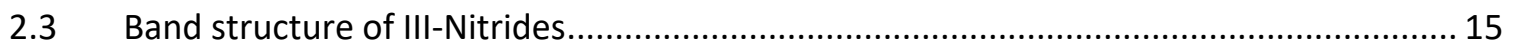

2.3.1 Potential profiles and bandgap bowing parameter ............................................. 15

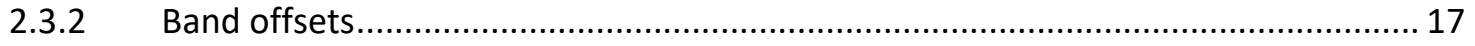

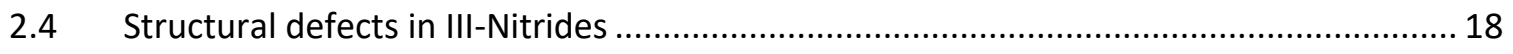

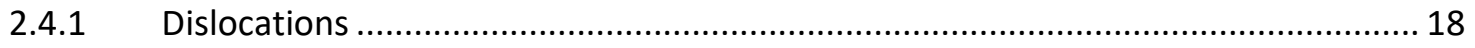

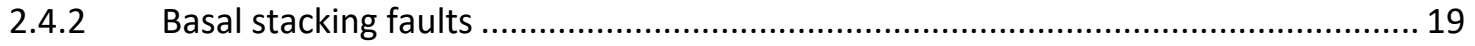

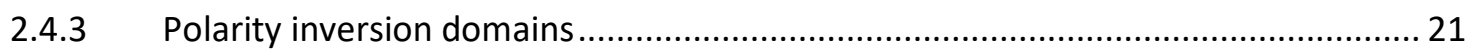

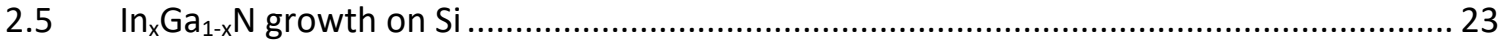

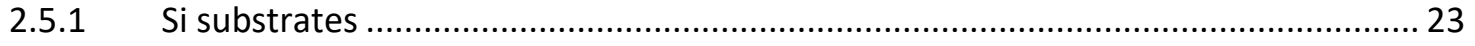

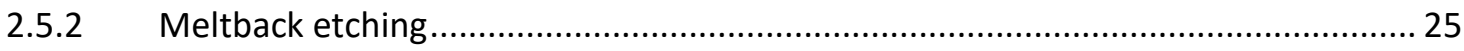

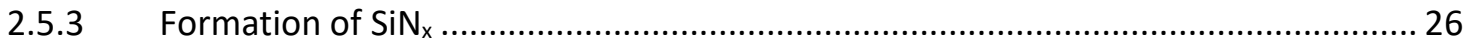

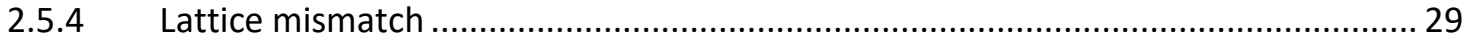

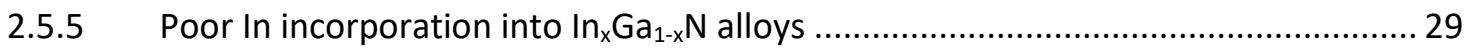

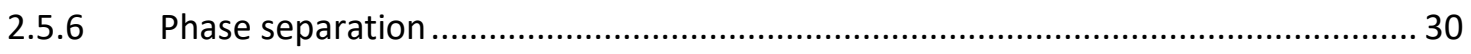

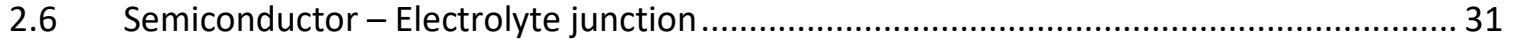




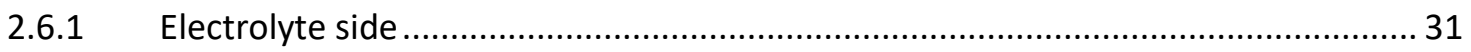

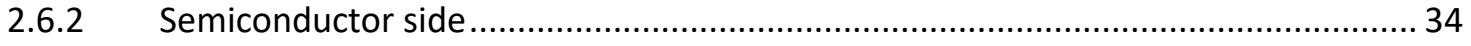

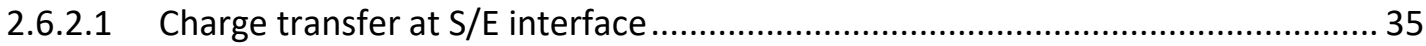

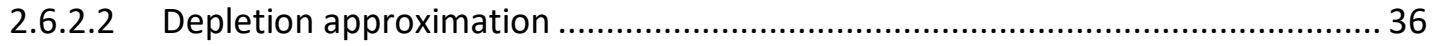

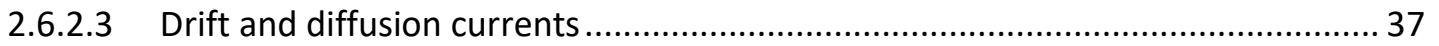

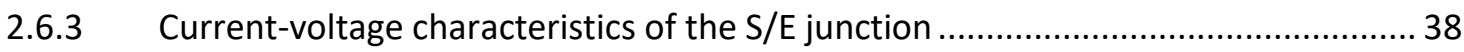

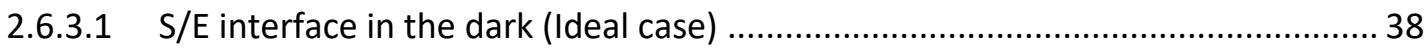

2.6.3.2 S/E interface under illumination (Ideal case) ...................................................... 41

2.6.3.3 Competition between charge transfer and recombination ................................ 42

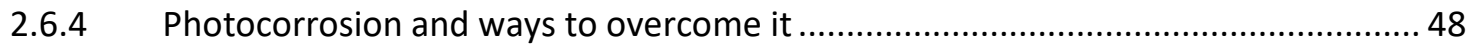

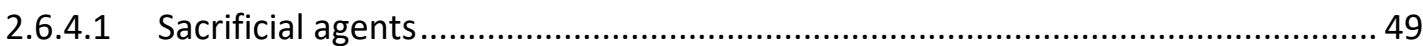

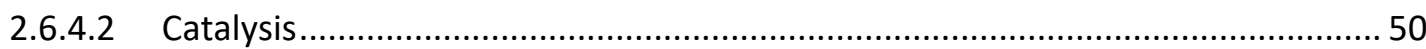

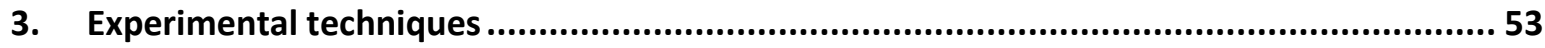

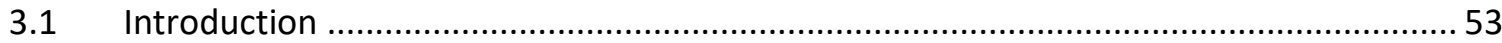

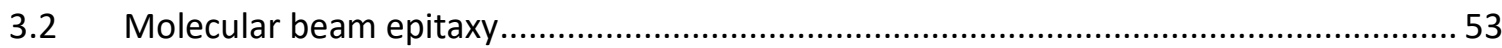

3.2.1 Description of the MBE system components ................................................... 54

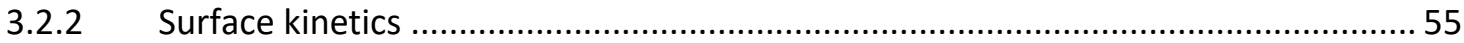

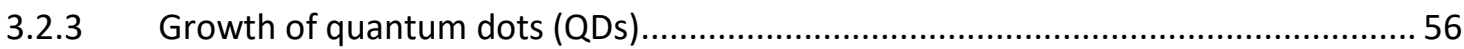

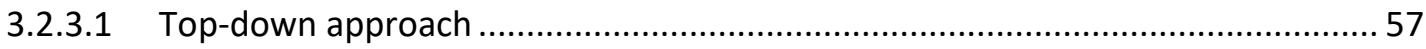

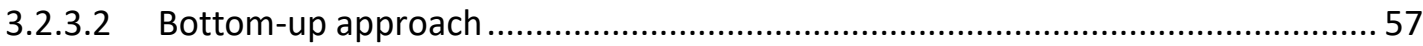

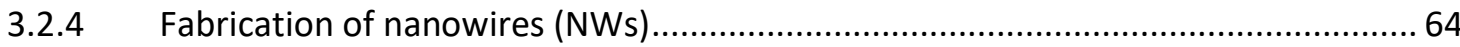

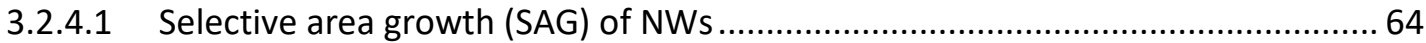

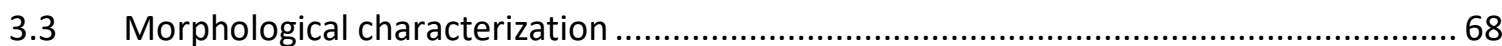

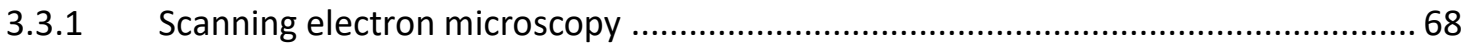

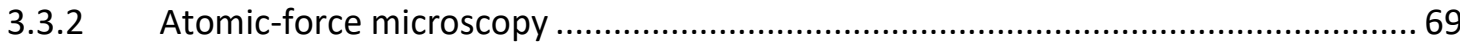

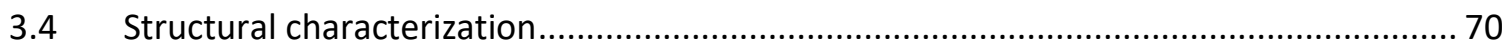

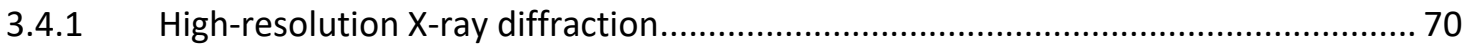

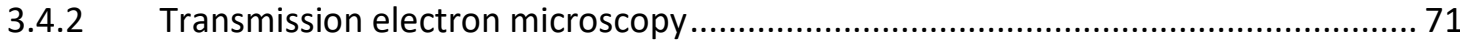

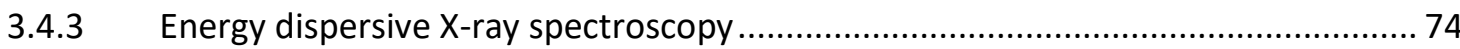

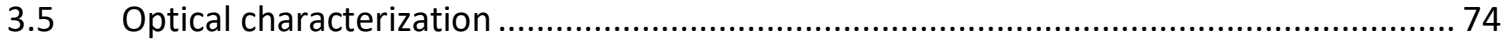

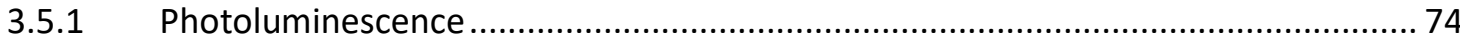

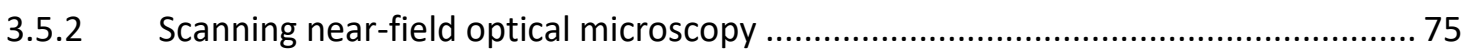

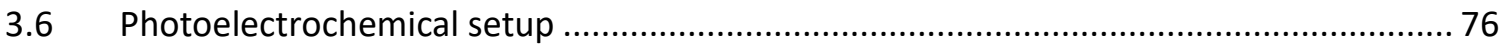




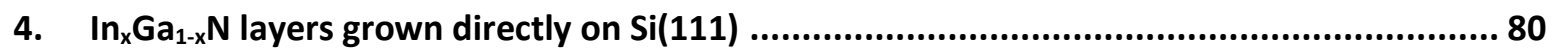

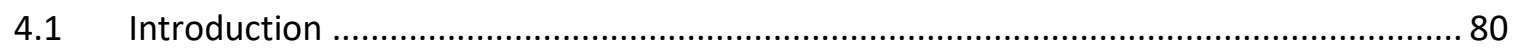

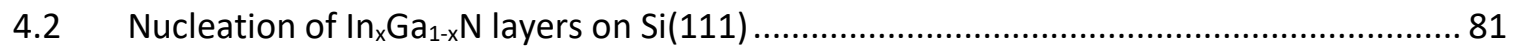

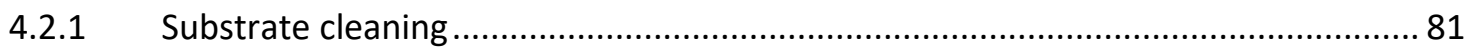

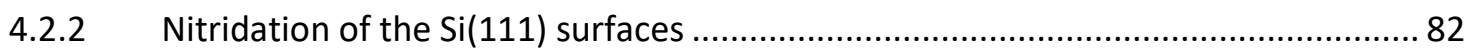

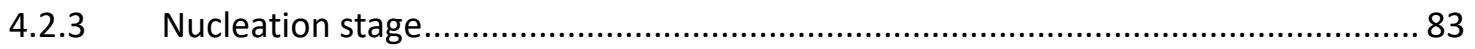

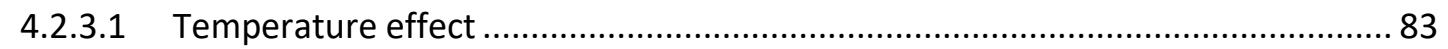

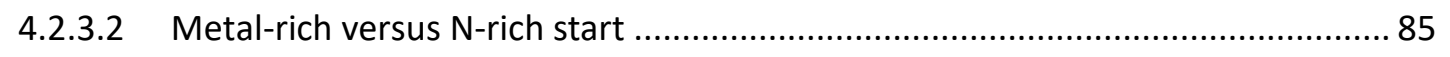

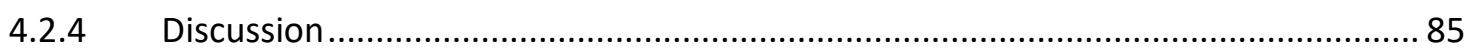

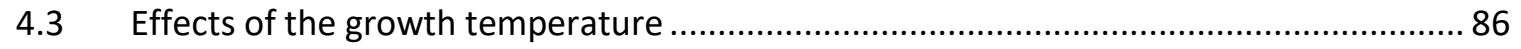

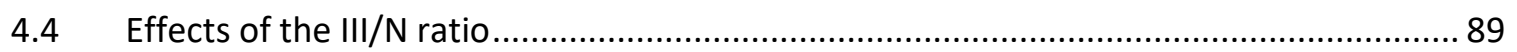

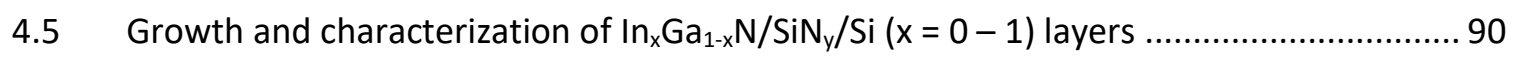

4.5.1 High-resolution X-ray diffraction measurements .................................................. 91

4.5.2 Transmission electron microscopy measurements................................................93

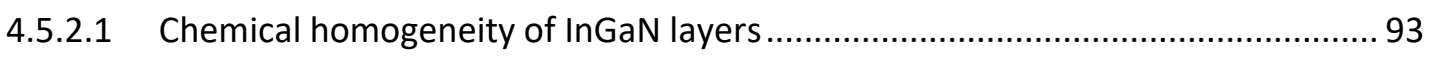

4.5.2.2 Structural quality of $\operatorname{InGaN}$ and its epitaxial relation to $\mathrm{Si}$................................... 95

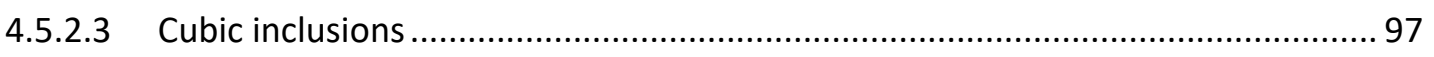

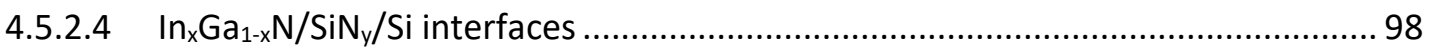

4.5.3 Photoluminescence and bowing parameter ...................................................... 100

4.5.4 Scanning near-field optical microscopy ............................................................ 101

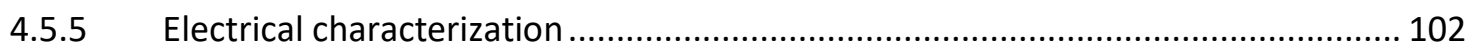

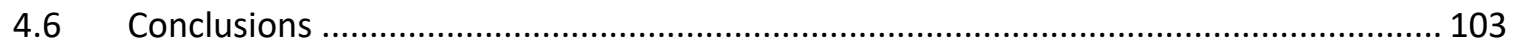

5. Growth of InN QDs via low temperature droplet epitaxy .......................................... 104

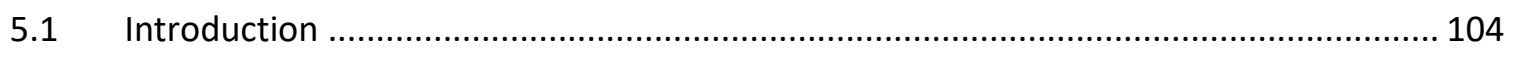

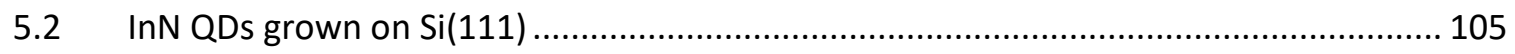

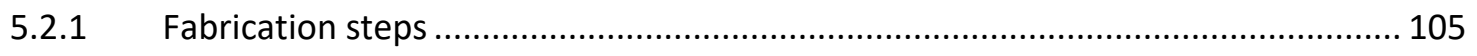

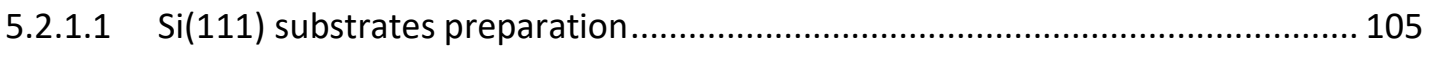

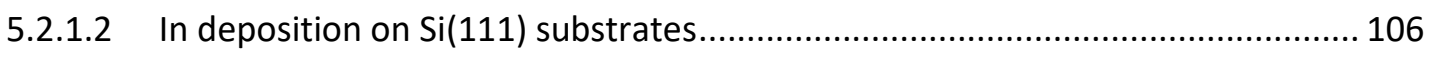

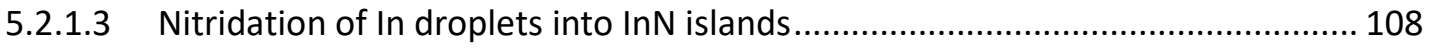

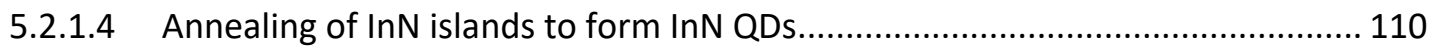

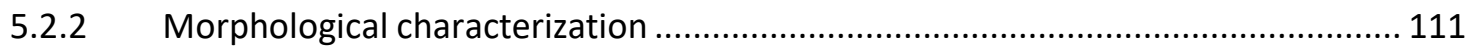

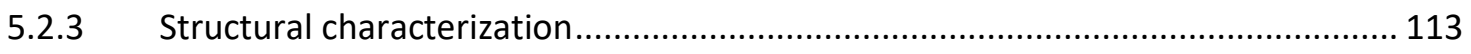




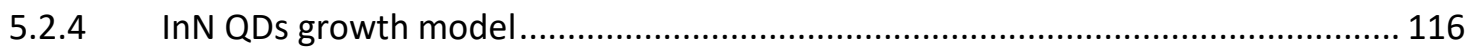

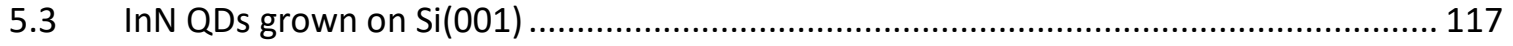

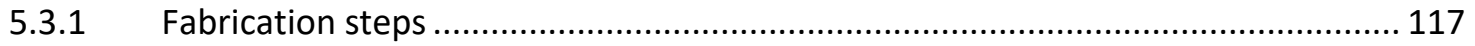

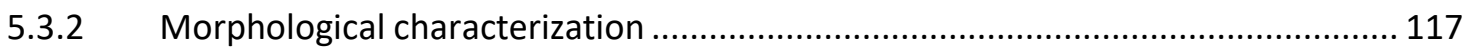

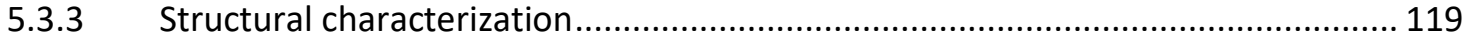

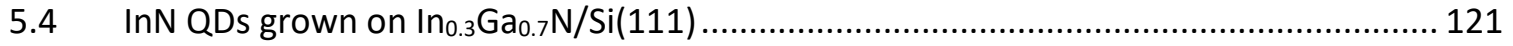

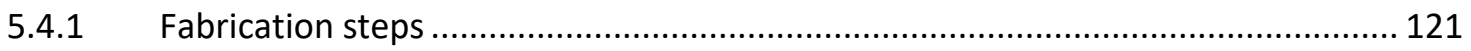

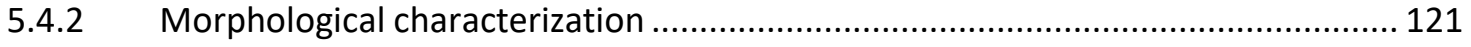

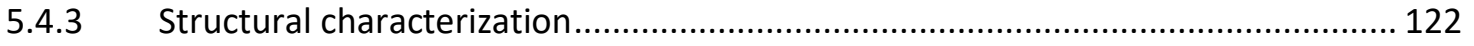

5.5 Comparison between InN QDs grown on $\ln _{0.3} \mathrm{Ga}_{0.7} \mathrm{~N} / \mathrm{Si}$ and bare Si ............................ 123

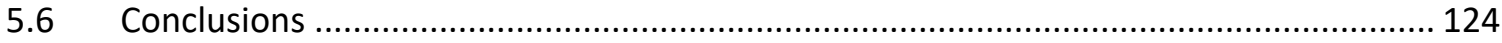

6. Selective area growth (SAG) of GaN nanowires (NWs) on various templates ................. 125

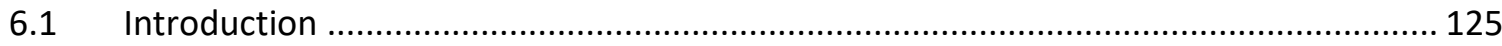

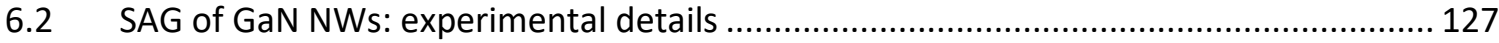

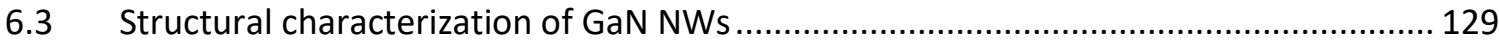

6.3.1 Dislocation filtering effect in NWs grown on a GaN/Si template.......................... 129

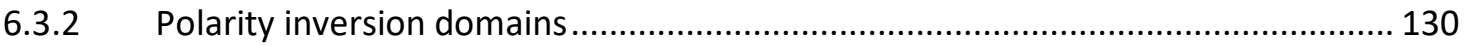

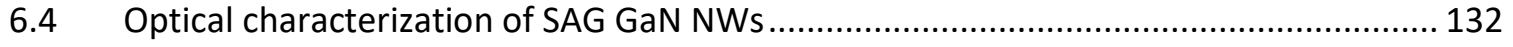

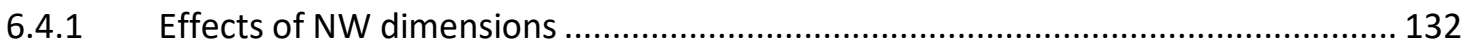

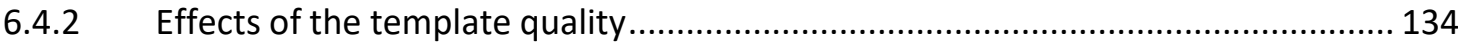

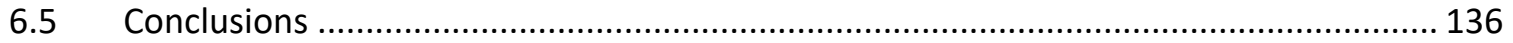

7. Photoelectrochemical evaluation of InGaN layers, InN QDs and NiO protection layers .... 137

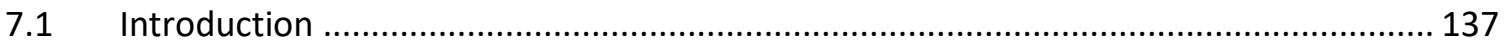

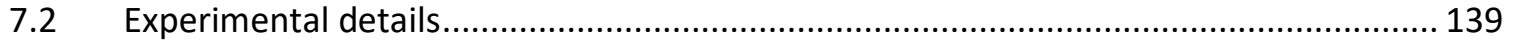

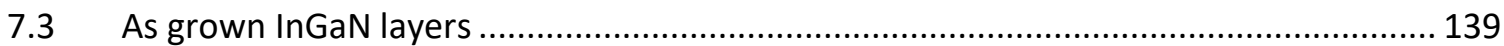

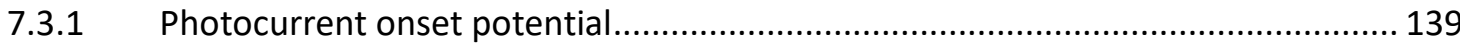

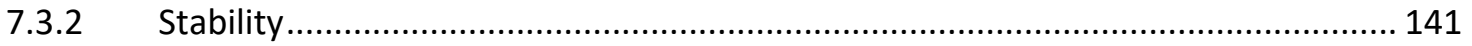

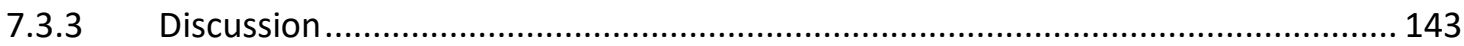

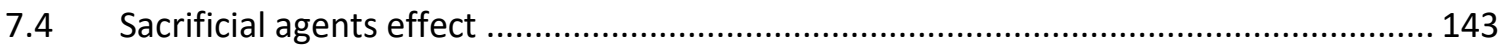

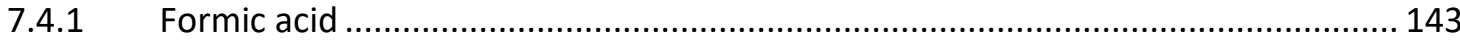

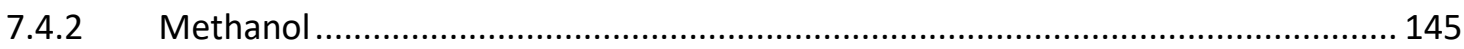

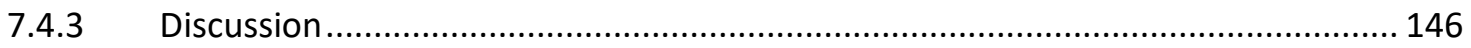




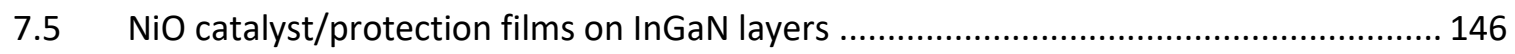

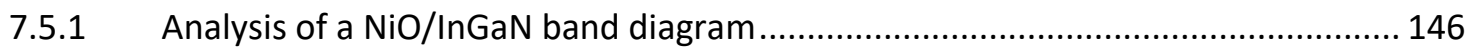

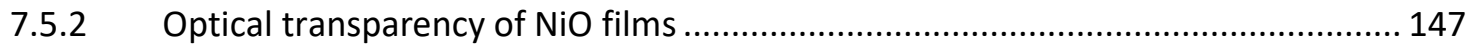

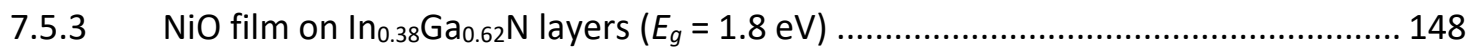

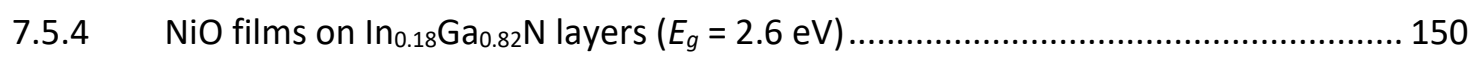

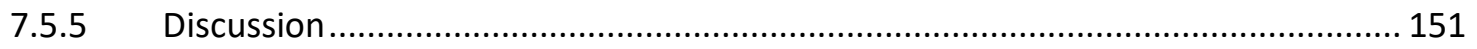

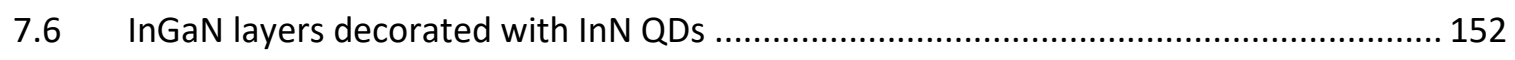

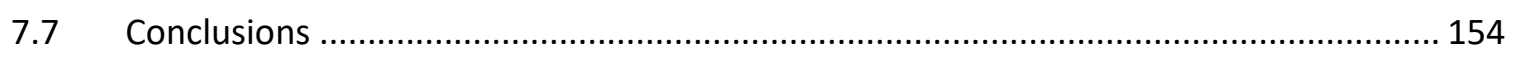

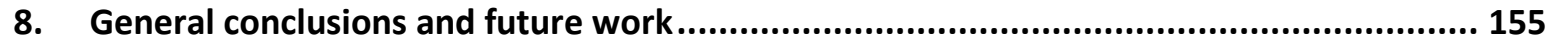

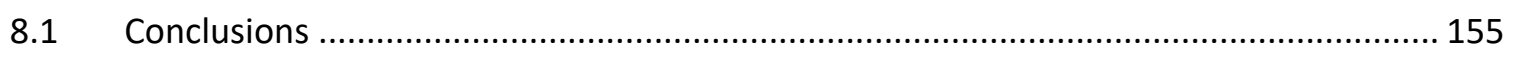

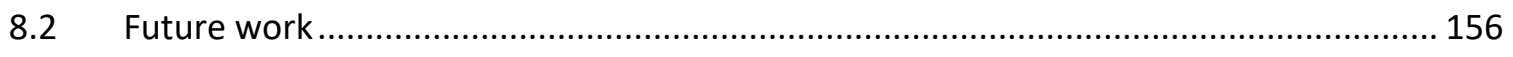

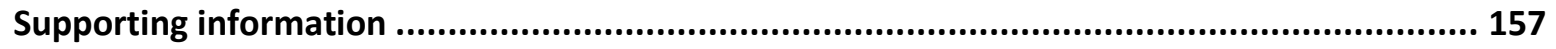

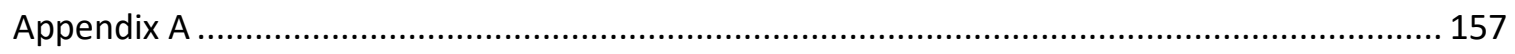

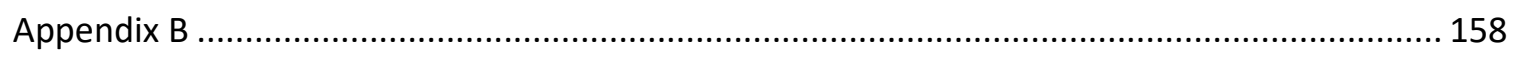

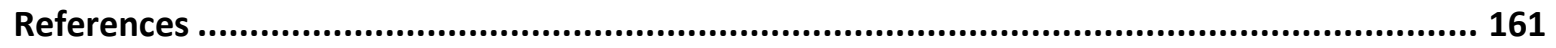




\section{Acknowledgements}

I would like to thank Prof. Dr. Enrique Calleja Pardo for supervising this thesis and providing his valuable feedback to obtained results along with spending many hours discussing and correcting the papers published in the framework of this work. Also, many thanks to my co-supervisor Prof. Dr. Žarko Gačević for his guidance in the lab and paper writing, as well as for our fruitful scientific and non-scientific discussions.

I want to thank Prof. Dr. Richard Noetzel for his support in getting this position and initial supervision. Also many thanks to my colleagues: Dr. Paul Soto Rodriguez for countless hours spent in the lab growing and characterizing samples, Victor Jesus Gomez for his unconditional guidance through various characterization tools and Spanish ways of living, Prof. Dr. Naveed ul Hassan Alvi for introducing me into the field of water splitting, and Dr. Praveen Kumar for help restoring molecular beam epitaxy machine.

Thanks to ISOM team of technicians Maika, Alicia, Fernando, Oscar and David for help with sample processing and general maintenance. Moreover, I would like to thank the rest of the ISOM personal with whom I was connected during this thesis work.

Special thanks to my collaborators Prof. Dr. Francisco Miguel Morales Sánchez, Dr. José Manuel Mánuel Delgado, and Juan Jesús Jiménez Ríos for performing countless TEM characterizations of the samples grown during this work and for, in general, broad support for my scientific carrier.

I want to thank other collaborators Prof. Dr. Christoph Lienau and Dr. Alexander Senichev for their SNOM measurements and discussions, as well as Prof. Dr. Almudena Torres-Pardo and Prof. Dr. Jose M. González-Calbet for additional TEM characterizations.

I also would like to thank Prof. Dr. Brian Seger, Prof. Dr. Peter Vesborg, and Prof. Dr. Ib Chorkendorff for giving me the opportunity to perform internship in their laboratory as well as to Dr. Dowon Bae and Martin Jesper Bækbo for their assistance on various topics during this internship.

My thanks also go to Prof. Dr. Sophia Haussener and Yannick Gaudy for many hours of discussion of fundamentals of water splitting cells.

My deepest thanks to Prof. Dr. Jose Calleja for his tremendous support when I was initially moving to Spain and during the whole later stay.

I wish to express my deepest gratitude to my wife Ekaterina for encouraging me to start the PhD career and was always supporting me. Finally, I would like to thank my parents, whose love is always warming me. 


\section{Abstract (in English)}

During this work the constituent functional blocks of a InGaN/Si-based solar-assisted water splitting cell were obtained by molecular beam epitaxy, which includes:

- Compact and chemically homogeneous InGaN layers were grown directly on Si substrates over the entire composition range. Such InGaN layers with the InN mole fraction above $30 \%$ grown directly on Si are demonstrated for the first time.

- InN quantum dots were grown on $\mathrm{Si}(111), \mathrm{Si}(001)$ and $\operatorname{lnGaN}(0001) / \mathrm{Si}(111)$ substrates by near room temperature droplet epitaxy method.

- Selective area grown GaN nanowires with well controlled geometry and structural/optical quality were fabricated by selective area growth method on different quality templates. A dislocation filtering effect was studied in detail.

All grown samples were extensively characterized by large variety of tools to confirm their high quality. The photoelectrochemical performance of InGaN-on-Si layers was evaluated and ways to enhance it were proposed, such as improving its stability via NiO protection layers and boosting their performance by $\ln N$ quantum dots decoration. Overall, despite many challenges, it was demonstrated that InGaN is a very promising material for realization of tandem (with Si cells) water splitting cells. 


\section{Resumen (en Español)}

Durante este trabajo se obtuvieron los bloques funcionales constitutivos de una célula para la disociación de agua mediante energía solar basada en heteroestructuras de InGaN/Si crecidas por epitaxia de haces moleculares, que incluye:

- Crecimiento de capas de InGaN compactas y químicamente homogéneas directamente sobre sustratos de Si cubriendo todo el rango de composiciones. Se ha demostrado por vez primera el crecimiento de capas de InGaN con fracciones molares de InN superiores al 30\% y crecidas directamente sobre Si.

- Crecimiento de puntos cuánticos de $\ln N$ sobre sustratos de $\mathrm{Si}(111), \mathrm{Si}(001)$ e InGaN(0001)/Si(111) mediante epitaxia sobre gotas a temperaturas cercanas a la ambiente.

- Crecimiento de nanocolumnas de GaN con control de su geometría y elevada calidad cristalina y estructural mediante crecimiento localizado y selectivo utilizando diferentes substratos. Estudio en detalle del efecto de filtrado de dislocaciones.

Todas las muestras crecidas se caracterizaron extensamente mediante una gran variedad de herramientas para confirmar su elevada calidad. Se evaluó el rendimiento fotoelectroquímico de capas de InGaN sobre Si y se han propuesto modos de mejorarlo, tales como aumentar su estabilidad con capas de protección de NiO y mediante la decoración con puntos cuánticos de InN. En general, a pesar de los muchos desafíos, se ha demostrado que las aleaciones de InGaN constituyen un sistema material muy prometedor para la realización de células tándem (junto con células Si) para la disociación de agua. 


\section{List of Publications}

\section{First author:}

[1] Aseev, P. | Gačević, Ž. | Torres-Pardo, A. | González-Calbet, J.M. | Calleja, E., Improving optical performance of GaN nanowires grown by selective area growth homoepitaxy: Influence of substrate and nanowire dimensions. Applied Physics Letters $^{\text {IF } 3.30}, 108,253109$ (2016).

[2] Aseev, P. | Soto, P. | Gómez, V.J. | Alvi, N.H. | Mánuel, J.M. | Morales, F.M. | Jiménez, J.J. | García, R. | Senichev, A. | Lienau, C. | Calleja, E. | Nötzel, R., Nearinfrared emitting In-rich InGaN layers grown directly on Si: Towards the whole composition range, Applied Physics Letters IF 3.30, 106(7), 072102 (2015).

[3] Aseev, P. | Soto, P. | Kumar, P. | Gomez, V.J. | Alvi, N.H. | Manuel, J.M. | Morales, F.M. | Jimenez, J.J. | Garcia, R. | Calleja, E. | Noetzel, R., Uniform Low-to-High In Composition InGaN Layers Grown on Si. Applied Physics Express IF 2.57, 6, 1155031-4 (2013).

\section{Coauthor:}

[4] Gómez, V.J. | Gačević, Ž. | Soto, P. | Aseev, P. | Nötzel, R. | Calleja, E. | Sánchez-García, M.A., Comparative study of single InGaN layers grown on Si(111) and GaN(0001) templates: The role of surface wetting and epitaxial constraint. Journal of Crystal Growth IF $1.69,447,48-54$ (2016).

[5] Alvi, N.H. | Soto, P. | Aseev, P. | Gómez, V.J. | Alvi, A.H. | Hassan, W.U. | Willander M. | Nötzel, R., InN/InGaN quantum dot photoelectrode: Efficient Hydrogen generation by water splitting at zero voltage. Nano Energy ${ }^{\text {IF } 10.21}, 13,291-297$ (2015).

[6] Soto, P. | Nash, V.C. | Aseev, P. | Gómez, V.J. | Kumar, P. | Alvi, N. H. | Sánchez, A. | Villalonga, R.| Pingarrón, J.M. | Nötzel, R., Electrocatalytic oxidation enhancement at the surface of InGaN films and nanostructures grown directly on Si(111). Electrochemistry Communications ${ }^{\text {IF } 4.85}, 60,158-162$ (2015).

[7] Soto, P. | Aseev, P. | Gómez, V.J. | Kumar, P. | Alvi, N.H | Calleja, E. | Mánuel, J.M. | Morales F.M. | Jiménez, J.J. | García, R. | Senichev, A. | Lienau, C. | Nötzel, R., Stranski-Krastanov InN/InGaN quantum dots grown directly on Si(111). Applied $\underline{\text { Physics Letters }}^{\text {IF } 3.30}, 106(2), 023105$ (2015).

[8] Alvi, N.H. | Soto P. | Kumar, P. | Gómez, V.J. | Aseev, P. | Alvi, A.H. | Alvi, M. A. | Willander, M.| Nötzel, R., Photoelectrochemical water splitting and hydrogen generation by a spontaneously formed InGaN nanowall network. Applied Physics Letters $^{\text {IF } 3.30}, 104(22), 223104$ (2014). 
[9] Nechaev, D.V. | Aseev, P. | Jmerik, V.N. | Brunkov, P.N. | Kuznetsova, Y.V. | Sitnikova, A.A. | Ratnikov, V.V. | Ivanov, S.V., Control of threading dislocation density at the initial growth stage of AIN on c-sapphire in plasma-assisted MBE. Journal of Crystal Growth IF $1.69,378,319-322$ (2013).

[10] Jmerik, V.N. | Mizerov, A.M. | Nechaev, D.V. | Aseev, P. | Sitnikova, A.A. | Troshkov, S.I. | Kop'ev, P.S. I Ivanov, S.V., Growth of thick AIN epilayers with droplet-free and atomically smooth surface by plasma-assisted molecular beam epitaxy using laser reflectometry monitoring. Journal of Crystal Growth ${ }^{\text {IF } 1.69}$, 354(1), 188-192 (2012).

\section{Research featured in Magazines/Newspapers:}

2015 SemiconductorToday Magazine (March, 18, 2015).

Direct growth of In gallium nitride on silicon substrate http://www.semiconductor-today.com/news items/2015/mar/maxplanck 180315.shtml

2015 SemiconductorToday Magazine (January, 30, 2015).

In nitride surface quantum dots on silicon substrates

http://www.semiconductor-today.com/news items/2015/jan/updm 300115.shtm 


\section{Acronyms}

2B two-beam

$A^{0} X \quad$ Acceptor-bound excitons

ABF Annular bright field

AFM Atomic force microscopy

AM1.5g The global standard spectrum with air mass 1.5 (atmosphere thickness)

BEP Beam equivalent pressure

BF Bright-field

BSF Base stacking fault

CA Chronoamperometry

$\mathrm{CB} \quad$ Conduction band

CCD Charge-coupled device

CE Counter electrode

$\mathrm{CH} \quad$ Crystal-field split hole

$\mathrm{C}_{\mathrm{H}} \quad$ Capacitance of the Helmholtz layer

$\mathrm{C}_{\mathrm{SC}} \quad$ Capacitance of the semiconductor

CTEM Conventional TEM

CV Cyclic voltammetry

DBE Donor-bound excitons

DC Diffraction contrast

DE Droplet epitaxy

DERI Droplet elimination by radical beam irradiation

DF Dark-field

DI Deionized

DW Dewetting

EBL Electron beam lithography

$E_{c} \quad$ Conduction band energy position

EDS Energy dispersive X-ray spectroscopy

$\mathrm{E}_{\mathrm{f}} \quad$ Fermi energy

$E_{g} \quad$ Bandgap energy

EQE External quantum efficiency

$\mathrm{E}_{\mathrm{v}} \quad$ Valance Band Energy position

FFT Fast fourier transform

$\mathrm{F}_{\mathrm{Ga}} \quad$ Gallium flux

$F_{\text {In }} \quad$ Indium flux

FM Frank-van der Merve

$\mathrm{F}_{\mathrm{N}^{*}} \quad$ Active nitrogen flux

FWHM Full width at half maximum
FX Free exciton

$\mathrm{FX}_{\mathrm{A}} \quad$ A-exciton

$\mathrm{FX}_{\mathrm{B}} \quad$ B-exciton

$\mathrm{FX}_{\mathrm{C}} \quad \mathrm{C}$-exciton

HAADF High-angle annular dark field

HER Hydrogen evolution reaction

HEMT High-electron-mobility transistor

$\mathrm{HH}$ Heavy hole

HRTEM High-resolution TEM

HRXRD High-resolution X-ray diffraction

HVPE Hydride vapor phase epitaxy

ID Inversion domain

IDB Inversion domain boundary

IHP Inner Helmholtz plane

IPCE Incident power conversion efficiency

IR Infrared

ISOM Instituto de Sistemas Optoelectrónicos y Microtecnología

IV Current-voltage

$k_{\text {rec }} \quad$ First-order rate constant of charge recombination

$\mathrm{k}_{\mathrm{tr}} \quad$ First-order rate constant of charge transfer

LD Laser diode

LED Light-emitting diode

LH Light holes

LPE Liquid phase epitaxy

LT Low temperature

MBE Molecular beam epitaxy

MD Misfit dislocation

MEE Migration-enhanced epitaxy

MLs Monolayers

MME Metal modulated epitaxy

MOVPE Metal organic vapor phase epitaxy

NCs Nanocolumns

NWs Nanowires

OED Optical emission detector

OER Oxygen evolution reaction

OHP Outer Helmholtz plane

OM Optical microscopy 


\begin{tabular}{|c|c|c|c|}
\hline PA MBE & Plasma assisted MBE & $S / E$ & Semiconductor/electrolyte \\
\hline \multirow[t]{2}{*}{ PDDA } & \multirow{2}{*}{$\begin{array}{l}\text { Poly diallyl-dimethylammonium } \\
\text { chloride }\end{array}$} & SEM & Scanning electron microscopy \\
\hline & & SF & Stacking fault \\
\hline PEC & Photoelectrochemical & SHE & Standard hydrogen electrode \\
\hline pIDB & Polarity inversion domain boundary & SK & Stranski-Krastanow \\
\hline PL & Photoluminescence & SNOM & \multirow{2}{*}{$\begin{array}{l}\text { Scanning near-field } \\
\text { microscopy }\end{array}$} \\
\hline PMT & Photomultiplier tube & & \\
\hline PS & Poly-styrene & SSL & Solid state lighting \\
\hline PSS & Poly-styrene sulfonate & STEM & Scanning TEM \\
\hline PVD & Physical vapor deposition & STH & Solar-to-hydrogen efficiency \\
\hline PVTEM & Plan-view TEM & STM & Scanning tunneling microscopy \\
\hline QD & Quantum dot & TD & Threading dislocation \\
\hline QMS & Quadrupole mass spectrometer & TEM & Transmission electron microscopy \\
\hline Qs & Surface charge & TES & Two-electron satellite \\
\hline QW & Quantum well & TSRs & Tetrahedral-shaped recesses \\
\hline RE & Reference electrode & UHV & Ultra high vacuum \\
\hline RF & Radio frequency & UV & Ultraviolet \\
\hline \multirow[t]{2}{*}{ RHEED } & \multirow{2}{*}{$\begin{array}{l}\text { Reflection high-energy electron } \\
\text { diffraction }\end{array}$} & UX & Unknown exciton \\
\hline & & VB & Valence band \\
\hline RMS & Root mean square & $\mathrm{V}_{\mathrm{H}}$ & \multirow{2}{*}{$\begin{array}{l}\text { Potential drop at the Helmholtz } \\
\text { layer }\end{array}$} \\
\hline RSM & Reciprocal space map & & \\
\hline RT & Room temperature & VLS & Vapor-liquid-solid \\
\hline SA & Self-assembled & $V_{\text {onset }}$ & Photocurrent onset potential \\
\hline SAED & Selected area electron diffraction & VW & Volmer-Weber \\
\hline SAG & Selective area growth & WE & Working electrode \\
\hline \multirow[t]{2}{*}{ SCCM } & \multirow{2}{*}{$\begin{array}{l}\text { Standard cubic centimeters per } \\
\text { minute }\end{array}$} & WL & Wetting layer \\
\hline & & XTEM & Cross-sectional TEM \\
\hline SCE & Saturated calomel electrode & YB & Yellow band \\
\hline SCR & Space charge region & $\Delta \phi_{\mathrm{sc}}$ & Potential drop across SCR \\
\hline
\end{tabular}




\section{Introduction}

\subsection{Development of the III-Nitride technology}

III-Nitrides are recognized as one of the most promising semiconductor families for fabricating optical devices. This is mainly because their direct bandgaps $\left(E_{g}\right)$ together with a high excitonic binding energy that provides high emission efficiency. In addition, this material system is characterized by a wide bandgap energy variation, from infrared (IR) (InN, $0.65 \mathrm{eV})$ to ultraviolet (UV) (AIN, $6.2 \mathrm{eV}$ ), offering a unique possibility to cover the entire visible spectrum with a single material system. The initial success of III-Nitrides came from the light-emitting-diode (LED) technology, due to the development of high-brightness blue InGaN/GaN-based LEDs and the achievement of efficient white emission for solid state lighting (SSL) [Fini\&Nakamura2005].

The first attempt to grow epitaxial GaN layers started back in the 1969, when Maruska and Tietjen [Maruska\&Tietjen1969] demonstrated single-crystalline GaN with a direct bandgap of about 3.39 $\mathrm{eV}$ at room temperature (RT). However, these layers had poor crystal quality as a consequence of large lattice and thermal mismatches between GaN and the sapphire substrate used. In the 1980s Akasaki and Amano [Amano1986] proposed to use a low temperature (LT) AIN buffer layer on sapphire, which drastically improved the GaN layers crystal quality. It also led to a significant reduction of the residual electron concentration, from $10^{19}$ to less than $10^{15} \mathrm{~cm}^{-3}$ [Amano1988, Akasaki1989]. Consequently, the work on technologically demanding p-type doping could finally be started.

After several trials with different materials, Mg was finally proposed as a relatively shallow acceptor in III-Nitrides [Amano1989]. It was later shown that it is essential to activate Mg acceptors by releasing hydrogen during a thermal annealing in order to achieve p-type conductivity [Nakamura1992a, Nakamura1992b, Vechten1992].

Once high crystal quality and both $\mathrm{n}$ - and p-type doping control were achieved, the first GaN p-n junction ultra-violet LED was developed [Amano1989], followed by blue LEDs commercialization [Nakamura1994]. Exploiting the InGaN quantum wells (QWs) as the active layer increased the LED efficiency by almost an order of magnitude [Nakamura1995a, Amano1995]. Nowadays, blue LEDs based on this design are widely used in SSL [Yamada2002].

Thus, there are three major technological breakthroughs, which led to a great success of the InGaN/GaN-based blue LEDs:

- Use of a low temperature AIN buffer layer.

- $\quad$ Achievement of efficient p-type doping with Mg.

- Use of InGaN QWs as an active layer.

Isamu Akasaki, Hiroshi Amano and Shuji Nakamura were awarded the Nobel Prize in Physics in 2014 for their GaN/InGaN related work and demonstration of efficient white light emission. 


\subsection{Further development of III-Nitride epitaxy}

While the initial work on $\mathrm{GaN} / \mathrm{In}_{\mathrm{x}} \mathrm{Ga}_{1-\mathrm{x}} \mathrm{N}$ growth was done by hydride vapor phase epitaxy (HVPE) [Maruska\&Tietjen1969], further advances were achieved by metal organic vapor phase epitaxy (MOVPE) [Amano1986, Nakamura1992b]. Sapphire(0001) was chosen as substrate because of its chemical and thermal stability, rather low price and quality. However, both MOVPE and sapphire pose serious limitations to possible InGaN-based devices. The first one is because of the high temperatures involved that hinder the incorporation of high $\ln N$ mole fractions (decomposition). The second one is with sapphire because its insulating nature prevents the fabrication of vertical devices. Thus, plasma-assisted molecular beam epitaxy (PA MBE) emerged as an alternative method allowing to grow III-Nitrides on conductive Si substrates (direct growth of GaN on Si by MOVPE is quite problematic).

Currently there are several major directions to further develop the $\ln _{x} \mathrm{Ga}_{1-\mathrm{x}} \mathrm{N}$ technology (the main focus of this work):

- In-rich $\ln _{x} \mathrm{Ga}_{1-\mathrm{x}} \mathrm{N}$ alloys to extend emission wavelengths to the IR region.

- Use of Si(111) substrates to enable vertical devices and integration with Si technology.

- Use of nanostructures as new crystal quality and geometry control standards for novel devices.

All of these research paths are explored in this thesis.

\subsection{Novel potential application: solar-assisted water splitting}

The last century revolutionized mankind history due to a rapid industrialization, increase in world population, and overall living standards growth. As a consequence of such large scale changes, mankind is experiencing a dramatic surge in energy demands and industrial waste generation. The main energy source nowadays is fossil fuel, being non-renewable and exhaustible in the mid-term. Moreover, its extraction and utilization are continuously threatening the Earth's ecology, being the reason why in the recent years an increasing attention is paid to search sustainable, clean, and renewable energy sources. Solar energy, of which our planet receives in enormous quantities (23000 TW per year [Perez2009]), greatly exceeding our energy needs estimated at 17TW for 2014 [BP2014], is a very promising solution.

One way to make use of solar energy is to convert it into storable hydrogen fuel through photoelectrochemical (PEC) water splitting. In this process, a semiconductor material immersed in an electrolyte is used as a light absorber to generate photoexcited charge carriers. Then charges are transported to the electrolyte to start a reaction in which species in the solution become either oxidized (electron loss) or reduced (electron gain). Fig. 1.3.1 shows a schematic PEC cell with a semiconductor photoanode and metal electrode as a cathode. In this scheme, the photoanode performs an oxygen evolution reaction (OER) and the cathode a hydrogen evolution reaction (HER). Depending on the electrolyte these reactions can be expressed as follows:

In acidic electrolyte:

$\begin{array}{ll}\text { Cathode (reduction } \rightarrow \text { HER): } & 2 H^{+}+2 e^{-} \rightarrow H_{2}, \quad \text { at } \mathrm{E}_{0}=0.00 \mathrm{~V} \text { vs. RHE, } \\ \text { Anode (oxidation } \rightarrow \text { OER): } & 2 \mathrm{H}_{2} \mathrm{O}+4 h^{+} \rightarrow 4 H^{+}+\mathrm{O}_{2} \text {, at } \mathrm{E}_{0}=1.23 \mathrm{~V} \text { vs. RHE, }\end{array}$

In alkaline electrolyte: 
Cathode (reduction $\rightarrow \mathrm{HER}$ ): $\quad 2 \mathrm{H}_{2} \mathrm{O}+e^{-} \rightarrow \mathrm{H}_{2}+2 \mathrm{OH}^{-}$, at $\mathrm{E}_{0}=0.00 \mathrm{~V}$ vs. RHE,

Anode (oxidation $\rightarrow \mathrm{OER}$ ): $\quad 4 \mathrm{OH}^{-}+4 h^{+} \rightarrow \mathrm{O}_{2}+2 \mathrm{H}_{2} \mathrm{O}$, at $\mathrm{E}_{0}=1.23 \mathrm{~V}$ vs. RHE,

The redox potentials ( $E_{0}$ - a measure of the tendency of any chemical substance to lose or gain electrons) of the above reactions, expressed in volts, are given versus a reversible hydrogen electrode (RHE) as reference electrode (Described in more details in section 2.6.2). The overall water splitting reaction is:

$$
\mathrm{H}_{2} \mathrm{O} \rightarrow \mathrm{H}_{2}+1 / 2 \mathrm{O}_{2}, \Delta \mathrm{E}_{\text {cell }}=1.23 \mathrm{~V}
$$

Where $\Delta \mathrm{E}_{\text {cell }}$ is a minimum potential difference between electrodes in the water splitting cell (at $25^{\circ} \mathrm{C}$ and $1 \mathrm{~atm}$ standard conditions) required to drive the reaction from a thermodynamic point of view. The Gibbs free energy change associated to this reaction is $\Delta \mathrm{G}=237.2 \mathrm{~kJ} / \mathrm{M}$, suggesting that the overall water splitting reaction is an "uphill" one that cannot be driven spontaneously.

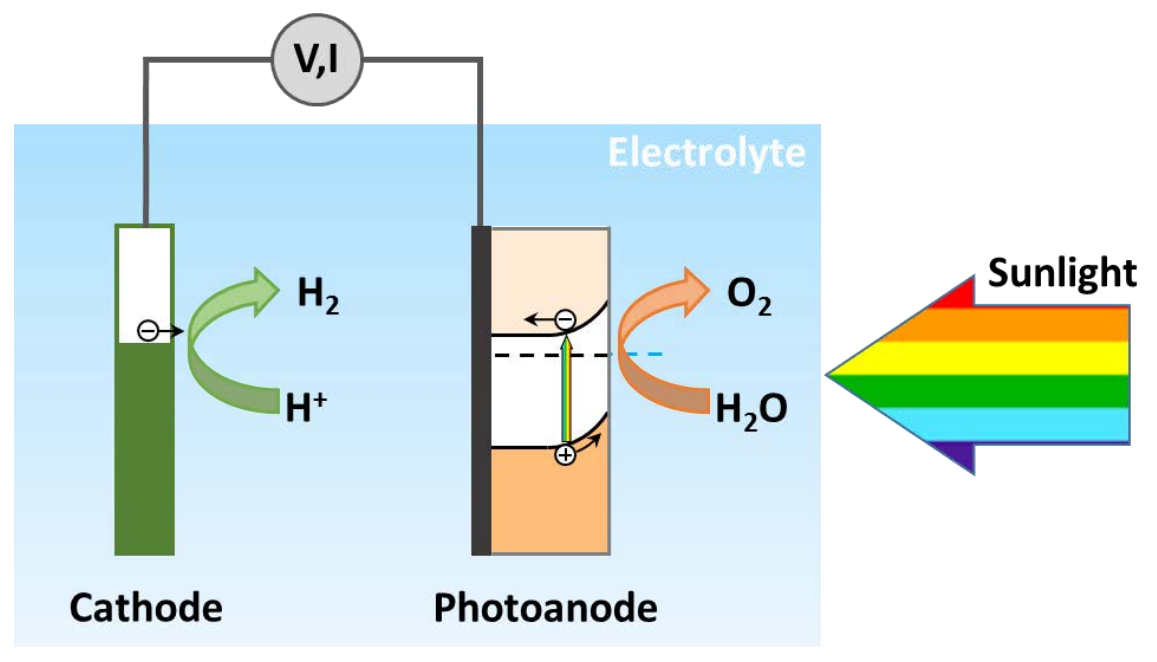

Fig. 1.3.1. A schematic diagram of a PEC cell under operation.

In practice, an extra potential is necessary to provide feasible OER and HER rates (due to slow kinetics). This potential difference between the thermodynamically defined reduction potential and the experimentally observed value is called overpotential (there are two overpotentials, for HER and OER half-cell reactions). Therefore, the actual potential required to drive the water splitting process in practical devices is typically of $\sim 1.8 \mathrm{~V}$ [Hu2013].The PEC water splitting cell is based on a semiconductor/electrolyte (S/E) interface acting similarly to a Schottky junction, thus leading to the formation of a built-in potential upon which photovoltage $\left(V_{p h}\right)$ can be generated at the junction under solar illumination (for more details refer to section 2.6.3.2). If enough photovoltage is generated by the S/E junction, it can be used to drive the water splitting reaction without external electrical bias. A single wide bandgap semiconductor with a bandgap higher than $2.0 \mathrm{eV}$ would be sufficient to drive the water splitting process, accounting for potential losses due to carrier recombination at the $\mathrm{S} / \mathrm{E}$ interface.

Since a semiconductor absorbs photons with energy $\left(E_{p h}\right)$ equal to or higher than its bandgap, a rather low absorption efficiency (in terms of number of photons) over the solar spectrum is expected for wide bandgap materials. Reducing the bandgap $\left(E_{g}\right)$ increases the number of absorbed photons, but photon with energy higher than $E_{g}$ loose part of their energy $\Delta E=E_{p h}-E_{g}$ as heat, 
and thus decreasing the absorption efficiency of the device. This interplay places a limit on the maximum solar conversion efficiency, the so called Shockley-Queisser efficiency limit [Shockley1961]. In an ideal case (no losses on recombination or catalysis), a semiconductor with $E_{g}$ $=2.0 \mathrm{eV}$ can provide a photocurrent density of about $15 \mathrm{~mA} / \mathrm{cm}^{2}$, corresponding to a solar-tohydrogen efficiency (STH) of $18.5 \%$. Considering that competing technologies, such as hybrid photovoltaic+electrolyzer cells, currently demonstrate higher energy conversion efficiency, the use of a single semiconductor for PEC water splitting would not be a viable approach for commercialization.

To overcome the Shockley-Queisser efficiency limit, dual photo-absorber (also called tandem) cell approach can be exploited, where the cell power output is optimized when high energy photons are absorbed by a top semiconductor with a $2.0 \mathrm{eV}$ bandgap, and low energy photons by a bottom cell with a bandgap of $1.2 \mathrm{eV}$ [Henry1980]. This configuration can result in a theoretical STH value as high as $31.1 \%$ [Hu2013].

The overall water splitting reaction with a tandem cell would take place only if the combined (added) photovoltage $\left(V_{p h}\right)$ from the photocathode and photoanode is above $1.23 \mathrm{~V}$, then the overall STH is determined by the operating current density $\left(J_{o p}\right)$ that can be estimated from the I-V curves intersection, as shown in Fig. 1.3.2.

The highest efficiency attainable with a tandem device will occur only when this intersection is close to the individual curves maximum power points, i.e. having a high fill factor. STH values exceeding $10 \%$ were reported for $\mathrm{p}-\mathrm{Ga}_{0.5} \mathrm{In}_{0.5} \mathrm{P} / \mathrm{pn}$-GaAs cells [Turner1998], though these cells have a very high production cost because of pn-GaAs substrates. This can be solved by using cheaper Si substrates.

Recently, significant advances were made in the field of silicon-based devices, with a silicon cell either as a standalone device [Seger2013] or in a junction with larger bandgap semiconductors [Abdi2013]. A bandgap of $1.1 \mathrm{eV}$, low price and high thermal conductivity make silicon a near ideal

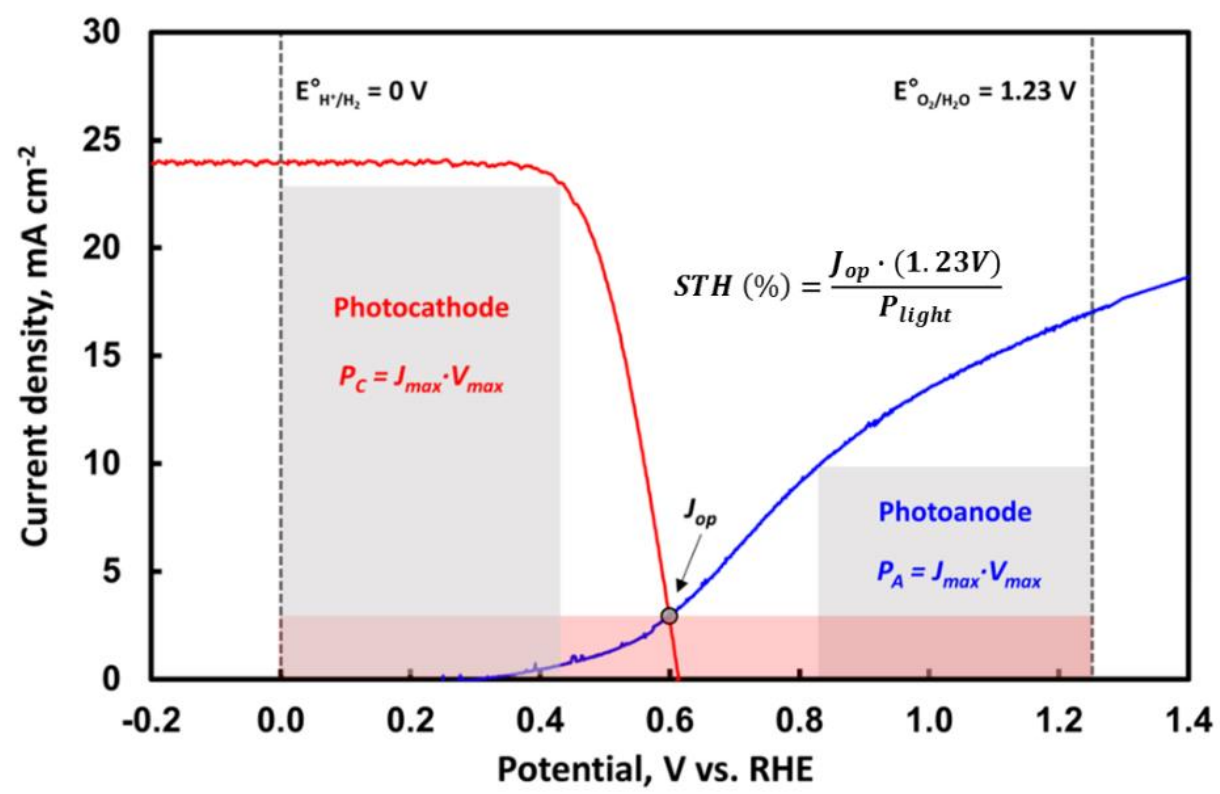

Fig. 1.3.2. Current-voltage (I-V) characteristics of the photocathode (red) and photoanode (blue). Combined power generated $P_{\text {tandem }}=J_{o p} \cdot(1.23 \mathrm{~V})$ is marked with the red filling. Adapted from [Bae2015]. 
low energy photon absorber (bottom cell) in a tandem cell design [Hsu\&Walukiewicz2008]. Thus, the search for materials compatible with silicon becomes of great importance. A very promising candidate as top cell in a Si-based tandem PEC device is the $\ln _{x} G_{1-x} N$ alloy. [Walukiewicz2011] having a bandgap adjustable to the required value of $2.0 \mathrm{eV}(\mathrm{x} \sim 0.4)$.

Moreover, a n-type $\ln _{x} \mathrm{Ga}_{1-\mathrm{x}} \mathrm{N}$ with $\mathrm{x}>0.4$ forms a type-Il heterojunction with p-type $\mathrm{Si}$, which facilitates the formation of an ohmic junction between them. Additionally, the $\ln _{x} G_{1-x} N$ direct bandgap nature results in a high absorption coefficient of $\sim 10^{6} \mathrm{~cm}^{-1}$ [Muth1997], which allows $95 \%$ of light to be absorbed in the first $300 \mathrm{~nm}$ of the layer $(99 \%$ in $500 \mathrm{~nm})$. Fig. 1.3.3 shows one of the possible designs of a PEC cell based on a tandem $n-\mathrm{In}_{x} \mathrm{Ga}_{1-\mathrm{x}} \mathrm{N} / \mathrm{pn}-\mathrm{Si}$ heterojunction.

Although all these properties make the $\ln _{x} G a_{1-x} N / S i$ material combination very promising for solar energy applications, so far, only low-In-composition $(x<0.3)$ layers were successfully grown on $\mathrm{Si}$ [Romanyuk2009]. No growth method was proposed for high-In-composition $(x>0.3) \ln _{x} G_{1-x} N$ layers on Si with the required quality for PEC applications.

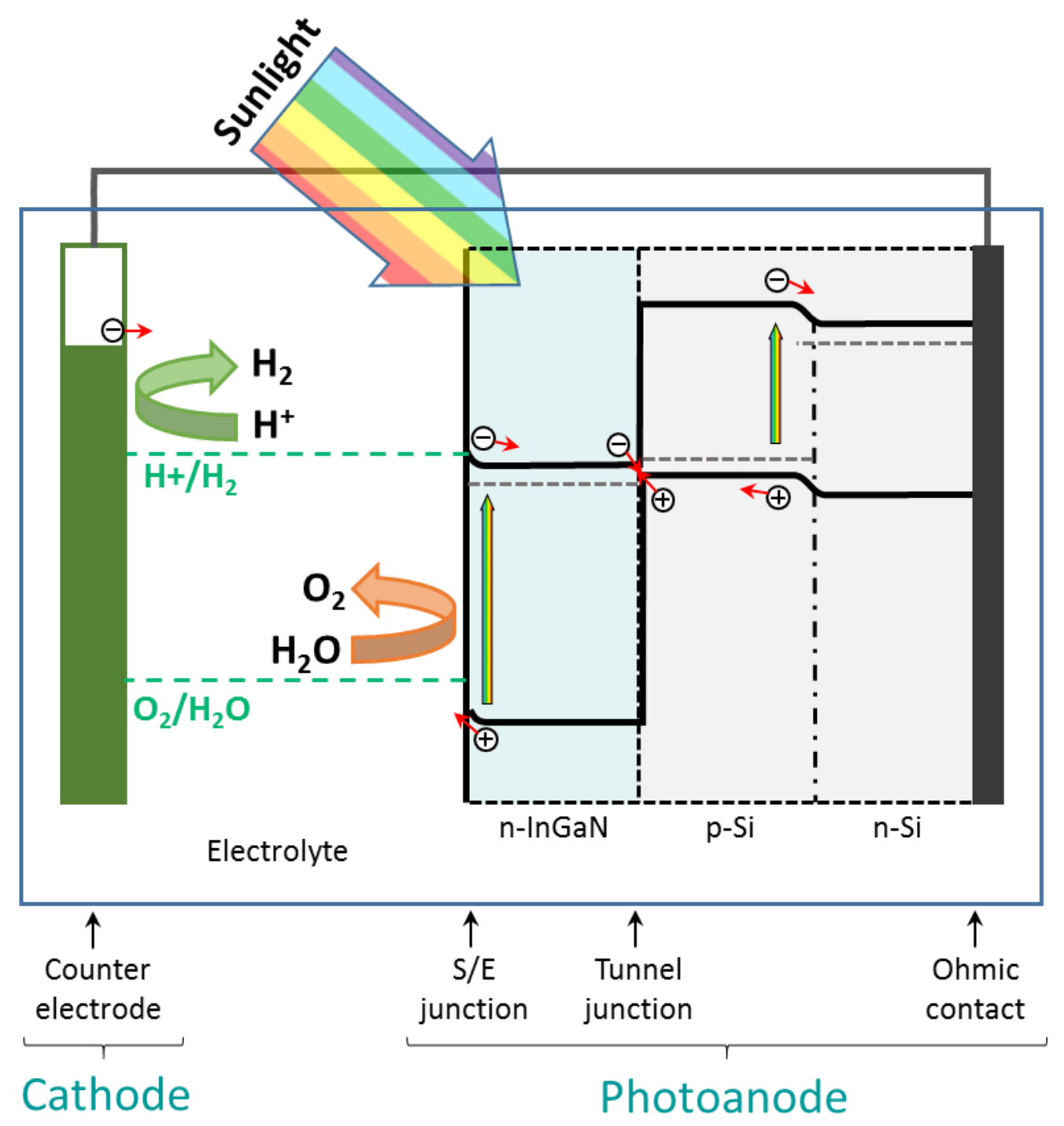

Fig. 1.3.3. A schematic diagram of the tandem $n-\operatorname{In}_{\times} \mathrm{Ga}_{1-\times} \mathrm{N} / \mathrm{pn}-\mathrm{Si}$ PEC cell. Adapted from [Walukiewicz2011]. 
Aside from the PEC water splitting process, the electrodes themselves may be involved in the reaction as a result of dissolution or corrosion. When the corrosion reaction is induced by the photogenerated charges in the photoelectrode it is called photocorrosion. For hydrogen production this is a less than desirable effect. Unfortunately, many semiconductor materials, including $\ln _{\mathrm{x}} \mathrm{Ga}_{1-\mathrm{x}} \mathrm{N}$ alloys, are prone to photocorrosion in acidic and alkaline electrolytes [Finken2015].

A protection layer that stands self-oxidation (reduction) while allowing charge transfer from the photoanode (cathode) into the electrolyte can be used to inhibit photocorrosion. A NiO protective coating was recently very successfully applied to various semiconductors including InP [Sun2015] and Si [Mei2014] to prevent the photocorrosion in alkaline solutions. It was also used to protect InGaN NWs structures [Benton2013], thus showing a great potential to prevent photocorrosion of $\mathrm{In}_{\mathrm{x}} \mathrm{Ga}_{1-\mathrm{x}} \mathrm{N}$ layers as well.

In practical PEC devices, the STH conversion efficiency is not as high as predicted by theoretical calculations. Once the semiconductor is immersed in the electrolyte, kinetic and thermodynamic barriers arising at the S/E interface limit the STH value. However, a proper electrode surface modification can effectively reduce these barriers [Guijarro2015], like decorating the electrode surface with quantum dots (QDs) that enhance both the electrochemical properties of the S/E interface and the optical properties (absorption) of the semiconductor itself [Yang2014]. In case of $\ln _{x} \mathrm{Ga}_{1-x} \mathrm{~N}$ layers it was reported that decoration with InN QDs can improve efficiency drastically [Alvi2015]. However, because of the typical $\ln _{x} G_{1-x} N$ layer surface roughness when grown on $\mathrm{Si}$, the Stranski-Krastanow mode to grow QDs based on lattice mismatch [Widmann1998] is both difficult to implement and to study. Thus, other QDs fabrication techniques allowing growth on rough surface must be proposed.

Another approach would be the use of nanowires (NW) to further enhance the performance of PEC water splitting devices [Yahyaie\&Lewis2011, Kibria\&Mi2016]. Compared to thin films, NWs have a higher surface to volume ratio, thus the photo-generated carriers will travel less distance to reach the surface with a much lower recombination probability [Osterloh2013]. Moreover, due to their specific shape, NWs drastically increase absorbance and reduce reflectance at the surface of the photoelectrode [Parkinson\&Turner2013]. The use of NWs can then significantly improve the performance of photoelectrodes made of materials with short carrier lifetime and low mobility [Osterloh2013]. A tremendous progress has been done in fabricating high quality self-assembled (SA) InGaN NWs [Kishino1997, Sanchez-Garcia1998, Calleja2000], though SA NWs suffer from a poor shape and distribution control, making it hard to tune their properties to meet the desired requirements. The selective area growth (SAG) method, recently developed [Sekiguchi2008, Kishino2009, Bengoechea2011], adds huge advantages as the precise control of NWs position, height, diameter, and orientation. Most works on SAG were done on GaN/sapphire substrates [Albert2014], while for a tandem water splitting cells a Si substrate is preferable. Until now no systematic study was done on how the crystal quality of different substrates, as well as the NWs dimensions, affect the optical and crystal quality of the last when grown by SAG. 


\subsection{Objectives and methodology}

The goal of this doctoral thesis is the PA MBE growth and characterization of constituent functional blocks of an $\ln _{x} G_{1-x} N / S i$-based solar-assisted water splitting cell. Aside from the PA MBE growth, the thesis covers a wide range of related issues on optical, structural, and electrochemical characterization of the $\ln _{x} \mathrm{Ga}_{1-x} \mathrm{~N}$ layers, QDs, and NWs structures. The motivation behind the work, background information, and description of the experimental techniques used are presented in Chapters 1 to 3, respectively. The results of the work are divided into four packages presented in Chapters 4 to 7, with general conclusions and future work in Chapter 8. The description of these packages is presented below:

1. Growth and characterization of compact, thick, homogeneous, In-rich InGaN layers directly on Si(111) substrates.

Chapter 4 addresses the MBE growth of $\ln _{x} G_{1-x} N$ layers over the entire composition range ( $x=0$ 1) directly on Si. Particular attention is paid to the optimization of the growth parameters to obtain compact, thick $(\sim 500 \mathrm{~nm})$, and chemically homogeneous InGaN layers. The silicon substrate preparation is addressed first, which includes optimization of the substrate cleaning, degassing and nitridation procedures. The impact of the growth temperature, In/Ga flux ratios, and the III/N ratio on the layer morphological, optical and structural properties is then studied. The most challenging task is to extend the $\ln _{x} G_{1-x} N$ alloy compositions over the entire range. Layers morphology is characterized by scanning electron microscopy (SEM) and atomic force microscopy (AFM). Their structural properties and $\mathrm{InN}$ mole fraction are assessed by high-resolution X-ray diffraction (HRXRD) and their optical properties and corresponding bandgap emission is determined by photoluminescence (PL) and scanning near-field optical microscopy (SNOM) measurements. The InGaN/Si(111) interface is studied with atomic detail by various transmission electron microscopy (TEM) techniques. Their electrical properties are established from current-voltage measurements.

Based on the results from the mentioned characterization, the bandgap (InN mole fraction), thickness, and morphology of the InGaN layers can be adjusted to fit the requirements of efficient InGaN/Si- based water splitting electrodes.

\section{Growth and characterization of InN QDs on $\mathrm{Si}(111)$, $\mathrm{Si}(001)$ and $\ln \mathrm{GaN}(0001) / \mathrm{Si}(111)$ substrates.}

The growth of $\operatorname{lnN} Q D$ s on rough surfaces (root mean square $>5 \mathrm{~nm}$, without atomically flat plateaus) is explored in Chapter 5 by a novel method of droplet epitaxy below RT. To facilitate the structural study, the InN QDs are first grown on atomically flat Si substrates. This also allows to determine how the atomic arrangement is transferred from the substrate to QDs for two substrate orientations, $\mathrm{Si}(111)$ and (001) having a 3-fold (more compatible with the wurtzite InN 6-fold symmetry) and 4-fold symmetry, respectively. A series of $\mathrm{InN}$ nanodots are grown with varying deposition temperature, nitridation time, and annealing temperature/time in order to find the growth parameters resulting in a uniform distribution of small QDs ( $<30 \mathrm{~nm}$ in diameter). The InN QDs morphology and crystalline structure is examined by AFM and HRTEM. The optimized InN QDs growth procedure is transferred to $\ln _{0.3} \mathrm{Ga}_{0.7} \mathrm{~N}$ layers (bandgap of $\sim 2 \mathrm{eV}$ ) to fabricate surfacemodified InN-QDs//n $\mathrm{n}_{0.3} \mathrm{Ga}_{0.7} \mathrm{~N} / \mathrm{Si}$ photoanodes. 


\section{Growth and characterization of SAG GaN NWs on different templates.}

Chapter 6 addresses the study of the effects of NWs dimensions (diameter, height) and the quality of the substrate used (GaN/Si, GaN/AIN/Si, GaN/sapphire) on the SAG GaN NWs structural properties and optical performance. Various III/N ratios and substrate temperatures are tested to optimize the growth conditions and to ensure the SAG growth regime. Series of GaN NWs are grown on all three mentioned templates with varying length (controlled by growth time) and diameter (controlled by lithographic nanoholes mask patterning). NWs morphology is characterized by SEM; their crystal structure and extended defects (dislocations) are studied by HRTEM and their optical quality characterization is performed by PL. Results from these experiments shine light on the fundamental properties of NWs and their dependence on growth conditions, dimensions, and underlying layers. Ultimately, it provides enough knowledge about the limitations of SAG GaN NWs when $\mathrm{Si}$ is used as a substrate instead of sapphire, which is important for optimizing these structures for solar water splitting tandem cells.

\section{PEC characterization of bare and InN QDs decorated InGaN layers as photoanodes.}

Both, bare and InN QDs-decorated InGaN layers on $\mathrm{Si}$, are examined with PEC characterization tools to determine their potential for water splitting applications and presented in Chapter 7 . These tools include cyclic voltammetry (CV) in the dark and under illumination (Xe-lamp mimicking sunlight) and chronoamperometry (CA). Various electrolytes with different $\mathrm{pH}$ values are used in order to check their effects on voltammetry measurements. In addition, electrolytes with organic hole-scavengers (formic acid, methanol) are used to check the kinetic limitations of the water oxidation of InGaN photoanodes. Corrosion of the samples in different electrolytes is studied by CV, CA, and SEM measurements. In addition, the effect of magnetron sputtered NiO-film catalyst on InGaN electrodes efficiency and corrosion resistance is studied. 


\section{Background}

\subsection{Introduction}

The structural, mechanical, and optical properties of III-nitride binaries, GaN, InN and AIN, together with the ternary $\ln _{x} G_{1-x} N$ alloy, are briefly summarized in this chapter. While significant progress has been recently achieved (such as the determination of the actual bandgap value of InN [Davydov2002]), the knowledge about III-Nitride material system is still incomplete. Thus, in some cases the properties of ternary alloys are linearly interpolated between the corresponding binaries. When necessary, a more precise quadratic interpolation (taking into account the so called bowing parameters) is applied.

Because one of the main goals of this thesis is to grow $\ln _{x} G a_{1-x} N$ on $\mathrm{Si}(111)$ substrates, the basic properties of Si crystals are also briefly presented.

Finally, an overview of the semiconductor/electrolyte (S/E) junction (the key component of the semiconductor-based water splitting cells) is given, both for an ideal and a close to real cases. The most common modifications of the S/E junction behavior, such as the use of sacrificial agents and catalysts, are also described.

\subsection{Structural properties of III-Nitrides}

The material properties of semiconductors are largely determined by their crystallographic structure. In this section, the structural and mechanical properties of III-Nitrides are briefly reviewed.

\subsubsection{Crystalline structure}

Group III-Nitrides are known to crystallize in three different phases:

(i) hexagonal wurtzite $\alpha$-phase structure (most common GaN-like, Fig. 2.2.1 (a)).

(ii) cubic zinc blende $\beta$-phase structure (diamond-like, Fig. 2.2.1 (b)).

(iii) cubic rocksalt structure (Fig. 2.2.1 (c)).

Only the hexagonal wurtzite structure is thermodynamically stable at room temperature. The zinc blende phase has crystallographic similarities to the wurtzite phase leading to a small energy difference $\Delta E_{W Z-Z B}=-9.88 \mathrm{meV} /$ atom between them for GaN [Yeh1992]. This fact can lead to the incorporation of zinc blende phases into wurtzite crystals, a "defect" which is called basal plane stacking fault (BSFs). On the other hand the rock salt structure is only achieved (and stable) under very high pressures [Xia1993].

The description of the crystalline structure of III-Nitrides is focused on the GaN case, though it is easily extendable to the other III-Nitrides by changing the metallic group-III element. 

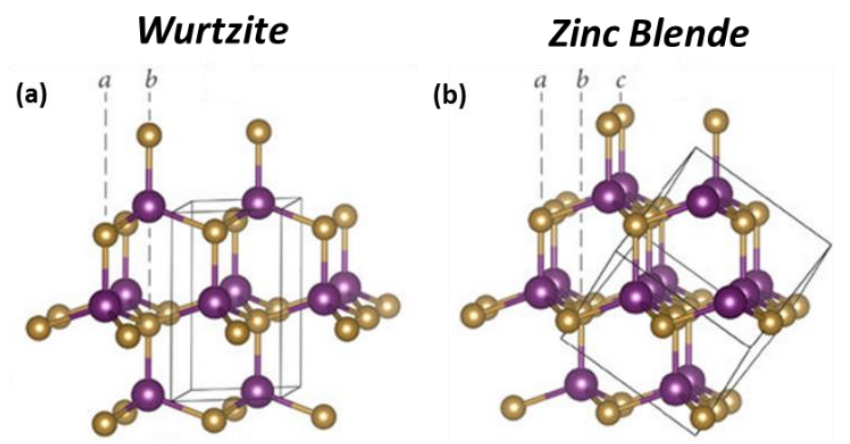

\section{Rocksalt}

(c)

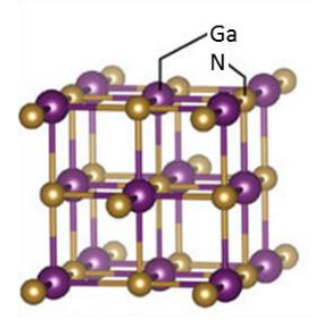

Fig. 2.2.1. Ball-and-stick models of the GaN wurtzite (a), zinc blende (b), and rocksalt (c) structures unit cells. Adapted from [Balachandran2015].

The atomic arrangement in a wurtzite GaN crystal is formed by hexagonal double layers (in epitaxy usually called monolayers (MLs)), one consisting of nitrogen and the other of gallium atoms. Each atom is bound by four $\mathrm{sp}^{3}$ hybridized atomic orbitals to its nearest neighbor, resulting in a tetrahedral 4-fold coordination.

The basic unit cell of wurtzite GaN (Fig. 2.2.1 (a)) contains six atoms of each type (Ga, N). It has a hexagonal structure and can be described by two lattice constants $\boldsymbol{c}$ and $\boldsymbol{a}$. The primitive lattice vectors (Fig. 2.2.2 (a,b)) in the Cartesian coordinate system are:

$$
a_{1}=a\left(\begin{array}{c}
1 / 2 \\
\sqrt{3} / 2 \\
0
\end{array}\right), a_{2}=a\left(\begin{array}{c}
1 / 2 \\
-\sqrt{3} / 2 \\
0
\end{array}\right), c=c\left(\begin{array}{l}
0 \\
0 \\
1
\end{array}\right)
$$

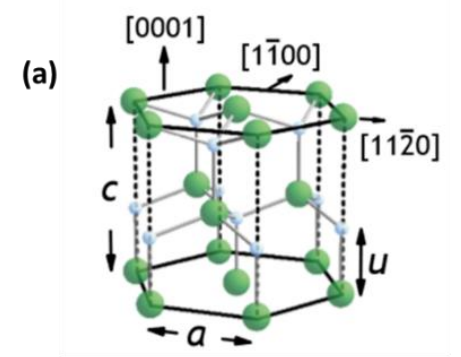

(b)

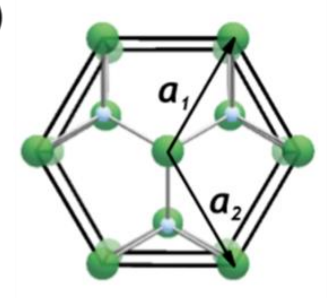

(c)

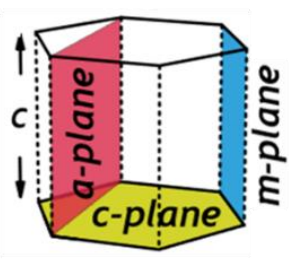

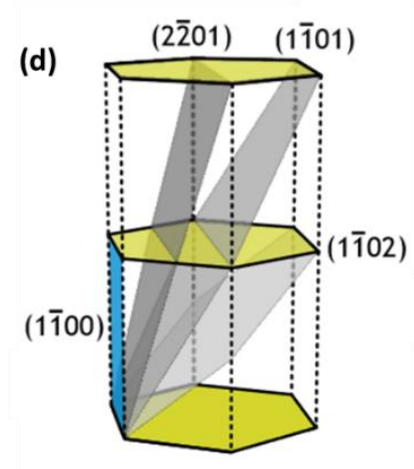

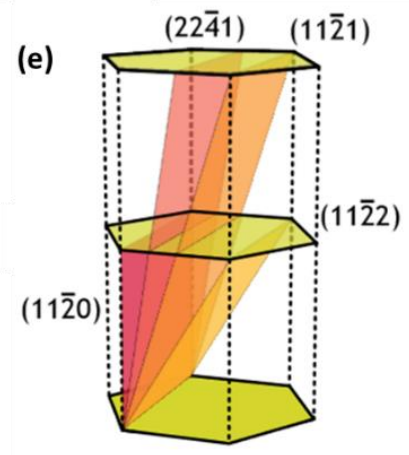

Fig. 2.2.2. Crystalline directions in Ga-polar wurtzite $G a N(a)$. Top-view projection of along the c-axis (b). Polar c-plane, non-polar $\boldsymbol{m}$ - and $\boldsymbol{a}$-planes (d), and low-index semi-polar planes inclined towards either $\mathbf{m}$ (d) or a-planes (e). Adapted from [Urban2013]. 
Besides that, the wurtzite structure is characterized by the internal parameter $\boldsymbol{u}$, which describes the bond-length of $\mathrm{Ga}$ and $\mathrm{N}$ atoms along the $c$-axis. The "ideal" wurtzite structure is characterized by $u=0.375 c$ and $\frac{c}{a}=\sqrt{\frac{8}{3}}$. However, because of the mixed ionic-covalent nature of III-Nitride bonds and lack of inversion symmetry in the wurtzite crystal structure, the actual $u$ parameter deviates from its "ideal" value. This effect causes the tetrahedral angles to be distorted, which induces a spontaneous polarization field along the c-direction [Bernardini1997, Lahnemann2012].

The lattice parameters of the InN, GaN, and AIN binaries are listed in Table I as well as in Fig. 2.2.3, where their bandgap is plotted against lattice parameters. The Si in-plane (111) lattice parameter $a_{(111)}=\frac{1}{\sqrt{2}} a_{(001)}$ is also presented for comparison. The mismatch to Si(111) is calculated as follows:

$$
\varepsilon_{\text {to Si(111) }}=\frac{a_{S i(111)}-a_{I n N}}{a_{S i(111)}},
$$

The lattice parameters of the $\ln _{x} \mathrm{Ga}_{1-\mathrm{x}} \mathrm{N}$ alloy ( $\mathrm{x}$ being the $\mathrm{InN}$ molar fraction) can be interpolated between the corresponding binaries using a linear relationship called Vegard's law:

$$
\begin{aligned}
& a_{I n_{x} G a_{1-x} N}=x \cdot a_{I n N}+(1-x) \cdot a_{G a N}, \\
& c_{I n_{x} G a_{1-x}}=x \cdot c_{I n N}+(1-x) \cdot c_{G a N},
\end{aligned}
$$

The Miller-Bravais indices in the form of [ $\boldsymbol{h} \boldsymbol{k} \boldsymbol{i} \boldsymbol{l}]$ and $(\boldsymbol{h} \boldsymbol{k} \boldsymbol{i} \boldsymbol{l})$ are commonly used to designate different crystal directions and planes respectively in wurtzite structures (Fig. 2.2.2). The first three indices (hki) belong to the same crystal plane and, thus, they are not independent $(h+\underline{k}+i=0)$. The polar (0001) c-plane is perpendicular to the basal plane and is the typical direction for epitaxial growth of III-Nitrides. Other widely used non-polar planes are the $\boldsymbol{a}$ - $(11 \overline{2} 0)$ and the $\boldsymbol{m}$ - (11 $\overline{1} 00)$ planes (Fig. 2.2.2 (c)). All planes in between the non-polar and polar plane(s), with nonzero $h, k$, $i$, and $I$ indices, are called semi-polar (Fig. 2.2.2 (d,e)). Fig. 2.2.4 shows the theoretical inclination of selected semi-polar planes with respect to the $c$-plane, together with an example of how these planes can be experimentally observed.

The lack of central symmetry in wurtzite GaN (Fig. 2.2.1 (a)) makes its c-direction, either Ga-polar [0001] or N-polar [0001] ], depending on the starting atomic layer, either Ga or N, in the c-direction.

Table I. Lattice parameters of binary III-Nitrides at room temperature (From [Morales2009]). *The Si inplane (111) lattice parameter $a_{(111)}$ is presented for comparison (from [Adachi2009]). **The mismatch is calculated according to Eq.2.2.2.

\begin{tabular}{lcccc} 
Material & $\boldsymbol{a}[\AA]$ & $c[\AA]$ & $u$ [a. u.] & $\begin{array}{c}\text { Mismatch to Si [\%] } \\
* *\end{array}$ \\
$\boldsymbol{\alpha}-\operatorname{InN}$ & 3.5377 & 5.7037 & $0.380 c$ & $\sim 8 \%$ \\
$\boldsymbol{\alpha - G a N}$ & 3.1884 & 5.1850 & $0.376 c$ & $\sim 17 \%$ \\
$\boldsymbol{\alpha}-\mathrm{AIN}$ & 3.1120 & 4.9809 & $0.377 \mathrm{c}$ & $\sim 19 \%$ \\
$\operatorname{Si(111)*}$ & 3.8403 & - & - & - \\
\hline
\end{tabular}




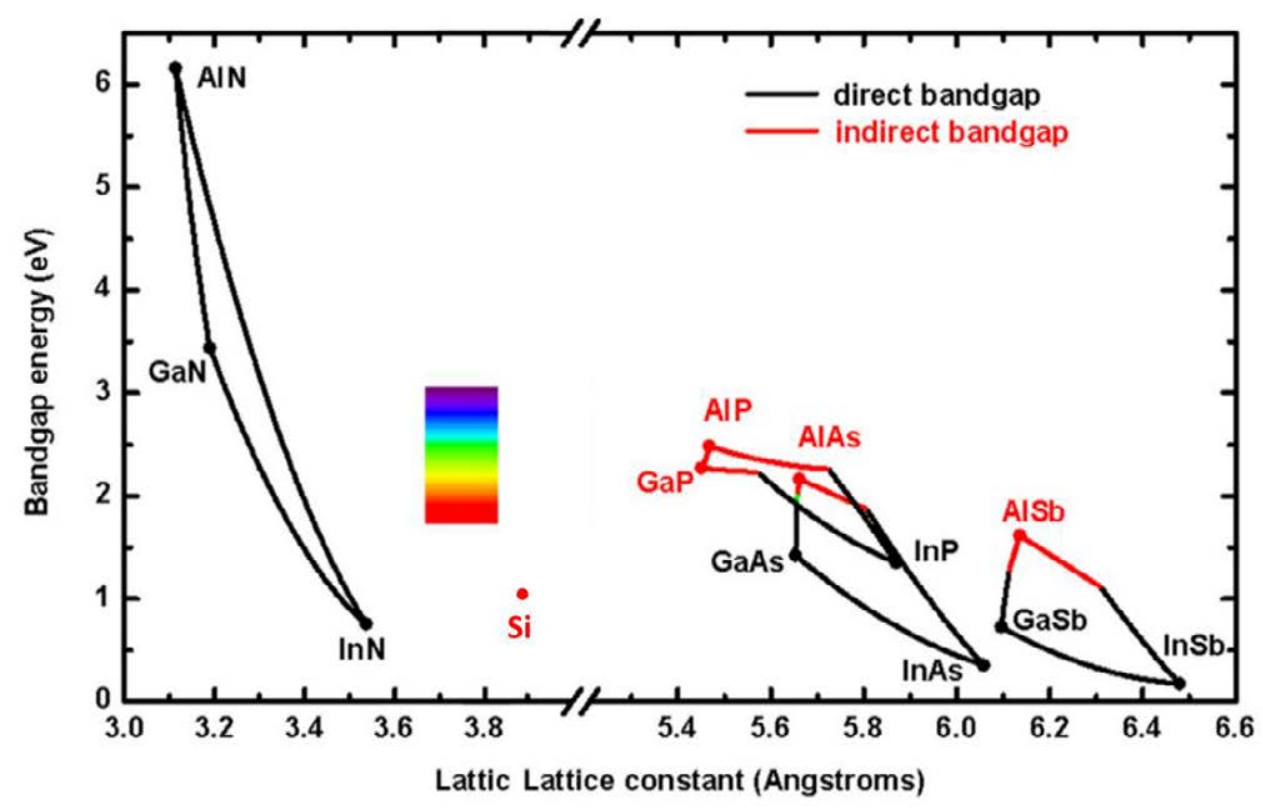

Fig. 2.2.3. Bandgap energies at $300 \mathrm{~K}$ of $\mathrm{III}-\mathrm{V}$ compound semiconductors, plotted against their lattice constants. Si $\left(a_{(111)}\right)$ is marked for comparison. Adapted from [Zhu2013].

\begin{tabular}{ccc}
$\begin{array}{l}\text { Miller-Bravais } \\
\text { index (hkil) }\end{array}$ & $\begin{array}{l}\text { Angle vs c- } \\
\text { plane in deg. }\end{array}$ & Nomenclature \\
\hline$(1 \overline{1} 00)$ & $90.0^{\circ}$ & m-plane \\
$(1 \overline{1} 01)$ & $62.0^{\circ}$ & s-plane \\
$(1 \overline{1} 02)$ & $43.2^{\circ}$ & r-plane \\
$(1 \overline{1} 03)$ & $32.1^{\circ}$ & \\
$(1 \overline{1} 04)$ & $25.2^{\circ}$ & \\
$(11 \overline{2} 0)$ & $90.0^{\circ}$ & a-plane \\
$(11 \overline{2} 1)$ & $72.9^{\circ}$ & \\
$(11 \overline{2} 2)$ & $58.4^{\circ}$ & \\
$(11 \overline{2} 3)$ & $47.3^{\circ}$ & n-plane \\
\hline
\end{tabular}

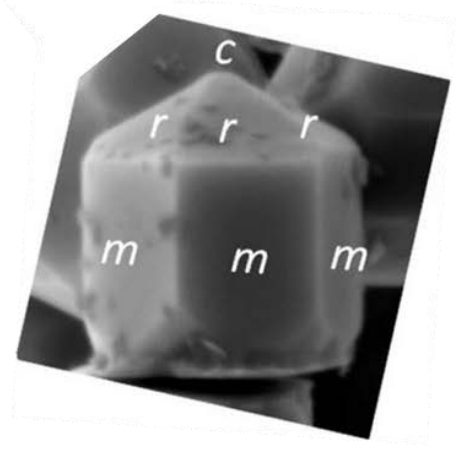

Fig. 2.2.4. Nomenclature of non-polar and semi-polar planes in wurtzite $\mathrm{GaN}$ with their inclination angles to the $c$-plane (adapted from [Urban2013]) (left). SEM image of the Ga-polar GaN nanocrystal exhibiting both semi-polar $(r)$ and non-polar $(m)$ planes (Adapated from [Gacevic2015]). 


\subsubsection{Strain}

Strain in the crystal is defined as the amount of deformation of its unit cell that typically appears in epitaxial layers grown on a substrate because of:

(i) in-plane lattice parameter difference.

(ii) thermal expansion coefficient difference.

The cause (i) usually occurs during the growth and increases as a function of the film thickness, while the cause (ii) originates during the cooling process and increases as a function of the temperature variation. When the accumulated strain energy exceeds the energy needed to form a certain type of crystalline imperfection (i.e. dislocation) the atoms rearrange into an energetically favorable form to relieve strain partially or fully in a so called relaxation process.

The in-plane strain $\left(\varepsilon_{x x}, \varepsilon_{y y}\right)$ and out-of-plane strain $\left(\varepsilon_{z z}\right)$ are defined as:

$$
\begin{aligned}
& \varepsilon_{x x}=\varepsilon_{y y}=\frac{a-a_{\text {relax }}}{a_{\text {relax }}}, \\
& \varepsilon_{z z}=\frac{c-c_{\text {relax }}}{c_{\text {relax }}},
\end{aligned}
$$

where $a, c, a_{\text {relax }}$ and $c_{\text {relax }}$ denote strained (actually measurable) and fully relaxed (usually known values) in-plane and out-of-plane lattice parameters of the epilayer, respectively.

Depending on the in-plane strain sign it can be compressive $\left(a_{\text {relax }}>a\right)$ or tensile $\left(a_{\text {relax }}<a\right)$. For example, if strain is caused by (i) then it is compressive if the in-plane lattice parameter of the substrate is smaller than that of the epilayer (Fig. 2.2.5 (a)) and tensile in the opposite case (Fig. 2.2.5 (b)). According to elasticity theory, biaxial strain is usually accompanied by uniaxial strain of opposite sign. This relation can be formally described by Hook's law relating a deformation (strain) in a crystal $\varepsilon_{k l}$ responding to an internal or external force (stress) $\sigma_{i j}$ through the forth order elastic tensor $C_{i j k l}$ :

$$
\sigma_{i j}=\sum_{i j k l} C_{i j k l} \cdot \varepsilon_{k l}
$$

(a) Compressive

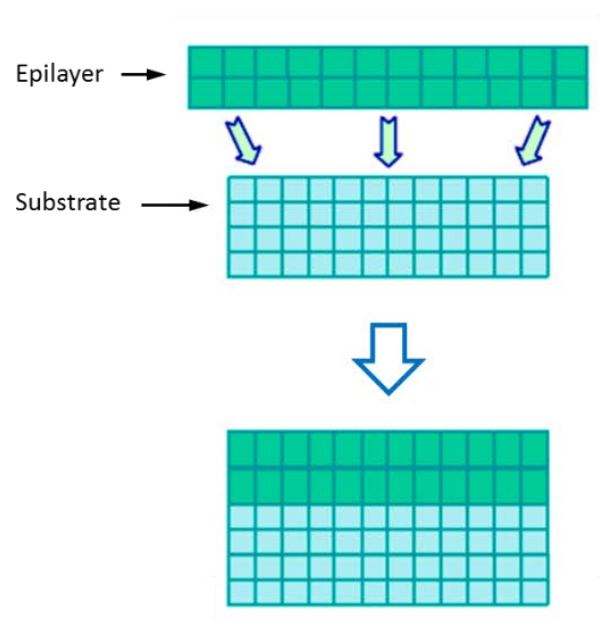

(b) Tensile
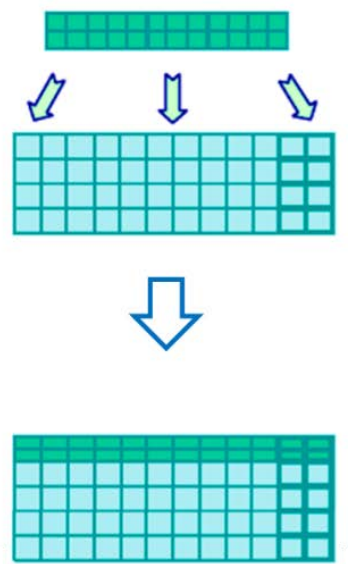

Fig. 2.2.5. A schematic diagram of an epilayer accommodation to a substrate with a different in-plane lattice parameter: (a) compressive and (b) tensile strain. Adapted from [Gacevic2012]. 
This equation can be written in a matrix form taking into account the high symmetry of III-Nitrides $\left(C_{6 v}^{4}\right.$ space group in the Schoenflies notation) and applying the Voight's notation ( $\mathrm{xx}=1, \mathrm{yy}=2, \mathrm{zz}=$ $3, y z, z y=4, z x, x z=5, x y, y x=6$ ) [Piprek2007]:

$$
\left[\begin{array}{l}
\sigma_{1} \\
\sigma_{2} \\
\sigma_{3} \\
\sigma_{4} \\
\sigma_{5} \\
\sigma_{6}
\end{array}\right]=\left[\begin{array}{cccccc}
C_{11} & C_{11} & C_{11} & 0 & 0 & 0 \\
C_{11} & C_{11} & C_{11} & 0 & 0 & 0 \\
C_{11} & C_{11} & C_{11} & 0 & 0 & 0 \\
0 & 0 & 0 & C_{11} & 0 & 0 \\
0 & 0 & 0 & 0 & C_{11} & 0 \\
0 & 0 & 0 & 0 & 0 & \frac{C_{11}-C_{11}}{2}
\end{array}\right] \cdot\left[\begin{array}{c}
\varepsilon_{1} \\
\varepsilon_{2} \\
\varepsilon_{3} \\
\varepsilon_{4} \\
\varepsilon_{5} \\
\varepsilon_{6}
\end{array}\right]
$$

In the case of epitaxial growth there is only biaxial and uniaxial strain $\left(\varepsilon_{4}=\varepsilon_{5}=\varepsilon_{6}=0\right.$ ), while stress is only biaxial $\left(\sigma_{3}=\sigma_{4}=\sigma_{5}=\sigma_{6}=0\right.$ ). Taking into account that the in-plane stress is uniform in both $\mathrm{x}$ and $\mathrm{y}$ directions $\left(\sigma_{1}=\sigma_{2}\right)$ the final relation of biaxial and uniaxial strain is:

$$
\begin{gathered}
\varepsilon_{1}=\varepsilon_{2} \\
\varepsilon_{3}=-2 \frac{C_{13}}{C_{33}} \varepsilon_{1},
\end{gathered}
$$

The elastic constants of the InN, GaN, and AIN binaries are listed in Table II. The corresponding values for ternary InGaN alloys can be interpolated with the linear Vegard's law:

$$
\begin{aligned}
& C_{13}^{I n_{x} G a_{1-x} N}=x \cdot C_{13}^{I n N}+(1-x) \cdot C_{13}^{G a N}, \\
& C_{33}^{I n_{x} G a_{1-x} N}=x \cdot C_{33}^{I n N}+(1-x) \cdot C_{33}^{G a N},
\end{aligned}
$$

Since the elastic constants are positive numbers the in-plane and out-of-plane strains are always of opposite sign. This means that compressing/expanding the epilayer in-plane lattice to fit the substrate one provokes the out-of-plane lattice to be expanded/compressed as shown in Fig. 2.2.5. The above description corresponds to the case of a substrate being much thicker than the epilayer, for instance $500 \mathrm{~nm}$-thick InGaN layers grown on a $300 \mu \mathrm{m}$-thick Si substrate.

Table II. Elastic coefficients of InN, GaN and AIN compounds measured in GPa units [Morales2009].

$\begin{array}{cccc}- & \alpha-\operatorname{InN} & \alpha-G a N & \alpha-A I N \\ C_{11} & 237 & 374 & 395 \\ C_{12} & 106 & 138 & 137 \\ C_{13} & 85 & 101 & 107 \\ C_{33} & 236 & 395 & 404 \\ C_{44} & 53 & 98 & 117\end{array}$




\subsection{Band structure of III-Nitrides}

Bringing atoms together to form a crystal, forces the electron wavefunctions to overlap leading to the formation of energy bands (allowed states), which in semiconductors are separated by bandgaps (forbidden states). From a Bloch description of wavefunctions in a periodic medium, the system can be completely characterized by the behavior in a primitive cell with translational symmetry in a reciprocal space ( $k$-space), namely the Brillouin zone. Wurtzite III-Nitrides have the VB maximum and the CB minimum located at the center of the Brillouin zone ( $\Gamma$-point) what makes them direct bandgap semiconductors.

\subsubsection{Potential profiles and bandgap bowing parameter}

The potential profile of the $\mathrm{CB}$ in $\mathrm{GaN}$ is mainly determined by the Ga s-orbitals leading to a parabolic dispersion. The VB maximum with its $p$-like character is formed by the $\mathrm{N}$ p-orbitals. The VB splits into three bands, called A, B and C (optical nomenclature), due to spin-orbit coupling as well as from crystal field splitting derived from the non-ideal GaN wurtzite structure. These bands in wurtzite GaN can be taken as heavy-hole $(\mathrm{HH})$, light-hole $(\mathrm{LH})$, and crystal-field split hole $(\mathrm{CH})$ bands. In zinc blende structures, only the spin-orbit splitting is present. Fig. 2.3.1 shows the GaN band structure close to the $\Gamma$-point with a magnified detail of the VB splitting. In principle, all three excitonic emissions can be observed, though with intensities decreasing from $A$ to $C$, making $C$ excitons difficult to observe even in high quality GaN layers [Leroux1999] or NWs [Calleja2000]. In samples with lower quality the emission spectrum is dominated by donor-bound excitons (DBE) or defect emission lines like BSFs or inversion domain boundaries (IDBs).

The experimental determination of the $A$ to $C$ free-exciton lines in $\ln N$ is complicated because of the non-intentional very high electron density, from $10^{17}$ to high $10^{20} \mathrm{~cm}^{-3}$ [Wu2004a] [Li2005], so that their appearance strongly depends on the growth method used. This high electron density results in a Moss-Burstein shift of the optical absorption edge because of the CB states occupation [Wu2004a] what leads to an InN bandgap overestimation when measured by optical absorption.

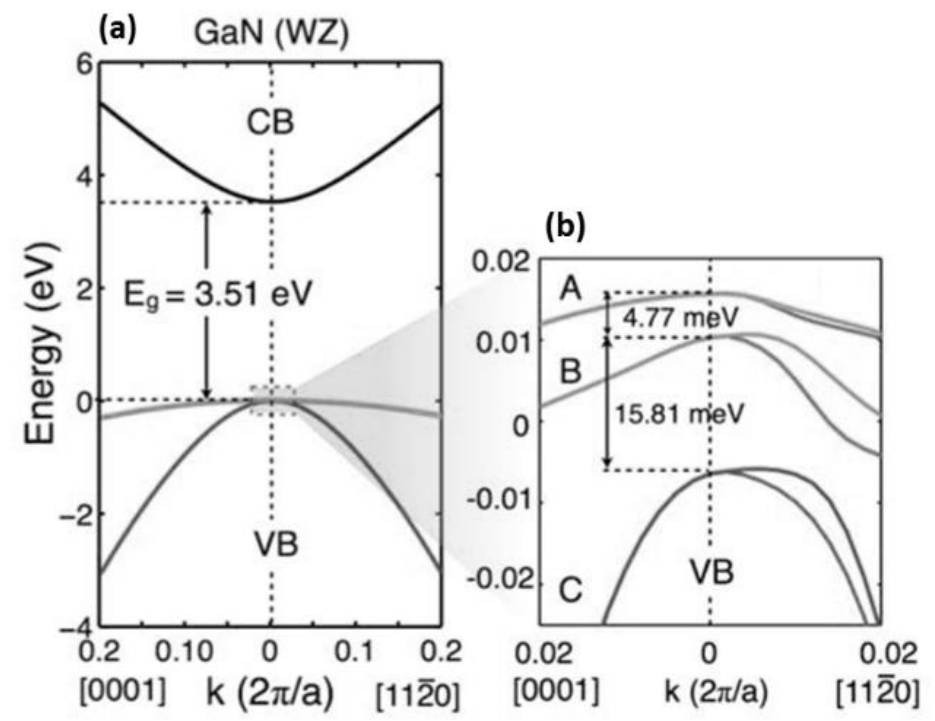

Fig. 2.3.1. Calculated electronic band structure of wurtzite GaN at OK (a). Magnified VB details include spinorbit and crystal-field interactions (b). Adapted from [Amloy2013]. 
The actual InN bandgap was finally determined to be $0.65 \mathrm{eV}$ [Davydov2002, Wu2009]. Table III gives the updated bandgap values of $\operatorname{lnN}, \mathrm{GaN}$, and AIN binaries.

When alloying $\mathrm{GaN}$ and $\operatorname{InN}$ the bandgap energy of the resulting $\ln _{x} \mathrm{Ga}_{1-\mathrm{x}} \mathrm{N}$ alloy changes gradually from GaN to InN depending on the alloy composition $\mathrm{x}$. In this case a quadratic Vergard's law with a bowing parameter $b$ is used for a most precise estimation:

$$
E_{I n_{x} G a_{1-x} N}=x \cdot E_{I n N}+(1-x) \cdot E_{G a N}-b \cdot x \cdot(1-x),
$$

A strong spread of the bowing parameter value in $\ln _{x} \mathrm{Ga}_{1-\mathrm{x}} \mathrm{N}$, from 1 to $4.4 \mathrm{eV}$, has been reported ([Dridi2003] and references therein). This discrepancy, that depends strongly on the optical technique used, can be partially attributed to a very large energy difference between absorption edge and emission (Stocks shift) in $\ln _{x} \mathrm{Ga}_{1-x} \mathrm{~N}$ reaching up to $0.5 \mathrm{eV}$ at $\mathrm{x}=0.5$ [Wu2002a]. This explains why a value of $b=2.5 \mathrm{eV}$ was reported from PL measurements [Davydov2002] while a value of $b=1.4$ was determined by absorption measurements [Wu2002a]. Another reason for these strong discrepancies is that PL spectroscopy is affected by a distribution of smaller-gap regions that have larger-than-average In concentrations [Martin1999, Ferhat2002]. Thus, depending on these In composition fluctuations (localization effect) and the temperature at which PL spectra are considered, the average value of the measured bandgap can be underestimated.

In contrast, optical absorption measurements overestimate the bandgap values due to the MossBurstein effect, which is especially strong in In-rich $\operatorname{In}_{x} G_{a} a_{1-x} N$ layers [Wu2004a]. A summary of literature data on the $\ln _{x} G_{1-x} N$ bandgap versus composition, estimated by both absorption and $\mathrm{PL}$ measurements, is presented in Fig. 2.3.2. Most reports on optical properties of $\ln _{x} G_{1-x} N$ related to layers grown on GaN/sapphire substrates, with the exception of a work by Romanyuk et al. [Romanyuk2009] where $\ln _{\mathrm{x}} \mathrm{Ga}_{1-\mathrm{x}} \mathrm{N}$ layers $(\mathrm{x}<0.3)$ were grown on $\mathrm{Si}$.

In this work the bowing parameter over the entire composition range of $\ln _{x} G_{1-x} N$ layers grown on Si was estimated by RT PL (for bandgap), combined with HRXRD (RSM) (for In composition) measurements. For more details on measurement techniques refer to Chapter 3.

Table III. Bandgap values of III-Nitride binaries [Wu2009].

\begin{tabular}{ccc} 
Material & \multicolumn{2}{c}{ Bandgap [eV] } \\
& 0K & RT \\
\hline $\boldsymbol{\alpha}-\operatorname{InN}$ & 0.69 & 0.64 \\
$\boldsymbol{\alpha}-\mathrm{GaN}$ & 3.51 & 3.43 \\
$\boldsymbol{\alpha}-\mathrm{AIN}$ & 6.25 & 6.14
\end{tabular}




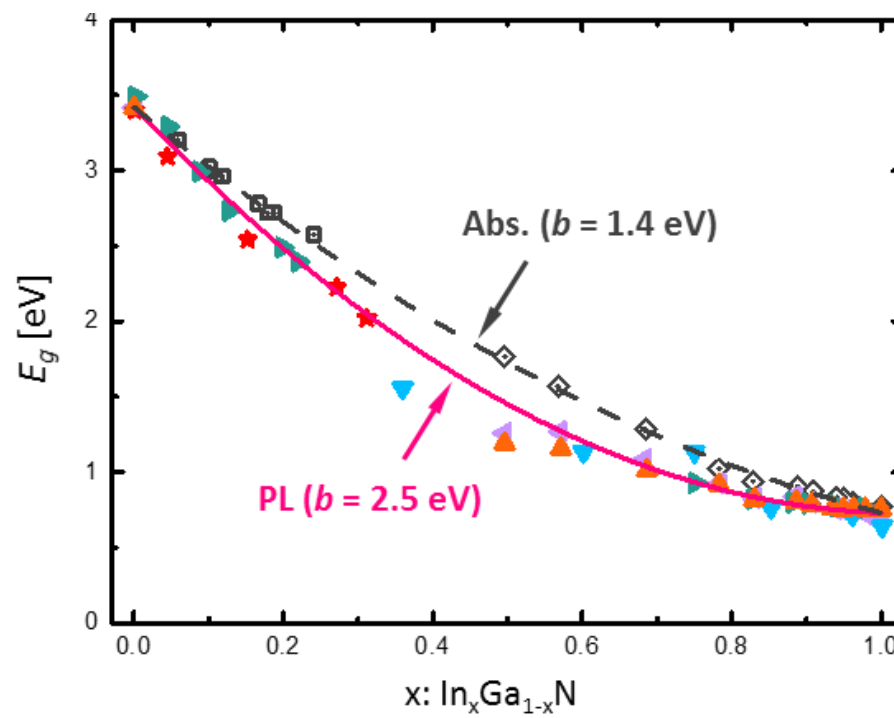

Photoluminescence:

A PL, RT [Wu2002]

$\nabla \mathrm{PL}, 77 \mathrm{~K}$ [Davydov2002]

$4 \mathrm{PL}, 11 \mathrm{~K}[\mathrm{Wu} 2002]$

PL, 10K [Moret2009]

* PL, 11K (on Si) [Romanyuk2009]

\section{Absorption:}

v Abs., RT [Pereira2001]

$\diamond$ Abs., RT [Wu2002]

\section{Quadratic fit:}

$-b=2.5$

$--b=1.4$

Fig. 2.3.2. In $\mathrm{n}_{\mathrm{G}} \mathrm{a}_{1-\mathrm{x}} \mathrm{N}$ bandgap estimated by absorption (grey) and PL (color). A quadratic Vergard's law fit gives $\boldsymbol{b}$ values of 1.4 and 2.5 , respectively.

\subsubsection{Band offsets}

The knowledge on the actual and precise band alignments in heterostructures is needed in optoelectronics and even more in photoelectrochemical applications to determine if the material of interest is appropriately aligned to redox potentials of $\mathrm{H}_{2} \mathrm{O} / \mathrm{O}_{2}$ and $\mathrm{H}^{+} / \mathrm{H}_{2}$.

An early work on photoelectrochemical characterization of $\mathrm{GaN}$ revealed that its bandgap straddles the redox potential of water with sufficient overpotentials to allow water splitting without external bias [Kocha1995].

The band alignment diagram for $\ln _{x} G_{1-x} N$ over the entire composition range was later determined by Moses et al. [Moses2010] as shown in Fig. 2.3.3.

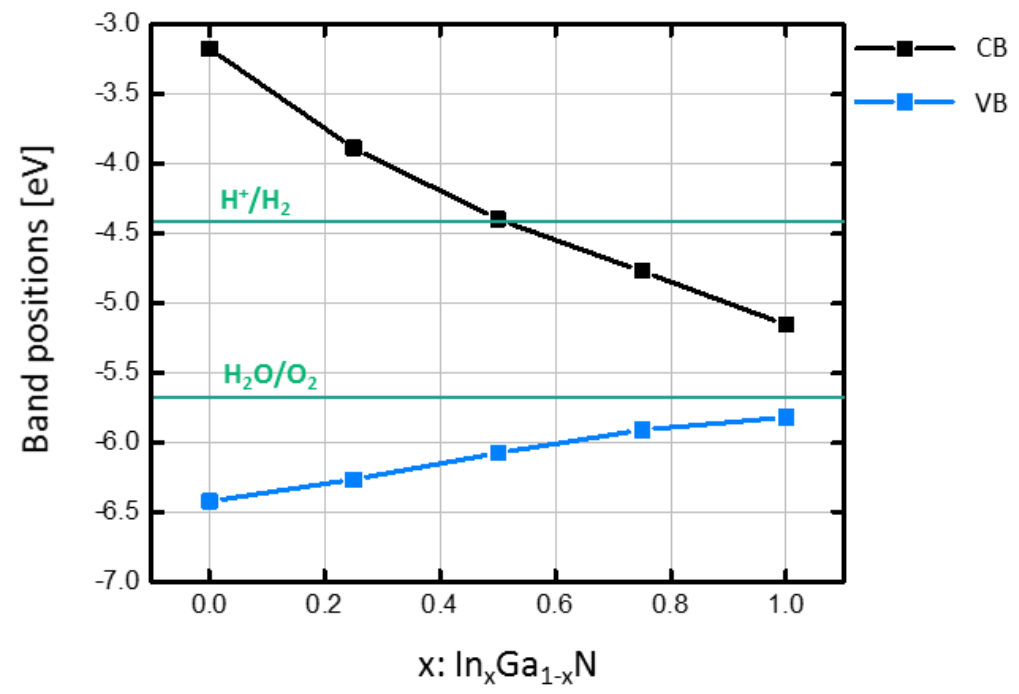

Fig. 2.3.3. $\ln _{x} \mathrm{Ga}_{1-\mathrm{x}} \mathrm{N}$ band alignment relative to vacuum level as a function of the In content $(\mathrm{x})$ for a fully relaxed film. $\mathrm{H}^{+} / \mathrm{H}_{2}$ and $\mathrm{H}_{2} \mathrm{O} / \mathrm{O}_{2}$ redox potentials are marked with green lines. Adapted from [Moses2010]. 


\subsection{Structural defects in III-Nitrides}

The defects in crystals are typically classified by their dimension. Point defects (OD) include: vacancies (unoccupied lattice sites), interstitials (atoms incorporated at non-substitutional lattice sites), antisites (exchanged anion-cation lattice sites), impurity point-defect complexes and Frenkel pairs (vacancy interstitial complexes). Extended defects include: linear (1D) defects (dislocations) and planar (2D) defects such as: interfaces, grain boundaries, stacking faults, polarity inversion domains boundaries, and micro-cracks. Finally, the three dimensional (3D) defects include among others: precipitates, voids (including nanopipes), surface hillocks and pits (so called pinholes). The density of intrinsic point defects in III-Nitrides can be very high and may significantly affect the physical properties of the material.

The most common extended defects in III-Nitrides layers and NWs, such as dislocations (1D), stacking faults (2D) and polarity inversion domain boundaries (2D) are briefly described below.

\subsubsection{Dislocations}

There are two main types of dislocations: edge and screw. Additionally, dislocations may generate as a composition of these two types and are commonly referred to as "mixed dislocations".

The dislocations are characterized by two parameters:

(i) $\vec{t}-$ a dislocation line representing the line of propagation of the dislocation.

(ii) $\vec{b}$ - the Burgers vector describing the magnitude and direction of the lattice distortion. It is defined as the vector difference between two closed lattice circuits: one in the deformed crystal (surrounding the dislocation line) and the other one in the crystal without deformation. Its module brings some valuable information about the shift magnitude and the strain energy released by its formation.

An edge dislocation is formed when, due to strain accumulation, an extra half-plane of atoms is introduced, distorting nearby planes of atoms. A schematic diagram depicting an edge dislocation is represented in Fig. 2.4.1 (a). In an edge dislocation, the Burgers vector is perpendicular to the dislocation line direction, $\vec{b} \perp \vec{t}$ Fig. 2.4.1 (c). If the edge dislocation originated at the heterointerface between two layers with different lattice constant, then it is called misfit dislocation (MD). It only propagates at the heterointerface. If the edge dislocation originated at the edge (angled to the heterointerface plane) of the missing plane generated by MD, then it is a threading dislocation (TD). It propagates through the bulk of the layer to it surface where it is blocked or form a loop so that the two ends of the dislocation can join and annihilate.

A screw dislocation can be visualized as a spiral of connected atomic planes around the dislocation vector $\vec{t}$ (Fig. 2.4.1 (b)). In pure screw dislocations the Burgers vector $\vec{b}$ is parallel to the dislocation line direction $\vec{b} \| \vec{t}$ Fig. 2.4.1 (d). Screw dislocations are always threading.

One way to actually see the threading dislocations is to use cross-sectional TEM. For example, sample can be imaged by TEM under two-beam (2B) conditions, such that particular types of threading dislocations become visible under the different diffraction-contrast (DC) conditions. An example of this technique is shown in Fig. 2.4.1 (e,f) for the case of an InN layer grown on top of a GaN/sapphire(0001) template [Loitsch2013]. 
It is well known that in III-V semiconductors, such as GaAs and InP, or II-VI such as ZnSe dislocations are electrically active "charged tubes" hampering carrier mobility. Dislocations may not be per-se lifetime "killers", however, they come always accompanied (decorated) by point defects which are responsible for luminescence degradation. In that respect, dislocations are generally regarded as non-radiative recombination centers [Sugahara1998, Rosner1997], and the luminescence of the devices based on these materials can be completely inhibited when the dislocation density reaches the order of $10^{4} \mathrm{~cm}^{-2}$ [Nakamura1995b, Ueda1997, Petroff1976, Guha1993]. In contrast, optical devices based on $\ln _{x} \mathrm{Ga}_{1-x} \mathrm{~N}$ alloys have a quite high light emission efficiency despite of the presence of a high dislocation density of the order of $10^{8}-10^{11} \mathrm{~cm}^{-2}$ [Nakamura1995b, Lester1995]. The interpretation is that In clustering in $\ln _{x} \mathrm{Ga}_{1-\mathrm{x}} \mathrm{N}$ alloy localizes electron-hole pairs (or excitons) far away from dislocations [Chichibu2006]. However, this works only for low In compositions $(x<0.2)$, for which the density of defects (intrinsic and extrinsic) is still moderate. Therefore, for In-rich InGaN layers alternative solutions to increase device optical emission efficiency must be found. A very promising method is to use the NWs morphology where the dislocation density is reduced drastically due to the filtering effect [Kishino2015].

\section{Type of dislocations}

Edge

(a)

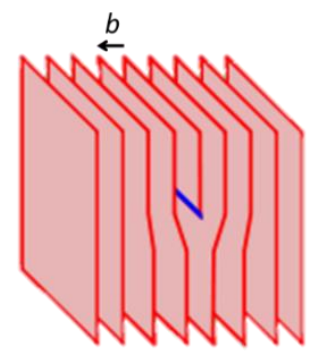

(c)

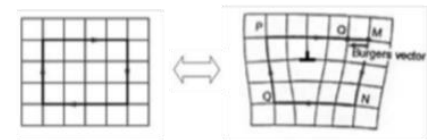

(e)

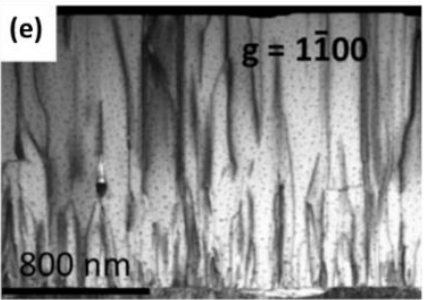

Screw

(b)

(d)
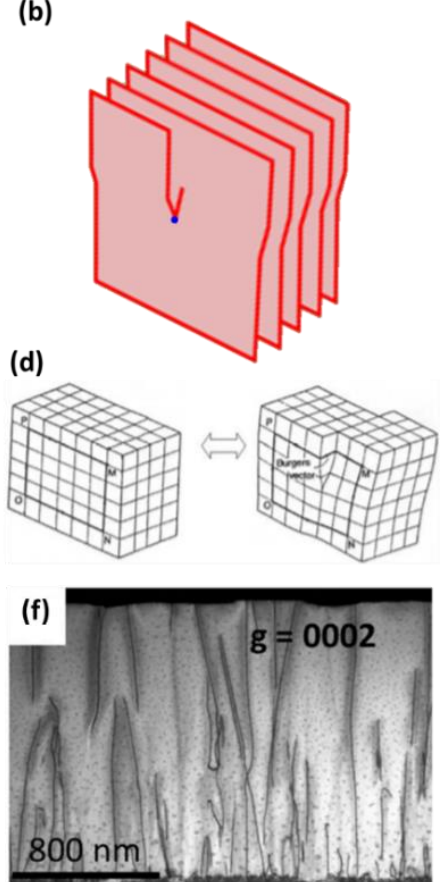

Fig. 2.4.1. Schematic representation of edge $(a, c)$ and screw $(b, d)$ dislocations. Examples of cross-sectional TEM images of InN layer ([11 $\overline{2} 0]$ zone axis) taken at 2B-DC conditions using $g=1 \overline{1} 00$ sensitive to edge-type TDs (e) and g=0002 sensitive to screw-type TDs (f). Adapted from [Loitsch2013].

\subsubsection{Basal stacking faults}

As it was shown in section 2.2.1, GaN crystals may present polytipism in their structures ranging between the two: equilibrium wurtzite with a plane stacking sequence of $A B A B$... (hexagonal closepacked) and metastable zinc blende with $A B C A B C$... sequence (face-centric cubic). The local changes in the stacking sequence of the crystal, i.e. inclusions of zinc blende segments in a wurtzite matrix 
of GaN crystal, are called basal stacking faults (BSFs). These inclusions can be intrinsic if BSFs are formed by the change from one hexagonal close-packed lattice to another one (i.e. $A B$ to $B C$ or $A C$ ), or extrinsic if the insertion of an extrinsic layer is involved (i.e. layer $C$ into $A B$ sequence). Based on these definitions there are three types of BSFs distinguished in the literature [Lahnemann2014]:

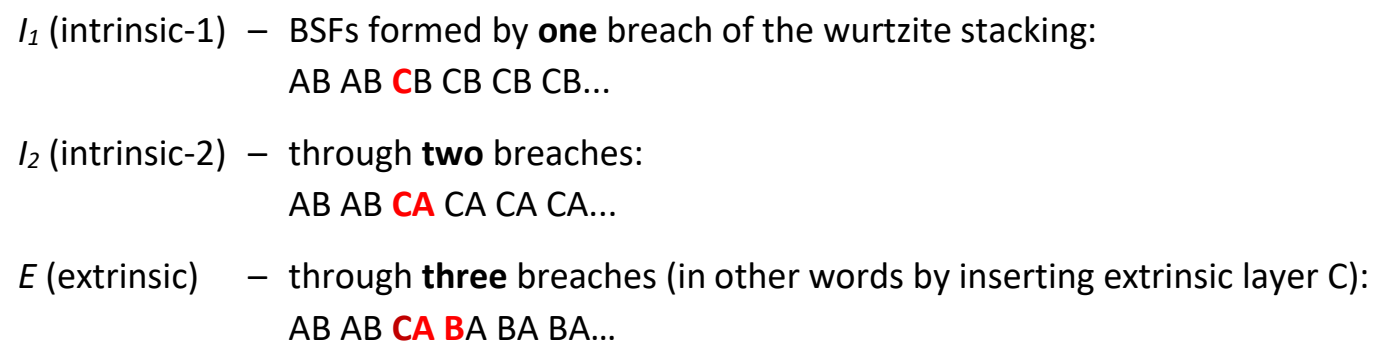

A schematic representation of these BSF types is presented in Fig. 2.4.2 (a), while HRTEM images of a real GaN crystal lattice containing BSFs are shown in Fig. 2.4.2 (b,c,d). In Fig. 2.4.2 (a) the in-plane stacking $\mathrm{A} \rightarrow \mathrm{B}, \mathrm{B} \rightarrow \mathrm{C}$ or $\mathrm{C} \rightarrow \mathrm{A}$ are marked by $\Delta$, and their opposites are marked by $\nabla$, according to the operator notation from Frank et al. [Frank1953]. Thus, the wurtzite stacking is a $\Delta \nabla$ alternation and the zinc blende stacking is represented by a sequence of the same operator. In this notation, BSFs can be characterized by the number of breaches of the wurtzite stacking rule as $I_{1}$ $(1 \Delta), I_{2}-(2 \Delta)$, and $E-(3 \Delta)$. When there are more breaches, then it becomes simply a zinc blende segment. According to calculations, the formation probability of a given BSF decreases with the
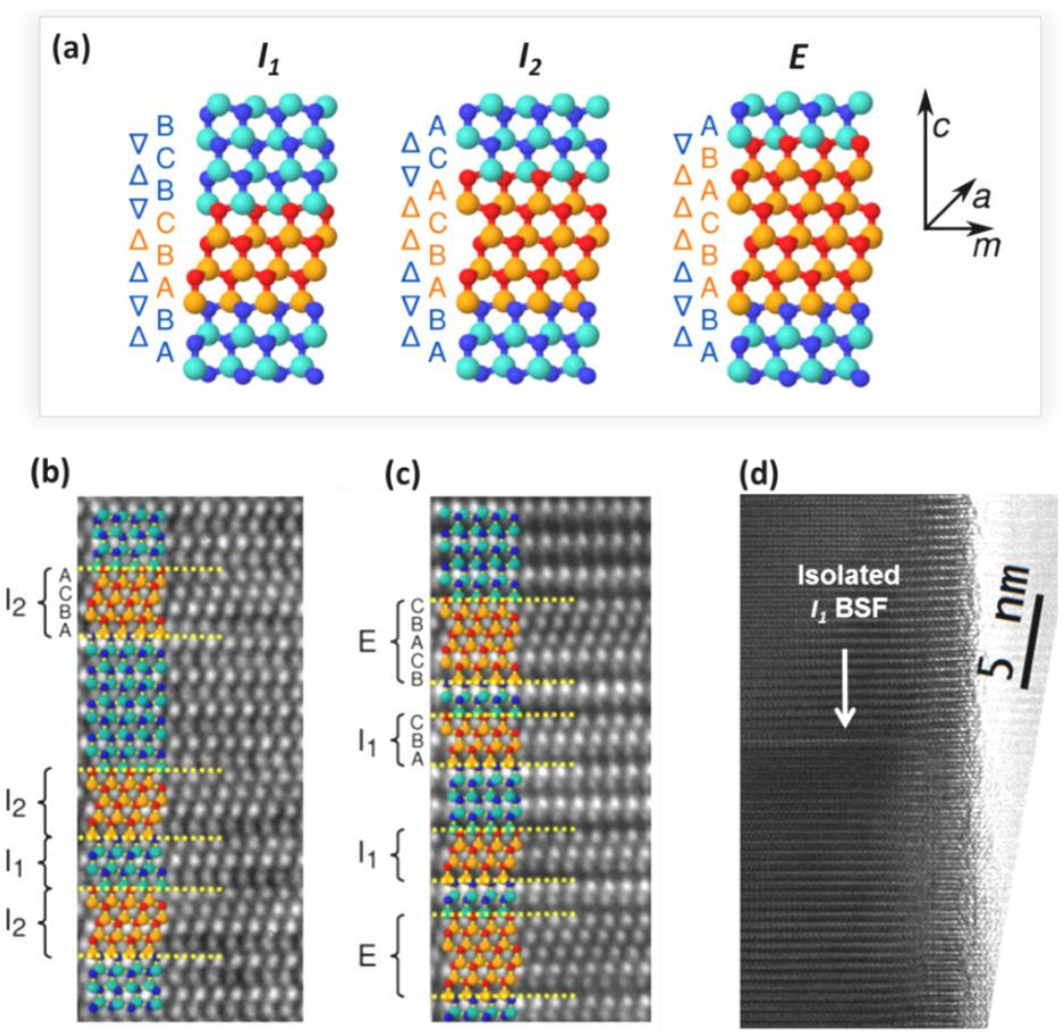

Fig. 2.4.2. Stacking sequences for the intrinsic $I 1$ and $I 2$ as well as the extrinsic $E$ stacking faults (a) (From [Lahnemann2014]). The layers for which the zinc blende stacking sequence is upheld are highlighted, while in the operator notation the breaches to the wurtzite stacking rule are highlighted. HRTEM images of all mentioned types of BSFs (b, c) (From [Lahnemann2014]). HRTEM image of an isolated BSF in GaN NW (d). 
amount of breaches involved, which makes $I_{1}$ the most common type of BSFs [Frank1953, Stampfl1998].

BSFs are optically active defects which induce a bound state within the gap of the host crystal where excitons can be localized. Due to the two-dimensional nature of these planar defects, BSFs act as ideal QWs, which makes them highly luminescent, even at low densities or in the presence of other high density non-radiative centers within the lattice [Lahnemann2012, Korona2014, Corfdir2014a]. Typically reported BSF associated PL emission lines are $I_{1}-3.40-3.43 \mathrm{eV}$ [Liu2005, Korona2014, Corfdir2014a], $I_{2}-3.32-3.36 \mathrm{eV}$ [Liu2005, Tischer2011], and $E-\sim 3.29 \mathrm{eV}$ [Liu2005, Lahnemann2014]. $I_{1}$ is expected to be the most intense line, according to its occurrence probability [Stampfl1998]. It must be noted that these emission energies can be shifted by several factors, including spontaneous polarization fields, electronic coupling of bundled BSFs, coupling between BSFs and adjacent point defects, strain induced shifts, etc. [Lahnemann2014]. An example of a $\mu-P L$ spectrum from a GaN layer containing a high density of all mentioned BSFs types is presented in Fig. 2.4.3.

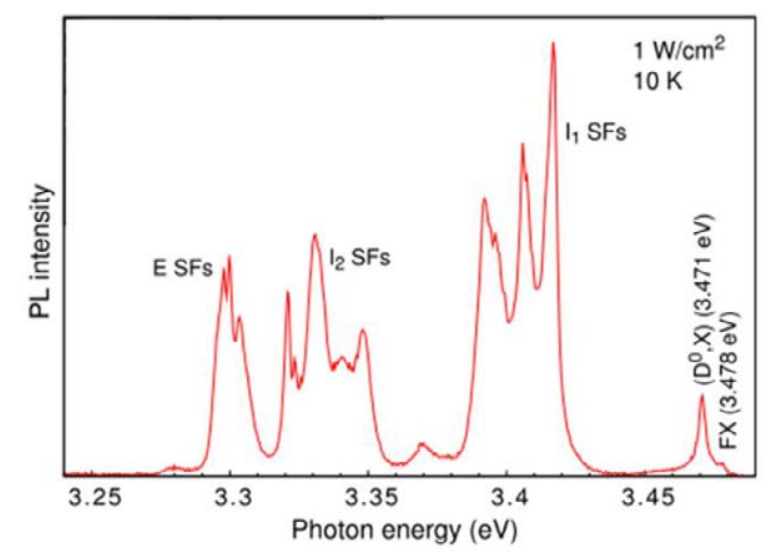

Fig. 2.4.3. $\mu-P L$ spectrum of a single GaN crystal at low-excitation density. Individual BSFs and BSF bundles emerge as sharp lines right below the energies of the three BSF types. From [Lahnemann2014].

\subsubsection{Polarity inversion domains}

As it was mentioned in section 2.2.1, the wurtzite structure of III-Nitrides has a polar axis parallel to the $c$-direction of the crystal lattice which can have two non-equivalent orientations: (0001) and $(000 \overline{1})$. In the case of GaN these two orientations are referred to as Ga-face (polarity), if Ga atoms in the $\mathrm{Ga}-\mathrm{N}$ bilayers forming the crystal point to the sample surface and $\mathrm{N}$-face (polarity) in the opposite case. In case when Ga-face and $\mathrm{N}$-face domains coexist side by side on the same layer, the boundary between them forms a special type of structural defect, named polarity inversion domain boundary (pIDB). Several atomic configurations of pIDBs are possible [Cherns1998, Dimitrakopulos2001], one of which is illustrated in Fig. 2.4.4.

Early reports on pIDBs in GaN bulk layers showed that the boundary between two adjacent polarity domains is a very efficient radiative recombination center, with luminescence intensities exceeding that from bulk regions by more than an order of magnitude [Schuck2001, Stutzmann2001]. The emission energy range assigned to pIDB of 3.45-3.46 eV coincides with that tentatively assigned to the two-electron satellite (TES) of DBE (exciton bound to a neutral donor recombines and leaves the donor in an excited state) [Monemar1997, Corfdir2014a], which often hinders a proper identification of the pIDB optical signature, making the study of pIDBs optical properties challenging. 


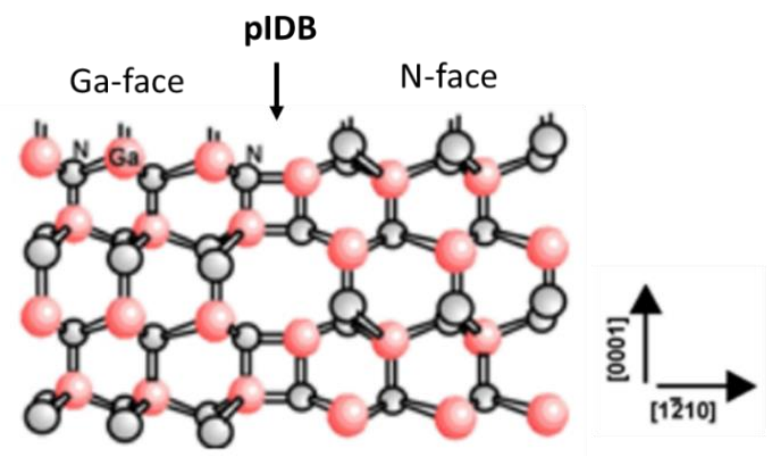

Fig. 2.4.4. Atomic structure of a polarity inversion domain boundary. From [Stutzmann2001].

The identification of pIDBs and the assignment of their corresponding emission lines can be done either indirectly by comparing optical and structural characteristics of the crystal, or directly by spatially resolved PL measurements (see Fig. 2.4.5) and HRTEM.

The role of pIDBs is particularly interesting in GaN NWs, where it was found that the emission at $\sim 3.45 \mathrm{eV}$ can be much more intense than the DBE peak [Calleja2000], which directly discards the assignment of this emission to TES. Since then, the origin of such a strong $3.45 \mathrm{eV}$ emission in GaN NWs is under debate, being the reason to label this emission line as unknown exciton "UX". The UX/DBE intensity ratio was observed to increase when reducing the NW diameter [Calleja2000] pointed to a NW surface related emission. In addition, it was observed a doublet $3.450-3.456 \mathrm{eV}$ where the energy distance of 6 meV coincides with $\mathrm{FX}_{A}-\mathrm{FX}_{\mathrm{B}}$ [Calleja2000, Corfdir2014b].

Recently, Auzelle et al. [Auzelle2015] using a combination of HRTEM and $\mu$-PL, claimed that the UX line observed in self-assembled (SA) GaN NWs is due to pIDBs. In the current work we gathered experimental evidence (by combining HRTEM with PL measurements) that this is also the case for the selective area grwon (SAG) GaN NWs (refer to Chapter 6).

(a)

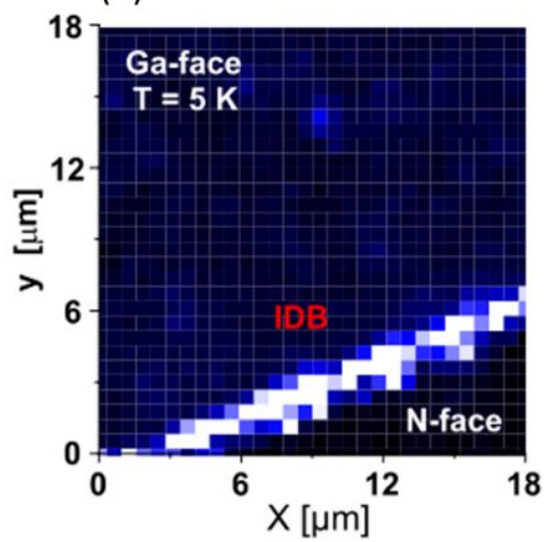

(b)

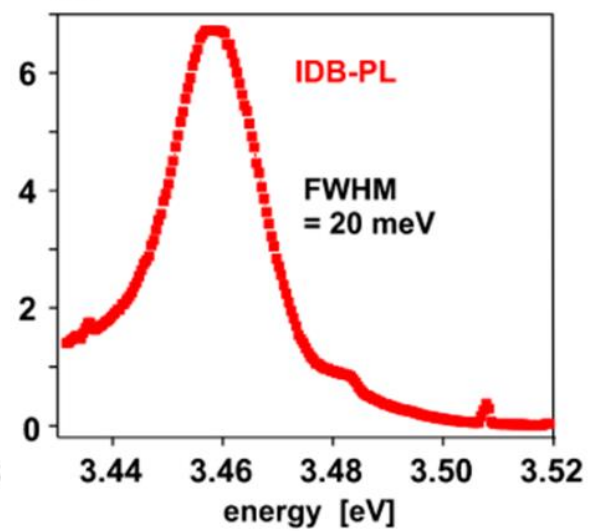

Fig. 2.4.5. Emission intensity map of a pIDB between adjacent $\mathrm{N}$ - and Ga-face bulk regions resolved by low temperature (5K) $\mu$-PL. Radiative recombination occurs predominantly at the pIDB (a). The emission energy of pIDB in such structures is close to $3.46 \mathrm{eV}(\mathrm{b})$. From [Stutzmann2001]. 


\section{$2.5 \ln _{x} \mathrm{Ga}_{1-\mathrm{x}} \mathrm{N}$ growth on $\mathrm{Si}$}

The growth of $\operatorname{In}_{\mathrm{x}} \mathrm{Ga}_{1-\mathrm{x}} \mathrm{N}$ alloys has proven to be extremely challenging and even more when grown on Si substrates due to:

- Meltback etching.

- Formation of $\mathrm{SiN}_{\mathrm{x}}$.

- Lattice mismatch.

- Poor In incorporation into $\ln _{x} \mathrm{Ga}_{1-\mathrm{x}} \mathrm{N}$ alloy.

- Phase separation.

All of these points are reviewed in detail in the following sections.

\subsubsection{Si substrates}

Crystalline $\mathrm{Si}$ is a group IV semiconductor with a diamond-like (cubic) structure. Each Si atom has four neighbors tetrahedrally coordinated. The space group for the diamond structure is $O_{h}^{7}$ in Schoenflies notation. The lattice constant of the Si cubic primitive cell is $a_{(001)}=5.43 \AA$, while the shortest distance between the atoms located on a (111)-plane is $a_{(111)}=\frac{1}{\sqrt{2}} a_{(001)}=3.84 \AA$, as shown in Fig. 2.5.1.

Si substrates can be prepared in various crystalline orientations, being the Si(001) the most common for electronic applications. However, the 4-fold symmetry of this Si surface leads to two differently aligned domains when III-Nitrides layers (6-fold symmetry) are grown on it. For that reason a 3-fold symmetric $\mathrm{Si}(111)$ orientation is preferable.

Semiconductor surfaces have a high density of dangling bonds that increase the total energy. This excess energy can be minimized by surface reconstructions that occur when the unshared electrons of the dangling bonds of neighboring atoms overlap and rearrange decreasing the number of dangling bonds. This surface reconstruction usually involves a change in the lateral surface periodicity and/or reduction in the surface symmetry. Wood's notation is the common method of specifying reconstructed surface structures.

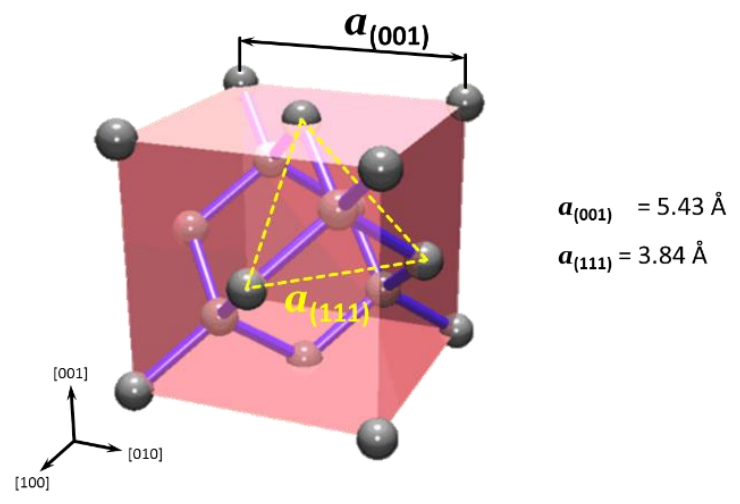

Fig. 2.5.1. Diamond-like (cubic) crystalline structure of Si. 
$(1 \times 1)$

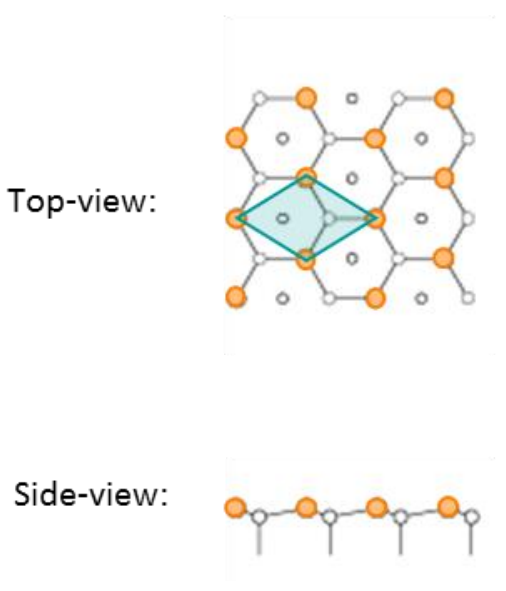

$(7 \times 7)$

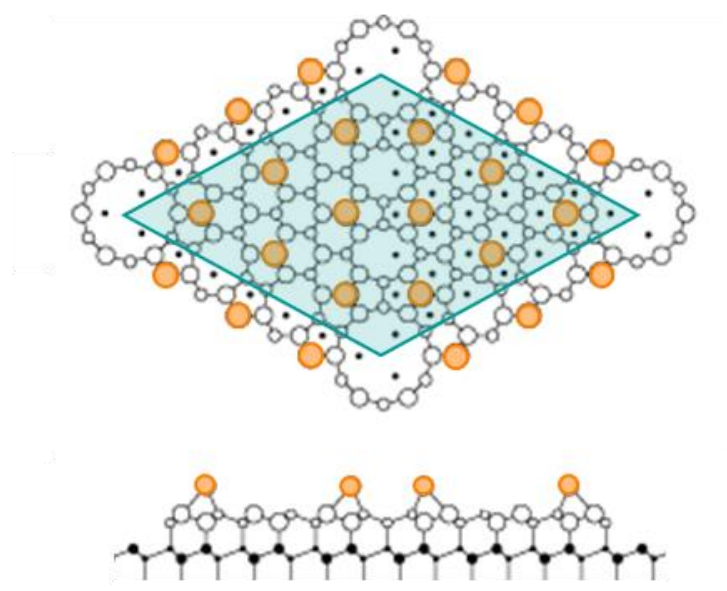

Fig. 2.5.2. (1x1) (a) and (7x7) (b) surface reconstructions of $\mathrm{Si}(111)$. The topmost layer of $\mathrm{Si}$ atoms is orange colored. The unit cell of surface reconstructions is green colored. Adapted from [Takayanagi1987].

Si surface reconstructions significantly affect surface morphology, adsorbates sticking coefficients, defect generation, surface diffusion and surface electronic structure [Dabrowski2000]. Fig. 2.5.2 (a,b) show unreconstructed $\operatorname{Si}(111)-(1 \times 1)$ and reconstructed $\operatorname{Si}(111)-(7 \times 7)$ surfaces, the last having a lower free energy (19 vs. 49 dangling bonds, respectively), that is why at RT clean $\mathrm{Si}$ is usually $(7 \times 7)$ reconstructed (Fig. 2.5.3). The reversible transition from $(7 \times 7)$ to $(1 \times 1)$ occurs upon decreasing the substrate temperature below $827 \pm 15^{\circ} \mathrm{C}$ (determined by pyrometry [Telieps1985]). This reference point is used to calibrate the thermocouple temperature readings. Notice that for this transition to be observed a native oxide layer must be removed from the Si surface (by high temperature annealing under UHV conditions).

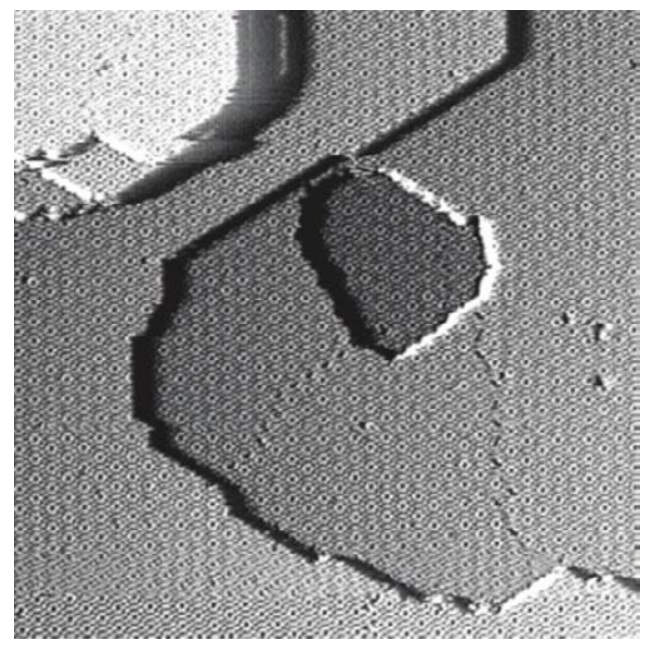

Fig. 2.5.3. A clean stepped Si(111)-(7x7) surface imaged by scanning tunneling microscopy (STM) at RT. Adapted from [Kraus1998]. 


\subsubsection{Meltback etching}

Historically sapphire was chosen as a substrate for growing III-Nitrides because of its stability at high temperature and being chemically inert [Maruska1969]. Si in contrast is a very reactive material, particularly with both active $\mathrm{N}$ plasma and group-III metals that lead to surface nitridation and eutectic reaction respectively. Group-III metals react with $\mathrm{Si}$ reducing drastically its melting temperature leading to a liquid metal-Si mixture. Thus, the accumulation of group-III metals can significantly damage a clean Si surface creating voids filled with eutectic compound (usually of triangular prism form dictated by the Si(111) surface 3-fold symmetry [Ishikawa2011]) in a process called meltback etching. The temperature at which such reaction starts is called eutectic temperature, which is $29^{\circ} \mathrm{C}$ for both Ga [Olesinski1985a] and In [Olesinski1985b], and $577^{\circ} \mathrm{C}$ for Al [Murray1984].

This poses a significant problem to grow III-Nitrides on clean Si surfaces because the meltback etched voids can generate "hillocks" [Kaiser1999, Ishikawa2011, Sobanska2012] and polarity inversion domains [Kaiser1999, Walther2012]. Etched Si can also be incorporated into the growing film forming additional defects ( $\mathrm{SiN}_{\mathrm{x}}$ inclusions) and acting as unintentional dopant [Walther2012]. An example of such effects is given in Fig. 2.5.4 (a) for an AIN buffer grown on a Si(111) substrate grown at $850^{\circ} \mathrm{C}$ under Al-rich regime (Al/N flux ratio $>1$ ) [Kaiser1999]. Similar effects were reported for GaN [Ishikawa1998] and InN ([Walther2012] Fig. 2.5.4 (b)).

There are several methods to reduce the probability of meltback etching, such as:

(i) Use of low temperature buffer layers [Kaiser1999].

(ii) Use of a buffer layer grown under $\mathrm{N}$-rich conditions (III/N flux ratio $<1$ ), also called $\mathrm{N}$ rich start in this work [Ishikawa2011].

(iii) Nitridation of the $\mathrm{Si}$ surface to form a $\mathrm{SiN}_{\mathrm{x}}$ thin film that inhibits metal diffusion [Gwo2003].

(a)

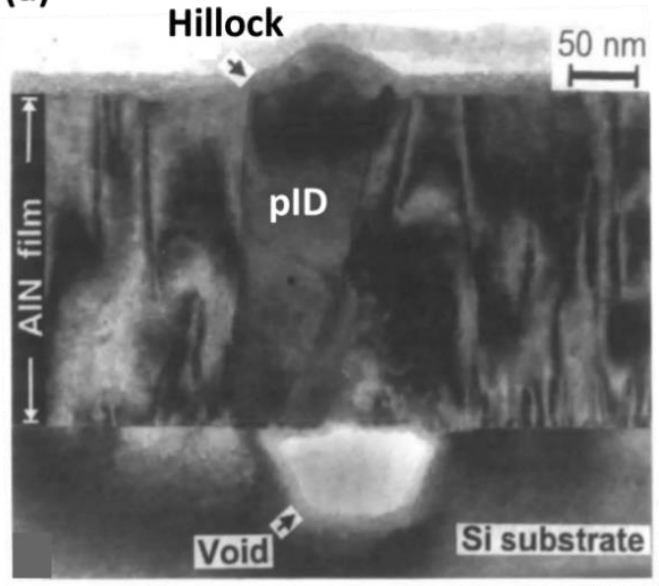

(b)

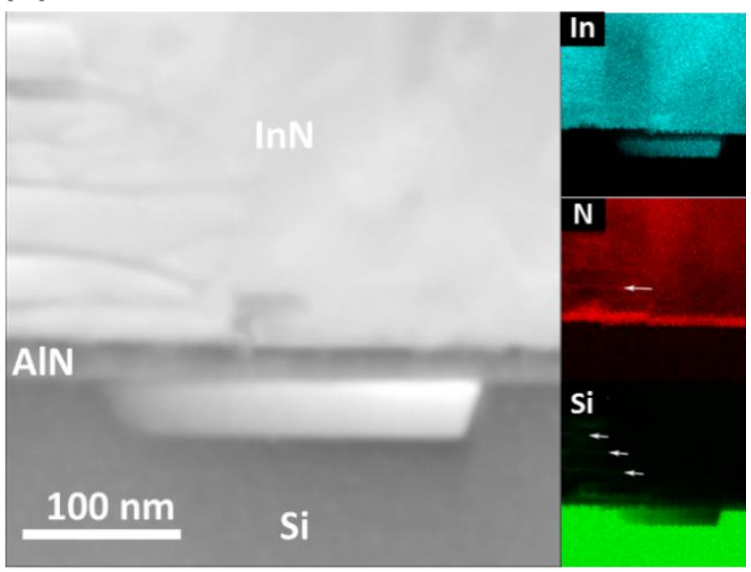

Fig. 2.5.4. Examples of severe meltback etching during AIN (a) and InN/AIN (b) epitaxy on Si(111) substrates measured by BF-CTEM and HAADF+EDX, respectively. In (a) hillocks and polarity inversion domains (pID) generated by meltback damage are marked. In (b), elemental In, N and Si distributions are shown. White arrows indicate segmented In/SiN $\mathrm{N}_{\mathrm{x}}$ inclusions. AIN and InN images are adapted from [Kaiser1999] and [Walther2012], respectively. 
Based on the III-metals eutectic temperatures, method (i) should be more effective for AIN, rather than GaN or InN. However, due to a poor wetting of III-Nitrides on Si, if an AIN buffer is used to inhibit meltback etching it should be thick enough (hundreds of $\mathrm{nm}$ ) to ensure its compactness and avoiding exposure of any small area of bare Si to metals Fig. 2.5.4 (b) [Kaiser1999].

The (ii) method allows to avoid metal accumulation on the Si surface and thus reduce the chances of the eutectic reaction [Ishikawa2011].

Gwo et al. originally proposed the method (iii) to form a crystalline $\beta-\mathrm{Si}_{3} \mathrm{~N}_{4}$ film acting as a diffusion inhibiting layer which protects Si from reacting with group-III metals during the growth of III-Nitride films [Gwo2003, Wu2002b]. This method is discussed in detail in the following section.

All of these methods can be combined together to achieve better results. For instance, high quality InN layers were reported to be grown directly on Si by combining methods (i) and (ii) [Kehagias2013]. In the present work, a combination of all three methods is applied to grow high quality $\ln _{x} \mathrm{Ga}_{1-\mathrm{x}} \mathrm{N}$ layers directly on $\mathrm{Si}$.

\subsubsection{Formation of $\mathrm{SiN}_{x}$}

When a bare $\mathrm{Si}(111)$ surface is exposed to the active $\mathrm{N}$ flux, even simultaneously with metal fluxes, the strong reactivity of $\mathrm{Si}$ to $\mathrm{N}$ produces a thin $\mathrm{SiN}_{\mathrm{x}}$ film [Calleja1999, Gangopadhyay2006]. In the case of AIN, this can be avoided if growth is started with Al pre-deposition [Stevens1994, Calleja1998, Calleja1999]. The $\mathrm{SiN}_{\mathrm{x}}$ film formation is common to many epitaxial techniques, including MOCVD, plasma- and $\mathrm{NH}_{3}$-assisted MBE. Simultaneous III-Nitrides nucleation and formation of a $\mathrm{SiN}_{\mathrm{x}}$ film can result in a chemically inhomogeneous III-Nitride/Si interface and ,thus, can lead to formation of defects, like intermixing hexagonal and cubic III-Nitride phases [Ploog1998]. In order to avoid this, either an AIN buffer, or intentional and controlled nitridation can be used, to completely inhibit, or form a homogeneous $\mathrm{SiN}_{\mathrm{x}}$ respectively. Gwo et al. [Gwo2003] proposed to use a combination of both methods to reduce both the chemical inhomogeneity of III-Nitride/Si interface and probability of meltback etching. In this work, only intentional nitridation was used to avoid any resistivity increase of III-Nitride/Si interface due to AIN buffers.

Considering MBE epitaxy, it was shown that when a Si(111) surface is exposed to nitrogen plasma or $\mathrm{NH}_{3}$ flux a self-limited 2-3 nm thick $\mathrm{SiN}_{\mathrm{x}}$ film develops ([Wu2004b, Ahn2001] and references therein). According to Bahari et al. report [Bahari2005] this $\mathrm{SiN}_{\mathrm{x}}$ film has an amorphous/crystalline structure if produced below/above $500^{\circ} \mathrm{C}$ respectively. In case of crystalline films (high substrate temperatures) their formation process can be monitored by RHEED, as demonstrated in Fig. 2.5.5. Both in situ RHEED (Fig. 2.5.5 (c)) and ex situ STM (Fig. 2.5.6) measurements indicate the conversion of $\mathrm{Si}(111)$ into a crystalline $\beta-\mathrm{Si}_{3} \mathrm{~N}_{4}(0001)$ layer (or "flakes" when nitridation is not complete) upon reaction with active nitrogen or $\mathrm{NH}_{3}$ [Ahn2001, Wu2002b]. It was also reported that the $\beta$ $\mathrm{Si}_{3} \mathrm{~N}_{4}(0001) / \mathrm{Si}(111)$ interface is lattice matched [Ahn2001]. Mansurov et al. [Mansurov2015] proposed to control the completeness of the $\beta-\mathrm{Si}_{3} \mathrm{~N}_{4}$ coverage by monitoring the RHEED pattern spots intensity: its maximum corresponding to a maximum coverage.

In contrast, HRTEM measurements on III-Nitrides grown on intentionally nitridated Si(111) substrates reveal an amorphous $2 \mathrm{~nm}$ thick $\mathrm{SiN}_{\mathrm{x}}$ interlayer no matter what temperature was used. This contradiction caused a long-standing debate about the $\operatorname{SiN}_{x}$ interlayer crystallinity and 
(a)

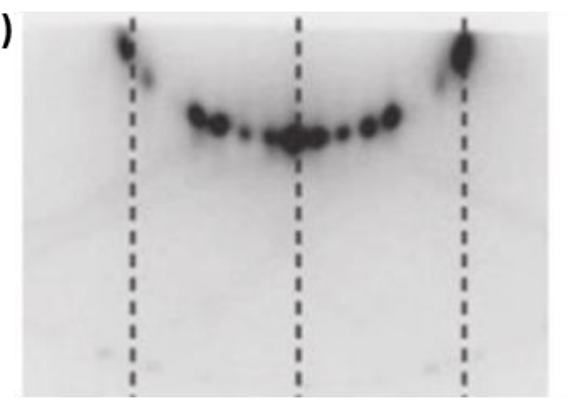

Clean Si surface

below $827^{\circ} \mathrm{C} \rightarrow(7 \times 7)$

\section{Si(111) substrate}

(b)

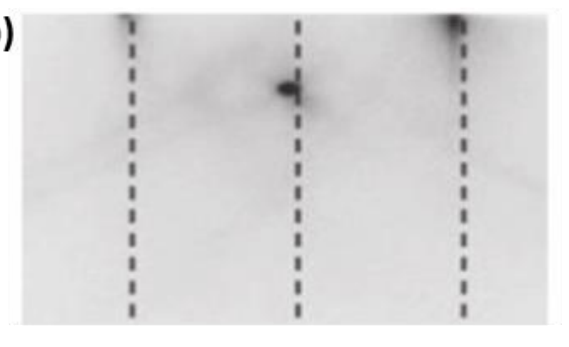

(c)
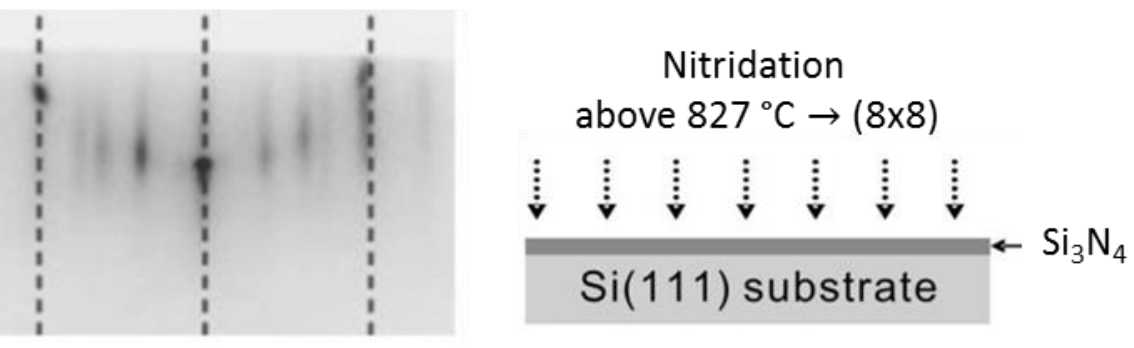

Fig. 2.5.5. Evolution of the RHEED pattern of Si(111) from a $(7 \times 7)$ surface reconstruction taken below $827^{\circ} \mathrm{C}$ (a) to a (1x1)-Si(111) reconstruction upon heating above $827^{\circ} \mathrm{C}(\mathrm{b})$ and then to $(8 \times 8)$ for $\beta-\mathrm{Si}_{3} \mathrm{~N}_{4}(0001)$ layer formed upon thermal nitridation under N plasma. Adapted from [Lastras-Martinez2015].

questioned how epitaxial relation is transferred from Si to III-Nitrides through an amorphous $\mathrm{SiN}_{\mathrm{x}}$ layer. Rawdanowicz et al. [Rawdanowicz2005] suggested that the $\mathrm{SiN}_{\mathrm{x}}$ layer amorphization occurs post-growth during the $\mathrm{Ar}^{+}$ion milling preparation of TEM specimens. To check these possibilities two GaN samples were grown on clean $\mathrm{Si}(111)$ (with nonintentional nitridation) and then prepared HRTEM specimens in two different ways: by (i) ion milling and (ii) mechanical cleaving. In both cases HRTEM revealed presence of a $2 \mathrm{~nm}$ thick $\mathrm{SiN}_{\mathrm{x}}$ interlayer at the interface, but amorphous in the first case (Fig. 2.5.7 (a)) and polycrystalline in the second one (Fig. 2.5.7 (b)). These results are consistent with other reports of $\mathrm{Ar}^{+}$ion milling induced damage on ultra-thin crystalline films during TEM preparation [Chao1987, Wang2001].

Another growth model was proposed by Tamura et al. [Tamura2001] to explain why there is an epitaxial relation between the III-Nitride and Si substrate, while the HRTEM results show a presence of an amorphous $\mathrm{SiN}_{\mathrm{x}}$ layer. In this model the $\mathrm{SiN}_{\mathrm{x}}$ amorphous layer had a partial coverage on the $\mathrm{Si}(111)$ surface, thus leaving some "pin-holes" which act as nucleation centers for the III-Nitride film and allows it to growth laterally from the bare substrate. This is called a pin-holes model. However, to the best of our knowledge, no experimental evidence of the existence of these "pin-holes" was ever reported. 
Notice that because the (0001) surface of crystalline III-Nitrides is less energetic among others, the appearance of polar III-Nitrides should not be a surprise even on top of non-crystalline materials, and similar interface have been previously reported for other polar materials, such as ZnO [Byrne2011].
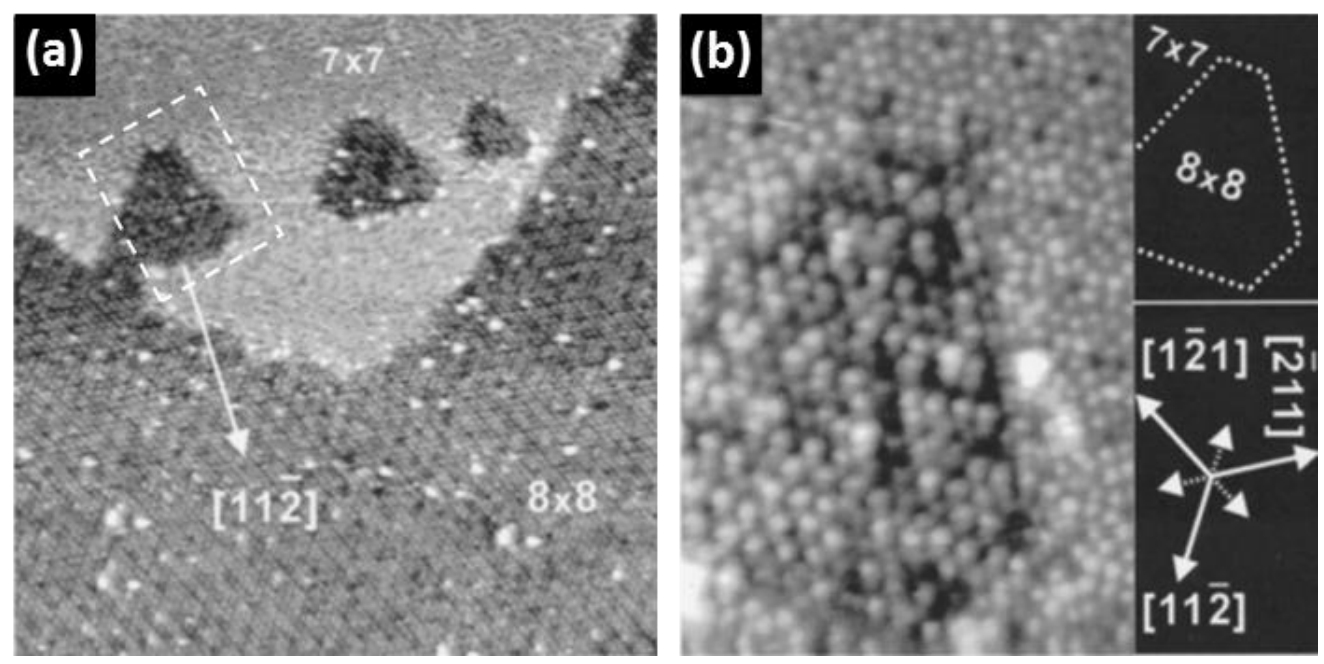

Fig. 2.5.6. STM image of a $\mathrm{Si}(111)$ surface featuring both $(7 \times 7)$ and $(8 \times 8)$ surface reconstructions of $\mathrm{Si}(111)$ and $\beta-\mathrm{Si}_{3} \mathrm{~N}_{4}(0001)$ surfaces, respectively, after a short thermal annealing under $\mathrm{NH}_{3}$ flux at $900^{\circ} \mathrm{C}$ (a). Zoomin STM image of a triangular (dark) "flake" of $\beta-\mathrm{Si}_{3} \mathrm{~N}_{4}(0001)$ (b). Adapted from [Wu2002b].

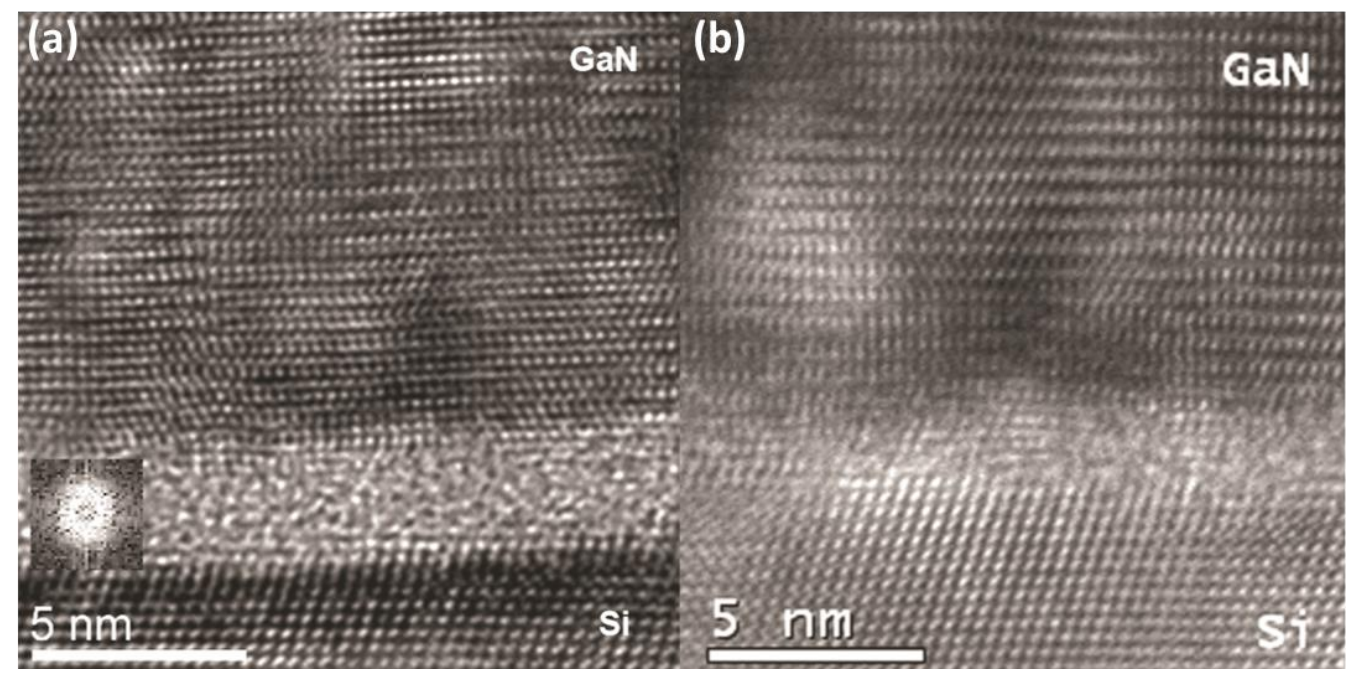

Fig. 2.5.7. HRTEM images of a GaN/Si(111) sample viewed from the $\langle 110\rangle$ direction of the Si showing the differences at the interface of ion-mill (a) and mechanical cleave specimen preparation (b). The inset (a) is a 64×64 FFT to verify that the $\mathrm{SiN}$ is amorphous. Adapted from [Rawdanowicz2005]. 


\subsubsection{Lattice mismatch}

The growth of InGaN on Si is challenged by a large lattice mismatch between them. GaN(0001) and $\ln N(0001)$ wurtzite structures have a planar misfit of $\sim 17 \%$ and $\sim 7 \%$, respectively with the diamond-like structure of $\mathrm{Si}(111)$ along the $<110\rangle$ direction at the interface [Adachi2009] (refer to Table I). This builds up a large biaxial tensile strain at the $\ln _{x} \mathrm{Ga}_{1-\mathrm{x}} \mathrm{N} / \mathrm{Si}$ interface which usually relaxes by generating a large density of misfit dislocations. This tensile strain can also relax by film cracking (in contrast to InGaN films grown on sapphire where, due to compressive strain, cracking is less likely to occur).

In addition, the difference in thermal expansion coefficients of GaN $\left(3.2 \times 10^{-6} \mathrm{~K}^{-1}\right)$ and $\operatorname{InN}$ $\left(3.8 \times 10^{-6} \mathrm{~K}^{-1}\right)$ versus $\mathrm{Si}\left(2.6 \cdot 10^{-6} \mathrm{~K}^{-1}\right)$ results in tensile stress and the subsequent crack formation in the $\operatorname{In}_{x} G_{1-x} N$ epilayer upon post-growth cooling [Zamir2001, Krost\&Dadgar2002]. However, this effect is more pronounced in MOCVD grown samples because of the much higher growth temperatures involved compared to $\mathrm{MBE}\left(800-1100{ }^{\circ} \mathrm{C}\right.$ vs. $500-700^{\circ} \mathrm{C}$, respectively).

Intentional nitridation of $\mathrm{Si}$ to form a homogeneous $\mathrm{SiN}_{\mathrm{x}}$ interlayer [Calleja1998, SanchezGarcia1998, Calleja1999, Huang2002b, Gwo2003], AIN buffer [Calleja1999, LeLouarn2009] and their combination [Wu2003, Gw02003] were reported to be effective in decreasing the dislocation density in III-Nitride films grown on Si. In this work, intentional nitridation of $\mathrm{Si}$ is going to be used for that purpose.

\subsubsection{Poor In incorporation into $\ln _{x} \mathrm{Ga}_{1-\mathrm{x}} \mathrm{N}$ alloys}

The reasons behind a poor In incorporation into $\ln _{x} \mathrm{Ga}_{1-\mathrm{x}} \mathrm{N}$ alloys and, thus, difficulties to obtain $\mathrm{In}$ rich $\ln _{x} G_{1-x} N$ layers, is mainly the very low formation enthalpy of $\operatorname{InN}$ compared to $\mathrm{GaN}(-36 \mathrm{~kJ} / \mathrm{M}$ [Saitoh2008] vs. $-156 \mathrm{~kJ} / \mathrm{M}$ at $298 \mathrm{~K}$ [Ranade2000], respectively), which leads to the fact that $\mathrm{InN}$ starts to decompose already at $\sim 550{ }^{\circ} \mathrm{C}$, while the optimum growth temperature for $\mathrm{GaN}$ is $\sim 750{ }^{\circ} \mathrm{C}$ (in MBE).

The key to growth $\operatorname{In}$-rich $\ln _{x} \mathrm{Ga}_{1-x} \mathrm{~N}$ layers is a relatively low growth temperature close or below $\operatorname{InN}$ decomposition $\left(\sim 550^{\circ} \mathrm{C}\right)$, which allows incorporation of In more efficiently. Both MOCVD and ammonia-MBE, however, have an inherent disadvantage, because conflicting temperature requirements of $\mathrm{NH}_{3}$ pyrolysis $\left(\mathrm{T}>550^{\circ} \mathrm{C}\right)$ and prevention of $\operatorname{InN}$ dissociation $\left(\mathrm{T}<550{ }^{\circ} \mathrm{C}\right)$ must be satisfied [Grandjean2001]. In contrast, $\mathrm{N}_{2}$ radio-frequency plasma source of PA MBE provides active $\mathrm{N}^{*}$ atomic species regardless of the growth temperature. This is the main reason for a great success of PAMBE in fabrication of $\operatorname{In}_{x} G_{1-x} N$ films over the entire composition range $(x=0-1)$ on sapphire(0001) substrates without any buffers [Kurouchi2004], and with GaN [Singh1995, Iliopoulos2006, Bailey2008, Gacevic2013], AIN [Wu2002a, Lu2001], InN [Nanishi2003] buffers. Eventually, $\ln _{x} \mathrm{Ga}_{1-\mathrm{x}} \mathrm{N}(\mathrm{x}=0-1)$ layers were also grown by the MOCVD technique on GaN/sapphire(0001) by exploiting low growth temperatures as well [Liu2013a]. 


\subsubsection{Phase separation}

Besides the lack of native substrates to grow $\ln _{x} \mathrm{Ga}_{1-\mathrm{x}} \mathrm{N}$ layers, additional complications arise from:

(i) Very high miscibility (solubility) gap between $I n N$ and $G a N$ that makes $\ln _{x} G a_{1-x} N$ prone to phase separation and spinodal decomposition.

(ii) Strong differences in the optimal growth temperatures for the two binaries.

A detailed explanation of phase separation and spinodal decomposition, based on thermodynamic description, was proposed by Ho \& Stringfellow [Ho\&Stringfellow1996] where an In solubility in GaN below $6 \%$ was calculated at $800{ }^{\circ} \mathrm{C}$ (typical temperature for InGaN growth temperatures in MOCVD). Many experimental evidences of this effect were collected since then from both MOCVD and MBE grown samples at temperatures above $600{ }^{\circ} \mathrm{C}$ [El-Masry1998, Doppalapudi1998]. An example of the phase separation in InGaN layers is shown in Fig.2.5.8.

Using lower growth temperatures $\left(<600^{\circ} \mathrm{C}\right)$ allows growing thermodynamically unstable $\ln _{x} \mathrm{Ga}_{1-x} \mathrm{~N}$ alloy compositions by kinetically "freezing" the phase separation, but usually at the cost of a reduced crystal quality. This freezing effect is utilized in this work to obtain $\ln _{x} G_{1-x} N$ alloys over the entire composition range.

(a) Phase separation
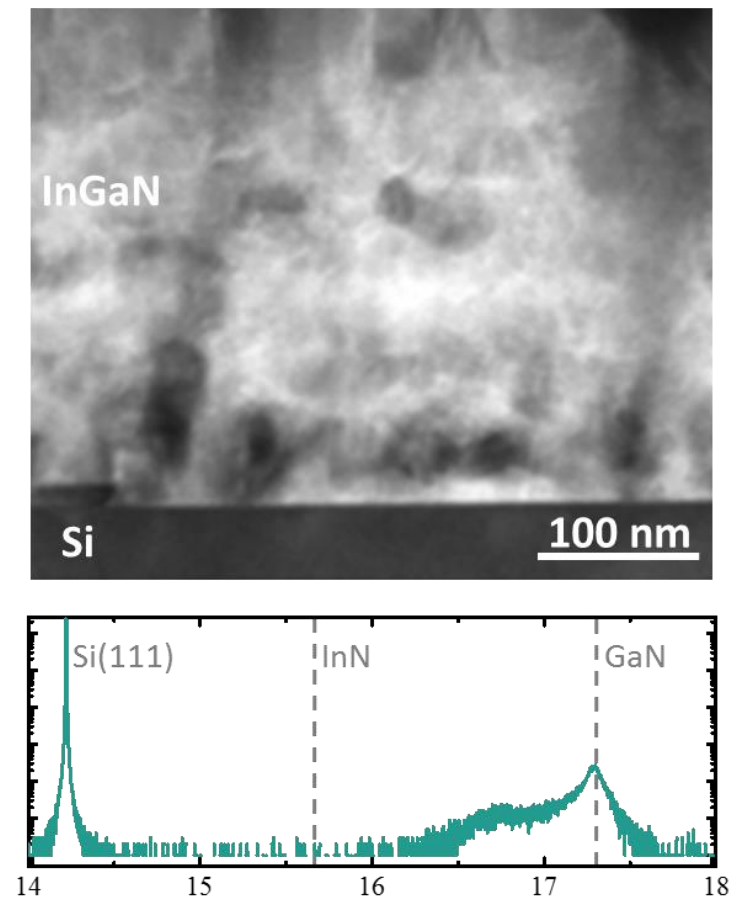

(b) Homogeneous alloy
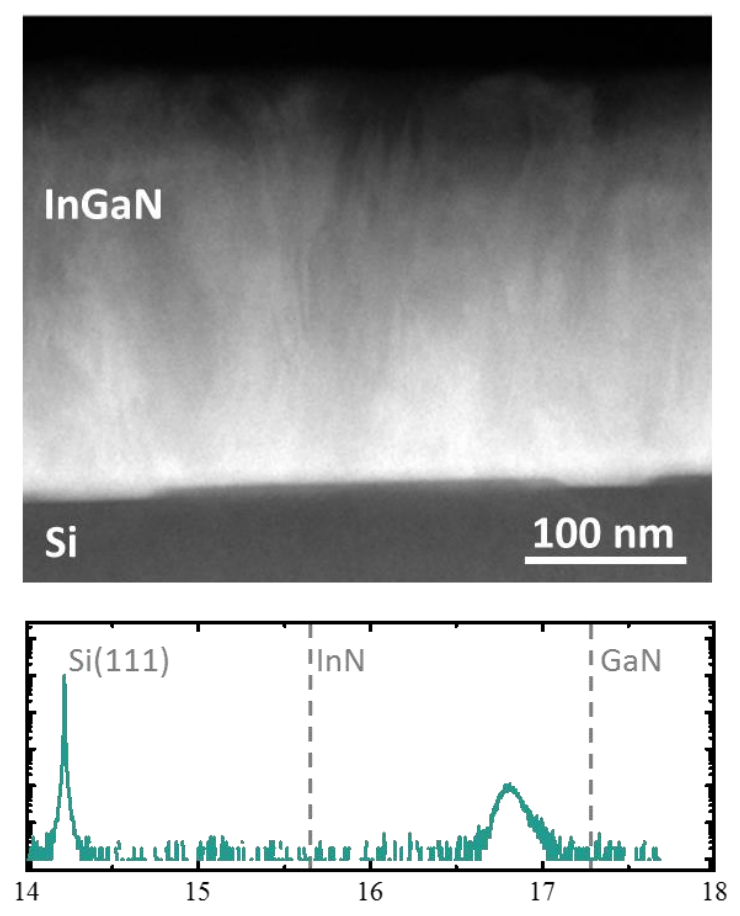

Fig. 2.5.8. Phase separated (a) and homogeneous (b) InGaN layers on $\mathrm{Si}(111)$ as evidenced by TEM HAADF (top) and $\omega-2 \theta$ HRXRD (bottom). Note that in the former case In composition ranging from pure GaN to $40 \%$, while in the latter one it is a single peak around $25 \%$ of In composition. Adapted from [Soto2013]. 


\subsection{Semiconductor - Electrolyte junction}

To understand the working principles of semiconductor-based photoelectrochemical (PEC) water splitting the semiconductor/electrolyte (S/E) interface must be first considered. This interface is one among the three most common interfaces in inorganic physics, the other two being the semiconductors homo/heterojunctions ( $\mathrm{S} / \mathrm{S}$ ) and the semiconductor/metal (S/M). There are certain similarities and differences between these interfaces which are addressed in detail in the following sections.

\subsubsection{Electrolyte side}

When a semiconductor is immersed in an aqueous solution, $\mathrm{H}^{+}$and $\mathrm{OH}^{-}$ions in it continuously adsorb/desorb to/from its surface establishing a dynamic equilibrium, which determines the charge distribution at the $\mathrm{S} / \mathrm{E}$ interface and thus the potential distribution. The structure of the S/E interface in case of n-type semiconductors is illustrated in Fig. 2.6.1 (a). The charges at the surface are electrons trapped at surface states plus the adsorbed ions. The counter charges are provided by ionized donors (acceptors in case of a p-type semiconductor) in the solid, plus an accumulation of opposite charge ions in the solution. Due to the large dipole moment of water $\left(\mathrm{H}_{2} \mathrm{O}\right.$ is a polar molecule) all the ions in the solution are surrounded by a solvation cloud of water molecules. This cloud prevents them from approaching the surface closer than a few $\AA$. The region between the adsorbed ions and the closest distance to the surface of ions still in the solution is called the Helmholtz layer [Helmholtz1853].

The potential drop across the Helmholtz layer (Fig. 2.6.1 (b)) is given by:

$$
V_{H}=\frac{Q_{s} d}{\varepsilon_{0} \varepsilon_{r}}
$$

where $Q_{s}$ stands for the surface charge, $d$ is the width of the Helmholtz layer, $\varepsilon_{0}$ the permittivity of vacuum, and $\varepsilon_{r}$ a relative permittivity of water (dielectric constant).

The typical width of the Helmholtz layer is 2 to $5 \AA$ and on such a small length scale the dielectric constant of water is estimated to be 6 , i.e., much smaller than its bulk value of 80 [Bockris2001]. For example, considering a typical surface charge of $Q_{s}=10^{13} \mathrm{~cm}^{-2}$, the potential drop across the Helmholtz layer is in the order of 0.1 to $0.5 \mathrm{~V}$, resulting in a Helmholtz capacitance $\left(Q_{S} / \mathrm{V}_{H}\right)$ of 10 to 20 $\mu \mathrm{F} \cdot \mathrm{cm}^{-2}$ [Bockris2001]. It must be noted that the Helmholtz potential changes with $-59 \mathrm{mV}(2.3 \mathrm{kT} / e)$ per pH unit at $25^{\circ} \mathrm{C}$, the so called Nernstian slope (for more details, refer to [Gratzel2012]).

Due to the large voltage drop across a very small distance in the electrolyte, the $S / E$ interface might be seen as very similar to a $\mathrm{S} / \mathrm{M}$ one. Indeed, the number of available states per unit energy in the electrolyte solution (e.g. $\sim 10^{23} \mathrm{~cm}^{-3}$ for $1 \mathrm{M}$ ion concentration) exceeds by far the states in a semiconductor (almost no states within the bandgap). References in the literature have shown that a S/E interface can be successfully described by means of a Schottky contact model, introduced by Gärtner [Gartner1959] (the best example of the Schottky contact model to describe the real electrode behavior in GaN/electrolyte interfaces can be found in [Gaudy2016]).

This is of course a very simplified model of the actual liquid contact layer, though it is good enough to understand the basic principles of a S/E interface, necessary in this work. 


\section{Helmholtz layer model}

(a)

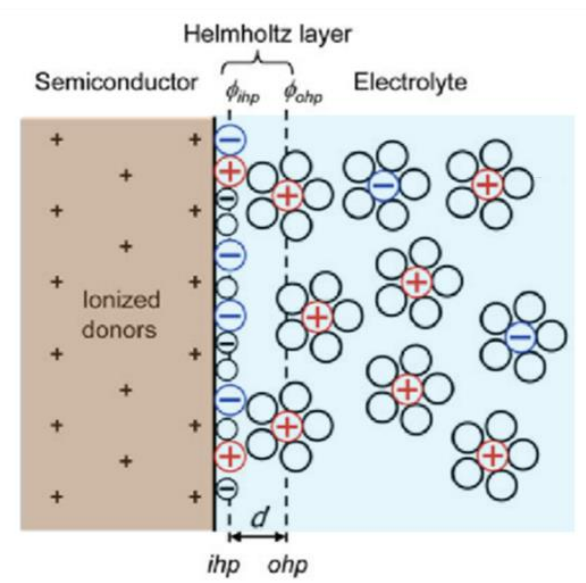

(b)

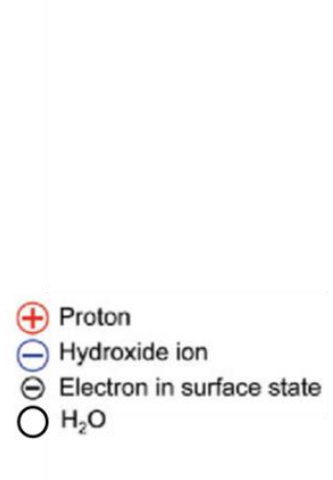

Fig. 2.6.1. Schematic model of the $S / E$ interface: Helmholtz layer (a) and voltage drop across it (b). The inner Helmholtz plane (ihp) consists of $\mathrm{H}^{+}$and $\mathrm{OH}^{-}$ions that are adsorbed at the semiconductor surface. The outer Helmholtz plane (ohp) determines the closest distance to the surface of ions still in the solution. (Adapted from [Gratzel2012]).

In order to build the energy diagram of the S/E interface, the energy distribution of the ions in the electrolyte must be considered. According to thermodynamics, when two phases are brought in contact their potential energies must be equilibrated. In our case it is an equilibrium of the electrochemical potentials, which is described here for the simplest case of the contact between a n-type semiconductor and a solution containing an electron acceptor $(A)$ and an electron donor $\left(A^{-}\right.$ ) called a redox couple $A / A^{-}$(they actually are the ions above mentioned) that are governed by the following reversible chemical reaction:

$$
A+e^{-} \leftrightarrow A^{-}
$$

In an electrolyte solution containing a redox couple $A / A^{-}$, the electrochemical potential $\left(E_{\text {redox }}\left(A / A^{-}\right)\right.$measured in $\left.\mathrm{eV}\right)$ is defined by solution redox potential $\left(\mathcal{E}_{\text {redox }}\left(A / A^{-}\right)\right.$, measured in $\mathrm{V})$ which is the tendency of any chemical substance to lose or gain electrons, by simply multiplying it by the electron charge $(q)$ :

$$
E_{\text {redox }}\left(A / A^{-}\right)=q \cdot \varepsilon_{\text {redox }}\left(A / A^{-}\right),
$$

In analogy with the Fermi level in a semiconductor, the redox potential (level) of the solution is simply described as the energy at which the probability for a given species to give an electron away (oxidation) equals the probability for another species to accept an electron (reduction).

From now on, it will be equivalent to talk about electrochemical or redox potential. Finally, the electrochemical potential (which represents how easy or difficult it is to add more of a given species to a specific location) in this case is defined by the Nernst equation as follows:

$$
E_{\text {redox }}\left(A / A^{-}\right)=E_{\text {redox }}^{0}\left(A / A^{-}\right)+\frac{k T}{n_{e}} \ln \frac{[A]}{\left[A^{-}\right]},
$$

where $E_{\text {redox }}^{0}\left(A / A^{-}\right)$is the stardard redox potential (taken at 1 atm with concentrations of the redox species at $1 \mathrm{M} / \mathrm{L})$ of the redox couple $\left(A / A^{-}\right), n_{e}$ is the number of electrons per molecule of 
redox couple that are exchanged during the reaction, and $[A]$ and $\left[A^{-}\right]$are the respective concentrations of the acceptor and donor species.

The next step is to define the density of states of the redox species as the number of ions with a certain energy level per interval of energy. As it was already mentioned all ions in the solution are surrounded by a solvation cloud of water molecules, thanks to its polar nature (Fig. 2.6.1 (a)). In addition to these nearest-neighbor solvent molecules in the so called inner sphere (involving covalently bonded solvent molecules), the ion also has Coulomb interactions with polar solvent molecules and other ions that are further away in the outer sphere (involving molecules adsorbed solely by Coulomb electrostatic force). The energy level of the central ion fluctuates because of the thermal motion of the solution. Moreover, after the reduction or oxidation of the ion, the surrounding solvent molecules and ions will rearrange themselves to accommodate the different charge at the ion. The electrostatic energy required for this reorganization is given by $2 \lambda$, where $\lambda$ is called the reorganization energy. It can be shown that the probability for a reduced or oxidized species to have a certain energy $E$ is given by [Gerischer1969]:

$$
\begin{gathered}
W_{o x}(E)=\frac{1}{\sqrt{4 \pi \lambda k T}} \operatorname{Exp}\left(\frac{-\left(E-E_{\text {redox }}+\lambda\right)^{2}}{4 k T \lambda}\right), \\
W_{\text {red }}(E)=\frac{1}{\sqrt{4 \pi \lambda k T}} \operatorname{Exp}\left(\frac{-\left(E-E_{\text {redox }}-\lambda\right)^{2}}{4 k T \lambda}\right),
\end{gathered}
$$

Here, $E_{\text {redox }}$ is the redox potential for the species involved given by Eq. 2.6.1.4. The density of states $D_{o x}\left(D_{r e d}\right)$ depends on the concentration of the concentration of the ox $\left(\right.$ red) species $c_{o x}\left(c_{r e d}\right)$ according to:

$$
\begin{aligned}
& D_{o x}=c_{o x} W_{o x}, \\
& D_{\text {red }}=c_{\text {red }} W_{\text {red }},
\end{aligned}
$$

Fig. 2.6.2 illustrates the probability distributions of these energy levels fluctuation. The maximum values of $D_{o x}$ and $D_{\text {red }}$ occur at $E_{o x}=E_{\text {redox }}+\lambda$ and $E_{\text {red }}=E_{\text {redox }}-\lambda$. It should be emphasized that because it is a probability distribution (it should not be confused with the density of states in a semiconductor, which is a fundamentally different concept) only one level exists at a time for every ion, so optical transitions between these redox levels are not possible. The reorganization energy values typically lies in the range from $0.3 \mathrm{eV}$ to more than $1 \mathrm{eV}$. At room temperature the width of each distribution curve (at half height) is given by $\Delta E \cong 0.53 \sqrt{\lambda}(\mathrm{eV})$.

For semiconductors, charge transfer takes place via either the top of the valence band (VB) or the bottom of the conduction band (CB) for n-type or $\mathrm{p}$-type, respectively. Though surface statesmediated charge transfer can also occur. An important conclusion derived from Fig. 2.6.2 is that the probability of electron transfer actually decreases if $E_{\text {redox }}$ is too far above $E_{V B}$. This is markedly different from the behavior of metal electrodes which show a continuous increase of current with applied potential. 


\section{Redox species energy distribution}
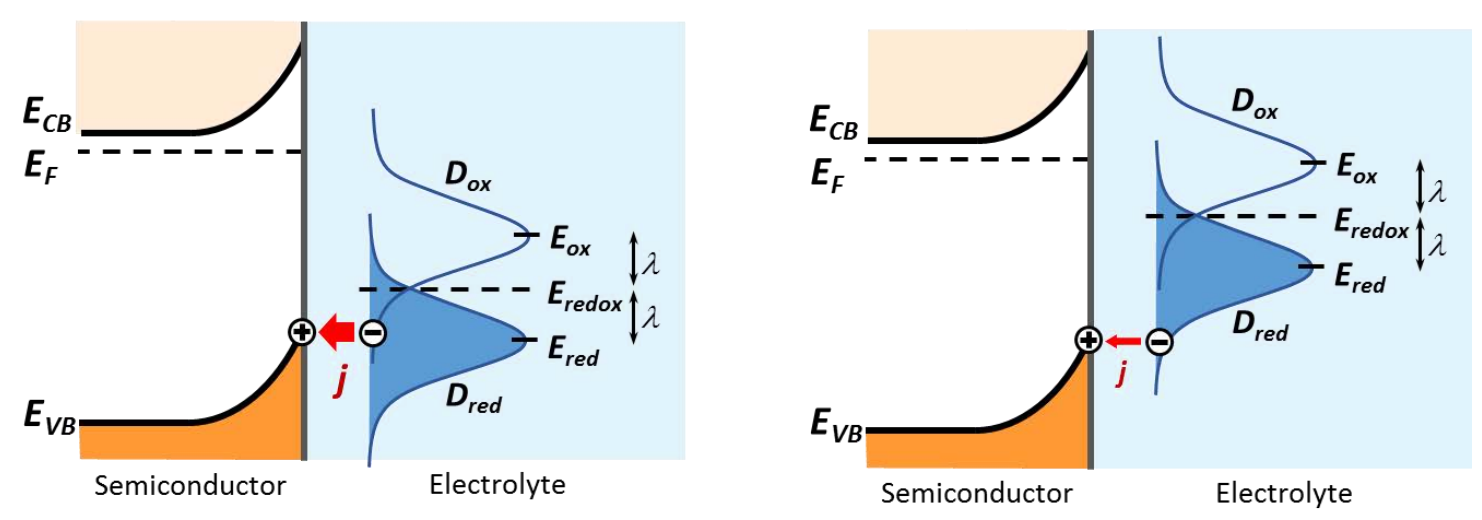

Fig. 2.6.2. Energy level model for electron transfer from a redox species in the electrolyte to the semiconductor valence band (only when photo-generated holes are present). A large overlap of the redox energy distribution with the energy of the hole (left) gives higher currents than a small overlap (right). (Adapted from [Gratzel2012]).

Everything above described is related to a simple scenario involving a single redox pair electrolyte. However in this work aqueous electrolytes are used (mixture of acid or base with water), which contains two redox couples: $\mathrm{H}^{+} / \mathrm{H}_{2}$ and $\mathrm{O}_{2} / \mathrm{H}_{2} \mathrm{O}$. In this case the description is similar, but the redox level of the solution (also called the Fermi level of it) depends on the relative $\mathrm{H}_{2}$ to $\mathrm{O}_{2}$ concentrations and lies between the $\mathrm{H}^{+} / \mathrm{H}_{2}$ and $\mathrm{O}_{2} / \mathrm{H}_{2} \mathrm{O}$ redox potentials. In order to fix it, $\mathrm{H}_{2}$ or $\mathrm{O}_{2}$ gas can be bubbled through the solution, which shifts the redox level of water to $\mathrm{H}^{+} / \mathrm{H}_{2}$ or $\mathrm{O}_{2} / \mathrm{H}_{2} \mathrm{O}$ level, respectively.

\subsubsection{Semiconductor side}

The electrochemical potential in semiconductors is called the Fermi level $\left(E_{F}\right)$, formally defined in statistical mechanics as the energy level at which probability of finding an electron is one-half. In the case of non-degenerate doped n-type semiconductors the Fermi level position can be derived from the Fermi-Dirac distribution resulting in:

$$
E_{F}=E_{i}-k T \ln \frac{n}{n_{i}},
$$

where $n_{i}$ is the intrinsic carrier density, $n$ is the electron density, $E_{i}$ is the Fermi level position for intrinsic semiconductors $\left(n=n_{i}\right)$, that corresponds to the midgap if electron and hole masses are equal, $T$ is the temperature, and $k$ the Boltzmann constant.

Traditionally, the Fermi level in semiconductors is measured versus the vacuum level (thus, as negative values) while the redox potential is measured versus the Reversible Hydrogen Electrode (RHE) which is an ideal electrode that defines zero potential, and does not depend, neither on $\mathrm{pH}$, nor on temperature (positive values). Thus, in order to compare them one must recalculate their values by using the following expression (in standard conditions at $25^{\circ} \mathrm{C}$ and $\mathrm{pH}=0$ ):

$$
E_{v s \text { vaccum }}=-E_{v S R H E}-4.44 \mathrm{eV},
$$


where $E_{v s \text { vaccum }}$ and $E_{v S R H E}$ are the same potential measured from the vacuum level and a RHE, respectively. This relation between the two scales is illustrated in Fig. 2.6.3.

\section{Potential scales}

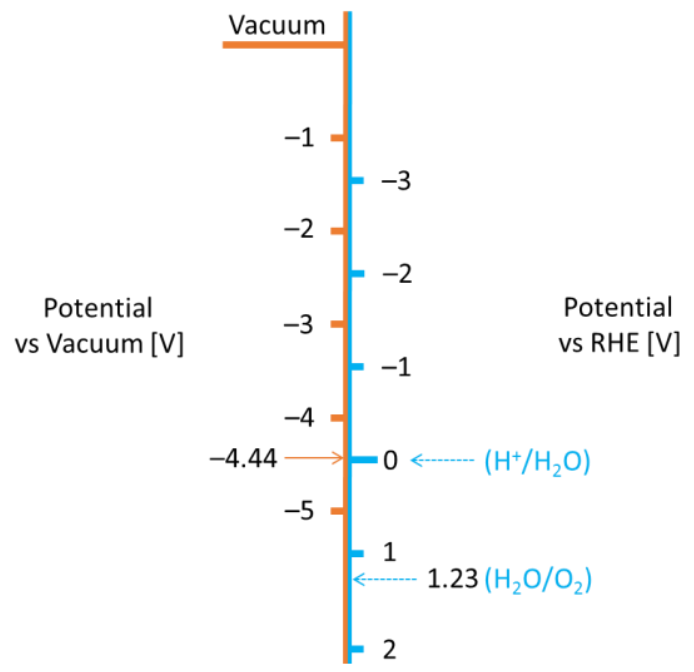

Fig. 2.6.3. Comparison of potential scales versus vacuum (left) and reversible hydrogen electrode (right). Note that these two scales have reversed polarities.

\subsubsection{Charge transfer at S/E interface}

Once both electrochemical potentials of the semiconductor and electrolyte are properly defined, it is possible to analyze what happens when they are interfaced (S/E). Whenever two materials of different electrochemical potentials are brought into contact, electrons, in order to minimize their energy, travel from the material with more negative electrochemical potential to the one with more positive electrochemical potential (vs. RHE) (see Fig. 2.6.4 (a)). As a result, positive (negative) charge will accumulate at the surface of the material with more negative (positive) electrochemical potential, thus changing the band bending at the interface. Such charge transfer will continue until the electric field, building up at the S/E interface by these charges, cancels out the initial difference in the electrochemical potentials between the semiconductor and electrolyte and thermodynamic equilibrium is reached (see Fig. 2.6.4 (b)). This equilibrium electric field is called built-in potential $\left(V_{b i}\right)$ and determines the band bending. The total barrier height for electrons to jump from the semiconductor Fermi level (by thermionic emission) to the electrolyte is then $V_{b}$ (equivalent to the Schottky barrier height, $\left.\phi_{B}\right)$.

If an external reverse bias is applied to this junction it will be equilibrated by changing the fixed positive charge (ionized donors in n-type) within the semiconductor close to the surface (space charge region, SCR) and establishing a new dynamic equilibrium (see Fig. 2.6.4 (c)). If an external forward bias is applied the SCR width (or depletion width) $W$ reduces, as well as the fixed positive charge eventually leading to the so called flatband condition (flat CB and VB profiles in Fig. 2.6.4 (a)) when the external bias equals $V_{b i}$. Beyond that point, electrons move with no barrier from the semiconductor to the electrolyte, which results in a high current across the junction. 
These charge transfer processes across the S/E interface are accomplished through forward and reverse reactions described by $E q$. 2.6.1.2. A forward reaction represents the reduction of the acceptors $(A)$ in the electrolyte by electron transfer from the semiconductor, while a reverse reaction represents the oxidation of the donors $\left(A^{-}\right)$in the electrolyte by electron injection into the semiconductor (or hole transfer from the semiconductor).
(a) Before equilibrium
(b) In equilibrium
(c) Applying external
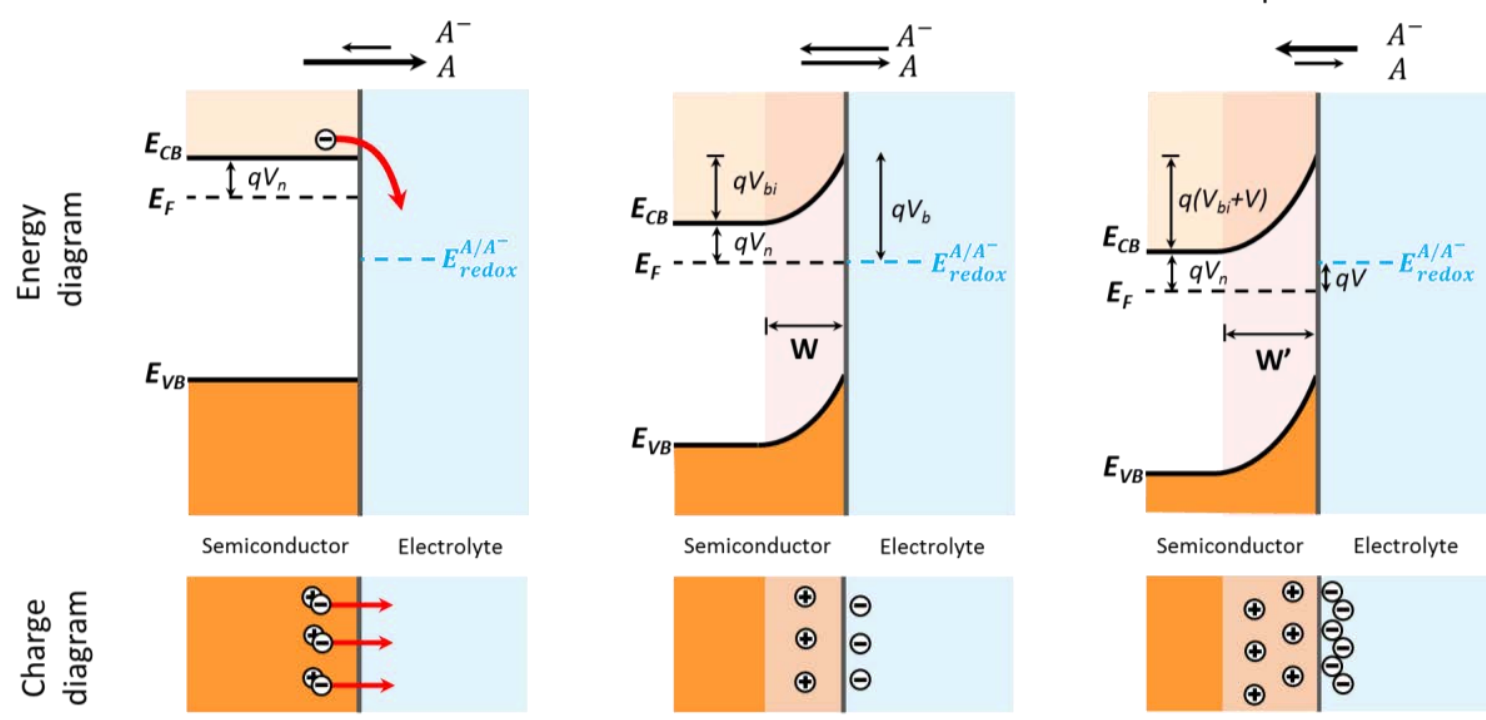

Fig. 2.6.4. Energy diagram of the S/E interface and the corresponding charge transfer processes under different external bias conditions.

\subsubsection{Depletion approximation}

Following the equivalence with a Schottky barrier, the built-in potential $\left(V_{b i}\right)$ induced by the initial charge transfer to reach equilibrium (Fig. 2.6.4 (a)) can be expressed as the difference between the initial electrochemical potentials of the semiconductor $\left(E_{F}\right)$ and the electrolyte $\left(E_{\text {redox }}\left(A / A^{-}\right)\right)$:

$$
V_{b i}=\left|E_{F}-E_{\text {redox }}\left(A / A^{-}\right)\right| / q,
$$

being the main driving force for charge separation in PEC cells based on S/E interfaces.

The energy diagram of the S/E interface can be built assuming that all the potential drop occurs at the semiconductor side due to the electrolyte much higher density of states, along with the depletion approximation [Neudeck1989]: that is, the built-in electric field is confined to the junction region within the SCR (also called depletion region) and there is no electric field outside it (flat band beyond $W$ ). If a constant donor density $N_{d}$ is considered in an n-type semiconductor, the fixed (positive) charge density $(\rho)$ within the depletion region width $W$ will also be constant:

$$
\rho=q N_{d},
$$

here $N_{d}$ is considered in a first approximation as the majority carrier density at room temperature, i.e. all donors are ionized and no other fixed charge is present in the semiconductor. 
The band bending profile (built-in potential profile) across the depletion region in the semiconductor can be expressed as follows:

$$
V_{b i}^{p r o f i l e}(x)=\int \frac{q N_{d}}{\varepsilon_{s}} x \cdot d x=\frac{q N_{d}}{2 \varepsilon_{s}} x^{2}
$$

where $\varepsilon_{s}$ is the semiconductor dielectric constant. The potential changes as a quadratic function of the distance from the neutral region in the semiconductor bulk (no ionized donors) to the S/E interface where it reaches its maximum value (semiconductor surface):

$$
V_{b i}=\frac{q N_{d}}{2 \varepsilon_{s}} W^{2}
$$

Combining Eq. 2.6.2.3 and 2.6.2.6 results in the expression for the depletion region width (or SCR) in equilibrium:

$$
W=\sqrt{\frac{2 \varepsilon_{s} V_{b i}}{q N_{d}}}=\frac{1}{q} \sqrt{\frac{2 \varepsilon_{s}\left|E_{F}-E_{\text {redox }}\left(A / A^{-}\right)\right|}{N_{d}}},
$$

If an external potential $(V)$ is applied to the system (e.g. from the potentiostat) the equilibrium is altered and the new value of the depletion width is:

$$
W^{\prime}=\sqrt{\frac{2 \varepsilon_{s}\left(V_{b i}+V\right)}{q N_{d}}},
$$

However, as it is going to be shown later in the text (refer to section 2.6.3.3.3), the presence of surface states pinning the Fermi level may lead to a non-linear relation between the applied potential and the potential drop across the space charge region, so instead of $V_{b i}$ which is derived from a linear model, the term $\Delta \phi_{s c}$ must be used (It is going to be explained later in this chapter). Then it is better to write:

$$
W=\sqrt{\frac{2 \varepsilon_{S} \Delta \phi_{S C}}{q N_{d}}},
$$

Knowing this parameter, together with the redox level of the electrolyte, allows determining the band diagram of the S/E junction as shown in Fig. 2.6.4.

\subsubsection{Drift and diffusion currents}

As it was discussed in section 1.3, the photon absorption and photogenerated charge separation are the main functions of the semiconductor in the PEC cell. If the incoming light penetrates deep into the semiconductor, so that photon absorption occurs far from the SCR, the photo-generated charges may eventually recombine, either directly or defect-mediated. If charges are photogenerated inside the SCR they can be effectively separated by the internal electric field, thus adding to the total current if not recombined (this contribution is called drift current). Another possibility for charges to be separated occurs if they are generated close enough (but not inside) to the SCR because electrons and holes can diffuse there. The generated excess carriers may in principle diffuse in both directions, but while holes can move towards the SCR, electrons cannot due to the electric field, which makes preferential movement of charge, thus current (this contribution is called diffusion current). While for majority carriers the probability of recombination is very low (there are 
almost no minority carriers around), for minority carriers it is very high. Thus, the minority carrier (holes) diffusion length $L_{h}$ determines whether photo-generated carriers will contribute by diffusion to the total current or not.

$$
L_{h}=\sqrt{D_{h} \tau_{h}},
$$

where $D_{h}$ is the hole diffusion coefficient and $\tau_{h}$ the hole lifetime. $D_{h}$ relates to the hole flux through its concentration gradient, while $\tau_{h}$ tells how long holes can move before recombining with electrons or getting trapped by defects (in that sense, is a measure of how good the semiconductor crystal quality is).

The carrier diffusion length is very important for indirect bandgap semiconductors with low absorption coefficient where light penetrates hundreds of microns away from the SCR and thus, minority carriers must travel a considerable distance without recombining in order to contribute to the output current. To make this possible, the semiconductor has to be of very high crystal quality (long minority carrier lifetime). In this case, almost all the current is determined by the diffusion component. However, drift current is more important in direct bandgap semiconductors with high absorption coefficient where most carriers are generated within the SCR. In this case if the material quality is not that good (a short bulk lifetime), i.e. with a high defect density (dislocation densities at or above $10^{9} \mathrm{~cm}^{-2}$ are typical for $\mathrm{GaN}$ ), then there is only a very weak (negligible) diffusion component. But due to an efficient charge separation within the SCR, most generated holes are able to reach the S/E interface, even if their bulk lifetime is short. The key point is that lifetime of holes is determined by the electron (majority carrier) concentration in SCR. The majority carrier concentration at the surface of an n-type semiconductor (for a photo-anode) is given by:

$$
n_{\text {surf }}=n_{\text {bulk }} \cdot \exp ^{-\frac{\Delta \phi_{S C}}{k T}},
$$

It follows that increasing $\Delta \phi_{s c}$ by, for instance, $600 \mathrm{mV}$, lowers $n_{\text {surf }}$ by ten orders of magnitude, and this gives minority carriers that move towards the surface the chance to live long enough to take part in the rather slow reactions involved in water splitting. Terefore, the total current is going to be determined by the drift component. However, the SCR is usually quite small (depending on doping and surface potential) thus, only direct band semiconductors with high absorption coefficients (absorbing $95 \%$ of light in few hundreds of $\mathrm{nm}$ ) can benefit from such scheme.

\subsubsection{Current-voltage characteristics of the S/E junction}

The current-voltage characteristics of a junction (S/E interface) with the corresponding band diagram are crucial to understand how the absorbed photons are converted into chemical fuels in a semiconductor-based PEC cell. The ideal case of a photoanode in contact with the electrolyte is first discussed and then some specific implications, such as a slow carrier transport rate and the presence of surface states are introduced and analyzed.

\subsubsection{S/E interface in the dark (Ideal case)}

In the dark an ideal S/E junction (no surface states, no kinetic limitations) exhibits the typical diode behavior characterized by an exponential cathodic current rise at cathodic bias (forward biased diode) associated with the transfer of majority carriers from the semiconductor to the electrolyte. It must be noted that in electrochemical notation the diode is forward biased when negative bias is applied to an n-type semiconductor. 
In a Schottky diode, which is a good approximation of a S/E interface [Gaudy2016], the dark current can be driven by three main mechanisms [Sze2007]. The first one is a thermionic emission of carriers above the Schottky barrier (including field assisted thermionic). Analytically the thermionic emission in an ideal case is described by the Shockley diode equation [Gartner1959]:

$$
J=-J_{0}\left(\exp ^{-\frac{q(V)}{k T}}-1\right)
$$

where $J_{0}$ is the saturation current in the dark, $k$ the Boltzmann constant and $T$ the temperature.

A second component is due to diffusion/drift of carriers across the interface. The diffusion/drift current is associated with minority carriers moving or swept towards the junction interface due to, either diffusion mechanism, or the SCR electric field, respectively. The diffusion/drift components are linearly proportional to the minority carriers density, which is negligible in the dark (with exception of high reverse bias described later) due to extremely low intrinsic minority carrier concentrations (typically 4 to 6 orders of magnitude lower than majority carriers).

The third component is due to quantum-mechanical tunneling through the barrier (direct or defect assisted). Quantum-mechanical tunneling through the barrier takes into account the wave-nature of the electrons, allowing them to penetrate through thin barriers. The tunneling current is obtained from the product of the carrier charge, velocity and density, thus it is the dominating current component in highly doped semiconductors (and responsible for ohmic behavior of a contact).

For a more detailed discussion of the dark current mechanisms reader is referred to [Sze2007].

A combination of all three mechanisms could exist in one junction, depending on bias, temperature and semiconductor doping level, though usually only one of them dominates the current. The thermionic emission theory postulates that only carriers with energies comparable or above $\mathrm{V}_{\text {bi }}$ will contribute to the forward current flow (majority carrier). This mechanism is dominant in moderately doped semiconductors. Thus, in this work it is reasonable to assume that thermionic emission is the main contribution to the S/E junction current-voltage characteristic.

Fig. 2.6.5 represents a typical current-voltage characteristic of the photo-anode under dark conditions measured in a 3-electrode configuration. The potential of the working electrode (photoanode) is measured against the reference electrode and then recalculated vs. RHE by using a known and fixed potential of the reference electrode. Current is measured between the working and counter electrodes. A scheme of the measurement setup is given in experimental section 3.6. The advantage of such 3-electrode over a 2-electrode configuration is that it eliminates any polarization effects on the counter electrode, effectively making it an ideal counter electrode (with zero resistivity) [Hodes2012]. This allows evaluating the performance of photoanode independently from the counter electrode.

For simplicity let us assume that the potential of the electrolyte is equal to the $\mathrm{H}^{+} / \mathrm{H}_{2} \mathrm{O}$ redox potential, which is a reasonable assumption for the experiments where the aqueous electrolyte is bubbled with hydrogen gas.

The current-voltage characteristic shown in Fig. 2.6.5 can now be well described. Notice that the sign of current and voltage is reversed in respect to a Schottky diode, to accommodate to the electrochemical notation. The forward (dark) current starts to flow slowly (exponentially) from zero bias to the threshold voltage $V_{h}$ getting larger beyond this point. Series resistance effects may exist at these high current values in real junctions. Flat band condition might happen for a given forward (cathodic) bias at high current values. This forward current is the majority carrier one injected into the electrolyte though the S/E interface, where the reaction with the ions in the solution takes place initiating the hydrogen evolution reaction (HER). 
At anodic bias (reverse polarization) the SCR increases to account for the increase in built-in potential (or $\Delta \phi_{s c}$ ), as was shown in Fig. 2.6.4. In this region the reverse current is small, most likely due to electron tunneling processes from the electrolyte (redox level) to both $C B$ and VB (Fig. 2.6.5 (d)). At even higher anodic bias the reverse current increases because more energetic electrons from the electrolyte can also jump over the barrier (thermionic mechanism) to the semiconductor CB (Fig. 2.6.5 (e)). The ratio between tunneling and thermionic components depends very much on the barrier height, electric field and temperature.

In moderately doped semiconductors, breakdown usually occurs at high reverse bias and its primary cause is avalanche multiplication, i.e. carriers inside the SCR under strong electric field are rapidly accelerated and begin to collide and ionize other atoms (impact ionization) to produce electronhole pairs (causing further ionization, so called "avalanche" effect), so promptly multiplying the amount of free charge carriers and thus increasing substantially the reverse current. In highly doped semiconductors, breakdown occurs at low reverse bias and is mainly caused by thermionic-field emission (also called Zener effect in pn-junctions), because at high doping the SCR is very narrow with strong electric field even at low reverse voltages which allows tunneling of electrons into the semiconductor. A more detailed discussion can be found elsewhere [Sze2007, Pierret1996, Gomes1981].

\section{S/E interface in the dark}

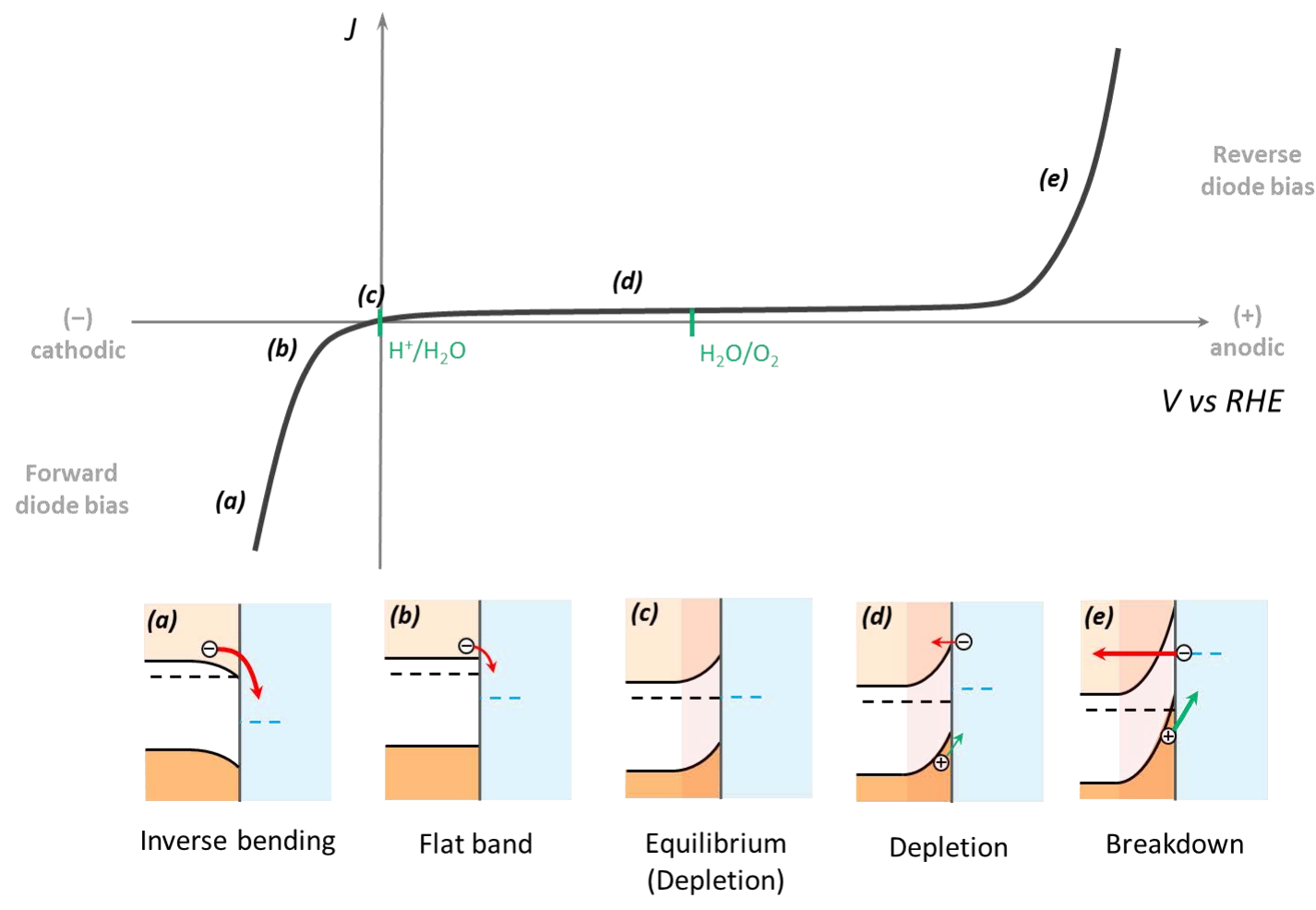

Fig. 2.6.5. Dark current-voltage characteristics of a semiconductor photo-anode immersed in a redox electrolyte solution, together with their corresponding band diagrams. Red and green arrows represent majority and minority carrier current components. Note that forward diode bias corresponds to negative potentials according to electrochemical notation. 


\subsubsection{S/E interface under illumination (Ideal case)}

Illumination of a semiconductor with photons of energy near, or above, its bandgap generates electron-hole pairs, which can contribute to the current through the S/E interface. The contribution by electrons to the overall current is very small, even in moderately doped photo-anodes (n-type) where there is already a high density of free electrons, thus the addition of a few more will not have any significant effect. Because of that, the majority carrier contribution (mainly by thermionic emission) to the overall current will be basically the same, either under illumination or in the dark. However the situation is quite different for minority carriers, having a very low density in the dark (n-type semiconductor) that can become significant under illumination and thus largely contribute to the overall current.

As it was mentioned in the previous section, the minority carrier current is driven by diffusion and drift mechanisms, being proportional to the minority carrier density. To estimate this current, the amount of photo-generated charge must be determined. In this work, direct bandgap semiconductors with relatively short minority carrier lifetimes are used. Thus, in a first approximation, diffusion contributions can be neglected because of strong absorption and $L_{h} \ll W$. Then, in a simple case of a semiconductor thin film with reflectance $R$ under monochromatic incident light of frequency $v$ and power density $P_{i n}$, the amount of photo-generated carriers per second can be expressed in terms of current $\left(j_{\text {photo }}\right)$ as follows:

$$
j_{\text {photo }}=-\frac{q(1-R) P_{\text {in }}}{h v}\left(1-\exp ^{-\alpha W}\right),
$$

where $\alpha$ is a semiconductor optical absorption coefficient. In case of a more complex light spectrum, it must be integrated over the frequency range used.

In an ideal case where no recombination occurs within the SCR and the transfer across the S/E interface is fast, all of these photo-generated carriers are contributing to the measured total current $\left(j_{\text {total }}\right)$, which can be expressed as:

$$
j_{\text {total }}=j_{\text {dark }}+j_{\text {photo }},
$$

Notice that $j_{\text {photo }}$ is indirectly (and weakly) dependent on the applied potential through $W \sim \sqrt{V}$ (Eq. 2.6.2.9).

For anodic bias (Fig. 2.6.6 (a)) the forward majority current is dominant both in the dark and under illumination, since a quite reduced SCR value means negligible photo-generated carriers. However, at reverse bias the current due to photo-generated carriers will dominate (before breakdown occurs) being almost constant (for semiconductors with moderate doping and high absorption coefficient) following Eq. 2.6.3.2 (See Fig. 2.6.6 (d2) (ideal)). Photo-generated holes reach the interface and initiate, either the oxygen evolution reaction (OER), or the semiconductor oxidation (corrosion), depending on which reaction is energetically favorable and kinetically allowed.

This simple photocurrent description based on a Schottky barrier model was introduced by Gärtner [Gartner1959]. His calculation of the photocurrent was carried out for a S/M interface in contact with an inert gas, neglecting the influence of the semiconductor surface and the properties of the electrical double layer at the semiconductor-solution interface. It was widely adopted by the electrochemical research society, and the Schottky barrier model was further developed by Laser \& Bard [Laser\&Bard1976], Gerischer [Gerischer1977], and Butler \& Ginley [Butler\&Ginley1980]. 
However, this simple model cannot account for certain effects observed in actual phototelectrodes. The surface of a semiconductor in contact with an electrolyte solution is non-ideal and contains a high density of surface states (with only very few exceptions, such as specially prepared Si and Ge surfaces) which play a very important role in determining the current-voltage characteristic of S/E interfaces. The effects related to non-ideal junctions is described in the following section.

\section{S/E interface under the illumination}

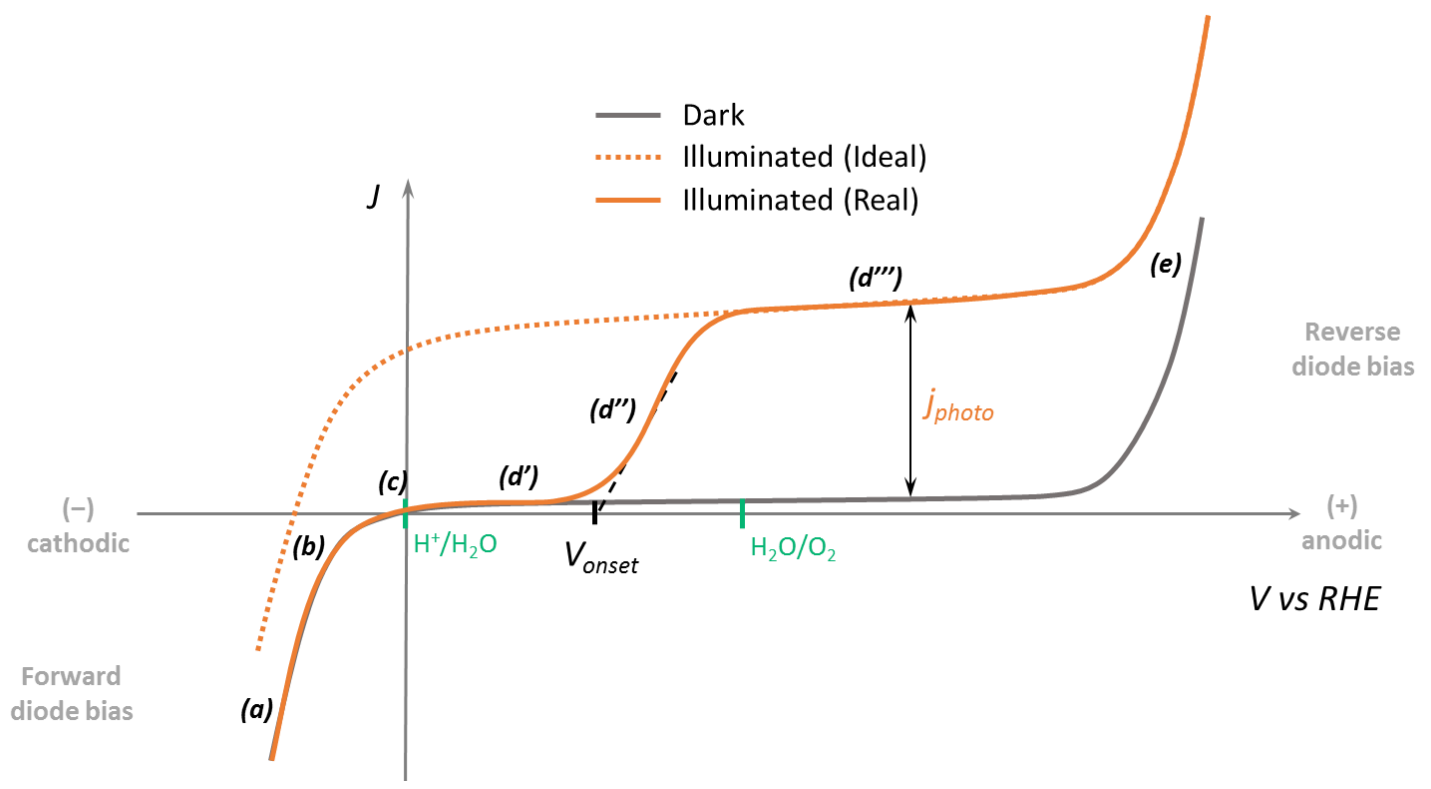

Fig. 2.6.6. Current-voltage characteristics under illumination of a semiconductor photo-anode immersed in a redox electrolyte solution. Both the ideal (dashed line) and actually measurable (solid line) behaviors are presented.

\subsubsection{Competition between charge transfer and recombination}

The current voltage curve of a real S/E junction is more complex than in the previously described ideal case. The actual shape of these characteristics as measured looks as the solid orange curve shown in Fig. 2.6.6, clearly differing from the ideal one in reverse polarization (anodic bias). The reason for this discrepancy is that, while carries are photo-generated their transport rate across the space charge region can be different from the rate at which they react with ions at the interface. So far, only a thermodynamic description of the processes at the S/E interface was given, while kinetic transport and surface non-idealities also play a major role.

As it was discussed above for direct bandgap semiconductors and short bulk lifetime, it is only in the SCR where a substantial change in minority carrier concentration occurs under illumination. However, in this case the charge is photo-generated very close to the S/E interface (small SCR) which is usually non-ideal. Semiconductor lack of stoichiometry and the presence of dangling bonds at its surface, as well as chemical species formed by interaction with the electrolyte, generate electron energy levels that lie within the bandgap, the so called surface states. These surface states can promote a very efficient electron-hole recombination (surface recombination) [Reichman1980, Peter1984, Li1985, Li1986]. Even if there were no surface states before illumination they may be 
formed under illumination by reaction of minority carriers and the electrolyte [Li1984]. Therefore, the desired photo-anode reaction by hole transfer from the semiconductor to the electrolyte (more precisely electron transfer from the electrolyte) has to compete with surface recombination. The longer the time minority carriers remain at the surface to take part in water splitting, the higher the chance that they will be lost by recombination. This means that a slow electron transfer leads to a low water-splitting efficiency. This is the main difference between PEC devices and conventional $p$ $\mathrm{n}$ junction or Schottky barrier photovoltaic devices.

Additionally, surface states can pin the Fermi level inside the semiconductor leading to a non-linear dependence of the potential drop across the $\operatorname{SCR}\left(\Delta \phi_{S C}\right)$ on applied bias $(V)$. This also largely affects the current voltage characteristic of S/E junctions.

This section addresses the water-splitting efficiency drop caused by the rate-limiting effect of charge transfer kinetics enhancing recombination and the Fermi level pinning associated with the presence of surface states.

\subsection{Kinetic limitations related to surface recombination}

The effect of surface states enhancing surface recombination can be described by a simple analytical model proposed by Ponomarev \& Peter [Ponomarev\&Peter1995, Peter2013] considering that recombination within the SCR is negligible and the surface electron-hole recombination is formulated in terms of the surface majority carrier concentration in the dark. More complex and precise models have been developed by Wilson [Wilson1977], Reichmann [Reichman1980], and El Guibaly \& Colbow [Guibaly\&Colbow1982], though a simplified phenomenological analysis gives enough understanding of the effect in order to explain the observed results.

The basic processes considered in case of an illuminated photo-anode are illustrated in Fig. 2.6.7, which shows that the holes generated in the semiconductor (jphoto, defined by Eq. 2.6.3.2) upon reaching the surface may, either accept electrons from the electrolyte in an oxidation process $\left(j_{t r}\right.$, transfer current), or recombine with electrons through surface states ( $j_{\text {rec }}$, recombination current). The simplifying assumption that kinetics of interface charge transfer and of surface recombination can be described in terms of surface hole concentrations gives the following expressions for transfer $\left(j_{\text {tr }}\right)$ and recombination $\left(j_{\text {rec }}\right)$ currents:

$$
\begin{aligned}
& j_{t r}=q k_{\text {tr }} p_{\text {surf }}, \\
& j_{\text {rec }}=q k_{\text {rec }} p_{\text {surf }},
\end{aligned}
$$

where $k_{t r}$ and $k_{\text {rec }}$ are first-order rate constants $\left(\mathrm{s}^{-1}\right)$ for charge transfer and surface recombination, respectively.

To understand what happens at the S/E interface upon illumination, a kinetic equation of the timedependent hole concentration must be considered. It is determined by the difference between the rate of arrival of holes at the surface ( $j_{\text {photo }}$, defined by Eq. 2.6.3.2) and the rate of their removal by transfer $\left(j_{\text {tr }}\right)$ and recombination $\left(j_{\text {rec }}\right)$.

$$
q \frac{d p_{\text {surf }}}{d t}=j_{\text {photo }}-j_{\text {tr }}-j_{\text {rec }}=j_{\text {photo }}-\left(k_{\text {tr }}+k_{\text {rec }}\right) p_{\text {surf }},
$$




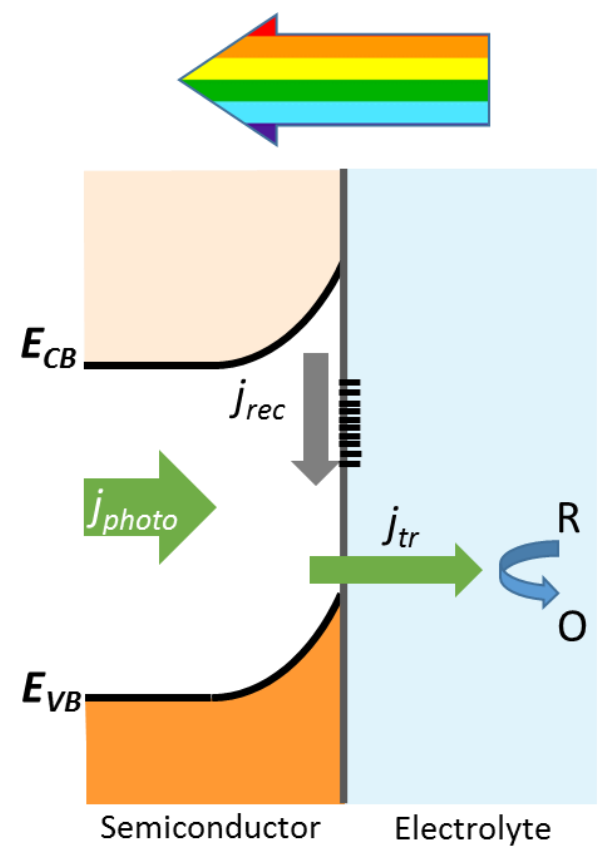

Fig. 2.6.7. Simple kinetic scheme describing the competition between charge transfer and surface recombination. (See text for definitions). Adapted from [Peter2013].

Upon the moment of illumination $(t=0)$, the change in hole surface density is basically determined by the photo-generated holes flux, $j_{\text {photo, }}$, because $\left.p_{\text {surf }}\right|_{t=0} \rightarrow 0$ and thus $\left.j_{t r}\right|_{t=0}=\left.j_{r e c}\right|_{t=0}=0$. Therefore, the measured current is associated with the holes reorganization within the semiconductor, in other words, the surface capacitance charging rather than an actual oxidation reaction.

In a steady-state regime, once the dynamic equilibrium is reached $\left(\left.\frac{d p_{\text {surf }}}{d t}\right|_{t=\infty}=0\right)$, the surface hole concentration is given by:

$$
\left.p_{\text {surf }}\right|_{t=\infty}=\frac{j_{\text {photo }}}{k_{\text {tr }}+k_{\text {rec }}},
$$

Therefore the output steady-state transfer current is given by:

$$
\left.j_{t r}\right|_{t=\infty}=j_{\text {photo }} \frac{k_{t r}}{k_{t r}+k_{r e c}}
$$

while the recombination current, which does not contribute to the output measured current, is given by:

$$
\left.j_{\text {rec }}\right|_{t=\infty}=j_{\text {photo }} \frac{k_{r e c}}{k_{t r}+k_{r e c}},
$$

From here it is clear that in case of a very fast transfer rate $\left(k_{t r} \gg k_{r e c}\right)$, which is a property of the S/E interface (weakly dependent on applied external potential [Wilson1977]), all photo-generated holes will reach the redox species and the electrochemical reaction will proceed. However, this is not the typical case, but the opposite, that is, a very slow charge transfer rate $\left(k_{t r} \ll k_{r e c}\right)$, especially 
for such complex reactions as water splitting, involving at least 4 holes [Reichman1980]. Slow transfer rates mean that photo-generated holes will be queuing-up near the S/E interface and may recombine before transfer is completed, and if so, no output current will be measured. The recombination rate depends on the electron concentration at the semiconductor surface (Eq. 2.6.2.11), because once holes reach the surface they recombine with surface states filled with electrons (those below the Fermi level) and they need to be recharged with electrons from the CB in order to keep the recombination process. In that sense, surface states act as pure recombination centers. This also means that recombination rates depend on the semiconductor band bending $\left(\Delta \phi_{\mathrm{sc}}\right)$.

$$
k_{r e c}=\sigma v_{t h} n_{\text {surf }}=\sigma v_{t h} n_{b u l k} \exp ^{-\frac{\Delta \phi_{S C}}{k T}} .
$$

Unfortunately, close to zero bias $\Delta \phi_{S C}$ is rather small, so the recombination rate is much higher than the transfer one $\left(k_{t r} \ll k_{r e c}\right)$, which means almost all photoegenerated carriers will recombine and only negligible output current can be measured (Fig. 2.6.6 (c)). Applying a small reverse bias (leading to only slight increase of $\Delta \phi_{s c}$ ) will almost does not change $k_{\text {rec }}$ and thus minority carriers will still recombine efficiently, resulting in negligible output current (Fig. 2.6.6 (d')). A substantial external reverse bias (called onset potential $V_{\text {onset }}$ ) is needed to increase $\Delta \phi_{s c}$ for the transfer rate to be comparable to the recombination rate $\left(k_{t r} \sim k_{r e c}\right)$, so that holes can complete transfer

(Fig. 2.6.6 (d')). Increasing the reverse bias will eventually lead to recover the whole output current to its maximum value ( $j_{\text {photo }}$ in Fig. 2.6.6 ( $\left.\mathbf{d}^{\prime \prime \prime}\right)$ ) because the recombination rate will become negligible according to Eq. 2.6.3.10, and the S/E interface behavior becomes close to ideal.

\subsection{Light-induced Fermi level pinning}

To improve the model presented above one must also include the effect of accumulation of charges at the interface due to a slow transfer rate in the transition region of current voltage curves (Fig. 2.6.6 (d')). Indeed, at that point the hole recombination rate becomes slower than transfer rate, but because the transfer rate is still slow (it does not substantially change with reverse bias), holes will start queuing-up near the S/E interface. This can be viewed as charging a photo-induced capacitance at the S/E interface, which results in a potential drop across the Helmholtz layer:

$$
\Delta V_{H}=\frac{q p_{\text {surf }}}{C_{H}+C_{S C}}
$$

where $C_{H}$ and $C_{S C}$ are capacitances of Helmholtz layer and semiconductor surface, respectively. At constant applied potential, this increase in potential drop across the Helmholtz layer has to be balanced by a corresponding decrease in $\Delta \phi_{s c}$. Thus, in this potential region dependence between applied bias $(V)$ and potential drop across the SCR $\left(\Delta \phi_{S C}\right)$ is not linear anymore. Without this effect ( $k_{t r}$ is high) the transition region would be quite small ( $\sim 0.2 \mathrm{~V}$ at room temperature, according to Eq. 2.6.3.8 and 2.6.3.10), but it will be substantially increased with reducing $k_{t r}$ as schematically shown in Fig. 2.6.8. This effect is called light-induced Fermi level pinning. 


\section{Light-induced Fermi level pinning}

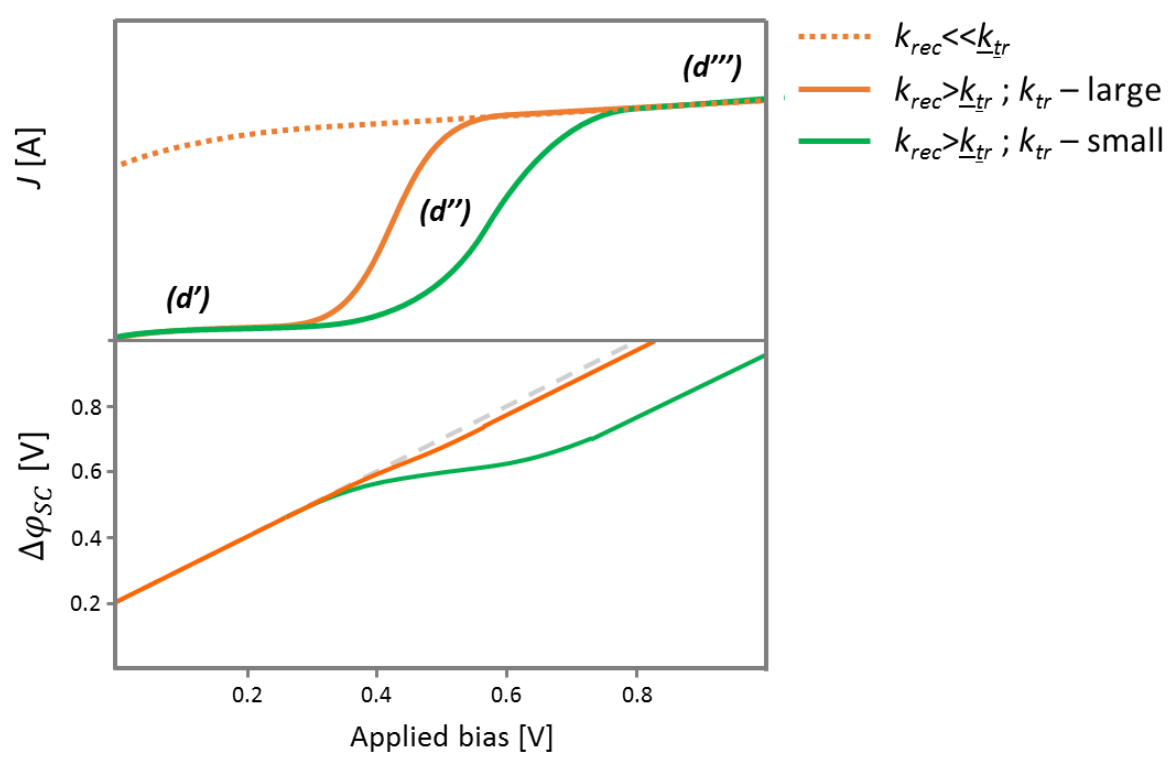

Fig. 2.6.8. Schematic dependence of output cell current $J$ (top) and potential drop in the semiconductor $\Delta \phi_{s c}$ (bottom) on applied bias illustrating light-induced Fermi level pinning due to slow transfer rate and consequent holes accumulation at the S/E interface. Different regions on the graph are marked according to Fig. 2.6.6 notation. Adapted from [Peter2013].

\subsection{Dark Fermi level pinning}

In addition to kinetical limitations of charge transfer at the S/E interface, the current voltage curve is also affected by charging of surface states when the Fermi level is moving through them, so called dark Fermi level pinning. "Dark" is referring to the fact that the mechanism behind this effect is independent from illumination conditions. To understand this phenomenon one must compare the band diagram change vs. applied bias when no (Fig. 2.6.9) or high density (Fig. 2.6.10) of surface states are present at the S/E interface.

When no surface states are present at the S/E interface, almost all applied bias drops across the semiconductor SCR ( $\Delta \phi_{S C}$ ) (Fig. 2.6.9). Thus, $\Delta \phi_{S C}$ depends linearly on the applied bias, as schematically shown in Fig. 2.6.11 (a). 


\section{NO surface states}

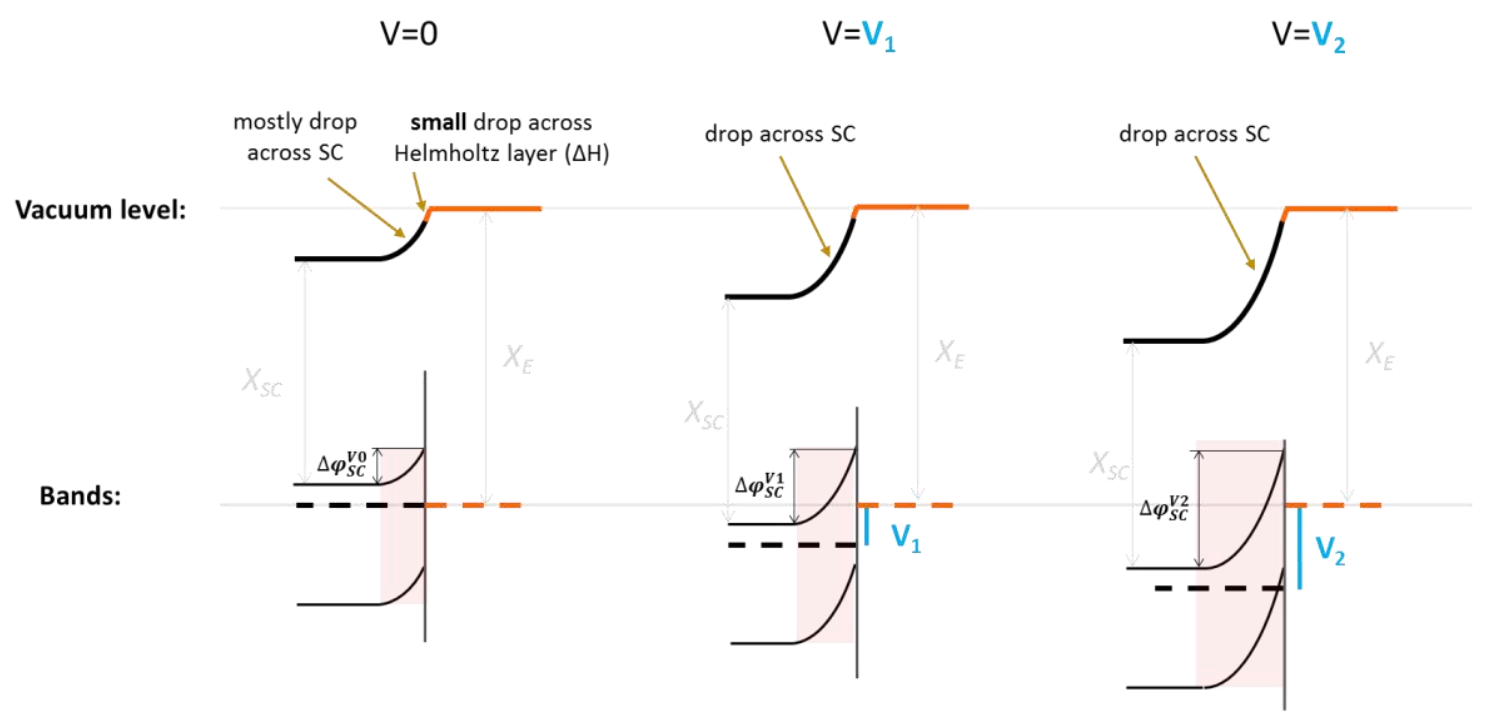

Fig. 2.6.9. Band profiles of a S/E junction when no surface states are present in the semiconductor. Most of the potential drop occurs at the semiconductor side.

When there is a high density of surface states at the S/E interface the situation is different. If, for example, at zero applied potential the Fermi level in the semiconductor is within the potential distribution of surface states (Fig. 2.6.10, at $V=0$ ), the charge accumulated at the surface states (below Fermi level) must be compensated by a variation in the potential drop across the Helmholtz layer in the redox solution in order to keep charge neutrality. Thus, an increase in the applied reverse bias drops across the Helmholtz region instead of the semiconductor SCR, because of surface states discharge, acting as a surface capacitor (Fig. 2.6.10 $\left.\left(\mathrm{V}=\mathrm{V}_{1}\right)\right)$. Only when surface states are fully

\section{Surface states}

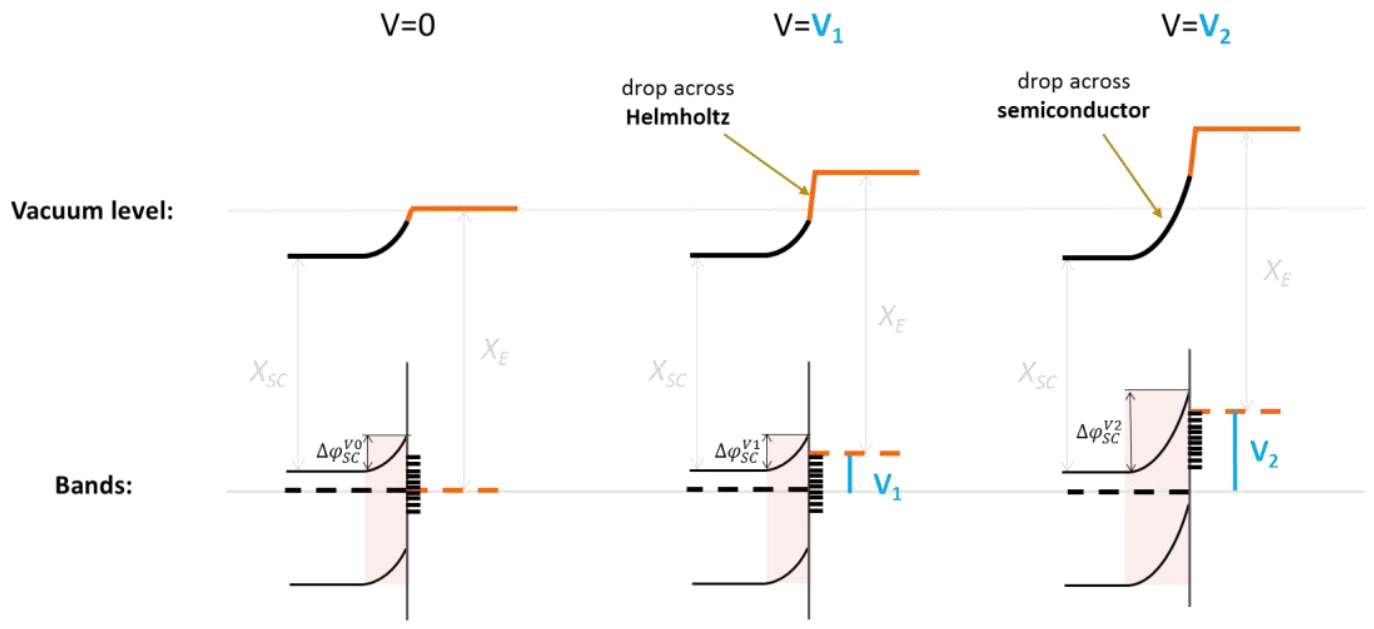

Fig. 2.6.10. Band profiles of a S/E junction when surface states are present in the semiconductor. Most of the potential drop occurs at the Helmholtz layer until discharge of surface states finishes. 
discharged the applied potential starts to drop across the SCR again (Fig. 2.6.10 $\left(V=V_{2}\right)$ ), because there is no more additional charge stored at the interface. As a result, $\Delta \phi_{s c}$ dependence on applied potential deviates from a linear behavior when the Fermi level sweeps through the surface state distribution as schematically shown in Fig. 2.6.11 (b). This phenomenon is called dark Fermi level pinning.

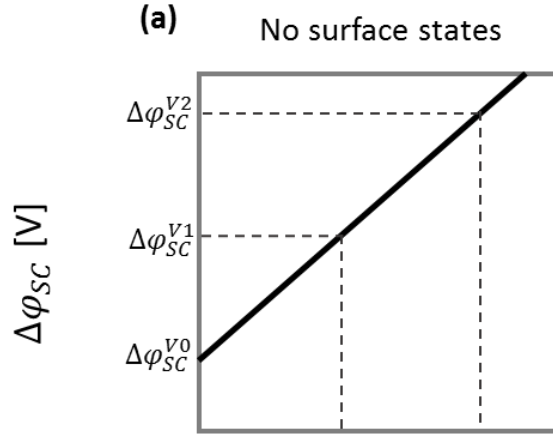

Applied bias [V] (b) Surface states

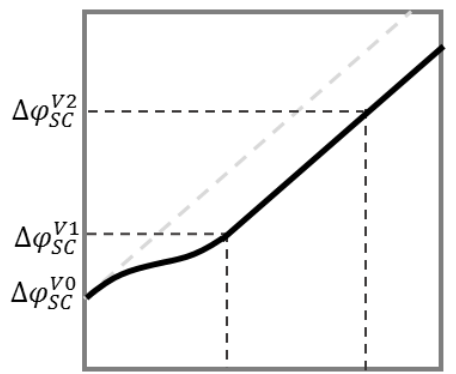

Applied bias [V]

Fig. 2.6.11. Dependence of $\Delta \varphi_{s c}$ with the applied voltage in the two described cases of no surface states (a) and a high density of surface states (b).

\subsection{Conclusion on non-ideality of S/E interface}

To summarize, there are three possible origins for non-ideality of the current-voltage characteristic of a S/E junction, that usually occur simultaneously:

(i) Enhanced surface recombination due to slow holes transfer at the S/E interface.

(ii) The effect of hole accumulation that acts as a charged capacitor due to slow holes transfer at the S/E interface (light-induced Fermi level pinning).

(iii) The Fermi level pinning at surface states inside the semiconductor bandgap (dark Fermi level pinning).

The discussed S/E junction model is by no means complete, as there are still open questions under debate in the scientific community about the surface states involvement in surface chemistry, despite so many years of research on this field (first mention of surface states largely controlling the surface kinetics were proposed in 1959 by Green [Green1959] and later evaluated by Uosaki \& Kita [Uosaki\&Kita1985]).

\subsubsection{Photocorrosion and ways to overcome it}

Another problem related to slow surface kinetics, which is even more severe than performance degradation, is the loss of semiconductor material due to its oxidation. When holes accumulate at the surface due to a slow transfer rate they may start oxidizing the semiconductor itself, instead of the redox species (water molecules in case of water splitting). If the semiconductor surface oxide is soluble, it can dissolve into the solution and the semiconductor oxidation process starts again. Even 
if the semiconductor oxide is not soluble, it can passivate the semiconductor surface and significantly reduce the performance of the S/E junction. This process is commonly referred to as photocorrosion. The fact that corrosion competes with water oxidation can be expressed in form of rate constants as follows:

$$
k_{t r} \rightarrow k_{o x}+k_{c o r r}
$$

Photocorrosion cannot be detected by measuring the current-voltage characteristics because it also contributes to the output current. An in situ method to indirectly quantify the corrosion rate is to measure the performance of the photoelectrode over a long period of time. A decay of photocurrent can be related to the loss of material (soluble oxide) or sample surface passivation (insoluble oxide) both induced by photocorrosion. AFM or SEM morphology studies can also be used ex situ to reveal if the surface was corroded. Both methods are going to be used in this work in Chapter 7.

From the desecription of corrosion it can be concluded that photocathodes, where the surface accumulates electrons instead of holes, are more corrosion resistant. However, as the main goal of this work is to use S/E junctions to avoid the need of InGaN p-doping, a way to avoid corrosion of photoanodes must be found. There are two frequently used methods for this purpose: (i) adding hole scavengers (sacrificial agents) to the electrolyte, which substitute "slow" water oxidation reaction by "faster" oxidation of the sacrificial agent, and (ii) decorating the semiconductor surface with metal or metal oxide catalysts, which can greatly enhance the rate of water oxidation and thus, kinetically suppressing corrosion. Both of them are going to be reviewed in the following sections.

\subsubsection{Sacrificial agents}

Slow kinetics at the S/E interface is generally related to the fact that the water splitting is a complex multistep reaction involving (at least) four electrons. One can substitute a slow water oxidation reaction by another faster (more energetically favorable) oxidation reaction of some other compound (with much lower redox potential), which in this case are called sacrificial agent. Because this new reaction is fast, then photogenerated holes can be removed from the semiconductor faster than they have a chance to recombine or oxidize the semiconductor, thus, suppressing both holes recombination and semiconductor photocorrosion. This process is called holes scavenging and the sacrificial agent in this case is called hole scavenger. Furthermore, because usually sacrificial agents are organic compounds, their oxidation process results in the formation of $\mathrm{CO}_{2}$ gas (instead of $\mathrm{O}_{2}$ in water oxidation), thus the back reaction to produce water is suppressed, increasing the $\mathrm{H}_{2}$ yield and avoiding a subsequent gas separation stage. The main drawback of such solution to corrosion problem is that since hole scavengers are consumed in the photocatalytic reaction their continuous addition is required to sustain $\mathrm{H}_{2}$ production (that is why they are called sacrificial agents). Additionally, the process in which sacrificial agents are involved cannot be called water splitting anymore but rather, for example, organic compound reforming in case of organic hole scavengers.

Organic compounds such as alcohols, organic acids, salts, and hydrocarbons can act as efficient hole scavengers (i.e., as electron donors) for the photo-catalytic $\mathrm{H}_{2}$ generation. In particular, methanol is frequently used as sacrificial reagent. For practical applications, the utilization of methanol will only be environmentally sensible provided that it is derived from biomass or from toxic residues that must be disposed of [Seger2009]. 
A good example of hole scavenger is formic acid $(\mathrm{HCOOH})$ with its oxidation reaction:

$$
\mathrm{HCOOH}(a q)+h^{+} \rightleftharpoons \mathrm{CO}_{2}(g)+2 H^{+}+2 e^{-}, \quad E_{\text {red }}=-0.11 \mathrm{~V} \text { vs. RHE }
$$

This reaction is much likely to occur instead of water oxidation because it has much lower standard redox potential $(-0.11$ to $+1.23 \mathrm{~V}$ vs. $\mathrm{RHE})$. This drastically speeds up holes extraction from the semiconductor, increasing the PEC cell efficiency while reducing corrosion. However, in that case as almost all holes consumed to oxidize $\mathrm{HCOOH}$, the water oxidation reaction is slowed down.

Some commonly used electrolytes for water splitting also act as sacrificial agents. For example, aqueous electrolytes based on $\mathrm{HBr}(\mathrm{aq})$ contain $\mathrm{Br}^{-}$ions which can undergo the following reaction:

$$
2 \mathrm{Br}^{-}+2 h^{+} \rightleftharpoons \mathrm{Br}_{2}(a q), \quad E_{\text {red }}=+1.08 \mathrm{~V} \text { vs. RHE }
$$

Thus, it also alters the overall reaction and it is impossible to refer to PEC cells using such electrolytes as pure water splitting cells. Very similar effects happen in case of $\mathrm{HCl}(\mathrm{aq})$.

While for practical applications sacrificial agents are rarely seen as a viable option, from a fundamental point of view they can be used to check for carrier accumulation at the semiconductor surface. Indeed, as it was previously described, three causes of high onset potential, namely (i) enhanced surface recombination, (ii) light-induced Fermi level pinning, and (iii) dark Fermi level pinning can be divided into two groups by their origin: slow transfer kinetics ((i) and (ii)) and high density of surface states (iii) related. Thus adding a sacrificial agent (eliminating slow transfer kinetics) can affect the onset potential of photocurrent $\left(V_{\text {onset }}\right)$ in the following ways:

1) Greatly improve it - meaning that a slow transfer at the interface was the limiting factor for photocurrent onset.

2) Leave it without change - meaning the high density of surface states and associated Fermi level pinning were the limiting factor for photocurrent.

This can be used as a fast probe of the surface kinetics.

\subsubsection{Catalysis}

Despite the still somehow poor understanding of the S/E interface, various attempts to improve its performance were made. A way to reduce the photocurrent onset $\left(V_{\text {onset }}\right)$ is the use of electrocatalysis: that is, the semiconductor decoration with noble or transition metal-based catalysts. A catalyst is a substance, which enhances the rate of a reaction without being consumed during the process, thus increasing the transfer rate $\left(k_{t r}\right)$ while reducing the corrosion contribution. Catalysts may be divided into two groups: homogenous (same phase, i.e. liquid-liquid) and heterogeneous (different phases, i.e. solid-liquid). Heterogeneous catalysis is of particular interest for PEC cells.

Catalysts work by localizing the reactants in space (properly aligning them) which drastically increases the probability of reaction. Thus, the catalyst provides an alternative route for the reaction with lower activation energy, but the overall thermodynamics are the same. Once the reaction starts, the byproducts must be released from the catalyst to renew the cycle. In general, a good catalyst must adsorb the reactant species strongly enough for them to react, but not so strongly that the product of reaction cannot be released from the surface. Silver is not a good catalyst because it does not allow reactant molecules to attach strongly enough. Tungsten is neither a good catalyst because it adsorbs the reactant species too strongly. Metals like platinum and nickel are 
good catalysts because they adsorb the reactant species strongly enough to hold and activate the reactants, but not so strongly that the byproducts cannot break away.

In case of PEC cells the effects on photocurrent onset due to modifications of the semiconductor surface, usually by metals, is called "photoelectrocatalysis". It was first discovered by Tsubmura et al. [Nakato1976] and treated in an early paper by Gerischer et al. [Kautek1980] (see also Ohashi et al. [Ohashi1977]). The addition of nanoparticles of noble metals to the semiconductor surface gave rise to a substantial ( $>10$ times) increase in the rate of hydrogen/oxygen production by photoelectrochemical reactions. Since then, many catalysts, such as noble metals ( $\mathrm{Pt}, \mathrm{Pd}, \mathrm{Ru}, \mathrm{Rh}$ ), transition metals $\left(\mathrm{Ti}, \mathrm{Ni}\right.$, etc.), and their oxides $\left(\mathrm{RuO}_{2}, \mathrm{TiO}_{2}, \mathrm{NiO}\right.$, etc.), deposited on the semiconductor surface were used to catalyze redox reactions, for instance, to reduce the surface recombination and corrosion processes. However, it is rather difficult to prove a real catalytic effect because the deposition of a metal layer leads usually to the formation of a rectifying Schottky junction at the S/M interface, which significantly alters the semiconductor band diagram [Memming2015].

One of the first studies to show that catalysts can reduce corrosion by enhancing the reaction rate was reported by Heller [Heller1981] for single crystalline $\mathrm{n}-\mathrm{GaAs}$ electrodes in alkaline solutions of the redox system $\mathrm{Se}^{2-} / \mathrm{Se}_{n}^{2-}$. In these experiments the photocurrent transition region (region (d') in the I-V curve in Fig. 2.6.6) became steeper after the electrode had been dipped in a solution of $\mathrm{RuCl}_{3}$ (leading to deposition of Ru catalyst) right before it was used in the cell. This effect was interpreted as a reduction of surface recombination by passivating surface states with Ru. Further investigations by Tan et al. [Tan1991] confirmed these results and also made clear that there is not only hole transfer from the valence band to the redox system, but also hole consumption at the semiconductor surface due to photocorrosion. A chemical analysis of the byproducts formed under photoanodic conditions showed that the oxidation rate of the redox system $\left(k_{o x}\right)$ was increased with respect to that of the corrosion $\left(k_{c o r r}\right)$ upon surface modification by $\mathrm{Ru}$. This result implies that $\mathrm{Ru}$ catalyzes the hole transfer from GaAs to $\mathrm{Se}^{2-}$ and proves that the improvement is rather due to enhanced catalytic activity, than by the formation of a Schottky junction.

Another approach to slow down corrosion rate using catalysts is to deposit metal-oxide protection layers on the semiconductor. This allows photogenerated holes to be transferred to the protection layer, thus charge will accumulate, not at the S/E interface, but at the catalyst/electrolyte interface where the reaction rate is faster and corrosion cannot occur because the metal oxide layers is already in the most oxidized form. Both of these effects significantly reduce corrosion. However, there are two requirements for this scheme to work successfully in photoanodes:

(i) There should be no potential barrier between the semiconductor VB and the metal oxide film, in other words, the VB top should lie below the VB of the metal oxide.

(ii) Catalyst layers should be thin enough to be transparent for holes and also allow a fast carriers transfer without severe recombination losses.

A good example of such system is an array of $\mathrm{ZnO}$ nanowires as photo-anode with $1 \mathrm{~nm}$ thick $\mathrm{TiO}_{2}$ film deposited by atomic layer deposition, as shown in Fig. 2.6.12 [Liu2013b]. In this work a similar scheme using $\mathrm{NiO}$ as a catalyst and protection coating on top of InGaN layers is going to be considered. 


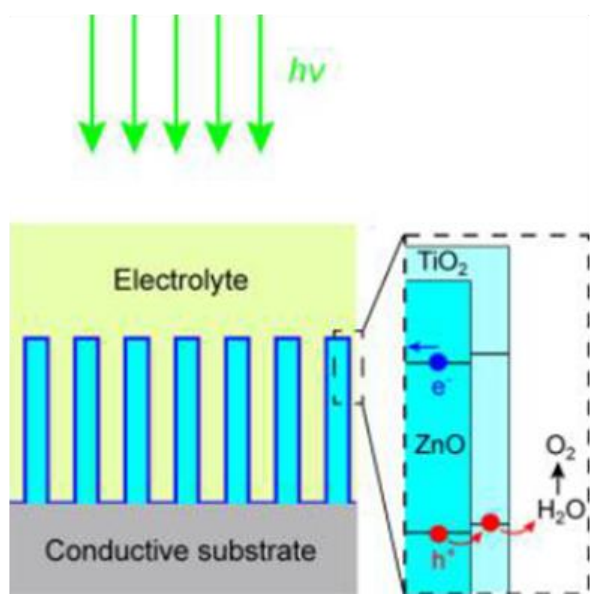

Fig. 2.6.12. A metal oxide $\mathrm{TiO}_{2}$ catalyst protective coating on $\mathrm{ZnO}$ nanowires. Adapted from [Liu2013b].

Metal nanoparticles can also be used as catalysts, deposited on the semiconductor surface. A pioneering work by Tsubomura et al. [Nakato1987a] showed that the performance of a p-type Si photo-cathode was greatly enhanced by depositing Pt nanoparticles (<10 nm) (Fig. 2.6.13), due to the simultaneous presence of S/E and catalyst/electrolyte interfaces and quantum confinement effects in nanoparticles. The same electrode covered with a Pt film formed an ohmic junction with Pt and exhibited a "metallic" behavior: currents in the dark and under illumination are the same (no photoresponse). In the experiments with n-Si photo-anodes covered with a Pt film, islands, and nanoparticles a similar photo-voltage enhancement was observed [Nakato1987b, Nakato2004].

In summary, decorating semiconductor photo-electrodes with catalysts increases their performance and reduces the corrosion rate, both due to lowering kinetic limitations at the S/E junction.

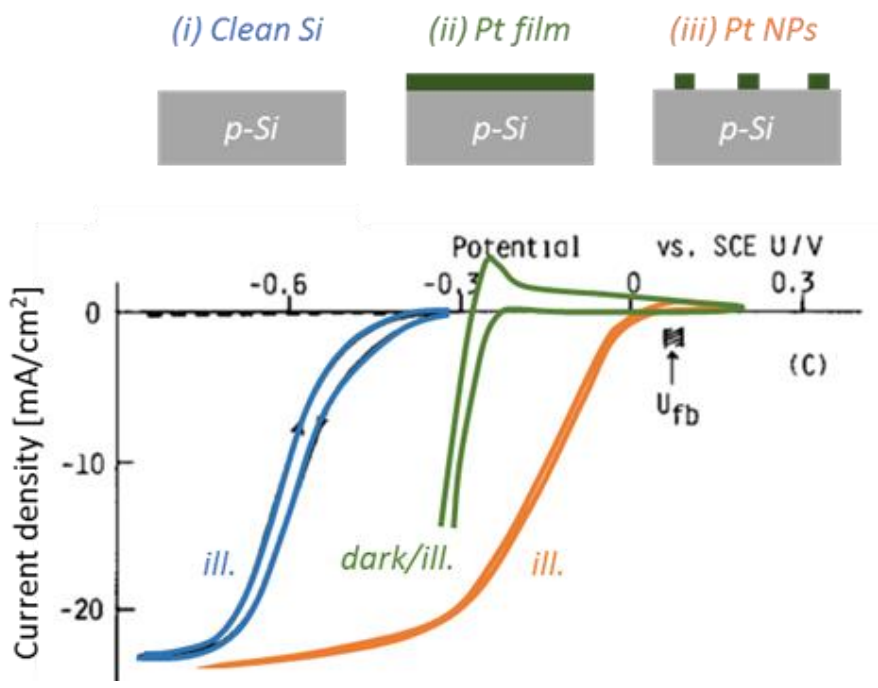

Fig. 2.6.13. Use of metal nanoparticles (NPs) as a catalyst to enhance a p-Si photocathode performance. Three cases are shown: (i) clean p-Si photo-cathode, (ii) p-Si covered with a continuous Pt film, and (iii) pSi covered with Pt nanoparticles. In case of (i) and (iii) dark currents are negligible in examined potential range. The electrode in case (ii) showed metallic Schottky behavior: current is the same both in dark and under illumination. Only the use of nanoparticles (iii) results in a greatly enhanced performance. Potential is measured against a saturated calomel electrode (SCE). Adapted from [Nakato1987]. 


\section{Experimental techniques}

\subsection{Introduction}

This chapter is dedicated to the experimental techniques used for the realization of this doctoral thesis. A major experimental part was done in the clean room facilities of the Institute of Optoelectronic Systems and Microtechnology (ISOM) located at the Escuela Técnica Superior de Ingenieros de Telecomunicación (ETSIT), at the Universidad Politécnica de Madrid (UPM). It includes a PA MBE system used for the epitaxial growth of all samples studied in this work, as well as ultraviolet (UV) and infra-red (IR) photoluminescence (PL) for optical characterization, scanning electron microscopy (SEM) and atomic-force microscopy (AFM) for morphological, and high-resolution X-ray diffraction (HRXRD) for structural one.

TEM analyses of $\ln _{x} \mathrm{Ga}_{1-\mathrm{x}} \mathrm{N}$ layers and $\mathrm{InN}$ nanostructures was performed by external collaborators Prof. Dr. Francisco M. Morales Sánchez, Dr. José M. Mánuel, Juan J. Jiménez, and Prof. Dr. Rafael García at the Departamento de Ciencia de los Materiales e Ingeniería Metalúrgica y Química Inorgánica, Universidad de Cádiz and Instituto Universitario de Investigación en Microscopía Electrónica y Materiales (IMEYMAT) (Cádiz, Spain).

TEM analyses of SAG GaN NWs was performed by external collaborators Prof. Dr. Almudena TorresPardo and Prof. Dr. Jose M. González-Calbet at the Centro Nacional de Microscopía Electrónica, Universidad Complutense de Madrid (Madrid, Spain).

SNOM measurements of $\ln _{\mathrm{x}} \mathrm{Ga}_{1-\mathrm{x}} \mathrm{N}$ layers were performed by external collaborators Prof. Dr. Christoph Lienau and Dr. Alexander Senichev at the Max Planck Institute of Microstructure Physics (Halle, Germany) and Institute of Physics and Center of Interface Science, Carl von Ossietzky Universität Oldenburg (Oldenburg, Germany).

PEC measurements, together with NiO deposition were performed in the Physics Department at Danmarks Tekniske Universitet (Copenhagen, Denmark) under the guidance of Prof. Dr. Brian Seger, Prof. Dr. Peter C. K. Vesborg, and Prof. Dr.lb Chorkendorff.

All techniques used in this thesis are briefly described in the following sections.

\subsection{Molecular beam epitaxy}

The term "epitaxy" refers to the deposition a crystalline layers on a crystalline substrate. If a layer is deposited on a substrate of the same composition, the process is called homoepitaxy, otherwise it is called heteroepitaxy. An example of homoepitaxy could be a growth of GaN nanowires on $\mathrm{GaN} /$ sapphire or GaN/Si template. Examples of heteroepitaxy include GaN grown on Sapphire $\left(\mathrm{Al}_{2} \mathrm{O}_{3}\right)$, silicon $(\mathrm{Si})$ or silicon carbide $(\mathrm{SiC})$. 
There are several widely used epitaxial methods, such as metal organic vapor phase epitaxy (MOVPE), hydride vapor phase epitaxy (HVPE), liquid phase epitaxy (LPE), molecular beam epitaxy (MBE), among others. In this work a plasma-assisted MBE system was used to grow the samples, the main advantage of it being the use of a radio frequency nitrogen plasma source, allowing to grow samples at any temperature, as no cracking (pyrolisis) of any chemical compound is involved. Other advantages of the MBE technique are: high purity of constituent materials, ability to form very abrupt interfaces, excellent control of thickness, composition and doping, and the possibility to monitor the growth in real time by in situ techniques.

\subsubsection{Description of the MBE system components}

In this work, a MECA 2000 MBE system was used (Fig. 3.2.1 (a)), with a growth chamber described schematically in Fig. 3.2.1 (b).

A typical MBE system is equipped with at least two chambers: introduction (load-lock) and growth ones. The substrates are first loaded in the introduction chamber where they are outgassed under high vacuum (pressure $\sim 10^{-8}$ torr) at $400^{\circ} \mathrm{C}$ to remove water vapor and organic contaminants. After that the substrate is transferred to the growth chamber maintained under ultra high vacuum (UHV) (pressure $<10^{-10}$ torr), where growth proceeds under a molecular beam regime, in which the scattering of flowing atoms by residual gas molecules is negligible. In both chambers high vacuum conditions are typically reached with turbomolecular, cryogenic and ion pumps. Since standard ion pumps are not efficient to pump molecular nitrogen, cryogenic or turbomolecular pumps with high pumping speed are needed, especially during the growth when the chamber pressure reaches $10^{-5}$ torr due to the input of $\mathrm{N}_{2}$. The growth chamber is also equipped with cryo-panels filled with liquid $\mathrm{N}_{2}$ during the growth, which also help to reduce the background pressure.

The pressure in both chambers is usually measured with a Bayard Alpert ionization gauge. An additional retractable Bayard Alpert ion gauge (beam flux monitor) in the growth chamber can be placed at the substrate position to accurately measure the impinging fluxes in beam equivalent

(a)

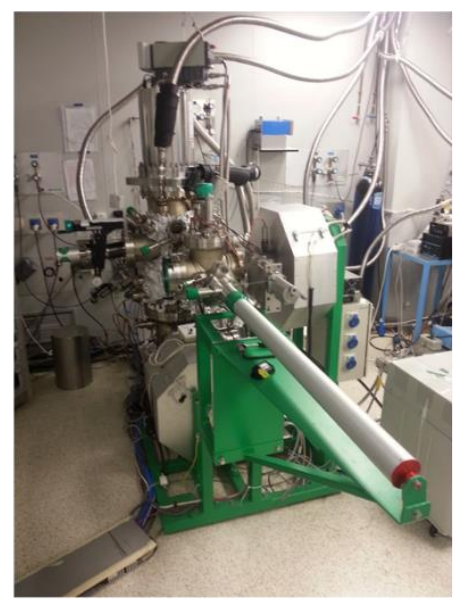

(b)

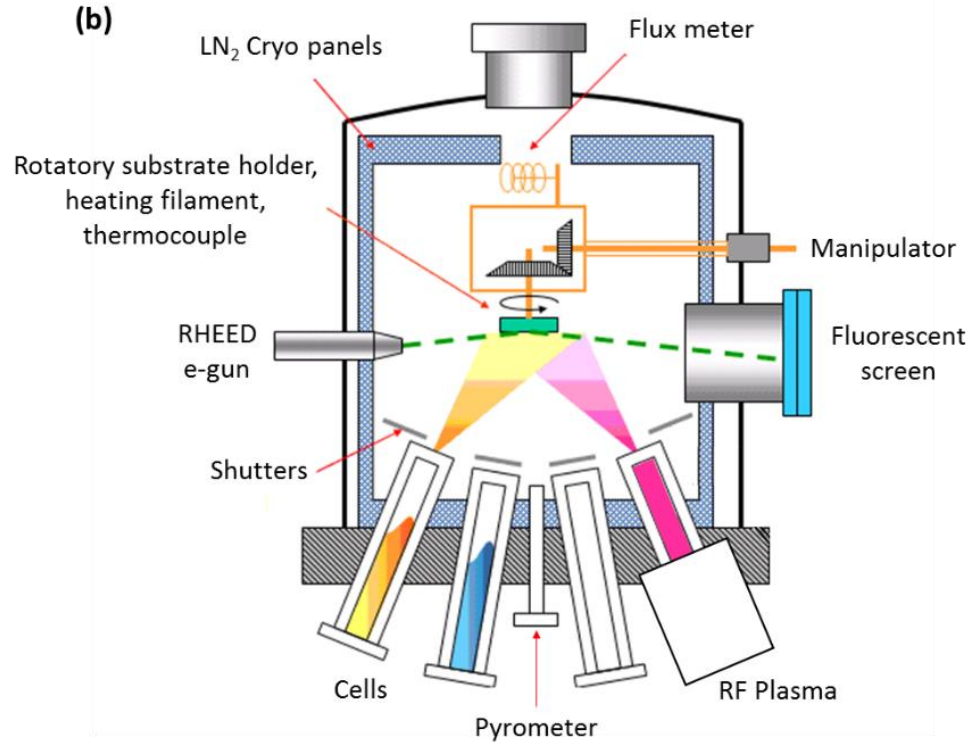

Fig. 3.2.1. Photo of the MECA 2000 MBE system (a) and a schematic view of its growth chamber (b). 
pressure (BEP) units (torr). The partial pressures of different residual gasses can be assessed with a quadrupole mass analyzer (QMS).

The substrate is fixed on a molybdenum holder (molyblock) placed in the manipulator with a heating filament (radiation) and a thermocouple to monitor its temperature. Temperature can also be measured by means of an optical pyrometer. The molyblock rotates during the growth to achieve an homogeneous molecular beams distribution.

The sources of molecular/atomic beams for III-group metals ( $\mathrm{Ga}, \mathrm{In}, \mathrm{Al}$ ) as well as dopant species (Si, Mg) are called Knudsen cells. A Knudsen cell comprises a pyrolytic boron nitride crucible containing the solid or liquid ultra pure material, which is heated with a filament (placed around the crucible) until it evaporates. Mechanical shutters, placed in front of each Knudsen cell, provide a very fast interruption of the molecular beam, facilitating thus the formation of abrupt interfaces and growth control down to a monolayer scale. BEP values for each cell can be calibrated as described in Appendix A.

For the nitrogen flux, a plasma source is used where low reactive nitrogen molecules $\left(\mathrm{N}_{2}\right)$ are dissociated into very reactive atomic form ( $\mathrm{N}^{*}$, also called "active" nitrogen) by means of radio frequency (RF) excitation. A high purity $\mathrm{N}_{2}$ (99.999999 \%) flux into the plasma source is controlled by a mass flow controller and is commonly expressed in standard cubic centimeters per minute (sccm) units. The active nitrogen flux is proportional to the source power and can be monitored by measuring the emission signal from specific atomic nitrogen emission lines by means of an optical emission detector (OED). OED values can be calibrated to give the actual atomic flux of $\mathrm{N}^{*}$ measured in atoms $/ \mathrm{cm}^{2} / \mathrm{s}$ as described in Appendix A.

In addition, the growth chamber of the MECA 2000 MBE system is equipped with a reflection highenergy electron diffraction (RHEED) system which allows in situ monitoring the surface structure (reconstruction) and growth rate. In a RHEED system an electron gun generates a beam of highenergy electrons ( $30 \mathrm{keV}$ ) striking the sample surface at a grazing angle (1-2 degrees). The incident electrons diffract at atomic planes close to the surface and a small fraction of the diffracted electrons interfere constructively at specific angles (Bragg condition) and form regular patterns on the fluorescent screen. The electrons interfere according to the position of atoms on the sample surface, so the diffraction pattern provides information of the processes taking place at the surface. More details on RHEED imaging and applications was given in section 2.5.3.

\subsubsection{Surface kinetics}

Once the MBE system is prepared for growth (i.e. the impinging fluxes and the substrate temperature are stabilized), the cells shutters open and the III and V atoms impinge on the substrate surface, where the growth starts. Growth proceeds under a kinetic regime, far from the thermodynamic equilibrium, in contrast to other epitaxial methods such as MOCVD and HVPE. The substrate temperature, the Knudsen cell temperatures (III-group metal fluxes) and active nitrogen flux determine the surface kinetics of the adsorbed atoms (adatoms), i.e. their surface mobility, diffusion length, desorption and incorporation rates, as well as the decomposition rate of the growing crystal.

The surface kinetics is briefly explained in the case of GaN growth, though this description is applicable to other III-Nitrides by changing the metallic group-III element. Atoms from an impinging 
Ga flux get adsorbed (adatoms) at the surface (physisorption). Once adsorbed, adatoms diffuse over the surface and depending on the adatom kinetic energy, strength of surface-to-adatom interaction, and $\mathrm{Ga} / \mathrm{N}$ availability, three scenarios are possible as shown in Fig. 3.2.2:

(i) The adatom incorporates into a crystal lattice site by forming a Ga-N chemical bond of mixed ionic-covalent nature (chemisorption).

(ii) The adatom can thermally desorb ("re-evaporate") if having enough kinetic energy (determined by the substrate temperature) to escape from the surface.

(iii) The adatoms can form Ga-Ga metallic bonds and start droplet accumulation (typical when growing with metal excess) if the substrate temperature is too low for $\mathrm{Ga}$ to be desorbed.

Additionally, atoms already incorporated into the crystal can escape from it to become again adatoms and continue experiencing the previously described kinetic processes. This process is called decomposition (Fig. 3.2.2). Due to the extremely high equilibrium vapor pressure of $\mathrm{N}_{2}$ compared to Ga, decomposition of GaN leaves behind only Ga adatoms, while $\mathrm{N}$ atoms immediately desorb from the surface.

At the initial stage of a heteroepitaxial growth, adatoms interact with other adatoms to form very small nuclei that, once a critical volume is exceeded to ensure stability (no decomposition), get bigger and eventually coalesce with other nuclei and the formation of a continuous layer. Depending on the surface kinetics, a complete coalescence may happen quickly, slowly, or not at all, defining thus the so called growth mode. The initial stage of the film formation is very similar to that of nanostructures, thus it is discussed in more details in the next section.

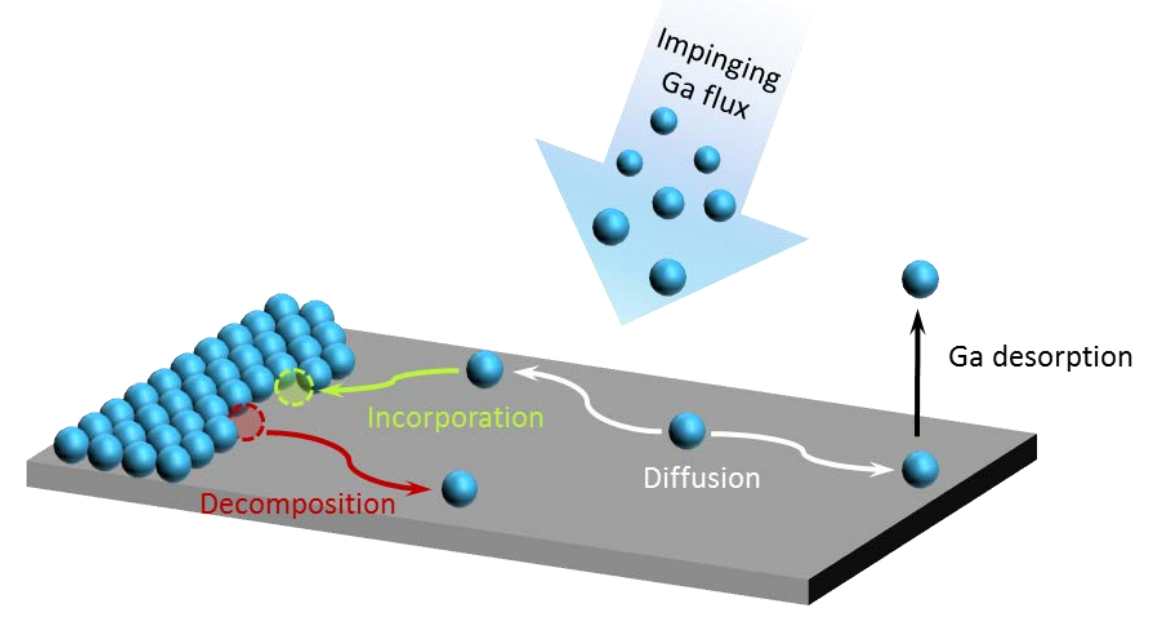

Fig. 3.2.2. A schematic diagram of kinetic processes on the substrate surface during the MBE growth.

\subsubsection{Growth of quantum dots (QDs)}

Since the 1980 s considerable efforts were devoted to fabricate heterostructures at nanometer scale. In general, there are two main approaches, commonly referred to as "top-down" and "bottom-up" techniques. The "top-down" approach implies the use of lithographic and etching techniques to remove mask-defined parts of bulk material leaving nanostructures behind, while "bottom-up" methods consist of growing the nanostructures from elementary compounds. In this 
section an overview of the most important techniques developed up to now, regarding both approaches, is given. Their applicability to InN Quantum Dots (QDs) fabrication is discussed.

\subsubsection{Top-down approach}

Historically this is the first developed technique to fabricate QDs for which there is a good understanding of all main stages, but also well-known limitations. For a very long time, patterning quantum wells (QWs) by lithography and etching was considered the most straightforward way for QDs fabrication. A schematic representation of this process for GaAs QDs fabrication is shown in Fig. 3.2.3. The main advantages of this method are:

(i) Exceptional control of lateral size, shape and arrangement of QDs (depending on the resolution of the patterning system).

(ii) Huge variety of available processing techniques with a very high degree of optimization.

(iii) Scalability of the process (though not for all lithographic systems).

The most commonly used fabrication procedures [Forchel1988, Bimberg1999] comprise optical, Xray, electron and focused ion beam lithography techniques, together with holography, scanning tunneling microscopy, etc. Many of them are industrial standards nowadays.

Despite many advantages of optical lithography for QDs fabrication, it was quickly realized that there are significant limitations, such as a down-limit on QDs size, inheriting defects existing in the initial 2D film, and generation of a high amount of defects at etched surfaces (sometimes even crystal layer amorphization). All these drawbacks, especially after the development of direct bottom-up epitaxial techniques, forced the above-mentioned lithographic methods to play a secondary role in the fabrication of QDs.

\section{"Top-down" QDs fabrication}
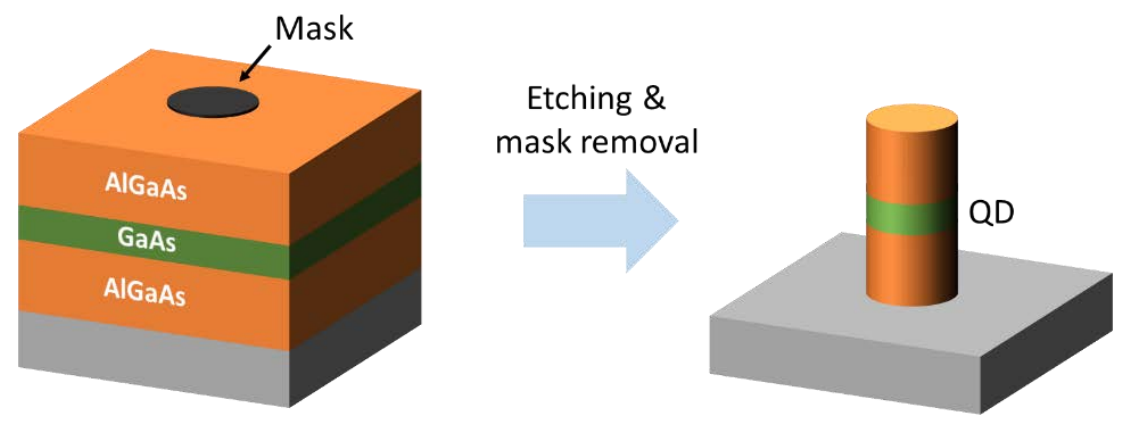

Fig. 3.2.3. Schematic representation of the "top-down" QDs fabrication through patterning of thin film structures.

\subsubsection{Bottom-up approach}

The bottom-up approach refers to the atom-by-atom QD formation, from elementary compounds provided during the epitaxial growth. QDs in this case can be obtained directly by self-organization or using a mask on the substrate surface. One of the first fabrication methods was based on the discovery of colloidal QDs formation in a glass matrix by Ekimov et al. [Ekimov1982, Ekimov1985] and later in colloidal solutions by Brus et al. [Brus1984]. These discoveries catalyzed an extensive 
research in this field, leading eventually to a wide variety of new bottom-up fabrication techniques. The most important ones are described hereafter, with an emphasis on those used in this work.

One of the widely used fabrication methods relies on quantum well (QW) thickness and/or composition fluctuations causing potential fluctuations that may confine excitons in the three spatial directions [Christen1990], thus leading to the formation of QD-like states. The drawbacks of such method are its poor controllability and reproducibility, as well as a typical very shallow localization energy of the confined carriers [Kop'ev1986].

\subsection{Epitaxial methods based on strain relaxation}

Another direct way to fabricate QDs with a given size (average) and composition, without catalyst or random fluctuations, is based on strain relaxation that takes place when growing a thin 2D film lattice mismatched with the substrate. The nucleation and initial growth of a thin epilayer is strongly governed by the interaction between the deposited material and the underlying crystalline substrate. Depending on how strong this interaction is, three growth modes can be distinguished: Frank-van der Merve (FM), Volmer-Weber (VW), and Stranski-Krastanow (SK) [Bauer1958] (Fig. 3.2.4). In all of them, the elemental fluxes participating in the crystal growth are supplied simultaneously to a heated substrate (in contrast to a droplet epitaxy technique, explained later in the text). Both VM and SK modes can be used to fabricate QDs, while the FM regime, commonly referred to as "layer-by-layer" growth is the preferential mode for 2D layers growth (also applicable to QWs ).

In the FM regime the adatoms attach preferentially to surface sites (dangling bonds) due to a strong adatom-to-surface interaction. It is typical in systems where there is no lattice mismatch, specifically in homoepitaxial growth. If there is a small mismatch, the growing epilayer builds up energy until a threshold thickness value (critical thickness), at which the accumulated energy is higher than that needed to generate a dislocation. Then strain is relaxed "plastically", by dislocation generation, and

(a) Frank-van der Merwe

2D

$\Theta<1 \mathrm{ML}$

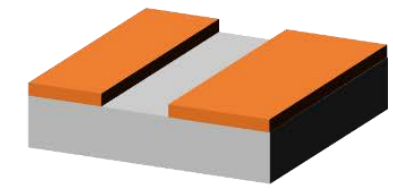

$1<\Theta<2 \mathrm{ML}$

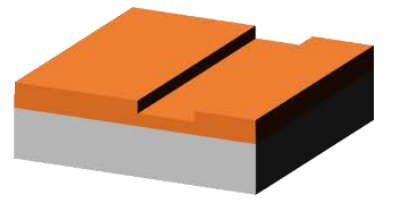

$\Theta>2 \mathrm{ML}$ (b) Stranski-Krastanow 2D/3D
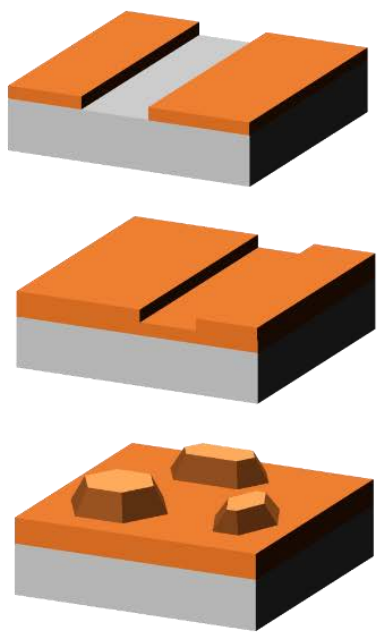

(c) Volmer-Weber 3D
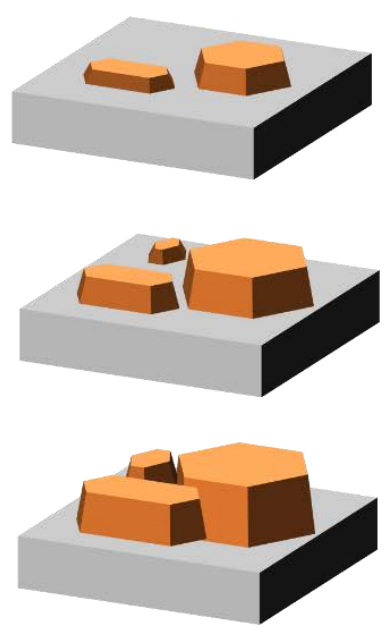

Fig. 3.2.4. Schematic representation of epitaxial methods based on strain relaxation. 
the epilayer keeps growing with its own (in-plane) lattice parameter (metamorphic regime). The resulting layer may still be essentially 2D, though in many cases with increasing roughness [Matthews1979]. Notice that for a really small mismatch this critical thickness may become quite large, even more than the actual film thickness, so that no relaxation takes place (pseudomorphic regime). A schematic representation of this mode is given in Fig. 3.2.4 (a).

In VW mode (Fig. 3.2.4 (c)) the adatom-to-adatom interaction is stronger than the adatom-tosurface one, leading to the formation of islands (3D) directly on the substrate surface. Generally, for the growth to proceed in this way, the epilayer-to-substrate lattice mismatch must be substantial. One way to picture this mode is by considering the strong lattice mismatch that is rapidly (instantaneously) relieved or minimized by islands formation which have a higher surface to volume ratio. A VM growth mode can also be intentionally induced by a chemical treatment of the substrate surface with "anti-surfactants" in order to inhibit diffusion of growth species and thus force islands formation. It must be noted, that when strain is not fully relaxed through additional free surface relaxation, then an undesirable generation of high density intra-dot structural defects is likely to happen [Moustakas2006].

In SK heteroepitaxial growth mode the first few monolayers of the epi-film grow 2D, adapting to the in-plane substrate lattice constant (either compressive or tensile) until the critical thickness is reached, leading to an "elastic" strain relaxation via islands formation. Thus, in SK typically a very thin 2D layer (wetting layer) coexists with 3D islands (Fig. 3.2.4 (b)). The SK mode can be viewed as a hybrid-mode between VW and FM ones, in which the epilayer critical thickness is of the order of several monolayers (for VM mode it is close to zero and for FM one it is much higher, actually "infinite" for pure homoepitaxial growth). SK growth typically results in the formation of coherent, i.e. defect-free islands of higher crystalline quality [Simmonds2012] compared to their VW counterparts.

Notice that the "SK growth" definition in its present form is a fairly new concept, since the same term was coined long ago referring to "strained heteroepitaxy", where the strain accumulated in the islands was relaxed through misfit dislocations [Bauer1958]. Moreover, an original publication by Stranski and Krastanow was referring to the islands formation mechanism in lattice matched ionic crystals that had different charges [Stranski1937]. In the present thesis, the SK growth refers exclusively to the current view on the growth mode as described in the previous paragraph.

Recently, a significant progress has been achieved in SK growth of GaN QDs [Daudin1997, Miyamura2002, Gogneau2003], low InN mole fraction InGaN QDs [Adelmann1999, Adelmann2000] and InN QDs [Norenberg2002, Ruffenach2005, Laboutin2008]. The SK mode generally requires the use of flat and moderately lattice-mismatched substrates, while for many applications of InN QDs addressed in this thesis (as in the case of photoelectrodes) only rough and/or highly mismatched substrates are available and/or preferred.

\subsection{Droplet epitaxy}

A possible way to overcome the requirement of flat morphology for QDs fabrication by SK is to use the so called "droplet epitaxy" (DE) technique. The main idea of this method is to form metallic droplets on the substrate surface (for example In), followed by their conversion into a solid alloy (for example $\ln N$ ) by supplying the second element of the compound (N). A schematic 
representation of the DE process is presented in Fig. 3.2.5. This method requires the desired material to be, at least, a binary compound. The main advantages of DE technique over SK are:

1) The DE method does not depend on QD-substrate lattice mismatch; it is suitable for both tensile and compressive strained systems and even for lattice-matched ones [Lee1998].

2) The DE method does not require atomically "flat" substrates; both "flat" and "rough" surfaces can be used.

3) QDs without wetting layer can be achieved (however, substrate surface still undergo modification during the process).

4) Size of QDs can normally be controlled over a much wider range.

5) High quality QDs can be grown at relatively low temperatures.

The DE technique was originally developed for closely lattice matched InSb QDs on CdTe substrates at the Research Institute for Metals in Tsukuba, Japan [Koguchi1991]. Later it was applied to GaAs $Q D s$, where a sulfur surface treatment was used to prevent any two-dimensional growth [Koguchi1993]. Samples not treated with sulfur exhibited conventional 2D GaAs growth, which can be called "migration-enhanced epitaxy". However, sulfurous treatment was apparently responsible for contamination of such QDs and, consequently, the degradation of their optical quality. Later Lee et al. suggested using a LT-AIGaAs buffer containing As excess [Lee1998], which, they claimed, limited the surface migration of Ga adatoms and favored a 2D to 3D transition. Finally, Watanabe et al. [Watanabe2000] proposed a modified DE method, where GaAs QDs were grown without using sulfurous treatments or specially prepared buffers. Instead, they were relying on a low substrate temperature $\left(150^{\circ} \mathrm{C}\right)$ and a high As-flux to convert Ga droplets into GaAs islands avoiding a 2D layer. They attributed this 2D to 3D transition to the suppression of Ga diffusion by both carefully optimized As flux and substrate temperature. It must be pointed out that the measurements of the substrate temperature and elemental fluxes (thus, their precise determination) are machine dependent.

Currently, any process involving metallic droplets formation (first constituent element) followed by their conversion to 3D islands (under the flux of the second constituent element) can be referred to as $\mathrm{DE}$ method. As mentioned before, the $2 \mathrm{D}$ to $3 \mathrm{D}$ transition can be stimulated by various growth

\section{Droplet epitaxy process}

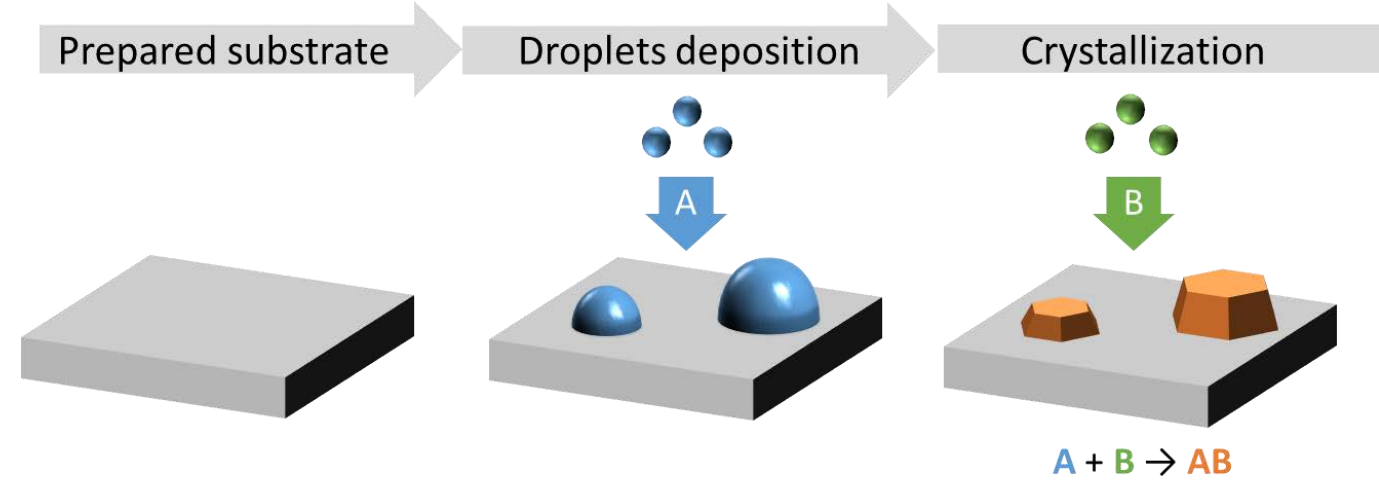

Fig. 3.2.5. Schematic representation of the droplet epitaxy process. A and B are elemental components fluxes, and $A B$ is the final compound semiconductor. 
factors including the use of a low growth temperature, special surface treatments, or modification of buffer layers surface stoichiometry. While the detailed mechanisms of DE process are not fully understood yet, it is clear that limiting the surface migration of adatoms plays a major role.

Finally, it must be emphasized that the main difference between SK/VW versus DE growth modes is that in SK/VW the 3D islands formation and the semiconductor material growth proceed "in parallel", while in the DE they proceed in two consecutive steps (i.e. in series): formation of metallic $3 \mathrm{D}$ islands and material conversion from metals into semiconductors.

There are several applications of the DE technique to grow III-N QDs. The most successful is the growth of GaN QDs on AlGaN substrates, which was demonstrated by various groups using different epitaxial techniques, including MOVPE [Tanaka1996, Gherasimova2004], $\mathrm{NH}_{3}$-assisted MBE [Kawasaki2001, Ponce2002, Maruyama2007], and PA MBE [Wu2004b, Kondo2006, Yu2014]. To decrease the Ga adatoms surface migration, two main methods have been reported: low substrate temperature [Kawasaki2001] and pre-treatment of the surface with Si [Tanaka1996]. However, the "anti-surfactant" properties of Si pre-treatment are still under debate, since the final formation of QDs can also be explained via the formation of an inhomogeneous $\mathrm{SiN}_{\mathrm{x}}$ mask on the substrate surface, which ultimately triggers the 2D to 3D transition [Tanaka1996]. This is also supported by experiments where the substrate was initially pre-treated with $\mathrm{SiO}_{2}$ which indeed triggered the 2D to 3D transition [Dimitrakis2013]. The DE method has also been applied to grow low In-content InGaN QDs [Oliver2008, Chin2013].

\subsection{Solid state dewetting}

Another interesting way to fabricate nanostructures is by solid state dewetting (DW), where a crystalline or polycrystalline film deposited on a substrate changes its shape to the one dictated by the thermodynamic equilibrium conditions (i.e. the total energy per unit volume minimization criterion) [Thompson2012]. This fabrication technique has been demonstrated for metals [Serrano2010, Sun2013] and for certain semiconductor films on various substrates, such as Si on $\mathrm{SiO}_{2}$ [Legrand2000, Jahan2005].

The driving force of the DW process, similarly to the strain induced transformations described in the previous section, is the minimization of the total interfacial energy of the free surfaces of the film $\left(\gamma_{f}\right)$, the substrate $\left(\gamma_{s}\right)$, and that of the film-substrate interface $\left(\gamma_{f_{s}}\right)$. In the case of a single crystalline island, a non-equilibrium "flat" island is deposited on the substrate at low temperature (kinetically limited deposition) as shown in Fig. 3.2.6 (a). Then the substrate temperature is raised to the point when kinetic limitations are negligible and the system can reach its thermodynamic equilibrium shape. The final shape of the island is determined by the Young's Equation:

$$
\gamma_{s}=\gamma_{f s}+\gamma_{f} \cos \theta
$$

where $\theta$ is the equilibrium contact angle (Fig. 3.2.6 (b)). If $\gamma_{s} \leq \gamma_{f s}+\gamma_{f}$, then $\theta \leq 0$ meaning that the film is going to stay $2 \mathrm{D}$ and will not dewet. If $\gamma_{s} \geq \gamma_{f s}+\gamma_{f}$, then $\theta \geq 0$ meaning then the film will collapse into a droplet shape (Fig. 3.2.6 (b)). 
Single crystalline

(a)

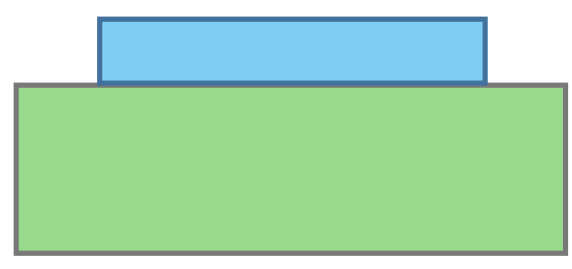

(b)

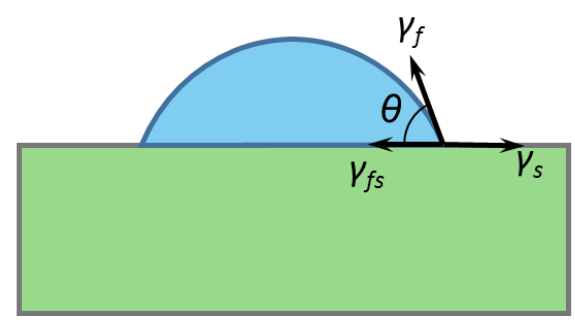

Polycrystalline

(c)

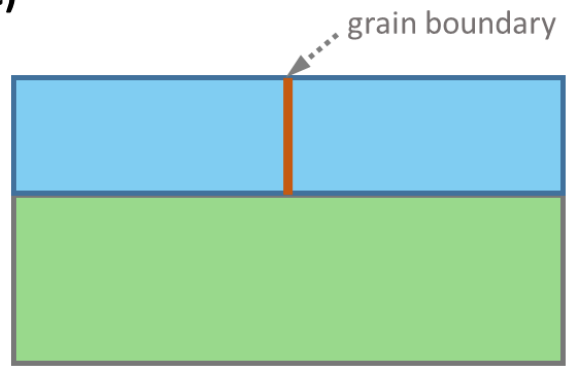

(d)

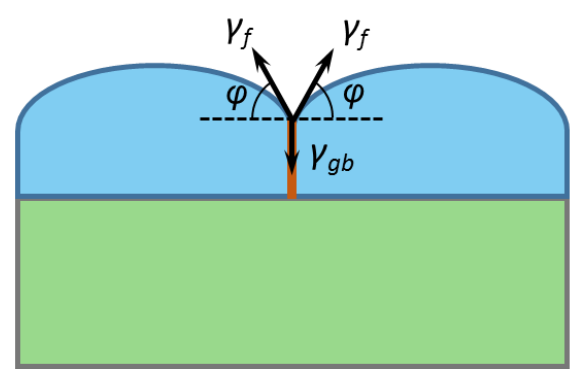

Fig. 3.2.6. Dewetting model for an initially flat single crystalline $(a, b)$ and polycrystalline (with grain boundaries) (c,d) islands. For more details refer to the text. Adapted from [Thompson2012].

If the initial island is polycrystalline with grain boundaries, their interfacial energy $\left(\gamma_{g b}\right)$ must also be accounted for. An example of such an island is shown in Fig. 3.2.6 (c). After rising the temperature, its thermodynamic equilibrium shape near a grain boundary is defined by the following equation:

$$
\gamma_{g b}=2 \gamma_{f} \cdot \sin \varphi,
$$

where $\varphi$ is the equilibrium curvature of the surface at the grain boundary (Fig. 3.2.6 (d)). In this case, increasing $\gamma_{g b}$ will result in a "groove" formation with increasing "depth". At certain values of $\gamma_{g b}$ the groove will reach the substrate surface and then the island will be split into a group of smaller islands.

This is an oversimplified model that does not take into account the fact that "deepening" the grove reduces the area of the grain boundary and thus reduces its interfacial energy. However, this model gives a rough idea on how polycrystalline islands containing grain boundaries can dewet into a group of single crystal islands. More complex models of this effect shows that there is a critical diameter of the initial island below which it will not form any groove, even in the presence of grain boundaries, but instead rearrange its atomic structure to form a single crystalline island [Srolovitz1986]. For more information refer to a review by Thompson [Thompson2012].

It was also shown both theoretically [Srolovitz1986] and experimentally [Genin1994] that even without grain boundaries, an initial island of finite size can dewet into a group of islands. In this case, 
the dewetting originates from the island periphery, which can also be viewed as an unstable region, so called Rayleigh-like instability (originally developed for liquids [Rayleigh1879]). Moreover, the strain induced by differential thermal expansion in heated films can play a significant role in the evolution of a film structure [Thompson2000]. Using such effects one could create a pattern of 3D nanostructures from initial 2D plateaus as schematically shown in Fig. 3.2.7.

(a)

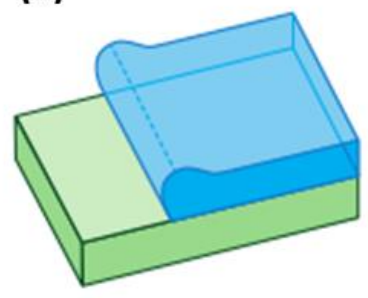

(d)

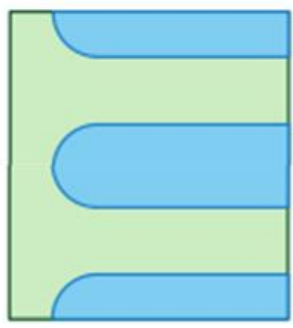

(b)

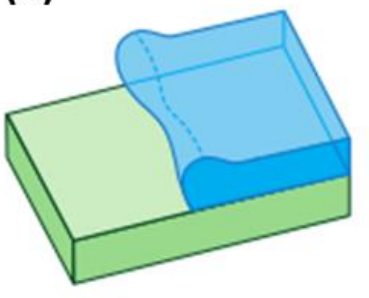

(e)

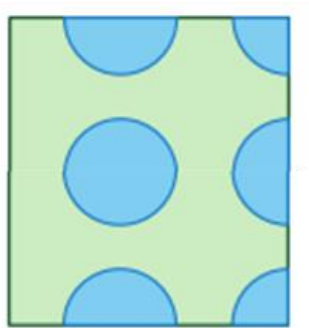

(c)

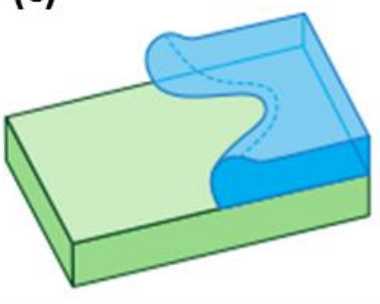

(f)

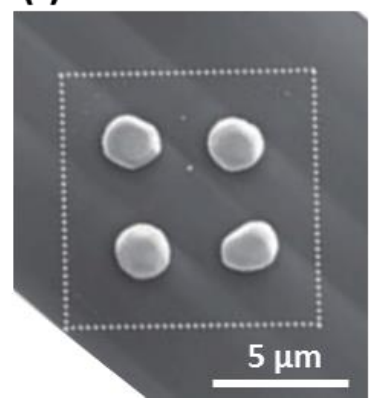

Fig. 3.2.7. Step-by-step formation of hemispherical structures from an initial 2D film via solid state dewetting driven by Rayleigh-like instability (model (a-e) from [Thompson2012] and experiment (f) from [Kim2009]). A retracting edge undergoing a fingering instability (a), then a perturbation develops in the rim of the retracting edge (b). Where the film is thinner, the edge retraction rate increases (c). Top view of the fingers that are formed (d). Top view of islands that form when the fingers break up due to a RayleighPlateau-like instability (e). SEM images of structures formed upon dewetting of an initial $30 \mathrm{~nm}$ thick Au square patterned (dashed white line) film (f).

Everything described above relates to materials with isotropic surface energy, in which the equilibrium shape is spherical. However, surface energy in crystals is anisotropic and the equilibrium shape is not spherical anymore, but defined by the so called Wulff construction. Example of a Wulff plot for a 2D crystal is given in Fig. 3.2.8 (a), where the surface energy is represented as a vector and the orientation of the crystal is represented by angles of rotation of that vector with respect to the crystal lattice [Winterbottom1967]. This $\gamma$-plot has several cusps (sharp points) at which the surface energy is minimized, which correspond to facets on the crystal equilibrium shape. The equilibrium shape can be determined by finding the envelope of lines drawn normal to the crystallographic orientation vectors at each point on the $\gamma$-curve, resulting in a faceting of the crystal surface as shown in Fig. 3.2.8 (b). This construction can also be generalized for 3D crystals [Taylor1988]. Thus, the crystalline film undergoing solid state dewetting will collapse into a single faceted island (in contrast to hemispherical droplet in isotropic materials) if its size is smaller than the critical one for groove formation, or into an agglomeration of faceted islands if it is big enough for a groove 
formation. In both cases, if equilibrium is reached (enough energy/time is supplied to the system) the final shape of the nanostructures is dictated by its Wulff plot. For III-Nitrides the Wulff plots have been fully described in the literature [Li2015, Wang2016].

(a)

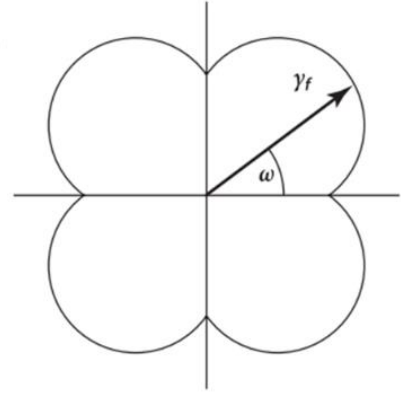

(b)

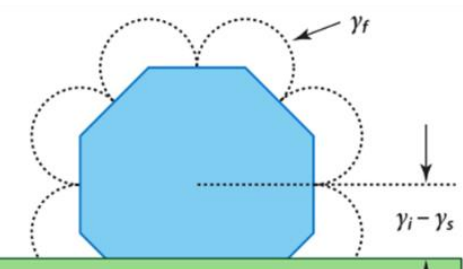

Fig. 3.2.8. A typical Wulff plot (a) and the final equilibrium shape (b) of a semiconductor crystal with anisotropic surface energy. Adapted from [Thompson2012].

\subsubsection{Fabrication of nanowires (NWs)}

Similarly to QDs, NWs can be produced either by "top-down" or "bottom-up" approaches. Taking into account that everything said about the "top down" approach for QDs can be applied to NWs, including the mentioned limitations and drawbacks (refer to section 3.2.3.1), this section is devoted to the "bottom-up" approach.

One of the earliest "bottom-up" techniques to grow NWs was the vapor-liquid-solid (VLS) epitaxy, in which NWs grow from catalyst seeds that collect the precursor species and induce a preferential growth along a given direction [Wagner1964]. Then it was extended to $\ln P, \operatorname{In} A s$, GaAs and other III-V NWs ([Wacaser2009] and references therein).

However, the field of III-Nitrides NWs attracted significant attention only after the pioneering work by Kishino and Calleja groups [Yoshizawa1997, Sanchez-Garcia1998] demonstrating that GaN NWs can be grown in a self-assembled (SA) mode by PA MBE without the need of any catalyst. The simplicity of the SA growth method and the possibility to grow defect- and strain- free GaN NWs heteroepitaxially on Si substrates with a much brighter PL emission (orders of magnitude) than 2D GaN layers [Calleja1999], triggered a great interest in this field. However, because of the random nature of the SA growth mode, such NWs show a significant spread in tilt and twist [Calleja1999, Geelhaar2011] and their size and diameter are difficult to control [Sanchez-Garcia1998, Calleja2000]. There is also a crucial drawback in terms of processing complications with such NWs for devices like LEDs.

\subsubsection{Selective area growth (SAG) of NWs}

To overcome the controllability problem of SA NWs, the selective-area growth (SAG) method (mask controlled) was developed for GaN NWs. SAG of GaN NWs was rather easy to develop for epitaxial techniques where chemical reactions between constituents were involved, such as hydride vapor phase epitaxy (HVPE) [Usui1997], metal-organic vapor phase epitaxy (MOVPE) [Nakamura1997], metal-organic molecular beam epitaxy (MO-MBE) using triethyl gallium [Guha1999] and gas-source MBE using ammonia [Tang2006]. On the other hand SAG by PA MBE has been proven rather difficult 
because of the use of the elemental fluxes. Nevertheless, Sekiguchi et al. [Sekiguchi2008], followed later by Bengoechea et al. [Bengoechea2011], demonstrated the homoepitaxial site-selective growth of GaN NWs on GaN/sapphire templates using a TiN-mask. The main factor behind SAG is the difference between Ga desorption rates from the GaN template and the TiN mask. If it is much higher on a TiN mask, a preferential nucleation of GaN will take place on the mask openings exposing the GaN template surface [Bengoechea2011]. This growth process differs considerably from the SA one, where the main driving force for the spontaneous formation of NWs is the lattice mismatch between the substrate and the NWs material [Ristic2008, Fernandez-Garrido2009].

In order to reach a SAG regime the template temperature should be high enough to inhibit the nucleation of GaN on the mask, while providing a low enough desorption rate on GaN. Another factor that depends on the growth temperature is the capability of metal adatoms to diffuse on the surface (diffusivity) until reaching a mask opening. Moreover, the active $\mathrm{N}$ flux should also be high enough to allow GaN growth within the mask opening at a reasonable rate, but low enough to avoid GaN nucleation on the mask. What this means is that too much active N will "pin" Ga atoms on the mask before they have the chance to, either reach a mask opening, or desorb. The mask openings arrangement (geometry), in terms of diameter and pitch, has substantial effects in the selection of temperature growth and active $\mathrm{N}$ flux to be used. This can be understood because a longer pitch would need a faster adatoms diffusion (temperature), while very small (or big) openings diameters would change the local III/ $\mathrm{V}$ ratio leading to morphological differences in NWs, as it is studied in detail in the following sections. A very precise control of NWs site, density and dimensions is achieved through the SAG technique [Albert2012, Gacevic2015a, Gacevic2015b]. The SAG technique has also been used later to grow ordered arrays of InGaN NWs covering the whole composition range [Albert2013, Bengoechea2014], axial and core-shell NWs nanoLEDs [Albert2015], and dot-in-a-wire nanostructures [Chernysheva2015, Lazic2015]. In the following sections the SAG technique to grow GaN NWs is described in detail.

\subsection{SAG: mask fabrication}

The first step towards SAG of NWs is the deposition of a nanohole mask on the chosen substrate. Considering that the mask material must stand rather high growth temperatures, $\mathrm{Ti}, \mathrm{SiO}_{2}$ and $\mathrm{Si}_{3} \mathrm{~N}_{4}$ are the most commonly used. The mask openings can be fabricated by different lithography techniques, including optical, e-beam, colloidal and nanoimprint lithography. In this work the SAG of GaN NWs is performed by means of colloidal lithography and Ti masking. Though not achieving a perfect periodicity, as can be seen in Fig. 3.2.9, colloidal lithography is a very easy method allowing to process wide areas ( 2 inch or more) in a relatively short time, as compared to other lithography methods, like e-beam. The thermal stability of Ti masks is achieved by nitridation to produce TiN, which is the final mask material.

The mask fabrication process developed at ISOM [Bengoechea2011] involves four main steps:

(i) Preparation of the template surface.

(ii) Deposition of colloidal nanobeads and their size reduction.

(iii) Ti coverage by metal evaporation.

(iv) Mask openings by "lift-off" (removing nanobeads).

In step (i) the GaN surface is pre-treated by a sequential immersion in solutions of negatively and positively charged poly-electrolytes, namely: poly-styrene sulfonate (PSS) and poly diallyl- 
dimethylammonium chloride (PDDA), respectively. The result is a tri-layer of PSS/PDDA/PSS with thickness of a few nanometers, covering the GaN surface.

In step (ii) a $4 \%$ solution (in weight) of negatively charged poly-styrene (PS) colloidal nanobeads is deposited on the pre-treated GaN surface by spin coating, resulting in a one monolayer coverage of hexagonally close-packed nanobeads of the GaN surface. It is schematically shown in Fig. 3.2.9 (a) and the SEM image of the real PS arrangement is presented in Fig. 3.2.9 (e). Further, an oxygen plasma treatment reduces the diameter of the nanobeads (Fig. 3.2.9 (b)).

In step (iii) a Ti layer 5 to $7 \mathrm{~nm}$ thick is deposited by e-beam evaporation (Fig. 3.2.9 (c)). It is worth to notice that such thin mask was chosen to avoid clogging the holes before nucleation takes place during the initial stage of the growth.

Finally, in step (iv) the nanobeads are mechanically removed (lift-off process) from the surface and cleaned in isopropanol (Fig. 3.2.9 (d)). Fig. 3.2.9 (f) shows the SEM image of an actual mask on GaN after completing the process described here.
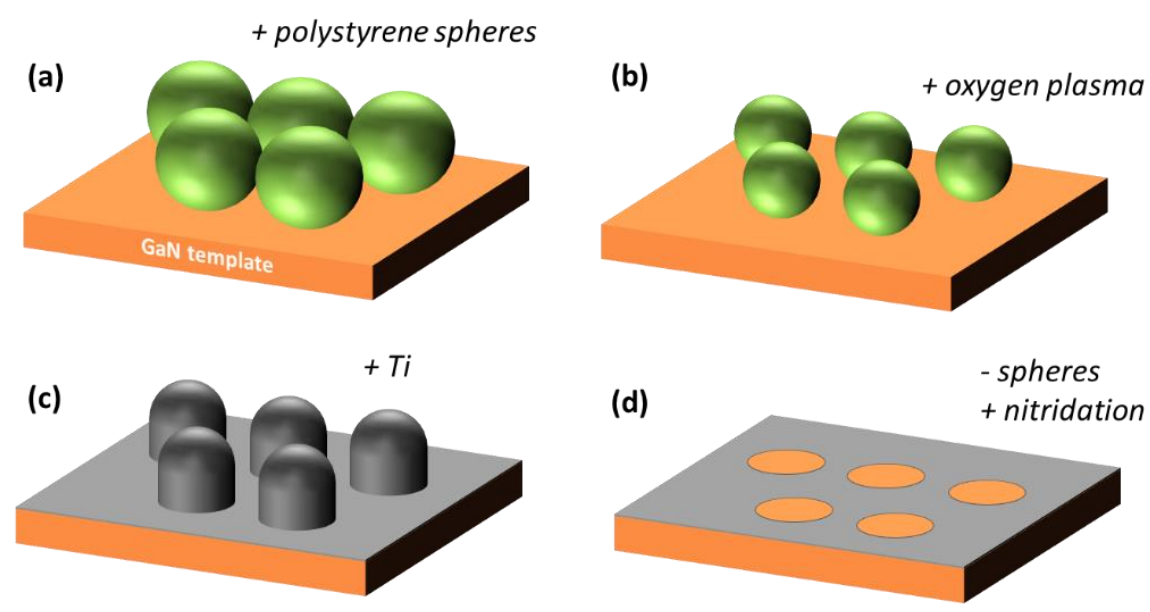

(e)

(f)
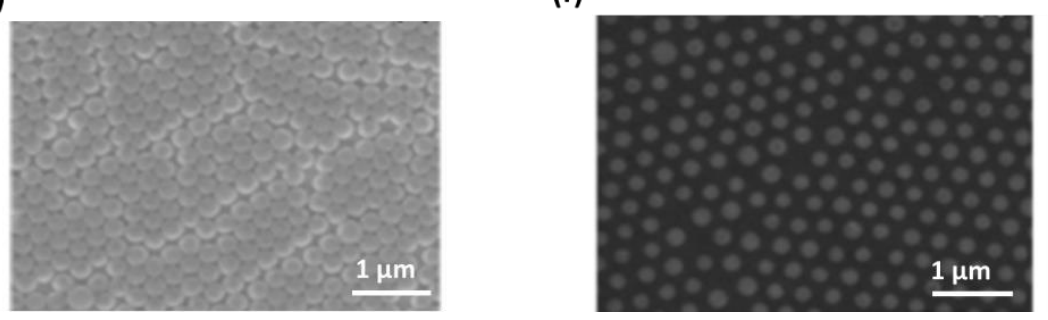

Fig. 3.2.9. Schematic mask preparation by colloidal lithography (a-d) and SEM images of the process (e-f). Pre-treated GaN surface is spin coated with PS nanobeads, forming a monolayer with hexagonal symmetry (a). SEM images taken from [Albert2014].

\subsection{SAG: growth procedure}

Once the Ti mask is prepared, the sample is loaded into the introduction PA MBE chamber and degassed at a moderate temperature $\left(300^{\circ} \mathrm{C}\right)$ for several hours. Then, the sample is transferred into the growth chamber and nitridated at $450^{\circ} \mathrm{C}$ for $30 \mathrm{~min}$ under $\mathrm{N}$ plasma $(0.5 \mathrm{sccm}, 350 \mathrm{~W})$. Next, the sample is heated up to the growth temperature $\left(\sim 765^{\circ} \mathrm{C}\right)$ still under nitrogen flux. As mentioned before, the goal of nitridation is to convert $\mathrm{Ti}$ into $\mathrm{TiN}$ to obtain a thermally stable material capable 
to stand the growth temperature (above $700{ }^{\circ} \mathrm{C}$ ). Failing to do this, the Ti mask will degrade and form a rough and irregular islands pattern making SAG impossible.

As it was mentioned before, the growth temperature for SAG must be optimized for a given material ( GaN, AIN, InGaN) and nanohole pattern (diameter and pitch), as well as depending on the mask material whereupon the Ga adatoms may have different diffusivity. All these rather strict conditions establish a narrow "growth window". Once the selected growth temperature is reached, the III/V ratio is set to Ga-rich nominal conditions. This is so when considering the impinging fluxes on the template surface, however the actual III/N ratio at the NWs top could be different due to the diffusion of $\mathrm{Ga}$ from the mask up to the NWs sidewalls and due to a high Ga desorption rate [Sekiguchi2008, Bengoechea2011, Albert2014]. The best selectivity of the growth only inside the mask is achieved at high temperatures where, due to high Ga desorption rate, the NWs vertical growth proceeds under stoichiometric conditions [Bengoechea2011].

Two main stages are considered for GaN NWs SAG, as shown in Fig. 3.2.10. The first one, kinetically driven, is the initial nucleation of very small nuclei preferentially along the nanohole inner periphery (i), followed by the nuclei "coalescence onset", yet along the nanohole inner periphery (ii), until coalescence produces a single nanocrystal that occupies the entire nanohole surface (iii). In the second growth stage, driven by free-surface-energy minimization, the formed nanocrystal undergoes a morphological evolution, from an initial cylindrical-like shape (iv), intermediate dodecagonal shape $(v)$, to a thermodynamically stable hexagonal shape (vi) [Gacevic2015a]. At this point the NW vertical growth proceeds while keeping a stable hexagonal shape.

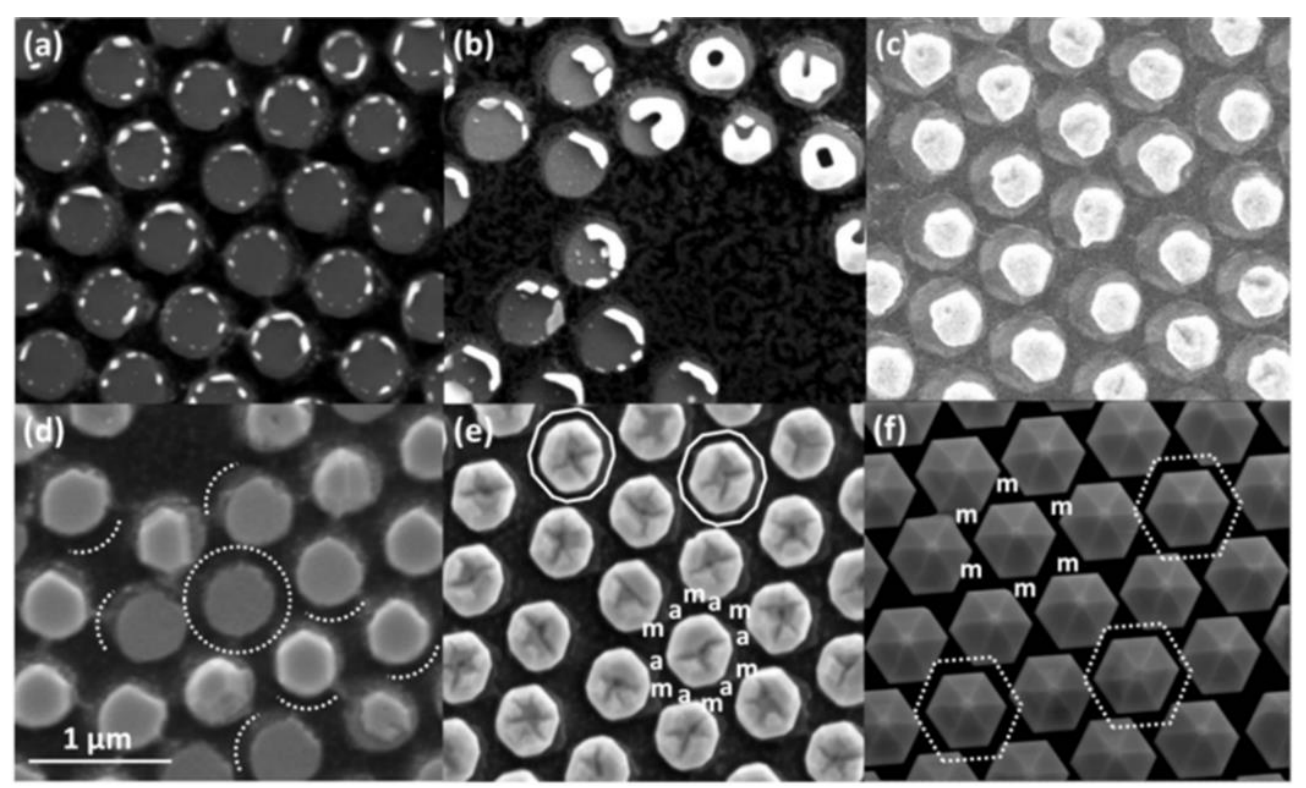

Fig. 3.2.10. Growth stages during the GaN NWs SAG evidenced by SEM. First stage: $i$ - nucleation (a); $i i$-coalescence onset (b); iii-full coalescence (c). Second stage: iv-cylindrical-like shape (d); $v$-dodecagonal shape (e); vi-hexagonal shape (f). Adapted from [Gacevic2015a]. 


\subsection{Morphological characterization}

The typical dimensions of morphology features in the samples studied in this work are in the range from tens of nanometers to several microns. Thus, high-resolution microscopy techniques, such as SEM and AFM, were used to study them and are briefly described in the following sections.

\subsubsection{Scanning electron microscopy}

In contrast to optical microscopy (OM) that relies on photons to perform a sample imaging, scanning electron microscopy (SEM) relies on a beam of high energy electrons, implying orders of magnitude better focusing and a higher resolution and magnification than OM.

A schematic representation of a SEM system is shown in Fig. 3.3.1. Its working principle is based on an electron gun emitting electrons (a tungsten filament is commonly used) which are then accelerated towards the anode to an energy ranging from $1-50 \mathrm{keV}$. The electron beam is focused by condenser lenses to a spot less than $10 \mathrm{~nm}$ in diameter. Finally, the beam passes through scanning coils that move it along $x$ and $y$ axes scanning over a rectangular area of the sample surface. When the primary electron beam interacts with the sample, the electrons lose their energy and the energy exchange mainly results in emission of:

1) Backscattered electrons (reflection of high-energy electrons by elastic scattering).

2) Secondary electrons (produced by inelastic scattering).

3) Electromagnetic radiation (X-rays, cathodoluminescence).

Each of whem can be detected by specialized detectors.

\section{Scanning Electron Microscopy}

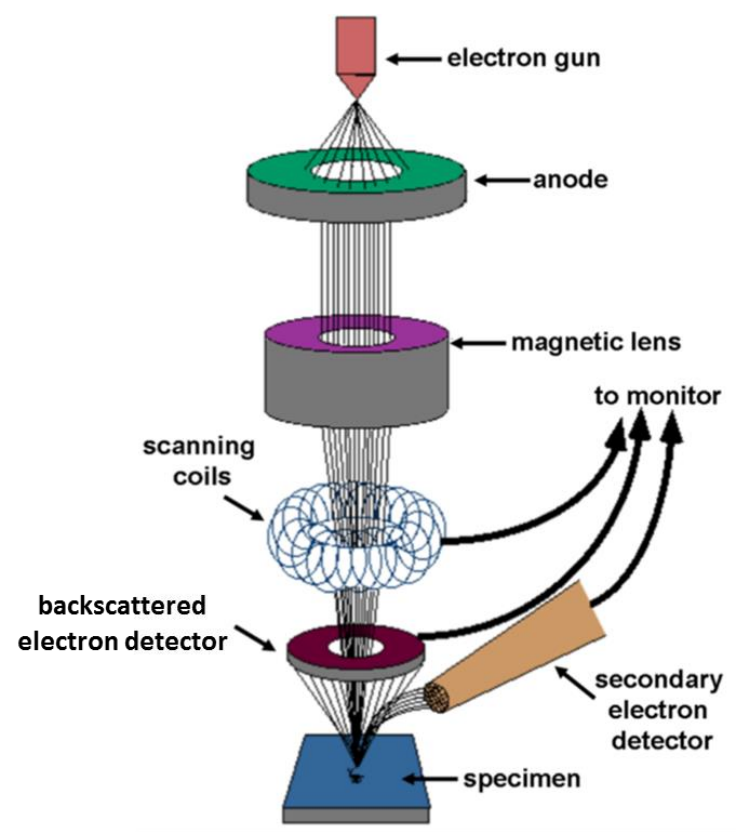

Fig. 3.3.1. Principle scheme of a scanning electron microscopy. Adapted from https://www.purdue.edu/ehps/rem/rs/sem.htm 
Secondary electrons aalyses were mainly used in this work, which allows probing the sample surface topology because secondary electrons originate from the very sample surface (10 to $100 \mathrm{~nm}$ deep). Positions like kinks and edges from which electrons can escape easily are especially highlighted on the image. Thus, very precise (resolution of several nanometers) topological maps of the sample can be constructed. For SEM analysis a FEI Inspect F50 system has been used.

\subsubsection{Atomic-force microscopy}

Atomic-force microscopy (AFM) is a high-resolution topology mapping technique based on the measurement of the force between the probe and the sample surface. The probe, also called cantilever, has a sharp tip with the radius of curvature of the order of ten nanometers, usually made of $\mathrm{Si}$ or $\mathrm{Si}_{3} \mathrm{~N}_{4}$. In an AFM setup the sample is placed on a piezoelectric tube that can move it in 3 axes with a very high precision. When a sample surface is brought into proximity of the tip, forces between the sample and the tip lead to a deflection of the cantilever according to Hooke's law. This can be detected by a photodiode measuring the reflection of a laser beam, as shown in Fig. 3.3.2. In most cases this force is kept constant by a feedback mechanism which results in a constant distance between the two along the $z$ direction while the scan goes in the $(x, y)$ plane. The resulting map of the area $z=f(x, y)$ represents the topography of the sample.

There are three common AFM operation modes: contact, non-contact and tapping. In this work tapping mode was employed. In this regime the cantilever is driven to oscillate near its resonant frequency with typical amplitudes of 100 to $200 \mathrm{~nm}$. The amplitude change due to the force interaction with the sample surface can be detected and used to map the surface topology.

AFM measurements in this thesis have been performed in tapping-mode with a Digital Instruments MMAFM-2 AFM system. Obtained results were analyzed in Gwyddion v2.45 software.

\section{Atomic Force Microscopy}

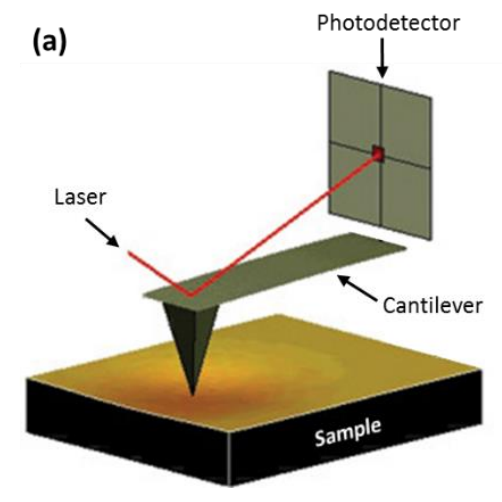

(b)

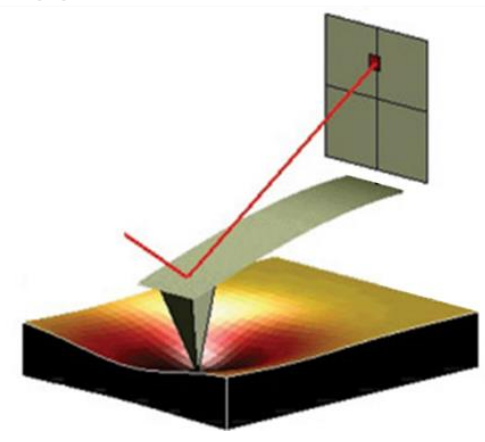

(c)

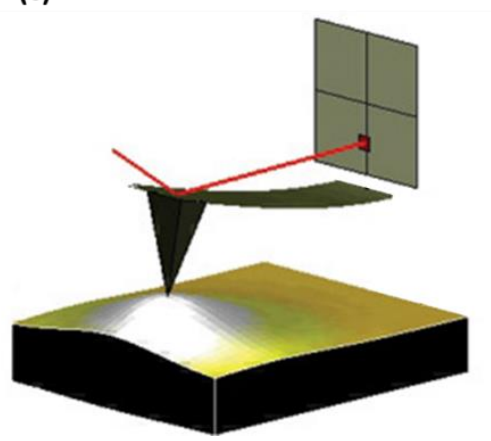

Fig. 3.3.2. Basic principles of atomic force microscopy showing change of the reflected laser beam position on the photodetector upon resolving surface curvature. Adapted from https://www.asylumresearch.com/Applications/PFMAppNote/PFMAppNote.shtml 


\subsection{Structural characterization}

\subsubsection{High-resolution $X$-ray diffraction}

High-resolution X-ray diffraction (HRXRD) is a powerful technique to characterize structural properties of crystalline films, such their composition and strain.

Crystals are regular three dimensional arrays of atoms and when excited by X-rays, they produce secondary spherical waves that interfere constructively at specific angles. A schematic diagram of $X$-ray diffraction on crystalline planes is shown in Fig. 3.4.1. Constructive interference takes place when the optical path length difference between the beams (with incident angle $\theta$ ) diffracted from two consecutive crystal planes (with the spacing $d$ ) equals to integer number of incident beam wavelengths $(n \lambda)$ :

$$
2 d \sin \theta=n \lambda,
$$

This is known as Bragg's diffraction law. Thus knowing the X-ray wavelength and the incident angle the crystal planes separation i.e. the lattice constant can be determined.

In case of wurtzite crystals, one-dimensional $\omega / 2 \theta$ HRXRD measurements around a symmetric plane, such as [0002], provides information about the $c$ lattice parameter. If measurements are performed around an asymmetric plane, such as [1015], then information about both $c$ and $a$ lattice parameters is obtained by using linear elasticity theory (refer to section 2.2.2). In this case, the coordinates of the [1015] peak maximum $\left(q_{x}, q_{y}\right)$ are related to $a$ and $c$ parameters as follows:

$$
\begin{aligned}
& a=\frac{\sqrt{3}}{3} \frac{\lambda}{q_{x}}, \\
& c=\frac{5}{2} \frac{\lambda}{q_{y}},
\end{aligned}
$$

The $a$ and $c$ parameters of ternary alloys can be estimated by Vegard's law from the binary ones as:

$$
\begin{aligned}
& a(x)=x \cdot a_{\text {InN }}+(1-x) \cdot a_{G a N}, \\
& c(x)=x \cdot c_{\text {InN }}+(1-x) \cdot c_{G a N} .
\end{aligned}
$$

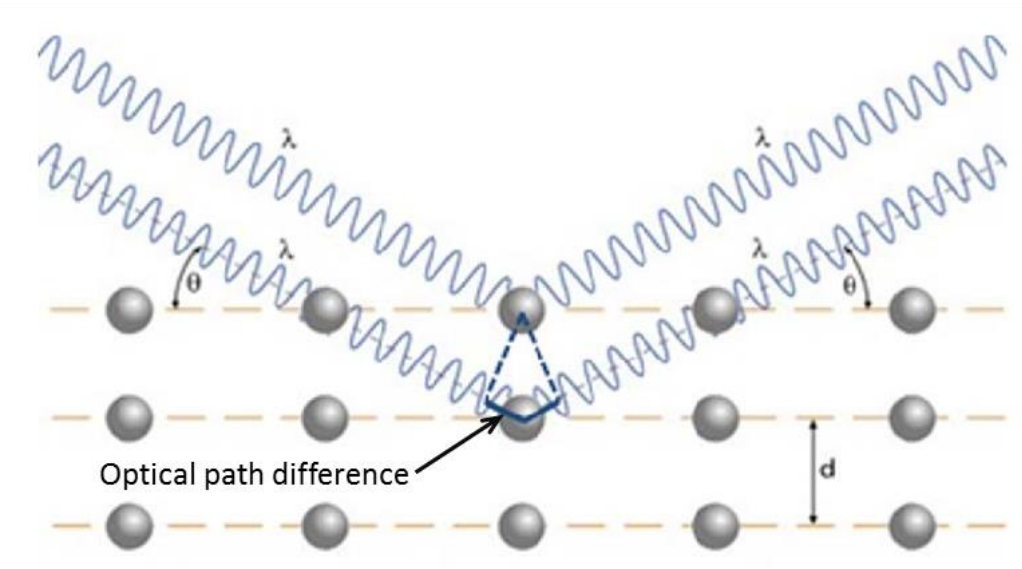

Fig. 3.4.1. Schematic diagram of X-ray diffraction. Adapted from [Gacevic2012]. 
Making use of linear elasticity theory and numerically solving Eq. 2.2.10 from section 2.2.2, which for convenience is presented here:

$$
\frac{c-c_{\text {relax }}(x)}{c_{\text {relax }}(x)}=-2 \frac{C_{13}(x)}{C_{33}(x)} \frac{a-a_{\text {relax }}(x)}{a_{\text {relax }}(x)},
$$

the alloy composition $x$ and strain in the layer can be determined. Note that $C_{13}(x)$ and $C_{33}(x)$ are estimated according to Eq. 2.2.11 and Eq. 2.2.12.

\subsubsection{Transmission electron microscopy}

Similarly to SEM, transmission electron microscopy (TEM) operates by using an electron beam, but in transmission mode, so the electrons go through the material with little to no interactions, due to their short mean free path. If a collection of parallel electrons interact continuously with the sample (TEM) it may imply some differences that if a rastering convergent beam scans the region of interest, called then scanning-transmission electron microscopy (STEM). Thus, the sample must be specially thinned in order to be studied by TEM, usually by a mechanical thinning process, down to $1 \mu \mathrm{m}$, followed by Arion milling until the layer is about $100 \mathrm{~nm}$ thick. In addition, compared to SEM, higher acceleration voltages are used $(80-300 \mathrm{keV})$ in TEM leading to the picometer range of the De Broglie electrons wavelength which allows to study solids down to nanoscale (typical resolution of $\sim 0.1 \mathrm{~nm}$ ).

TEM is based on the scattering of electrons within the crystal atomic structure. Diffraction patterns (reciprocal space) and images (real space) can be recorded at the diffraction plane (back focal plane of the electromagnetic objective lens) and at the object plane, as shown in Fig. 3.4.2, which defines diffraction and imaging modes, respectively. Samples can be oriented so that one or more particular crystallographic planes interact stronger with the beam increasing the amount of electrons in the corresponding "excited" and diffracted feature (ring, spot, etc.), which would allow to study in more detail the material, particularly regarding to such family of planes. In this way TEM results are sensitive to the differences in sample thickness, distortion of crystal lattices due to structural defects, strain, bending and composition. The focused image or diffraction pattern will be later magnified at a phosphor screen or CCD camera by a series of intermediate and projector lenses that are further located down through the TEM column.

There are two main modes to be considered when working with a (S)TEM microscope:

- TEM. In this mode, a wide incidence of parallel electron beams and its Bragg's refraction with a sample are used to register diffraction patterns or contrast imaging modes. Hereafter, among many possibilities, only the techniques used in our studies are briefly described: CTEM and HRTEM micrographs and SAED patterns.

- Conventional TEM (CTEM). It allows to form images by selecting one particular beam, which have either passed through the material without interacting with it (directly transmitted beam), or been elastically scattered, under Bragg conditions, by the electronic charges in the material (diffracted beams). If the direct beam is the only one selected, the contrast in the image is proportional to the density of the materials themselves. If a diffracted beam, that could be excited depending on the crystal orientation, is selected at the same time that the direct beam, an image that highlight certain atomic planes or structural defects, will be formed. This is the so-called two-beams diffraction-contrast mode. This way of operation 
promotes micrographs with a clear background and dark contrasts associated to densities, interfaces, extended structural defects and other present crystalline features, being called bright-field CTEM mode (BF). It is possible in diffraction-contrast techniques to exclude the (000) transmitted beam to pass through the objective aperture, thus forming micrographs with a shade background and enhanced bright features associated to the excited diffraction spots, which is the dark-field (DF) mode.

○ High-resolution TEM (HRTEM). In this mode, also known as phase-contrast imaging, a micrograph is formed by the acquisition of several, if not all, transmitted beams (direct and diffracted), using a large objective aperture. Since transmitted electrons experience different forces depending on their passing distance to the sample atoms nuclei, their wave phase differences can be used to form contrasts in an atomically resolved image, i.e. a very highresolution (down to $0.1 \mathrm{~nm}$ ). Therefore, phase-contrast images are interpreted as images of the atomic columns in a single crystalline portion of a material. HRTEM has been extensively and successfully used for analyzing crystal structures and lattice imperfections of various kinds in materials at bond length scale. It can be used to characterize point defects, stacking faults, dislocations, precipitates, grain boundaries, and surface structures.

- Selected area electron diffraction (SAED): Any image has a related electron diffraction (ED) pattern that can be registered if the mentioned back focal plane of intersection of converging beams is focused and magnified. If a diaphragm is used to allow the event of diffraction of a certain region of interest in prepared specimen, selected-area electron diffraction (SAED) patterns will arise. As a result, diffuse discs (amorphous), rings (polycrystalline) or periodical two-dimensional arrangements of spots (single-crystalline) may give information about crystal geometry, lattice parameters, presence of defects, etc.

- Scanning TEM (STEM). In this technique an incident electron beam is focused (as the conical beam of the SEM probe) into a narrow spot which is scanned over the sample surface. Similarly to parallel beam TEM, the data is collected after the electron beam has passed through the material. In this way, chemical and structural information of the material can be extracted with a high spatial resolution. Both image (BF and DF) and spectroscopic techniques were applied in this mode.

- Annular Bright Field (ABF): An annular detector is placed within the Bright Field cone which, in cases of an aberration-corrected microscope, might allow a direct imaging of light elements. Because it can detect both light and heavy elements, this technique is ideal to show, with images, the polarity of some crystals.

- High Angle Annular Dark Field (HAADF): Imaging mode in Dark Field conditions with an annular detector of very high angles (about $25 \mathrm{mrad}$ ) at which Rutherford electrons (unelastically scattered, at larger angles than diffracted beams) can be exclusively collected. The intensity of the signal is proportional to $\sim t \cdot Z^{2}$, where $t$ is the thickness of the sample and $Z$ the average atomic number, so the contrast can give compositional information. However, due to the brightness promoted by heavy elements nearby, even in an aberration-corrected microscope, light elements, such as nitrogen, are difficult to detect by this technique.

- Energy dispersive X-ray spectroscopy (EDX): This technique is not exclusive to STEM microscopes, and can be also utilized in SEM and TEM. Thus, it is discussed in the next section as a separate characterization tool. 


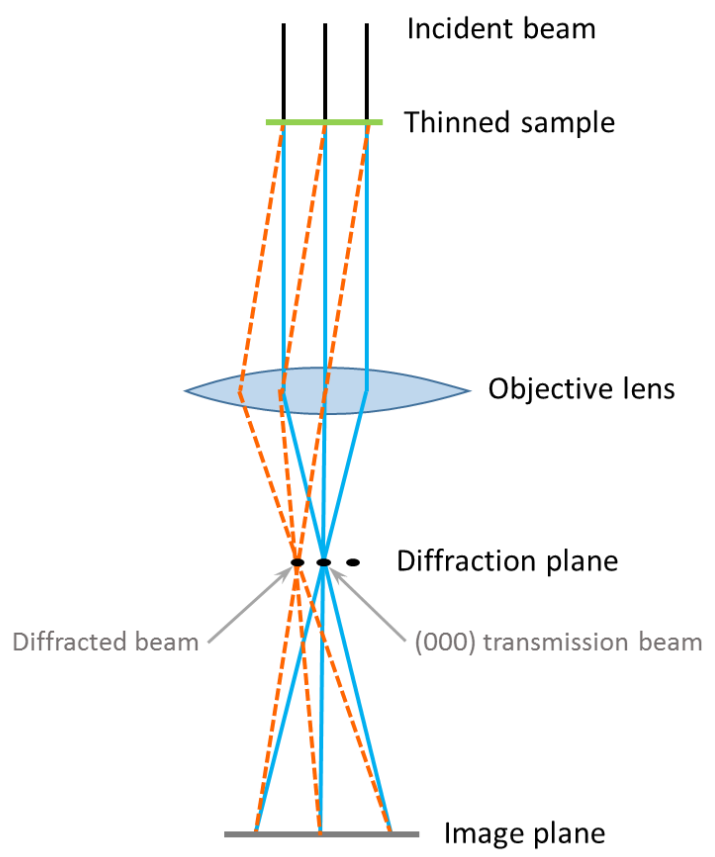

Fig. 3.4.2. Rays diagram of the electron beam paths during TEM operation. Incident (black solid line), transmitted (blue solid line) and Bragg scattered (orange dotted line) electron beams are indicated. Diffraction/imaging modes correspond to measuring in back focal/object planes. Objective is an electromagnetic lens. Adapted from [Williams1996].

In this work detailed structural and chemical analyses of $\ln _{x} \mathrm{Ga}_{1-x} N$ layers and $\operatorname{InN}$ QDs were performed using a JEOL-1200EX microscope with electrons emitted by thermionic effect (with a tungsten filament) and accelerated at a $120 \mathrm{kV}$ voltage, and a JEOL-2010F, with electrons obtained through a $\mathrm{LaB}_{6}$ Field Effect Gun and accelerated at $200 \mathrm{kV}$. The achievable spatial resolution with the JEOL-2010F is $0.19 \mathrm{~nm}$. For a TEM analysis the samples were prepared in two different orientations: cross-section (XTEM) in which the growth and one of the basal directions could be observed, and plain-view (PVTEM) in which the whole basal plane is perpendicularly observed. The samples were thinned to electron transparency by mechanical grinding, polishing, and Ar ion milling at $3 \mathrm{keV}$ with a Gatan low-voltage precision ion polishing system. CTEM and SAED experiments were performed in the JEOL 1200EX microscope, while HRTEM and STEM results were collected in the JEOL 2010-F microscope operated at $200 \mathrm{keV}$.

Structural TEM characterization of GaN NWs was performed with a JEOL JEMARM200cF electron microscope (cold emission gun) operating at $200 \mathrm{kV}$, equipped with a spherical aberration corrector in probe (current density $\sim 1.4 \times 10^{-9} \mathrm{~A}$ and probe size of $\sim 0.08 \mathrm{~nm}$ ). Polarity was monitored by imaging $\mathrm{Ga}$ and $\mathrm{N}$ atomic columns by atomically-resolved annular bright field scanning TEM (ABFSTEM) with a nominal camera length of $60 \mathrm{~mm}$ and inner and outer collection semi-angles of 11 and 22 mrad, respectively. 


\subsubsection{Energy dispersive X-ray spectroscopy}

Energy dispersive X-ray spectroscopy (EDX) is another technique that provides information on the sample chemical composition and it can be performed both in SEM and STEM systems. The EDX is based on the detection of X-ray emission generated from the sample subjected to a high energy electron beam. The incident beam may excite an electron from an atom inner shell creating a hole at the location of the left electron. An electron from an outer shell is able to fill in the hole, and the difference in energy between the higher-energy shell and the lower energy shell is released as $X$ rays. The intensity and energy of the emitted X-rays can be measured by an energy-dispersive spectrometer. Different atoms in the sample are identified by a unique set of peaks of their X-ray emission, because each atom in the periodic table of elements has a unique atomic structure. Composition of alloys then can be easily identified simply by comparing the intensities of constituent atoms on EDX spectra.

In this work, EDX measurements were done in a INCAPentaFET-x3 module attached to the JEOL2010F microscope. A $1 \mathrm{~nm}$-sized high-resolution STEM probe with a scanning area of the sample of $5 \times 5 \mathrm{~nm}^{2}$ was used.

\subsection{Optical characterization}

The two main optical characterization techniques used in this work were photoluminescence (PL) spectroscopy and scanning near-field microscopy (SNOM). PL was used to assess the optical bandgap, while SNOM determined spatial homogeneity of PL signal with high resolution.

\subsubsection{Photoluminescence}

$\mathrm{PL}$ is the process in which photons from a sample are emitted upon excitation (absorption) with monochromatic photons with above bandgap energy from an external source (laser). During the process of light absorption electron-hole pairs are generated. These excited carriers thermalize to the corresponding band edges by phonon interaction in a very fast process $\left(10^{-15}\right.$ to $\left.10^{-12} \mathrm{~s}\right)$. Carriers will remain in the ground state for a longer period $\left(10^{-9}-10^{-6} \mathrm{~s}\right)$ until they recombine, either radiatively or non-radiatively. Radiative recombination can be directly band-to-band leading to emission of photon with energy close to material bandgap or mid-gap energy states mediated (due to the presence of impurities or defects in the crystal). Non-radiative (detrimental) recombination happens when excited carriers recombine without emission of any photons solely through interaction with phonons on defect states (poor material quality).

PL gives information about the crystal quality, composition and optically active defects. Usually, low temperature (LT) is used during PL measurements to enhance excitonic emissions because the nonradiative recombination processes are highly reduced, while RT measurements are needed to estimate the radiative channels efficiency (LT to RT intensity ratio).

A typical PL setup is schematically shown in Fig. 3.5.1 and in this thesis two separate setups were used to measure in UV-Vis and IR ranges. The excitation source used in UV-Vis PL was a continuous He-Cd laser emitting at $325 \mathrm{~nm}$ with $30 \mathrm{~mW}$ power, whereas a $1 \mathrm{~W}$ diode laser emitting at $781 \mathrm{~nm}$ was used for IR PL. The light emitted from the sample is collected by series of two lenses and then analyzed using a Jobin-Yvon THR 1000 monochromator and Horiba Jobin-Yvon DPM-HV 
photomultiplier for UV-Vis PL and a SPEX 1702/04 monochromator and Ge detector for IR PL. The sample is mounted in a cryostat (Sumitomo CH-204SFF with a HC-4A compressor) which allows performing measurements at temperatures ranging from 7 to $300 \mathrm{~K}$.

\section{Photoluminescence setup}

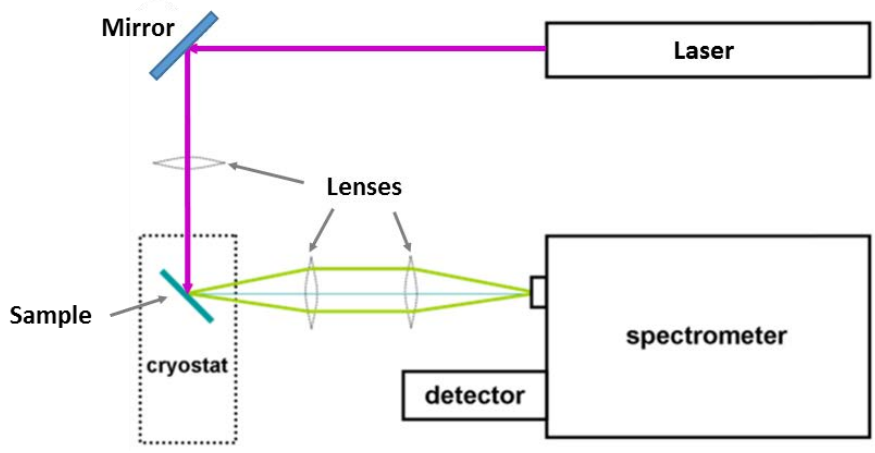

Fig. 3.5.1. Principle scheme of the PL setup. Adapted from [Albert2015b].

\subsubsection{Scanning near-field optical microscopy}

The ability to resolve two distinct objects by traditional optical microscopy (OM) is fundamentally limited by the diffraction of light because it is collected in a far field. Resolution of standard OM is determined by $\lambda / 2 N_{A}$, where $\lambda$ is a wavelength of light and $N_{A}$ is optical aperture of the microscope. This parameter cannot be improved by improving quality of the lenses involved.

In 1928 E.H. Synge [Synge1928] proposed a method to overcome the diffraction limit by measuring the evanescent waves with very thin optical fiber probe. This method is based on that if the probe's aperture is smaller than $\lambda$, then its photons collection area has a very narrow lateral size in close proximity to it (near-field) compared to longer distance (far-field), as schematically shown in Fig. 3.5.2 (a). When the sample is scanned with such probe in a close distance to the surface then, even if surface emission centers are placed at a lateral distance closer than $\lambda$, their PL signal still can be resolved with a resolution determined by the probe's aperture size (typically $50-100 \mathrm{~nm}$ ) (Fig. 3.5.2 (b)). This technique is called scanning near-field optical microscopy (SNOM) and it is based on the AFM setup with a specially prepared cantilever which has a hole inside of a small diameter connected to a photodetector through an optical fiber. This method allows simultaneous highresolution measurements of surface topology and spatial PL emission.

In this work, SNOM measurements were performed at $10 \mathrm{~K}$ under excitation of a $543 \mathrm{~nm} \mathrm{He}-\mathrm{Ne}$ laser coupled into an uncoated single mode near-field fiber probe resulting in an excitation power of $3.9 \mu \mathrm{W}$. The PL signal was collected through the same fiber, dispersed in a monochromator and recorded with a liquid-nitrogen-cooled InGaAs detector, providing a spatial resolution of $\sim 150 \mathrm{~nm}$. Further details about the SNOM setup can be found in [Behme1997, Intonti2001]. 


\section{Scanning Near-Field Optical Microscopy}
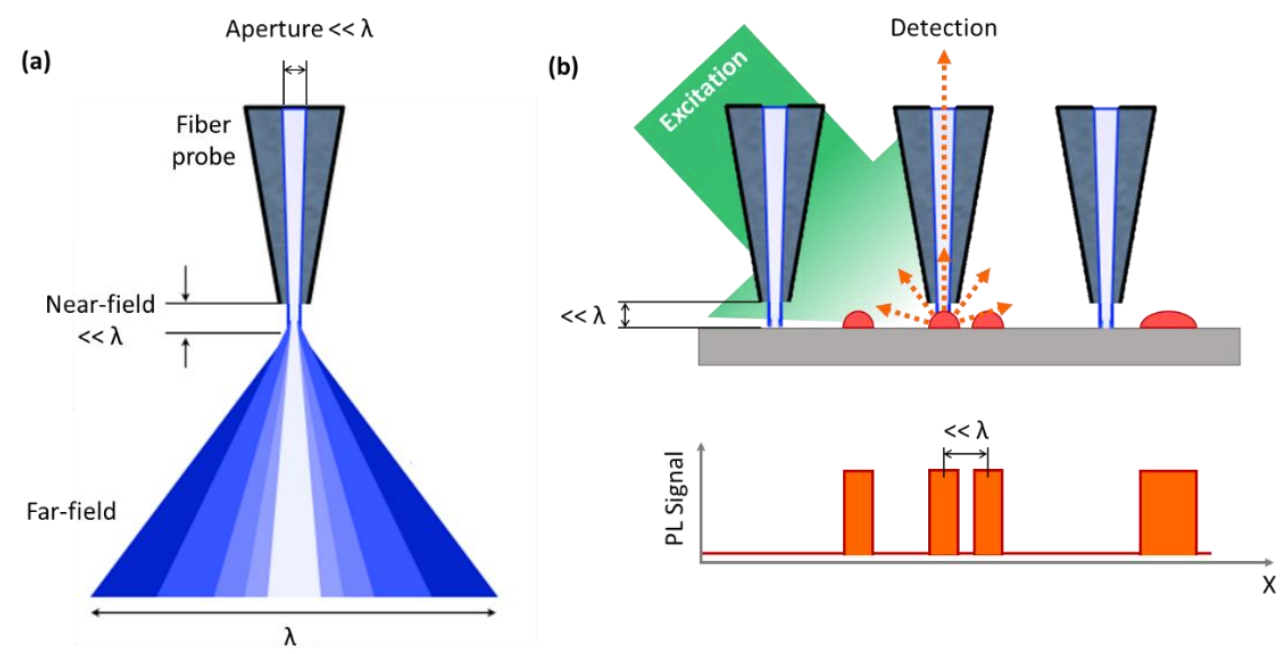

Fig. 3.5.2. Working principles of SNOM imaging. Photons collection area (blue) of a fiber probe (a). Scanning with SNOM across the sample under constant external excitation (b) where PL signal from the emission centers (red dots) can enter the fiber probe only when it is originated inside the probe's collection area. Note that for simplicity amplitude of PL signal from the emission centers is presented as constant. Adapted from [Herzog2011].

\subsection{Photoelectrochemical setup}

The PEC water splitting process is a combination of solar energy harvesting and the electrolysis of water in one device. In a real semiconductor-based water splitting device both anode and cathode electrodes should be semiconductors, each enhancing the corresponding half-reaction under illumination. However, in this case it is impossible to determine the anode and cathode efficiency separately. Thus, the use of a 3-electrode setup consisting of a working, counter and reference electrodes allows evaluating the efficiency of the working electrode independently from the counter electrode by fixing the potential of the former versus a known value of the reference electrode potential. For example, for a semiconductor-based photo-anode such setup should also contain a metal-based counter electrode and a reference electrode suitable for the chosen electrolyte solution. Using a potentiostat, the working electrode is set at a fixed potential versus the reference electrode and the current is measured at this potential. To obtain a current-voltage plot of the photo-electrode, the potential versus the reference electrode is scanned and the current monitored as a function of this potential scan. In this type of measurement, the counter electrode performance is not important. The potentiostat maintains the potential of the photoelectrode at the desired value and reads out the current at that potential. It supplies whatever voltage between the photoelectrode and counter electrode is necessary in order to maintain that current.

In this work, all of the samples are n-type semiconductors, thus, according to the theory of S/E interfaces discussed in section $\mathbf{2 . 6}$ they are all photoanodes. The schematics of a laboratory PEC cell are shown in Fig. 3.6.1. A Pt mesh is used as counter electrode was used, being the most frequently 


\section{Photoelectrochemical setup}

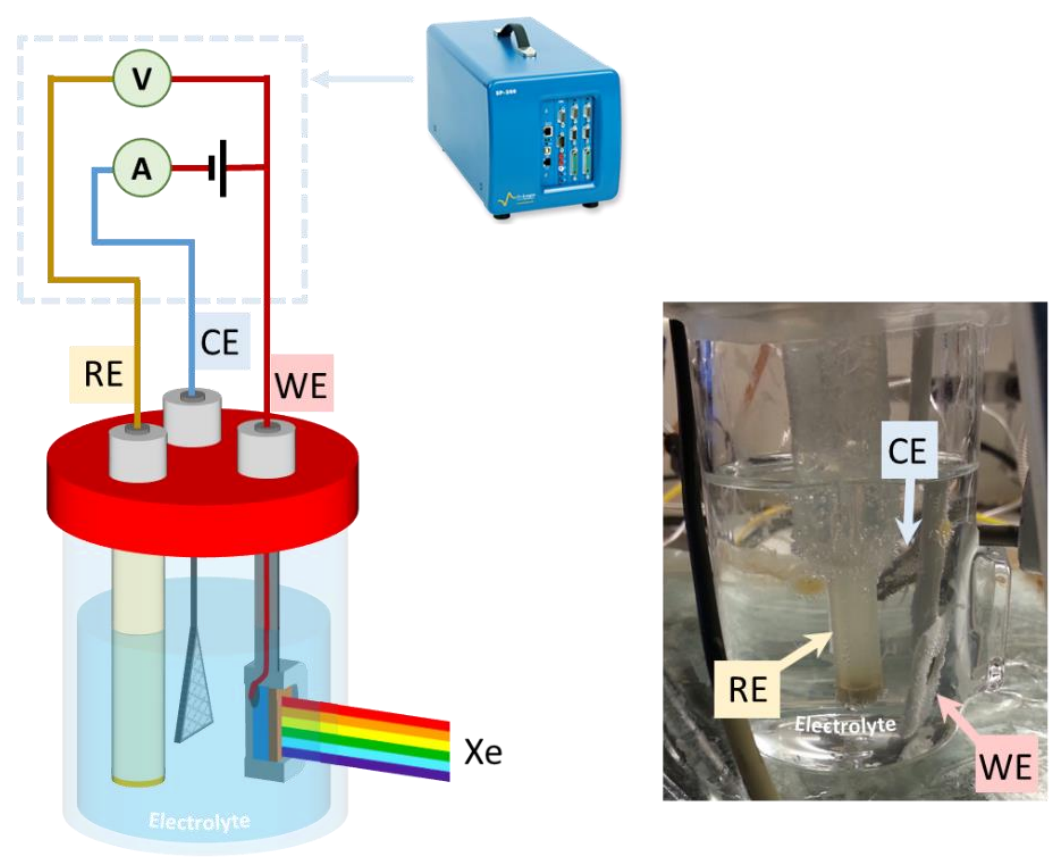

Fig. 3.6.1. Schematic representation of the 3-electrode phototelectrochemical setup (left) with the actual picture of it (right). RE, CE, and WE - are reference, counter, and working electrodes, respectively.

used counter electrode due to its exceptional corrosion resistance. A mesh, instead of a wire, was used to increase the surface area of the Pt counter electrode available for hydrogen evolusiaction (HER) to proceed. Depending on the electrolyte used, different reference electrodes were considered (to be discussed in detail later). The system consists of a PEC cell and a potentiostat (BioLogic VSP, EC lab software) as bias source.

In a three electrode setup, the potential of the working electrode is measured versus the reference one $\left(E_{W E} v s\right.$ Ref $)$. For an easier comparison of the results to those one from the literature, the potential should be referenced to the reversible hydrogen electrode (RHE), $E_{W E} v S R H E$ using the following equation:

$$
E_{W E v s R E}=E_{W E v s e f}+E_{R e f}+0.059 \cdot p H=E_{W E v s R e f}+E_{R e f_{-} p H}
$$

where $E_{\text {Ref }}$ is the reference electrode versus RHE at pHO and $E_{\text {Ref_pH }}$ the reference electrode potential versus $\mathrm{RHE}$ including the $\mathrm{pH}$ dependence correction. In the case of acidic electrolytes like $\mathrm{Hg} / \mathrm{Hg}_{2} \mathrm{SO}_{4}$ (VWR International) reference electrodes were used. For PEC experiments in alkaline electrolytes, the reference was a Teflon incased $\mathrm{Hg} / \mathrm{HgO}$ (Koslow Scientific Company). Table IV contains detailed information on the potentials of these reference electrodes. 
Cyclic voltammetry (CV) and Chronoamperometry (CA) measurements in this work were done with $\mathrm{H}_{2}$ gas bubbling of working electrode compartment to fix the redox potential of the electrolyte around HER potential.

In alkaline media, glass corrodes and the arising contaminants and/or poisoning of the electrodes (mainly due to silica and lead) [Mayrhofer2008] can significantly influence the hydrogen oxidation reaction activity therefore a quartz glass cell was used for alkaline electrolytes. Before starting each experiment all glassware was cleaned using a freshly prepared Aqua regia solution $\left(\mathrm{HCl}: \mathrm{HNO}_{3}=3: 1\right.$ volume ratio). In all experiments requiring the preparation of aqueous solutions, deionized (DI) water (Millipore water, $18.2 \mathrm{M} \Omega$ ) was used.

Table IV. Potential of the reference electrodes used in this work. $E_{\text {Ref }}$ - potential vs. RHE at pHO, $E_{\text {Ref_pH }}-$ potential vs. RHE including pH dependence correction.

\begin{tabular}{cccc} 
Electrode & Filling solution & $\boldsymbol{E}_{\text {Ref }}$ & $\boldsymbol{E}_{\text {Ref_pH }}$ \\
\hline \hline $\mathrm{Hg} / \mathrm{HgO}$ & $4 \mathrm{M} \mathrm{KOH}^{\text {poH }}$ & 0.054 & $0.88^{\text {pH14 }}$ \\
$\mathrm{Hg} / \mathrm{Hg}_{2} \mathrm{SO}_{4}$ & $0.5 \mathrm{M} \mathrm{Hg}_{2} \mathrm{SO}_{4}$ & 0.680 & $0.68^{\mathrm{pHO}}$ \\
$\mathrm{Ag} / \mathrm{AgCl}$ & $3 \mathrm{M} \mathrm{KCl}$ & 0.210 & $0.21^{\mathrm{pHO}}$
\end{tabular}

A 1000 W Xenon lamp (Oriel) was used as a light source, with an AM1.5G filter to simulate the standard solar spectrum for all experiments in this work. Before each experiment, the light intensity reaching the sample surface through the optical fiber was measured with a spectrometer (International Light Technologies Inc, RPC 900-R) and its integrated irradiance was adjusted (via change of the focal length) to match that of the white light of the AM1.5G solar spectrum (100 $\mathrm{mW} \mathrm{cm}{ }^{-2}$ ). An example of this calibration is given in Fig. 3.6.2 (a) where the irradiance of a Xenon lamp is plotted together with the AM1.5G solar spectrum. In order to convert the irradiance $(E)$ into the theoretical maximum photocurrent density which can be obtained at a given wavelength $(\lambda)$ the photon flux $\left(N_{p}\right)$ must be calculated first:

$$
N_{p}(\lambda)=\frac{E(\lambda) \cdot \lambda}{h \cdot c}
$$

where $h=6.63 \times 10^{-34} \mathrm{~W} \mathrm{~s}^{2}$ is the Plank constant and $c=2.998 \times 10^{10} \mathrm{~cm} \mathrm{~s}^{-1}$ is speed of light. Then the theoretical maximum photocurrent density at a given wavelength can be calculated from

$$
J_{\max }(\lambda)=\int_{0}^{\lambda} \frac{N_{p}(x)}{q} d x
$$

where $q=6.24 \times 10^{18} \mathrm{C}$ is the elemental electric charge. The curve of theoretical maximum photocurrent for the Xenon lamp used in this work is presented in Fig. 3.6.2 (b).

In this work, because of the use of samples with different bandgaps, the integral external quantum efficiency (EQE) values rather than the actual photocurrent densities must be used to compare various samples. EQE is defined as the ratio between the number of collected carriers (measured photocurrent density) and the number of incident photons with wavelength below the absorption edge $\left(\lambda_{a b s}\right)$ of the photoanode:

$$
E Q E=\frac{J_{p h}}{J_{\max }\left(\lambda_{a b s}\right)}
$$


Thus, EQE values can be easily recalculated from the photocurrent densities if the spectral irradiance distribution of the light source is known in the experiments.

\section{Light source calibration}
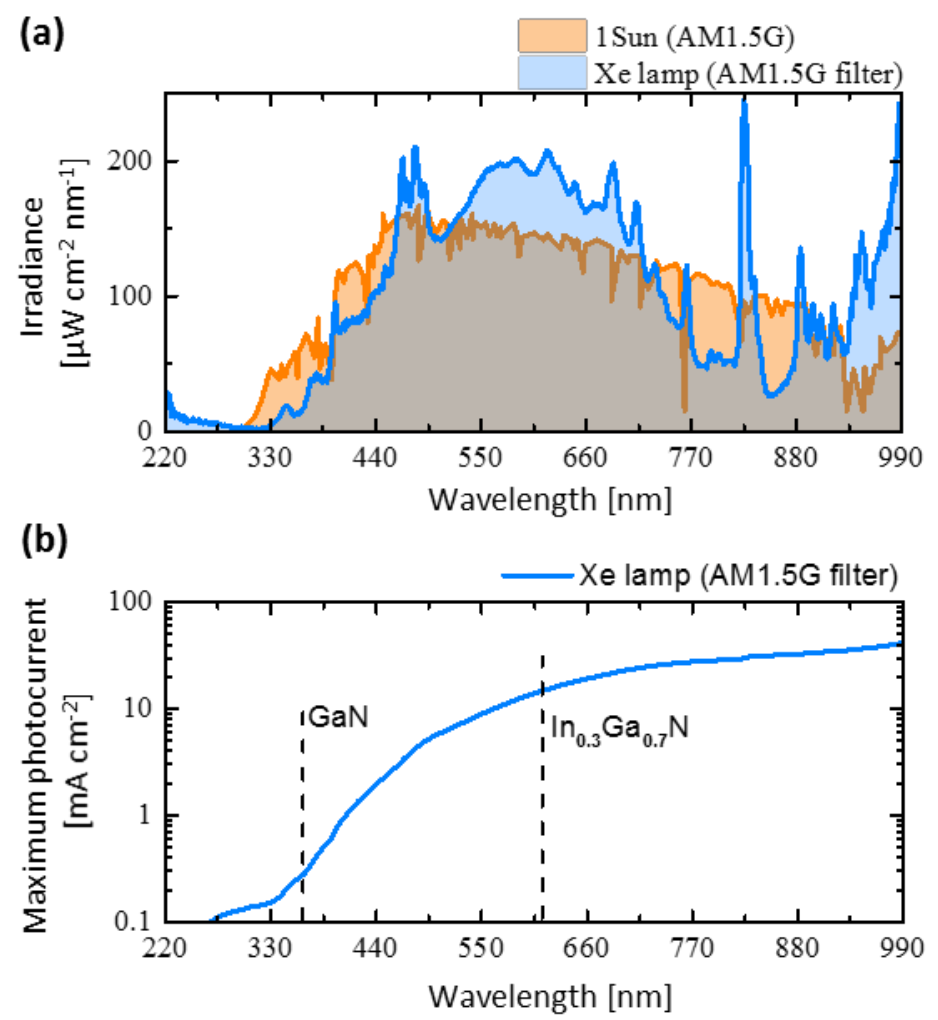

Fig. 3.6.2. Spectral irradiance distribution for the Xenon lamp with a AM1.5G filter used in this work (blue line) plotted together with the standard sunlight at ASTM G-173 AM 1.5 (orange line) (https://www.astm.org/Standards/G173.htm) (a). Integral intensity of the Xe lamp spectrum is $100 \mu \mathrm{W} \mathrm{cm}{ }^{-2}$. Calculated maximum photocurrent density vs. absorption edge wavelength for the Xenon lamp (b). Positions of GaN and In ${ }_{0.3} \mathrm{Ga}_{0.7} \mathrm{~N}$ absorption edges are marked with dashed lines. 


\section{$\ln _{\mathrm{x}} \mathrm{Ga}_{1-\mathrm{x}} \mathrm{N}$ layers grown directly on $\mathrm{Si}(111)$}

\subsection{Introduction}

Historically, sapphire was chosen as the main substrate to grow III-Nitride films because of its hexagonal surface symmetry and the fact that it is both thermally and chemically stable (i.e. it is not reacting with ammonia in MOCVD systems) [Maruska1969]. However, sapphire is a highly insulating material, a problem that remains a major technological issue since it prevents the fabrication of vertical devices. In addition, its thermal conductivity is quite poor, what hinders its use for power electronic devices. Silicon, on the other hand, is a conductive material that also possesses other attractive qualities such as a high thermal conductivity, low price, availability of variety of substrate sizes and crystal quality. Thus, there is a great interest in integrating III-Nitrides with Si technology. An overview of the recent achievements in that direction is presented in this section focusing on the $\mathrm{In}_{\mathrm{x}} \mathrm{Ga}_{1-\mathrm{x}} \mathrm{N}$ alloy case, as a material of interest of this thesis.

The growth of thick, compact, and chemically homogeneous $\ln _{x} G_{1-x} N(x=0-1)$ films, especially on Si substrates, is considered an extremely challenging task. As it was discussed in section 2.5 there are various reasons for that, which can be summarized into two groups:

(i) InGaN film growth (chemical homogeneity, large difference in optimal growth conditions for GaN and $\ln N$ ).

(ii) InGaN/Si interface (meltback etching, formation of $\mathrm{SiN}_{\mathrm{x}}$ interlayer, lattice mismatch).

During the past several decades a substantial progress in understanding and overcoming problems related to point (i) was achieved mainly for InGaN single crystals grown on sapphire(0001) substrates. Initially, high quality GaN and Ga-rich InGaN layers were obtained by MOCVD technique [Amano1988, Nakamura1993], but the incorporation of large fractions of InN into thick InGaN films remained as a great challenge [Matsuoka1990] until the PA MBE technique become more mature, which allowed growth at lower temperatures $\left(T<550^{\circ} \mathrm{C}\right)$. This is the main reason for a great success of PA MBE to grow $\ln _{x} G_{1-x} N$ films over the entire composition range $(x=0-1)$ on sapphire(0001) without any buffers [Kurouchi2004], as well as with various buffers, including GaN [Singh1995, Iliopoulos2006, Bailey2008, Gacevic2013], AIN [Wu2002a, Lu2001], and InN [Nanishi2003]. Eventually, $\ln _{x} G_{1-x} N(x=0-1)$ layers were also grown by MOCVD on GaN/sapphire(0001) by exploiting low growth temperature [Liu2013a].

Further advances, such as the introduction of the droplet elimination by radical beam irradiation (DERI) method, first proposed for PA MBE growth of InN [Nanishi2009] and then extended to $\ln _{x} \mathrm{Ga}_{1-x} \mathrm{~N}$ alloys [Yamaguchi2013], allowed the fabrication of high quality p-type doped $\ln _{x} G a_{1-x} N$ layers over the entire composition range on GaN/sapphire(0001) [Wang2013].

Compared to sapphire substrates, much less research work has been done on III-Nitrides binary and ternary compounds growth on Si substrates. While the growth of high quality AIN [Morita1981, 
Schenk1999] and GaN [Stevens1994, Ohtani1994, Calleja1999] layers on Si was established quite long time ago, the progress on InN films was much slower. Only in the 2000 s, InN films with relatively flat morphology and good structural quality were grown directly on Si [Gwo2004, Grandal2005, Hsiao2005, Kehagias2013], as well as on AIN/Si [Yamaguchi2003, Hsiao2005, Wu2006] and GaN/AIN/Si [Sakalauskas2010] substrates. InN NWs were also grown on Si [Hsiao2005, Grandal2005, Ajagunna2009, Le2014]. Eventually, even the very challenging tasks of Mg doping and fabrication of p-i-n structures were achieved in InN NWs directly grown on Si [Le2014, Zhao2016].

As for ternary $\ln _{x} \mathrm{Ga}_{1-\mathrm{x}} \mathrm{N}$ alloys on $\mathrm{Si}$, only a moderated In content was demonstrated so far for films grown by PA MBE using different buffer strategies, namely: $x<0.32$ on GaN/AIN/Si [Reichertz2008, Gherasoiu2010] and $x<0.45$ on AIN/Si [Ager2009]. $\ln _{x} G_{1-x} N$ on Si with $x<0.49$ was demonstrated by MOCVD on AIN/Si substrates [Yamamoto2013]. Concerning the direct growth of $\operatorname{In}_{x} G_{1-x} N$ on $S i$ over the entire composition range $(x=0-1)$ there were only reports for nanocolumnar structures fabricated by HVPE [Kuykendall2007] and PA MBE [Vajpeyi2009] techniques. For compact $\ln _{x} G_{1-x} N$ films directly grown on $\mathrm{Si}$, to the best of our knowledge, there are only few reports with just moderate In content $(x<0.4$, by PA MBE) [Romanyuk2009, Hoffbauer2013]. Thus, the growth of Inrich $\ln _{x} \mathrm{Ga}_{1-\mathrm{x}} \mathrm{N}$ films directly on $\mathrm{Si}$ is vastly unexplored.

In this chapter, the method to grow thick, compact, and chemically homogeneous $\ln _{\mathrm{x}} \mathrm{Ga}_{1-\mathrm{x}} \mathrm{N}$ films over the entire composition range $(x=0-1)$ directly on Si substrates is presented. The importance of the Si substrate preparation is discussed. Growth parameters are optimized to obtain the best relation between the layers surface flatness and their structural/optical quality. An extensive analysis of the morphological (SEM), optical (PL, SNOM), structural (HRXRD, HRTEM), chemical (EDX), and electrical (IV) properties of the layers confirm their great optical and structural quality. Ways to improve the films morphology are also proposed.

\subsection{Nucleation of $\ln _{x} \mathrm{Ga}_{1-x} \mathrm{~N}$ layers on $\mathrm{Si}(111)$}

It was reported by many authors that the nucleation stage of III-Nitrides on Si largely determines the resulting layer quality [Calleja1998, Sanchez-Garcia1998, Hashimoto1997, Kehagias2013]. Therefore, in this section the Si substrate preparation and InGaN film nucleation conditions are studied in order to determine the optimum initial growth parameters to obtain compact and relatively flat InGaN layers on $\mathrm{Si}$.

\subsubsection{Substrate cleaning}

Prior to any growth experiments the $\mathrm{Si}$ substrate surface must be cleaned from organic contaminants and a native oxide layer. The former is done by pre-annealing the Si substrate at $450^{\circ} \mathrm{C}$ for several hours in the introduction chamber of the PA MBE system under high vacuum $\left(\sim 10^{-8}\right.$ torr). The latter is done by high temperature annealing at $850^{\circ} \mathrm{C}$ for $30 \mathrm{~min}$ inside the UHV growth chamber ( $\sim 10^{-10}$ torr). In both cases the temperature is measured by thermocouples located behind the substrate holder. Removal of the native oxide layer is confirmed by RHEED observation of the $(1 \times 1) \mathrm{Si}(111)$ surface reconstruction and then a transition to a $(7 \times 7)$ one upon decreasing the substrate temperature below $830^{\circ} \mathrm{C}$, as shown in Fig. 4.2.1. This reversible transition occurring at $827 \pm 15^{\circ} \mathrm{C}$ [Telieps1985] is used for calibration of the thermocouple readings. 

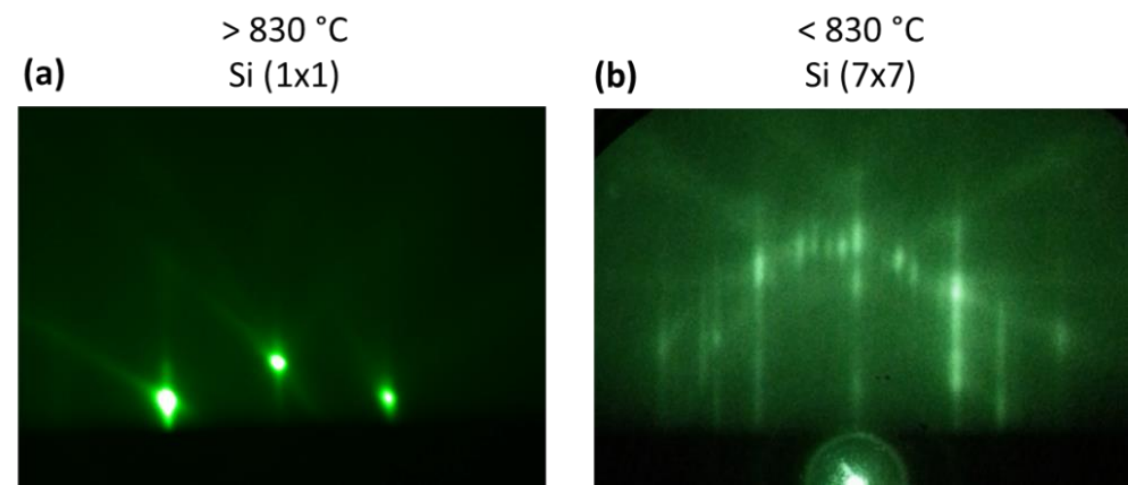

Fig. 4.2.1. RHEED pattern of a clean $\mathrm{Si}(111)$ surface once the native oxide is removed showing a (1x1) surface reconstruction (a) that changes to a ( $7 \times 7)$ one (b) upon decreasing the substrate temperature below $\sim 830{ }^{\circ} \mathrm{C}$.

\subsubsection{Nitridation of the Si(111) surfaces}

In this work an intentional nitridation of the Si surface was used to avoid chemical inhomogeneity of the InGaN/Si interface and reduce the probability of Si meltback etching (refer to section 2.5.2). Nitridation of a clean $\mathrm{Si}(111)$ surface is performed at $850^{\circ} \mathrm{C}$ with the nitrogen plasma set to $P=350 \mathrm{~W}$ and $F_{N 2}=0.6 \mathrm{sccm}$. Such high nitridation temperature was chosen to ensure the formation of a continuous crystalline $\beta-\mathrm{Si}_{3} \mathrm{~N}_{4}$ film (self-limited to 2-3 nm thickness) instead of an amorphous layer [Bahari2005].

The nitridation process was monitored by RHEED observation. Upon nitridation, the initial (1x1) surface reconstruction of a clean Si(111) surface (Fig. 4.2.2 (a)) changed to (8 $\times$ 8) (Fig. 4.2.2 (b)), which suggests the formation of crystalline $\beta-\mathrm{Si}_{3} \mathrm{~N}_{4}(0001)$ interlayer [Lastras-Martinez2015, Wu2003, Ahn2001, LeLouarn2009]. In order to ensure the completeness of the nitridation process,

(a)

\section{Annealing}

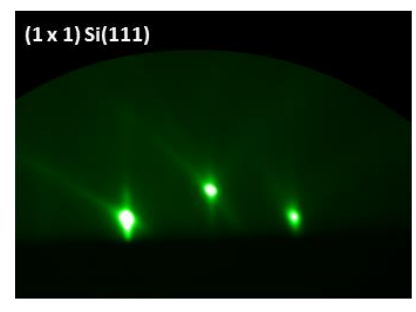

(b)

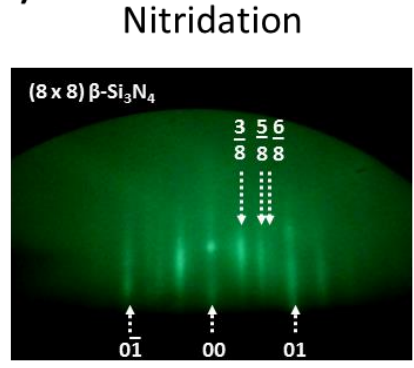

$\sim 2 \mathrm{~nm} \mathrm{SiN}$

Si substrate

\section{Si substrate}

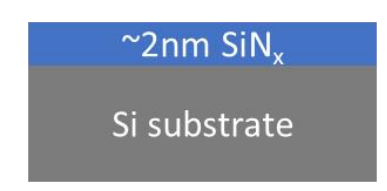

(c)

III-Nitride growth

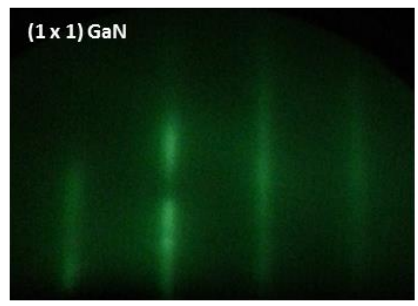

III-Nitride

$\sim 2 \mathrm{~nm} \mathrm{SiN}$

Si substrate

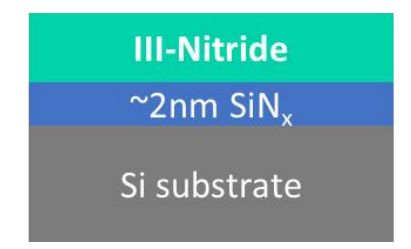

Fig. 4.2.2. RHEED patterns of clean $\mathrm{Si}(111)-(1 \times 1)$ surface right after annealing at $850{ }^{\circ} \mathrm{C}$ for $30 \mathrm{~min}(\mathrm{a})$, $\mathrm{Si}(111)$ surface after nitridation at $850^{\circ} \mathrm{C}$ for $1 \mathrm{~min}$ (exhibiting (8x8) reconstructions of crystalline $\beta$ $\left.\mathrm{Si}_{3} \mathrm{~N}_{4}(0001) / \mathrm{Si}(111)\right)$ (b), and $\mathrm{GaN}(000 \overline{1})$ surface after 1 min of growth at $750^{\circ} \mathrm{C}$ (c). 
the plasma source was turned off only once the intensity of the RHEED $(03 / 8)$ fractional spot reaches its maximum and starts to decay. This process usually takes $5 \mathrm{~min}$ at $850{ }^{\circ} \mathrm{C}$. Then the substrate temperature was lowered to the growth one. Upon initiating the epilayer growth, the RHEED pattern changed rapidly (less than $10 \mathrm{~nm}$ of deposited material) to that corresponding to the III-Nitride crystal, as shown in Fig. 4.2.2 (c) for 1 min growth of a GaN layer.

\subsubsection{Nucleation stage}

The aim of this section is to determine how the nucleation of $\ln _{x} G_{1-x} N$ layers on $\mathrm{Si}(111)$ depends on the substrate temperature and III/N ratio. This should allow finding the optimal conditions for growing compact, flat, and chemically homogeneous InGaN layers on $\mathrm{Si}$, which is one of the main goals of this thesis. Before any growth, the calibration of the $\mathrm{Ga}$, In, and $\mathrm{N}$-plasma fluxes (denoted as $F_{G a}, F_{l n}$, and $F_{N^{*}}$, respectively, with the III/N ratio as $\left.\left(F_{G a}+F_{l n}\right) / F_{N^{*}}\right)$ was carried out according to the standard procedure described in Appendix A.

\subsubsection{Temperature effect}

A series of InGaN layers were grown on nitridated $\mathrm{Si}(111)$ at different substrate temperatures under N-rich conditions (III/N 0.9) with $F_{G a}=2.64 \times 10^{14}$ atoms $/ \mathrm{cm}^{2} / \mathrm{s}, F_{I n}=1.30 \times 10^{14}$ atoms $/ \mathrm{cm}^{2} / \mathrm{s}$, and $F_{N^{*}}=4.38 \times 10^{14}$ atoms $/ \mathrm{cm}^{2} / \mathrm{s}$ (@350 W, $0.6 \mathrm{sccm}$ ) supplied simultaneously during 1 hour. Such conditions corresponds to the formation of $\ln _{0.33} \mathrm{Ga}_{0.67} \mathrm{~N}$ layer at $450^{\circ} \mathrm{C}$, however, the exact In composition can vary with used growth temperature (lower In at higher temperatures). Plan view (top) and cross section (bottom) SEM images of these samples grown at 350,480 , and $500{ }^{\circ} \mathrm{C}$ are shown in Fig. 4.2.3 (a,b,c), respectively. In all cases some defects can be observed in these films (marked with red arrows), which are called macro-defects in this work due to their lateral size (usually above $1 \mu \mathrm{m}$ in diameter). At a low substrate temperature of $350^{\circ} \mathrm{C}$ the InGaN layer has a coalesced columnar morphology (to be discussed in more detail in section 4.3). Cross-section SEM images of the macro-defects in this case (Fig. 4.2.3 (a)) show that they originate by lifting of the InGaN layer, attributed to the accommodation of the compressive stress accumulated in the epilayer. However, the origin of this stress is not clear.

At a substrate temperature of $480{ }^{\circ} \mathrm{C}$ there are clear indications of meltback etching (refer to section 2.5.2), such as pits at the Si surface with triangular prism shape (marked with white arrows in Fig. 4.2.3 (b) inset and cross section). On top of these pits there are macro-defects formed with near circular shape (marked with red arrow). The density of these macro-defects is of $9 \times 10^{5} \mathrm{~cm}^{-2}$, which is almost 1 order of magnitude lower than in the previous case $\left(7 \times 10^{6} \mathrm{~cm}^{-2}\right)$.

At higher substrate temperature of $500{ }^{\circ} \mathrm{C}$, metallic droplets start to form on the surface. SEM measurements revealed that there are macro-defects in the InGaN layer underneath each of the droplets (Fig. 4.2.3 (c), red arrow). Their density is of $~ 8 \times 10^{6} \mathrm{~cm}^{-2}$, which is very close to the previous case. However, their size is increased significantly as can be seen by comparing plan view SEM images in Fig. 4.2.3 (b) and (c).

Results from samples of this series indicate that the formation of macro-defects (detrimental to sample morphology) cannot be avoided by just changing the growth temperature in the studied range of $350-500{ }^{\circ} \mathrm{C}$. The possible origin of these macro-defects and ways to avoid their formation are going to be discussed later, together with the III/N ratio effect on the InGaN nucleation stage. 

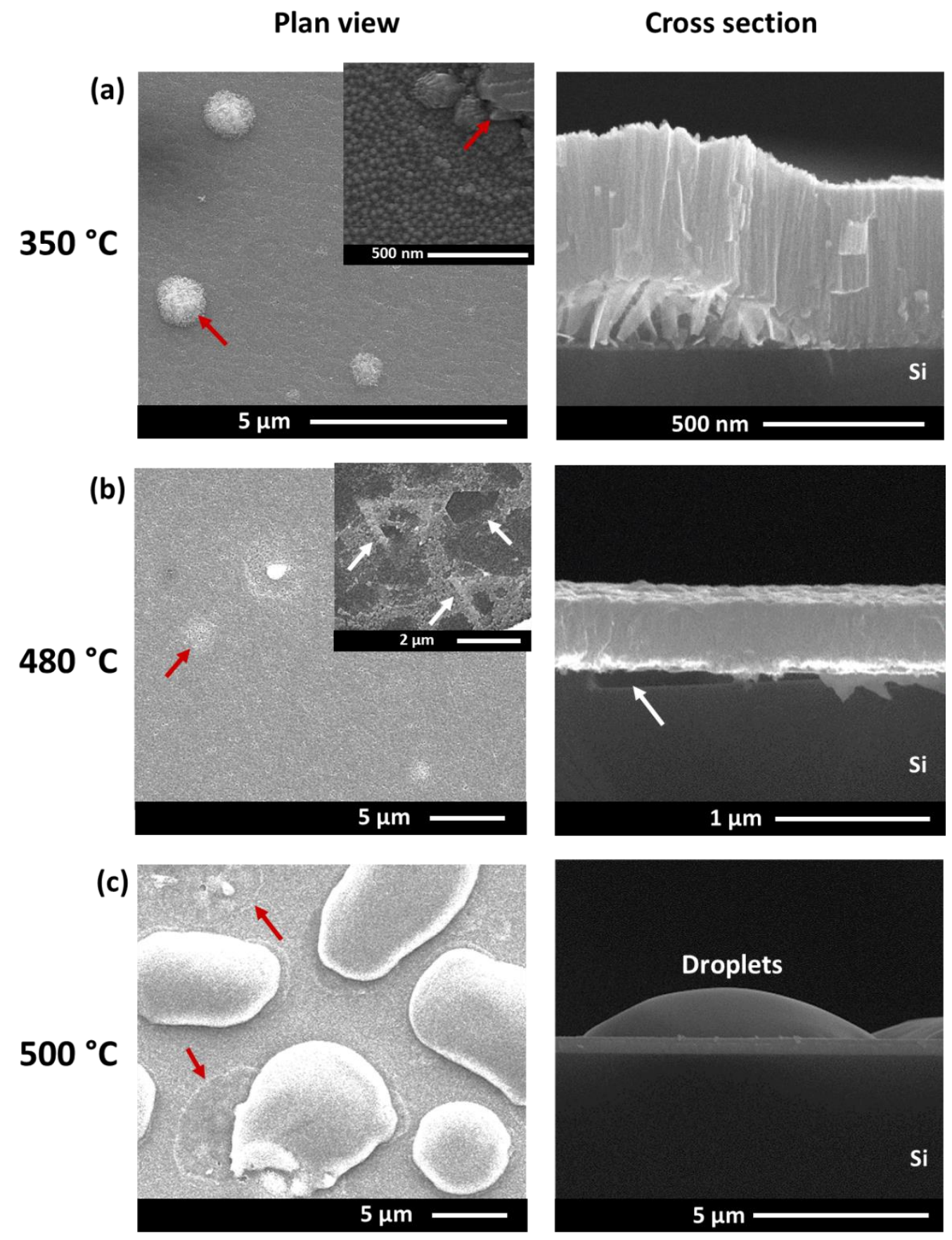

Fig. 4.2.3. Macro-defects in $\mathrm{In}_{0.33} \mathrm{Ga}_{0.67} \mathrm{~N}$ layers grown on $\mathrm{Si}$ as evidenced by SEM (marked by red arrows). The inset in (a) shows zoomed in morphology of sample grown at $350{ }^{\circ} \mathrm{C}$. The inset in (b) shows the Si surface underneath the InGaN layer (a small part of the InGaN layer was detached from the Si during cleaving). Pits with a triangular prism shape on the Si surface (b), most likely originated from meltback etching are marked with white arrows. 


\subsubsection{Metal-rich versus N-rich start}

To test the effect of the III/ $\mathrm{N}$ ratio on the $\operatorname{InGaN}$ nucleation stage two $\ln _{0.33} \mathrm{Ga}_{0.67} \mathrm{~N} / \mathrm{Si}$ samples were grown using metal-rich $(\mathrm{III} / \mathrm{N}>1)$ and $\mathrm{N}$-rich $(\mathrm{III} / \mathrm{N}<1)$ conditions for the initial stage of the growth (5 minutes). After that, the growth proceeded under similar conditions for both samples during 1 hour: slightly $\mathrm{N}$-rich regime $(\mathrm{III} / \mathrm{N} \sim 0.9)$, substrate temperature of $450{ }^{\circ} \mathrm{C}$, $F_{G a}=2.64 \times 10^{14}$ atoms $/ \mathrm{cm}^{2} / \mathrm{s}, F_{I n}=1.30 \times 10^{14}$ atoms $/ \mathrm{cm}^{2} / \mathrm{s}$, and $F_{N^{*}}=4.38 \times 10^{14}$ atoms $/ \mathrm{cm}^{2} / \mathrm{s}(350 \mathrm{~W}$, $0.6 \mathrm{sccm})$.

In order to achieve metal-rich conditions, the In cell shutter was opened for $5 \mathrm{~s}$ prior to sending the $\mathrm{N}$ plasma and $\mathrm{Ga}$ fluxes. Then the growth continued as described above. Plan view and cross section SEM images of the resulting layer (Fig. 4.2.4 (a)) reveal that the InGaN film had a 3D morphology with macro-defects and metallic droplets covering almost the entire surface.

For $\mathrm{N}$-rich start, the first $5 \mathrm{~min}$ of the growth proceeded with the same metal fluxes as before with an increased $F_{N^{*}}=5.12 \times 10^{14}$ atoms $/ \mathrm{cm}^{2} / \mathrm{s}(410 \mathrm{~W}, 0.6 \mathrm{sccm})$ corresponding to III/N=0.77, while the second growth step (55 $\mathrm{min}$ ) continued under the conditions described above. Such a simple method resulted in a compact and relatively flat $\operatorname{In}_{0.33} \mathrm{Ga}_{0.67} \mathrm{~N}$ film with no macro-defects across the whole surface, as evidenced by SEM images in (Fig. 4.2.4 (b)).

These results suggest that the metal accumulation during the initial stages of the growth is detrimental to the resulting layer morphology and should be avoided.

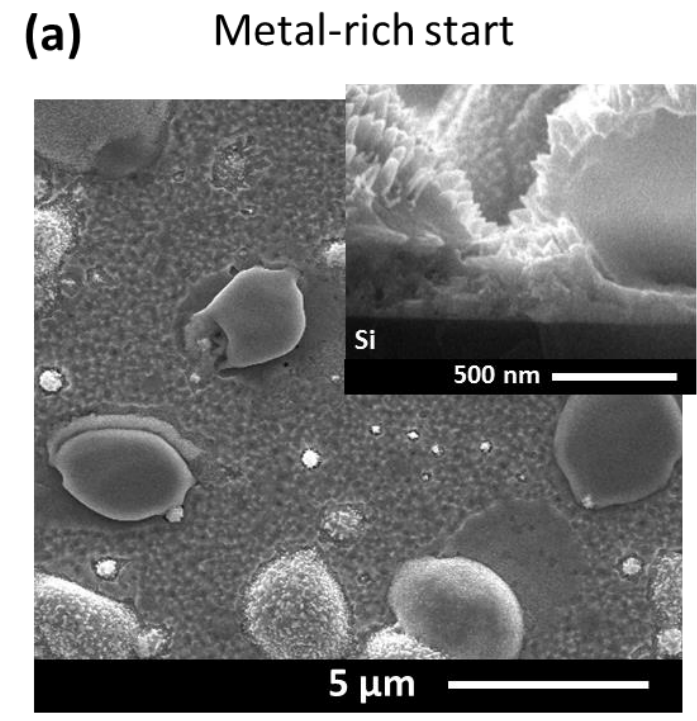

(b) $\quad \mathrm{N}$-rich start

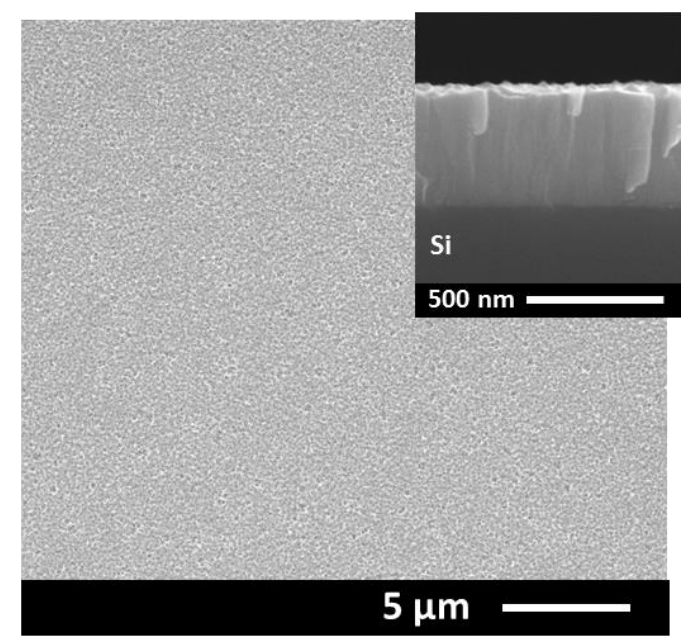

Fig. 4.2.4. Comparison of $\operatorname{In}_{0.33} \mathrm{Ga}_{0.67} \mathrm{~N}$ layer morphology (plan view) using two different starting conditions: metal pre-deposition (a) and N-rich start (b). Insets show cross section SEM images.

\subsubsection{Discussion}

In this section the Si substrate cleaning procedure was described. Removal of the native oxide was monitored by observing RHEED patterns change from $\mathrm{Si}(111)-(1 \times 1)$ to $\mathrm{Si}(111)-(7 \times 7)$ surface reconstructions at $830^{\circ} \mathrm{C}$. After exposure of a clean $\mathrm{Si}(111)$ surface to active $\mathrm{N}$ plasma at $850^{\circ} \mathrm{C}$, RHEED patterns changed to a $(8 \times 8)$ reconstructions, which suggests the formation of a crystalline $\beta-\mathrm{Si}_{3} \mathrm{~N}_{4}(0001)$ film. 
The nucleation stage is mainly influenced by the $\mathrm{III} / \mathrm{N}$ ratio, rather than by the substrate temperature. Varying the initial starting growth conditions, showed that metal accumulation during the initial growth stage has a detrimental impact on the resulting InGaN layer morphology, such as 3D nucleation and formation of macro-defects. The fact that even under $\mathrm{N}$-rich conditions (III/N 0.9) macro-defects are formed suggests that there is a InGaN nucleation time onset during which a meltback process is happening (similarly to the GaN-on-Si case reported in the literature [Furtmayr2008]). The use of N-rich starting conditions avoids these effects and results in compact and relatively flat InGaN layers without macro-defects. These results are in agreement with reports on meltback etching of the Si(111) surface being responsible for the formation of macro-defects ("hillocks") [Kaiser1999] (refer to section 2.5.2). Similarly to the obtained results in this section, it was also reported that the use of strong N-rich conditions during the initial growth stages of GaN and AIN on $\mathrm{Si}(111)$ is successfully inhibiting the formation of macro-defects [Ishikawa2011, Sobanska2012].

In summary, the growth of InGaN layers is strongly influenced by the initial III/N ratio. N-rich starting is proposed to obtain compact InGaN films with relatively flat morphology and without macrodefects. This method is used in all samples grown from now on studied in this chapter.

\subsection{Effects of the growth temperature}

Following the optimized initial conditions that deliver thick and compact InGaN layers, the study of the growth temperature effect on their morphology was addressed in a series of $\ln _{0.33} \mathrm{Ga}_{0.67} \mathrm{~N}$ layers grown in the 280 to $500{ }^{\circ} \mathrm{C}$ temperature range (using growth conditions as in section 4.2.3.2 with $\mathrm{N}$-rich start). Such conditions correspond to the formation of an $\ln _{0.33} \mathrm{Ga}_{0.67} \mathrm{~N}$ layer at $450{ }^{\circ} \mathrm{C}$, however, the exact In composition can vary with the used growth temperature (lower In composition at higher temperatures). SEM images of these samples in plan-view and cross section are shown in Fig. 4.3.1 (left and right, respectively). The morphology evolution with the growth temperature is as follows: (i) films grown at $280^{\circ} \mathrm{C}$ exhibit a coalesced columnar morphology that becomes more evident with well-defined hexagonal facets at $350{ }^{\circ} \mathrm{C}$; (ii) at $400{ }^{\circ} \mathrm{C}$ the columnar morphology coexists with a more compact phase, that becomes dominant with quite rough surfaces at $450{ }^{\circ} \mathrm{C}$; (iii) increasing the temperature to $480{ }^{\circ} \mathrm{C}$ significantly reduces the surface roughness, but a further increase to $500{ }^{\circ} \mathrm{C}$ leads to the formation of metallic droplets due to $\ln N$ thermal decomposition. It must be noted that none of the samples exhibited thermal cracking.

The thickness of these samples decreased upon increasing the growth temperature (Fig. 4.3.2), in agreement with the morphology change from columnar structures to compact layers (empty space in between columns makes up for the additional thickness). For samples grown above $400{ }^{\circ} \mathrm{C}$ the measured thickness of $366 \mathrm{~nm}(\sigma=23 \mathrm{~nm})$ is very close to the expected value for the used parameters (360 nm). 


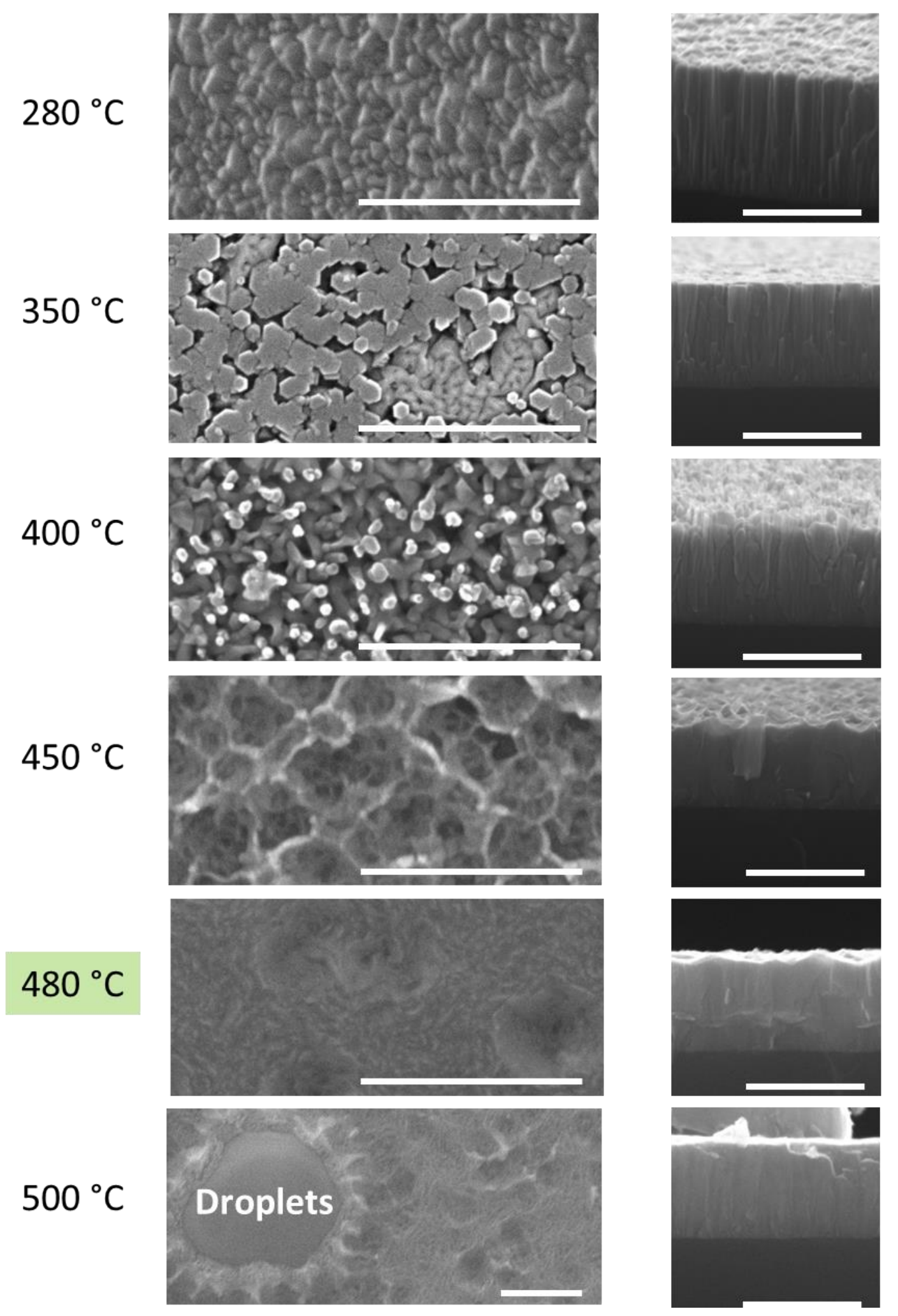

Fig. 4.3.1. In ${ }_{0.33} \mathrm{Ga}_{0.67} \mathrm{~N}$ layer morphology evolution upon increasing the growth temperature studied by plan view (left) and cross section (right) SEM. Scale bar is $500 \mathrm{~nm}$. 
In order to understand why the growth temperature provokes these strong morphology changes it is worth to remember the mechanisms governing the growth of self-assembled (SA) III-Nitride NWs. According to recent models [Ristic2008, Debnath2007] the reason behind the GaN NW growth on Si substrates, instead of compact layers at $700{ }^{\circ} \mathrm{C}$ is the suppression of metal adatoms surface diffusion under high $\mathrm{N}$-plasma flux. However, metal adatoms surface diffusion is already thermally suppressed at low temperatures, automatically leading to the NW morphology. In case of low enough growth temperatures, NWs nucleation is expected to happen even under metal-rich conditions. Indeed, this was confirmed by growing $\ln _{0.33} \mathrm{Ga}_{0.67} \mathrm{~N}$ layers with a $\mathrm{III} / \mathrm{N}=1.3$ ratio at $350{ }^{\circ} \mathrm{C}$, that exhibited both metal droplets and NWs (refer to Fig. 4.4.1).

PL measurements from samples studied in this section revealed that, despite the typical high optical quality of a NW morphology [Sanchez-Garcia1998], samples grown below $430{ }^{\circ} \mathrm{C}$ showed no emission at RT and, in general, a very limited optical performance compared to samples grown at higher temperatures. This indeed points to defect generation at low temperatures independently of the NW morphology. Then, higher substrate temperatures $\left(>450^{\circ} \mathrm{C}\right)$ bring both an improved crystal quality (as evidenced by PL measurements) and the desired compact morphology (as evidenced by SEM).

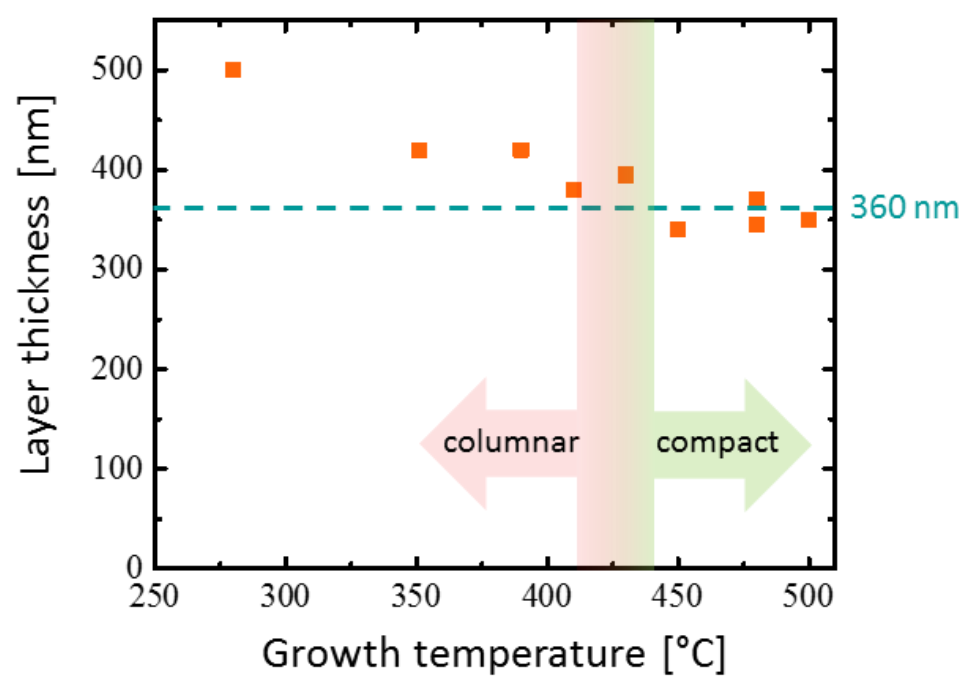

Fig. 4.3.2. In $\ln _{0.33} \mathrm{Ga}_{0.67} \mathrm{~N}$ layers thickness dependence on the growth temperature. Growth time is 1 hour, III/V=0.9, $F_{N^{*}}=4.38 \times 10^{14}$ atoms $/ \mathrm{cm}^{2} / \mathrm{s}$. The dashed line indicates the expected thickness under such growth conditions. 


\subsection{Effects of the III/N ratio}

A detailed growth map reflecting the morphology dependence on the $\mathrm{III} / \mathrm{N}$ ratio and growth temperature was constructed (Fig. 4.4.1), based on a large set of samples grown with fixed $F_{G a}=2.64 \times 10^{14}$ and $F_{N^{*}}=4.38 \times 10^{14}$ atoms $/ \mathrm{cm}^{2} / \mathrm{s}$. The total III/N ratio was varied by adjusting the In flux $F_{I n}$ from 0.52 to $7.75 \times 10^{14}$ atoms $/ \mathrm{cm}^{2} / \mathrm{s}$. The substrate temperature was varied in the $350-555^{\circ} \mathrm{C}$ range. For a III/N=0.9 ratio and $450{ }^{\circ} \mathrm{C}$ growth temperature these growth conditions result in a $\operatorname{In}_{0.33} \mathrm{Ga}_{0.67} \mathrm{~N}$ film. The actual In composition depends on $\mathrm{II} / \mathrm{N}$ ratio and substrate temperature used.

As was discussed previously growth temperatures below $430^{\circ} \mathrm{C}$ yield columnar morphologies (independently of the III/ $\mathrm{N}$ ratio) and poor crystal quality, so that, this regime must be avoided. At growth temperatures between 500 and $525^{\circ} \mathrm{C}$, metallic In droplets were observed for samples grown even under nominally $\mathrm{N}$-rich conditions (III/ $\mathrm{N}=0.9)$ due to $\mathrm{InN}$ decomposition. Also, grooves

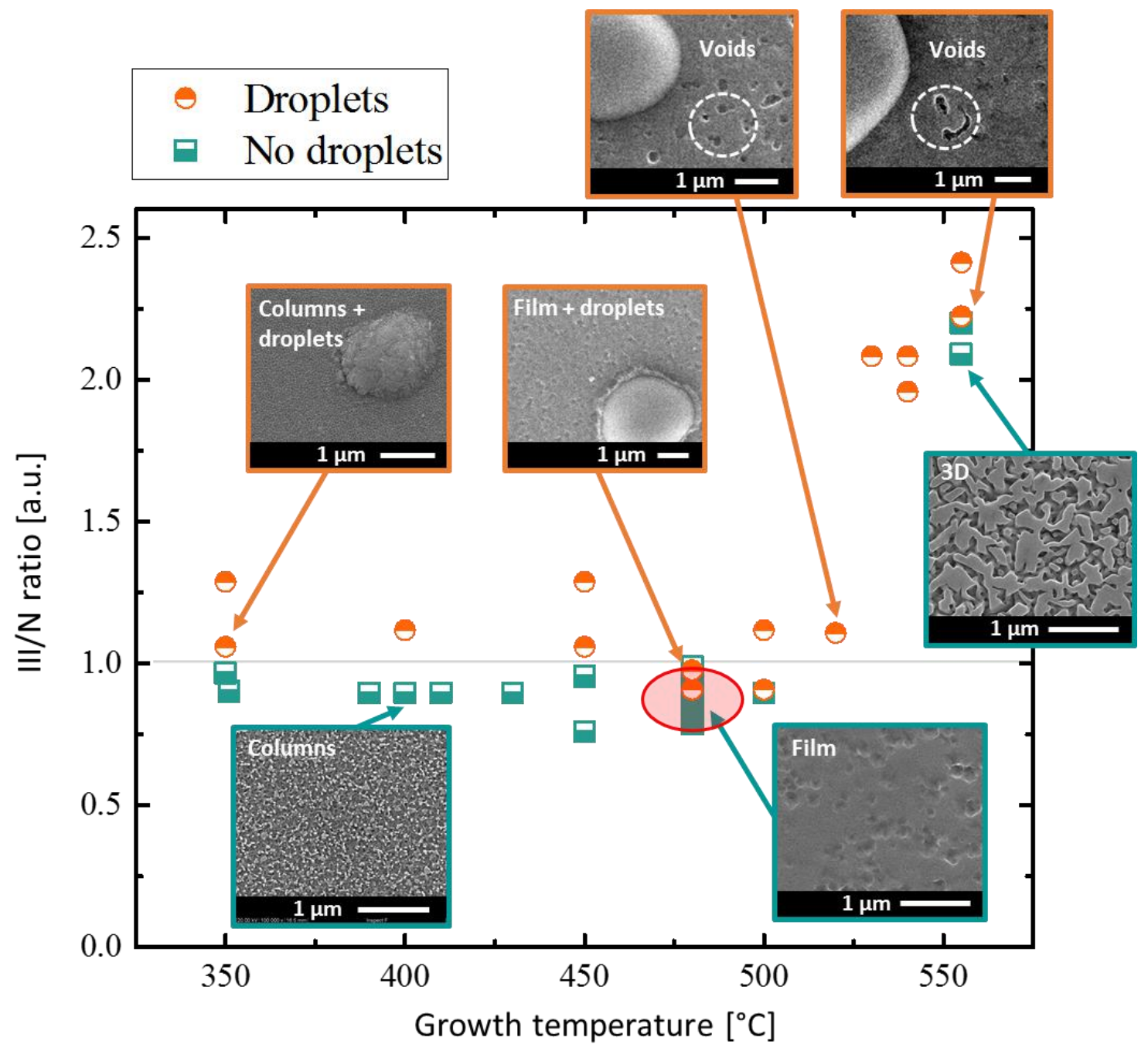

Fig. 4.4.1. Morphology dependence on the $\mathrm{III} / \mathrm{N}$ ratio and growth temperature. $F_{G a}=2.61 \times 10^{14}$ and $F_{N^{*}}=4.38 \times 10^{14}$ atoms $/ \mathrm{cm}^{2} / \mathrm{s}$ were fixed, while the total III/N flux ratio was varied by adjusting the In flux $F_{I n}$ $=0.52-7.75 \times 10^{14}$ atoms $/ \mathrm{cm}^{2} / \mathrm{s}\left(\operatorname{In}_{0.33} \mathrm{Ga}_{0.67} \mathrm{~N}\right.$ is the expected composition for III/N=0.9). An optimized growth window is marked with a red oval. 
were found in such layers despite In metal accumulation, which usually leads to smooth morphology. At these temperatures the sample morphology is very difficult to control because any small variation in the growth temperature or III/ $\mathrm{N}$ ratio can lead to a significant metal accumulation. This is in a good agreement with reports on a similar case of InGaN films grown on sapphire substrates [Valdueza-Felip2014]. At even higher temperatures (above $525^{\circ} \mathrm{C}$ ) In desorption starts to be noticeable. The interplay between $\ln N$ decomposition and In desorption processes is especially critical at temperatures as high as $550^{\circ} \mathrm{C}$ where a small increase in III/N ratio from 2.10 to 2.25 leads to a drastic morphology change from a 3D layer without metal on the surface to a film with droplets and voids. In addition to morphology deterioration, severe $\operatorname{InN}$ decomposition can introduce metallic In into the growing layer leading to an increase of unintentional doping (even up to metallic behavior) according to [Ivanov2014]. A 3D morphology (either NW or rough surface with grooves) of the InGaN layers is caused by the reduced metal adatoms surface mobility due to either the temperature decrease or the relative $F_{N^{*}}$ increase derived from In losses at high temperatures.

Therefore, based on the morphology diagram, growth temperatures around $480^{\circ} \mathrm{C}$ and $\mathrm{III} / \mathrm{N} \leq 0.9$ were determined as optimum growth parameters (marked with a red oval in Fig. 4.4.1) where compact and relatively flat InGaN layers without metallic droplets can be grown in a highly controllable manner. The only drawback of such technique is that the morphology of the obtained layers is relatively rough and it is difficult to improve it by simply adjusting growth parameters [Valdueza-Felip2014]. One possible solution could be to use a more elaborated technique such as migration-enhanced epitaxy (MEE), which might allow independent control of layer morphology and crystal quality [Horikoshi1988, Hooper1996], however it is out of the scope of this thesis.

\subsection{Growth and characterization of $\ln _{x} \mathrm{Ga}_{1-\mathrm{x}} \mathrm{N} / \mathrm{SiN}_{\mathrm{y}} / \mathrm{Si}(\mathrm{x}=0-1)$ layers}

Following the optimized growth procedure, a series of $\sim 360 \mathrm{~nm}$ thick $\ln _{x} \mathrm{Ga}_{1-\mathrm{x}} \mathrm{N}$ layers with $\mathrm{x}$ covering the whole composition range, from 0 to 1 , was grown to check for their structural and optical quality. $\ln _{x} \mathrm{Ga}_{1-\mathrm{x}} \mathrm{N}$ layers with $\mathrm{x}$ below 0.5 were grown under the optimum conditions determined in the previous section (growth temperature of $480^{\circ} \mathrm{C}$ (mostly), $I I / / N=0.9$, $F_{N^{*}}=4.38 \times 10^{14}$ atoms $/ \mathrm{cm}^{2} / \mathrm{s}$ (@350 W, $\left.0.6 \mathrm{sccm}\right)$ ). The samples with $\mathrm{x}$ above 0.5 were grown at a reduced temperature of $450^{\circ} \mathrm{C}$ to prevent metal accumulation due to the combined effect of a higher $F_{I n}$ and the InN decomposition. In both cases the InN mole fraction was controlled by adjusting the $F_{l n} /\left(F_{G a}+F_{l n}\right)$ ratio. The growth parameters and $\ln N$ molar fractions (indirectly estimated from HRXRD-RSM using the linear elasticity theory) of different InGaN layers are summarized in Table $\mathbf{V}$.

The samples were extensively characterized by various techniques in order to determine their structural/optical/electrical properties together with their chemical composition and homogeneity. The results of this evaluation are presented in the following sections, each corresponding to the certain characterization tool used. 
Table V. Growth parameters and InN molar fractions of the InGaN layers grown and characterized in this work. In all cases a $F_{N^{*}}=4.38 \times 10^{14}$ atoms $/ \mathrm{cm}^{2} / \mathrm{s}$ (@350 W, $0.6 \mathrm{sccm}$ )) was used.

\begin{tabular}{|c|c|c|c|c|c|}
\hline Sample & $\begin{array}{c}\text { Growth } \\
\text { temperature }\end{array}$ & $F_{l n}$ & $F_{G a}$ & $\mathrm{III} / \mathrm{N}$ ratio & $\begin{array}{c}\text { HRXRD, } \\
\text { RSM }\end{array}$ \\
\hline & ${ }^{\circ} \mathrm{C}$ & $\begin{array}{c}\mathrm{x} 10^{14} \\
\text { atoms } / \mathrm{cm}^{2} / \mathrm{s}\end{array}$ & $\begin{array}{c}\times 10^{14} \\
\text { atoms } / \mathrm{cm}^{2} / \mathrm{s}\end{array}$ & & $\% \operatorname{InN}$ \\
\hline GaN & 600 & - & 4.30 & 0.95 & 0.0 \\
\hline M86 & 480 & 1.12 & 4.00 & $1.17^{*}$ & 9.5 \\
\hline M64/LC1 & 480 & 0.98 & 2.90 & 0.90 & 25.7 \\
\hline M125/LC2 & 480 & 0.98 & 2.90 & 0.90 & 26 \\
\hline M129/LC3 & 480 & 0.98 & 2.90 & 0.90 & 25.4 \\
\hline M120 & 480 & 0.98 & 2.90 & 0.90 & 26.4 \\
\hline M133 & 450 & 1.30 & 2.64 & 0.90 & 32.0 \\
\hline M148 & 450 & 1.30 & 2.64 & 0.90 & 34.1 \\
\hline M126 & 480 & 1.37 & 2.64 & 0.92 & 34.9 \\
\hline M113 & 480 & 1.49 & 2.60 & 0.93 & 37.5 \\
\hline M69 & 450 & 1.82 & 1.40 & 0.74 & 51.2 \\
\hline M227 & 450 & 1.64 & 1.74 & 0.77 & 55.0 \\
\hline M208/HC1 & 450 & 3.12 & 1.04 & 0.95 & 72.2 \\
\hline M217 & 450 & 3.79 & 0.66 & $1.02 *$ & 88.3 \\
\hline $\ln N$ & 400 & 4.10 & - & 0.70 & 100 \\
\hline
\end{tabular}

\subsubsection{High-resolution X-ray diffraction measurements}

The composition of the $\ln _{x} G_{1-x} N$ layers was determined from high-resolution X-ray diffraction (HRXRD) scans using a commercial XPert PRO PANalytical MRD system equipped with a Ge(220) hybrid monochromator and using the $\mathrm{Cu} K \alpha 1$ line $\left(\lambda_{\kappa \alpha 1}=1.5406 \AA\right.$ ) [Morales2009, Gomez2016]. Fig. 4.5.1 (a) shows symmetric $\omega / 2 \theta$ scans (absolute values) around the InGaN(0002) Bragg reflection for samples covering the entire composition range. Peak positions of $\mathrm{Si}(111)-14.22^{\circ}, \operatorname{InN}$ $-15.71^{\circ}$, and $\mathrm{GaN}-17.30^{\circ}$ are marked with dashed lines. The single diffraction peaks of very similar intensity and width confirm the absence of phase separation for the whole composition range. The peaks broadening for In contents around 50\% is typical for random ternary alloys [Mattila1999].

The InN molar fraction values (\%) placed next to each InGaN peak in Fig. 4.5.1 (a) were estimated from asymmetric scans around the $\operatorname{InGaN}(10 \overline{15}) / \mathrm{Si}(224)$ Bragg reflections, the so called reciprocal space maps (RSMs), making use of the linear elasticity theory described in section 3.4.1. Compared to $\omega / 2 \theta$, RSM scans give more precise composition values because of accounting for strain present in the sample. Examples of RSM scans are shown in Fig. 4.5.1 (b,c,d) for $\ln _{0.35} G_{0.75} N(M 126)$, 
$\ln _{0.55} \mathrm{Ga}_{0.45} \mathrm{~N}(\mathrm{M} 227)$ and $\ln _{0.73} \mathrm{Ga}_{0.28} \mathrm{~N}$ (M208), respectively. The dashed line represents the theoretical position of relaxed $\ln _{x} G_{1-x} N$ peaks. As can be seen, thick $\ln _{x} \mathrm{Ga}_{1-x} N$ layers grown on $\mathrm{Si}$ substrates are almost fully relaxed (in all analyzed cases a $<3 \%$ strain was found) as expected for this system. Residual biaxial strain is most likely of thermal origin [Leszczynski1994, Calleja1998, Sanchez-Garcia1998, Calleja1999].

Additionally, epitaxial relationships as $\operatorname{lnGaN}(0001)|| \operatorname{Si}(111)$ and $\operatorname{lnGaN}(10 \overline{1} 0)|| \operatorname{Si}(11 \overline{2})$ were determined by measuring the $\psi-\phi$ and $\omega / 2 \theta-\phi$ type pole figures around the $\mathrm{Si}(220)$ and InGaN $(10 \overline{1} 2)$ planes. These results confirm the 3-fold symmetry of the Si (220) planes and the 6-fold symmetry of the InGaN (10 12$)$ ones, as expected for face-centered cubic and hexagonal closely packed crystal structures, respectively.
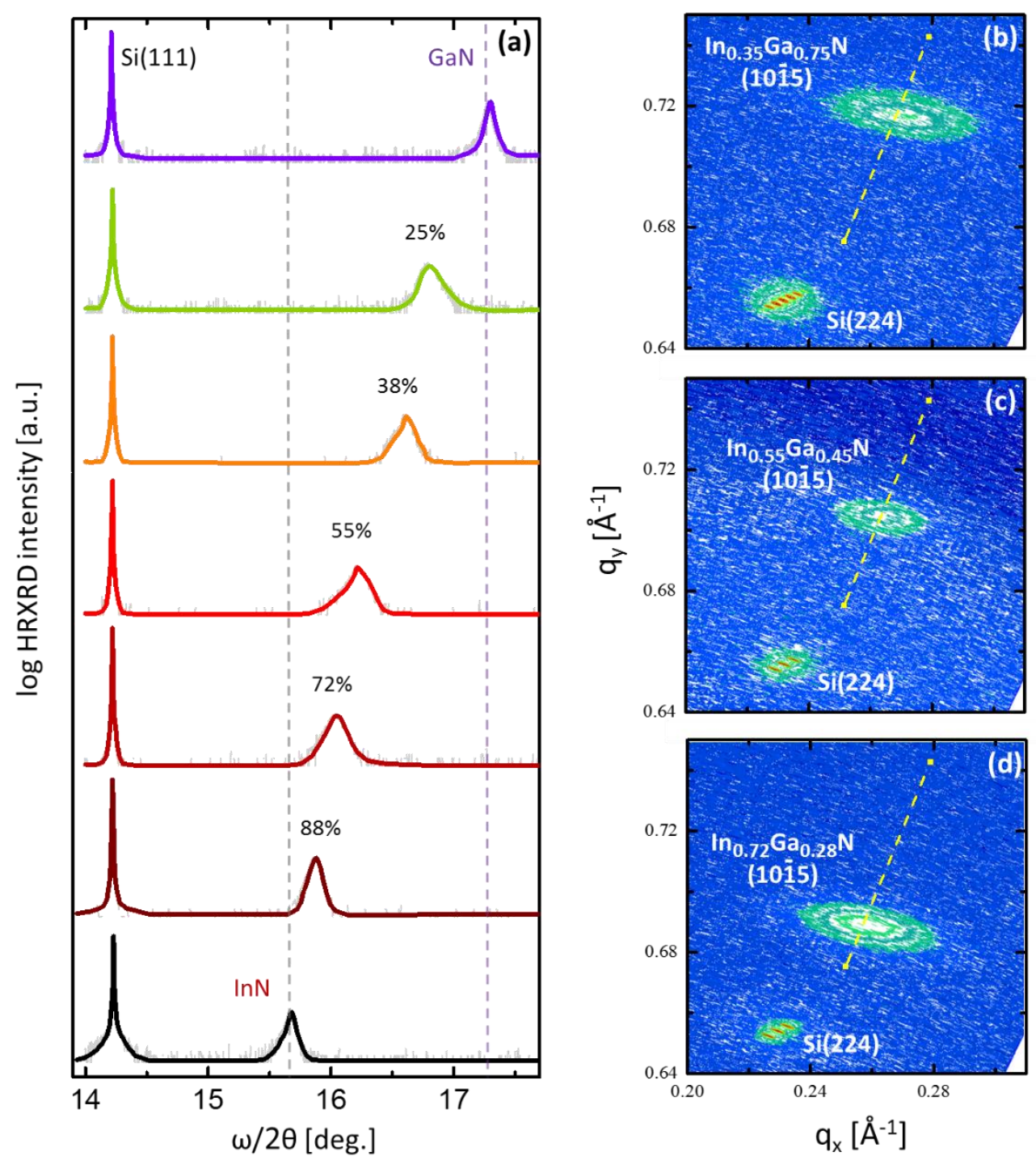

Fig. 4.5.1. HRXRD $\omega / 2 \theta$ spectra recorded around the symmetric InGaN(0002)/Si(111) Bragg reflection (a) and RSM scans around the asymmetric InGaN(1015)/Si(224) Bragg reflection (b, c, d) for various InGaN/Si samples. The positions of relaxed $\ln _{\mathrm{x}} \mathrm{Ga}_{1-\mathrm{x}} \mathrm{N}$ alloy compositions are marked with a yellow dashed line. 


\subsubsection{Transmission electron microscopy measurements}

A study by TEM aimed to (i) checking the chemical composition homogeneity of the InGaN layers (HAADF, EDX), (ii) assessing the structural quality and epitaxial relation of the InGaN layers in respect to the Si substrate (SAED, CTEM), and (iii) examining the interfacial SiN $_{x}$ layer (HRTEM). In the lowIn-composition range three InGaN/Si samples (marked as LC\# in Table V) with $x^{\sim 0} 0.25$ grown under identical conditions were examined to confirm the reproducibility of the InGaN growth. For the highIn-composition range, an InGaN/Si sample (marked as $\mathrm{HC1}$ in Table V) with $\mathrm{x}^{\sim 0.72}$ was characterized. Table VI shows a comparison of the $\operatorname{InN}$ mole fraction values measured by HRXRD, TEM-EDX, and SAED (with lattice parameters $(a, c)$ and strain $\left(\varepsilon_{x x}, \varepsilon_{z z}\right)$ calculated from the diffractograms), which is going to be discussed later. Notice that while the HRXRD technique gives average In compositions over a large scale $(\sim 1 \mathrm{~cm})$, the SAED technique is more local (confined to a region of $\sim 400 \mathrm{~nm}$ in diameter). The performed EDX is in between of these techniques, because it measures atomic In/Ga ratio point by point but at different locations, which can be separated as far as $1 \mathrm{~mm}$ and the presented value is an average.

Table VI. Comparison of In composition values measured by XRD and TEM

\begin{tabular}{|c|c|c|c|c|c|c|c|}
\hline \multirow[t]{2}{*}{ Sample } & RSM, & EDX, TEM & \multicolumn{5}{|c|}{ SAED, TEM } \\
\hline & $\% \ln N$ & $\% \ln N$ & $a(\AA ̊)$ & $c(\AA ̊)$ & $\varepsilon_{x x}(\%)$ & $\varepsilon_{z z}(\%)$ & $\% \operatorname{InN}$ \\
\hline LC1 (M64) & 25.7 & $24.8(\sigma=0.5)$ & 3.249 & 5.329 & -0.71 & 0.39 & 26.0 \\
\hline LC2 (M125) & 26.0 & $24.8(\sigma=1.0)$ & 3.255 & 5.343 & -0.74 & 0.41 & 26.2 \\
\hline LC3 (M129) & 25.4 & $25.9(\sigma=0.8)$ & 3.249 & 5.315 & -0.52 & 0.28 & 22.2 \\
\hline HC1 (M208) & 72.2 & $75.0(\sigma=2.6)$ & 3.441 & 5.570 & -0.10 & 0.07 & 73.4 \\
\hline
\end{tabular}

\subsubsection{Chemical homogeneity of InGaN layers}

The chemical uniformity of InGaN-on-Si samples was assessed by HAADF imaging and EDX spectroscopy. HAADF images of a cross-section (XTEM) preparation of low- and high-In-composition $\operatorname{In}_{x} \mathrm{Ga}_{1-\mathrm{x}} \mathrm{N}$ layers are shown in Fig. 4.5.2 $(\mathrm{a}, \mathrm{c})$ and $(\mathrm{b}, \mathrm{d})$, respectively. In both cases, the contrast continuity reveals a highly uniform composition, as the intensity of HAADF images is approximately proportional to the square of the material effective atomic mass (thus, InN-rich regions would be much brighter than GaN-rich regions). The slight contrast variations are due either to thickness differences during sample preparation, as shown in zoomed-out images in Fig. 4.5.2 (a,b)), or to strain fields associated with threading dislocations (TDs), as seen in zoomed-in images in (Fig. 4.5.2 (c,d)).

To further confirm this chemical homogeneity, EDX measurements were made at 10 to 30 points at different positions of each sample (separated up to tens of $\mu \mathrm{m}$ in one preaparation). Representative spectra are shown in Fig. 4.5.2(e)). In all cases an exceptional chemical homogeneity was confirmed, as the InN molar fraction standard deviation was lower than $2.5 \%$ for each examined InGaN layer. Moreover, results from the atomic Ga/In ratio measured by EDX are in excellent agreement with the estimations performed by SAED, HRXRD and RSM measurements as shown in Table VI. Notice that EDX measurements are independent of strain, because it accesses sample chemical composition directly. 

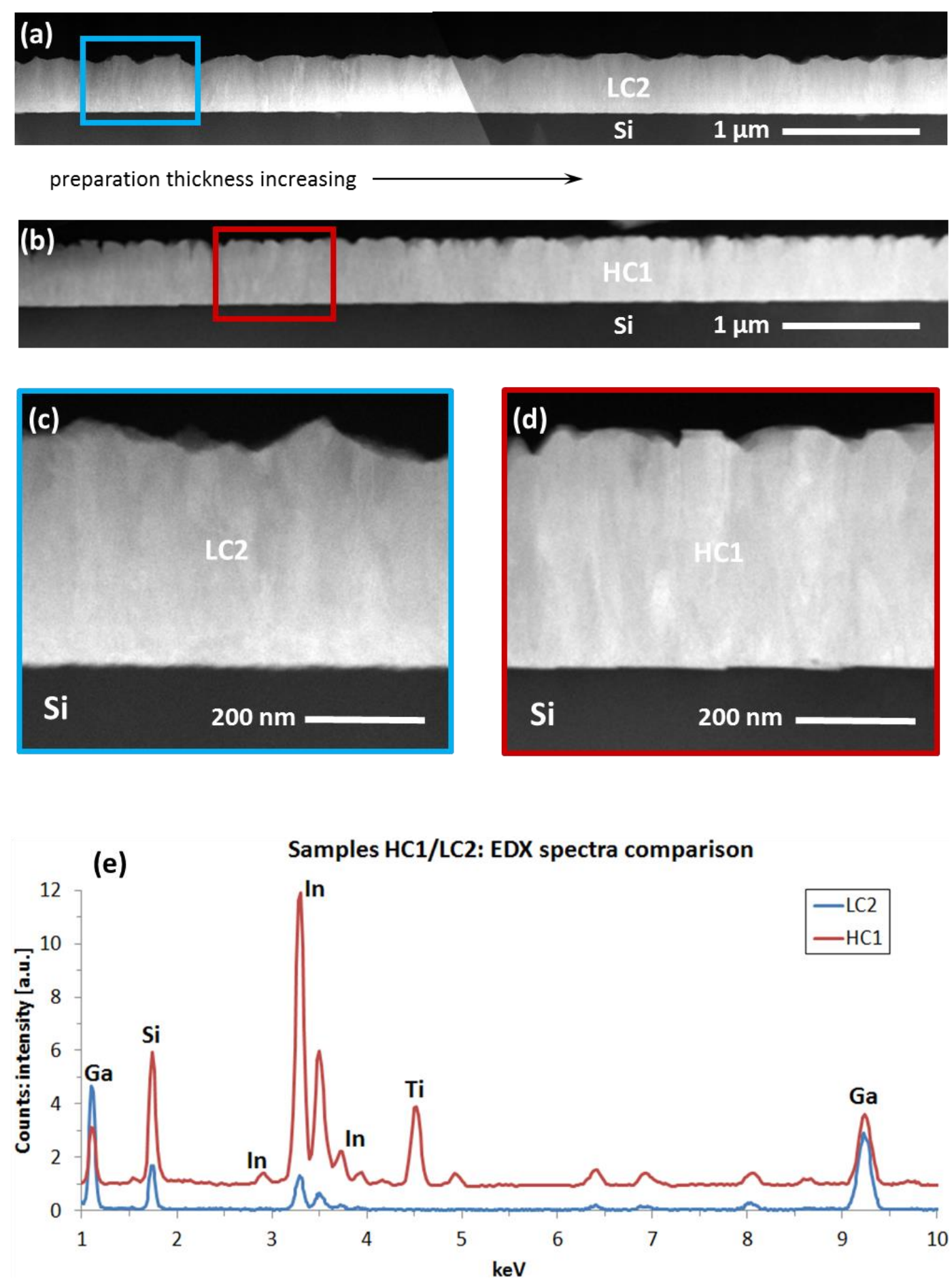

Fig. 4.5.2. Chemical composition uniformity of GaN-rich (LC2, $x=0.26$ from RSM) and InN-rich (HC1, $x=0.72$ from RSM) InGaN/Si samples assessed by HAADF (a-d) and EDX (e, representative spectra). In HAADF images LC2 and HC1 samples are oriented near the [0110] and [11 20$]$ InGaN zone axis, respectively. The Ti signal in HC1 is due to a Ti supportive grid used for the TEM preparation of this sample. 


\subsubsection{Structural quality of InGaN and its epitaxial relation to Si}

The epitaxial relationships between low-In-composition ( $L C 1, \operatorname{In}_{0.25} \mathrm{Ga}_{0.75} \mathrm{~N}$ ) and high-In-composition $\left(\mathrm{HC1}, \mathrm{In}_{0.72} \mathrm{Ga}_{0.28} \mathrm{~N}\right.$ ) samples and Si substrates were analyzed from SAED patterns (Fig. 4.5.3 left and

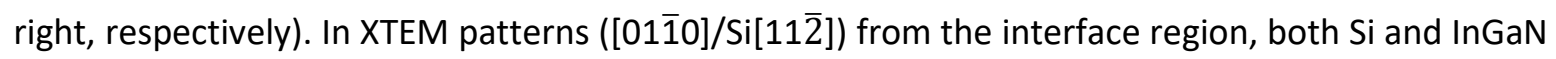
associated diffraction networks are concentric and their related spots are aligned, which implies that the InGaN crystalline structure is epitaxially grown on the Si lattice. The fact that this effect is

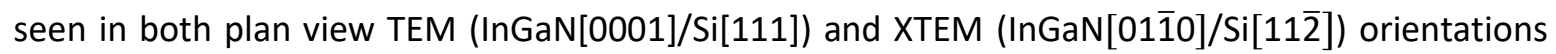
confirms both axial InGaN(0001) ||Si(111) and in-plane $\operatorname{InGaN}(01 \overline{1} 0)|| \mathrm{Si}(11 \overline{2})$ epitaxial relations. The slight arcing of InGaN reflections indicates that the alloy structure is composed of pseudodomains, mostly epitaxial, but some of them still having some small tilt and twist with respect to the growth direction. Although Si-associated spots are not visible in the PVTEM patterns, the InGaN diffraction data was collected when electron beam was parallel to the [111] Si zone axis. Lattice parameters extracted from SAED patterns of all four samples are presented in Table VI. Based on that, strain values and InN molar fractions were calculated, showing an excellent agreement with values obtained from HRXRD RSM measurements. Only sample LC3 has significant deviation of SAED results from HRXRD and EDX, which is probably related to smaller regions of SAED measurements (area of $\sim 400 \mathrm{~nm}$ in diameter selected with an aperture).

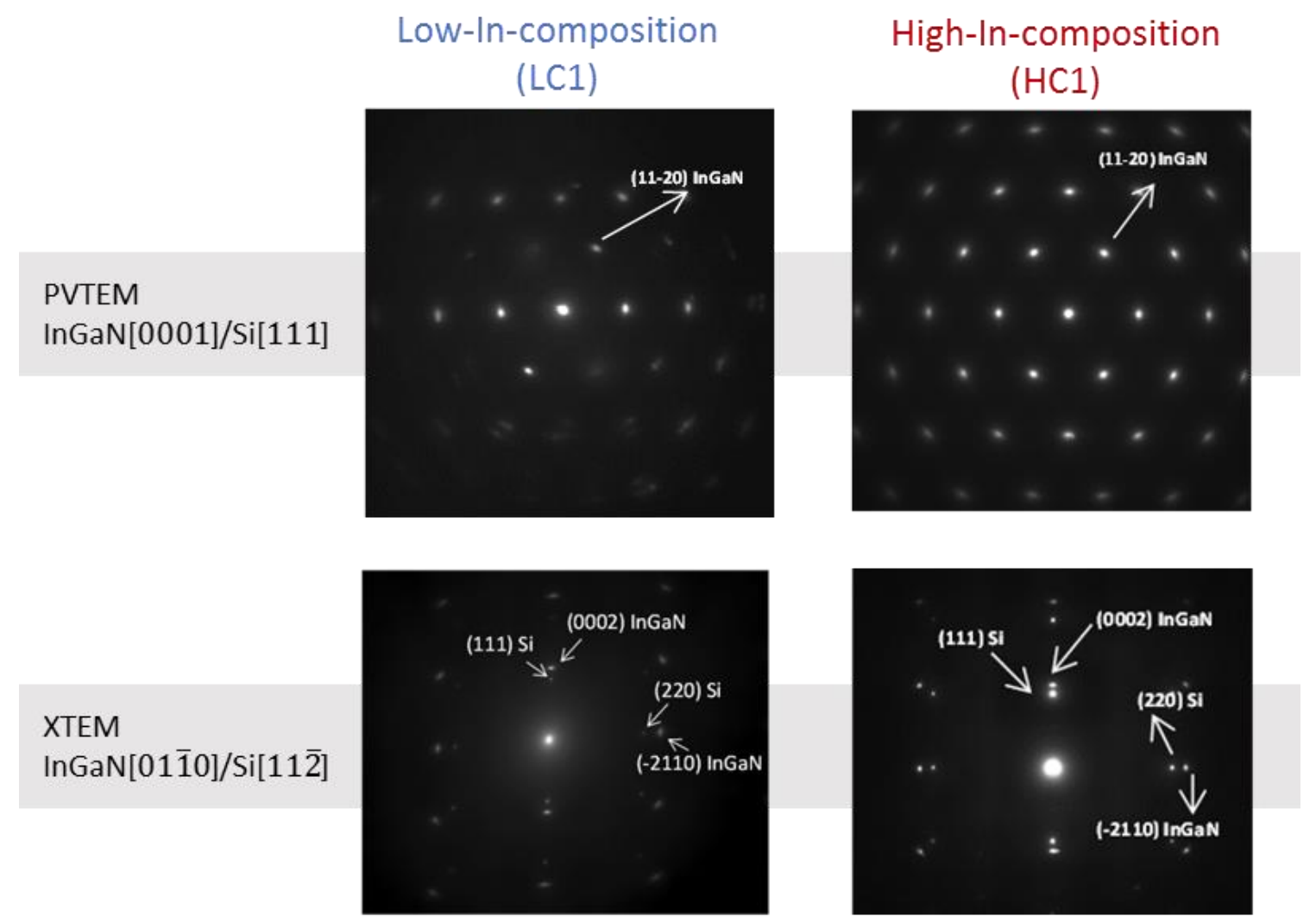

Fig. 4.5.3. SAED patterns of low-In-composition (LC1) and high-In-composition (HC1) samples taken in PVTEM (top row) and XTEM (bottom row) orientations. For both samples full epitaxial relationships as

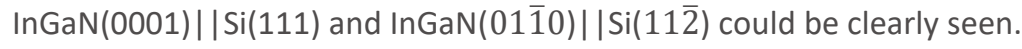


Further, the crystal quality of the low- (LC2) and high-In-composition (HC1) samples were examined by conventional TEM (CTEM) micrographs recorded along the InGaN[01 $\overline{1} 0] / \mathrm{Si}[11 \overline{2}]$ zone axes, as shown in Fig. 4.5.4, which indicate that the $\operatorname{lnGaN}$ film is, at micrometric scale, a single-crystalline layer. However, a noticeable density of extended defects, mostly threading dislocations (TDs) expected for such a high lattice mismatch, is present being similar to that found in other III-Nitrides heteroepitaxially grown on non-native substrates [Manuel2012].
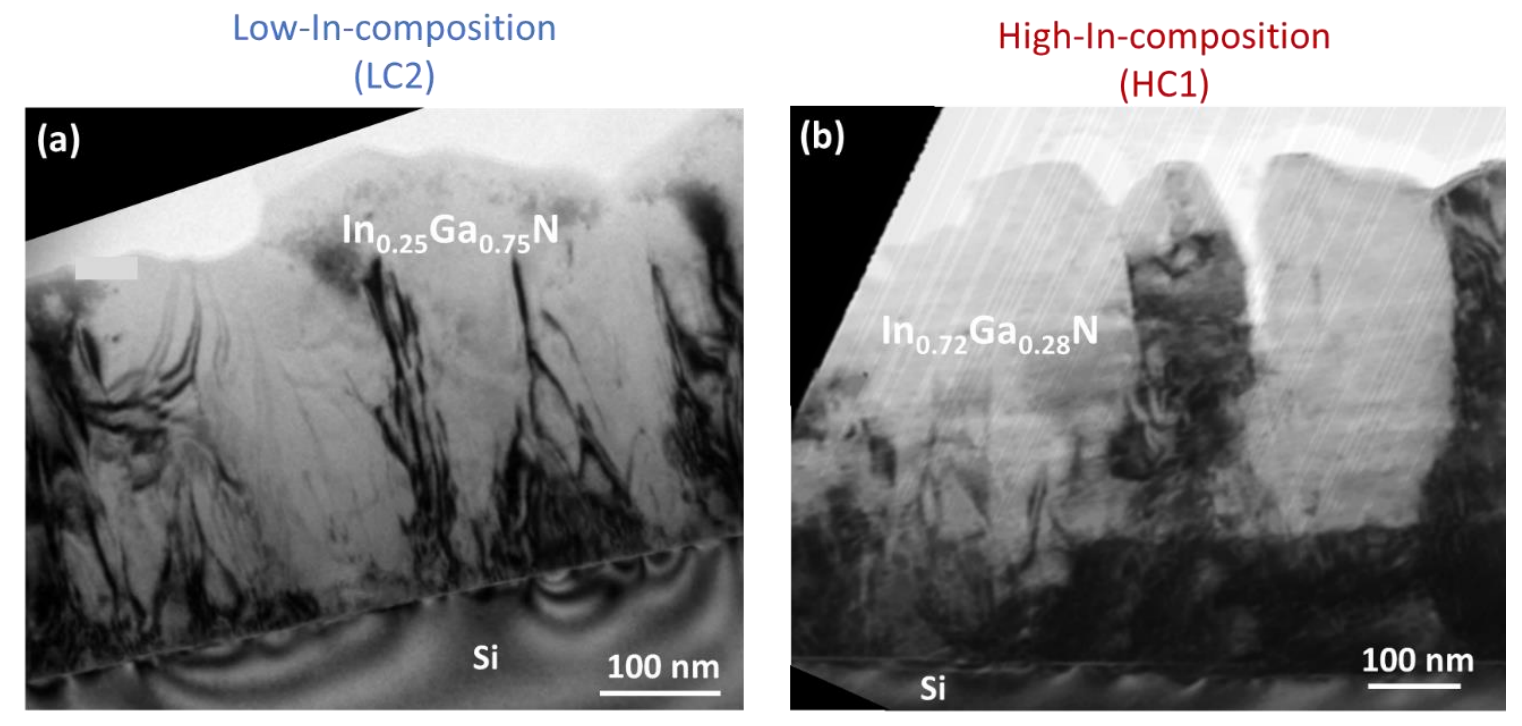

Fig. 4.5.4. CTEM micrographs of low (a) and high In composition (b) samples.

The LC2 sample was additionally examined by Bright Field (BF) mode XTEM in a 2-Beam diffraction contrast (2B-DC) under different reflections near the [0110] InGaN zone axis. To estimate a screw/edge TDs ratio two panoramic micrographs of the same sample area were acquired for perpendicular reflections $g 0002$ (Fig. 4.5.5 (a)) and g2 $\overline{1} \overline{10}$ (Fig. 4.5 .5 (b)), respectively. A high TDs accumulation appears always at very localized regions, what makes the quantification of TDs densities very difficult [Manuel2012]. Interesting to note that very pronounced and somehow periodic loop contours can be seen in the Si substrate, as an indirect image of strain fields accumulated at the Si near the interface. It seems that TDs mainly originate at the intersections of these loops with the interface. This suggest that InGaN grows on $\mathrm{Si}(111)$ in a domain-like way (in agreement with SAED data) with TDs mainly generated at pseudo-grains coalescence boundaries where strain is released. This results suggest that InGaN on $\mathrm{Si}(111)$ grows in a similar way to GaN (as expected), which is well known to grow on non-native substrates as an ensemble of crystallites, or grains (mosaic structure), that coalesce into a compact film [Akasaki1989, Hiramatsu1991, Lebedev2007]. 


\section{(a)}

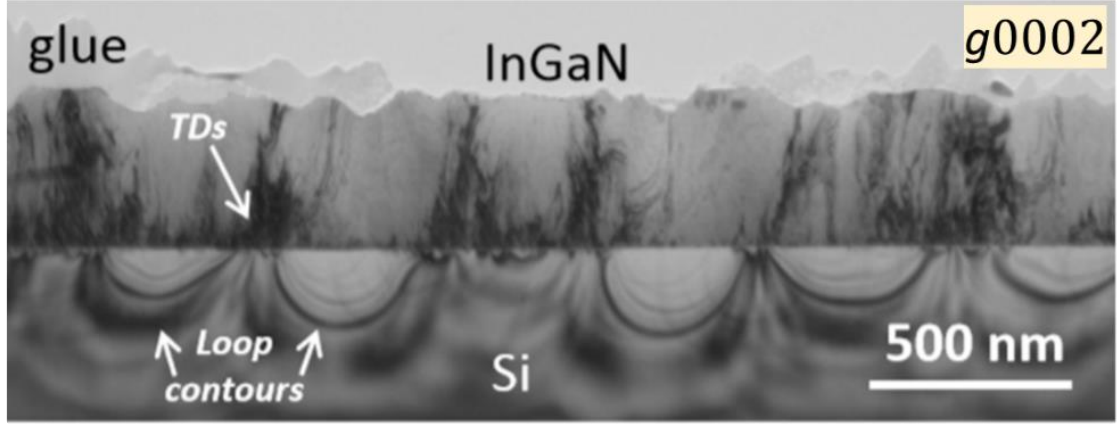

(b)

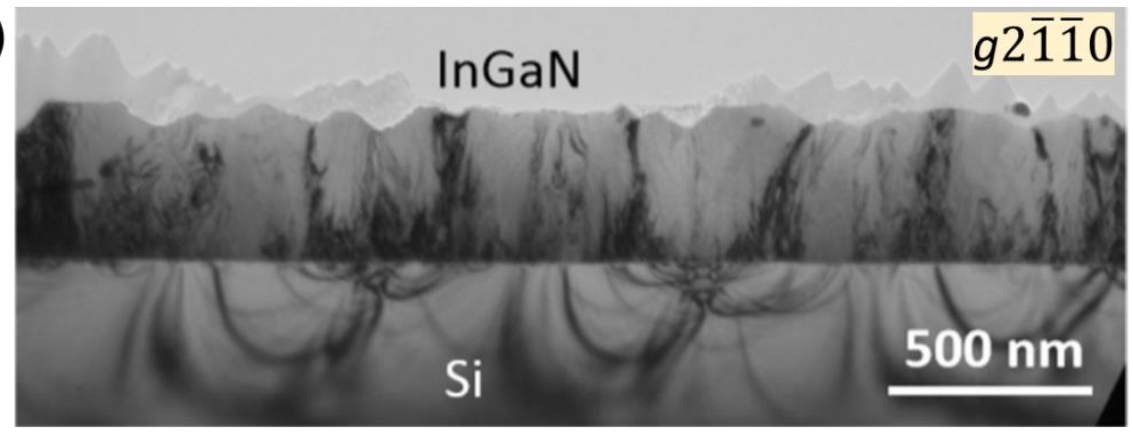

Fig. 4.5.5. DC-2B BF XTEM panoramic micrographs of the same area of a sample (Ino.25Ga0.75N, LC2) taken at perpendicular reflections $g 0002$ (a) and $g 2 \overline{110}$ (b), aimed to look for screw- and edge-type threading dislocations (TDs), respectively. Strain field loop contours in Si and accumulation of TDs near their ends are marked with white arrows.

\subsubsection{Cubic inclusions}

Although not observed at lower magnifications, the presence of relatively small regions with cubic structure, randomly distributed in the InGaN layer, was detected in HRTEM images of sample LC2 (Fig. 4.5.6) where most of the crystal exhibit the wurtzite structure (region 1). The corresponding Fast Fourier Transform (FFT) image of this region FFT(1) confirms an hexagonal symmetry. In contrast, the atomic columns arrangement of region 2 clearly indicates a cubic phase, which is confirmed by the FTT(2) analysis of this region. Finally, regions with a mixture of cubic+hexagonal structures were also found (region 3 with the corresponding FTT(3)).

Cubic inclusions have been reported many times in III-Nitrides near the interface with the substrate [Calleja1998, Calleja1999, Albert2014]. However, in our case, such inclusions were only observed in one (LC2) out of four examined samples. This could be due to a limited ability of HRTEM technique to examine large areas of the sample. The fact that neither HRXRD nor SAED techniques revealed the presence of cubic phases in all samples suggests that their occurrence is indeed quite small. 


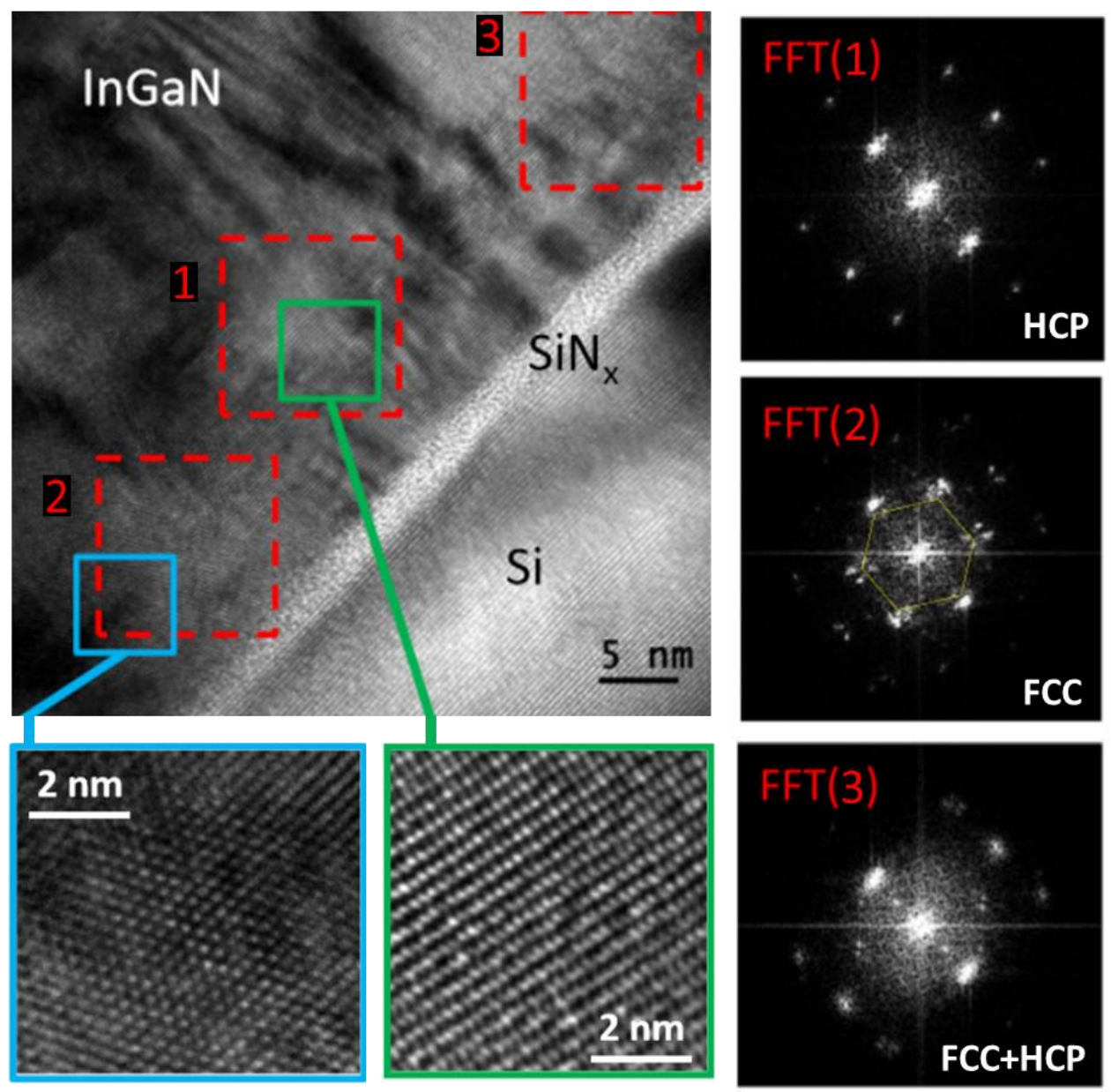

Fig. 4.5.6. Cubic and cubic/hexagonal mixture phases evidenced by HRTEM in LC2 sample. Three representative regions containing hexagonal (1), cubic (2), and hexagonal/cubic mixed (3) phases are marked and their corresponding FFT images presented.

\subsubsection{4 $\ln _{x} \mathrm{Ga}_{1-x} \mathrm{~N} / \mathrm{SiN}_{\mathrm{y}} / \mathrm{Si}$ interfaces}

The structure of the $\operatorname{lnGaN} / \mathrm{SiN}_{\mathrm{x}} / \mathrm{Si}$ interfaces was examined by HRTEM with images as those from low-In (LC1, oriented along the [011̄0] InGaN zone axis) and high-In (HC1, oriented along the [11 $\overline{2} 0]$ InGaN zone axis) samples shown in Fig. 4.5.7 (a,b), respectively. Aside from occasional localized defects, clear projections of atomic columns are observed for both InGaN layers and Si substrates, confirming an excellent InGaN(0001) || Si(111) epitaxial relation.

In both samples a 2-3 $\mathrm{nm}$ thick continuous $\mathrm{SiN}_{\mathrm{x}}$ interlayer appears to be amorphous on HRTEM images, since no atomic columns could be resolved. This agrees well with reports in the literature as no crystalline $\mathrm{SiN}_{\mathrm{x}}$ layer was ever reported in HRTEM studies of III-Nitride/Si interface (samples prepared by ion milling). However, it contradicts the in situ RHEED measurements performed during the growth that indicated the formation of crystalline $\beta-\mathrm{Si}_{3} \mathrm{~N}_{4}$ (Fig. 4.2.2 (b)). As it was described in section $\mathbf{2 . 5 . 3}$, this contradiction is most likely to arise from damage during the ion milling TEM preparation [Rawdanowicz2005]. 
(a) Low-In-composition

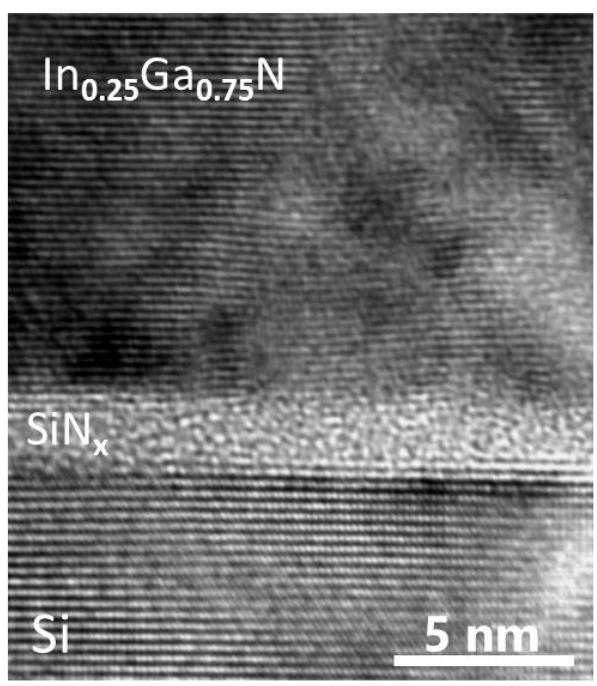

(b) High-In-composition

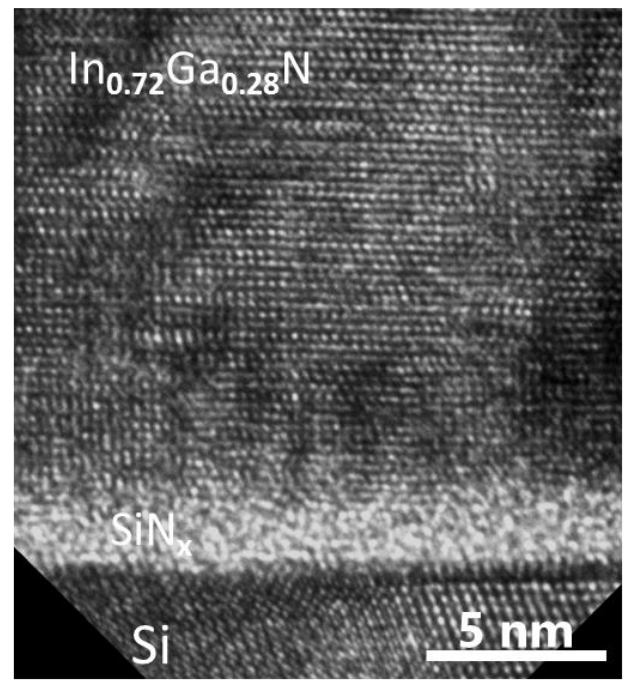

Fig. 4.5.7. Structure of the $\ln \mathrm{GaN}_{\mathrm{SiN}} / \mathrm{Si}$ interfaces examined by HRTEM in low-In (LC1, oriented along the [0110] InGaN zone axis) and high-In-composition (HC1, oriented along the [1120] InGaN zone axis) samples.

The $\mathrm{SiN}_{\mathrm{x}}$ layer examination on a larger scale (several microns) revealed a continuous layer over the examined areas in all inspected samples, even at existing $\mathrm{Si}$ substrate steps, as shown in Fig. 4.5.8 $(a, b, c)$ for sample HC1. Notice that CTEM images of LC3 sample revealed that high TDs densities are associated with these Si steps (Fig. 4.5 .8 (d)).
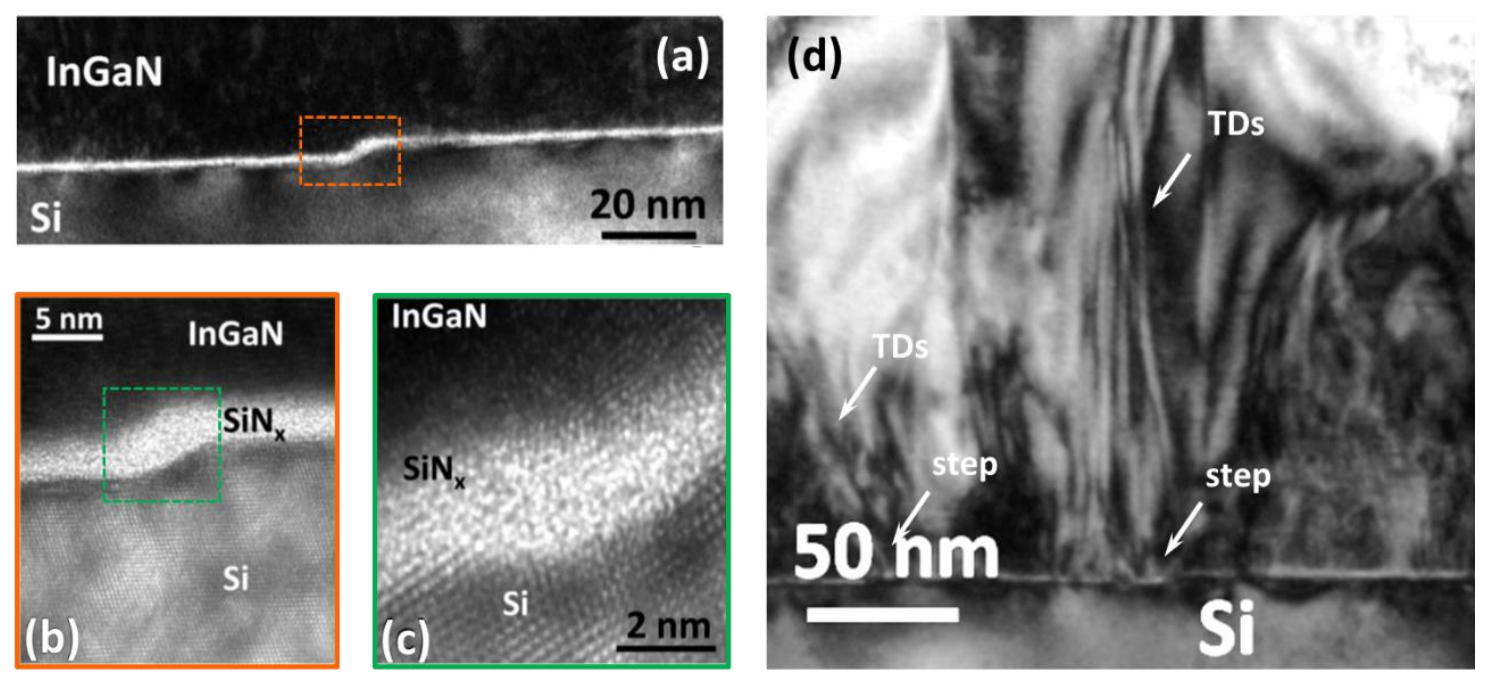

Fig. 4.5.8. Formation of a continuous $\mathrm{SiN}_{\mathrm{x}}$ layer, even on Si surface steps as evidenced by CTEM (a) and $\operatorname{HRTEM}(b, c)$ of sample HC1. CTEM image of sample LC3 (d) clearly shows a high density of TDs at Si steps. 


\subsubsection{Photoluminescence and bowing parameter}

The InGaN layers optical quality was assessed by photoluminescence (PL) measurements at both low $(8-15 \mathrm{~K})$ and room temperature, as shown in Fig. 4.5.9 (a,b), respectively. The regions where detectors reach their detection limits are marked with a grey hatching. Pure InN samples were excluded from this study as the Ge detector cannot be reliable. Once plotted together in an absolute scale, spectra were normalized to the maximum amplitude of the brightest sample and scaling factors assigned to each spectrum in Fig. 4.5.9. The ratio between low- and RT PL intensities scale is 95:1. Most of the PL spectra exhibit a single peak with intensity varying by a factor of 4 among the samples, thus pointing to a comparable optical quality over the entire composition range, as well as the absence of significant phase separation (refer to section 2.5.6). Some broadening is seen for low-In-content samples, which is probably due to the fact that the growth temperature is too low for such Ga-rich samples or due to lattice pulling effect [Stringfellow2010].

By combining RT-PL energies and the In content determined from HRXRD RSM data, the bandgap dependence versus composition was derived, as shown in Fig. 4.5.10, plotted against the AM1.5g solar spectrum to show the overlap. From this dependence, the bowing parameter $b$ of $2.5 \mathrm{eV}$ was estimated by a quadratic Vergard's equation (orange line) (Eq. 2.3.1 in section 2.3.1). The bowing parameter determined in the present study is in the range of $1.2-4.5 \mathrm{eV}$ reported for the InGaN layers grown on sapphire [Adachi2009].

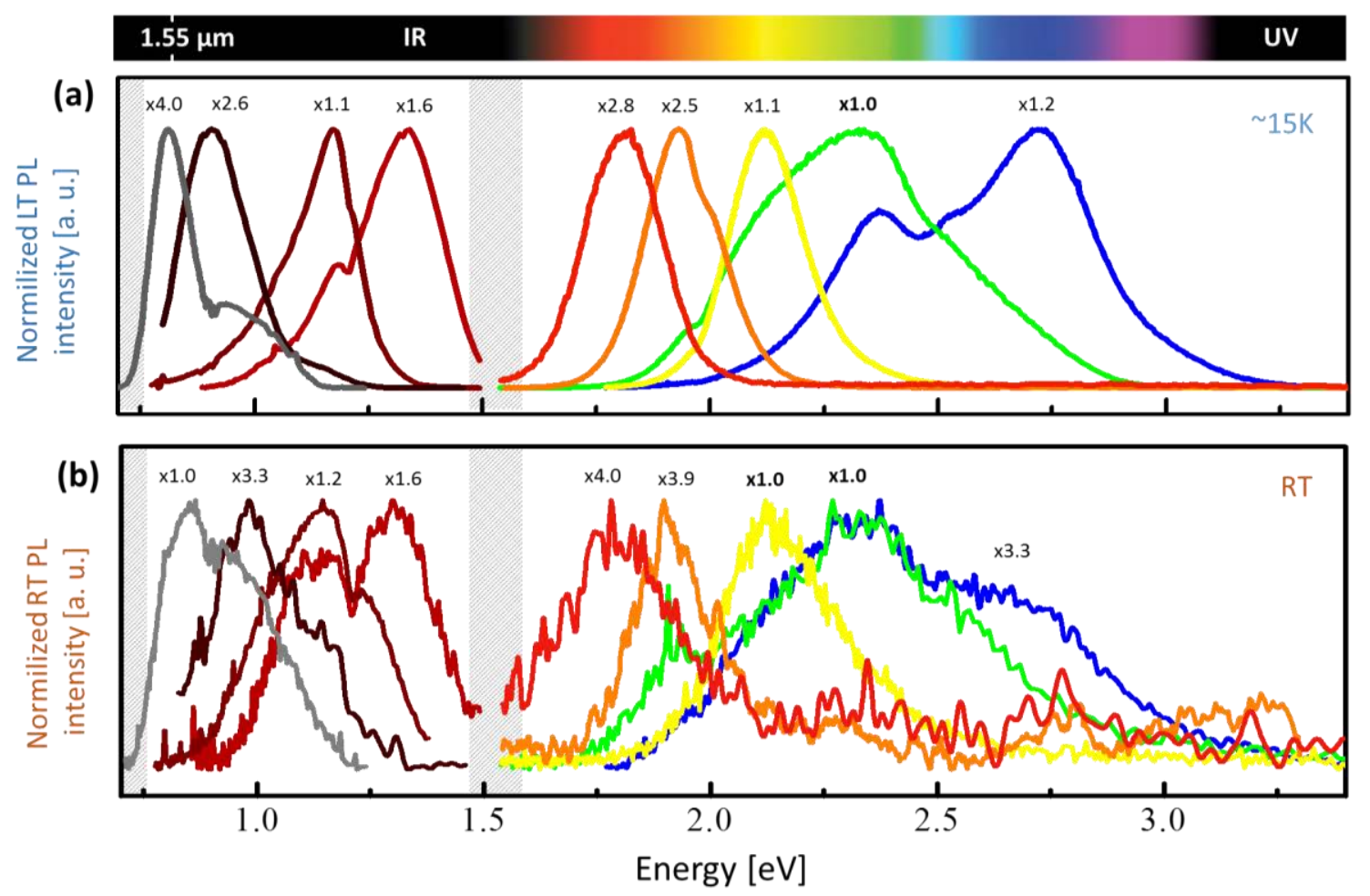

Fig. 4.5.9. Normalized PL spectra of the InGaN layers taken at $15 \mathrm{~K}$ (a) and RT (b). The relative PL intensity scaling factors are indicated. The low- to RT PL intensities scale is 95:1. 


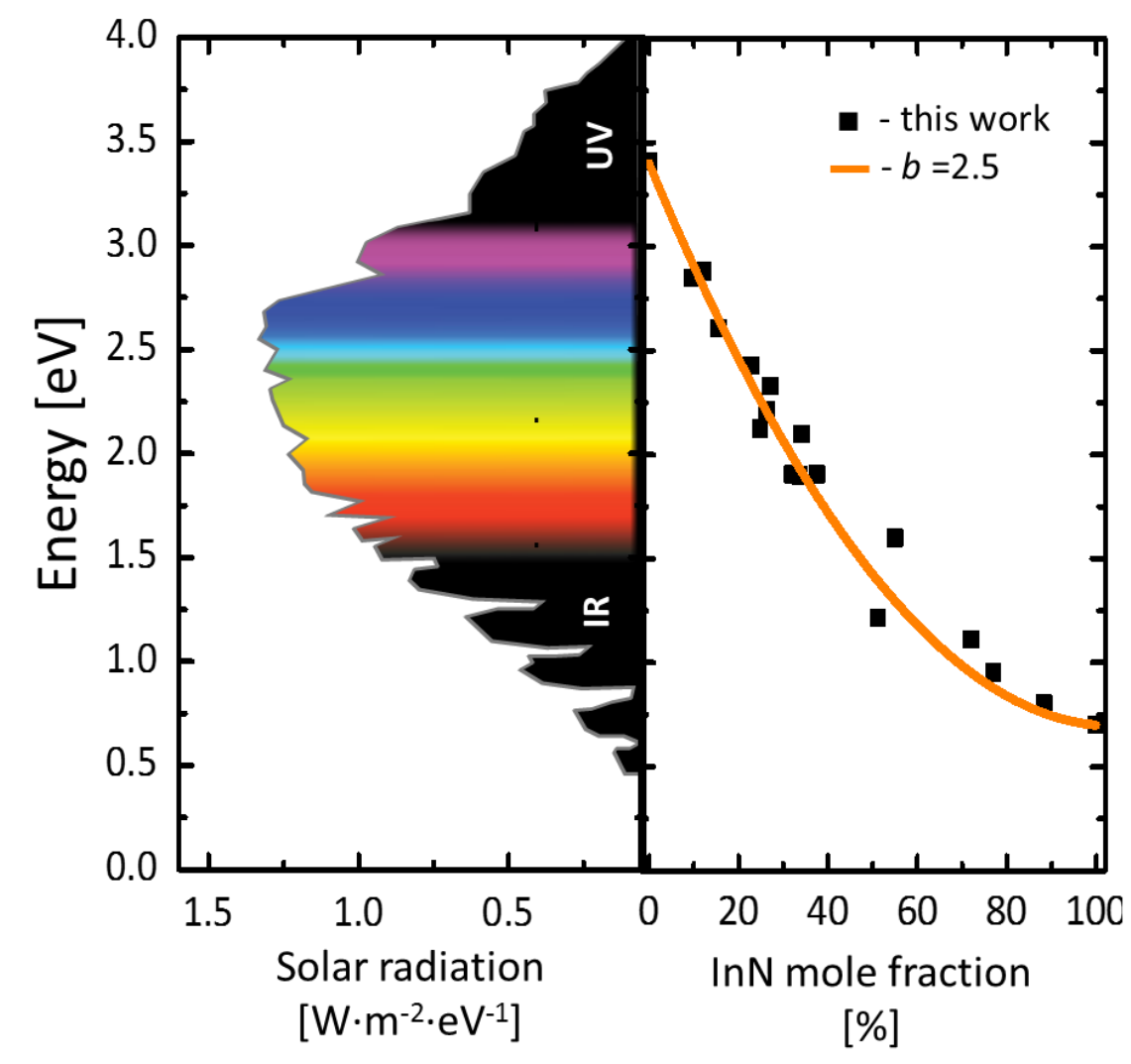

Fig. 4.5.10. In $\mathrm{n}_{\mathrm{x}} \mathrm{a}_{1-\mathrm{x}} \mathrm{N}$ bandgap energy (from RT-PL) dependence with In content (from HRXRD RSM). AM1.5g solar spectrum is plotted as a reference.

\subsubsection{Scanning near-field optical microscopy}

Scanning near-field optical microscopy (SNOM) is an ideal tool to assess the spatial distribution of the emission wavelength in a sample. SNOM allows determining any sign of phase separation at a scale of $\sim 150 \mathrm{~nm}$ or lower. Thus, spatially resolved optical properties of an $\ln _{0.72} \mathrm{Ga}_{0.2} 8 \mathrm{~N}$ layer (M208/HC3) (best potential case for phase separation) were studied by SNOM at 10K.

Fig. 4.5.11 (a) shows a $4 \times 4 \mu \mathrm{m}^{2}$ near-field PL peak intensity map, revealing a spatially homogeneous distribution with a standard deviation of intensity values of about $\sigma_{I}=15 \%$ (histogram in Fig. 4.5.11 (a)). High intensity red regions are most likely corresponding to weak In fluctuations which localize carriers and, thus, emit light more efficiently. Representative near-field PL spectra taken at randomly chosen locations are presented in Fig. 4.5.11 (b), that shows that the peak energy lies in a narrow energy range around $1.24 \mathrm{eV}$ with a standard deviation of $\sigma_{E}=9 \mathrm{meV}$. This roughly corresponding to $73 \% \operatorname{InN}$ molar fraction with standard deviation of $0.3 \%$, based on data in Fig. 4.5.10, which is consistent with a high chemical uniformity assessed by TEM and HRXRD in the previous sections. 

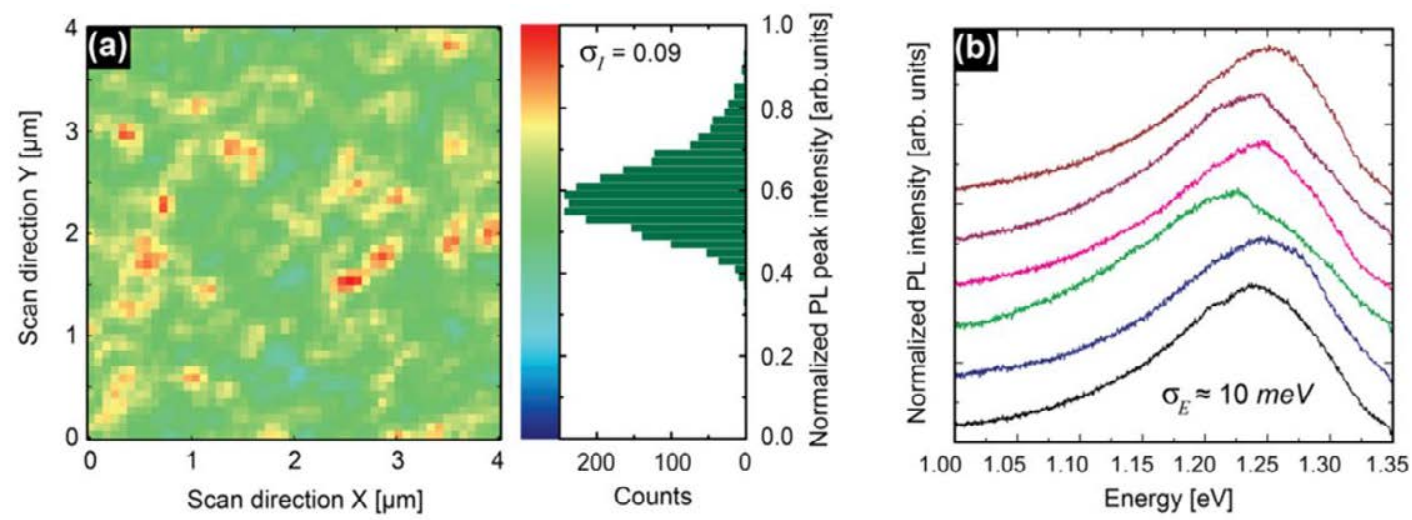

Fig. 4.5.11. SNOM map of the $10 \mathrm{~K}$ normalized PL peak intensity from an $\ln 0 .{ }_{72} \mathrm{Ga}_{0.28} \mathrm{~N}$ (HC1) layer with a histogram of the intensity distribution (a). Near-field PL spectra taken at randomly selected positions (b).

\subsubsection{Electrical characterization}

The electrical properties of the InGaN/Si heterointerfaces were assessed by current-voltage (I-V) measurements. For that $\mathrm{Ti} / \mathrm{Al} / \mathrm{Ni} / \mathrm{Au}$ top contacts were used for InGaN layers [Fontsere2011], while contact to the back side of the p-type Si substrate was done by indium soldering. The I-V characteristic from a medium In-content $\operatorname{In}_{0.37} \mathrm{Ga}_{0.63} \mathrm{~N}$ sample (M139), shown in Fig. 4.5.12 (a), reveals top-to-top contacts quasi-ohmic behavior but the vertical conductivity is rather rectifying (though leaky). This may be due to a Schottky-like top contact as residual n-type conductivity of the $\mathrm{In}_{0.37} \mathrm{Ga}_{0.63} \mathrm{~N}$ layer is not high enough.

For In contents above $50 \%$ the InGaN layers are highly n-type conductive, most likely due to $\mathrm{O}_{2}$ contamination or $\mathrm{N}$-vacancies [King2007]. Therefore, ohmic Ti/Al/Ni/Au top contacts are easily obtained. The I-V characteristic for a high-In-content $\operatorname{In}_{0.53} \mathrm{Ga}_{0.47} \mathrm{~N}$ sample top-to-top contacts shows low resistivity ohmic behavior (inset in Fig. 4.5.12 (b)). Most important, the vertical (top-to-bottom) $\mathrm{I}-\mathrm{V}$ characteristic also shows a near-ideal ohmic behavior of the $\mathrm{n}-\mathrm{In}_{0.53} \mathrm{Ga}_{0.47} \mathrm{~N} / \mathrm{p}$-Si heterointerface (Fig. 4.5.12 (b)). This behavior agrees well with the theoretical predictions by [Hsu\&Walukiewicz2008] that establish a 45 to $50 \%$ In composition as the onset for a strong residual n-type conductivity in $\operatorname{InGaN}$ layers (i.e. $\ln _{x} G a^{1-x} \mathrm{~N}$ layers are highly conducive above $50 \%$ In composition and resistive below).
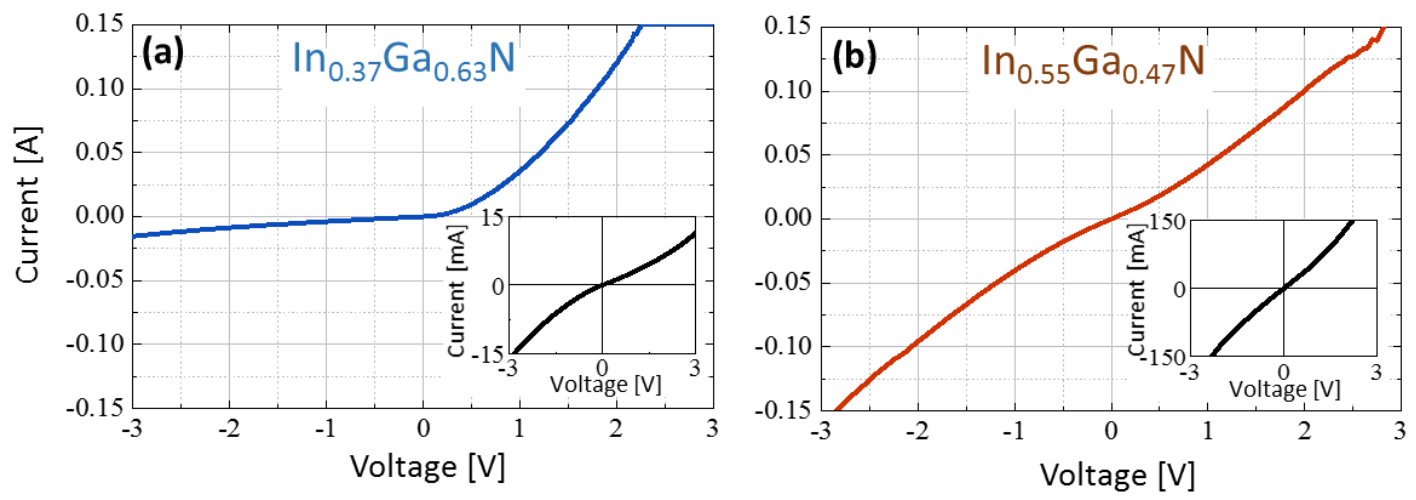

Fig. 4.5.12. I-V curves measured for top to bottom contacts from InGaN/Si samples with below (a) and above (b) 50\% of In-content. I-V curves for top-top contacts are shown in insets. 


\subsection{Conclusions}

In this chapter thick, compact, and chemically uniform $\ln _{x} G_{1-x} N$ layers of high optical and structural quality with $x$ covering the entire composition range $(x=0-1)$ were grown directly on nitridated $\mathrm{Si}(111)$ by PA MBE. Excellent chemical homogeneity and absence of significant phase separation in In-rich $\operatorname{In}_{x} G_{1-x} N(x=0.25-1$ ) layers was confirmed by HRXRD, EDX, HAADF, PL, and SNOM measurements. Their wurtzite crystalline structure was confirmed by HRXRD and TEM SAED techniques. A relatively small amount of cubic inclusions close to the $\ln G a N / S_{N} N_{x} / S i$ interface detected in one of four samples examined by HRTEM, suggests that the cubic phase is exceptionally rare. Both axial $\operatorname{lnGaN}(0001)|| \mathrm{Si}(111)$ and in-plane $\ln \mathrm{GaN}(01 \overline{1} 0)|| \mathrm{Si}(11 \overline{2})$ epitaxial relations were confirmed by HRXRD, SAED, and HRTEM measurements. Similarly to the well described in literature case of GaN growth on Si [Akasaki1989], CTEM and SAED measurements revealed that InGaN is growing on Si as slightly misaligned grains (mosaic structure), which later coalesce into a compact layer.

An exceptionally wide tuneability of the $\ln _{x} G_{1-x} N$ layers emission wavelength was achieved, spanning from UV to near-IR regions from 356 to $\sim 1800 \mathrm{~nm}$ (pure InN emission, expected at $\sim 1850 \mathrm{~nm}$ was not measured due to the Ge detector range limitations), as confirmed by measuring $\mathrm{PL}$. Based on RT PL and HRXRD RSM measurements, $\ln _{\mathrm{x}} \mathrm{Ga}_{1-\mathrm{x}} \mathrm{N}^{\prime} \mathrm{s}$ bandgap versus In composition dependence was constructed and a bowing parameter of $2.5 \mathrm{eV}$ was estimated, which is within the range of literate values of $1.2-4.5 \mathrm{eV}$ [Adachi2009].

Additionally, the vertical conductivity of InGaN-on-Si was confirmed by I-V measurements, on two samples, with low ( 37\%) and high ( $55 \%)$ InN molar fraction of $\ln _{x} \mathrm{Ga}_{1-x} \mathrm{~N}$ layers.

To achieve these results, the substrate preparation, nucleation procedure and the following growth were carefully optimized. The key factors determining the successful InGaN growth on $\mathrm{Si}(111)$ were identified as: (i) intentional nitridation of the Si substrate to form a crystalline $\beta-\mathrm{Si}_{3} \mathrm{~N}_{4}$ layer on $\mathrm{Si}(111)$, which promotes a chemically homogeneous $\ln _{x} \mathrm{Ga}_{1-x} \mathrm{~N} / \beta-\mathrm{Si}_{3} \mathrm{~N}_{4} / \mathrm{Si}$ interface, (ii) moderately $\mathrm{N}$-rich start (III/N 0.77) during the first $5 \mathrm{~min}$ of the III-Nitride growth to avoid metal accumulation and formation of macro-defects, and (iii) bulk growth under slightly $\mathrm{N}$-rich conditions (III/N 0.9) and at relatively low substrate temperatures $\left(450-480^{\circ} \mathrm{C}\right)$ to form compact and chemically homogeneous films without phase separation. The only drawback of such technique is that the morphology of obtained layers is relatively rough and difficult to improve by simply adjusting growth parameters [Valdueza-Felip2014]. The use of a more elaborated techniques such as migrationenhanced epitaxy (MEE) [Horikoshi1988] was suggested to overcome this issue, which is proposed for future work.

For some applications where an enlarged surface area is of great importance, a rough morphology is an advantage, for example, for water splitting or chemical sensing. In the case of water splitting, the obtained results are particularly exciting, as ability to tune the InGaN bandgap over an exceptionally wide range that allows optimizing the optical properties of InGaN-based photoelectrodes. This issue is addressed in detail in Chapter 7.

Results from the work presented in this chapter were published in [Aseev2013, Aseev2015]. 


\section{Growth of InN QDs via low temperature droplet epitaxy}

\subsection{Introduction}

Semiconductor quantum dots (QDs) have been studied extensively because of their unique physical properties and potential for device applications. Due to quantum confinement effects the emission wavelength of QDs can be directly controlled by changing their size. These effects can be measured if the QDs size is uniform, because, otherwise, a broad distribution of sizes "smears" the density of states, producing a behavior similar to that of bulk material. Thus, the fabrication of small and uniform in size nanostructures becomes of great interest.

In the last decade, III-Nitrides QD structures became crucially important for various applications in electronic and photoelectric devices, such as infrared photodetectors [Hsu2014], solar cells [Deng2011, Rashid2013], single photon sources [Chernysheva2015, Lazic2015], chemical- and biosensors [Lu2007, Alvi2012], etc. Moreover, in respect to water splitting it was recently demonstrated that decorating an InGaN photoanode with InN QDs can nearly double its maximum incident photon-to-current conversion efficiency (IPCE) [Alvi2015]. However, the fabrication of IIINitride QDs is still a challenge, particularly InN QDs.

InN QDs are usually fabricated through Volmer-Weber (VW), Stranski-Krastanow (SK) and Droplet epitaxy (DE) methods (refer to section 3.2.3). The VW mechanism is mostly reported in samples grown by MOCVD systems [Chang2008, Wang2009], and very rarely for PA MBE growth [Moustakas2008]. However, the size of InN nanostructures in case of VW growth mechanism is in the order of hundreds of nanometers, which makes them not feasible for applications requiring "real QDs" (i.e. 3D carrier confinement). In contrast, in case of the SK growth mecahnism nanometer-sized InN nanostructures were obtained on various substrates, including GaN/sapphire(0001) [Norenberg2002, Dimakis2005, Ruffenach2007], GaN/SiC(001) [Ng2002, Cao2003], AIN/Si(111) [Shen2006], and Si(111) [Kumar2011]. As explained in section 3.2.3.2.1, the SK growth mode initiates by a 2D strained wetting layer further developing into 3D islands upon strain relaxation, implying that the method is essentially useful only for "flat" substrates (with atomically flat surface). However, many devices relying on more complex 3D surface morphologies, often intentionally used to enhance their performance (such as sensing or energy harvesting devices) may also require QD incorporation for further performance boost. In the case of complex morphologies without any "flat" regions the application of the SK method becomes very complicated if not impossible. Thus, in order to introduce InN QDs into these devices a novel fabrication method allowing growth of InN QDs on rough surfaces must be developed.

Requirement of "flat" surface can be negated if droplet epitaxy (DE) is used (refer to section 3.2.3.2.2). While the current understanding of DE predicts that it would be feasible, so far 
only results using "flat" substrates were reported, including GaN/sapphire(0001) [Krishnamurthy2012], AIN/Si(111) [Kumar2013], and Si(111) [Kumar2010, Qi2013, Chen2016]. Moreover, almost all of these reports are restricted to a morphological analysis of the InN QDs but no comprehensive studies of their formation mechanisms and/or their crystalline structure were addressed. Thus, it is safe to say that DE of InN QDs is still largely under development.

In this work, InN nanostructures (or nanodots in more general term) are called island or QDs, depending on their low or high aspect ratio (Height/Diameter below or above 0.1). First, the low temperature (below RT) droplet epitaxy formation mechanisms of InN islands and QDs were studied on $\mathrm{Si}(111)$ and $\mathrm{Si}(001)$ substrates, which flat surface allowed a relatively easy structural characterization. In addition, the Si (111) vs. (001) different crystal planes allowed for comparative study, in particular tracing the evolution of the $\mathrm{InN} /$ substrate epitaxial relation at the interface. Once the growth procedure was understood, it was finally successfully applied transferred to InGaN/Si(111) layers with rough surface ( $\mathrm{rms}>10 \mathrm{~nm}$, with no atomically flat regions) to grow $\mathrm{InN}$ QDs of exceptional crystalline quality, with high aspect ratio, nanometer size, and moderate density.

\subsection{InN QDs grown on $\mathrm{Si}(111)$}

Before trying to grow InN QDs directly on rough InGaN layers, a relatively simpler case of $\mathrm{Si}(111)$ substrate was chosen as a "testing ground", because of its flat surface and well known surface properties, which significantly facilitate the characterization of the grown samples, but still allowing to get insights into the formation mechanisms of InN QDs. In the following section, the main steps of InN QDs growth are examined in detail.

\subsubsection{Fabrication steps}

The fabrication process of InN QDs is described Step by step in the following subsections.

\subsubsection{Si(111) substrates preparation}

Substrates must be properly prepared before depositing metallic In. As it was mentioned before in section 3.2.3, the use of "antisurfactants" can significantly modify the surface diffusion barriers and yield totally different wetting patterns on the same substrate [Koguchi1993]. The presence of additional gases, even inert ones, during the deposition of the In droplets can also affect their size and density [Ruffenach2007]. That is why a special care was taken about the substrate preparation in our case.

For all experiments in this section, $\mathrm{Si}(111)$ substrates were cleaned as follows:

1. Degassing in the MBE introduction chamber at $400{ }^{\circ} \mathrm{C}$ at high vacuum $\left(10^{-8}\right.$ torr) for 1 hour to remove any water and contaminants.

2. Degassing in the $\mathrm{MBE}$ growth chamber at $850^{\circ} \mathrm{C}$ at ultra-high vacuum $\left(10^{-10}\right.$ torr) for $30 \mathrm{~min}$ to remove native oxides (confirmed by RHEED observation of (1x1) to (7x7)-Si (111) reconstructions transition).

3. Cooling down to the deposition temperature (at least for 8 hours, overnight) keeping ultra-high vacuum $\left(10^{-10}\right.$ torr). 
During this process, all Knudsen cells were kept at stand-by temperatures and the $\mathrm{N}_{2}$ plasma source turned off in order to prevent any unexpected contamination of the Si surface. Si(111) 7x7 surface reconstruction was preserved during the overnight cooling.

Another important effect which must be taken into account during the In droplets deposition is the additional effect of change of the surface adatom mobility due to the presence of heat sources within the MBE growth chamber (sometimes referred to as "thermal coupling") [Bickford2008]. Because of high surface mobility of In even at low temperatures and the proximity of the sample surface temperature to In melting point $\left(156.6^{\circ} \mathrm{C}\right)$ any additional heat source can drastically modify the distribution of In droplets on the substrate surface [Pocza1969, Bickford2008]. For example, nitrogen plasma has a potential to increase the surface temperature above the In melting point (without affecting thermocouple readings) which would result in a much higher In surface mobility and, consequently, much larger In droplets with lower density. This effect is machine dependent. This may explain the scatter in results from different groups reporting on In droplets size and density on similar substrates and deposited at nominally equal temperatures [Kim2004, Lee2009]. In order to minimize the effect of heat sources and MBE walls on In deposition procedure, the cleaned substrates were kept overnight cooling with all the Knudsen cells at stand-by.

\subsubsection{In deposition on Si(111) substrates}

The next step in InN QDs growing process is to deposit In droplets. In the original works on lowtemperature DE of III-As QDs, the control of metal droplets size and density was achieved by substrate temperature variations during the metal deposition. Thus, in this work, a series of In depositions on clean $\mathrm{Si}(111)$ substrates at different temperatures was performed. After the substrate cleaning and overnight cooling, it was heated (the heater switched on) or cooled (the heater switched off and the MBE internal cryopanels kept cold) to the desired deposition temperature. Cooling rate was measured to be of $\sim 10^{\circ} \mathrm{C} /$ hour (when below $60{ }^{\circ} \mathrm{C}$ ), while In deposition time was set to $\sim 10 \mathrm{~s}$, which ensures that experiments were done at a stable surface temperature.

Several In depositions were done at substrate temperatures varying from $-10^{\circ} \mathrm{C}$ to $110^{\circ} \mathrm{C}$. Fig. 5.2 .1 presents an AFM scan of a Si(111) substrate after being covered with $\ln \left(T_{\ln }=770^{\circ} \mathrm{C}\right.$, corresponding to In flux of $3.2 \times 10^{14}$ atoms $/ \mathrm{cm}^{2} / \mathrm{s}$, deposition time $t_{\text {dep }}=10 \mathrm{~s}$ ) at a substrate temperature of $53{ }^{\circ} \mathrm{C}$, showing very dense $\left(\sim 1 \times 10^{11} \mathrm{~cm}^{-2}\right)$, small droplets with average diameter of $\sim 15 \mathrm{~nm}$ and height of $\sim 1.5 \mathrm{~nm}$, with standard deviations of $\sigma_{D}=5 \mathrm{~nm}$ and $\sigma_{h}=1.2 \mathrm{~nm}$, respectively. It must be noted, that the AFM can significantly overestimate the diameter of such small objects. The change in substrate temperatures did not have any noticeable effect on the In droplets size distribution (this issue is further commented hereafter). This makes $D E$ of $\operatorname{InN}$ on $\mathrm{Si}(111)$ significantly different from the original DE of III-As QDs, where III-element droplets size and density can be controlled through the substrate temperature.

Temperatures above the In melting point were also used, resulting in In droplets with exceedingly and non-suitable low density $\left(\sim 8 \times 10^{4} \mathrm{~cm}^{-2}\right)$ and a much larger size $(1-10 \mu \mathrm{m})$. Moreover, higher temperatures promoted an eutectic reaction between In and Si. Fig. 5.2.2 illustrates the damage of the sample surface due to the eutectic etching for the sample with In droplets formed at $400^{\circ} \mathrm{C}$ and then nitridated for $3 \mathrm{~min}(0.5 \mathrm{sccm}, 350 \mathrm{~W})$. 
In droplets on $\mathrm{Si}(111)$

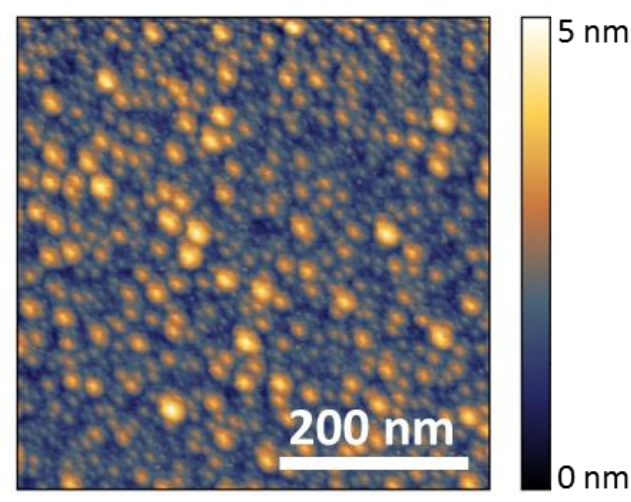

Fig. 5.2.1. AFM scans of $\mathrm{Si}(111)$ after In droplets deposition at a substrate temperature of $53^{\circ} \mathrm{C}$.

Thus, only the low temperature In deposition was used in the further experiments because (i) it yields very small In droplets, as needed for DE of InN nanostructures about $10 \mathrm{~nm}$ in size, and (ii) it can drastically reduce the eutectic reaction between metallic In and the clean, unprotected Si surface (meltback etching).

While In droplets formation below its melting point $\left(156.6^{\circ} \mathrm{C}\right)$ seems surprising, however, recent experimental studies of below RT deposition of various metals including In on Si surfaces [Tringides2010, Lunceford2012, Qi2013] and other substrates like MoS 2 and C [Pócza1969] confirmed unusually high metal adatom mobility which is largely governed by deposition rate and time rather than substrate temperature. Numerical simulations [Ratsch1994] and theoretical works [Stroscio1994] on islands nucleation in non-equilibrium cases also predict similar behavior. Thus, taking into account results of this work and published reports, it could be speculated that at temperature below melting point of In its surface diffusion is determined rather by kinetic energy supplied by impinging flux than substrate temperature. Additional experiments are necessary to clarify this.

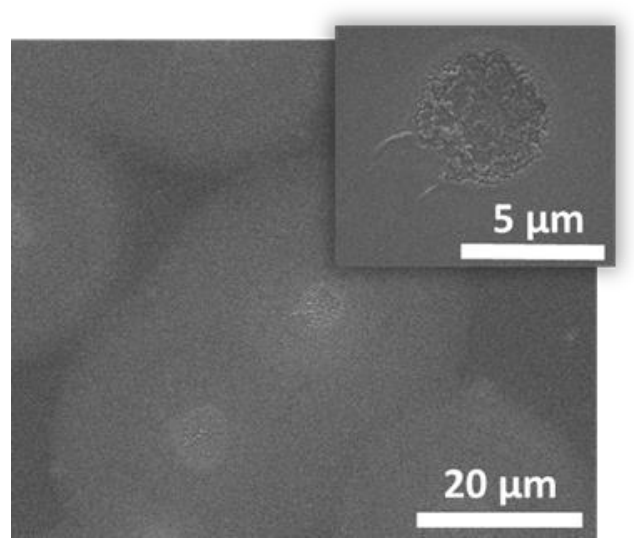

Fig. 5.2. SEM image illustrating the surface damage due to eutectic reaction between In and Si. 


\subsubsection{Nitridation of In droplets into InN islands}

The following step for InN DE is the nitridation of In droplets to form InN islands by exposing the sample to the active nitrogen from the plasma source at the same substrate temperature $\left(T_{\text {sub }}\right)$ as that for In droplets deposition. The plasma parameters were chosen to be identical to those for optimized InGaN growth, as discussed in the previous chapter, namely: $350 \mathrm{~W}$ and $0.5 \mathrm{sccm}$. The $\mathrm{N}_{2}$ plasma was turned on in less than 1 min after the In droplets deposition. In order to minimize any surface modifications during the $\mathrm{N}_{2}$ plasma switching on, the substrate manipulator was positioned in a way that the substrate is not facing neither the plasma nor the In cells.

Initial tests performed on In droplets deposited in the $15-110^{\circ} \mathrm{C}$ temperature range and further nitridated for 10 min revealed that, opposite to III-As DE (e.g. [Kim2004]), the In droplets conversion into InN islands does not happen "one-to-one". SEM images of samples A1 to A3 in Table VII clearly show that the resulting InN islands have a lateral size of $\sim 300 \mathrm{~nm}$ and density $\sim 3 \times 10^{6} \mathrm{~cm}^{-2}$, much higher and lower, respectively, compared to that of the In droplets, $\sim 15 \mathrm{~nm}$ and $\sim 1 \times 10^{11} \mathrm{~cm}^{-2}$ (for $T_{\text {ln }}$ $=770^{\circ} \mathrm{C}$ ). All the InN islands have a triangular-like shape with a dip in the center. Similar morphologies were reported by Tringides et al. for In islands on Si(111) deposited at low temperature [Tringides2010]. Moreover, the fact that a triangular shape is a characteristic of meltback etching of Si (refer to section 2.5.2) suggests that these effects can be related.

The morphology of sample A4 nitridated at $15^{\circ} \mathrm{C}$ is markedly different (See Table VII, A4), showing a significant size reduction $(\sim 50 \mathrm{~nm})$ and a density increase $\left(3.8 \times 10^{9} \mathrm{~cm}^{-2}\right)$ of the $\operatorname{InN}$ islands, compared to samples grown at higher temperatures. This strongly suggests that, at least in our PA MBE system configuration, substrate temperatures above $60^{\circ} \mathrm{C}$ are not suitable for $\mathrm{DE}$ of InN. A more detailed morphological and structural analysis of sample A4 is given in sections 5.2.2 and 5.2 .3 , respectively.

While it is unknown that is the exact reason behind revealed difference in $\ln$-to-InN conversion process compared to III-As case, once can speculate that it may be related to the fact that in PA MBE the nitrogen plasma source can supply additional energy to In adatoms and increase their mobility before converting into InN [Neugebauer2003, Mittemeijer2014]. In the case of III-As, the V-element (As) is generally supplied from a solid source (thermal beam) which should not have such great influence. Moreover, similar behaviour was reported for Ag nanoparticles deposited on a Si surface which undergo a plasma-induced Oswald ripening, leading to a nanoparticle size increase and a density decrease upon plasma treatment [Tang2011, Shi2015]. It seems that a similar process likely occurs in the $\mathrm{In} / \mathrm{Si}$ system under nitrogen plasma used in this work. 
Table VII. In droplets deposited during $10 \mathrm{~s}$ and nitridated for $10 \mathrm{~min}$ at different substrate and In cell temperatures. Nitridated islands of sample A3 are marked with white arrows.

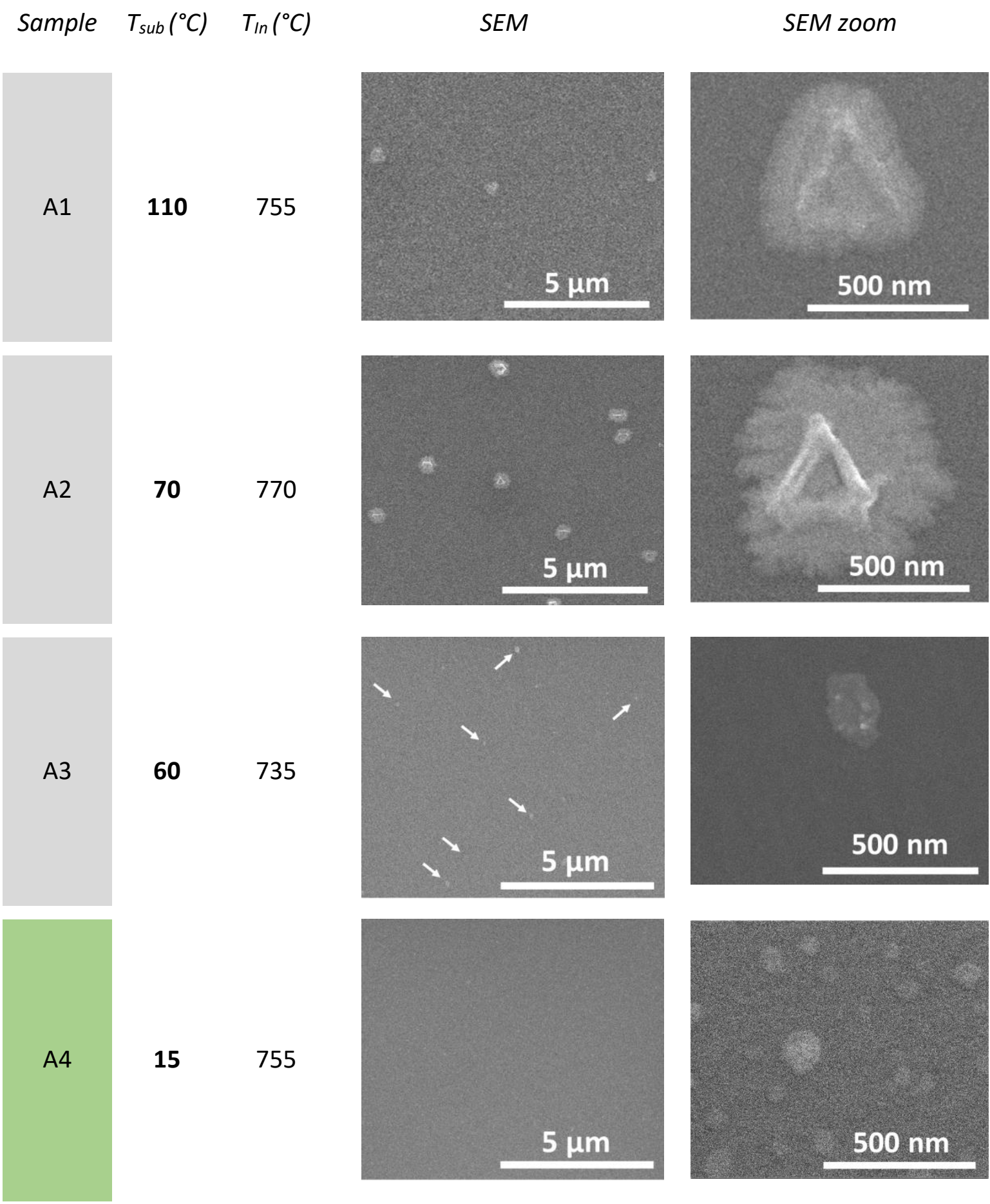




\subsubsection{Annealing of InN islands to form InN QDs}

Next, InN islands were annealed with aiming to increase their crystal quality and reshape them into QDs that are more compact. For that, another sample (A5) was grown under identical conditions as A4, but after the nitridation step the plasma source was kept going while the substrate temperature was slowly rising $\left(4^{\circ} \mathrm{C} / \mathrm{min}\right)$ up to $300^{\circ} \mathrm{C}$ and then kept there for a $30 \mathrm{~min}$ annealing. After annealing the sample was cooled to RT. The nitrogen plasma source switched on during the whole process to suppress a loss of material due to decomposition of $\ln N$. The substrate was rotated during the whole procedure (at 20 rotation per minute).

After nitridation and annealing processes, a drastic morphology change was observed in which the low aspect ratio (height/diameter $<0.1$ ) InN islands (Fig. 5.2.3, left) were converted into quasihemispherical shaped QDs (Fig. 5.2.3, right). A more detailed morphological and structural analysis of sample $A 5$ is given in section 5.2.2 and 5.2.3, respectively.

Annealing at different temperatures was also tested. Identical to described above annealing conditions, but at $100{ }^{\circ} \mathrm{C}$, resulted in no change of sample morphology. At $400{ }^{\circ} \mathrm{C}$ substantially less InN material left on the surface, probably due to enhanced decomposition of $\ln N$ and desorption of In. Thus, annealing temperature of $300^{\circ} \mathrm{C}$ showing the best results was chosen as an optimal one.

Revealed transformation of InN islands into InN QDs agrees well with the description of solid state dewetting process for polycrystalline islands (refer to section 3.2.3.2.3), where crystal islands deposited on a substrate reach their thermodynamic equilibrium shape if given enough energy/time [Thompson2012].

\section{InN morphology change upon annealing}

InN islands (A4)

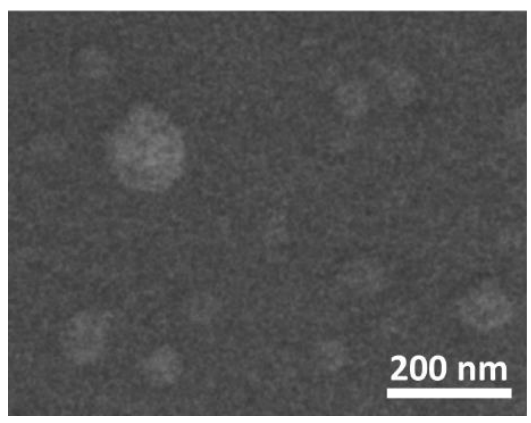

InN QDs (A5)

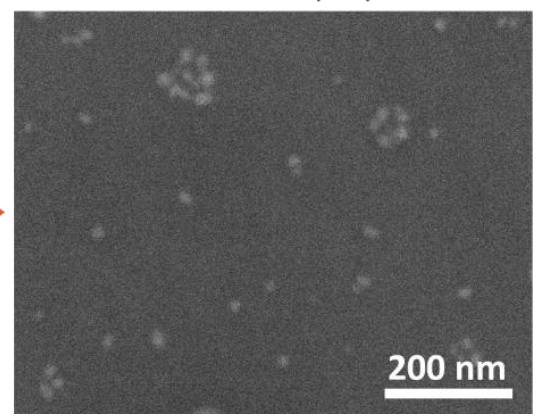

Fig. 5.2.3. InN islands transformation into InN QDs upon annealing at $300^{\circ} \mathrm{C}$ under nitrogen plasma for 30 min as evidenced by SEM. 


\subsubsection{Morphological characterization}

\section{InN islands on $\mathrm{Si}(\mathbf{1 1 1})$.}

The morphology of sample A4 with InN islands was examined by AFM and SEM in more details (Fig. 5.2.4 (a)). An AFM scan of an individual InN island presented in Fig. 5.2.4 (b) reveals its hexagonal shape. From the AFM images it seems that the crystal facets of different InN islands are all aligned (with only slight misorientation) which most likely indicates an epitaxial relation between the $\operatorname{InN}(0001)$ nanostructures and $\mathrm{Si}(111)$ substrate underneath. The measured surface density of InN islands is $3.8 \times 10^{9} \mathrm{~cm}^{-2}$. The InN islands height is about $1.8 \mathrm{~nm}$ on average with a standard deviation of $0.6 \mathrm{~nm}$ (Fig. $\mathbf{5 . 2 . 4}$ (c)). Their average diameter is $60 \mathrm{~nm}$ with a standard deviation of $22 \mathrm{~nm}$ (Fig. 5.2.4 (d)). Thus, InN islands have an aspect ratio (H/D) of 0.03.

Apart from InN islands, AFM images sometimes reveal the presence of some other small round surface features (bright spots in Fig. 5.2.4 (a)). The most probable origin of these objects is carbon contamination, because they also appear on clean Si substrates (without InN deposition). Thus, the possibility that these objects are associated with In or InN was excluded.

\section{InN QDs on Si(111).}

The morphology of sample A5 with InN QDs (converted from InN islands upon annealing) was also studied by AFM and SEM (Fig. 5.2.4 (e)). Two types of QDs can be distinguished: single QDs (blue circle) and clusters of QDs (green circle). Their corresponding AFM scans are presented in Fig. 5.2.4 $(\mathbf{f}, \mathbf{g})$, respectively. Based on these findings, the surface density of InN QDs was estimated using two different approaches:

(i) Both single QDs and each QD within clusters were counted as individual objects.

(ii) Each single QD, as well as each QDs cluster, was taken as individual objects.

The resulting surface density is $1.06 \times 10^{10} \mathrm{~cm}^{-2}$ for (i) and $4.6 \times 10^{9} \mathrm{~cm}^{-2}$ for (ii). The fact that the (ii) approach gives a value very close to the surface density of $\mathrm{InN}$ islands before annealing $\left(3.8 \times 10^{9} \mathrm{~cm}^{-2}\right)$ suggests that upon annealing small $\mathrm{InN}$ islands transform into single QDs and big ones into clusters of QDs. This transformation can be well described in terms of solid state dewetting process (refer to section 3.2.3.2.3).

The height distribution of InN QDs (QDs inside agglomerates were treated as individual QDs with their own height and diameter) was measured by AFM (Fig. 5.2.4 (g)) yielding an average value of $5.2 \mathrm{~nm}$ (later confirmed by HRTEM) with a standard deviation of $1.2 \mathrm{~nm}$. The diameter histogram of InN QDs presented in Fig. 5.2.4 (h) was estimated by using SEM images, instead of AFM, because of its better lateral resolution, thanks to sufficient material and height contrast. AFM measurements significantly overestimate the lateral size of such small objects, probably due to limited tip sharpness. However, SEM also overestimates the actual QD diameter because of operating at is resolution limit. Thus, the InN QDs average aspect ratio (H/D) of 0.65 was measured based on a series of HRTEM images (8 images of different QDs), since this technique allows measuring height and diameter of QDs simultaneously and with greater precision than AFM or SEM. Based on the QDs HRTEM derived aspect ratio and AFM derived height distribution, the actual QDs average diameter was estimated to be $\sim 8 \mathrm{~nm}$. It is worth to notice that, to the best of our knowledge, a H/D value of 0.65 is the largest aspect ratio reported so far for InN QDs. 


\section{Morphology of InN on Si(111)}

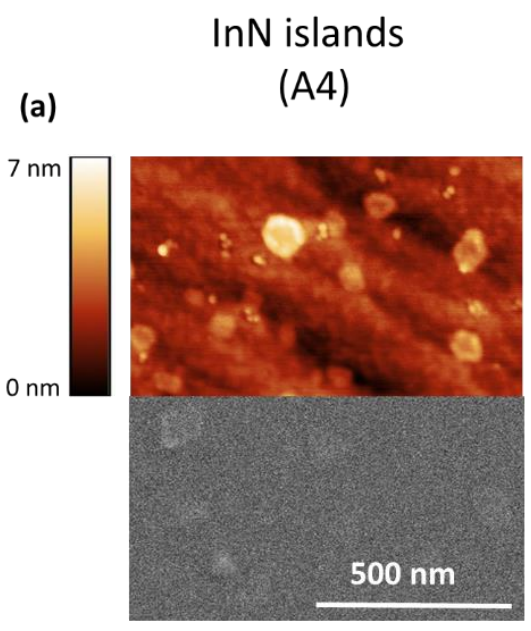

(b)

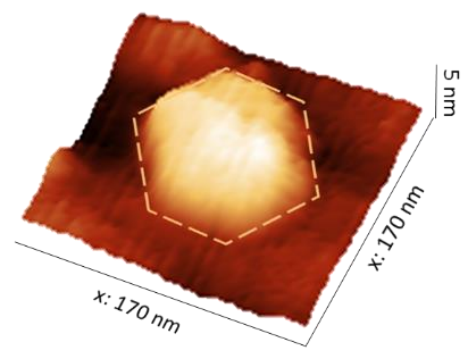

(c)

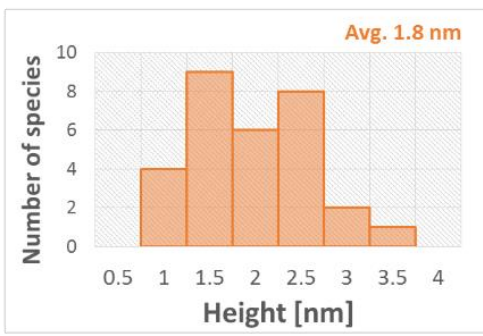

(d)

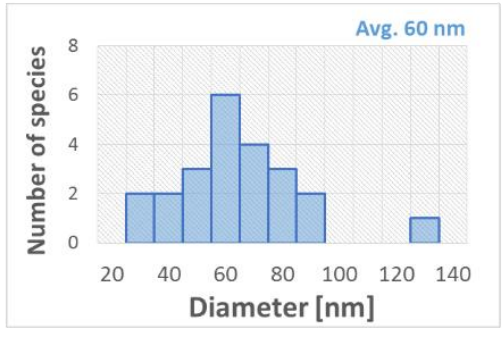

InN QDs

(e)

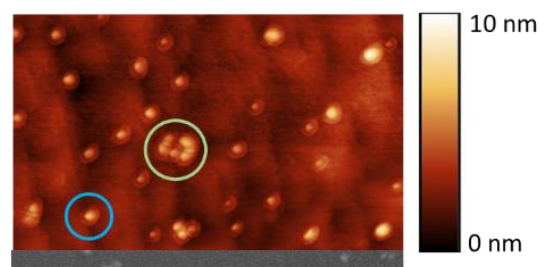

Annealing

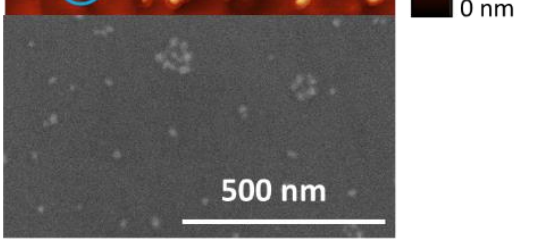

(f)

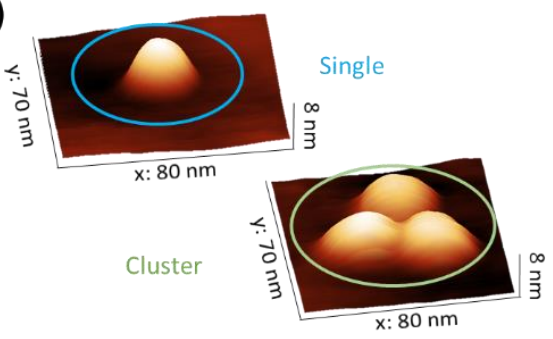

(g)

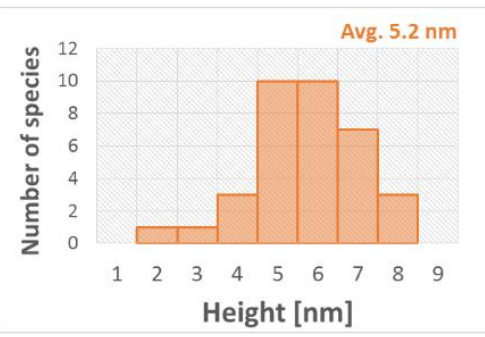

(h)

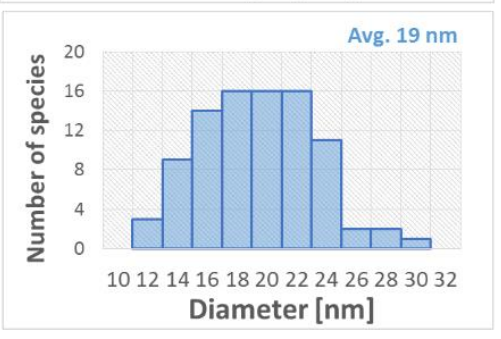

Fig. 5.2.4. Morphology change upon annealing of InN islands (left) to InN QDs (right) evidenced by AFM and SEM. AFM/SEM scans of A4 sample surface (a) and an individual InN island (b) after In droplets conversion into InN crystals (nitridation). Histograms of InN islands height (c) and diameter (d). AFM/SEM scans of InN QDs of sample A5 sample after annealing under plasma at $300^{\circ} \mathrm{C}$ for $30 \mathrm{~min}$ (e). AFM scans of single (blue circle) and clusters (green circle) of QDs (f). Histograms of InN QDs height (e) and diameter (f) distributions. 


\subsubsection{Structural characterization}

To exclude the possibility that the $\operatorname{InN}$ islands of sample A4 and InN QDs of sample A5 may consist of metallic In instead of $\operatorname{InN}$, both of them were dipped in a concentrated $\mathrm{HCl}$ solution (36\%) for $10 \mathrm{~min}$ in an ultrasonic bath. No difference in sample morphology was evidenced by AFM before and after this procedure (Fig. 5.2.5). If these objects were metallic In or In-Si eutectic, they would have been immediately etched away.

Next the structural properties of the samples A4 and A5 surface objects were further studied by HRTEM individually.

\section{Acid etch test}

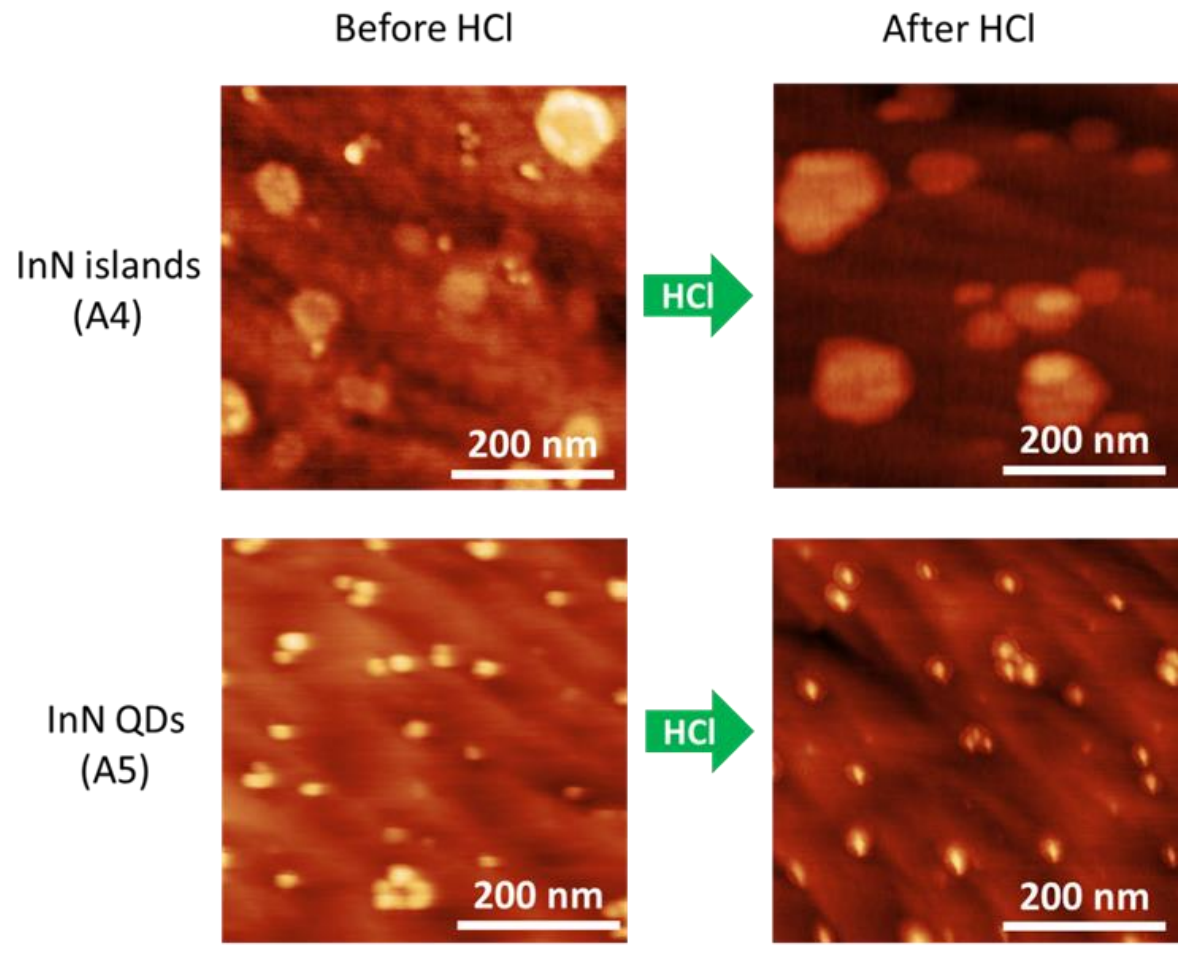

Fig. 5.2.5. AFM scans of sample A4 (top) and A5 (bottom) before and after dipping in $\mathrm{HCl}$ solution ( $36 \%$ ) for $10 \mathrm{~min}$. No significant change in samples morphology suggest that observed objects are not of metallic origin.

\section{InN islands on $\mathrm{Si}(\mathbf{1 1 1})$.}

The Bright Field XTEM micrograph in Fig. 5.2.6 (a) indicates the presence of InN islands with similar lateral sizes and surface density, as described in section 0 . The high contrast between the $\mathrm{Si}$ substrate and the $\mathrm{InN}$ islands confirms their different chemical composition. A HRTEM micrograph of one of these islands is shown in Fig. 5.2.6 (b), revealing a thickness of $\sim 2 \mathrm{~nm}$ (no more than 5-6 monolayers) and a lateral size of $\sim 46 \mathrm{~nm}$, which are in a good agreement with the AFM results. It is also clear that these $\mathrm{InN}$ islands have grainy structure (polycrystalline-like), with slightly different crystal orientations of grains (grain boundaries are marked with red lines in Fig. 5.2.6 (b)). This, together with the islands small thickness, made impossible to accurately measure any interplanar spacing from HRTEM images. 


\section{Crystalline structure of InN islands on $\mathrm{Si}(111)$}
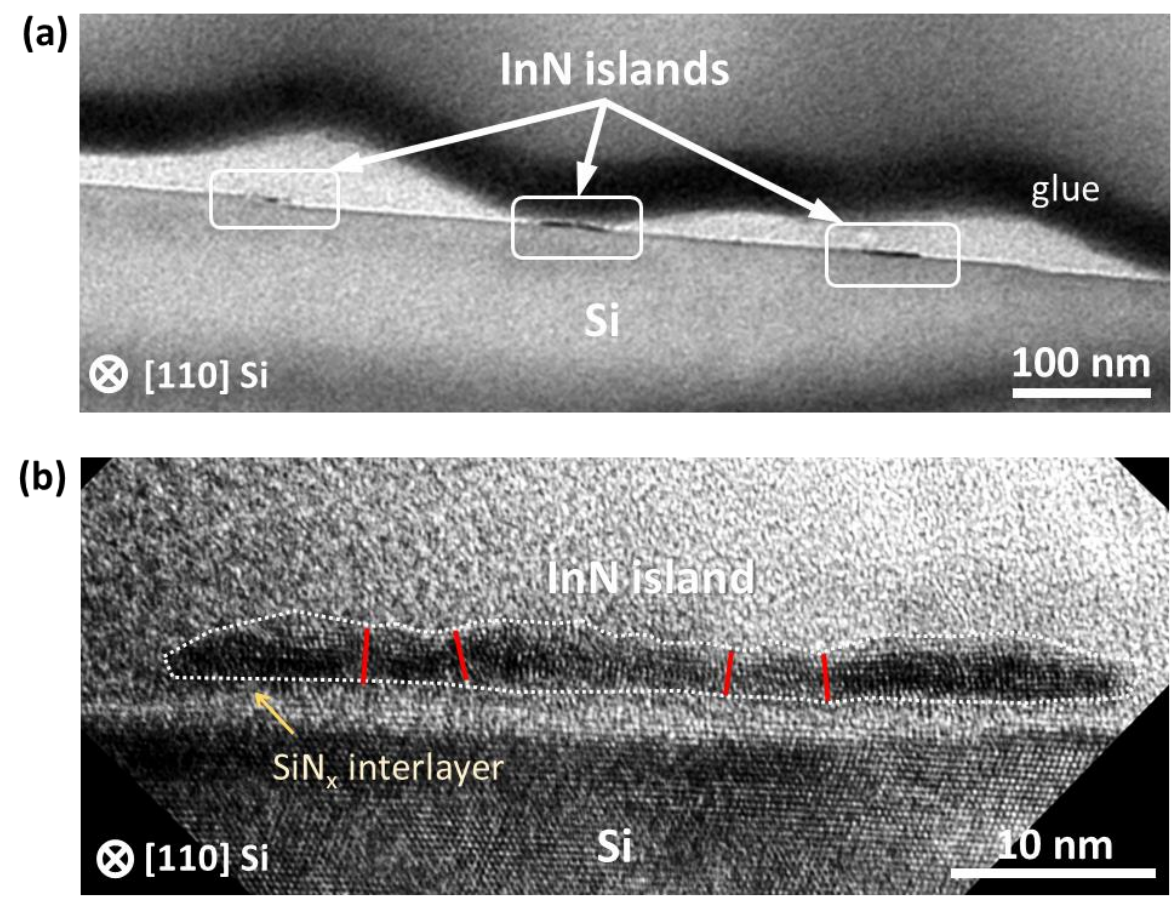

Fig. 5.2.6. Structural analysis of InN islands on $\mathrm{Si}(111)$ surface (A4) done by STEM-HAADF (a) and HRTEM (b) techniques, with Si substrate oriented along the [011] zone axis. InN islands have granular structure (grain boundaries are marked with red lines).

The obtained $\ln N$ islands have a polycrystalline structure most probably due to the low growth temperature used, which kinetically suppressed any crystal structural re-arrangement [Witten1981]. A similar polycrystalline-like structure formation was reported for GaN structures grown at room temperatures [Yamazaki2009].

In addition, an amorphous thin (1-2 nm) layer of $\mathrm{SiN}_{\mathrm{x}}$ was observed at the $\ln \mathrm{N} / \mathrm{Si}$ interface in all examined cases.

\section{InN QDs on Si(111).}

InN QDs were analyzed by STEM-HAADF (Fig. 5.2.7 (a)) in which the Si/InN contrast is quite clear, since the intensity of HAADF images is roughly proportional to the square of the atomic number $\left(Z^{2}\right)$ of the material. In order to obtain these images the sample was tilted to avoid the channeling effect, meaning that the $\mathrm{Si}$ is oriented close to, but not exactly along its [110] zone axis.

HRTEM images of individual QDs (Fig. 5.2.7 (b,c)) undoubtedly show the ABAB... atomic ordering, expected for a wurtzite structure when observed along the [11 $\overline{2} 0]$ (or the equivalent, [2 $\overline{1} 10]$ ) zone axis. To obtain these images, the QDs with a [1120] basal direction matched to the Si [110] basal direction were needed, which does not happen in many cases since QDs are rather randomly twisted along the normal to the Si basal plane. Nevertheless, the QDs growth direction seems to be the polar one [0001] (i.e. thus, a vertical epitaxial relation of $\ln N[0001] / \mathrm{Si}[111]$ is present). 
Plane distances, measured in perpendicular directions (both parallel to the basal and growth directions in HRTEM images) are:

$$
\begin{aligned}
& d^{\text {basal }}=2.82 \AA \text {, attributed to (0002) with theoretical value of } 2.846 \AA \text {, } \\
& d^{\text {growth }}=3.04 \AA \text {, attributed to }(10 \overline{1} 0) \text { with theoretical value of } 3.059 \AA .
\end{aligned}
$$

This good match between measured and theoretical values indicate that the QDs are undoubtedly of wurtzite $\ln \mathrm{N}$ and almost fully relaxed.

Images in Fig. 5.2.7 (b,c) suggest that QDs sidewalls are composed of two clearly defined facets. Based on their angles to the basal plane, of $\sim 63^{\circ}$ and $\sim 28^{\circ}$, they were assigned to (10 $\left.\overline{1} 1\right)$ and (1014) planes, respectively.

\section{Crystalline structure of InN QDs on $\mathrm{Si}(111)$}

(a)

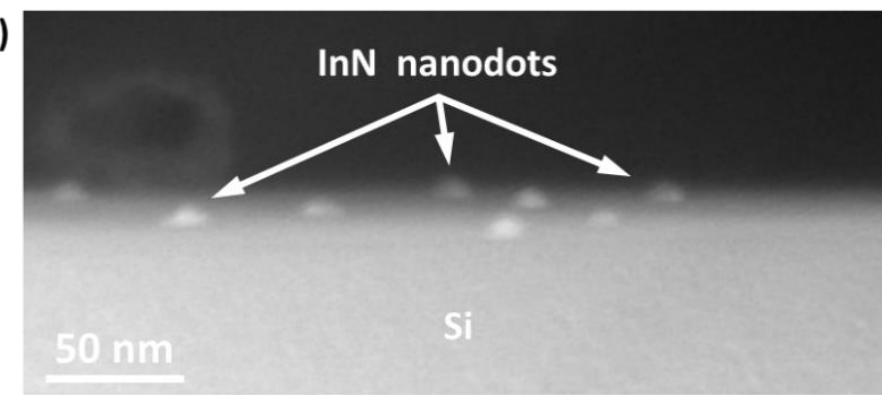

(b)

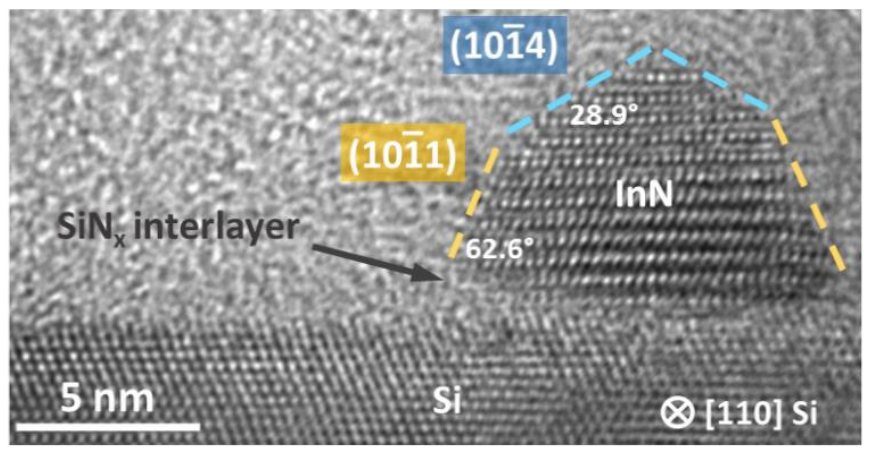

(c)

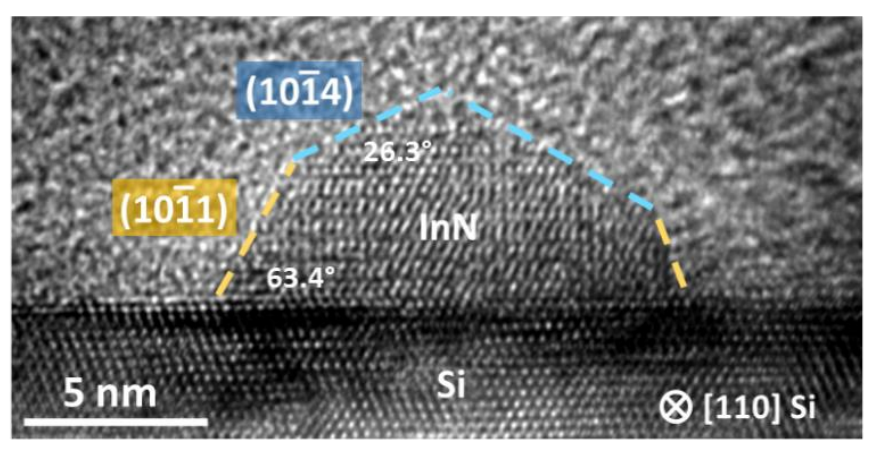

Fig. 5.2.7. Structural properties of InN QDs on $\mathrm{Si}(111)$ surface (A5) determined by STEM-HAADF (a) and HRTEM images (b, c), with the Si substrate oriented along the [110] zone axis. InN facets are marked with dashed lines. In both HRTEM images, the $\mathrm{SiN}_{\mathrm{x}}$ introlayer is present, but in (c) it cannot be seen due to the tilt angle at which the image was taken. 
Similarly to the previously described case of $\mathrm{InN}$ islands before annealing, there is a $1-2 \mathrm{~nm}$ thick $\mathrm{SiN}_{\mathrm{x}}$ amorphous layer between the InN QDs and the Si surface (Fig. 5.2.7 (b)). In some images, for example in Fig. 5.2.7 (c), the $\mathrm{SiN}_{\mathrm{x}}$ layer is hidden (due to the tilt angle to take the image) but still present.

HRTEM measurements did not reveal a presence of any InN wetting layer in both A4 nor A5 samples (usually present in the case of Stranski-Krastanow growth mode; Refer to section 3.2.3.2.1).

Combining together the morphological and structural analysis of obtained structures once can conclude that observed transformations could be well described in terms of solid state dewetting process for polycrystalline islands (refer to section 3.2.3.2.3). The theory of solid state dewetting predicts that for diameters of polycrystalline islands above a critical value it becomes energetically favorable to break up the island at the grain boundaries and form separate objects. In contrast, if the island diameter is smaller than the critical value, the islands adopt a well defined (by 3D Wullf plot) single shape [Thompson2012]. This is the behavior observed in this work.

\subsubsection{InN QDs growth model}

Based on the results and discussions from the previous sections, the fabrication of InN QDs can be divided into three well defined stages:

(i) In metal deposition (at near RT).

(ii) Nitridation: crystallization of In droplets into polycrystalline $\operatorname{InN}$ islands upon applying active nitrogen flux.

(iii) Annealing: recrystallization of InN islands into single QDs (or clusters) upon annealing at elevated temperatures for sufficiently long time (under active nitrogen flux).

They are schematically presented in Fig. 5.2.8. Transition from (i) to (ii) can be described in terms of droplet epitaxy, during which the active nitrogen converts metallic In droplets into InN islands. The polycrystalline nature of the $\mathrm{InN}$ islands is a consequence of the low growth temperature. Transition from (ii) to (iii) can be described in terms of solid state dewetting, during which polycrystalline InN islands are rearranged into compact (thermodynamically driven shape with high aspect ratio) single-crystalline wurtzite $\operatorname{InN} Q D$ s. Depending on the size of $\ln N$ islands they are converted into single QDs (<30 nm in diameter) and clusters of QDs (>30 nm in diameter).
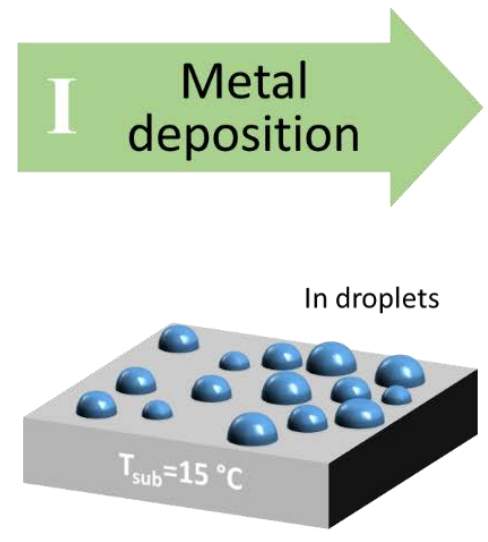

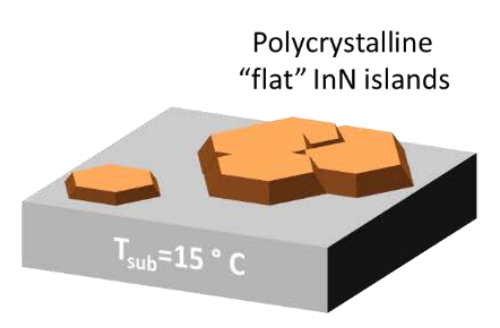

Nitridation

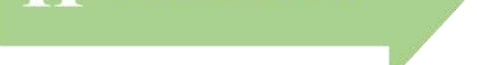

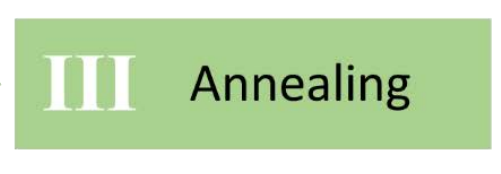

Single crystalline InN QDs

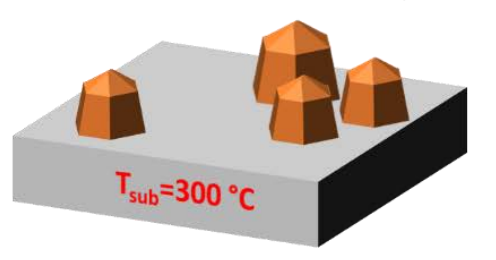

Fig. 5.2.8. Growth model of InN QDs. 


\subsection{InN QDs grown on $\mathrm{Si}(001)$}

Before applying the developed technique to grow QDs on InGaN/Si(111) templates, it was tested on $\mathrm{Si}(001)$ to trace the evolution of the $\mathrm{InN} /$ substrate epitaxial relation at the interface. According to the proposed model and general concepts of InN growth, it is expected that in the case of Si(001) substrates InN forms wurtzite QDs of similar morphology as those grown on Si(111), but without a defined epitaxial relation to $\mathrm{Si}(001)$. Confirmation of this assumption and more details of this study are presented in the following sections.

\subsubsection{Fabrication steps}

In order to perform fare comparison of substrate effect (Si(001) versus $\mathrm{Si}(111))$, two samples B1 (without annealing) and B2 (with annealing) were prepared in growth conditions outlined in the Table VIII. Note that these growth conditions are identical to the ones of samples grown on $\mathrm{Si}(111)$ (A4 and A5).

Detailed morphological and structural analyses of these samples and a comparison to their counterparts on $\mathrm{Si}(111)$ are given in the following sections.

Table VIII. Growth conditions of samples grown on Si(001).

\begin{tabular}{|c|c|c|c|c|c|c|}
\hline Sample & Substrate & In deposition & \multicolumn{2}{|l|}{ Nitridation } & \multicolumn{2}{|l|}{ Annealing } \\
\hline B1 & Si(001) & $T_{\text {sub }}=15^{\circ} \mathrm{C}$ & $T_{\text {sub }}=15^{\circ} \mathrm{C}$ & & none & \\
\hline islands & & $T_{\text {In }}=755^{\circ} \mathrm{C}$ & $N$ plamsa: & $350 \mathrm{~W}$, & & \\
\hline & & $\left(2.15 \times 10^{14}\right.$ atoms $\left./ \mathrm{cm}^{2} / \mathrm{s}\right)$ & $0.5 \mathrm{sccm} * *$ & & & \\
\hline & & $t=10 \mathrm{sec}$ & $t=10 \mathrm{~min}$ & & & \\
\hline B2 & $\mathrm{Si}(001)$ & $T_{\text {sub }}=15^{\circ} \mathrm{C}$ & $T_{\text {sub }}=15^{\circ} \mathrm{C}$ & & $T_{\text {sub }}=300^{\circ} \mathrm{C}$ & \\
\hline QDs & & $T_{\text {ln }}=755^{\circ} \mathrm{C} *$ & $N$ plasma: & $350 \mathrm{~W}$, & $N$ plasma: & $350 \mathrm{~W}$, \\
\hline & & $\left(2.15 \times 10^{14}\right.$ atoms $\left./ \mathrm{cm}^{2} / \mathrm{s}\right)$ & $0.5 \mathrm{sccm}$ & & $0.5 \mathrm{sccm}$ & \\
\hline & & $t=10 \mathrm{sec}$ & $t=10 \mathrm{~min}$ & & $t=30 \mathrm{~min}$ & \\
\hline
\end{tabular}

\subsubsection{Morphological characterization}

\section{InN islands on $\mathrm{Si}(001)$.}

The morphology of sample B1 with InN islands was examined by AFM and SEM in more details (Fig. 5.3.1 (a)). Shape and size distribution of islands grown on the $\mathrm{Si}(001)$ is more random compared to the $\mathrm{Si}(111)$ case. Moreover, an AFM scan of an individual InN island presented in Fig. 5.3.1 (b) shows that it has a close to hexagonal shape, but it is not clearly defined.

The InN islands surface density is $1.62 \times 10^{10} \mathrm{~cm}^{-2}$, having an average height of about $2.7 \mathrm{~nm}$ with a standard deviation $(\sigma)$ of $1.1 \mathrm{~nm}$ (Fig. 5.3.1 (c)). As for lateral size, in AFM/SEM images of sample B1 one can notice that there are two types of $\ln N$ islands, namely, a high density of small InN islands 


\section{Morphology of InN on Si(001)}

InN islands

(B1)

(a)

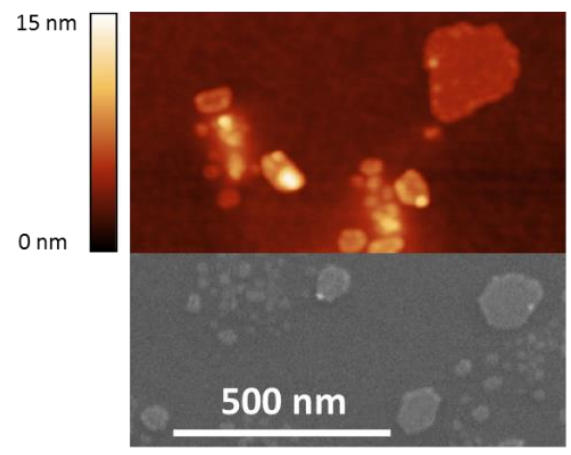

(b)

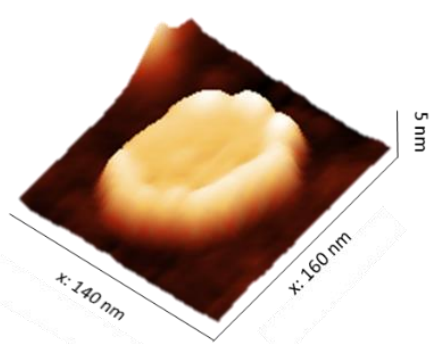

(c)

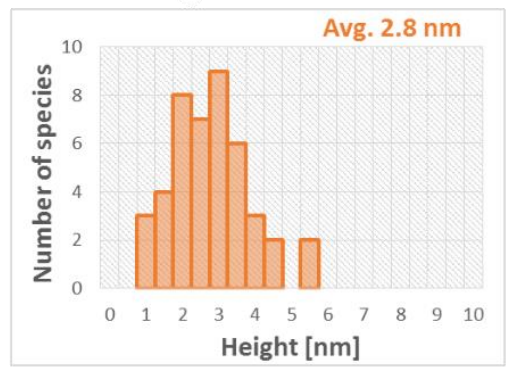

(d)

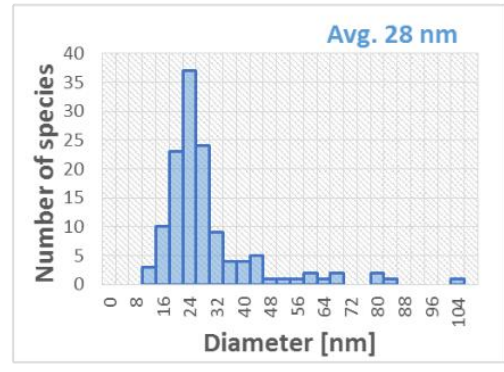

InN QDs

(B2)

(e)

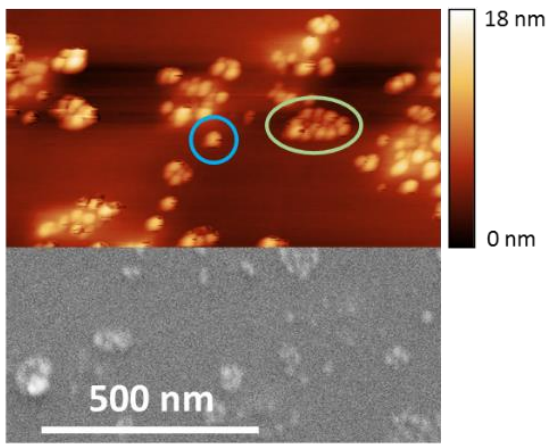

(f)

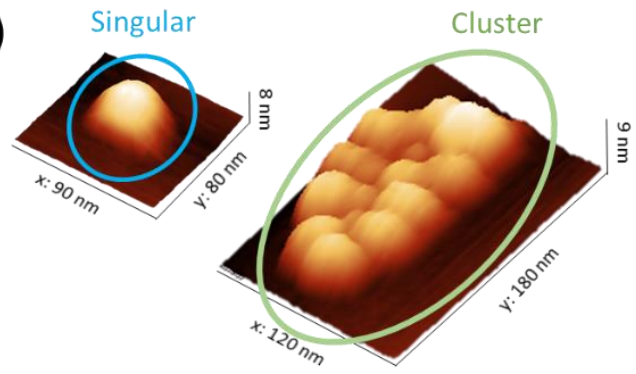

(g)

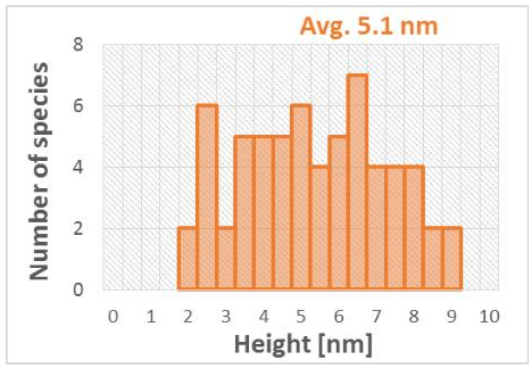

(h)

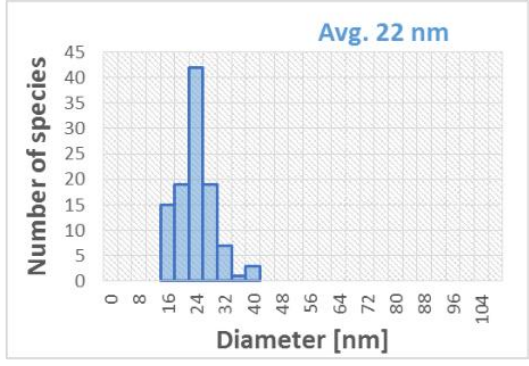

Fig. 5.3.1. Morphological analysis of InN nanostructures right after initial nitridation (sample B1) and after annealing (sample B2). AFM and SEM pictures of samples B1 (a) and B2 (e). Detailed AFM of single nanostructures of samples B1 (b) and B2 (f). Height and diameter distributions of samples B1 (c,d) and B2 (g,h). 
(diameter of $24 \mathrm{~nm}$ with $\sigma=7 \mathrm{~nm}$ ) and a scarce number of large ones (diameter of $67 \mathrm{~nm}$ with $\sigma=27 \mathrm{~nm}$ ) (Fig. 5.3.1 (a)). Histogram of their diameters, measured from SEM images (as discussed in section 5.2.2) is presented in Fig. 5.3.1 (d). Based on these values the estimated aspect ratio (heigh/diameter) yielded of 0.12 and 0.04 for "small" and "large" islands in sample B1, respectively. The values for "large" islands are very close to that on Si(111) substrate (A4), however "small" islands were absent in sample A4.

\section{InN QDs islands on Si(001).}

The morphology of sample B2 with InN QDs (converted from InN islands upon annealing) was also studied by AFM and SEM (Fig. 5.3.1 (e)). Similarly to the Si(111) case, single QDs (blue circle) and clusters of QDs (green circle) can be distinguished (Fig. 5.3.1 (f)). It is safe to assume that, upon annealing, small InN islands get transformed into single QDs and big ones into clusters of QDs, similarly to the Si(111) case.

The surface distribution of QDs in sample B2 is not as clear as in A5, thus the density of InN QDs was estimated by counting both single QDs and QDs within clusters as individual objects, yelding a value of $1.55 \times 10^{10} \mathrm{~cm}^{-2}$, very similar to the one measured in sample B1 (in contrast to A4 vs. A5 case). This is due to the fact that B1 sample has already a high density of "small" islands, and the increase in number of dots upon conversion of scarce "large" islands in B1 into clusters of QDs (B2) is negligible.

The InN QDs height distribution in sample B2 measured by AFM is shown in Fig. 5.3.1 (g). The average $\mathrm{InN}$ islands height increased to $5.1 \mathrm{~nm}$ with a standard deviation of $2.1 \mathrm{~nm}$ (confirmed by HRTEM), compared to sample B1 $(2.7 \mathrm{~nm}$ in diameter). An histogram of InN QDs lateral size measured by SEM (as discussed in section 5.2.2), shown in Fig. 5.3.1 (h), reveals an average diameter of $24 \mathrm{~nm}$ with a standard deviation of $7 \mathrm{~nm}$. This value is certainly overestimated because of the extremely small sizes of InN QDs. Thus, the aspect ratio estimation was done by HRTEM instead, yealding a value of 0.67 , based on which the actual QDs average diameter was estimated to be $7.5 \mathrm{~nm}$. Therefore, the InN QDs have very similar size and aspect ratio on both $\mathrm{Si}(001)$ and $\mathrm{Si}(111)$, as expected. The only difference is in their surface distribution randomness, which is probably attributed to difference in symmetry of $\mathrm{Si}(111)$ and $\mathrm{Si}(001)$ surfaces, namely 3-fold (more compatible with 6-fold symmetry of $\ln N$ ) and 4-fold symmetries, respectively.

\subsubsection{Structural characterization}

\section{InN islands on $\mathrm{Si}(001)$.}

Results from a structural analysis of sample B1 by HRTEM suggest that there is no clear epitaxial relationship between the $\mathrm{InN}$ islands and the $\mathrm{Si}(001)$ substrate. Only fringes (related to atomic planes, marked with yellow lines in Fig. 5.3.2 (a,b)) instead of atomic columns could be observed. Besides, since the orientation of the $\ln \mathrm{N}$ islands can be far away from any crystalline zone axis, the images obtained had a low-contrast.

From the angles of atomic fringes with the Si(001) surface (Fig. 5.3.2 (a,b)) it can be concluded that not only their crystalline structure is randomly oriented in respect to $\mathrm{Si}$ [001] direction, but also their internal structure consist of many small crystalline domains randomly oriented among them (polycrystalline-like structure with twisted domains). Very rarely, atomic fringes are parallel to the $\mathrm{Si}(001)$ surface, for instance in the region marked with a dotted white line in Fig. 5.3.2 (b). 
In the few cases where it was possible to measure interplanar distances the following values were extracted:

$$
\begin{aligned}
& d_{h k l}^{1}=1.77 \AA \text {, corresponding to [1120] with theoretical value of } 1.766 \AA \\
& d_{h k l}^{2}=2.77 \AA \text {, corresponding to [0002] with theoretical value of } 2.846 \AA
\end{aligned}
$$

This good match between measured and theoretical values indicates that InN islands of sample B1 have wurtzite structure and are almost fully relaxed.

\section{InN QDs on Si(001).}

HRTEM images of sample B2 revealed that InN QDs in that case form either clusters of QDs (Fig. 5.3.2 (c)) or single QDs (Fig. 5.3.2 (d,e)), in agreement with the results obtained by AFM/SEM. In contrast to sample B1, all measured QDs from sample B2 have a single-crystalline structure. In most cases the atomic fringes in the HRTEM images are parallel (or almost) to the substrate surface, whereas in some cases the fringes are $30^{\circ}-45^{\circ}$ tilted (See Fig. 5.3.2 (e)). But again, the fact that only atomic fringes and not columns were resolved, suggest that QDs are randomly rotated along the normal to the $\mathrm{Si}(001)$ surface. The following interplanar distances were measured in series of QDs:

$$
\begin{aligned}
& d_{h k l}^{1}=1.71 \AA \text {, corresponding to [1120] with theoretical value of } 1.766 \AA \text {, } \\
& d_{h k l}^{2}=2.85 \AA \text {, corresponding to [0002] with theoretical value of } 2.846 \AA \text {, } \\
& d_{h k l}^{3}=2.73 \AA \text {, corresponding to [10111] with theoretical value of } 2.695 \AA \text {, } \\
& d_{h k l}^{4}=3.04 \AA \text {, corresponding to [1010] with theoretical value of } 3.059 \AA \text {, }
\end{aligned}
$$

This again point to a perfect wurtzite InN QDs structure. As in all previous cases, 1-2 $\mathrm{nm} \operatorname{SiN}_{\mathrm{x}}$ interlayers were observed.

\section{Crystalline structure of $\operatorname{InN}$ on $\mathrm{Si}(\mathbf{0 0 1})$}
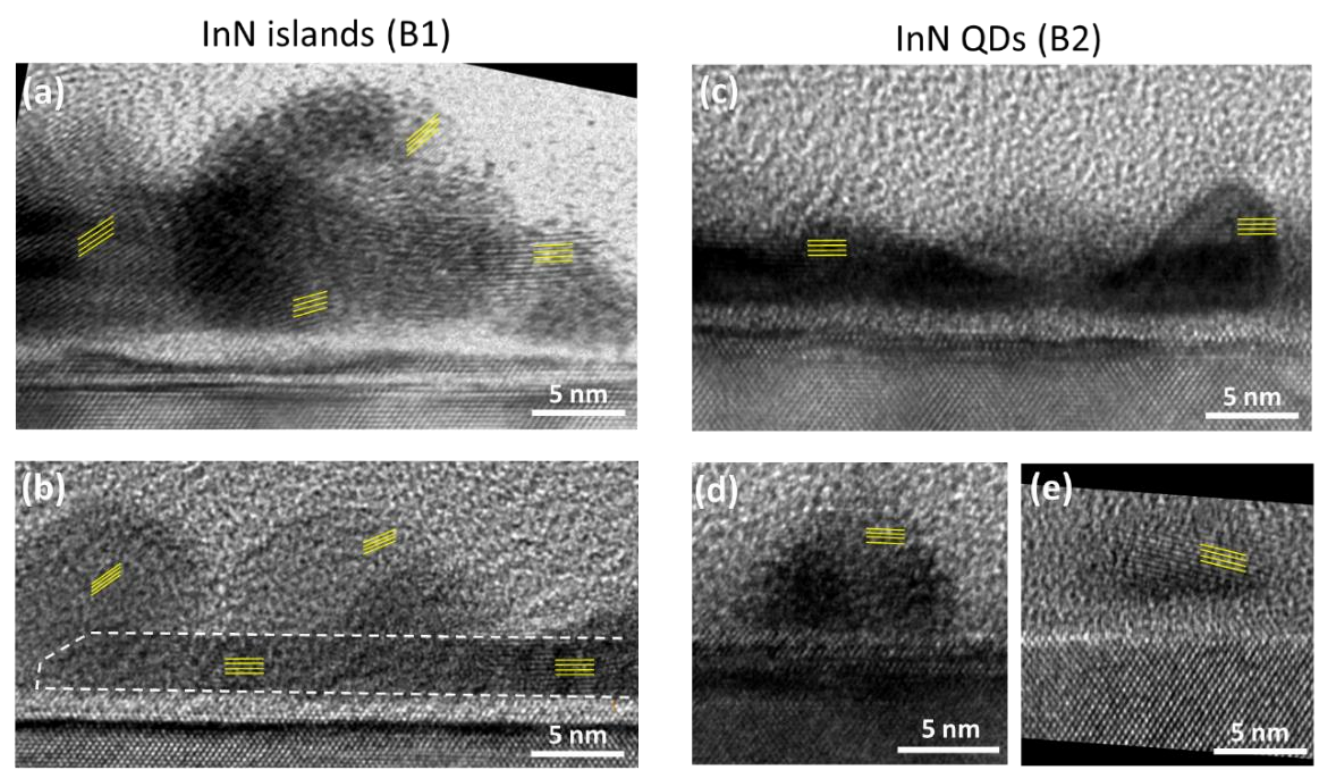

Fig. 5.3.2. Crystalline structure of InN islands after nitridation (a, b) and InN QDs after annealing ((c) cluster of QDs, and (d, e) - single QDs) evidenced by HRTEM. Distinguished crystalline planes (fringes) are marked with yellow lines. 


\subsection{InN QDs grown on $\operatorname{In}_{0.3} \mathrm{Ga}_{0.7 \mathrm{~N}} \mathrm{Si}(111)$}

The final part of this chapter is devoted to the fabrication of InN QDs on InGaN(0001)/Si(111) following the established procedure. It is expected that wurtzite InN QDs on wurtzite InGaN surface exhibit both vertical and in-plane epitaxial relations, in contrast to Si substrates. Although the complications related to the layers surface roughness, affecting the initial InN nucleation stage, are foreseeable, the resulting QDs are expected to have excellent structural quality and similar morphology.

\subsubsection{Fabrication steps}

An InGaN/Si(111) template with $30 \% \operatorname{lnN}$ mole fraction under the growth conditions established in Chapter 4: $\quad T_{\text {sub }}=450^{\circ} \mathrm{C}, \quad F_{G a}=2.64 \times 10^{14}$ atoms $/ \mathrm{cm}^{2} / \mathrm{s}, \quad F_{l n}=1.30 \times 10^{14}$ atoms $/ \mathrm{cm}^{2} / \mathrm{s}$, and $F_{N^{*}}=4.38 \times 10^{14}$ atoms $/ \mathrm{cm}^{2} / \mathrm{s}(350 \mathrm{~W}, 0.6 \mathrm{sccm})$, and $t_{\text {growth }}=1$ hour. The resulting InGaN layer was cooled down overnight to RT inside the UHV chamber. Next, the growth temperature was cooled down to $15^{\circ} \mathrm{C}$ and the InN QD growth proceeded following similar steps as for sample $\mathrm{A} 5$ ( $T_{\text {In }}=$ $755^{\circ} \mathrm{C}$, N-pasma: $350 \mathrm{~W} \& 0.5 \mathrm{sccm}, t_{\text {nitrid }}=10 \mathrm{~min} T_{\text {anneal }}=300^{\circ} \mathrm{C}, t_{\text {anneal }}=30 \mathrm{~min}$ ) but for the In deposition time, that in this case was $15 \mathrm{~s}$ instead of $10 \mathrm{~s}$, aiming to form slightly larger InN QDs to facilitate their morphological and structural characterization.

\subsubsection{Morphological characterization}

The formation stages of InN QDs on the $\ln _{0.3} \mathrm{Ga}_{0.7} \mathrm{~N} / \mathrm{Si}$ template were monitored by SEM, as shown in Fig. 5.4.1. The "as grown" InGaN layer, shown in Fig. 5.4.1 (a), exhibits a much rougher surface compared to the Si substrate used before, which is the main challenge to fabricate QDs. After the In metal deposition small droplets with $\sim 13 \mathrm{~nm}$ average diameter and a high density of $2.6 \times 10^{11} \mathrm{~cm}^{-2}$ were formed as shown in Fig. 5.4.1 (b).

SEM image in Fig. 5.4.1 (c) shows that InN QDs formed after nitridation and annealing have a larger size compared to the Si substrate case, with $\sim 26 \mathrm{~nm}$ average diameter with $\sigma=6 \mathrm{~nm}$ (Fig. 5.4.2) and a density of $1.06 \times 10^{10} \mathrm{~cm}^{-2}$ estimated from SEM images. Thanks to the relatively large size of QDs on the InGaN/Si tempalte, their lateral size estimations are much more trustworthy than in the previous cases of small QDs on Si substrates. The rough InGaN surface prevented a reliable AFM

As grown InGaN

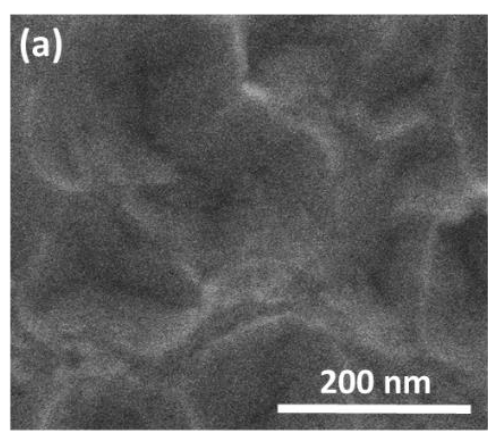

In droplets

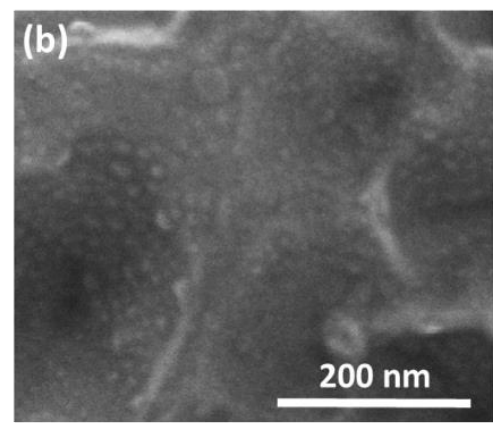

InN QDs

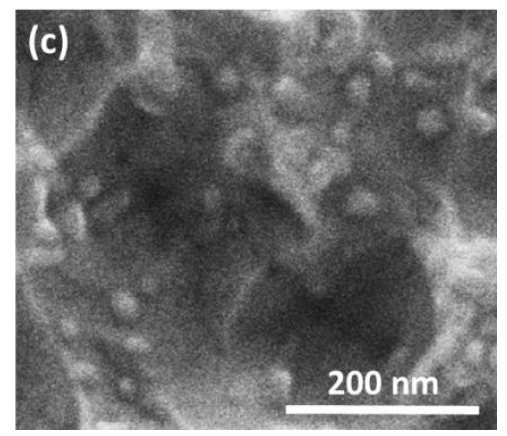

Fig. 5.4.1. Main steps during the InN QDs fabrication on InGaN/Si(111) evidenced by SEM. As grown surface of InGaN (a), In metal droplets deposition (b), and InN QDs (c). 
measurement of the QDs height, thus, the only option to measure the QDs aspect ratio was by HRTEM. Only very few images were obtained, from which the InN QDs average height of $13 \mathrm{~nm}$ and aspect ratio (H/D) of 0.38 were determined. Smaller aspect ratio of QDs grown on the InGaN compared to $\mathrm{Si}$ templates is attributed to a stronger chemical bond between $\operatorname{lnN} / \mathrm{InGaN}$ rather than between InN/Si.

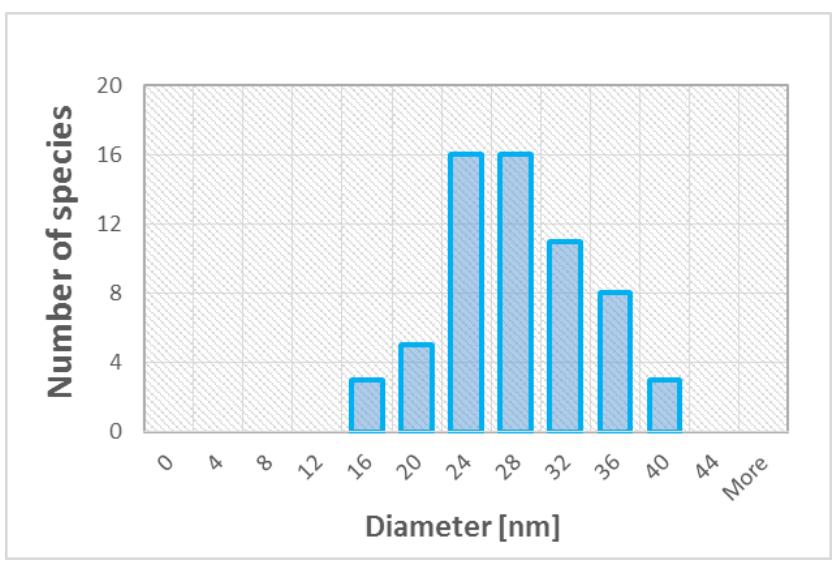

Fig. 5.4.2. Diameter distribution of InN QDs on InGaN/Si substrate.

\subsubsection{Structural characterization}

A structural study via CTEM and HRTEM was also complicated due to the rough morphology of the InGaN layer. Only very few images were obtained where InN QDs could be clearly distinguished from the InGaN layer, like that on Fig. 5.4.3 (a) with several InN QDs (marked with white arrows) on top of the InGaN layer.

An HRTEM image of one of the InN QDs is shown in Fig. 5.4.3 (b) where clear atomic columns and not only fringes are identified in both $\operatorname{lnGaN}$ and $\ln N$ regions, suggesting that there are vertical and in-plane epitaxial relations between the $\ln N(0001)$ and $\ln G a N(0001)$. $\ln N$ atomic columns corresponding to the hexagonal lattice were measured. They are slightly rotated in respect to the InGaN[0001] direction $\left(\sim 1^{\circ}\right)$. The $\operatorname{InN}$ atomic planes spacing were measured:

$$
\begin{aligned}
& d_{0002}=2.868 \AA \text {, corresponding to [0002] with theoretical value of } 2.846 \AA \\
& d_{11 \overline{2} 0}=1.758 \AA \text {, corresponding to [11̄이 with theoretical value of } 1.766 \AA
\end{aligned}
$$

thus indicating that the QDs are almost fully relaxed hexagonal $\operatorname{lnN}\left(\varepsilon_{a}=-0.61 \% ; \varepsilon_{c}=0.56 \%\right)$.

Angles between sidewall facets of this $\ln N Q D$ and the $\ln G a N(0002)$ plane were measured to be $32.1^{\circ}$ and $35.8^{\circ}$, which allows their assignment to the (1013) crystalline pyramidal planes (theoretical angle is $32.1^{\circ}$ ). The interface between $\operatorname{InN}$ and $\ln _{0.3} \mathrm{Ga}_{0.7} \mathrm{~N}$ is clearly heteroepitaxial, as expected. 
(a)

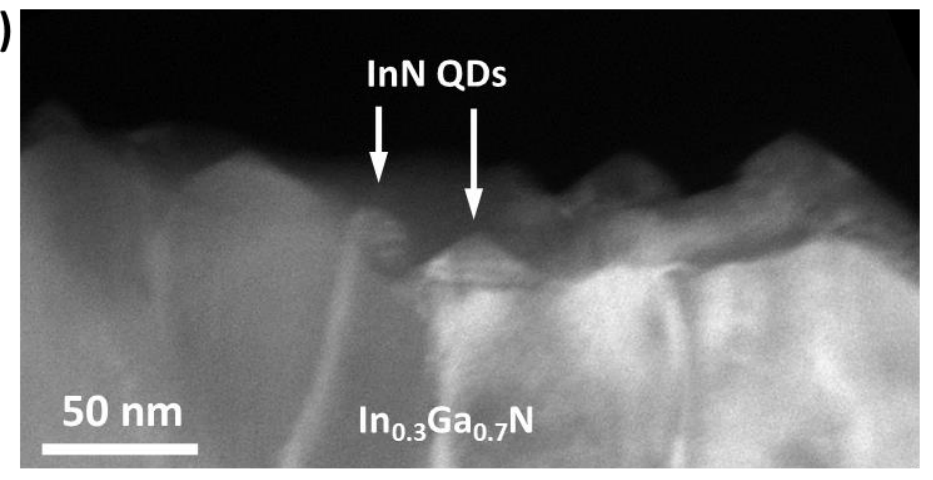

(b)

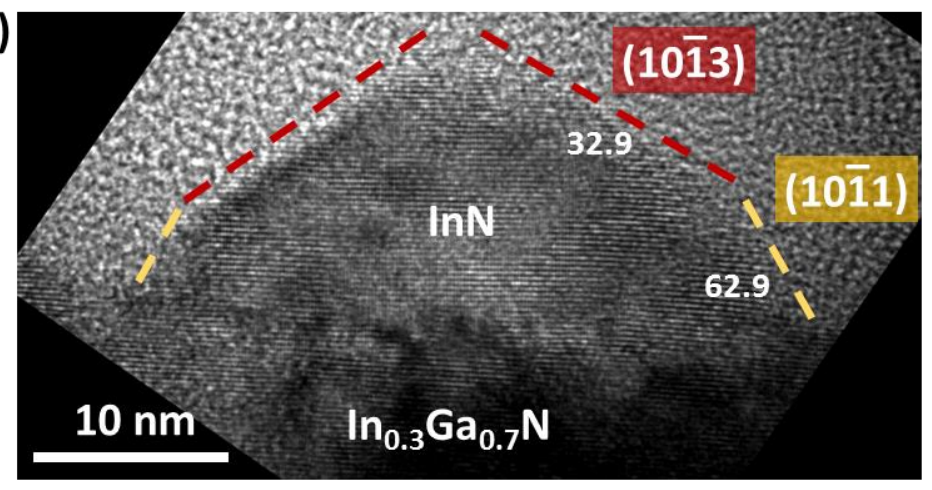

Fig. 5.4.3. Structural properties of InN QDs grown on an InGaN/Si template measured by CTEM (a) and HRTEM (b). InN QD facets are depicted with their angles vs. the (0001)-plane.

\subsection{Comparison between InN QDs grown on $\operatorname{In}_{0.3} \mathrm{Ga}_{0.7} \mathrm{~N} / \mathrm{Si}$ and bare $\mathrm{Si}$}

From results presented above it is clear that the formation mechanism of $\ln N$ QDs is very similar on both $\mathrm{Si}(111)$ and $\mathrm{Si}(001)$ as well as on InGaN/Si substrates. In droplets covered the substrate surface in a very similar way. Upon nitridation and following annealing the resulting InN QDs have a very similar morphology and crystalline structure (See Table IX). The smaller InN QDs aspect ratio when grown on InGaN ( 0.38) as compared to $\mathrm{Si}(111)(\sim 0.65)$ and $\mathrm{Si}(001)(\sim 0.67)$ can be attributed to a stronger epitaxial constrain between $\operatorname{lnN} / \mathrm{InGaN}$ compared to $\operatorname{lnN} / \mathrm{Si}$.

HRTEM measurements confirm that InN QDs are completely relaxed (as consequence of enlarged free surface area) wurtzite single crystals with the $\ln N$ [0001] axis almost aligned (with a slight twist) to the substrate surface normal in all examined cases. However, the in-plane epitaxial relation between the InN QDs and the underlying layer was only observed when grown on InGaN(0001)/Si(111) substrates, as it should be expected. A similar behavior was observed in GaN NWs, where the GaN[0001] axis is perpendicular to the Si surface independently from the Si orientation (either (001) or (111)) [Cerutti2006].

Rough comparison of the total volume per surface area of obtained InN QDs based on data presented in Table IX revealed that there approximately 10 times less material present in the case of growth on Si substrates (either (111) or (001)) compared to the case of InGaN/Si. This is most likely attributed to higher decomposition/desorption rates of InN/In from Si than from InGaN, because of weaker $\mathrm{InN} / \mathrm{Si}$ bonding versus $\operatorname{InN} / \mathrm{In}_{0.3} \mathrm{Ga}_{0.7} \mathrm{~N}$. This effect is also can be enhanced by active nitrogen plasma flux used during annealing, which can break the In-Si bond to form N-Si 
instead [Cho2006, Qi2013]. Moreover, the fact that the same behavior was reported for nitridation of Ga on a Si surfaces [Debnath2009] suggests that this effect is not indium specific, but rather related to the formation mechanisms of III-Si bonds.

Table IX. Morphological comparison of InN nanostructures grown on Si(111) and Si(001).

\begin{tabular}{|c|c|c|c|c|}
\hline Sample & Density & Height & Diameter & Aspect ratio \\
\hline & $\mathrm{cm}^{-2}$ & $n m$ & $n m$ & height/diameter \\
\hline Islands on $\mathrm{Si}(111)$ - A4 & $3.80 \cdot 10^{9}$ & 1.78 & 60 & 0.03 \\
\hline Islands on $\mathrm{Si}(001)$ - B1 & $1.06 \cdot 10^{10}$ & 2.75 & $24 / 67 *$ & $0.12 / 0.04^{*}$ \\
\hline QDs on $\mathrm{Si}(\mathbf{1 1 1})-\mathrm{A} 5$ & $1.55 \cdot 10^{10}$ & 5.23 & 8 & 0.65 \\
\hline QDs on $\mathrm{Si}(111)-\mathrm{B} 2$ & $1.62 \cdot 10^{10}$ & 5.05 & 7.5 & 0.67 \\
\hline QDs on $\ln _{0.3} \mathrm{Ga}_{0.7} \mathrm{~N} / \mathrm{Si}(111)^{* *}$ & $1.06 \cdot 10^{10}$ & 13.0 & 26 & 0.38 \\
\hline
\end{tabular}

\subsection{Conclusions}

In this chapter, the PA MBE growth of single-crystalline wurtzite InN quantum dots with a small size (average diameter and height are blow 26 and $13 \mathrm{~nm}$, respectively) was successfully demonstrated on atomically flat $\mathrm{Si}(111), \mathrm{Si}(001)$ substrates, and $\ln _{0.3} \mathrm{Ga}_{0.7} \mathrm{~N}(0001) / \mathrm{Si}(111)$ substrates with rough (RMS $>10 \mathrm{~nm}$ ) surface morphology. This achievement allows extending the application of InN QDs to rough morphologies, which is particularly desired in the applications where active surface area of the device must be enlarged, such as water splitting and chemical sensing.

In all cases the $\operatorname{InN}$ [0001] axis is almost aligned (with a slight twist) perpendicular to the substrate surface, however, in-plane epitaxial relation between the $\operatorname{InN}$ QDs and the underlying layer was observed only when grown on $\ln _{0.3} \mathrm{Ga}_{0.7} \mathrm{~N}(0001) / \mathrm{Si}(111)$ substrates, as expected. In addition, no evidence of an InN wetting layer was found based on HRTEM observations.

Three main InN QDs formation stages were identified: (i) In droplets deposition below RT, (ii) their conversion into polycrystalline-like $\mathrm{InN}$ islands upon nitridation, and (iii) following reshaping and recrystallization into single-crystalline InN QDs upon annealing. The transition from (i) to (ii) can be described in terms of droplet Epitaxy, while that from (ii) to (iii) follow a solid state dewetting mechanism.

Notice that the proposed method of InN QDs fabrication greatly deviates from the "standard" droplet epitaxy of InAs QDs, because conversion of In droplet to InN crystal does not necessarily happens 1-to-1. The difference was attributed to nitrogen plasma source being acting as a heat source and consequently enhancing In mobility during the nitridation. The same mechanism is not active in the case of InAs DE, because of different supplying mechanisms of atomic $\mathrm{N}$ and atomic As.

Obtained InGaN layers "decorated" with InN QDs are evaluated for their potential in water splitting applications in Chapter 7. 


\section{Selective area growth (SAG) of GaN nanowires (NWs) on various templates}

\subsection{Introduction}

A significant progress in the fabrication of nanowires (NWs) has been achieved in the recent years. For III-Nitrides NWs the first reports on self-assembled (SA) GaN NWs growth come from the late 1990s [Yoshizawa1997, Calleja2000]. The simplicity of the SA growth method, performed mainly heteroepitaxially on $\mathrm{Si}$, combined with the NWs extraordinary crystal quality, enabled the achievement of a wide variety of nanostructures and devices, such as: NW-based light emitting diodes (LEDs) [Guo2010, Nguyen2011, Yan2015], NW lasers [Frost2014], NW polariton lasers [Das2011], dot-in-a-wire single photon sources [Deshpande2013], and field-effect nanotransistors [Huang2002c, Blanchard2008, Gacevic2016]. Besides, new applications continue to appear every year in new fields, like biosensing, photovoltaics or solar-assisted water splitting. In the last field, a variety of materials, compositions and designs were used to increase the photoelectrodes conversion efficiency, including GaN NWs [AlOtaibi2013a, Wang2011], InGaN NWs [Kamimura2013], axial InGaN/GaN NWs [Benton2013], InGaN-core/GaN-shell NWs [AlOtaibi2013b], coaxial GaN NWs with InGaN/GaN MQWs [Ebaid2015], Si-core/InGaN-shell NWs [Hwang2012], or $\mathrm{np}-\mathrm{Si} / \mathrm{n}-\mathrm{GaN} /$ Tunnel Junction/p-InGaN axial NWs [Fan2015].

In parallel, a significant progress has been made in understanding NWs fundamental optical properties. An example of a PL spectrum from a GaN NW ensemble, showing all major transitions is given in Fig. 6.1.1, with more information about each line given in Table X. It has been undoubtedly

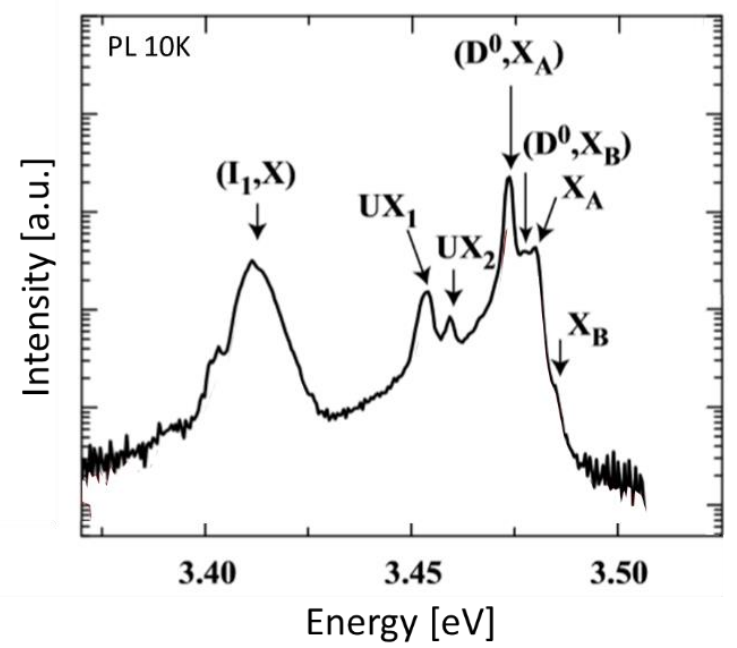

Fig. 6.1.1. Low temperature (10 K) PL spectra of a SA GaN NW ensemble grown on Si(111) with all major transitions labeled according to their origin. Adapted from [Corfdir2014a]. 
demonstrated that the change of GaN morphology from bulk to nanocolumnar structures leads to a dramatic enhancement of the near-bandgap emission, concomitant with a strong suppression of the below-bandgap luminescence (in particular the $2.2 \mathrm{eV}$ yellow band) [Yoshizawa1997, Calleja2000, Gacevic2015b]. The near-bandgap emission lines around 3.472-3.476 eV and 3.478$3.484 \mathrm{eV}$ have been associated to donor-bound (DBE) and free excitons, respectively [Lagerstedt1974, Calleja2000]. However, only recently, the 3.41-3.43 eV lines have been undoubtedly attributed to basal stacking faults (BSF) [Corfdir2014a, Urban2015]. Interestingly, the change of GaN morphology, from bulk to nanocolumnar, usually leads to a significant enhancement of the UX emission line at $3.45 \mathrm{eV}$ [Calleja2000, Reshchikov2005, Corfdir2014b], the origin of which is still a matter of debate, the interpretations include: two electron satellite (TES) of DBE [Corfdir2009], unidentified surface defects [Calleja2000, Sam-Giao2013], as well as polarity inversion domain boundaries (pIDB) [Auzelle2015, Robins2007].

Table X. Most common LT-PL emission lines from unintentionally doped GaN NWs

$\begin{array}{lll}\text { Label } & \begin{array}{l}\text { Peak positions } \\ {[\mathrm{eV}]}\end{array} & \begin{array}{l}\text { Description } \\ \text { FX }\end{array} \\ & 3.478\left(\mathrm{FX}_{\mathrm{A}}\right) & \text { Free excitons [Monemar1997], [Corfdir2014a] (refer to section 2.3.1). } \\ & 3.484\left(\mathrm{FX}_{\mathrm{B}}\right) & \\ & 3.502\left(\mathrm{FX}_{\mathrm{C}}\right)\end{array}$

DBE $\quad 3.472\left(D_{0}, X_{A}\right) \quad$ Donor bound exciton (most commonly involving Si and O impurities. $3.476\left(D_{0}, X_{B}\right) \quad$ [Corfdir2014a].

UX $3.452\left(U_{1}\right) \quad$ Polarity inversion domains [Auzelle2015] [Stutzmann2001] (refer to $\begin{array}{ll}3.458\left(\mathrm{UX}_{2}\right) & \begin{array}{l}\text { section 2.4.3), TES [Corfdir2009] (or surface related emission } \\ \text { [Calleja2000]) }\end{array}\end{array}$

BSF 3.40-3.43 $\left(I_{1}\right) \quad$ Basal stacking faults [Lahnemann2014] [Corfdir2014a] [Urban2015] 3.32-3.36 $\left(I_{2}\right) \quad$ (refer to section 2.4.2).

$\sim 3.29$ (E)

YL 2.2-2.3 Yellow band. Most likely a Ga Vacancy-Donor complex defect [Neugebauer1996, Lyons2010].

To overcome the poor controllability of SA NWs (diameter, height, density and orientation dispersion), as well as the concomitant inhomogeneous electrical and optical behavior, homoepitaxial SAG method was recently developed on both GaN/sapphire(0001) and GaN/Si(111)

templates [Sekiguchi2008, Kishino2009, Bengoechea2011, Gacevic2015a]. The huge advantages of SAG, such as a precise control of NWs sites, diameter, and orientation (tilt and twist), have led to further device developments, such as arrays of nano-LEDs [Sekiguchi2010a, Albert2014], single photon sources [Holmes2014, Chernysheva2015] and photonic crystals [Matsubara2008].

As mentioned before, a drastic improvement of GaN NWs optical and structural quality, compared to bulk layers, was a clear tendency since the beginning of NWs development [Yoshizawa1997, Calleja2000]. But why SAG GaN NWs are typically free of extended defects (or with a much lower 
density than in 2D layers), even when grown on low quality substrates? A vast amount of experimental data and numerical calculations [Colby2010, Sekiguchi2010b, Kishino2015, Coulon2016] suggest that the reason is the so called dislocation filtering effect occurring at a relatively early stage of the NWs growth. Once the NW starts to develop significant lateral free surface (sidewalls), dislocations that entered the NW from the template eventually bend towards the sidewalls and annihilate [Hersee2011]. Initially, this filtering effect was linked to the NWs aspect ratio [Colby2010], though later the NWs diameter was regarded as the main parameter affecting the dislocation filtering efficiency, since it determines the critical NWs height at which almost all dislocations are filtered out [Hersee2011, Kishino2015].

Most works describing the dislocation filtering effect were done in SAG GaN NWs grown on high quality GaN/sapphire templates [Colby2010, Sekiguchi2010b, Hersee2011, Coulon2016] with the only exception of a comprehensive study on Si substrates buffered with an AIN/GaN superlattice [Kishino2015].

While SAG NWs have yet to be checked for water splitting applications, it is clear that a precise morphology control inherent to SAG has a great potential for photoelectrochemical applications. Especially it would be useful in III-Nitrides/Si tandem cell devices, where the rather low quality of InGaN films (compared to those grown on sapphire) can be overcome with the use of SAG NWs with a significantly enlarged SCR region, which reduces the minority carrier transfer distance and, consequently, boosts the efficiency of the water splitting cell (refer to section 1.3). On the other hand, homoepitaxial SAG of NWs is mostly performed nowadays on commercial, high quality (in terms of dislocation densities, surface roughness and overall optical performance [Amano2015]) GaN/Sapphire(0001) templates, which are not suitable for vertical conduction in tandem cells (insulating material). There are only scarce reports on the use of GaN buffered Si substrates for NWs SAG [Albert2011] and even less about the effects of these templates on the quality of the NWs grown on them [Kishino2015].

This chapter addresses the study of the effect of NWs dimensions (diameter $160-500 \mathrm{~nm}$ and height $420-1100 \mathrm{~nm}$ ) and the crystal quality of the GaN template used (GaN/Si, GaN/AIN/Si, GaN/sapphire) on the SAG GaN NWs structural properties and optical performance. The aim of this study is to find a way to avoid or filter dislocations in NWs grown on a conductive substrate like $\mathrm{Si}(111)$ because dislocations are lifetime killers that may considerably affect the PEC photoanode performances.

\subsection{SAG of GaN NWs: experimental details}

Three different GaN templates were used for NWs SAG, namely: GaN/Si(111), GaN/AIN/Si(111), and GaN/sapphire(0001) with expectedly improving crystal quality. The temperature at which the GaN buffer was grown on Si(111) was estimated from the calibration point at which the surface reconstruction changes from $(1 \times 1)$ to (7x7) (Refer to section 2.5.1). In the case of GaN/sapphire(0001) templates, the temperature was estimated from Ga desorption experiments [Gacevic2015b, Koblmuller2007]. Si(111) substrates were prepared according to the procedure described in section 4.2.1 (degassing + nitridation) [Aseev2015].

For the first mentioned template, $500 \mathrm{~nm}$ thick GaN buffers were grown on $\mathrm{Si}(111)$ at $T_{\text {sub }}=750^{\circ} \mathrm{C}$. The $\mathrm{N}$ and $\mathrm{Ga}$ fluxes were calibrated as described in Appendix $\mathbf{A}$ and both adjusted to $5 \mathrm{~nm} / \mathrm{min}$ (in 
GaN units). For GaN/AIN/Si templates, a first $250 \mathrm{~nm}$ thick AIN layer was grown by the droplet elimination by radical beam irradiation (DERI) method [Jmerik2012, Nanishi2009], followed by a 250 $\mathrm{nm}$ thick GaN layer, both grown at $T_{\text {sub }}=750{ }^{\circ} \mathrm{C}$. The Al flux was set to $6 \mathrm{~nm} / \mathrm{min}$ aiming to a metalrich regime during the DERI process, while the $\mathrm{Ga}$ and $\mathrm{N}$ fluxes were kept the same as before $(5 \mathrm{~nm} / \mathrm{min})$. In agreement with the growth conditions, both templates were expected to be N-polar [Brubaker2016]. The GaN/sapphire templates were $4 \mu \mathrm{m}$-thick Ga-polar commercial MOVPE grown ones (Lumilog).

Ti masks were fabricated by colloidal lithography, according to the procedure described in section 3.2.4.1.1. PS nanobeads with 600,400 , and $300 \mathrm{~nm}$ in diameter were further reduced to 500,280 , and $170 \mathrm{~nm}$, respectively, by oxygen plasma treatment (3.5 min long, plasma power of 20W, 10 sccm of $\mathrm{O}_{2}$ flux). A $7 \mathrm{~nm}$ thick $\mathrm{Ti}$ layer was deposited on top by e-gun evaporation and the mask openings were achieved by lift-off. Prior to NWS SAG, the templates were degassed in the introduction chamber at $350^{\circ} \mathrm{C}$ for several hours and then in the UHV chamber at $450^{\circ} \mathrm{C}$ for $30 \mathrm{~min}$. Later, the Ti mask was nitridated under nitrogen plasma $(0.5 \mathrm{sccm}, 350 \mathrm{~W})$ for $30 \mathrm{~min}$ at the same temperature to form thermally stable TiN. Finally, the template was heated to the growth temperature still being under the nitrogen plasma flux.

For all GaN NWs SAG the $N$ and Ga fluxes were set to $F_{N^{*}}=5 \mathrm{~nm} / \mathrm{min}$ and $F_{G a}=15 \mathrm{~nm} / \mathrm{min}$, respectively (two Ga cells were used to achieve this flux), according to the previously established optimal flux values [Bengoechea2011].

In order to determine the optimal growth temperature, a series of SAG GaN NWs samples were grown on GaN/Si templates at different temperatures in the 700 to $800{ }^{\circ} \mathrm{C}$ range. SEM images of three representative samples grown at 740,765 , and $780^{\circ} \mathrm{C}$ are shown in Fig. 6.2.1. The GaN NWs sample grown at $740{ }^{\circ} \mathrm{C}$ reveals a parasitic grow on the mask (not fully selective). Reducing the growth temperature enhances this effect to the point where GaN grows everywhere (due to reduced Ga desorption from the mask). Increasing the temperature to $765^{\circ} \mathrm{C}$ results in a perfect selective growth of NWs having a clear hexagonal shape. At even higher temperatures $\left(780^{\circ} \mathrm{C}\right.$ and above), both parasitic growth on the mask and deterioration of NWs shape are evident (due to decomposition of GaN). Thus, the optimal growth temperature for SAG of GaN NWs was set to $765^{\circ} \mathrm{C}$ for the chosen $\mathrm{Ga}$ and $\mathrm{N}$ fluxes, where a perfect SAG regime is reached. The same optimized temperature resulted in SAG regime on GaN/AIN/Si(111) and GaN/sapphire(0001) templates.
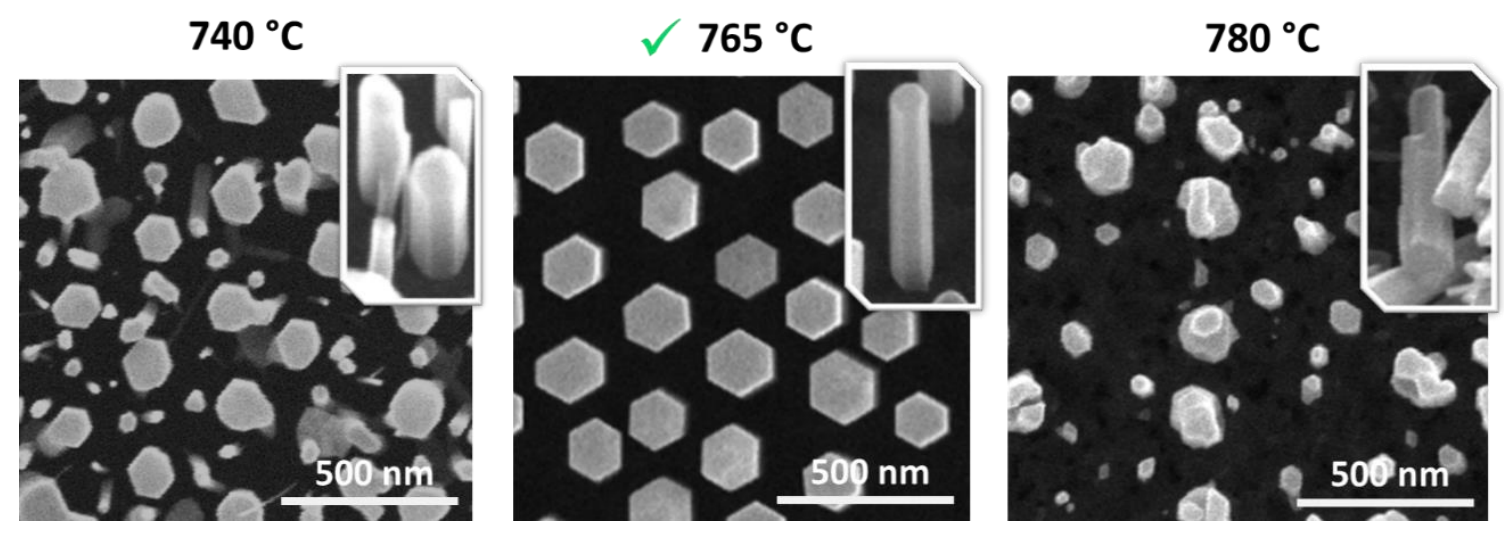

Fig. 6.2.1. Optimal growth temperature determination for GaN NWs SAG on GaN/Si templates using SEM. 
Following the optimal temperature determination, series of SAG GaN NWs were grown on each of the mentioned templates, labeled as NA\#, NB\# and NC\# (see Table $\mathbf{X I}$ ), under identical growth conditions $\left(F_{G a}=15 \mathrm{~nm} / \mathrm{min}, F_{N^{*}}=5 \mathrm{~nm} / \mathrm{min}, T_{\text {sub }}=765^{\circ} \mathrm{C}\right.$ ), but for the growth times varying between 1 and 4 hours aimed to NWs heights of 420 and $1100 \mathrm{~nm}$ (NWs dimensions estimated from SEM images are listed in Table XI).

Standard deviations of NWs diameters and heights were $12 \%$ and $5 \%$, respectively, in a very good agreement with previous results on the shape variations analysis of SAG NWs [Gacevic2015b].

The NWs growth rates estimated from SEM images and growth times reveal lower values for longer NWs, probably due to a reduced Ga flux along sidewalls to the NWs top as they get longer [Gacevic2015a]. The structural and optical properties of the NWs series are examined in the following sections.

Table XI. Series of GaN NWs grown on various templates

\begin{tabular}{lcc} 
Sample & Diameter, $\mathrm{nm}$ & Height, $\mathrm{nm}$ \\
\hline GaN/Si: & & \\
NA1 & 500 & 1100 \\
NA2 & 280 & 1100 \\
NA3 & 170 & 1100 \\
NA4 & 500 & 420 \\
NA5 & 170 & 420 \\
\hline GaN/AIN/Si: & & \\
NB1 & 500 & 1100 \\
NB2 & 280 & 1100 \\
NB3 & 170 & 1100 \\
\hline GaN/sapphire: & \\
\hline NC1 & 500 & 1100 \\
NC2 & 210 & 1100 \\
NC3 & 160 & 1100 \\
\hline
\end{tabular}

\subsection{Structural characterization of GaN NWs}

This section presents a TEM study of the structural properties of SAG GaN NWs grown on GaN/Si templates, as well as their comparison with NWs grown on the other templates.

\subsubsection{Dislocation filtering effect in NWs grown on a GaN/Si template}

TEM images of dispersed NWs from samples NA1 and NA3 with diameters of $\varnothing 500$ and $\varnothing 170 \mathrm{~nm}$, respectively, and the same height of $1100 \mathrm{~nm}$ (see Table XI) are shown in Fig. 6.3.1. In thick NWs ( $\varnothing 500 \mathrm{~nm}, \mathrm{NA1}$ ) the dislocations bend towards the NW sidewall along the whole NW height, while for thin ones ( $\varnothing 170 \mathrm{~nm}, \mathrm{NA3}$ ) almost all dislocations reach the NW sidewalls within the first $300 \mathrm{~nm}$ 

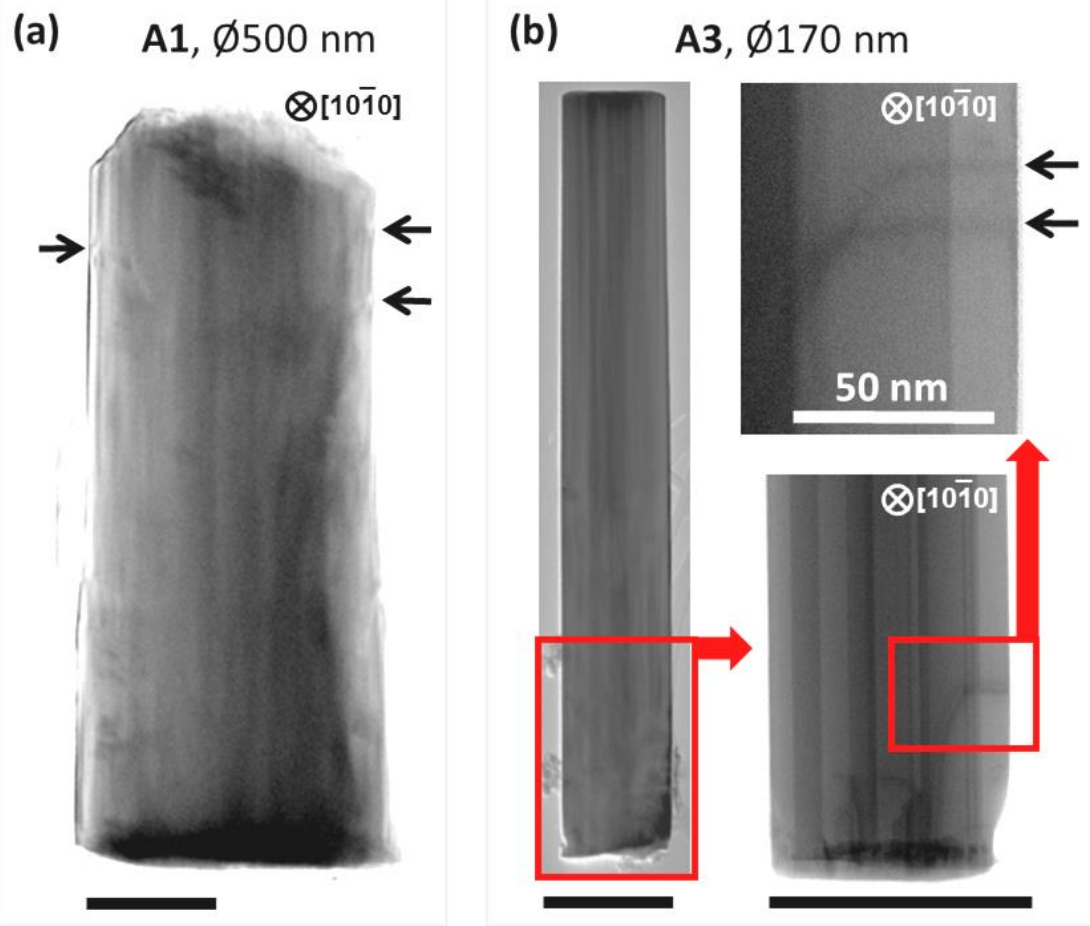

Fig. 6.3.1. Dislocation filtering effect on thick (a) and thin (b) GaN NWs grown on GaN/Si template, as observed by TEM. Black arrows indicate where bent dislocations reach the NWs surface. Bottom black scale bars are $200 \mathrm{~nm}$.

height. These results suggest that effective (critical) heights for full dislocation filtering are above $1100 \mathrm{~nm}$ and around $300 \mathrm{~nm}$, respectively for the two NW diameters considered. Bearing in mind that both samples were grown simultaneously on the same GaN/Si(111) template, i.e. under identical growth conditions, the change in critical height for dislocation filtering is exclusively correlated to their different diameters, defined by the nanohole mask.

The observed behavior is in a good qualitative agreement with previous reports on SAG GaN NWs grown on GaN/AIN/Si [Kishino2015] and on GaN/sapphire [Sekiguchi2010b, Hersee2011, Coulon2016] templates, despite the significant differences in growth method (MBE and MOVPE), quality of buffer layers, growth parameters (NW diameter, Ga/N fluxes, growth temperature and time), strain in the GaN buffer layers (tensile for Si substrates and compressive for sapphire), and NWs polarity (Ga and N polarity).

Therefore, from our results and previously reported ones it is concluded that in SAG GaN NWs there is a critical height, depending on the NW diameter, at which the dislocation density is drastically reduced, leaving most of the NW upper region volume free of dislocations. The smaller the NWs diameter, the smaller the critical height.

\subsubsection{Polarity inversion domains}

SEM imaging revealed that NWs grown on Si substrates (either GaN/Si or GaN/AIN/Si) have in general flat tops, but a significant fraction exhibit partial top faceting, as shown in Fig. 6.3.2 (a) (marked with blue arrows). The thicker the NW is, the more irregularly shaped its top becomes. On 
the contrary, all NWs grown on GaN/sapphire templates exhibit a perfect hexagonal pyramidal shape even for the thickest NWs (Fig. 6.3.2 (b)).

In order to understand the origin of these morphology features CTEM and ABF-STEM measurements were performed on two types of NWs found in sample NA3: (i) with completely flat tops and (ii) exhibiting partial faceting. Examination of flat top NWs (i) without faceting revealed that they are fully N-polar (as expected from a flat top morphology). In contrast, TEM of faceted NWs (ii) revealed that they also exhibit $(10 \overline{1} X)$ facets, normally corresponding to Ga-polar crystal (Fig. 6.3.2 (c)). Detailed ABF-STEM examination of the faceted NWs shown in Fig. 6.3.2 (d) confirms that the flat part of the NW is N-polar, while faceted part - Ga-polar. Consequently, the border between these regions contains a pIDB defect (Refer to section 2.4.3) that extend through the whole NW length, as it can be seen in Fig. $\mathbf{6 . 3 . 2}$ (c).

Thus, the top partial faceting is attributed to the presence of pIDs. Similar effects of partial faceting of SA [Chen2010, Auzelle2015b] and SAG [Chen2010] GaN NWs related to pIDBs were already reported. On the other hand, all NWs grown on GaN/Sapphire templates studied by TEM and ABF-STEM exhibit full Ga-polarity and perfect hexagonal pyramidal shape, in agreement with previous reports [Gacevic2015].

Morphology

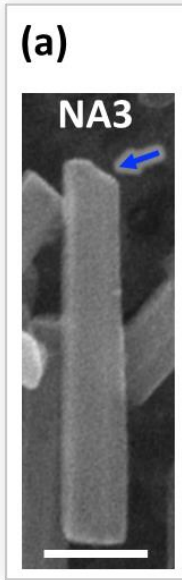

(b)

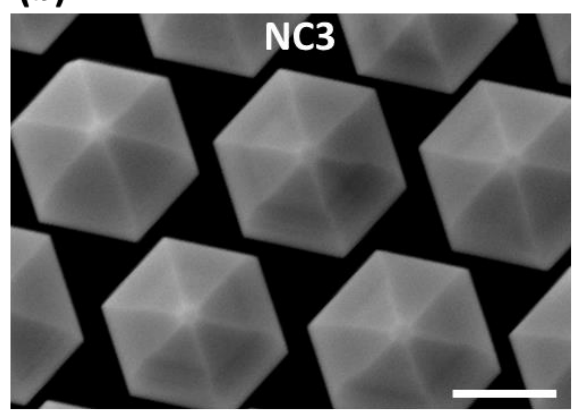

TEM of pID

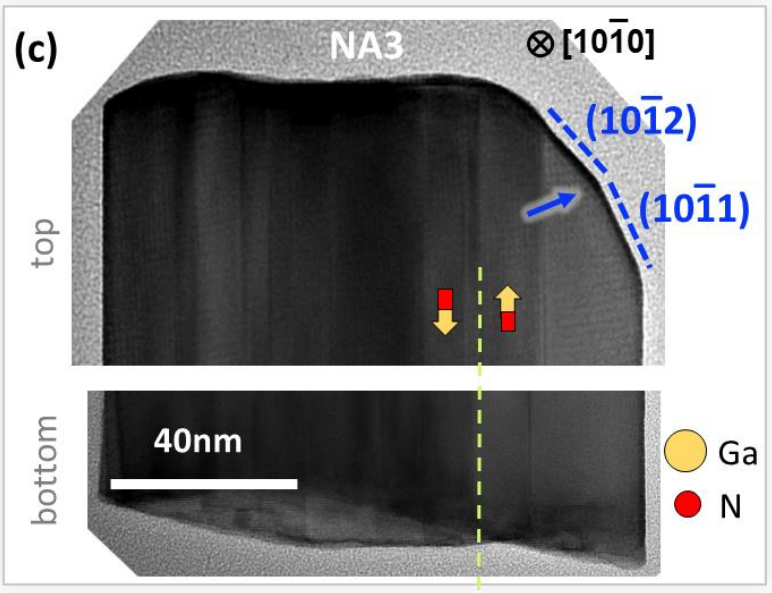

(d)

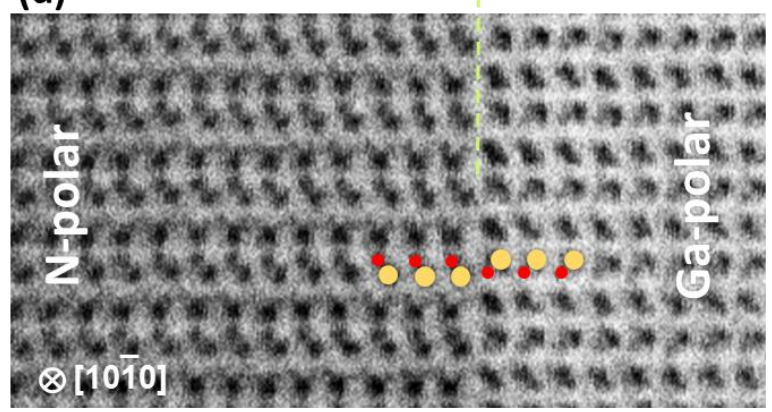

Fig. 6.3.2. NWs shape irregularities (marked with blue arrows) related to the presence of pIDBs in samples grown on both GaN/Si and GaN/AIN/Si templates, evidenced by SEM (a). SEM images of NWs grown on a GaN/sapphire template with a perfect hexagonal pyramidal shape (b). White scale bars on all SEM pictures are $300 \mathrm{~nm}$ long. TEM (c) and ABF-STEM (d) images of GaN NWs from sample NA3, revealing the presence of a pIDB. Polarity is monitored by imaging the heavy Ga and light $\mathrm{N}$ atomic columns (d). 
There are at least two possible origins of these pIDBs defects in SAG GaN NWs: (i) the propagation of existing pIDBs from the underlying GaN template into the NW and (ii) Ti-induced nucleation of pIDBs at the NW/template interface due to Ti diffusion from the mask [Kong2016]. The fact that in our experiments under identical growth conditions pIDBs were found only in NWs grown on Sibased templates strongly favors (i) explanation. The reason is probably increased roughness of the InGaN/Si interface due to meltback etching, which promotes formation of pIDBs. However, additional TEM experiments on not dispersed NWs (to examine the GaN NWs/GaN layer interface) must be performed to verify this hypothesis.

Notice that TEM did not reveal the presence of BSF-type defects in none of the examined samples, suggesting that they are quite rare compared to dislocations and pIDBs.

\subsection{Optical characterization of SAG GaN NWs}

Standard PL measurements were performed at 10K, excited by a He-Cd laser $\left(325 \mathrm{~nm}, 3 \mathrm{~W} \cdot \mathrm{cm}^{-2}\right)$. In order to avoid PL contributions from the GaN templates, NWs were mechanically transferred to an optically inactive Si substrate [Chernysheva2015] and up to $\sim 10^{4}$ transferred (by mechanical scratching of the NW sample against carrier substrate) NWs were simultaneously excited, yielding, thus, average optical properties of the NWs ensemble. For an easier comparison, all PL spectra were normalized to the most intense peak amplitude. No yellow band emission was observed for all NWs studied in this work, in agreement with previous reports [Gacevic2015b].

\subsubsection{Effects of NW dimensions}

LT-PL spectra with the corresponding SEM images of NWs with different diameters and heights grown on GaN/Si templates (series NA\#) are shown in Fig. 6.4.1. Spectra from thick $\varnothing 500 \mathrm{~nm}$ NWs (NA1, NA4, Fig. 6.4.1 (a,b)) are dominated by a broad below-bandgap emission, peaking at 3.41$3.43 \mathrm{eV}$, most likely related to BSFs [Corfdir2014a, Urban2015]. Only very weak DBE and UX emission lines are observed around $3.47 \mathrm{eV}$ and $3.45 \mathrm{eV}$, respectively. No significant change in the overall PL spectra shape was observed upon increasing the height of these thick NWs.

When the NW diameter is reduced to $\varnothing 170 \mathrm{~nm}$ (NA3, NA5, Fig. 6.4.1 (c,d)), PL spectra show sharper and far better resolved below-bandgap emissions. For short NWs (NA5) both $I_{1}(3.41 \mathrm{eV})$ and $I_{2}$ (3.33 eV) BSF associated emission lines are resolved (Refer to section 2.4.2). Moreover, the NW height increase from 420 to $1100 \mathrm{~nm}$, while keeping the same diameter (N5 to N3), results in a clear DBE emission intensity enhancement, being a signature of a higher crystal quality. The UX band emission also becomes more prominent, actually dominating the spectrum. High spectral resolution measurements shown in the inset of Fig. 6.4.1 (d) reveal a doublet at $3.451 \mathrm{eV}\left(\mathrm{UX}_{1}\right)$ and $3.457 \mathrm{eV}$ $\left(\mathrm{UX}_{2}\right)$, similarly to those reported in the literature [Calleja2000, Corfdir2015]. In addition, the BSF emission line becomes much less intense upon NW height increase.

Under the assumption of equal coverage, absolute intensities of transferred NWS can also be very roughly compared. In this case, scaling factors (representing the ratio of maximum absolute intensities of each sample to NA4) can be assigned to PL graphs in Fig. 6.4.1 (grey $x$ marks). This rough comparison suggests that long and thin NWs (NA3) exhibit superior optical quality as compared to other samples in this series in agreement to the previous discussion of the shape of corresponding PL spectra (Fig. 6.4.1 (d)). 


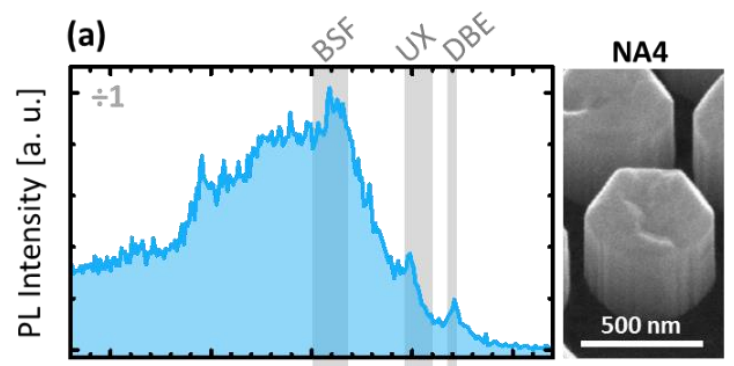

(c)

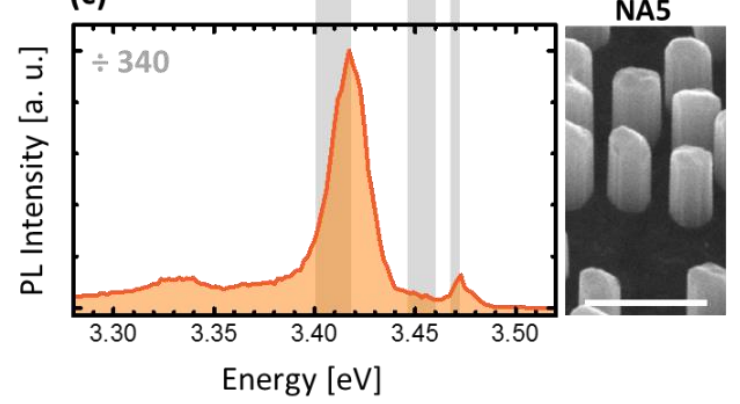

(b)

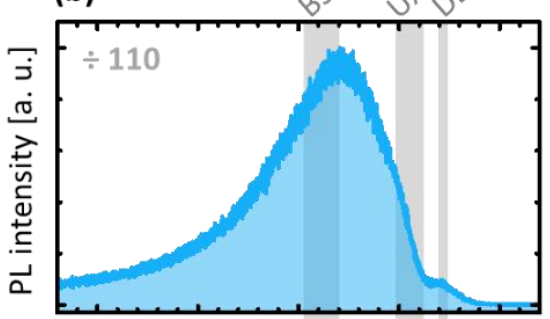

(d)

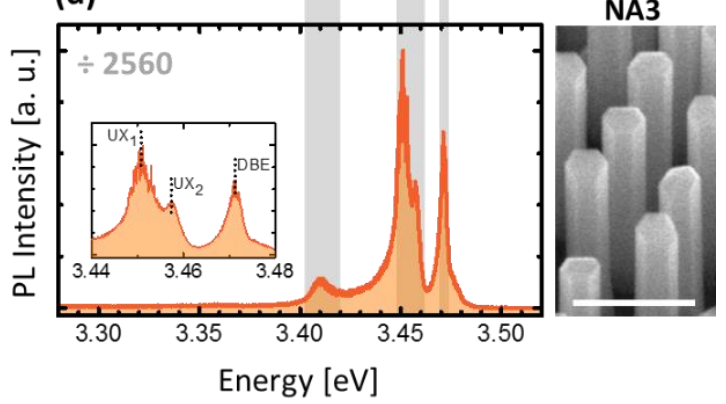

Fig. 6.4.1. Dependence of the PL performance at $10 \mathrm{~K}$ on NWs diameter and height for SAG GaN NWs ensembles transferred to a Si surface from samples NA4 (a), NA1 (b), NA5 (c), and NA3 (d). The positions of DBE, UX, and BSF emission bands are marked, for clarity. Bird's-eye view SEM images of NWs (before transfer) are placed next to each PL spectrum (scale bars are $500 \mathrm{~nm}$ ).

When considering the dislocation filtering effect and its dependence on NW diameter and height (section 6.3.1), the PL spectra sequence in Fig. 6.4.1 becomes clear. NA4 is the worst case (in terms of dislocation density affecting the NW volume) due to the largest diameter (high critical height) and shortest height. The PL spectrum broad lineshape cannot be built-up just from BSFs, UX and DBE contributions, thus pointing to the presence of many other (radiative) defects (from SFs, IDBs, etc. families), most likely related to the presence of a high dislocation density. Similarly, NA1 with the same diameter but 2.6 higher, has a bit better chances to filter dislocations, but still shows a broad PL spectrum, only slightly improved. The fact that in both cases the PL peak maximum is close to the BSF emission energy most likely reflects the QW-like nature of BSF defects, which makes their emission efficiency less affected by dislocations (strong carrier localization) as compared to DBE transitions (shallow donor) [Lahnemann2012, Korona2014, Corfdir2014a]. It is worth to mention that BSFs defects run parallel to the c-plane [Urban2015], thus, not expected to be filtered by NWs morphology changes.

In GaN NWs with a much smaller diameter (smaller critical height) (NA5, NA3), PL spectra are strongly improved in terms of both intensity and resolved peaks. A PL spectrum of sample NA5 shows distinct features like DBE and a dominant BSF signature (the same explanation regarding the QW-like nature of BSFs is applicable here). Upon increasing the NW height (sample NA3), there is a further improvement since DBE and UX lines are dominant over the BSFs one. Such a remarkable enhancement of the DBE intensity certainly is related to a lower dislocation density (confirmed by TEM) and their accompanying point defects.

In summary, the combination of PL and TEM results suggests a strong correlation between the SAG GaN NWs dimensions (diameter, height) and their optical performance. Short and/or thick NWs 
have a high dislocation density because of a rather inefficient filtering effect, which makes their PL spectra either featureless broad or dominated by BSFs emission. Long and thin NWs have a quite reduced dislocation density due to a strong dislocation filtering efficiency and consequently they show a dramatic enhancement of the DBE emission (a fingerprint of high crystal quality).

\subsubsection{Effects of the template quality}

Previous results on dislocation filtering efficiency should be qualitatively the same for templates of different quality (dislocations already existing in the template). Normalized PL spectra from SAG GaN NW samples grown on GaN/AIN/Si(111) (NB\#) and GaN/sapphire (NC\#) templates with expectedly increasing structural quality, are shown in Fig. $\mathbf{6 . 4 . 2}(\mathbf{b}, \mathbf{c})$ together with those from the abovementioned GaN/Si(111) case (NA\#) (Fig. 6.4.2 (a)). For comparison purposes, all NWs studied had the same height $(1100 \mathrm{~nm})$ but diameters in the range of 160-500 nm (for details on growth parameters refer to section 6.2).

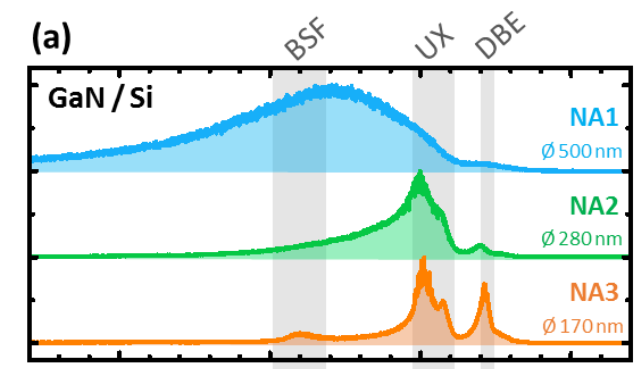

\section{(b)}

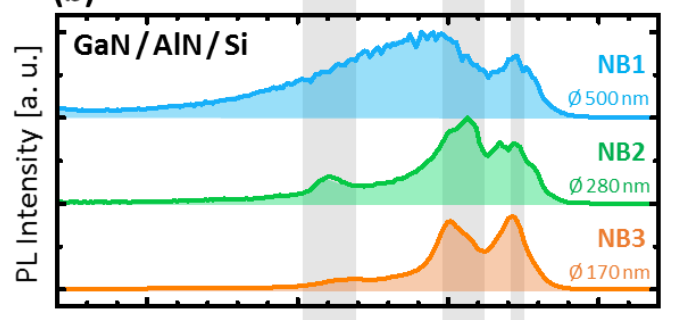

(c)
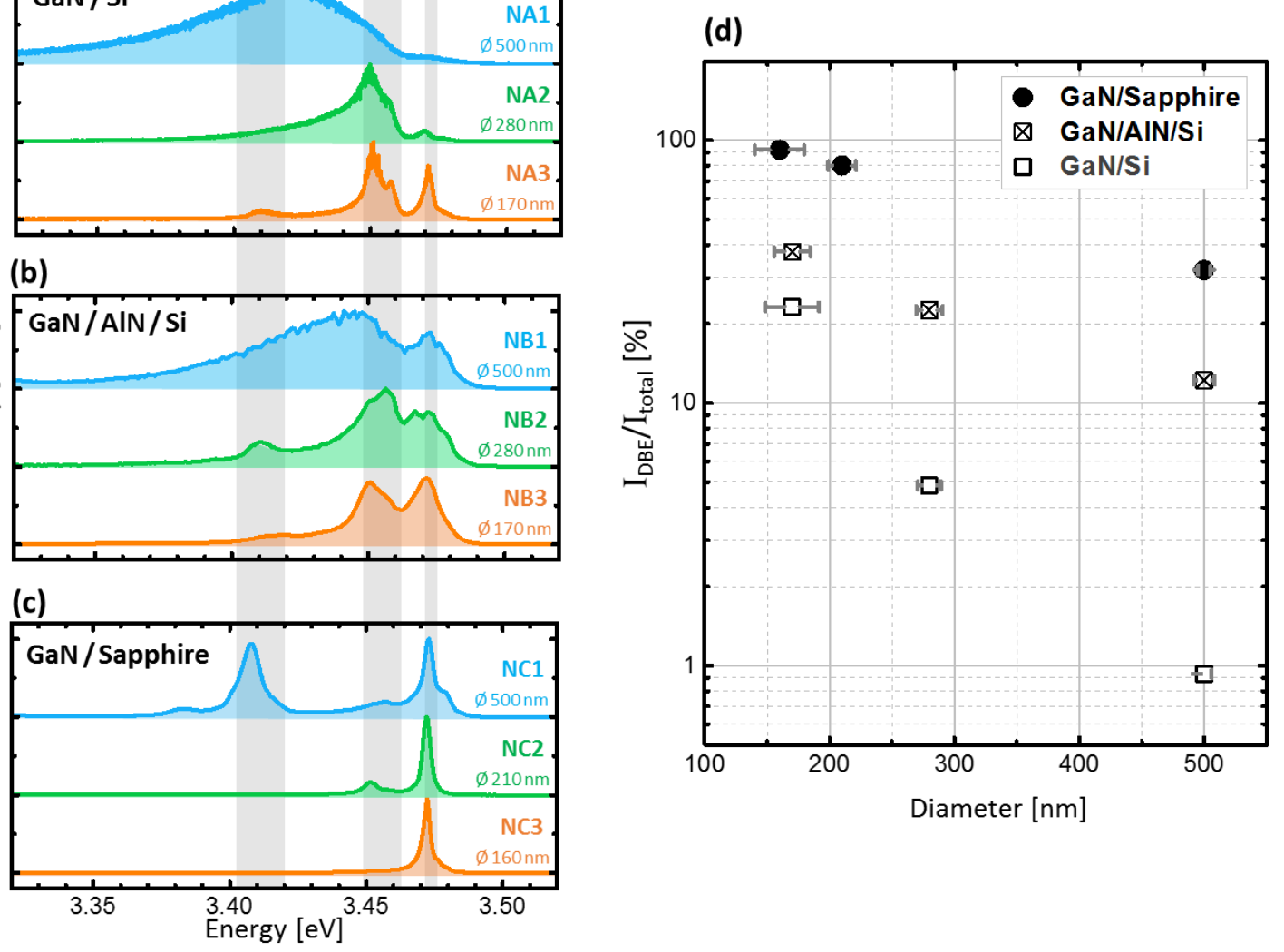

Fig. 6.4.2. Influence of the template quality on the optical performance of SAG GaN NWs grown on them: GaN/Si (a), GaN/AIN/Si (b) and GaN/sapphire (c). PL IDBE/Itotal ratio from NWs grown on the different templates as a function of the NW diameter (d). Horizontal error bars represent the diameter standard deviation. 
Fig. 6.4.2 (a) summarizes the results already discussed for NWs grown on GaN/Si(111) templates for samples with the same height $(1100 \mathrm{~nm})$ but different diameters. For completeness, a PL spectrum from sample NA2 (280 nm diameter) was added showing the trend that a lower NW diameter means less dislocations and enhanced quality.

PL spectra from samples in series NB\# (Fig. 6.4.2 (b)) show a similar trend with the NWs diameter, that is, a decrease of the NWs diameter leads to a progressively better resolution of below-bandgap emission into distinct lines, as well as the DBE emission enhancement.

Finally, PL spectra from samples in series NC\# (Fig. 6.4.2 (c)) are fully resolved into BSF and UX bands for NW diameters as big as $\varnothing 500 \mathrm{~nm}$ (NC1) and strongly improved for $\varnothing 160 \mathrm{~nm}$ (NC3), for which PL spectrum shows only an intense and narrow DBE emission. This is expected since the quality of the starting GaN template is much better (a much lower dislocation density) than in previous cases.

The optical quality changes in these three series of NWs can be estimated from the integrated intensity DBE/total PL ratio $\left(I_{D B E} / I_{\text {total }}\right)$, as shown in Fig. 6.4.2 (d). The $I_{D B E} / I_{\text {total }}$ ratio increase upon decreasing NW diameter from $\varnothing 500$ to $\varnothing 160 \mathrm{~nm}$ for NWs grown on the best GaN template (GaN/sapphire) lies within the same order of magnitude ( 25 to $90 \%$ ), having the thinnest ones $(\varnothing 160 \mathrm{~nm})$ a quasi ideal $I_{D B E} / I_{\text {total }}$ ratio $(90 \%)$ strongly suggesting an exceptional NWs crystal quality. On the other hand, NWs grown on Si-based templates start from much lower $I_{D B E} / I_{\text {total }}$ ratios for thick NWs $(\varnothing 500 \mathrm{~nm}$ ), but drastically improve it up to $30 \%$ upon decreasing their diameter (meaning up to two orders of magnitude enhancement for GaN/Si templates). The main message from these results is that by reducing the NW diameter, for a given height $(1100 \mathrm{~nm})$, the influence of the template quality on the optical performance of GaN NWs decreases significantly.

In addition, notice that strong UX emission was only observed in PL spectra of SAG GaN NWs grown on Si-based templates as seen in Fig. 6.4.2 (a,b). As was mentioned in section 6.1, there are several explanations of the origin of this emission were proposed in literature. From the PL spectrum of $\emptyset 170 \mathrm{~nm}$ NWs in Fig. 6.4.2 (a) it is clear that UX emission is not originated from two electron satellite (TES) of DBE, because its intensity is higher than DBE one. The other proposed origin related to emission from unidentified surface point defects [Calleja2000, Sam-Giao2013] is ruled out by comparing UX emission of NWs with the same dimensions (area): it is absent/present for NWs grown on sapphire-/Si-based templates, respectively (See Fig. 6.4.2 (a,b,c)). But the same occurrence behavior was reveled for polarity inversion domain boundaries (pIDBs) defects by TEM and SEM results described in section 6.3.2. Therefore, a strong $3.45 \mathrm{eV}$ UX band emission in the GaN NWs grown by SAG on Si-based templates is most probably attributed to radiative pIDBs defects. This finding agrees well with reports assigning the $3.45 \mathrm{eV}$ emission to the pIDB in both SA GaN NWs [Auzelle2015] and GaN bulk layers [Stutzmann2001]. 


\subsection{Conclusions}

In summary, a strong correlation between the GaN NWs structural properties, their optical performance, and the GaN templates quality has been established. The effects of the NWs dimensions (diameter and height) have also been determined. TEM data from NWs grown on GaN/Si templates confirmed that dislocation filtering is much more efficient in NWs with small diameters. $P L$ results revealed that a higher dislocation filtering efficiency results in a stronger enhancement of the DBE emission intensity (a fingerprint of high crystal quality), reaching a $I_{D B E} / I_{\text {total }}$ ratio as high as $90 \%$ for NWs with $\varnothing 160 \mathrm{~nm}$ and $1100 \mathrm{~nm}$ high grown on the best GaN template (GaN/sapphire). In addition, a proper selection of the NWs dimensions enhances the dislocation filtering to the point that high quality NWs can be grown on GaN templates with a relatively poor quality. This has strong implications when growing NWs for photovoltaic or water splitting applications, particularly for $\mathrm{Ga}(\mathrm{In}) \mathrm{N} / \mathrm{Si}$ tandem cells, because dislocations "kill" their performance. In other applications where the available surface is crucial, such as sensing applications, it is also advisable to enlarge the active surface by increasing the NWs length and density (smaller pitch) rather than by increasing their diameter.

Evaluation of obtained NWs (combined with InGaN layers and InN QD "decoration") in application to water splitting is reserved for the future work.

Results from the work presented in this chapter were published in [Aseev2016]. 


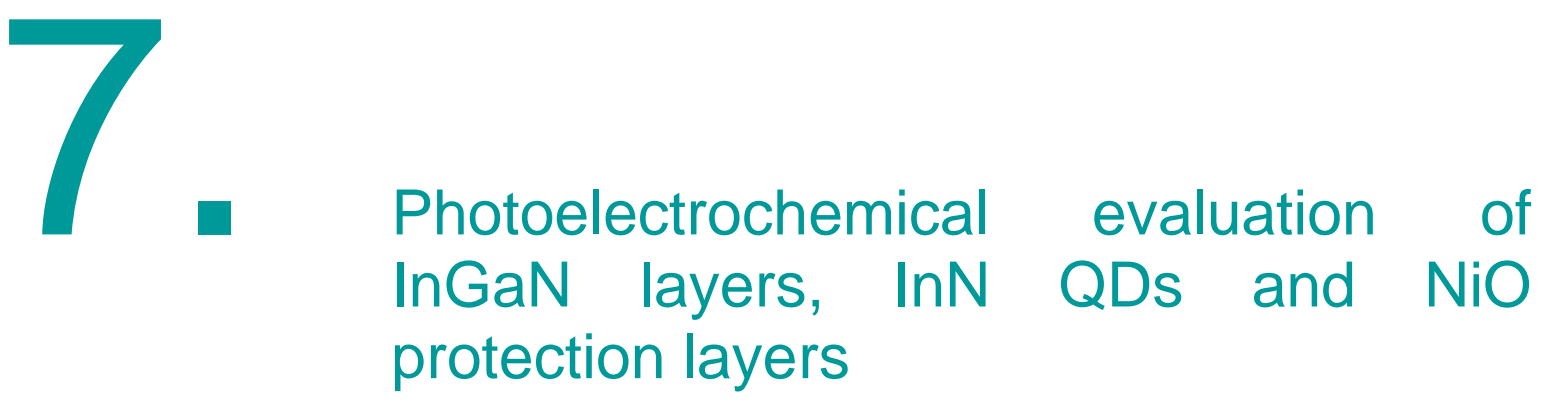

\subsection{Introduction}

Since the first report by Turner et al. [Kocha\&Turner1995] on n-GaN showing that its conduction (CB) and valence (VB) bands were well positioned in respect to the hydrogen and oxygen redox levels, thus allowing to drive water splitting reaction without external bias, a huge research effort has been devoted to this topic. Moving from binary GaN to ternary InGaN compounds opened unique possibilities to vary the bandgap over a very wide range $(0.7-3.4 \mathrm{eV})$, which combined with its high absorption coefficient ( $10^{6} \mathrm{~cm}^{-1}$ [Muth1997]) makes these alloys very good absorbers, a must for high performance photoelectrochemical (PEC) cells based on semiconductor electrodes. There is another relevant requirement which must be fulfilled to successfully use any material as electrode for PEC cells, namely: stability under operation conditions. However, there were many contradictions concerning InGaN-based photoanodes, depending on authors.

The idea of III-Nitrides superior chemical stability may originate from the experience in AIGaN highelectron-mobility transistors (HEMTs) where processing based on traditional wet etching was of limited use (Refer to a comprehensive review [Zhuang\&Edgar2005]). This stability concept was referenced by many researchers in PEC cells. Li et al. [Li2008] reported no visible physical degradation of $\operatorname{In}_{0.2} \mathrm{Ga}_{0.8} \mathrm{~N}$ and $\mathrm{In}_{0.4} \mathrm{Ga}_{0.6} \mathrm{~N}$ layers grown on $\mathrm{GaN} /$ sapphire substrates after several hours of PEC measurements in $1 \mathrm{M} \mathrm{HCl}$. Then Hwang et al. [Hwang2012] showed an enhanced performance of $\mathrm{Si} / \mathrm{InGaN}$ core/shell NWs compared to compact layers in $\mathrm{pH}_{3} \mathrm{H}_{2} \mathrm{SO}_{4}$ electrolyte with $0.5 \mathrm{M}$ of $\mathrm{Na}_{2} \mathrm{SO}_{4}$ salt. He claimed that no obvious photochemical etching or amorphous layer formation was evident after $15 \mathrm{~h}$ of illumination. AlOtaibi et al. [AlOtaibi2013a] showed that GaN NWs on $\mathrm{Si}(111)$ dipped in a $1 \mathrm{M} \mathrm{HBr}$ electrolyte can produce $\mathrm{H}_{2}$ for $5 \mathrm{~h}$, however nothing was mentioned about the $\mathrm{O}_{2}$ evolution. The same group also demonstrated [AlOtaibi2013b] an enhanced performance and stability of an InGaN/GaN core/shell NWs photoanode (grown on $\mathrm{Si}(111)$ ) in a $1 \mathrm{M} \mathrm{HBr}$ electrolyte, claiming that the $\mathrm{GaN}$ shell can protect the InGaN from corrosion and oxidation. In that case, a stable $\mathrm{H}_{2}$ production over $10 \mathrm{~h}$ was demonstrated but again nothing was mentioned about the $\mathrm{O}_{2}$ evolution. Later, Dahal et al. [Dahal2014] reported 7 days of stable $\mathrm{H}_{2}$ production (only $10 \%$ output decrease) using InGaN/GaN MQWs in a $1 \mathrm{M} \mathrm{HBr}$ electrolyte. It must be noted that the use of $\mathrm{HBr}$ electrolytes may difficult interpreting results on stability since $\mathrm{HBr}$ can act as a hole scavenger due to $\mathrm{Br}$ oxidation.

Many other groups reported photo-induced corrosion of InGaN photoanodes, like Minsky et al. [Minsky1996] that demonstrated laser-enhanced wet etching of GaN[0001]/sapphire using either dilute $\mathrm{HCl}: \mathrm{H}_{2} \mathrm{O}(1: 10)$ or $45 \% \mathrm{KOH}: \mathrm{H}_{2} \mathrm{O}(1: 3)$ electrolytes. Later Huygens et al. [Huygens 2000] studied 
photo-induced wet etching of $\mathrm{GaN}[0001]$ /sapphire in $1 \mathrm{M} \mathrm{KOH}$ and $1 \mathrm{M} \mathrm{H}_{2} \mathrm{SO}_{4}$ electrolytes showing that only holes contribute to photoetching of n-GaN. Moreover, Kamimura et al. [Kamimura2013] observed photoetching of $n$-type $\operatorname{In}_{0.42} \mathrm{Ga}_{0.58} \mathrm{NWs}$ on $\mathrm{n}$-Si(111) substrates by in situ $\mathrm{N}_{2}$ evolution instead of $\mathrm{O}_{2}$ at anodic potentials in a $0.5 \mathrm{M} \mathrm{H}_{2} \mathrm{SO}_{4}$ electrolyte. Juodkazyte et al. [Juodkazyte2014] attributed the photocorrosive effect in $\ln _{x} G_{1-x} N(x=0-1)$ photo-electrodes either in alkaline or acidic electrolytes to its oxidation under photoanodic conditions and the formation of $\mathrm{Ga}_{2} \mathrm{O}_{3}$ together with the $\mathrm{N}_{2}$ evolution. Later, this idea was expanded by Caccamo et al. [Caccamo2016] who showed that the formation of a thin amorphous oxide layer with a $\mathrm{pH}$-dependent chemical composition occurs on the InGaN surface under photoanodic conditions. Additionally, Finken et al. [Finken2015] performed deep investigations on the long-term stability of InGaN layers (grown on $\mathrm{GaN}$ /sapphire(0001)) with a vast variety of electrolytes, including $\mathrm{NaCl}, \mathrm{HCl}, \mathrm{HBr}, \mathrm{H}_{2} \mathrm{SO}_{4}, \mathrm{NaOH}$ and $\mathrm{KOH}$. He showed that only in $1 \mathrm{M} \mathrm{NaCl}$ and in $1 \mathrm{M} \mathrm{HBr}$ the InGaN surface is stable due to the presence of $\mathrm{Cl}^{-}$and $\mathrm{B}^{-}$ions and their corresponding reaction with photo-generated holes. This idea was proposed earlier by Luo et al. [Luo2008] who attributed the stability of $\operatorname{In}_{x} G_{a_{1-x}} N(0<x<0.2)$ layers (grown on $\mathrm{GaN} /$ sapphire(0001)) to $\mathrm{HBr}$ as an effective sacrificial agent. Similar experiments in $1 \mathrm{M}$ $\mathrm{HCl}$, and $0.5 \mathrm{M} \mathrm{H}_{2} \mathrm{SO}_{4}$ solutions resulted in photo-corrosion. For more information on InGaN application to water splitting refer to a comprehensive review by Kibria \& Mi [Kibria\&Mi2016].

There were also reports where the stability of InGaN photo-electrodes is enhanced by $\mathrm{NiO}$ protection layers, which were recently very successfully employed to improve stability of many photoanodes in alkaline solutions (to name just a few, n-Si [Mei2014], n-InP [Sun2015], n-CdS [Chan2011], n-CdTe [Sun2015b]). For example, Hayashi et al. [Hayashi2012] showed $110 \mathrm{~h}$ of stable $\mathrm{H}_{2}$ production at enhanced rate using $\mathrm{GaN}$ layers covered with a $\mathrm{NiO}$ catalyst layer, compared to only $10 \mathrm{~h}$ for layers without $\mathrm{NiO}$, both in $1 \mathrm{M} \mathrm{NaOH}$. Layers without $\mathrm{NiO}$ were severely corroded, while those with $\mathrm{NiO}$ were not. However the $\mathrm{H}_{2} / \mathrm{O}_{2}$ ratio was very low in that case suggesting that an additional reaction was involved. Benton et al. [Benton2013, Benton 2014] compared the InGaN/GaN NWs morphology after 180 min of PEC experiments in $1 \mathrm{M} \mathrm{NaOH}$ for samples with and without NiO nanoparticles from which it was evident that corrosion was drastically suppressed by $\mathrm{NiO}$ nanoparticles. The performance increase was attributed to enhanced electron-hole separation due to a band alignment of $\mathrm{NiO}$ with GaN. Ryu et al. [Kang2014] reported very similar conclusions but for a solution casted $\mathrm{NiO}$ protection layer on $\mathrm{GaN} /$ sapphire samples in $1 \mathrm{M} \mathrm{NaOH}$. Later, the same group [Kim2014a] suggested that the crystallinity and geometry of a NiO catalyst greatly affect the performance of a GaN photoanode and proposed a patterned NiO catalyst [Kim2014b] to improve water splitting efficiency and long-term stability. While the improved water splitting performance was attributed to the fast transport of photo-generated holes in the valence band from $\mathrm{GaN}$ to $\mathrm{NiO}$ and the efficient water reduction at the NiO/electrolyte interface, the significance of the use of $\mathrm{NiO}$ islands instead of a layer was not clearly stated.

In order to address all mentioned controversies and to determine how InGaN photoanodes behave under working conditions, several PEC experiments were performed in this work. First, "as grown" $\ln _{x} \mathrm{Ga}_{1-\mathrm{x}} \mathrm{N} / \mathrm{Si}(111)$ samples were tested in both acidic and alkaline electrolytes with and without sacrificial agents in order to evaluate and compare their performance and sustainability. Then, $\mathrm{In}_{x} \mathrm{Ga}_{1-\mathrm{x}} \mathrm{N} / \mathrm{Si}(111)$ samples covered with $\mathrm{NiO}$ catalyst were studied to identify if such surface treatment enhances the catalytic activity and corrosion resistivity of the $\ln _{\mathrm{x}} \mathrm{Ga}_{1-\mathrm{x}} \mathrm{N}$-based photoanodes. Additionally, $\ln _{x} \mathrm{Ga}_{1-x} \mathrm{~N} / \mathrm{Si}(111)$ samples decorated with InN quantum dots (QDs) were tested as a novel method to improve the $\ln _{x} \mathrm{Ga}_{1-x} N$ photoanodes performance enhancement. In all 
cases electrical contacts to the $\ln _{x} \mathrm{Ga}_{1-\mathrm{x}} \mathrm{N}$ layers was done through the Si substrate, which is one of the main requirements for tandem solar cells. The experimental details, results, and discussions are presented in the following sections.

\subsection{Experimental details}

All samples used in this work were cut into $5 \times 5 \mathrm{~mm}$ pieces to allow performing several experiments on the same sample, because of a relatively small size of the substrates used ( 1 inch diameter). Various $\operatorname{In}_{x} \mathrm{Ga}_{1-\mathrm{x}} \mathrm{N}$ layers $(\mathrm{x}=0-0.4)$ grown on highly conductive $\mathrm{p}^{+}$-Si substrates (to allow vertical conductivity and placing contact to the $\operatorname{lnGaN} / \mathrm{p}^{+}-\mathrm{Si}$ electrode on the back side), as described in Chapter 4, were used in this study. All InGaN layers were treated as photoanodes because of the unintentionally n-type doping. In PEC experiments, the InGaN layers (either with or without NiO protection layers, or decorated with InN QDs) were placed in direct contact to the electrolyte, while the back Si substrate was electrically contacted by metal wire and connected to a potentiostat. The wiring and the whole Si substrate (back side and edges) were chemically and electrically isolated from the electrolyte with epoxy. The potentiostat circuit was closed on the other side through the Pt mesh counter electrode (For experimental setup description refer to section 3.6). The physical vapor deposition (PVD) by magnetron sputtering technique was used to cover the InGaN layers with compact, polycrystalline $15 \mathrm{~nm}$ thick NiO films. The samples with InN QDs were grown according to the procedure described in Chapter 5. Further details regarding samples, chemicals, and setup preparations are given in Appendix B.

\subsection{As grown InGaN layers}

The aim of the work presented in this section was to gain understanding on the InGaN layers performance limiting factors as photoanodes. The photocurrent onset potential dependence on the bandgap of non-catalyzed InGaN layers is determined, and the stability of InGaN layers against corrosion is also evaluated.

\subsubsection{Photocurrent onset potential}

A series of InGaN layers grown on highly conductive $\mathrm{p}^{+}-\mathrm{Si}$ substrates were studied by cyclic voltammetry $(\mathrm{CV})$ in order to determine the change of onset potential upon bandgap variation. Both alkaline, $1 \mathrm{M} \mathrm{KOH}$ (Fig. 7.3.1 (a-c)) and acidic, $1 \mathrm{M} \mathrm{HClO}_{4}$ (Fig. 7.3.1 (d-f)) electrolytes were tested. CV measurements were recorded versus the reference electrode potential and then recalculated to values versus RHE (refer to section 2.6.2) in order to compare them independently of the electrolyte $\mathrm{pH}$ level. Each sample was used only once to avoid errors associated with their corrosion. Because of that, the results presented in Fig. 7.3.1 (a,d) correspond to two different GaN pieces. In Fig. 7.3.1 (c) the photocurrent was measured with a chopped light, to ensure that it is indeed photo-induced current. Because the maximum photocurrent value which can be generated by the sample depends on its bandgap, the photocurrent values were recalculated to external quantum efficiency (EQE) using data from Fig. 3.6.2 (b) (refer to section 3.6). All samples had similar maximum EQE values above $20 \%$ (except for $\mathrm{GaN}$ in acidic electrolyte with a much higher efficiency), which suggests that the samples have comparable quality. 
$\mathrm{In}_{\mathrm{x}} \mathrm{Ga}_{1-\mathrm{x}} \mathrm{N}$ in alkaline electrolyte

(1M KOH)

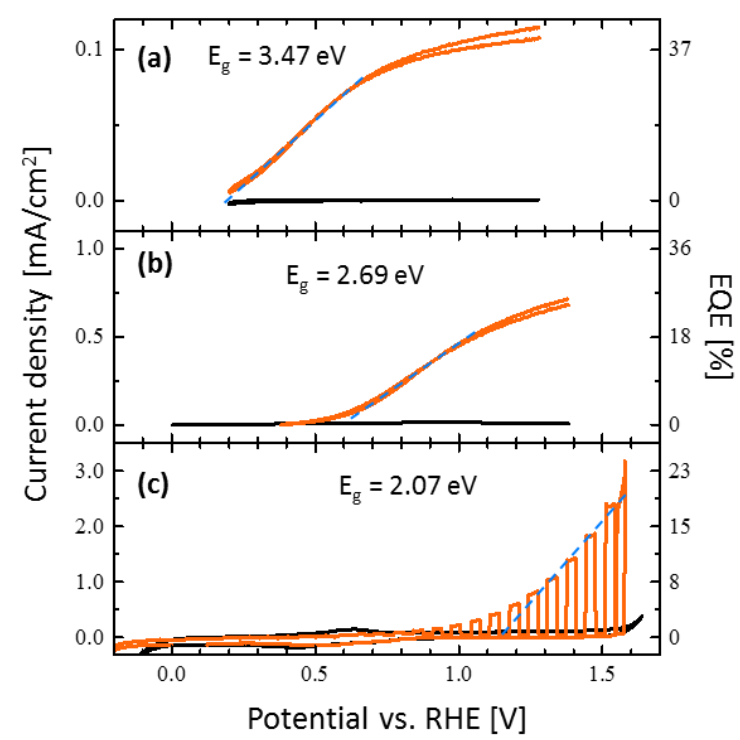

$\ln _{x} \mathrm{Ga}_{1-\mathrm{x}} \mathrm{N}$ in acidic electrolyte

$\left(1 \mathrm{M} \mathrm{HClO}_{4}\right)$

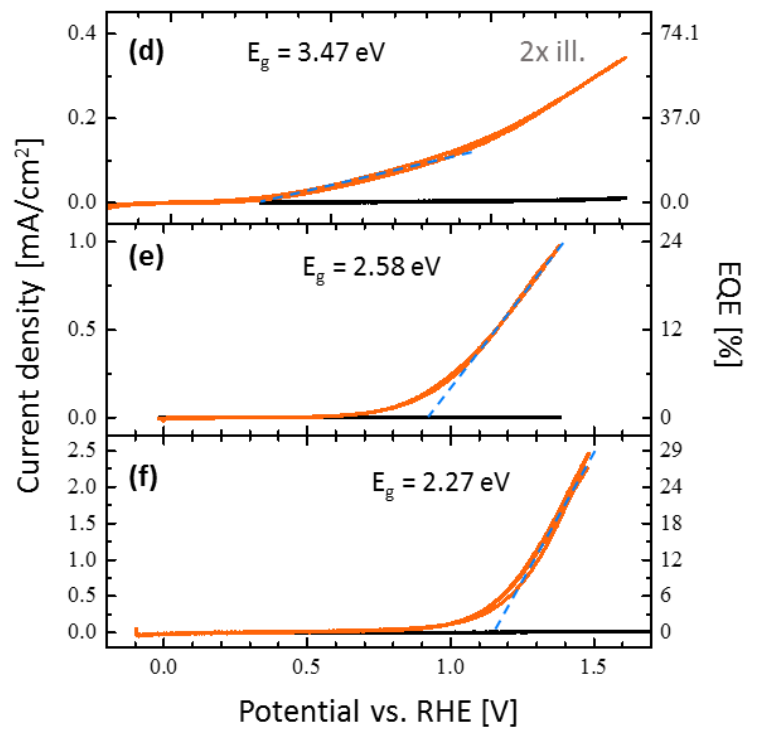

Fig. 7.3.1. Cyclic voltammetry study in the dark (black solid lines) and under 1 sun AM1.5G light illumination (orange solid lines) of series of InGaN layers with varying bandgaps in alkaline (a-c) and acidic (d-f) electrolytes. In (c), chopped light illumination was used to demonstrate that measured current is indeed photo-induced. In (d). a 2xsun illumination was used. Dashed lines in each graph demonstrate how the photocurrent onset potential was estimated.

The photocurrent rise for all graphs in Fig. 7.3.1 is very slow and no saturation regime was reached within the used range of potentials (except for the GaN layer in alkaline electrolyte, Fig. 7.3.1 (a)). This suggests a strong Fermi level pinning effect (either light-induced or dark) at the InGaN/electrolyte interface (see section 2.6.3). In case of "dark" Fermi level pinning effect, the surface states involved most likely have the same origin as the ones which induce the electron accumulation at the InGaN surface [Veal2006].

The photocurrent onset potential (red dots) dependence on the InGaN bandgap for all examined samples, measured both in alkaline and acidic electrolytes, (converted to RHE) are plotted in Fig. 7.3.2, together with the CB and VB edge offsets [Moses2010]. A similar trend was reported for the photocurrent onset potential of $\ln _{x} G_{1-x} N / G a N /$ sapphire samples grown by MOCVD and measured in a $1 \mathrm{M} \mathrm{HBr}$ electrolyte (using a $500 \mathrm{~W}$ Xe lamp as illumination source) [Luo2008].

A linearity of the photocurrent onset potential anodic shift with the InGaN bandgap (Fig. 7.3.2) could be due to the "dark" Fermi level pinning at the InGaN/electrolyte surface, as it also has a linear dependence on InGaN bandgap [Veal2006]. 


\section{$\mathrm{In}_{\mathrm{x}} \mathrm{Ga}_{1-\mathrm{x}} \mathrm{N}$ photocurrent onset potential vs bandgap}

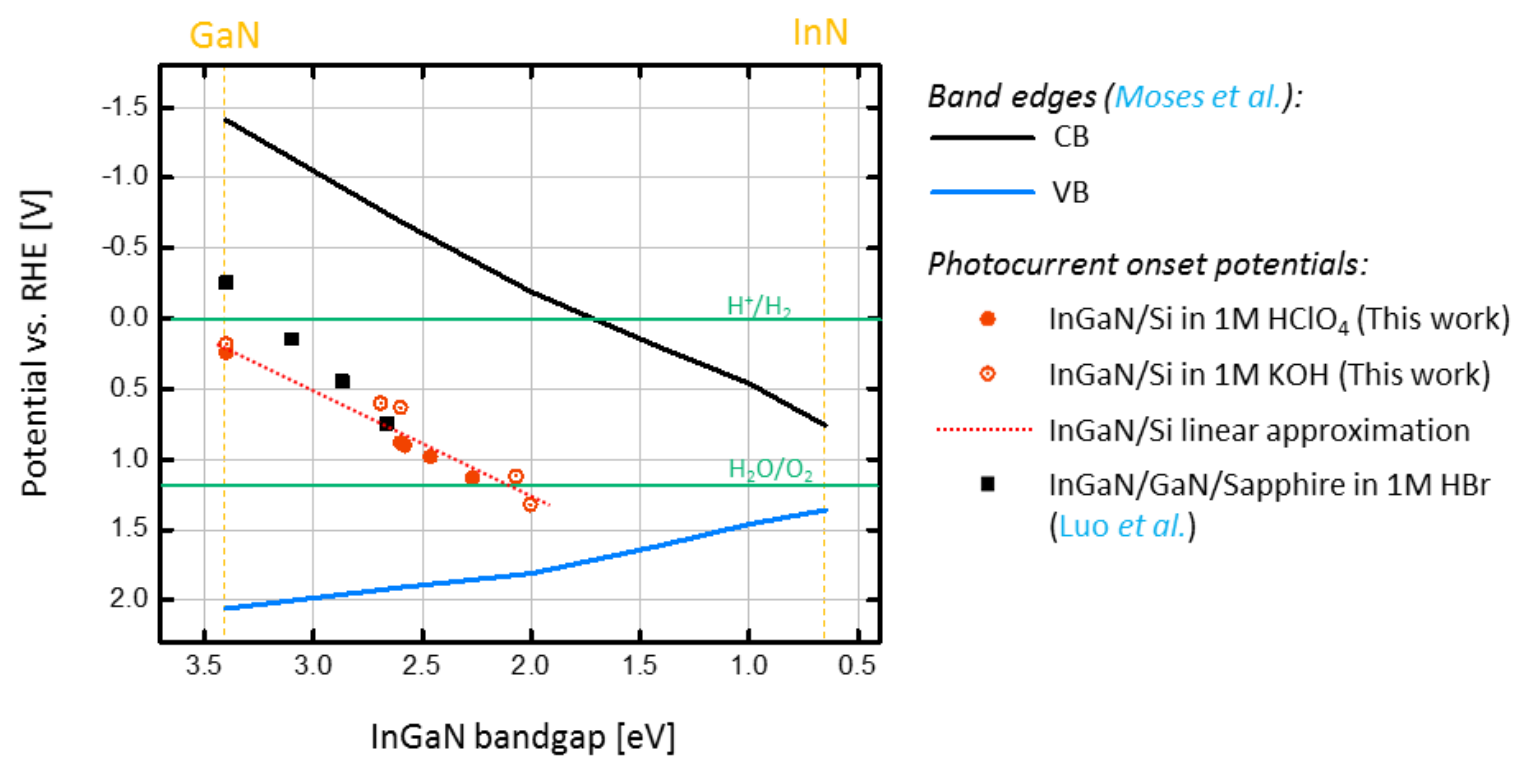

Fig. 7.3.2. The photocurrent onset potential dependence on InGaN bandgap values. A linear approximation (red dotted line) takes into account both sets of $\mathrm{HClO}_{4}$ and $\mathrm{KOH}$ electrolytes. Data for InGaN samples grown on GaN/sapphire templates measured in $1 \mathrm{M} \mathrm{HBr}$ electrolyte is extracted from [Luo2008]. HER and OER redox potentials are marked with green dashed lines. The values of CB and VB offsets are taken from [Moses2010] for fully relaxed InGaN layers. The bandgap of the binary compounds GaN and InN are marked with yellow dotted lines.

\subsubsection{Stability}

The stability of bare InGaN layers was tested by performing a series of chronoamperometry (CA) measurements at fixed potential under illumination simulated at 1 sun AM1.5G. Additionally CV curves (dark/ill.) were recorded before and after CA measurements in order to reveal performance degradation.

Stability test curves for $\ln _{0.3} \mathrm{Ga}_{0.7} \mathrm{~N}\left(E_{g}=2.0 \mathrm{eV}\right)$ layers in $1 \mathrm{M} \mathrm{KOH}$ electrolyte are shown in Fig. 7.3.3 (a,b). A rapid decrease of the photocurrent at $1.48 \mathrm{~V}$ vs. RHE from $0.125 \mathrm{~mA} / \mathrm{cm}^{2}$ to $0.01 \mathrm{~mA} / \mathrm{cm}^{2}$ over $22 \mathrm{~min}$ (Fig. 7.3.3 (b)) indicates a severe photoanode degradation. A CV curve taken after the CA measurement confirms this result, as it shows much smaller photocurrent over the whole potential range used (Fig. 7.3.3 (a)).

Similar results were obtained for $\ln _{0.17} \mathrm{Ga}_{0.83} \mathrm{~N}\left(E_{g}=2.6 \mathrm{eV}\right)$ layers in $1 \mathrm{M} \mathrm{HClO}_{4}$ electrolyte (Fig. 7.3.3 (c,d)). A relatively high photocurrent value of $0.8 \mathrm{~mA} / \mathrm{cm}^{2}$ diminished to $0.02 \mathrm{~mA} / \mathrm{cm}^{2}$ over 11 min (Fig. 7.3.3 (d)), which indicates an even faster degradation rate than in the previous case. Short light interruptions during CA experiment resulting in current drops to almost zero confirmed that the output current originates solely from the photo-generated carriers. Current spikes at the moment when the light is interrupted relate to capacitance-induced current caused by 


\section{Stability evaluation in acid and base}

$\ln _{0.3} \mathrm{Ga}_{0.7} \mathrm{~N}\left(\mathrm{E}_{\mathrm{g}}=2.0\right)$ film in $1 \mathrm{M} \mathrm{KOH}$

(a)

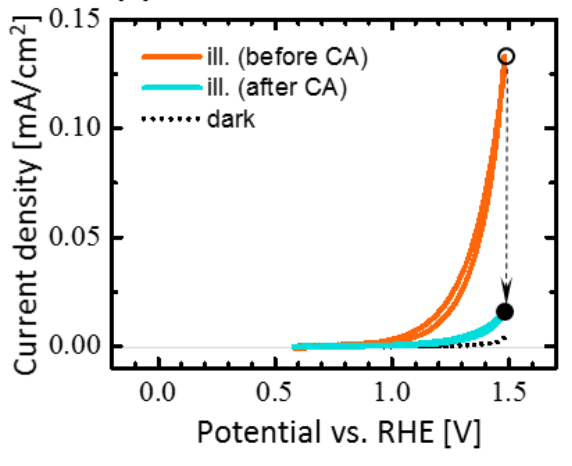

(b)

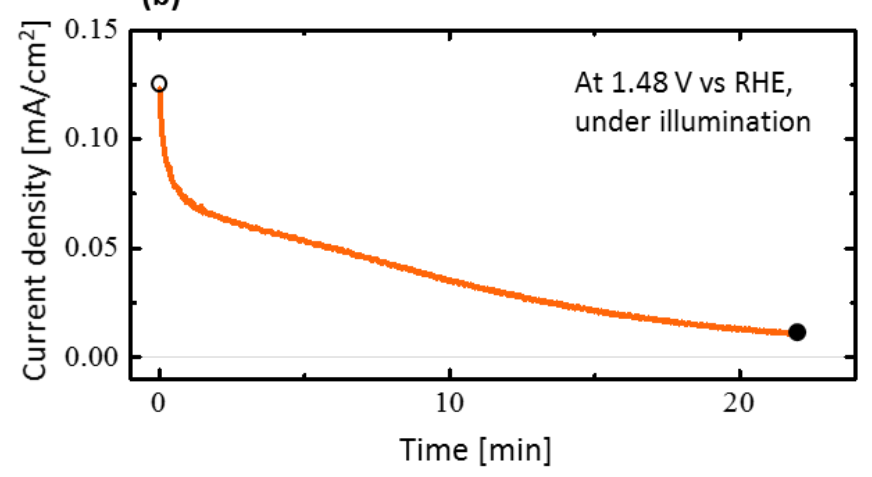

$\mathrm{In}_{0.17} \mathrm{Ga}_{0.83} \mathrm{~N}\left(\mathrm{E}_{\mathrm{g}}=2.6\right)$ film in $1 \mathrm{M} \mathrm{HClO}_{4}$

(c)

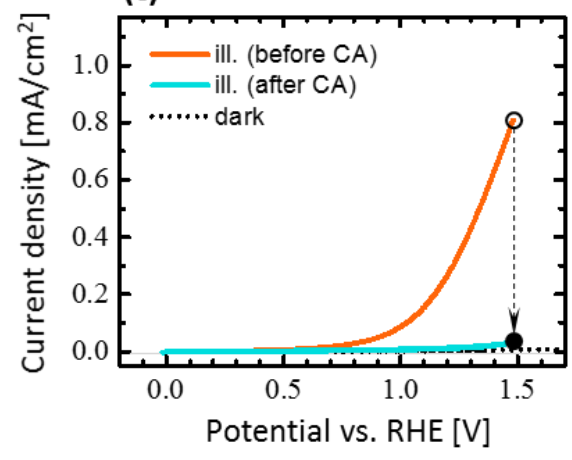

(d)

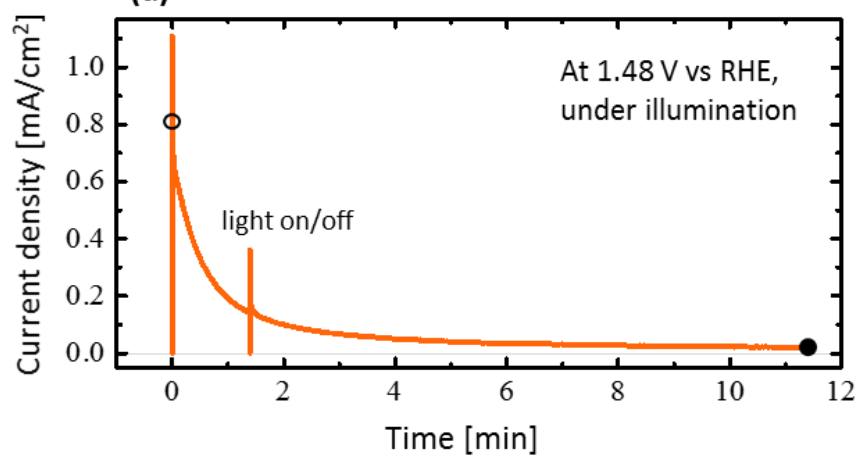

Fig. 7.3.3. Stability of $\operatorname{In}_{0.3} \mathrm{Ga}_{0.7} \mathrm{~N}\left(\mathrm{E}_{\mathrm{g}}=2.0 \mathrm{eV}\right.$ ) layers in $1 \mathrm{M} \mathrm{KOH}$ electrolyte and $\operatorname{In}_{0.17} \mathrm{Ga}_{0.83} \mathrm{~N}\left(\mathrm{E}_{\mathrm{g}}=2.6 \mathrm{eV}\right)$ layers in $1 \mathrm{M} \mathrm{HClO}_{4}$ electrolyte evaluated by $\mathrm{CV}$ (a,c, respectively) and CA (b, d, respectively). In both experiments 1 sun AM1.5G simulated light was used.

slow transfer kinetics at the S/E interface (refer to section 2.6.3.3). CV measurements have confirmed a performance degradation over the whole potential range used (Fig. 7.3.3 (c)).

For both samples examined the most probable cause of photocurrent decrease is the photo-induced oxidation of the InGaN surface (anodic photocorrosion) that passivates it and inhibits further reactions. This process also competes with the dissolution of the oxide layer in the electrolyte, resulting in material loss. Photocurrent drop is slowed down with time because there is less material left to absorb light and thus a lower amount of carriers is generated to continue the corrosion process. SEM analyses confirmed severe corrosion of the InGaN layers after prolonged illumination during CA experiments.

However, InGaN layers still possess an excellent chemical stability in the dark, as no surface morphology changes were revealed by SEM and AFM measurements in samples after 1 hour in various electrolytes (concentrated $\mathrm{HF}, \mathrm{HCl}, 1 \mathrm{M} \mathrm{NaOH}, 1 \mathrm{M} \mathrm{KOH}$ ) under open circuit conditions at RT. These results are in agreement with the reports on stability of GaN in acids and bases at RT 
[Zhuang\&Edgar2005] and on electrochemical etching of GaN layers in the dark where the etching starts only at very high potentials ( $7 \mathrm{~V}$ versus Pt electrode, in $0.5 \mathrm{M} \mathrm{H}_{2} \mathrm{SO}_{4}$ [Tseng2014]).

\subsubsection{Discussion}

PEC experiments revealed a modest catalytic activity of as-grown InGaN layers, exhibiting high photocurrent onset potential and weak (resistive) slope of the current-voltage characteristics. Both effects were attributed to the presence of a high density of surface states at the InGaN/electrolyte interface and a consequent strong Fermi level pinning [Veal2006]. Despite this fact, InGaN/Si samples used in this work show comparable performance in terms of EQE ( 30\% in [Luo2008]) and photocurrent onset potential behavior to those from the better studied InGaN/GaN/sapphire samples reported in the literature [Luo2008, Juodkazyte2014, Finken2015].

Stability of InGaN/Si samples measured by CV and CA revealed that while they are stable in the dark, under illumination they are corroded quite rapidly in both alkaline and acidic electrolytes, which was also confirmed by SEM observations. These findings correlate well with the previous reports on photocorrosion of InGaN/GaN/sapphire samples in various electrolytes covering the whole $\mathrm{pH}$ range [Finken2015, Juodkazyte2014, Huygens2000, Minsky1996]. Thus, the issue of the chemical stability of InGaN alloys in relation to PEC devices is still open. Ways to improve both performance and corrosion resistance are going to be proposed later in this chapter.

\subsection{Sacrificial agents effect}

In this section the effects on corrosion rate when a sacrificial agent is added to the electrolyte are discussed. Organic sacrificial agents were used, such as formic acid for an acidic electrolyte and methanol for an alkaline one (refer to section 2.6.4.1).

\subsubsection{Formic acid}

Stability tests for $\ln _{0.17} \mathrm{Ga}_{0.83} \mathrm{~N}\left(E_{g}=2.6 \mathrm{eV}\right)$ layers in $1 \mathrm{M} \mathrm{HClO}_{4}$ electrolyte with addition of formic acid $(10 \mathrm{mM})$ as sacrificial agent are shown in Fig. 7.4.1. The initial photocurrent small differences among samples, with (orange solid line in Fig. 7.4.1 (a)) and without (grey solid line in Fig. 7.4.1 (a)) sacrificial agent, are attributed to sample to sample difference, since these two curves were recorded from different pieces of the same sample. CA measurements at $1.38 \mathrm{~V}$ vs. RHE under simulated 1 sun AM1.5G illumination revealed no current output degradation for at least 44 min (Fig. 7.4.1 (b)). Short light interruptions during the CA experiments, resulting in a current drop to almost zero, confirmed that photocurrent originates from photo-generated carriers. Concomitant with CA results, the CV curve measured after stability tests reaches a higher value than before. This is a remarkable different behavior from that of a similar sample tested in $1 \mathrm{M} \mathrm{HClO}_{4}$ electrolyte without any sacrificial agent (refer to Fig. 7.3.3 (c,d)), where a severe performance degradation was evident.

The initial flat morphology of the sample shown in Fig. 7.4.2 (a) allowed a careful SEM study of corrosion after 44 of CA experiments. The sample tested in an electrolyte with sacrificial agent (Fig. 7.4.2 (b)) shows a surface with a high density of pits together with flat regions, while the one tested 
in an electrolyte without sacrificial agent (Fig. 7.4.2 (c)) reveals a severe corrosion and no flat regions.

\section{Stability test in $1 \mathrm{M} \mathrm{HClO}_{4}+$ sacrificial agent (Formic acid)}

(a)

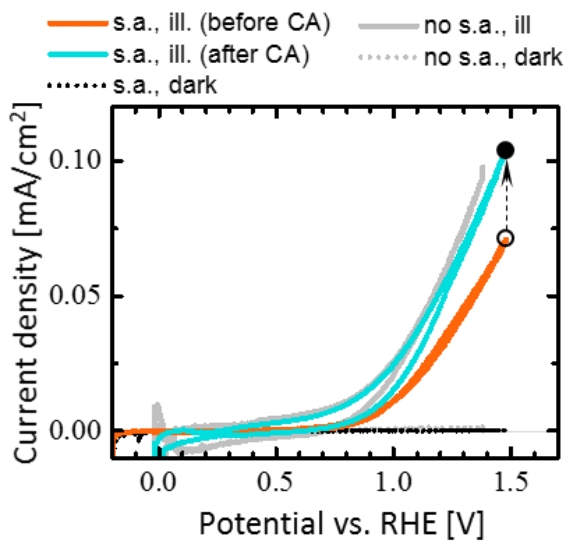

(b)

At $1.38 \mathrm{~V}$ vs. RHE, under illumination

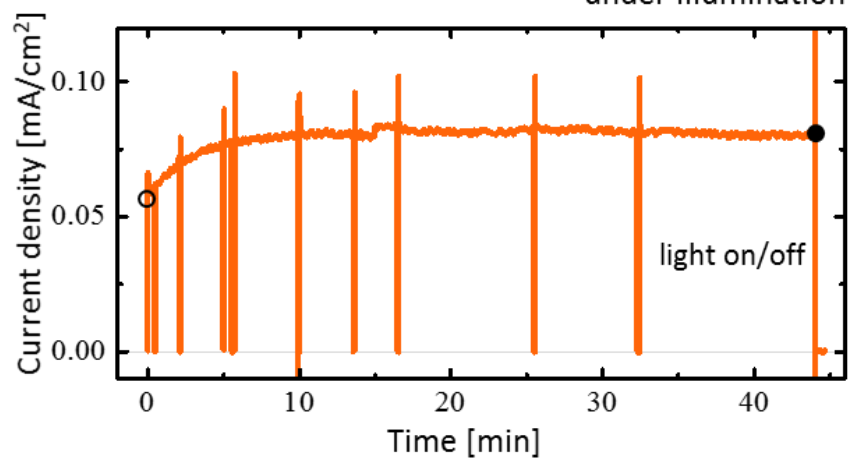

Fig. 7.4.1. Stability of an $\mathrm{In}_{0.17} \mathrm{Ga}_{0.83} \mathrm{~N}\left(E_{g}=2.6 \mathrm{eV}\right)$ layer in a $1 \mathrm{M} \mathrm{HClO}_{4}$ electrolyte with formic acid $(10 \mathrm{mM})$ as sacrificial agent measured by CV (a) and CA (b). Grey CV curves of the same sample in electrolyte without sacrificial agent are shown for reference. The photocurrent increase is indicated by open (before CA) and darkened (after CA) circles.

\section{Morphology change after stability tests}

as grown

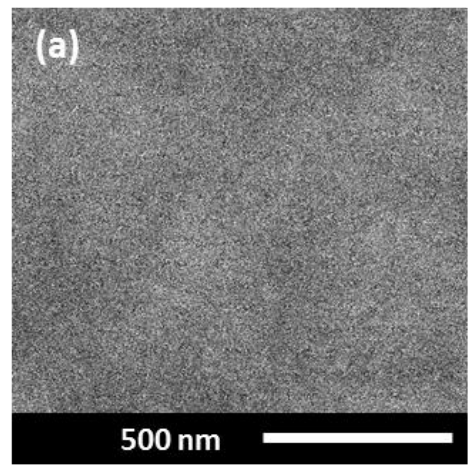

$1 \mathrm{M} \mathrm{HClO}_{4}+$ sacrificial agent(Formic acid)

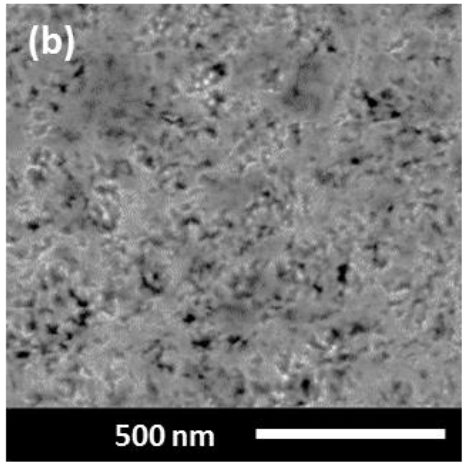

$1 \mathrm{M} \mathrm{HClO}_{4}$

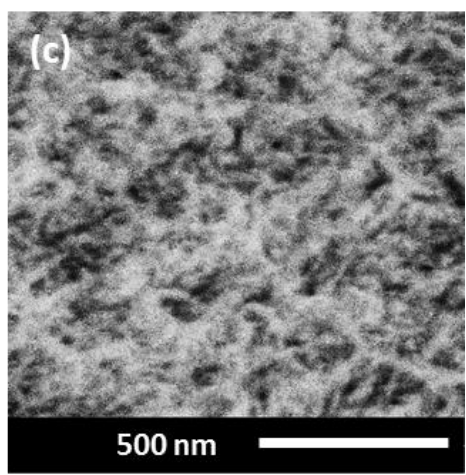

Fig. 7.4.2. Surface degradation of In $\ln _{0.18} \mathrm{Ga} a .82 \mathrm{~N}$ sample before $\mathrm{CA}(\mathrm{a})$, and after 44 min of $\mathrm{CA}$ under 1 sun 1.5AMG illumination at potential of $1.38 \mathrm{~V}$ vs. $\mathrm{RHE}$ in $1 \mathrm{M} \mathrm{HClO}_{4}$ electrolyte with sacrificial agent $(10 \mathrm{mM}$ formic acid) (b) and without (c). 


\subsubsection{Methanol}

Additionally, stability tests for $\operatorname{In}_{0.3} \mathrm{Ga}_{0.7} \mathrm{~N}\left(\mathrm{E}_{\mathrm{g}}=2.0 \mathrm{eV}\right.$ ) layers in $1 \mathrm{M} \mathrm{KOH}$ electrolyte were performed with methanol (5:1 mix) as a sacrificial agent. To increase the rate of methanol oxidation, the InGaN surface was decorated with Pt particles, as described in Appendix B. A significant boost in photocurrent at high potentials and a decrease of the onset potential is evident from the comparison of the CV curves for electrolytes with (orange solid line) and without (grey solid line) sacrificial agent (Fig. 7.4.3 (a)).

A peak at a potential of $\sim 0.7 \mathrm{~V}$ vs. RHE present both in the dark and under illumination for an electrolyte with sacrificial agent is related to the adsorption of methanol molecules on Pt particles and their consequent electrochemical oxidation [Mohapatra2008, Ji2015]. This was confirmed by measuring a CV curve of a Pt mesh as a working electrode in the same electrolyte, where a very similar peak appeared.

CA measurements at $1.38 \mathrm{~V}$ vs. RHE under simulated 1 sun AM1.5G illumination revealed stable photocurrent output (after an initial drop) for at least $20 \mathrm{~min}$ (Fig. 7.4.3 (b)). Short light interruptions during the CA experiment, resulting in current drop to almost zero, confirmed that photocurrent originates from photo-generated carriers. Consistently with CA results, a CV curve measured after the stability test showed only a small performance change (related to the CA initial current drop). This is a remarkably different behavior from the case of the same electrolyte without a sacrificial agent (refer to Fig. 7.3.3 (a,b)), where severe performance degradation was evident.

Unfortunately, the initial rough surface morphology of this sample did not allow SEM studies of the corrosion process.

\section{Stability test in $1 \mathrm{M} \mathrm{KOH}+$ sacrificial agent (Methanol)}

(a)

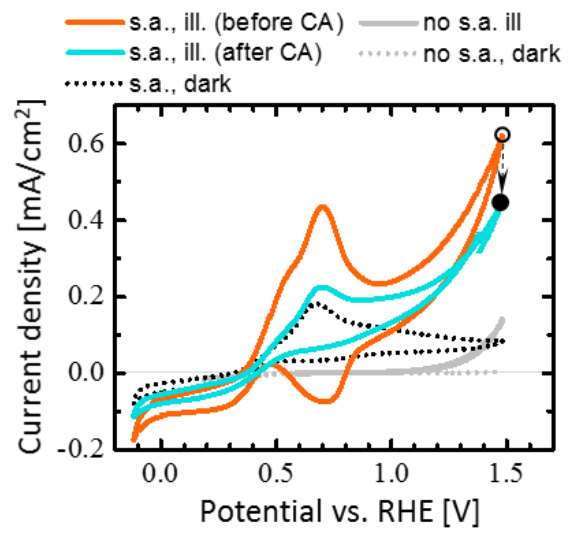

(b)

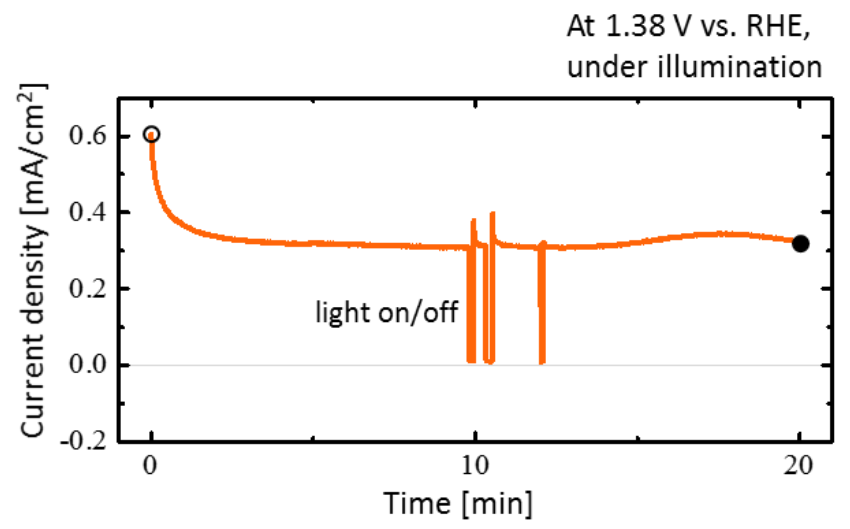

Fig. 7.4.3. Stability of an $\operatorname{In}_{0.3} \mathrm{Ga}_{0.7} \mathrm{~N}\left(\mathrm{E}_{\mathrm{g}}=2.0 \mathrm{eV}\right)$ layer in $1 \mathrm{M} \mathrm{KOH}$ electrolyte with methanol $(5: 1 \mathrm{mix})$ as sacrificial agent measured by CV (a) and CA (b). Grey CV curves of the same sample in electrolyte without sacrificial agent are shown for reference. The photocurrent change is indicated by open (before CA) and darkened (after CA) circles. 


\subsubsection{Discussion}

It can be concluded from the results presented above that the performance degradation of InGaN layers in electrolytes without sacrificial agents (presented in Fig. 7.3.3) is mainly attributed to severe photocorrosion. The addition of sacrificial agents to both acidic and alkaline electrolytes significantly reduces the corrosion rate (Fig. 7.4.1 and Fig. 7.4.3), though not fully inhibiting it (Fig. 7.4.2 (b)). The cause of such difference is attributed to an increased rate of hole transfer at the S/E interface by sacrificial agents, which results in a slower corrosion rate and reduced surface recombination [Mohapatra2008].

The photocurrent onset potential behavior was found different for the two studied cases. This could be related to the change in $\mathrm{pH}$ level of electrolytes due to the presence of sacrificial agents or to the difference in reaction between InGaN with formic acid and methanol. However, the origin of this dissimilarity is unclear and must be further studied.

While the increased performance of InGaN using methanol makes it a promising candidate for PEC hydrogen production from biomass derivatives (naturally containing methanol) and water [Lu2014, Seger2012], for water splitting applications other means of increasing stability must be found. In the following section the use of $\mathrm{NiO}$ protection layers to improve the InGaN photoanodes stability is discussed.

\subsection{NiO catalyst/protection films on InGaN layers}

In order to improve the corrosion resistance and performance of the InGaN photoanodes, decoration with a $\mathrm{NiO}$ film catalyst was performed. Before that, the $\mathrm{NiO} / \mathrm{InGaN}$ bands alignment was analyzed and optical transparency of $\mathrm{NiO}$ protection layer was checked to ensure that it is a suitable catalyst for the InGaN layers. Experimental results and discussion are presented in the following sections.

\subsubsection{Analysis of a NiO/InGaN band diagram}

As it was outlined in section 2.6.4.2, for a metal oxide catalyst to work properly there should be no potential barrier between the VBs at the semiconductor/metal oxide interface in case of a photoanode ( $C B s$ for a photocathode). Thus, the band diagram of $\ln _{x} \mathrm{Ga}_{1-x} \mathrm{~N} / \mathrm{NiO}$ must be constructed. Table XII presents the CB and VB potentials vs. vacuum level and RHE taken from [Moses2010] for $\operatorname{In}_{\mathrm{x}} \mathrm{Ga}_{1-\mathrm{x}} \mathrm{N}$ layers and from [Yip2012] for crystalline $\mathrm{NiO}$ films. The band alignments of $\mathrm{GaN}, \ln _{0.4} \mathrm{Ga}_{0.6} \mathrm{~N}$, and $\mathrm{InN}$ to $\mathrm{NiO}$ are shown in Fig. 7.5.1. It is clear that there is no potential barrier for hole transfer, and even more, the $\mathrm{NiO}$ can act as an electron blocking layer due to the presence of a high CB potential barrier.

However, for $\ln _{x} G_{1-x} N$ with compositions close to $\operatorname{In} N$ the $C B$ is only a few hundreds of meV above the $\mathrm{VB}$ of NiO resulting in a high probability of tunneling through the junction, thus making it transparent for majority carriers. Nevertheless, NiO fulfills the band requirements to be a suitable catalyst for $\ln _{x} G_{1-x} N$ only in the region of interest of this work $(x=0-0.5)$. Additionally, the fact that as-grown $\mathrm{NiO}$ is usually highly p-type unintentionally doped allows forming a pn-junction with $\mathrm{In}_{\mathrm{x}} \mathrm{Ga}_{1-\mathrm{x}} \mathrm{N}$ layers that additionally enhances the carrier separation efficiency. 
Table XII. CB and VB energy levels for $\mathrm{NiO}$ and $\operatorname{In}_{\mathrm{x}} \mathrm{Ga}_{1-\mathrm{x}} \mathrm{N}$.

\begin{tabular}{|cccc|}
\hline Material & Band & E vs vacuum & E vs RHE \\
\hline NiO & $C B$ & -1.8 & -2.64 \\
$E_{g}=3.60 \mathrm{eV}$ & $V B$ & -5.4 & 0.96 \\
\cline { 2 - 4 } & $\mathrm{V}$ & -3.03 & -1.41 \\
\hline $\mathbf{G a N}$ & $C B$ & -6.5 & 2.06 \\
$E_{g}=3.47 \mathrm{eV}$ & $V B$ & -4.34 & -0.1 \\
\hline $\ln _{0.4} \mathbf{G a}_{0.6} \mathbf{N}$ & $C B$ & -6.14 & 1.7 \\
$E_{g}=1.80 \mathrm{eV}$ & $V B$ & -5.2 & 0.76 \\
\hline $\ln \mathbf{N}$ & $C B$ & -5.9 & 1.46 \\
$E_{g}=0.7 \mathrm{eV}$ & $V B$ & & \\
\cline { 2 - 4 } & & &
\end{tabular}

$\mathrm{GaN} / \mathrm{NiO}$

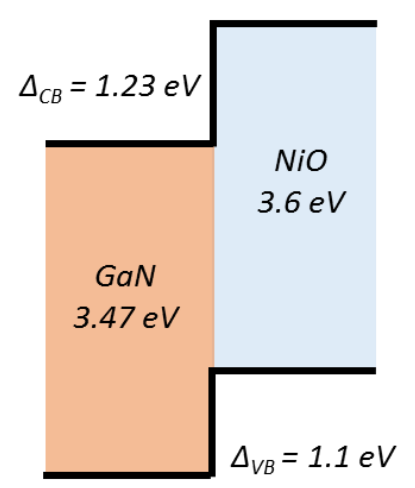

$\mathrm{In}_{0.4} \mathrm{Ga}_{0.6} \mathrm{~N} / \mathrm{NiO}$

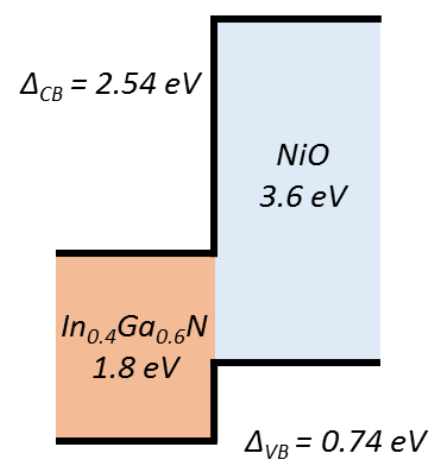

$\ln \mathrm{N} / \mathrm{NiO}$

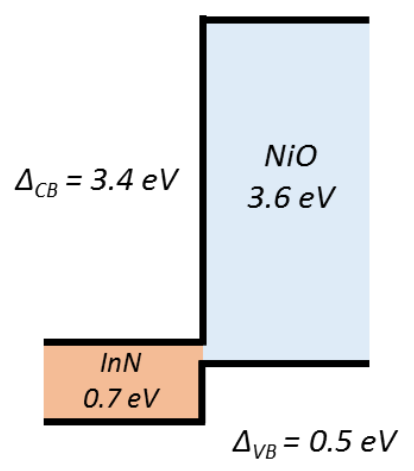

Fig. 7.5.1. Band alignment of $\mathrm{GaN}, \ln _{0.4} \mathrm{Ga}_{0.6} \mathrm{~N}$, and $\operatorname{InN}$ vs. $\mathrm{NiO}$ catalyst.

\subsubsection{Optical transparency of NiO films}

Metal oxide catalyst layers should be also optically transparent (refer to section 2.6.4.2). The NiO bandgap of $3.6 \mathrm{eV}$ suggests that the absorption edge is around $344 \mathrm{~nm}$. NiO films 15 and $100 \mathrm{~nm}$ thick were deposited by PVD magnetron sputtering on a Pyrex glass and measured by UV-Vis spectroscopy (Fig. 7.5.2), confirming that $\mathrm{NiO}$ is highly transparent in the region of interest of this work, namely in the 350-800 $\mathrm{nm}$ range. In order to minimize the absorption losses and avoid introducing additional resistivity to the S/E interface only thin (15 $\mathrm{nm}$ thick) NiO films were used in the experiments described further in this section. 


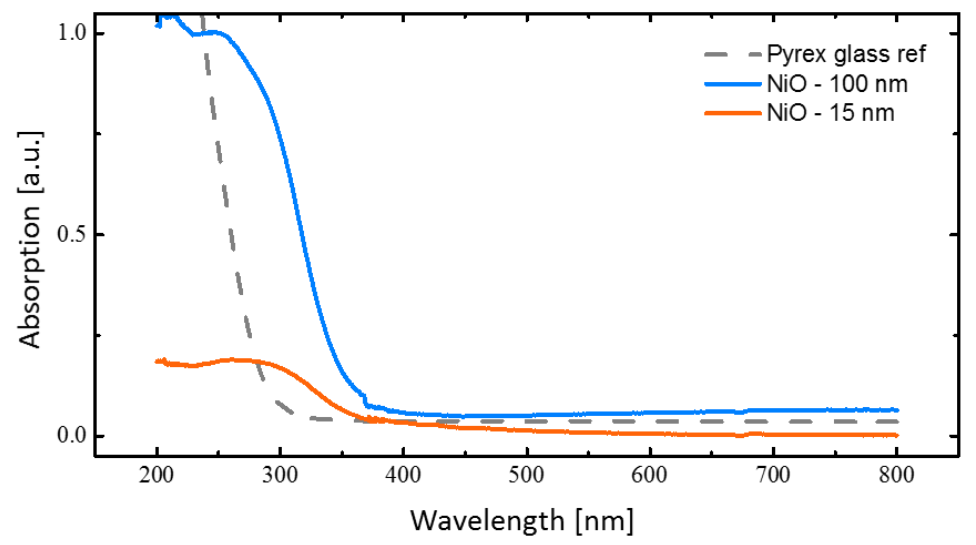

Fig. 7.5.2. Absorption spectra of 15 and $100 \mathrm{~nm}$ thick NiO films deposited on $2 \mathrm{~mm}$ Pyrex glass measured by UV-Vis spectroscopy.

\subsubsection{NiO film on In ${ }_{0.38} G_{0} .62 \mathrm{~N}$ layers $\left(E_{g}=1.8 \mathrm{eV}\right)$}

To test $\mathrm{NiO}$ catalytic and protective properties, a series of PEC measurements were performed on $\operatorname{In}_{0.38} \mathrm{Ga}_{0.62} \mathrm{~N}$ layers with $E_{g}=1.8 \mathrm{eV}$ (theoretical optimum value for a tandem solar cell) covered with a continuous $15 \mathrm{~nm}$ thick NiO film (with and without $3 \mathrm{~nm}$ Ni predeposition used to avoid the InGaN surface oxidation). CVs from this sample with Ni predeposition measured in $1 \mathrm{M} \mathrm{KOH}$ electrolyte in the dark and under light illumination (1 sun AM 1.5G solar) are shown in Fig. 7.5.3. Similar samples without Ni predeposition exhibited identical results, thus it is not related to InGaN oxidation during the $\mathrm{NiO}$ deposition. $\mathrm{NiO} / \mathrm{In}_{0.38} \mathrm{Ga}_{0.62} \mathrm{~N}$ photoanodes showed a negligible photoresponse, but instead a majority-carrier, "metallic" current-voltage characteristics (no photo-generated current, CV

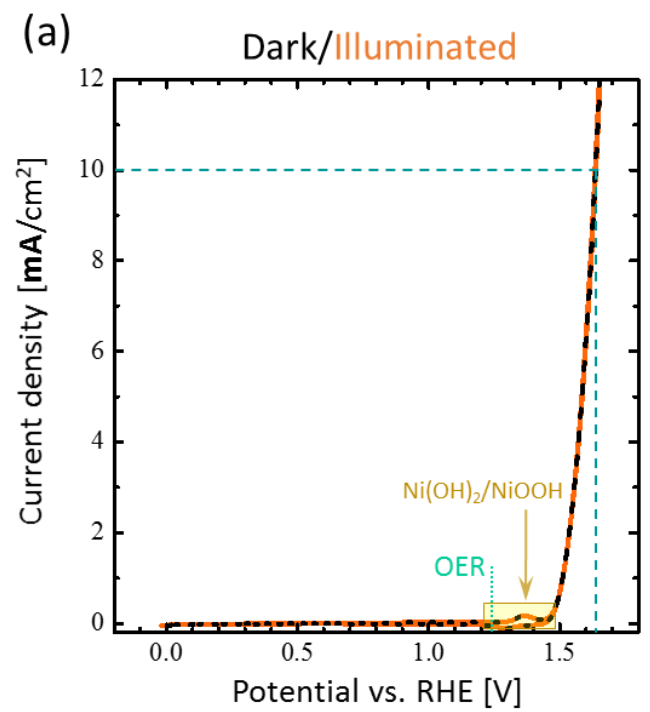

(b)

\begin{tabular}{|lc|}
\hline Material & $\begin{array}{l}\text { Overpotential } \\
\text { at J=10 } \mathrm{mA} / \mathrm{cm}^{2} \\
\text { for OER (1.23V vs. } \\
\text { RHE) }\end{array}$ \\
\hline $\mathrm{n}-\mathrm{InGaN} / \mathrm{NiO}$ & $400 \mathrm{mV}$ \\
\hline $\mathrm{n}-\mathrm{InP} / \mathrm{NiO}$ & $430 \mathrm{mV}^{(*)}$ \\
\hline $\mathrm{n}^{+}-\mathrm{InP} / \mathrm{NiO}$ & $390 \mathrm{mV}^{(*)}$ \\
\hline $\mathrm{p}^{+}-\mathrm{Si} / \mathrm{NiO}$ & $330 \mathrm{mV}^{(* *)}$ \\
\hline
\end{tabular}

Fig. 7.5.3. Cyclic voltammetry curve of an $\ln _{0.38} \mathrm{Ga}_{0.62} \mathrm{~N}$ layer covered with a $15 \mathrm{~nm}$-thick continuous film of $\mathrm{NiO}$ (a). The curve is the same in the dark (black line) and under 1 sun AM1.5G light illumination (orange dashed line). OER potential is marked with a dashed green line. The potential vs. OER (overpotential) yielding a current density of $10 \mathrm{~mA} / \mathrm{cm}^{2}$ is compared to literature values (b). (*[Sun2015], **[Sun2015b]) 
behavior is close to the case when $\mathrm{NiO}$ film is deposited on a metallic electrode). Typical for $\mathrm{NiO}$ films, CV peaks above OER potential related to oxidation reaction of $\mathrm{Ni}(\mathrm{OH})_{2} / \mathrm{NiOOH}$ are highlighted in yellow.

The observed behavior of $\mathrm{NiO} / \mathrm{In}_{0.38} \mathrm{Ga}_{0.62} \mathrm{~N}$ electrode suggests a strong tunneling at the their junction, probably, related to high unintentional doping concentration of $\operatorname{In}$-rich $\ln _{0.38} G_{0.62} \mathrm{~N}$ film. This results in a reduced band bending in the $\ln _{0.38} \mathrm{Ga}_{0.62} \mathrm{~N}$ layer and consequently the absence of photo-induced current. Majority carriers determine the output current instead. A very similar

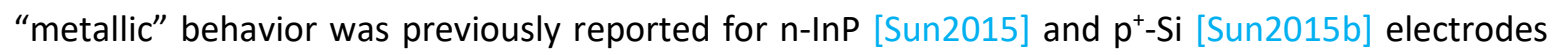
both covered with a NiO film. In fact, the measured overpotential value $(0.4 \mathrm{~V}$ vs. OER) is very close to that of the mentioned reported cases (Fig. 7.5.3 (b)).

Despite the absence of photocurrent, the NiO film still provides excellent protection properties as measured by CA at $1.68 \mathrm{~V}$ vs. RHE for 30 min under illumination (Fig. 7.5.4 (a)) and $24 \mathrm{~h}$ in the dark (Fig. 7.5.4 (b)). In both cases the current was stable with only small fluctuations around $10 \mathrm{~mA} / \mathrm{cm}^{2}$. Sharp current peaks are due to detachment of generated oxygen bubbles that was clearly observed by naked eye. SEM observations before (Fig. 7.5.4 (c)) and after (Fig. 7.5.4 (d)) the CA experiments confirm absence of any corrosion of the sample surface. Thus, NiO on In-rich InGaN layers is still an interesting combination if, for example, a buried pn-junciton is used to drive charge separation, while $\mathrm{NiO}$ solely acts as a protection layer.

(a)

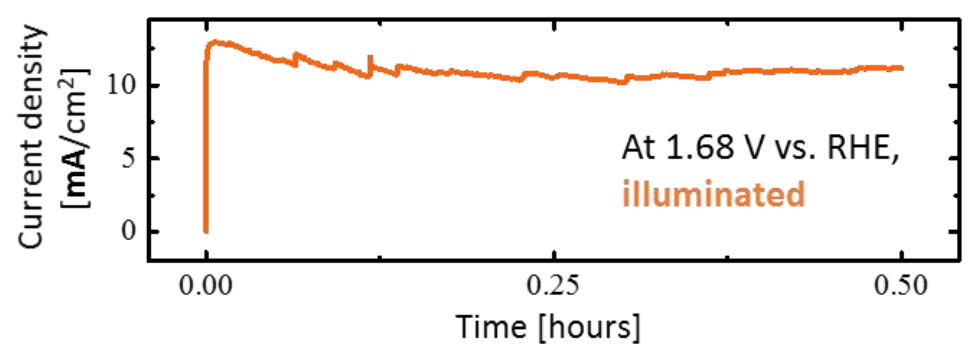

(b)

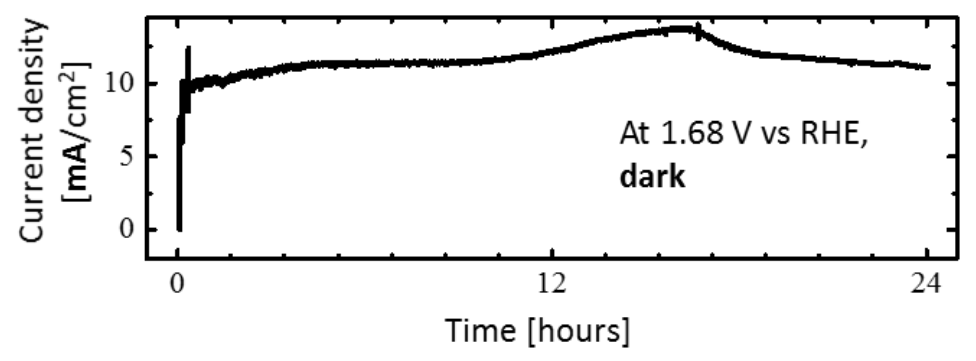

SEM

(c)

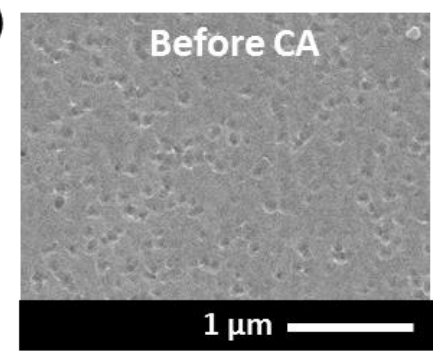

(d)

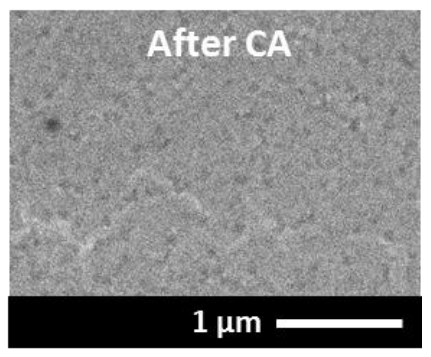

Fig. 7.5.4. Protection properties of the NiO film on In0.38Ga0.62 N layer measured by CA for 30 min under 1 sun AM1.5G illumination (a) and for $24 \mathrm{~h}$ in the dark (b). SEM images of the surface morphology before (c) and after (d) CA experiments. 


\subsubsection{NiO films on $\operatorname{In}_{0.18} \mathrm{Ga}_{0.82} \mathrm{~N}$ layers $\left(E_{g}=2.6 \mathrm{eV}\right)$}

Fig. 7.5.5 (a) shows CV curves measured on two pieces of the $\ln _{0.18} \mathrm{Ga}_{0.82} \mathrm{~N}$ layer sample with higher bandgap (2.6 eV), one as-grown (grey lines) and another one covered with a $15 \mathrm{~nm}$ thick NiO film with $3 \mathrm{~nm}$ Ni predeposition (orange, blue, and dark lines). While the photocurrent onset potential is the same for both samples, the photocurrent density increased by a factor of 2 for the NiOcovered sample.

It must be noted that the CV curve of the NiO-covered sample was recorded after 5 subsequent cycling over the same potential range during which the photocurrent values were slowly rising. This is most probably related to Iron ions incorporation into the $\mathrm{NiO}$ film from the $\mathrm{KOH}$ electrolyte and consequent improvement of its catalytic activity [Mei2014].

CA measurements from the NiO-covered sample, recorded at $1.08 \mathrm{~V} v \mathrm{vs}$. RHE under illumination, are presented in Fig. 7.5.5 (b). A large current overshoot ( 4 times) upon initiation of the CA scan (inset in Fig. 7.5.5 (b)) relates to capacitance-induced current caused by slow transfer kinetics at the S/E interface (refer to section 2.6.3.3). After this transient the photocurrent stabilizes at $0.11 \mathrm{~mA} / \mathrm{cm}^{2}$ for the rest of the measurements implying that the sample is stable for at least $20 \mathrm{~min}$.

These results suggest that there are no significant tunneling of the majority carriers at the $\mathrm{NiO} / \mathrm{In}_{0.18} \mathrm{Ga}_{0.82} \mathrm{~N}$ interface and, thus, photogenerated carriers can be effectively separated due to the band bending inside the InGaN layer. This is probably due to the reduced unintentional doping concentration in $\mathrm{In}_{0.18} \mathrm{Ga}_{0.82} \mathrm{~N}$ layer.

\section{Stability test of $\operatorname{In}_{0.18} \mathrm{Ga}_{0.82} \mathrm{~N}$ film $+\mathrm{NiO}$ catalyst}

(a)

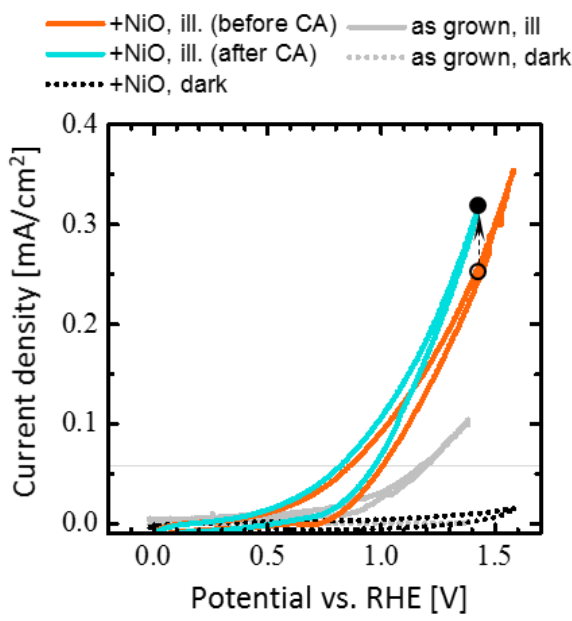

(b)

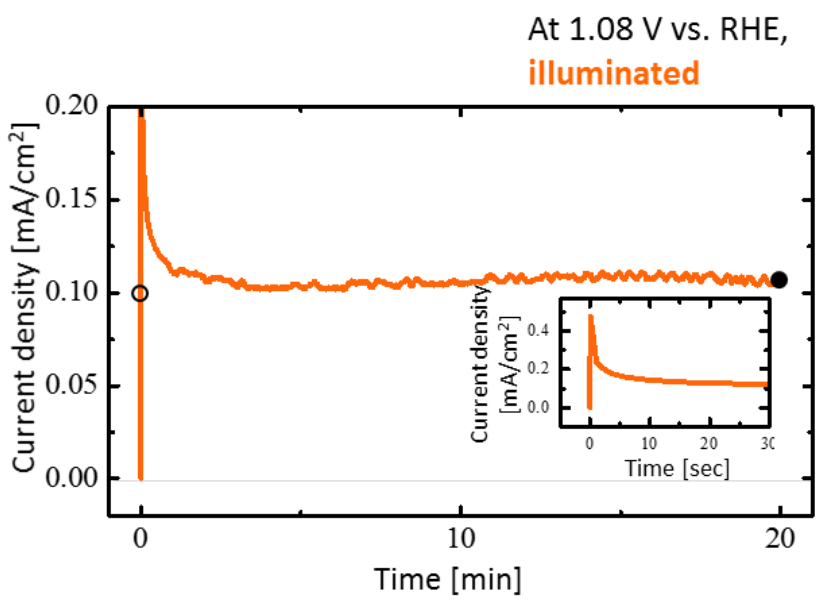

Fig. 7.5.5. Stability of a NiO-covered $\operatorname{In}_{0.18} \mathrm{Ga}_{0.82} \mathrm{~N}\left(E_{g}=2.6 \mathrm{eV}\right)$ layer in $1 \mathrm{M} \mathrm{KOH}$ electrolyte measured by $\mathrm{CV}$ (a) and CA (b). Grey CV curves represent the same sample without NiO. The photocurrent improvement in CV measurements is indicated by open (before CA) and darkened (after CA) circles. Inset depicts the initial 30 s of CA measurements. 
The sample morphology was studied by SEM before (Fig. 7.5.6 (a)) and after (Fig. 7.5.6 (b)) CA experiments, revealing that, despite the stable photocurrent output, the sample surface is modified suggesting that corrosion was not completely inhibited.
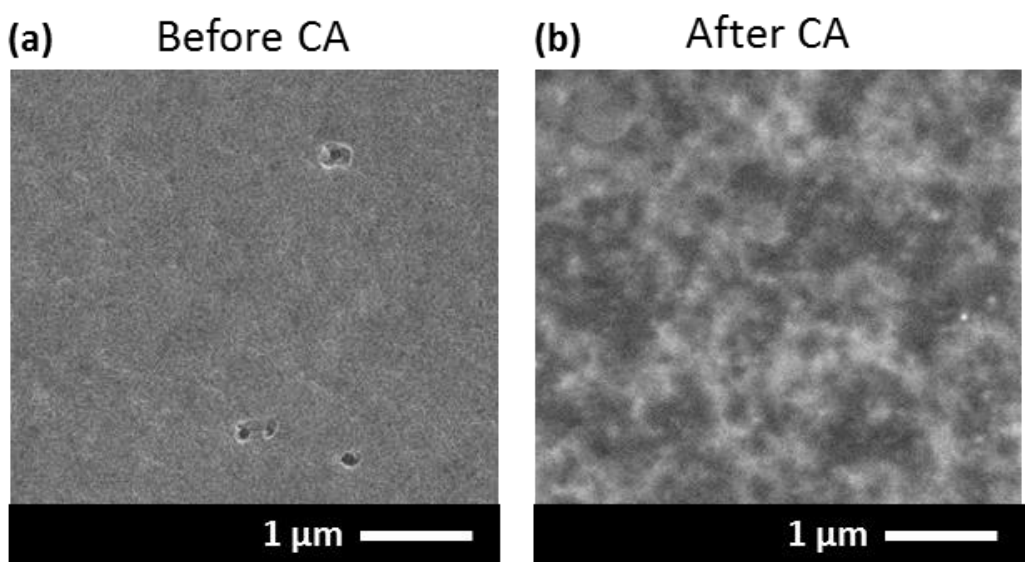

Fig. 7.5.6. SEM images of the surface morphology of NiO-covered $\operatorname{In}_{0.18} \mathrm{Ga}_{0.82} \mathrm{~N}$ layers before (a) and after 20 min of CA experiments at $1.08 \mathrm{~V}$ vs. RHE in $1 \mathrm{M} \mathrm{KOH}$ electrolyte under 1 sun $1.5 \mathrm{AMG}$ illumination.

\subsubsection{Discussion}

$\mathrm{In}_{\mathrm{x}} \mathrm{Ga}_{1-\mathrm{x}} \mathrm{N} / \mathrm{NiO}$ junctions were studied from both theoretical and experimental viewpoints. The theoretical analysis revealed that $\ln _{\mathrm{x}} \mathrm{Ga}_{1-\mathrm{x}} \mathrm{N}$ and $\mathrm{NiO}$ band edges form type II heterojunctions with a barrier for electrons over the whole composition range. However, for In-rich InGaN layers the CB edge is located only few hundreds of meV above the VB edge of NiO, which could promote the negative effect of majority carriers tunneling through the junction. Nevertheless, $\mathrm{NiO}$ band edges are properly positioned in respect to $\ln _{x} G_{1-x} N$ layers in the composition range of interest of this work $(x=0-0.5)$.

UV-Vis spectroscopy studies of NiO films determined their transparency to light for wavelengths above $350 \mathrm{~nm}$. This allows using $\mathrm{NiO}$ as a cover layer without blocking any significant fraction of light reaching the InGaN absorber placed beneath.

The catalytic and protection properties of magnetron sputtered $\mathrm{NiO}$ films deposited on $\ln _{\mathrm{x}} \mathrm{Ga}_{1-\mathrm{x}} \mathrm{N}$ layers with small $\left(E_{g}=1.8 \mathrm{eV}, \mathrm{x}=0.38\right)$ and large $\left(E_{g}=2.6 \mathrm{eV}, \mathrm{x}=0.18\right)$ bandgap values were studied by $\mathrm{CV}, \mathrm{CA}$ and $\mathrm{SEM}$. The $\mathrm{NiO} / \mathrm{In}_{0.38} \mathrm{Ga}_{0.62} \mathrm{~N}$ sample showed negligible photoresponse under simulated AM1.5G solar illumination but instead a majority carrier, "metallic" current-voltage behavior. Independently from illumination conditions current density was starting to rise at $1.48 \mathrm{~V}$ vs. RHE and reached $10 \mathrm{~mA} / \mathrm{cm}^{2}$ at $1.63 \mathrm{~V}$ vs. RHE (0.4 V vs. OER potential). This behavior is consistent with a high unintentional donor density in In-rich InGaN layers resulting in formation of tunnel junction with $\mathrm{NiO}$, and thus no significant photocurrent is obtained.

Prolonged CA measurements both in the dark and under illumination revealed an excellent chemical stability of the $\ln _{0.38} \mathrm{Ga}_{0.62} \mathrm{~N} / \mathrm{NiO}$ electrode. Despite the absence of photocurrent such structures still can be used together with a buried pn-juncton. In this case charge separation is determined by the pn-junction rather than S/E interface and, thus, tunneling at the interface between the 
semiconductor and metal oxide is no longer a problem. This was successfully demonstrated for pn-InP/NiO [Sun2015] and pn-Si/NiO [Mei2014] what suggests a high probability for success in the case of InGaN. However, p-type doping of In-rich InGaN layers is another very challenging task.

The $\mathrm{In}_{0.18} \mathrm{Ga}_{0.82} \mathrm{~N} / \mathrm{NiO}$ sample revealed a remarkably different behavior, namely, the photocurrent of the NiO-covered sample (CV measurements) increased by more than a factor of 2 as compared to the as-grown sample. The photocurrent onset potential, however, remained the same, most probably because of the "dark" Fermi level pinning effect (refer to section 2.6.3.3.3) and not due to slow transport kinetics at the interface. Therefore, the CV of such photoanode cannot be significantly improved by deposition of any catalysts, because catalysts affect primarily the transport kinetics. However, the NiO film still can reduce the recombination of holes by pushing them away from the InGaN to the $\mathrm{NiO} /$ electrolyte interface, where the electron concentration is expected to be lower.

In terms of stability, CA measurements from the $\ln _{0.18} \mathrm{Ga}_{0.82} \mathrm{~N} / \mathrm{NiO}$ sample under illumination revealed a stable photoresponse for at least $20 \mathrm{~min}$. This is a great improvement compared to similar samples without $\mathrm{NiO}$ protection under the same conditions, for which an exponential photocurrent decrease was evident during the CA experiments (refer to Fig. 7.3.3 (d)). However, SEM investigations still indicated NiO-covered sample morphology changes after the CA experiments, meaning that corrosion was reduced, though not fully suppressed.

Therefore, results suggest that $\mathrm{NiO}$ has potential to be used as a protection film for $\ln _{\mathrm{x}} \mathrm{Ga}_{1-\mathrm{x}} \mathrm{N}$ layers, either directly for $\ln _{x} G a_{1-x} N$ layers with $x<0.3$, or together with a buried pn-junction inside the InGaN for $x>0.3$. In terms of InGaN photoanodes performance, NiO films affect only the photocurrent slope steepness but not the onset potential. To decrease the photocurrent onset potential other techniques must be considered, such as the modification of the InGaN surface to reduce the dark Fermi pinning effect.

\subsection{InGaN layers decorated with InN QDs}

The effect of decorating InGaN with InN QDs on their performance as photoanodes was also tested. For that two $\ln _{0.3} \mathrm{Ga}_{0.7} \mathrm{~N}$ (Eg $=2.1 \mathrm{eV}$ ) layers were grown on the $\mathrm{p}^{+}-\mathrm{Si}(111)$ substrate under identical conditions, but with and without InN QDs at the surface (for InN QDs growth details refer to Chapter 5). CV and CA curves from these samples, taken in $1 \mathrm{M} \mathrm{KOH}$ electrolyte with added methanol as sacrificial agent (mix 50:1) to avoid rapid etching of InN QDs, are shown in Fig. 7.6.1 (a,b). Short light interruptions during the CA experiment, resulting in current drop to almost zero, confirmed that photocurrent originates from photogenerated carriers (Fig. 7.6.1 (b)).

Initially, both samples showed comparable performance (in CV), but after 20 min of CA (at $1.38 \mathrm{~V}$ vs. RHE, under illumination) the CV curve of the sample decorated with InN QDs improved remarkably (the photocurrent nearly tripled at $1.23 \mathrm{~V}$ vs. RHE), while the one without QDs deteriorated with time. Moreover, the photocurrent onset potential decreased drastically from $\sim 1$ $\mathrm{V}$ to $0.6 \mathrm{~V}$ vs. RHE Fig. 7.6.1 (a). Such delay in the improvement could be attributed to removing of oxide layer from InN QDs during the CA experiments and thus making them active. 


\section{InN QDs decoration effect}

(a)

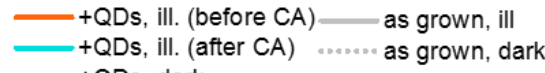
.......+QDs, dark

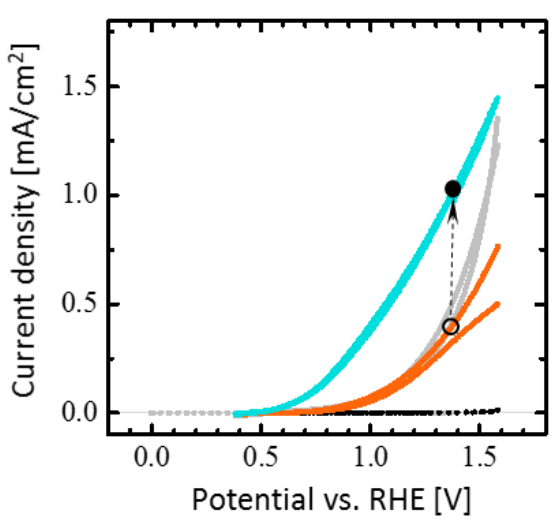

(b)

At $1.38 \mathrm{~V}$ vs. RHE, illuminated

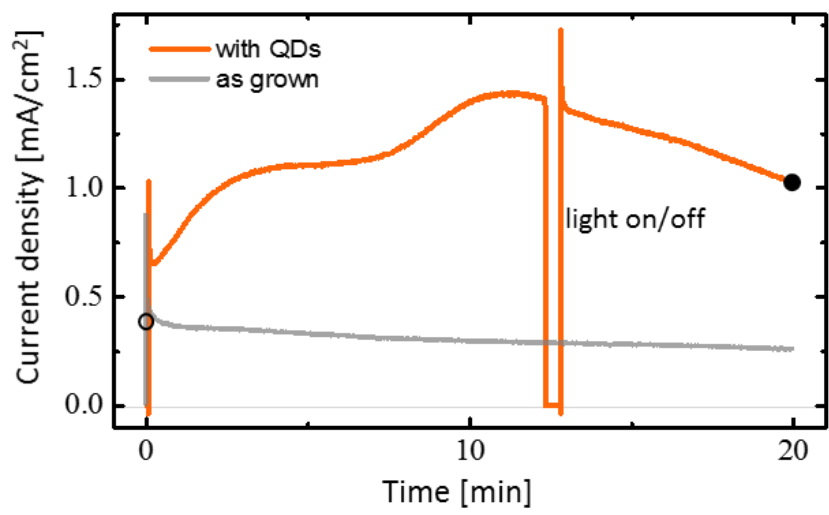

Fig. 7.6.1. Comparison of PEC performance ( $C V$ and $C A$ ) of an $\operatorname{In}_{0.3} \mathrm{Ga}_{0.7} \mathrm{~N}$ film with and without surface InN QDs done in $1 \mathrm{M} \mathrm{KOH}$ electrolyte with methanol as sacrificial agent (50:1 mix). The photocurrent increase is indicated by open (before CA) and darkened (after CA) circles.

While the exact mechanism responsible for the decrease in the photocurrent onset potential in the sample decorated with InN QDs is unknown, several reasonable guesses can be proposed:

(i) Increased band bending at the photoanode surface due to additional dipole at the InGaN/InN interface, which changes the Fermi level pinning energy. Similar effect was reported for a GaAs surface covered with an InAs film (p. 115 in [Williams1993]).

(ii) Increased band bending caused by re-pinning of the Fermi level closer to VB of InGaN on additional surface states formed by InN QDs. Similar effect was reported for a GaAs surface covered with InAs QDs [Walther2000].

(iii) InN QDs act as a sink for holes, sweeping them away from the InGaN surface and allowing OER to proceed without charging the surface states. This effectively negates the Fermi level pinning and, thus, reduces the photocurrent onset potential. Similar effect was reported for $\mathrm{Si}$ photoanodes covered with Pt nanoparticles [Nakato1992, Guijarro2015].

However, experiments presented in this work do not allow to determine the main mechanism of the observed improvement neither the. While (i) and (ii) should theoretically work for both QDs and thin film coverage, (iii) is only applicable to QDs. Thus, additional experiment comparing InN film vs. QDs coverage could help to resolve this issue. Besides, it must be taken into account, that InGaN surface was exposed to active nitrogen plasma for a prolonged period of time during the InN QDs fabrication (refer to Chapter 5), which also can modify its surface chemistry (in terms of surface state density) and affect the performance.

The results described above are different from these in another work on $\operatorname{InN} Q D s / \operatorname{InGaN}$ photoanodes, where QDs provided a significant current density increase without actually changing the onset potential [Alvi2015]. Additional experiments must be done in order to understand the QDs role in the InGaN photoanodes performance enhancement. 


\subsection{Conclusions}

The results presented in this chapter revealed a modest catalytic activity of as grown InGaN layers exhibiting high photocurrent onset potential values and weak slope of the current-voltage characteristics. These effects were attributed to the presence of a high density of surface states at the InGaN/electrolyte interface and a consequent strong Fermi level pinning [Veal2006]. Despite these effects, the InGaN/Si samples used in this work show a comparable performance in terms of EQE and photocurrent onset potential behavior to those from the better studied case of InGaN layers grown on GaN/sapphire substrates [Finken2015, Luo2008, Juodkazyte2014]. This is a great achievement on its own, as the structural and optical quality of $\operatorname{In}_{x} \mathrm{Ga}_{1-\mathrm{x}} \mathrm{N}$ grown on $\mathrm{Si}$ substrates are usually much lower than those from similar layers grown on GaN/sapphire templates.

Additionally, it was found that excellent stability of InGaN layers can be only referred to dark conditions, while under illumination corrosion takes place quite rapidly, as confirmed by CA and SEM measurements. A similar behavior was reported for InGaN/GaN/sapphire samples tested in various electrolytes covering the whole pH range [Finken2015, Juodkazyte2014, Huygens2000, Minsky1996]. The use of electrolytes with sacrificial agents confirmed the photo-assisted nature of this degradation. While both sacrificial agents used (formic acid and methanol) greatly reduced the corrosion rate (though not fully inhibited), only the latter one significantly enhanced the InGaN PEC performance. The origin of this dissimilarity is unclear and must be further studied.

The effect of magnetron sputtered NiO films on corrosion resistance and performance of the InGaN photoanodes was also studied. UV-Vis spectroscopy revealed that $\mathrm{NiO}$ is transparent for photons with wavelengths above $350 \mathrm{~nm}$. A theoretical analysis of a NiO/InGaN band diagram showed that $\mathrm{NiO}$ can be used as an electron blocking layer for Ga-rich InGaN. CV measurements from $\mathrm{In}_{x} \mathrm{Ga}_{1-\mathrm{x}} \mathrm{N} / \mathrm{NiO}$ samples in $1 \mathrm{M} \mathrm{KOH}$ electrolyte revealed majority carrier, "metallic" current-voltage behavior with a negligible photoresponse for $x>0.3$, attributed to high unintentional doping concentration of such $\ln _{x} G_{1-x} N$ layers and consequent tunnel junction with NiO. However, the excellent chemical stability of such $\mathrm{NiO}$ covered $\mathrm{In}$-rich $\mathrm{In}_{0.38} \mathrm{Ga}_{0.62} \mathrm{~N}$ sample suggests that it still can be used as a photoanode if a buried pn-junction is used.

In contrast, in sample with $x<0.3\left(\operatorname{In}_{0.18} \mathrm{Ga}_{0.82} \mathrm{~N}, E_{g}=2.6 \mathrm{eV}\right)$ the NiO film coverage nearly doubled the photocurrent density at $1.23 \mathrm{~V}$ vs. RHE. Moreover, the photocurrent became stable (for at least $20 \mathrm{~min}$ ) compared to a similar sample without a NiO protection, whose performance deteriorated exponentially with time. The different behavior was attributed to alower unintentional $\mathrm{n}$-type doping conductivity avoiding tunneling effects at the interface between $\mathrm{NiO}$ and $\ln _{0.18} \mathrm{Ga}_{0.82} \mathrm{~N}$ layer. Therefore, $\mathrm{NiO}$ films have a great potential as catalyst and protection against corrosion in $\ln _{x} \mathrm{Ga}_{1-\mathrm{x}} \mathrm{N}$ based photoanodes (for $\mathrm{x}<0.3$ ).

Additionally, PEC performance of an $\ln _{0.3} \mathrm{Ga}_{0.7} \mathrm{~N}\left(E_{g}=2.1 \mathrm{eV}\right)$ photoanode decorated with InN QDs nearly tripled (the photocurrent density) at $1.23 \mathrm{~V}$ vs. RHE compared to as grown layers. Moreover, it drastically decreased the photocurrent onset potential down to $\sim 0.4 \mathrm{~V}$. Some exploratory suggestions for this remarkable performance increase were proposed, though additional experiments are necessary to determine the actual reasons. 


\section{General conclusions and future work}

The most significant results obtained throughout this thesis and the future work, aimed to their further improvements, are briefly summarized in this chapter.

\subsection{Conclusions}

In the framework of this thesis constituent blocks of the tandem InGaN/Si-based water splitting device were realized, namely compact $\ln _{x} G_{a}$-x $N$ layers, quantum dots (QDs) and nanowires (NWs), all grown on $\mathrm{Si}(111)$ by plasma assisted molecular beam epitaxy (PA MBE).

- $\operatorname{In}_{x} \mathrm{Ga}_{1-\mathrm{x}} \mathrm{N}$ layers were grown over the entire composition range $(x=0-1)$ directly on nitridated $\mathrm{Si}(111)$, having wurtzite structure, composition uniformity, and reasonable structural and optical quality comparable to similar samples grown on GaN/sapphire templates. The study of the vertical conductivity of InGaN/p-Si heterointerfaces determined a Schottky behavior for low In\% but an ohmic behavior for high In content (In > 50\%). Moreover, use of highly p-type doped Si substrates allows fabricating vertically conductive devices over the entire In composition range.

- Single-crystalline wurtzite InN QDs with quasi-hemispherical shape and small size of few tens of nanometers were successfully grown by below room temperature droplet epitaxy followed by solid state dewetting step on atomically flat $\mathrm{Si}(111), \mathrm{Si}(001)$ and "rough" $(\mathrm{RMS}>10 \mathrm{~nm}) \ln _{0.3} \mathrm{Ga}_{0.7} \mathrm{~N}(0001) / \mathrm{Si}(111)$ substrates. This technique allows overcoming one of the main shortcomings of the widely used Stranski-Krastanow method, namely, the requirement of a flat substrate surface.

- Ordered arrays of GaN NWs were homoepitaxially grown by selective area growth (SAG) method on GaN/Si(111), GaN/AIN/Si(111) and GaN/sapphire(0001) templates. Dislocation filtering efficiency strongly depends on NWs geometry (diameter and height), being much higher in narrow and long NWs. Upon a proper selection of geometry, the resulting NWs can have a reasonable crystal quality even when grown on low quality templates. In addition, analysis of TEM, SEM, and PL results on the studied NWs suggested that the most probable origin of the UX excitonic emission in GaN NWs is related to polarity inversion domain boundaries defects.

- Photoelectrochemical (PEC) evaluation of compact $I_{n x} G_{a 1-x} N / S i(111)$ layers $(x=0-0.4)$ revealed their comparable external quantum efficiency (EQE), photocurrent onset potential and severe photo-induced corrosion as the $\mathrm{InGaN} / \mathrm{GaN} /$ Sapphire photoanode counterparts. In respect to NiO protection layers, they do not have any apparent effect on photocurrent onset potentials, but certainly improve stability of NiO-covered $I_{n x} G_{a 1-x} N / S i$ photoanodes, for $x<0.3$. For higher In compositions $(x>0.3)$ NiO-covered electrodes show metallic current- 
voltage behavior (no photoresponse). Additional optimization is needed to improve both performance and chemical stability of InGaN-based photoanodes.

- InGaN layers grown on Si(111) and "decorated" with InN QDs show a drastic photocurrent onset potential decrease and a consequent PEC performance boost. While the exact mechanism behind this improvement remains unknown, it is suggested that the presence of surface QDs led to a reduction of the Fermi level pinning, locally passivating the surface states.

\subsection{Future work}

This thesis addressed the critical points to develop InGaN/Si tandem water splitting devices, from the growth optimization to PEC evaluation. In terms of growth, there is still room for research. One important issue to be investigated is the reduction of the high unintentional n-type conductivity in In-rich $\operatorname{In}_{\mathrm{x}} \mathrm{Ga}_{1-\mathrm{x}} \mathrm{N}$ layers. In particular, using the metal modulated epitaxy (MME) that was reported to be a powerful tool to reduce unintentional $n$-type doping, allowing achievement of effective $p$ type conductivity in InGaN layers [Namkoong2008, Moseley2013].

Additional experiments must be performed on InN QDs to effectively control their density, for instance, by using different growth parameters during nitridation stage: changing nitrogen plasma flux and using even lower nitridation temperatures (as low as $-20^{\circ} \mathrm{C}$ ). Control of QDs density is expected to further enhance PEC efficiency of InGaN photoanodes "decorated" with InN QDs. Moreover, optical properties of such QDs must be studied, for which their capping with InGaN layer needs to be developed.

Recent advances in SAG NWs [Albert2013] should allow growing InGaN NWs on GaN/Si and InGaN/Si templates, needed for InGaN-based PEC cells. Moreover, methods to reduce the polarity inversion domains density in NWs grown on Si substrates should be developed, for instance, by means of insertion of InN interlayers [Koukoula2014]. This would provide a better control over the NWs surface properties which is crucial for their PEC performance, which also must be studied in detail.

From a PEC point of view, though InGaN photoanodes have great potential for solar-driven water splitting cells, this field is still largely under development. First of all, the actual $\mathrm{H}_{2}$ gas production should be quantified by gas chromatography. Moreover, work on determining the origin of large onset potentials of InGaN photoanodes is already in progress in collaboration with Prof. Sophia Haussener and Yannick Gaudy from LRESE EPFL, who previously developed a numerical model for GaN-based photoanodes [Gaudy2016]. Besides, other catalysts and protection layer instead of NiO must be studied, such as ion-permeable $\mathrm{NiOOH}$ or $\mathrm{Ni}(\mathrm{Fe}) \mathrm{OOH}$ catalysts, reported to form an "adaptive" junction (interface energy changes during operation as charge accumulates in the catalyst) at the S/E interface that drastically reduces photocurrent onset potentials [Lin2015, Nellist2016].

The specific aim of the proposed future work is to expand the achieved constituent blocks of the tandem InGaN/Si-based water splitting device towards the fabrication of a complete and optimized device [Walukiewicz2011] which could compete with the state-of-the-art tandem water splitting devices, such as $\mathrm{p}-\mathrm{Ga}_{0.5} \mathrm{In}_{0.5} \mathrm{P} / \mathrm{p}-\mathrm{n}$-GaAs cells [Khaselev\&Turner1998]. 


\section{Supporting information}

\section{Appendix A}

\section{Flux calibration}

An optical emission detector (OED) was used to calibrate the nitrogen plasma cell, which output signal (measured in $\mathrm{mV}$ ) is proportional to the active nitrogen flux. The map of OED values at varying molecular nitrogen gas flux $\left(F_{N_{2}}, \mathrm{sccm}\right)$ and power supply output $(P, W)$ was established (Fig. A1). Then a GaN layer was grown at a certain $P$ and $F_{N_{2}}$ under metal-rich conditions and its thickness was then measured from cross-sectional SEM images, to estimate growth rate corresponding to the selected parameters of the plasma source. Based on several of such measurements, the OED values were converted into growth rate values in atoms $/ \mathrm{cm}^{2} / \mathrm{s}$ (or $\mathrm{nm} / \mathrm{min}$ in $\mathrm{GaN}$ units). Initial set points for $\mathrm{Ga}$ and N plasma cells were used from a previous work by Gacevic et al. [Gacevic2013]. Once the calibration of the N-plasma source was complete, parameters of $P=350 \mathrm{~W}$ and $F_{N_{2}}=0.6 \mathrm{sccm}$ (corresponding to a growth rate of $4.38 \times 10^{14}$ atoms $/ \mathrm{cm}^{2} / \mathrm{s}$ or $6.0 \mathrm{~nm} / \mathrm{min}$ in $\mathrm{GaN}$ units) were chosen for all of the grown samples presented in this work.

For metal fluxes $\left(F_{G a}, F_{l n}\right)$ the cells calibration was done in terms of the beam equivalent pressure (BEP) dependence (in situ measured with a Bayard Alpert gauge) with the cell temperature is shown in Fig. A2 (a) for Ga and (b) In). Then, in case of Ga, BEP values were directly converted into growth rates from cross-sectional SEM images of GaN layers grown under N-rich conditions. Similarly for In, an $\ln _{\mathrm{x}} \mathrm{Ga}_{1-\mathrm{x}} \mathrm{N}$ layer grown under $\mathrm{N}$-rich conditions (with a known $F_{G a}$ ) was used. The growths were carried out at low temperatures $\left(600{ }^{\circ} \mathrm{C}\right.$ for $\mathrm{GaN}$ and $400{ }^{\circ} \mathrm{C}$ for $\left.\ln _{x} \mathrm{Ga}_{1-x} \mathrm{~N}\right)$ where thermal decomposition and metal desorption are negligible for both materials. It must be noted that whenever the III/N ratio is mentioned in this work, it was calculated by using measured fluxes in atoms $/ \mathrm{cm}^{2} / \mathrm{s}$.

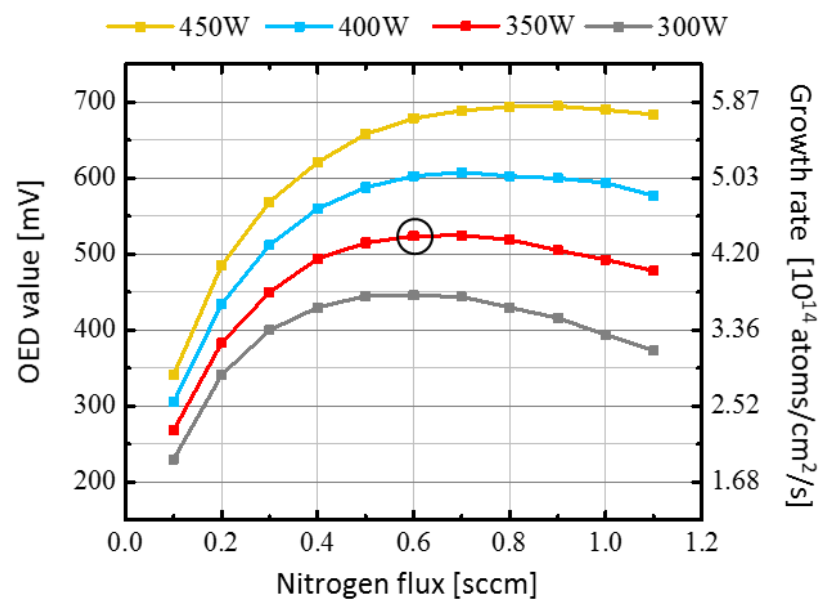

Fig. A1. Nitrogen plasma source calibration. OED values are converted into growth rates (in atoms $/ \mathrm{cm}^{2} / \mathrm{s}$ units). The chosen working point is marked with dark circle. 


\section{Ga flux}

(a)

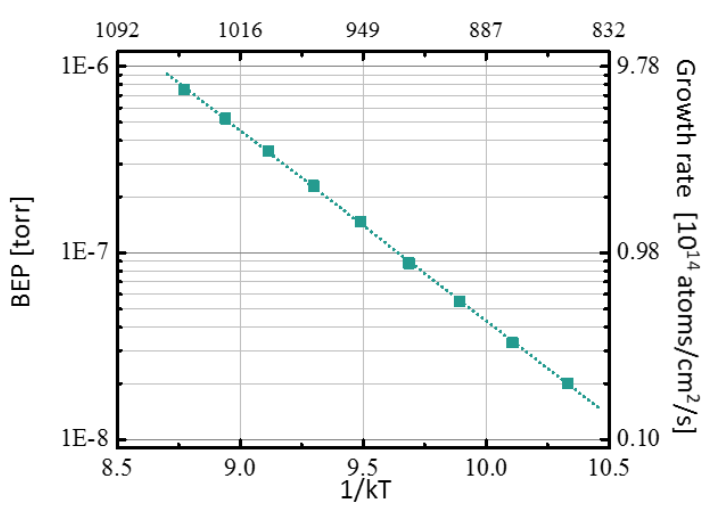

In flux

(b) Temperature $\left[{ }^{\circ} \mathrm{C}\right]$

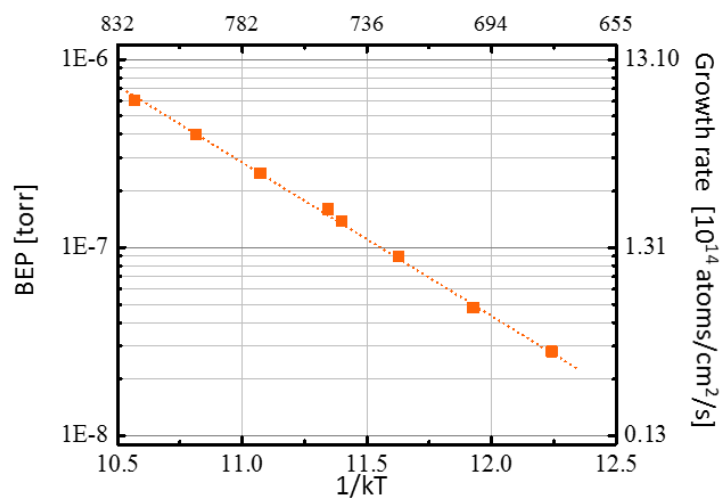

Fig. A2. Ga and In flux calibration by measuring beam equivalent pressures (BEP) and recalculated to atoms $/ \mathrm{cm}^{2} / \mathrm{s}$ flux values according to calibration growths.

\section{Appendix B}

This appendix section provides the experimental details on InGaN samples, chemicals, and setup preparations.

\section{$\mathrm{In}_{\mathrm{x}} \mathrm{Ga}_{1-\mathrm{x}} \mathrm{N} / \mathrm{Si}$ samples preparation}

All InGaN/Si samples, either as grown, with $\mathrm{NiO}$ film, or with InN QDs were prepared according to the following steps:

1. Scratch the back of Si with a diamond tool to remove the native oxide layer.

2. Put a droplet of liquid In-Ga eutectic solution onto the scratched Si back. This step ensures the ohmic contact between $\mathrm{Si}$ and $\mathrm{Cu}$ wire placed on the next step.

3. Prepare a $\mathrm{Cu}$ wire in a spiral shape, dip it in $5 \% \mathrm{HCl}$ solution to remove native oxide and then put it in contact with In-Ga covered Si back side (Fig. B1 (a)).

4. Cover the place where the $\mathrm{Cu}$ wire contacts the In-Ga eutectic with silver paint to ensure a good connection and protect the liquid In-Ga from spreading around (Fig. B1 (b)).

5. Let it dry for $10 \mathrm{~min}$.

6. Apply epoxy to isolate the wire and the whole Si substrate (back side and edges) from contacting the electrolyte, while leaving some part of the sample's front surface uncovered (active area of the photoanode). The $\mathrm{Cu}$ wire is additionally covered with thin glass tube before adding epoxy (Fig. B1 (c))

7. Dry epoxy in an oven at $60 \mathrm{C}$ for several hours.

In this procedure, In-Ga eutectic (Sigma-Aldrich) and Silver paint (VWR) ensures a good ohmic contact between the $\mathrm{Cu}$ wire and the Si back surface. The epoxy paste was prepared as a 2:1 mix of resin(1C) and hardener (EPKC) of Loctite ${ }^{\circledR}$ Epoxi-Patch $^{\circledR}$ Adhesive (Henkel Corporation). 
Once the samples were prepared, their opened active surface area (in $\mathrm{cm}^{2}$ ) was measured by image processing software ImageJ (https://imagej.nih.gov/ij/), based on their photos next to a ruler.

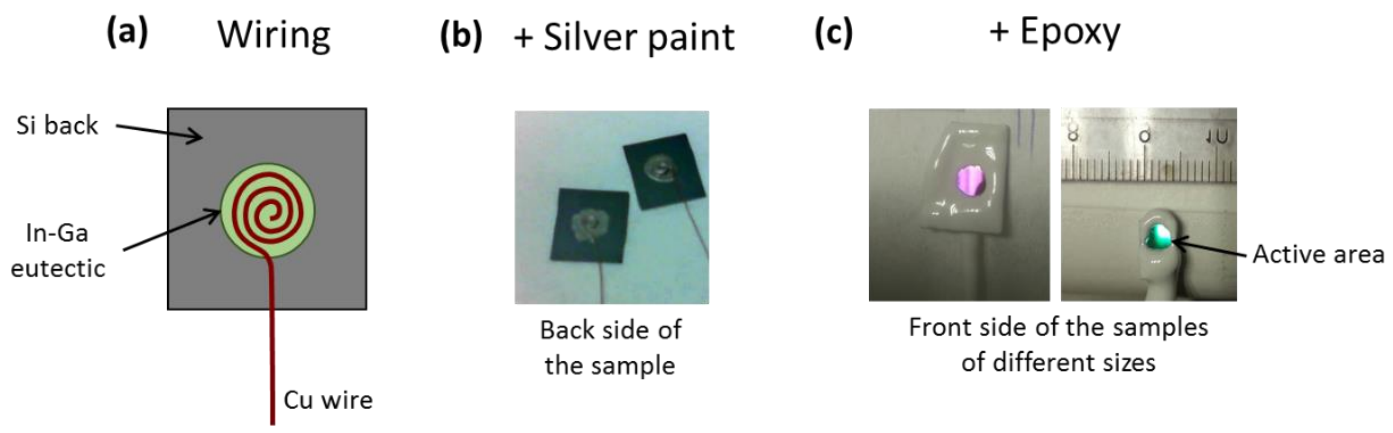

Fig. B1. InGaN/Si samples preparation steps. Schematic representation of Si back surface with an attached Cu wire using In-Ga eutectic (a). The Cu wire and In-Ga eutectic are covered with silver paint (b). The sample is finally covered with epoxy for chemical and electrical isolation (c).

\section{NiO film deposition}

Physical vapor deposition (PVD) by magnetron sputtering was used to deposit NiO films. Once the samples were sonicated in acetone for $10 \mathrm{~min}$ and washed with DI water, they were quickly loaded into the PVD chamber where $\mathrm{NiO}$ thin films were deposited according to the following procedure:

1. Sample surface was sputtered in Ar plasma ( $20 \mathrm{mTorr}, 50 \mathrm{sccm} \mathrm{Ar}, 35 \mathrm{~W}$, for $5 \mathrm{~min}$ ) at $200^{\circ} \mathrm{C}$.

2. A $3 \mathrm{~nm}$ Ni film was deposited in Ar plasma ( $5 \mathrm{mTorr}, 37 \mathrm{sccm} \mathrm{Ar}$ ) at $350{ }^{\circ} \mathrm{C}$ to avoid oxidation of the underlying Si.

3. A $15 \mathrm{~nm} \mathrm{NiO}$ film was deposited by reactive sputtering of $\mathrm{Ni}$ in $\mathrm{Ar} / \mathrm{O}_{2}$ plasma ( $5 \mathrm{mTorr}$, $37 \mathrm{sccm}-\mathrm{Ar}, 3 \mathrm{sccm}-02,65 \mathrm{~W})$ at $300^{\circ} \mathrm{C}$.

During this procedure the samples edges were protected by a mechanical mask to avoid short circuiting $\mathrm{NiO}$ films with the Si substrate. Once cooled, the samples were prepared for PEC experiments the same way as other InGaN samples.

\section{Electrolyte preapartion}

\section{Alkaline solutions}

To prepare a $1 \mathrm{M} \mathrm{KOH}\left(\mathrm{pH} 14\right.$ at $30{ }^{\circ} \mathrm{C}$ ) solution, semiconductor grade $(99.99 \%$ pure) $\mathrm{KOH}$ pellets (Sigma-Aldrich) with weight composition $>85 \%$ ( $15 \%$ water) were used. $\mathrm{KOH}$ molecular weight is $56.11 \mathrm{~g} / \mathrm{M}$. Thus, to prepare $1 \mathrm{~L}$ of $1 \mathrm{M} \mathrm{KOH}, 66.0 \mathrm{~g}$ of $\mathrm{KOH}$ pellets are needed $(1 \mathrm{M} / \mathrm{L} * 56.1 \mathrm{~g} / \mathrm{M} * 1 / 0.85$ $=66.0 \mathrm{~g} / \mathrm{L}$ ). The presence of $15 \%$ water were accounted in these calculations by introducing $1 / 0.85$ factor. DI water was poured into the measuring flask containing the calculated amount of $\mathrm{KOH}$ pellets to make it $1 \mathrm{~L}$.

The same procedure was used to prepare $1 \mathrm{M} \mathrm{NaOH}$ from semiconductor grade $\mathrm{NaOH}$ pellets (Sigma-Aldrich, weight composition $>98 \%$ ). $\mathrm{NaOH}$ molecular weight is $40.0 \mathrm{~g} / \mathrm{M}$. Thus, $40.8 \mathrm{~g}$ of $\mathrm{NaOH}$ pellets are needed to be diluted in $1 \mathrm{~L}$ of DI water $(1 \mathrm{M} / \mathrm{L} * 40 \mathrm{~g} / \mathrm{M} * 1 / 0.98=40.8 \mathrm{~g} / \mathrm{L})$. 


\section{Acid solutions}

To prepare a $1 \mathrm{M}$ Perchloric acid $\left(\mathrm{HCLO}_{4}\right)\left(\mathrm{pHO}\right.$ at $\left.30{ }^{\circ} \mathrm{C}\right)$ solution $70 \% \mathrm{HClO}_{4}(99.999 \%$ trace metals basis) with density of $1.664 \mathrm{~g} / \mathrm{mL}$ was used (Sigma-Aldrich). Its molecular weight is $100.46 \mathrm{~g} / \mathrm{M}$. This means that the Molarity of this solution is $0.7^{*} 1664 \mathrm{~g} / \mathrm{L} / 100.46 \mathrm{~g} / \mathrm{M}=11.59 \mathrm{M} / \mathrm{L}$. This solution has thus, $86 \mathrm{~mL}$ of $70 \% \mathrm{HClO}_{4}$ diluted in $1 \mathrm{~L}$ of DI water to obtain $1 \mathrm{M} \mathrm{HClO}_{4}$.

A similar procedure was followed to prepare a $1 \mathrm{M} \mathrm{HCL}\left(\mathrm{pHO}\right.$ at $30^{\circ} \mathrm{C}$ ) solution. For that $37 \% \mathrm{HCl}_{\mathrm{aq}}$ with $1.19 \mathrm{~kg} / \mathrm{L}$ density was used (Sigma-Aldrich). Molecular weight of $\mathrm{HCl}$ is $36.5 \mathrm{~g} / \mathrm{M}$. This means that the solution of $37 \% \mathrm{HCl}_{\mathrm{aq}}$ is $12 \mathrm{M} / \mathrm{L}(0.37 * 1190 \mathrm{~g} / \mathrm{L} / 36.5 \mathrm{~g} / \mathrm{M}=12 \mathrm{M} / \mathrm{L})$. Thus, $83 \mathrm{~mL}(1 \mathrm{~L} /$ $12 \mathrm{M} / \mathrm{L}$ ) of $37 \% \mathrm{HCL}_{\text {aq }}$ was diluted in $1 \mathrm{~L}$ of DI water to obtain $1 \mathrm{M} \mathrm{HCl}$.

\section{Sacrificial agents}

Formic acid $(\mathrm{HCOOH})$ was used as a sacrificial agent (hole scavenger) for the acidic electrolytes [Seger2012]. For that, a $10 \mathrm{mM}$ of $\mathrm{HCOOH}$ in $1 \mathrm{M} \mathrm{HCLO}_{4}$ solution was prepared using $50 \% \mathrm{HCOOH}$ (Sigma-Aldrich). For pure formic acid, molecular weight is $46.03 \mathrm{~g} / \mathrm{M}$ and density $1.22 \mathrm{~g} / \mathrm{mL}$. Thus, $1 \mathrm{~mL}$ of $50 \%$ formic acid contains $550 \mathrm{mg}$ of pure formic acid $(1 \mathrm{~g} /(1 \mathrm{~mL}+1 \mathrm{~g} / 1.22 \mathrm{~g} / \mathrm{mL})=550 \mathrm{mg} / \mathrm{mL}) .1 \mathrm{~L}$ of $10 \mathrm{mM} \mathrm{HCOOH}$ in $1 \mathrm{M} \mathrm{HCLO}_{4}$ solution contains 460 mg of pure $\mathrm{HCOOH}(10 \mathrm{mM} / \mathrm{L} \cdot 46.03 \mathrm{~g} / \mathrm{M}=460 \mathrm{mg} / \mathrm{L})$, which means that $0.83 \mathrm{~mL}$ of $50 \% \mathrm{HCOOH}$ $\left({ }^{460 \mathrm{mg}} / 550 \mathrm{mg} / \mathrm{mL}=0.83 \mathrm{~mL}\right)$ must be dissolved in $1 \mathrm{~L}$ of $1 \mathrm{M} \mathrm{HCLO}_{4}$.

For the alkaline electrolytes, methanol $\left(\mathrm{CH}_{3} \mathrm{OH}\right)$ was used as a hole scavenger [Wang2011]. For that, a 1:5 solution of $\mathrm{CH}_{3} \mathrm{OH}$ (Sigma-Aldrich, $>99.8 \%$ ) and $1 \mathrm{M} \mathrm{KOH}$ was prepared. 


\section{References}

[Abdi2013]

[Adachi2009]

[Adelmann1999]

[Adelmann2000]

[Ager2009]

[Ahn2001]

[Ajagunna2009]

[Akasaki1989]

[Albert2011]

[Albert2013]

[Albert2014]

[Albert2015]
Abdi, F. F., Han, L., Smets, A. H. M., Zeman, M., Dam, B. \& van de Krol, R. Efficient solar water splitting by enhanced charge separation in a bismuth vanadate-silicon tandem photoelectrode. Nat. Commun. 4, (2013).

Adachi, S. "Properties of Semiconductor Alloys: Group-IV, III-V and II-VI Semiconductors", John Wiley \& Sons, West Sussex, United Kingdom (2009).

Adelmann, C., Langer, R., Feuillet, G. \& Daudin, B. In incorporation during the growth of InGaN by molecular-beam epitaxy studied by reflection high-energy electron diffraction intensity oscillations. Appl. Phys. Lett. 75, 3518 (1999).

Adelmann, C., Simon, J., Feuillet, G., Pelekanos, N.T., Daudin, B. \& Fishman, G. Selfassembled InGaN quantum dots grown by molecular-beam epitaxy. Appl. Phys. Lett. 76,1570 (2000).

Ager, J.W., Reichertz, L.A., Cui, Y., Romanyuk, Y. E., Kreier, D., Leone, S. R., Yu, K. M., Schaff, W.J. \& Walukiewicz, W. Electrical properties of InGaN-Si heterojunctions. Phys. Status Solidi 6, S413-S416 (2009).

Ahn, H., Wu, C.L., Gwo, S., Wei, C.M. \& Chou, Y.C. Structure Determination of the $\mathrm{Si}_{3} \mathrm{~N}_{4} / \mathrm{Si}(111)-(8 \times 8)$ Surface: A Combined Study of Kikuchi Electron Holography, Scanning Tunneling Microscopy, and $a b$ initio Calculations. Phys. Rev. Lett. 86, 28182821 (2001).

Ajagunna, A.O., Adikimenakis, A., Iliopoulos, E., Tsagaraki, K., Androulidaki, M. \& Georgakilas, A. InN films and nanostructures grown on Si (111) by RF-MBE. J. Cryst. Growth 311, 2058-2062 (2009).

Akasaki, I., Amano, H., Koide, Y., Hiramatsu, K. \& Sawaki, N. Effects of ain buffer layer on crystallographic structure and on electrical and optical properties of $\mathrm{GaN}$ and $\mathrm{Ga}_{1-\mathrm{x}} \mathrm{Al}_{\mathrm{x}} \mathbf{N}$ $(0<0 x<0.4)$ films grown on sapphire substrate by MOVPE. J. Cryst. Growth 98, 209-219 (1989).

Albert, S., Bengoechea-Encabo, A., Lefebvre, P., Sanchez-Garcia, M.A., Calleja, E., Jahn, U. \& Trampert, A. Emission control of InGaN nanocolumns grown by molecular-beam epitaxy on Si(111) substrates. Appl. Phys. Lett. 99, 131108 (2011).

Albert, S., Bengoechea-Encabo, A., Sánchez-García, M.A, Kong, X., Trampert, A. \& Calleja, E. Selective area growth of $\ln (\mathrm{Ga}) \mathrm{N} / \mathrm{GaN}$ nanocolumns by molecular beam epitaxy on GaN-buffered Si(111): from ultraviolet to infrared emission. Nanotechnology 24 175303 (2013).

Albert, S., Bengoechea-Encabo, A. M., Barbagini, F., Lopez-Rormero, D., Sánchez-García, M.A., Calleja, E., Lefebvre, P., Kong, X., Jahn, U., Trampert, A., Müller, M., Bertram, F., Schmidt, G., Veit, P., Petzold, S., Christen, J., De Mierry, P. \& Zuñiga-Perez, J. Advances in MBE Selective Area Growth of III-Nitride Nanostructures: From NanoLEDs to Pseudo Substrates. Int. J. High Speed Electron. Syst. 23, 1450020 (2014).

Albert, S., Bengoechea-Encabo, A., Ledig, J., Schimpke, T., Sánchez-García, M.A., Strassburg, M., Waag, A. \& Calleja, E. Demonstration of (In, Ga)N/GaN Core-Shell Micro Light-Emitting Diodes Grown by Molecular Beam Epitaxy on Ordered MOVPE GaN Pillars. Cryst. Growth Des. 15, 3661-3665 (2015). 
[Albert2015b] Albert, S., "Self assembled and ordered group III-nitride nanocolumnar structures for light emitting applications", PhD Thesis, Universidad Politécnica de Madrid, Spain (2015).

[AlOtaibi2013a] AlOtaibi, B., Harati, M., Fan, S., Zhao, S., Nguyen, H.P.T., Kibria, M.G. \& Mi, Z. High efficiency photoelectrochemical water splitting and hydrogen generation using GaN nanowire photoelectrode. Nanotechnology 24, 175401 (2013).

[AlOtaibi2013b]

[Alvi2012]

[Alvi2015]

[Amano1986]

[Amano1988]

[Amano1989]

[Amano1995]

[Amano2015]

[Amloy2013]

[Aseev2013]

[Aseev2015]

[Aseev2016]

[Auzelle2015]
AlOtaibi, B., Nguyen, H.P.T., Zhao, S., Kibria, M.G., Fan, S. \& Mi, Z. Highly stable photoelectrochemical water splitting and hydrogen generation using a double-band InGaN/GaN core/shell nanowire photoanode. Nano Lett. 13, 4356-4361 (2013).

Alvi, N.H., Soto Rodriguez, P.E.D., Gómez, V.J., Kumar, P., Amin, G., Nur, O., Willander, M. \& Nötzel, R. Highly efficient potentiometric glucose biosensor based on functionalized InN quantum dots. Appl. Phys. Lett. 101, 153110 (2012).

Alvi, N.H., Soto, P., Aseev, P., Gómez Hernández, V.J., Alvi, A.H., Aliv, W.U., Willander, M. \& Nötzel, R. InN/InGaN quantum dot photoelectrode: Efficient Hydrogen generation by water splitting at zero voltage. Nano Energy 13, 291-297 (2015).

Amano, H., Sawaki, N., Akasaki I. \& Toyoda, T., Metalorganic vapor phase epitaxial growth of a high quality GaN film using an AIN buffer layer. Appl. Phys. Lett. 48, 353 (1986).

Amano, H., Akasaki, I., Hiramatsu, K., Koide, N. \& Sawaki, N., Effects of the buffer layer in metalorganic vapour phase epitaxy of GaN on sapphire substrate. Thin Solid Films $163,415-420$ (1988).

Amano, H., Kito, M., Hiramatsu, K. \& Akasaki I. P-type conduction in Mg-doped GaN treated with low-energy electron beam irradiation (LEEBI). Jpn. J. Appl. Phys. 28, L2112 (1989).

Amano H., \& Akasaki, I. Abstract at Int. Conf. Solid State Devices and Materials 7, 683 (1995).

Amano, H. Nobel Lecture: "Growth of GaN on sapphire via low-temperature deposited buffer layer and realization of p-type $\mathrm{GaN}$ by $\mathrm{Mg}$ doping followed by low-energy electron beam irradiation." Rev. Mod. Phys. 87, 1134-1138 (2015).

Amloy, S. "Polarization-resolved photoluminescence spectroscopy of III-nitride quantum dots", PhD Thesis, Linköping University (2013).

Aseev, P., Soto Rodriguez, P. E. D., Kumar, P., Gómez, V. J., Alvi, N. H., Manuel, J. M., Morales, F. M., Jimenez, J. J., Garcia, R., Calleja, E. \& Noetzel, R. Uniform Low-to-High In Composition InGaN Layers Grown on Si. Appl. Phys. Express 6, 115503-1-4 (2013).

Aseev, P., Rodriguez, P. E. D. S., Gómez, V. J., Alvi, N. U. H., Mánuel, J. M., Morales, F. M., Jiménez, J. J., García, R., Senichev, A., Lienau, C., Calleja, E. \& Nötzel, R. Near-infrared emitting In-rich InGaN layers grown directly on Si: Towards the whole composition range. Appl. Phys. Lett. 106, 72102 (2015).

Aseev, P., Gačević, Ž., Torres-Pardo, A., González-Calbet, J. M. \& Calleja, E. Improving optical performance of GaN nanowires grown by selective area growth homoepitaxy: Influence of substrate and nanowire dimensions. Appl. Phys. Lett. 108, 253109 (2016).

Auzelle, T., Haas, B., Den Hertog, M., Rouvière, J., Daudin, B. \& Gayral, B. Attribution of the $3.45 \mathrm{eV} \mathrm{GaN}$ nanowires luminescence to inversion domain boundaries. Appl. Phys. Lett. 107, 51904 (2015). 
[Auzelle2015b]

[Bae2015]

[Bahari2005]

[Bailey2008]

[Balachandran2015]

[Bauer1958]

[Behme1997]

[Bengoechea2011]

[Bengoechea2014]

[Benton2013]

[Benton2014]

[Bernardini1997]

[Bickford2008]

[Bimberg1999]

[Blanchard2008]

[Bockris2001]

[BP2014]
Auzelle, T., Haas, B., Minj, A., Bougerol, C., Rouvière, J.L., Cros, A., Colchero, J. \& Daudin, $B$. The influence of AIN buffer over the polarity and the nucleation of self-organized GaN nanowires. J. Appl. Phys. 117, 245303 (2015).

Bae, D., "Development of Silicon based Bottom Cell for Tandem Photoelectrochemical Water Splitting Device Structures ", PhD Thesis, Technical University of Denmark, Denmark (2015).

Bahari, A., Robenhagen, U., Morgen, P. \& Li, Z. S. Growth of ultrathin silicon nitride on Si(111) at low temperatures. Phys. Rev. B 72, 205323 (2005).

Bailey, L. R., Veal, T. D., King, P. D. C., McConville, C. F., Pereiro, J., Grandal, J., SánchezGarcía, M. a., Munoz, E. \& Calleja, E. Band bending at the surfaces of In-rich InGaN alloys. J. Appl. Phys. 104, 113716 (2008).

Balachandran, P. V., Theiler, J., Rondinelli, J. M. \& Lookman, T. Materials Prediction via Classification Learning. Sci. Rep. 5, 13285 (2015).

Bauer, E. Phaenomenologische Theorie der Kristallabscheidung an Oberflaechen.I. Z. Kristallogr. 110, 372 (1958).

Behme, G., Richter, A., Süptitz, M. \& Lienau, C. Vacuum near-field scanning optical microscope for variable cryogenic temperatures. Rev. Sci. Instrum. 68, 3458 (1997).

Bengoechea-Encabo, A., Barbagini, F., Fernandez-Garrido, S., Grandal, J., Ristic, J., Sanchez-Garcia, M.A., Calleja, E., Jahn, U., Luna, E. \& Trampert, A. Understanding the selective area growth of GaN nanocolumns by MBE using Ti nanomasks. J. Cryst. Growth 325, 89-92 (2011).

Bengoechea-Encabo, A., Albert, S., Lopez-Romero, D., Lefebvre, P., Barbagini, F., TorresPardo, A., Gonzalez-Calbet, J.M., Sanchez-Garcia, M.A. \& Calleja, E. Light-EmittingDiodes based on ordered InGaN nanocolumns emitting in the blue, green and yellow spectral range. Nanotechnology 25, 435203 (2014).

Benton, J., Bai, J. \& Wang, T. Significantly enhanced performance of an InGaN/GaN nanostructure based photo-electrode for solar power hydrogen generation. Appl. Phys. Lett. 103, 133904 (2013).

Benton, J. "Novel III-Nitride Semiconductors for Solar Hydrogen Production" PhD Thesis, The University of Sheffield, UK (2014).

Bernardini, F., Fiorentini, V. \& Vanderbilt, D. Spontaneous polarization and piezoelectric constants of III-V nitrides. Phys. Rev. B 56, 4 (1997).

Bickford, J.R. "Sparse gallium arsenide to silicon metal waferbonding for heterogeneous monolithic microwave integrated circuits" PhD Thesis, University of California, San Diego, USA (2008).

Bimberg, D., Grundmann, M., Ledentsov, N. N., "Quantum Dot heterostructures", Jhon Wiley\&Sons, England(1998).

Blanchard, P. T., Bertness, K. A., Harvey, T. E., Mansfield, L. M., Sanders, A. W. \& Sanford, N. A. MESFETs Made From Individual GaN Nanowires. IEEE Trans. Nanotechnol. 7, 760765 (2008).

Bockris, J.O.M., Reddy, A.K.N., Galboa-Aldeco, M.E. “Modern Electrochemistry 2A Fundamentals of Electrodics” Springer, New York (2001).

British Petroleum, BP statistical review of world energy. London: BP plc. , retrivew from www.bp.com/statisticalreview (2014). 
[Brubaker2016]

[Brus1984]

[Buffat 1976]

[Butler\&Ginley1980]

[Byrne2011]

[Caccamo2016]

[Calleja1998]

[Calleja1999]

[Calleja2000]

[Cao2003]

[Cerutti2006]

[Chan2011]

[Chang2008]
Brubaker, M. D., Duff, S. M., Harvey, T. E., Blanchard, P. T., Roshko, A., Sanders, A. W., Sanford, N. A. \& Bertness, K. A. Polarity-Controlled GaN/AIN Nucleation Layers for Selective-Area Growth of GaN Nanowire Arrays on Si(111) Substrates by Molecular Beam Epitaxy. Cryst. Growth Des. 16, 596-604 (2016). 6-604 (2016).

Brus, L.E. Electron-electron and electron-hole interactions in small semiconductor crystallites: The size dependence of the lowest excited electronic state. J. Chem. Phys. 80, 4403 (1984).

Buffat, P. \& Borel, J. P. Size effect on the melting temperature of gold particles. Phys. Rev. A 13, 2287-2298 (1976).

Butler, M. A. \& Ginley, D. S. Principles of photoelectrochemical, solar energy conversion. J. Mater. Sci. 15, 1-19 (1980).

Byrne, D., Fath Allah, R., Ben, T., Gonzalez Robledo, D., Twamley, B., Henry, M. O. \& McGlynn, E. Study of morphological and related properties of aligned zinc oxide nanorods grown by vapor phase transport on chemical bath deposited buffer layers. Cryst. Growth Des. 11, 5378-5386 (2011).

Caccamo, L., Cocco, G., Martín, G., Zhou, H., Fundling, S., Gad, A., Mohajerani, M. S., Abdelfatah, M., Estradé, S., Peiró, F., Dziony, W., Bremers, H., Hangleiter, A., Mayrhofer, L., Lilienkamp, G., Moseler, M., Daum, W. \& Waag, A. Insights into Interfacial Changes and Photoelectrochemical Stability of $\operatorname{In}_{x} \mathrm{Ga}_{1-\mathrm{x}} \mathbf{N}(0001)$ Photoanode Surfaces in Liquid Environments. ACS Appl. Mater. Interfaces 8, 8232-8238 (2016).

Calleja, E., Sánchez-García, M. A., Basak, D., Sánchez, F. J., Calle, F., Youinou, P., Muñoz, E., Serrano, J. J., Blanco, J. M., Villar, C., Laine, T., Oila, J., Saarinen, K., Hautojärvi, P., Molloy, C. H., Somerford, D. J. \& Harrison, I. Effect of Ga/Si interdiffusion on optical and transport properties of GaN layers grown on Si(111) by molecular-beam epitaxy. Phys. Rev. B 58, 1550-1559 (1998).

Calleja, E., Sánchez-García, M.A., Sánchez, F. J., Calle, F., Naranjo, F. B., Muñoz, E., Molina, S. I., Sánchez, A. M., Pacheco, F. J. \& García, R. Growth of III-nitrides on Si(111) by molecular beam epitaxy Doping, optical, and electrical properties. J. Cryst. Growth 201, 296-317 (1999).

Calleja, E., Sánchez-García, M. A., Sánchez, F. J., Calle, F., Naranjo, F. B., Muñoz, E., Jahn, U. \& Ploog, K. Luminescence properties and defects in GaN nanocolumns grown by molecular beam epitaxy. Phys. Rev. B 62, 16826-16834 (2000).

Cao, Y. G., Xie, M. H., Liu, Y., Ng, Y. F., Wu, H. S. \& Tong, S. Y. InN Island shape and its dependence on growth condition of molecular-beam epitaxy. Appl. Phys. Lett. 83, 5157-5159 (2003).

Cerutti, L., Ristić, J., Fernández-Garrido, S., Calleja, E., Trampert, a., Ploog, K. H., Lazic, S. \& Calleja, J. M. Wurtzite GaN nanocolumns grown on Si(001) by molecular beam epitaxy. Appl. Phys. Lett. 88, 213114 (2006).

Chan, X.H., Robert Jennings, J., Anower Hossain, M., Koh Zhen Yu, K. \& Wang, Q. Characteristics of $\mathrm{p}$-NiO Thin Films Prepared by Spray Pyrolysis and Their Application in CdS-sensitized Photocathodes. J. Electrochem. Soc. 158, H733 (2011).

Chang, W.-H., Ke, W.-C., Yu, S.-H., Lee, L., Chen, C.-Y., Tsai, W.-C., Lin, H., Chou, W.-C., Lee, M.-C. \& Chen, W.-K. Effects of growth temperature on InN/GaN nanodots grown by metal organic chemical vapor deposition. J. Appl. Phys. 103, 104306 (2008). 
[Chao1987]

[Chen2010]

[Chen2016]

[Cherns1998]

[Chernysheva2015]

[Chichibu2006]

[Chin2013]

[Cho2006]

[Christen1990]

[Colby2010]

[Corfdir2009]

[Corfdir2014a]

[Corfdir2014b]

[Corfdir2015]
Chao, S. S., Tyler, J. E., Tsu, D. V., Lucovsky, G. \& Mantini, J. J. Auger electron spectroscopy studies of silicon nitride, oxide, and oxynitride thin films: Minimization of surface damage by argon and electron beams. J. Vac. Sci. Technol. A 5, 1283 (1987).

Chen, X.J., Perillat-Merceroz, G., Sam-Giao, D., Durand, C. \& Eymery, J. Homoepitaxial growth of catalyst-free GaN wires on N-polar substrates. Appl. Phys. Lett. 97, 20102013 (2010).

Chen, H. J.-Y., Yang, D.-L., Huang, T.-W. \& Yu, I.-S. Formation and Temperature Effect of InN Nanodots by PA-MBE via Droplet Epitaxy Technique. Nanoscale Res. Lett. 11, 241 (2016).

Cherns, D., Young, W. T., Sanders, M., Steeds, J. W., Ponce, F. A. \& Nakamura, S. Determination of the atomic structure of inversion domain boundaries in $\alpha-\mathrm{GaN}$ by transmission electron microscopy. Philos. Mag. A 77, 273-286 (1998).

Chernysheva, E., Gačević, Ž., García-Lepetit, N., van der Meulen, H. P., Müller, M., Bertram, F., Veit, P., Torres-Pardo, a., González Calbet, J. M., Christen, J., Calleja, E., Calleja, J. M. \& Lazić, S. Blue-to-green single photons from InGaN/GaN dot-in-ananowire ordered arrays. Europhysics Lett. 111, 24001 (2015).

Chichibu, S. F., Uedono, A., Onuma, T., Haskell, B. A., Chakraborty, A., Koyama, T., Fini, P. T., Keller, S., DenBaars, S. P., Speck, J. S., Mishra, U. K., Nakamura, S., Yamaguchi, S., Kamiyama, S., Amano, H., Akasaki, I., Han, J. \& Sota, T. Origin of defect-insensitive emission probability in In-containing (Al,In,Ga)N alloy semiconductors. Nat. Mater. 5 , 810-816 (2006).

Chin, C., Hassan, Z., Yam, F. \& Ahmad, M. Growth of self-assembled InGaN quantum dots on Si (111) at reduced temperature by molecular beam epitaxy. Thin Solid Films 544, 33-36 (2013).

Cho, I. K., Kim, Y. K. \& Yeom, H. W. Temperature-dependent adsorption and dissociation behaviors of $\mathrm{NH3}$ on $\mathrm{Si}(111) 7 \times 7$ : A high-resolution core-level photoemission study. Phys. Rev. B 73, 115328 (2006).

Christen, J. \& Bimberg, D. Line shapes of intersubband and excitonic recombination in quantum wells: Influence of final-state interaction, statistical broadening, and momentum conservation. Phys. Rev. B 42, 7213-7219 (1990).

Colby, R., Liang, Z., Wildeson, I. H., Ewoldt, D. A., Sands, T. D., Garcia, R. E. \& Stach, E. A. Dislocation filtering in GaN nanostructures. Nano Lett. 10, 1568-1573 (2010).

Corfdir, P., Lefebvre, P., Ristić, J., Valvin, P., Calleja, E., Trampert, A., Ganière, J.-D. \& Deveaud-Plédran, B. Time-resolved spectroscopy on GaN nanocolumns grown by plasma assisted molecular beam epitaxy on Si substrates. J. Appl. Phys. 105, 13113 (2009).

Corfdir, P., Hauswald, C., Zettler, J. K., Flissikowski, T., Lähnemann, J., Fernández-Garrido, S., Geelhaar, L., Grahn, H. T. \& Brandt, O. Stacking faults as quantum wells in nanowires: Density of states, oscillator strength, and radiative efficiency. Phys. Rev. B 90, 195309 (2014).

Corfdir, P., Zettler, J. K., Hauswald, C., Fernández-Garrido, S., Brandt, O. \& Lefebvre, P. Sub-meV linewidth in GaN nanowire ensembles: Absence of surface excitons due to the field ionization of donors. Phys. Rev. B 90, 205301 (2014).

Corfdir, P., Feix, F., Zettler, J. K., Fernández-Garrido, S. \& Brandt, O. Importance of the dielectric contrast for the polarization of excitonic transitions in single GaN nanowires. New J. Phys. 17, 033040 (2015). 
[Coulon2016]

[Dabrowski2000]

[Dadgar2007]

[Dahal2014]

[Das2011]

[Daudin1997]

[Davydov2002]

[Debnath2007]

[Debnath2009]

[Deng2011]

[Deshpande2013]

[Dimakis2005]

[Dimitrakis2013]

[Dimitrakopulos2001]
Coulon, P., Alloing, B., Brändli, V., Vennéguès, P., Leroux, M. \& Zúñiga-Pérez, J. Dislocation filtering and polarity in the selective area growth of $\mathrm{GaN}$ nanowires by continuous-flow metal organic vapor phase epitaxy. Appl. Phys. Express 9, 15502 (2016).

Dabrowski J. \& Müssig, H.J. S"ilicon Surfaces and Formation of Interfaces", World Scientific, Singapore (2000).

Dadgar, A., Schulze, F., Wienecke, M., Gadanecz, A., Bläsing, J., Veit, P., Hempel, T., Diez, A., Christen, J. \& Krost, A. Epitaxy of GaN on silicon - Impact of symmetry and surface reconstruction. New J. Phys. 9, (2007).

Dahal, R., Pantha, B. N., Li, J., Lin, J. Y. \& Jiang, H. X. Realizing InGaN monolithic solarphotoelectrochemical cells for artificial photosynthesis. Appl. Phys. Lett. 104, 143901 (2014).

Das, A., Heo, J., Jankowski, M., Guo, W., Zhang, L., Deng, H. \& Bhattacharya, P. Room temperature ultralow threshold GaN nanowire polariton laser. Phys. Rev. Lett. 107, 15 (2011).

Daudin, B., Widmann, F., Feuillet, G., Samson, Y., Arlery, M. \& Rouvière, J. StranskiKrastanov growth mode during the molecular beam epitaxy of highly strained GaN. Phys. Rev. B 56, R7069-R7072 (1997).

Davydov, V.Y., Klochikhin, A.A., Emtsev, V.V., Kurdyukov, D.A., Ivanov, S.V., Vekshin, V.A., Bechstedt, F., Furthmüller, J., Aderhold, J., Graul, J., Mudryi, A.V., Harima, H., Hashimoto, A., Yamamoto, A. \& Haller, E.E. Band Gap of Hexagonal InN and InGaN Alloys. Phys. status solidi 234, 787-795 (2002).

Debnath, R. K., Meijers, R., Richter, T., Stoica, T., Calarco, R. \& Lüth, H. Mechanism of molecular beam epitaxy growth of GaN nanowires on Si(111). Appl. Phys. Lett. 90, 6770 (2007).

Debnath, R. K., Stoica, T., Besmehn, A., Jeganathan, K., Sutter, E., Meijers, R., Lüth, H. \& Calarco, R. Formation of GaN nanodots on Si (111) by droplet nitridation. J. Cryst. Growth 311, 3389-3394 (2009).

Deng, Q., Wang, X., Yang, C., Xiao, H., Wang, C., Yin, H., Hou, Q., Li, J., Wang, Z. \& Hou, X. Theoretical study on $\ln _{\mathbf{x}} \mathrm{Ga}_{1-\mathrm{x}} \mathrm{N} / \mathrm{GaN}$ quantum dots solar cell. Phys. $\mathrm{B}$ Condens. Matter 406, 73-76 (2011).

Deshpande, S., Heo, J., Das, A. \& Bhattacharya, P. Electrically driven polarized singlephoton emission from an InGaN quantum dot in a GaN nanowire. Nat. Commun. 4, 1675 (2013).

Dimakis, E., Iliopoulos, E., Tsagaraki, K., Kehagias, T., Komninou, P. \& Georgakilas, A. Heteroepitaxial growth of In-face InN on GaN (0001) by plasma-assisted molecularbeam epitaxy. J. Appl. Phys. 97, 113520 (2005).

Dimitrakis, P., Normand, P., Bonafos, C., Papadomanolaki, E. \& Iliopoulos, E. GaN quantum-dots integrated in the gate dielectric of metal-oxide-semiconductor structures for charge-storage applications. Appl. Phys. Lett. 102, 053117 (2013).

Dimitrakopulos, G., Komninou, P., Kioseoglou, J., Kehagias, T., Sarigiannidou, E., Georgakilas, a., Nouet, G. \& Karakostas, T. Structural transition of inversion domain boundaries through interactions with stacking faults in epitaxial GaN. Phys. Rev. B 64, 245325 (2001). 
[Doppalapudi1998]

[Dridi2003]

[Ebaid2015]

[Ekimov1982]

[Ekimov1985]

[El-Masry1998]

[Fan2015]

[Ferhat2002]

[Fernandez-Garrido2009]

[Fini\&Nakamura2005]

[Finken2015]

[Fontsere2011]

[Forchel1988]

[Frank1953]

[Frost2014]

[Furtmayr2008]
Doppalapudi, D., Basu, S. N., Ludwig, K. F. \& Moustakas, T. D. Phase separation and ordering in InGaN alloys grown by molecular beam epitaxy. J. Appl. Phys. 84, 1389 (1998).

Dridi, Z., Bouhafs, B. \& Ruterana, P. First-principles investigation of lattice constants and bowing parameters in wurtzite $\mathrm{Al}_{\mathrm{x}} \mathrm{Ga}_{1-\mathrm{x}} \mathrm{N}, \operatorname{In}_{\mathrm{x}} \mathrm{Ga}_{1-\mathrm{x}} \mathrm{N}$ and $\operatorname{In}_{\mathrm{x}} \mathrm{Al} \mathrm{l}_{1-\mathrm{x}} \mathrm{N}$ alloys. Semicond. Sci. ... 18, 850-856 (2003).

Ebaid, M., Kang, J.H., Lim, S.H., Cho, Y.H. \& Ryu, S.W. Towards highly efficient photoanodes: the role of carrier dynamics on the photoelectrochemical performance of InGaN/GaN multiple quantum well coaxial nanowires. RSC Adv. 5, 23303-23310 (2015).

Ekimov A.I., Onushchenko A.A., "Quantum size effect in the optical-spectra of semiconductor micro-crystals". Soviet Physics Semiconductors-USSR 16 (7): 775-778, (1982).

Ekimov, A. I., Efros, A. L. \& Onushchenko, A. A. Quantum size effect in semiconductor microcrystals. Solid State Commun. 56, 921-924 (1985).

El-Masry, N. A., Piner, E. L., Liu, S. X. \& Bedair, S. M. Phase separation in InGaN grown by metalorganic chemical vapor deposition. Appl. Phys. Lett. 72, 40-42 (1998).

Fan, S., AlOtaibi, B., Woo, S. Y., Wang, Y., Botton, G. a. \& Mi, Z. High Efficiency Solar-toHydrogen Conversion on a Monolithically Integrated InGaN/GaN/Si Adaptive Tunnel Junction Photocathode. Nano Lett. 15, 2721-2726 (2015).

Ferhat, M., Furthmüller, J. \& Bechstedt, F. Gap bowing and Stokes shift in InGaN alloys: First-principles studies. Appl. Phys. Lett. 80, 1394 (2002).

Fernández-Garrido, S., Grandal, J., Calleja, E., Sánchez-García, M. a. \& Lopez-Romero, D. A growth diagram for plasma-assisted molecular beam epitaxy of GaN nanocolumns on Si(111). J. Appl. Phys. 106, 126102 (2009).

Fini, P.T. \& Nakamura, S. High-Efficiency Nitride-Based Solid-State Lighting. US, Web. DOI:10.2172/877537, (2005).

Finken, M., Wille, A., Reuters, B., Heuken, M., Kalisch, H. \& Vescan, A. Investigations of the electrochemical stability of InGaN photoanodes in different electrolytes. Phys. status solidi 252, 895-899 (2015).

Fontserè, A., Pérez-Tomás, A., Placidi, M., Llobet, J., Baron, N., Chenot, S., Cordier, Y., Moreno, J. C., Gammon, P. M., Jennings, M. R., Porti, M., Bayerl, A., Lanza, M. \& Nafría, M. Micro and nano analysis of $0.2 \Omega \mathrm{mm} \mathrm{Ti} / \mathrm{Al} / \mathrm{Ni} / \mathrm{Au}$ ohmic contact to AIGaN/GaN. Appl. Phys. Lett. 99, 213504 (2011). 1).

Forchel, A., Leier H., Maile B.E., \& Germann, R., Festkorperprobleme, "Advances in Solid State Physics", 28, 99 (1988).

Frank, F. C. \& Nicholas, J. F. Stable dislocations in the common crystal lattices. London, Edinburgh, Dublin Philos. Mag. J. Sci. 44, 1213-1235 (1953).

Frost, T., Jahangir, S., Stark, E., Deshpande, S., Hazari, A., Zhao, C., Ooi, B. S. \& Bhattacharya, P. Monolithic Electrically Injected Nanowire Array Edge-Emitting Laser on (001) Silicon. Nano Lett. 14, 4535-4541 (2014).

Furtmayr, F., Vielemeyer, M., Stutzmann, M., Arbiol, J., Estradé, S., Peirò, F., Morante, J. R. \& Eickhoff, M. Nucleation and growth of GaN nanorods on Si (111) surfaces by plasma-assisted molecular beam epitaxy - The influence of Si- and Mg-doping. J. Appl. Phys. 104, 034309 (2008). 
[Gacevic2012]

[Gacevic2013]

[Gacevic2015a]

[Gacevic2015b]

[Gacevic2016]

[Gangopadhyay2006]

[Gartner1959]

[Gaudy2016]

[Geelhaar2011]

[Genin1994]

[Gerischer1969]

[Gerischer1977]

[Gherasimova2004]

[Gherasoiu2010]

[Ghosh2005]

[Gimenez2016]

[Gogneau2003]
Gačević, Ž. "Constituent blocks of planar III-Nitride microcavities grown by molecular beam epitaxy.", PhD thesis, Universidad Politecnica de Madrid, Spain (2012).

Gačević, Ž., Gómez, V. J., Lepetit, N. G., Soto Rodríguez, P. E. D., Bengoechea, A., Fernández-Garrido, S., Nötzel, R. \& Calleja, E. A comprehensive diagram to grow (0001)InGaN alloys by molecular beam epitaxy. J. Cryst. Growth 364, 123-127 (2013).

Gačević, Ž., Gómez Sánchez, D. \& Calleja, E. Formation Mechanisms of GaN Nanowires Grown by Selective Area Growth Homoepitaxy. Nano Lett. 15, 1117-1121 (2015).

Gačević, Ž., Bengoechea-Encabo, A., Albert, S., Torres-Pardo, A., González-Calbet, J. M. \& Calleja, E. Crystallographically uniform arrays of ordered (In)GaN nanocolumns. J. Appl. Phys. 117, 35301 (2015).

Gačević, Ž., López-Romero, D., Juan Mangas, T. \& Calleja, E. A top-gate GaN nanowire metal-semiconductor field effect transistor with improved channel electrostatic control. Appl. Phys. Lett. 108, 033101 (2016).

Gangopadhyay, S., Schmidt, T. \& Falta, J. N-plasma assisted MBE grown GaN films on Si(111). Phys. Status Solidi Basic Res. 243, 1416-1420 (2006).

Gärtner, W. W. Depletion-Layer Photoeffects in Semiconductors. Phys. Rev. 116, 84-87 (1959).

Gaudy, Y.K. \& Haussener, S. Utilizing modeling, experiments, and statistics for the analysis of water-splitting photoelectrodes. J. Mater. Chem. A 4, 3100-3114 (2016).

Geelhaar, L., Chèze, C., Jenichen, B., Brandt, O., Pfüller, C., Münch, S., Rothemund, R., Reitzenstein, S., Forchel, A., Kehagias, T., Komninou, P., Dimitrakopulos, G. P., Karakostas, T., Lari, L., Chalker, P. R., Gass, M. H. \& Riechert, H. Properties of GaN Nanowires Grown by Molecular Beam Epitaxy. IEEE J. Sel. Top. Quantum Electron. 17, 878-888 (2011).

Génin, F. Y., Mullins, W. W. \& Wynblatt, P. Capillary instabilities in polycrystalline metallic foils: Experimental observations of thermal pitting in nickel. Acta Metall. Mater. 42, 1489-1492 (1994).

Gerischer, H. Charge transfer processes at semiconductor-electrolyte interfaces in connection with problems of catalysis. Surf. Sci. 18, 97 (1969).

Gerischer, H. in "Semiconductor Liquid-Junction Solar Cells" (A. Heller, ed.), Electrochemical Society, Princeton, New Jersey (1977).

Gherasimova, M., Cui, G., Jeon, S. R., Ren, Z., Martos, D., Han, J., He, Y. \& Nurmikko, A. V. Droplet heteroepitaxy of GaN quantum dots by metal-organic chemical vapor deposition. Appl. Phys. Lett. 85, 2346-2348 (2004).

Gherasoiu, I., Yu, K. M., Reichertz, L. a., Kao, V. M., Hawkridge, M., Ager, J. W. \& Walukiewicz, W. High quality $\ln _{\mathbf{x}} \mathrm{Ga}_{1-\mathrm{x}} \mathrm{N}$ thin films with $\mathrm{x}>\mathbf{0 . 2}$ grown on silicon. Phys. Status Solidi 247, 1747-1749 (2010).

Ghosh, R. \& Basak, D. Quantum confinement of excitons in dendrite-like GaN nanowires. J. Appl. Phys. 98, 1-4 (2005).

Giménez, S. \& Bisquert, B., "Photoelectrochemical Solar Fuel Production From Basic Principles to Advanced Devices." Springer International Publishing, Switzerland (2016).

Gogneau, N., Jalabert, D., Monroy, E., Shibata, T., Tanaka, M. \& Daudin, B. Structure of GaN quantum dots grown under 'modified Stranski-Krastanow' conditions on AIN. J. Appl. Phys. 94, 2254-2261 (2003). 
[Gomes1981]

[Gomez2016]

[Grandal2005]

[Grandjean2001]

[Gratzel2012]

[Green1959]

[Guha1993]

[Guibaly\&Colbow1982]

[Guijarro2015]

[Guo2010]

[Gwo2003]

[Gwo2004]

[Ha1998]

[Hashimoto1997]

[Hayashi2012]

[Heller1981]

[Helmholtz1853]

[Henry1980]
Gomes, F.C.W.P. \& Dekeyser, W. "Photovoltaic and Photoelectrochemical Solar Energy Conversion", Plenum Press, New York (1981).

Gómez, V. J., Gačević, Ž., Soto-Rodríguez, P. E. D., Aseev, P., Nötzel, R., Calleja, E. \& Sánchez-García, M. A. Comparative study of single InGaN layers grown on Si(111) and GaN(0001) templates: The role of surface wetting and epitaxial constraint. J. Cryst. Growth 447, 48-54 (2016).

Grandal, J. \& Sánchez-García, M. A. InN layers grown on silicon substrates: effect of substrate temperature and buffer layers. J. Cryst. Growth 278, 373-377 (2005).

Grandjean, N., Damilano, B. \& Massies, J. Group-III nitride quantum heterostructures grown by molecular beam epitaxy. J. Phys. Condens. Matter 13, 6945-6960 (2001).

Gratzel, M., Krol, R. "Photoelectrochemical Hydrogen Production." Springer, New York (2012).

Green, M. Electrochemistry of the Semiconductor-Electrolyte Electrode. I. The Electrical Double Layer. J. Chem. Phys. 31, 200 (1959).

Guha, S., DePuydt, J. M., Haase, M. A., Qiu, J. \& Cheng, H. Degradation of II-VI based blue-green light emitters. Appl. Phys. Lett. 63, 3107 (1993).

El Guibaly, F. \& Colbow, K. Theory of photocurrent in semiconductor-electrolyte junction solar cells. J. Appl. Phys. 53, 1737 (1982).

Guijarro, N., Prévot, M. S. \& Sivula, K. Surface modification of semiconductor photoelectrodes. Phys. Chem. Chem. Phys. 17, 15655-15674 (2015).

Guo, W., Zhang, M., Banerjee, A. \& Bhattacharya, P. Catalyst-Free InGaN/GaN Nanowire Light Emitting Diodes Grown on (001) Silicon by Molecular Beam Epitaxy. Nano Lett. 10, 3355-3359 (2010).

S. Gwo, Method for growing group-III nitride semiconductor heterostructure on silicon substrate, Patent, US 7012016 B2, (2003)

Gwo, S., Wu, C. L., Shen, C. H., Chang, W. H., Hsu, T. M., Wang, J. S. \& Hsu, J. T. Heteroepitaxial growth of wurtzite $\ln \mathrm{N}$ films on Si(111) exhibiting strong near-infrared photoluminescence at room temperature. Appl. Phys. Lett. 84, 3765-3767 (2004).

Ha, J.S., Park, K.H., Yun, W. S., Lee, E. H. \& Park, S.J. Interaction of low-energy nitrogen ions with an Si(111)-7x7 surface: STM and LEED investigations. Appl. Phys. A Mater. Sci. Process. 66, 495-499 (1998).

Hashimoto, A., Aiba, Y. \& Motizuki, T. Initial growth stage of GaN on Si substrate by alternating source supply using dimethyl-hydrazine. J. Cryst. Growth 175/176, 129-133 (1997).

Hayashi, T., Deura, M. \& Ohkawa, K. High stability and efficiency of GaN photocatalyst for hydrogen generation from water. Jpn. J. Appl. Phys. 51, 6-9 (2012).

Heller, A. Conversion of sunlight into electrical power and photoassisted electrolysis of water in photoelectrochemical cells. Acc. Chem. Res. 14, 154-162 (1981).

Helmholtz, H. Ueber einige Gesetze der Vertheilung elektrischer Ströme in körperlichen Leitern mit Anwendung auf die thierisch-elektrischen Versuche. Ann. der Phys. und Chemie 165, 211-233 (1853).

Henry, C. H. Limiting efficiencies of ideal single and multiple energy gap terrestrial solar cells. J. Appl. Phys. 51, 4494 (1980). 
[Hersee2011]

[Herzog2011]

[Hiramatsu1991]

[Ho\&Stringfellow1996]

[Hodes2012]

[Hoffbauer2013]

[Holmes2014]

[Hooper1996]

[Horikoshi1988]

[Hsiao2005]

[Hsu\&Walukiewicz2008]

[Hsu2014]

[Hu2013]

[Huang2002b]

[Huang2002c]

[Huygens2000]

[Hwang2012]
Hersee, S.D., Rishinaramangalam, A.K., Fairchild, M.N., Zhang, L. \& Varangis, P. Threading defect elimination in GaN nanowires. J. Mater. Res. 26, 2293-2298 (2011).

Herzog J.B. "Optical spectroscopy of colloidal CdSe semiconductor nanostructures", PhD Thesis, University of Notre Dame, Indiana, USA (2011).

Hiramatsu, K., Itoh, S., Amano, H., Akasaki, I., Kuwano, N., Shiraishi, T. \& Oki, K. Growth mechanism of GaN grown on sapphire with A1N buffer layer by MOVPE. J. Cryst. Growth 115, 628-633 (1991).

Ho, I. \& Stringfellow, G. B. Solid phase immiscibility in GalnN. Appl. Phys. Lett. 69, 2701 (1996).

Hodes, G. Photoelectrochemical Cell Measurements: Getting the Basics Right. J. Phys. Chem. Lett. 3, 1208-1213 (2012).

Hoffbauer, M. A., Williamson, T. L., Williams, J. J., Fordham, J. L., Yu, K. M., Walukiewicz, W. \& Reichertz, L. A. In-rich InGaN thin films: Progress on growth, compositional uniformity, and doping for device applications. J. Vac. Sci. Technol. B Microelectron. Nanom. Struct. 31, 03 C114 (2013).

Holmes, M.J., Choi, K., Kako, S., Arita, M. \& Arakawa, Y. Room-temperature triggered single photon emission from a III-nitride site-controlled nanowire quantum dot. Nano Lett. 14, 982-6 (2014).

Hooper, S. E., Foxon, C. T., Cheng, T. S., Jeffs, N. J., Ren, G. B., Lacklison, D. E., Orton, J. W. \& Duggan, G. The Growth of GaN Films by Migration-Enhanced Epitaxy. MRS Proc. 449, 325 (1996).

Horikoshi, Y., Kawashima, M. \& Yamaguchi, H. Migration-Enhanced Epitaxy of GaAs and AIGaAs. Jpn. J. Appl. Phys. 27, 169-179 (1988).

Hsiao, C. L., Tu, L. W., Chen, M., Jiang, Z. W., Fan, N. W., Tu, Y. J. \& Wang, K. R.

Polycrystalline to single-crystalline InN grown on $\mathrm{Si}(111)$ substrates by plasma-assisted molecular-beam epitaxy. Japanese J. Appl. Physics, Part 2 Lett. 44, (2005).

Hsu, L. \& Walukiewicz, W. Modeling of InGaN/Si tandem solar cells. J. Appl. Phys. 104, 024507 (2008).

Hsu, L.H., Lin, C.C., Han, H.V., Lin, D.W., Lo, Y.H., Hwang, Y.C. \& Kuo, H.C. Enhanced photocurrent of a nitride-based photodetector with InN dot-like structures. Opt. Mater. Express 4, 2565 (2014).

Hu, S., Xiang, C., Haussener, S., Berger, A. D. \& Lewis, N. S. An analysis of the optimal band gaps of light absorbers in integrated tandem photoelectrochemical watersplitting systems. Energy Environ. Sci. 6, 2984-2993 (2013).

Huang, J., Ye, Z., Wang, L., Yuan, J., Zhao, B. \& Lu, H. Comparison of GaN epitaxial films on silicon nitride buffer and Si(111). Solid. State. Electron. 46, 1231-1234 (2002).

Huang, Y., Duan, X., Cui, Y. \& Lieber, C.M. Gallium Nitride Nanowire Nanodevices. Nano Lett. 2, 101-104 (2002).

Huygens, I. M., Strubbe, K. \& Gomes, W. P. Electrochemistry and Photoetching of $\mathbf{n}$ GaN. J. Electrochem. Soc. 147, 1797 (2000).

Hwang, Y. J., Wu, C. H., Hahn, C., Jeong, H. E. \& Yang, P. Si/InGaN Core/Shell Hierarchical Nanowire Arrays and their Photoelectrochemical Properties. Nano Lett. 12, 1678-1682 (2012). 
[Iliopoulos2006]

[Intonti2001]

[Ishikawa1998]

[Ishikawa2011]

[Ivanov2014]

[Jahan2005]

[Ji2015]

[Jmerik2012]

[Juodkazyte2014]

[Kaiser1999]

[Kamimura2013]

[Kang2014]

[Kautek1980]

[Kawasaki2001]

[Kehagias2013]
Iliopoulos, E., Georgakilas, A., Dimakis, E., Adikimenakis, A., Tsagaraki, K., Androulidaki, M. \& Pelekanos, N. T. InGaN(0001) alloys grown in the entire composition range by plasma assisted molecular beam epitaxy. Phys. status solidi 203, 102-105 (2006).

Intonti, F., Emiliani, V., Lienau, C., Elsaesser, T., Nötzel, R. \& Ploog, K. Near-field optical spectroscopy of localized and delocalized excitons in a single GaAs quantum wire. Phys. Rev. B 63, (2001).

Ishikawa, H., Yamamoto, K., Egawa, T., Soga, T., Jimbo, T. \& Umeno, M. Thermal stability of GaN on (111) Si substrate. J. Cryst. Growth 189-190, 178-182 (1998).

Ishikawa, H. \& Shimanaka, K. Reduction of threading dislocations in GaN on in-situ meltback-etched Si substrates. J. Cryst. Growth 315, 196-199 (2011).

Ivanov, S. V., Shubina, T. V., Komissarova, T. A. \& Jmerik, V. N. Metastable nature of InN and In-rich InGaN alloys. J. Cryst. Growth 403, 83-89 (2014).

Jahan, C., Faynot, O., Tosti, L. \& Hartmann, J. M. Agglomeration control during the selective epitaxial growth of Si raised sources and drains on ultra-thin silicon-oninsulator substrates. J. Cryst. Growth 280, 530-538 (2005).

Ji, W., Qi, W., Tang, S., Peng, H. \& Li, S. Hydrothermal Synthesis of Ultrasmall Pt Nanoparticles as Highly Active Electrocatalysts for Methanol Oxidation. Nanomaterials 5, 2203-2211 (2015).

Jmerik, V. N., Mizerov, A. M., Nechaev, D. V., Aseev, P. A., Sitnikova, A. A., Troshkov, S. I., Kop'ev, P. S. \& Ivanov, S. V. Growth of thick AIN epilayers with droplet-free and atomically smooth surface by plasma-assisted molecular beam epitaxy using laser reflectometry monitoring. J. Cryst. Growth 354, 188-192 (2012).

Juodkazytė, J., Šebeka, B., Savickaja, I., Kadys, A., Jelmakas, E., Grinys, T., Juodkazis, S., Juodkazis, K. \& Malinauskas, T. $\ln _{\mathbf{x}} \mathbf{G a}_{1-\mathbf{x}} \mathbf{N}$ performance as a band-gap-tunable photoelectrode in acidic and basic solutions. Sol. Energy Mater. Sol. Cells 130, 36-41 (2014).

Kaiser, U., Khodos, I. I., Jinschek, J., Richter, W., Festkorperphysik, W., Universitiit, F. \& Jena, D. A TEM study of local non-uniformities in epitaxial $2 \mathrm{H}$-AIN films on Si (111) substrate. J. Electron Microsc. (Tokyo). 48, 545-554 (1999).

Kamimura, J., Bogdanoff, P., Lahnemann, J., Hauswald, C., Geelhaar, L., Fiechter, S. \& Riechert, H. Photoelectrochemical properties of (In,Ga)N nanowires for water splitting investigated by in-situ electrochemical mass spectroscopy. J. Am. Chem. Soc. 135, 10242-10245 (2013).

Kang, J.-H., Kim, S. H., Ebaid, M., Lee, J. K. \& Ryu, S.-W. Efficient photoelectrochemical water splitting by a doping-controlled GaN photoanode coated with NiO cocatalyst. Acta Mater. 79, 188-193 (2014).

Kautek, W., Gobrecht, J. \& Gerischer, H. The Applicability of Semiconducting Layered Materials for Electrochemical Solar Energy Conversion. Ber. Bunsenges. Phys. Chemie 84, 1034-1040 (1980).

Kawasaki, K., Yamazaki, D., Kinoshita, A., Hirayama, H., Tsutsui, K. \& Aoyagi, Y. GaN quantum-dot formation by self-assembling droplet epitaxy and application to singleelectron transistors. Appl. Phys. Lett. 79, 2243 (2001).

Kehagias, T., Dimitrakopulos, G. P., Ajagunna, A. O., Koukoula, T., Tsagaraki, K., Adikimenakis, A., Komninou, P. \& Georgakilas, A. Growth mechanism and microstructure of low defect density $\ln N$ (0001) In-face thin films on Si (111) substrates. J. Appl. Phys. 114, 163519 (2013). 
[Khaselev\&Turner1998]

[Kibria\&Mi2016]

[Kim\&Choi2014]

[Kim2004]

[Kim2009]

[Kim2014a]

[Kim2014b]

[King2007]

[Kishino1997]

[Kishino2009]

[Kishino2015]

[Koblmuller2007]

[Kocha1995]

[Koguchi1991]

[Koguchi1993]

[Kondo2006]
Khaselev, O. \& Turner, J. A. A Monolithic Photovoltaic-Photoelectrochemical Device for Hydrogen Production via Water Splitting. Science 280, 425-427 (1998).

Kibria, M. G. \& Mi, Z. Artificial photosynthesis using metal/nonmetal-nitride semiconductors: current status, prospects, and challenges. J. Mater. Chem. A 4, 28012820 (2016).

Kim, T. W. \& Choi, K.-S. K.-S. Nanoporous BiVO4 Photoanodes with Dual-Layer Oxygen Evolution Catalysts for Solar Water Splitting. Science. 343, 990-994 (2014).

Kim, J. S. \& Koguchi, N. Near room temperature droplet epitaxy for fabrication of InAs quantum dots. Appl. Phys. Lett. 85, 5893-5895 (2004).

Kim, D., Giermann, A. L. \& Thompson, C. V. Solid-state dewetting of patterned thin films. Appl. Phys. Lett. 95, 251903 (2009).

Kim, S.H., Ebaid, M., Kang, J.H. \& Ryu, S.W. Improved efficiency and stability of GaN photoanode in photoelectrochemical water splitting by NiO cocatalyst. Appl. Surf. Sci. 305, 638-641 (2014).

Kim, S. H., Kang, J.-H. \& Ryu, S.-W. Enhanced Water Splitting Stability with Controlled NiO Co-Catalyst on GaN Photoanode. J. Nanosci. Nanotechnol. 14, 7903-7906 (2014).

King, P.D.C., Veal, T.D., McConville, C. F., Fuchs, F., Furthmüller, J., Bechstedt, F., Schley, P., Goldhahn, R., Schörmann, J., As, D.J., Lischka, K., Muto, D., Naoi, H., Nanishi, Y., Lu, H. \& Schaff, W.J. Universality of electron accumulation at wurtzite c- and a-plane and zinc-blende InN surfaces. Appl. Phys. Lett. 91, 92101 (2007).

Yoshizawa, M., Kikuchi, A., Mori, M., Fujita, N. \& Kishino, K. Growth of Self-Organized GaN Nanostructures on Al2O3(0001) by RF-Radical Source Molecular Beam Epitaxy. Jpn. J. Appl. Phys. 36, L459-L462 (1997).

Kishino, K., Sekiguchi, H. \& Kikuchi, A. Improved Ti-mask selective-area growth (SAG) by RF-plasma-assisted molecular beam epitaxy demonstrating extremely uniform GaN nanocolumn arrays. J. Cryst. Growth 311, 2063-2068 (2009).

Kishino, K. \& Ishizawa, S. Selective-area growth of GaN nanocolumns on Si(111) substrates for application to nanocolumn emitters with systematic analysis of dislocation filtering effect of nanocolumns. Nanotechnology 26, 225602 (2015).

Koblmüller, G., Gallinat, C. S. \& Speck, J. S. Surface kinetics and thermal instability of Nface InN grown by plasma-assisted molecular beam epitaxy. J. Appl. Phys. 101, 083516 (2007).

Kocha, S.S., Peterson, M.W., Arent, D.J., Redwing, J.M., Tischler, M.A. \& Turner, J.A. Electrochemical Investigation of the Gallium Nitride-Aqueous Electrolyte Interface. J. Electrochem. Soc. 142, L238 (1995).

Koguchi, N., Takahashi, S. \& Chikyow, T. New MBE growth method for InSb quantum well boxes. J. Cryst. Growth 111, 688-692 (1991).

Koguchi, N. \& Ishige, K. Growth of GaAs Epitaxial Microcrystals on an S-Terminated GaAs Substrate by Successive Irradiation of Ga and As Molecular Beams. Jpn. J. Appl. Phys. 32, 2052-2058 (1993).

Kondo, T., Saitoh, K., Yamamoto, Y., Maruyama, T. \& Naritsuka, S. Fabrication of GaN dot structures on Si substrates by droplet epitaxy. Phys. status solidi 203, 1700-1703 (2006). 
[Kong2016]

[Kop'ev1986]

[Korona2014]

[Koukoula2014]

[Kraus1998]

[Krishnamurthy2012]

[Krost\&Dadgar2002]

[Kumar2010]

[Kumar2011]

[Kumar2013]

[Kurouchi2004]

[Kuykendall2007]

[Laboutin2008]

[Lagerstedt1974]

[Lahnemann2012]

[Lahnemann2014]
Kong, X., Li, H., Albert, S., Bengoechea-Encabo, A., Sánchez-García, M. A., Calleja, E., Draxl, C. \& Trampert, A. Titanium induced polarity inversion in ordered (In,Ga)N/GaN nanocolumns. Nanotechnology 27, 65705 (2016).

Kop'ev, P.S. Ledentsov, N.N., Meltser, B. Ya., Uraltsev, I.N., Efros, Al. L. \& Yakovlev, D.R., Abstact in Proceedings of 18th International Coference on The Physics of Semiconductors, O. Engström (ed.), World Scientific, Singapore, p.219 (1986).

Korona, K. P., Reszka, A., Sobanska, M., Perkowska, P. S., Wysmołek, A., Klosek, K. \& Zytkiewicz, Z. R. Dynamics of stacking faults luminescence in GaN/Si nanowires. J. Lumin. 155, 293-297 (2014).

Koukoula, T., Kioseoglou, J., Kehagias, T., Ajagunna, A. O., Komninou, P. \& Georgakilas, A. Self-annihilation of inversion domains by high energy defects in III-Nitrides. Appl. Phys. Lett. 104, 141914 (2014).

Kraus, A., Hildebrandt, S., Kulla, R., Wilhelmi, G. \& Neddermeyer, H. In situ observation of thermal annealing processes of nanoholes on Si(111) $7 \times 7$. Appl. Phys. A Mater. Sci. Process. 66, S953-S957 (1998).

Krishnamurthy, D., Hasegawa, S., Tawil, S. N. M., Emura, S. \& Asahi, H. Growth of InN quantum dots by droplet epitaxy and their characterization. Phys. Status Solidi 9, 666669 (2012).

Krost, A. \& Dadgar, A. GaN-based optoelectronics on silicon substrates. Mater. Sci. Eng. B 93, 77-84 (2002).

Kumar, M., Roul, B., Bhat, T. N., Rajpalke, M. K., Sinha, N., Kalghatgi, a. T. \& Krupanidhi, S. B. Droplet Epitaxy of InN Quantum Dots on Si(111) by RF Plasma-Assisted Molecular Beam Epitaxy. Adv. Sci. Lett. 3, 379-384 (2010).

Kumar, M., Rajpalke, M. K., Bhat, T. N., Roul, B., Kalghatgi, a. T. \& Krupanidhi, S. B. Size dependent bandgap of molecular beam epitaxy grown $\operatorname{InN}$ quantum dots measured by scanning tunneling spectroscopy. J. Appl. Phys. 110, 114317 (2011).

Kumar, M., Roul, B., Bhat, T. N., Rajpalke, M. K. \& Krupanidhi, S. B. Substrate impact on the growth of InN nanostructures by droplet epitaxy. Phys. Status Solidi 10, 409-412 (2013).

Kurouchi, M., Araki, T., Naoi, H., Yamaguchi, T., Suzuki, A. \& Nanishi, Y. Growth and properties of In-rich InGaN films grown on (0001) sapphire by RF-MBE. Phys. status solidi (b) 241, 2843-2848 (2004).

Kuykendall, T., Ulrich, P., Aloni, S. \& Yang, P. Complete composition tunability of InGaN nanowires using a combinatorial approach. Nat. Mater. 6, 951-6 (2007).

Laboutin, O. A. \& Welser, R. E. Impact of GaN buffer layer on the growth and properties of InN islands. Appl. Phys. Lett. 92, 223103 (2008).

Lagerstedt, O. \& Monemar, B. Luminescence in epitaxial GaN:Cd. J. Appl. Phys. 45, 2266 (1974).

Lähnemann, J., Brandt, O., Jahn, U., Pfüller, C., Roder, C., Dogan, P., Grosse, F., Belabbes, A., Bechstedt, F., Trampert, A. \& Geelhaar, L. Direct experimental determination of the spontaneous polarization of GaN. Phys. Rev. B 86, 81302 (2012).

Lähnemann, J., Jahn, U., Brandt, O., Flissikowski, T., Dogan, P. \& Grahn, H. T. Luminescence associated with stacking faults in GaN. J. Phys. D. Appl. Phys. 47, 423001 (2014). 
[Lander1964]

[Laser\&Bard1976]

[Lastras-Martinez2015]

[Lazic2015]

[Le2014]

[Lebedev2007]

[Lee1998]

[Lee2009]

[Legrand2000]

[LeLouarn2009]

[Leroux1999]

[Lester1995]

[Leszczynski1994]

[Li1985]

[Li1986]

[Li1984]

[Li2005]
Lander, J. J. Chemisorption and ordered surface structures. Surf. Sci. 1, 125-164 (1964).

Laser, D. \& Bard, A. J. “Semiconductor Electrodes." J. Electrochem. Soc. 123, 1828

(1976).

Lastras-Martínez, L. F., Ulloa-Castillo, N. A., Herrera-Jasso, R., Balderas-Navarro, R. E., Lastras-Martínez, A., Pandikunta, M., Ledyaev, O., Kuryatkov, V. \& Nikishin, S.

Characterization of Si3N4/Si(111) thin films by reflectance difference spectroscopy. Jpn. J. Appl. Phys. 54, 21501 (2015).

Lazić, S., Chernysheva, E., Gačević, Ž., van der Meulen, H. P., Calleja, E. \& Calleja Pardo, J. $M$. Dynamic control of the optical emission from GaN/InGaN nanowire quantum dots by surface acoustic waves. AIP Adv. 5, 97217 (2015).

Le, B. H., Zhao, S., Tran, N. H. \& Mi, Z. Electrically injected near-infrared light emission from single InN nanowire p-i-n diode. Appl. Phys. Lett. 105, 231124 (2014).

Lebedev, V., Tonisch, K., Niebelschütz, F., Cimalla, V., Cengher, D., Cimalla, I., Mauder, C., Hauguth, S., Ambacher, O., Morales, F. M., Lozano, J. G. \& González, D. Coalescence aspects of III-nitride epitaxy. J. Appl. Phys. 101, (2007).

Lee, C. D., Park, C., Lee, H. J., Noh, S. K., Lee, K. S. \& Park, S. J. Formation of selfassembled GaAs/AIGaAs quantum dots by low-temperature epitaxy. Appl. Phys. Lett. 73, 2615-2617 (1998).

Lee, J. H., Wang, Z. M., Kim, N. Y. \& Salamo, G. J. Size and density control of In droplets at near room temperatures. Nanotechnology 20, 285602 (2009).

Legrand, B., Agache, V., Nys, J. P., Senez, V. \& Stievenard, D. Formation of silicon islands on a silicon on insulator substrate upon thermal annealing. Appl. Phys. Lett. 76, 3271 (2000).

Le Louarn, A., Vézian, S., Semond, F. \& Massies, J. AIN buffer layer growth for GaN epitaxy on (111) Si: Al or N first? J. Cryst. Growth 311, 3278-3284 (2009).

Leroux, M., Grandjean, N., Beaumont, B., Nataf, G., Semond, F., Massies, J. \& Gibart, P. Temperature quenching of photoluminescence intensities in undoped and doped GaN. J. Appl. Phys. 86, 3721 (1999).

Lester, S.D., Ponce, F.A., Craford, M.G. \& Steigerwald, D.A. High dislocation densities in high efficiency GaN-based light-emitting diodes. Appl. Phys. Lett. 66, 1249 (1995).

Leszczynski, M., Suski, T., Teisseyre, H., Perlin, P., Grzegory, I., Jun, J., Porowski, S. \& Moustakas, T.D. Thermal expansion of gallium nitride. J. Appl. Phys. 76, 4909 (1994).

Li, J. \& Peter, L.M. Surface recombination at semiconductor electrodes: Part III. Steadystate and intensity modulated photocurrent response. J. Electroanal. Chem. Interfacial Electrochem. 193, 27-47 (1985).

Li, J. \& Peter, L.M. Surface recombination at semiconductor electrodes: Part IV. Steadystate and intensity modulated photocurrents at $\mathbf{n}$-GaAs electrodes. J. Electroanal. Chem. Interfacial Electrochem. 199, 1-26 (1986).

Li, J., Peat, R. \& Peter, L.M. Surface recombination at semiconductor electrodes: Part II. Photoinduced 'near-surface' recombination centres in p-GaP. J. Electroanal. Chem. Interfacial Electrochem. 165, 41-59 (1984).

Li, S.X., Yu, K.M., Wu, J., Jones, R. E., Walukiewicz, W., Ager, J.W., Shan, W., Haller, E.E., Lu, H. \& Schaff, W.J. Fermi-level stabilization energy in group III nitrides. Phys. Rev. B 71,161201 (2005). 
[Li2008]

[Li2015]

[Lin2015]

[Liu2005]

[Liu2013a]

[Liu2013b]

[Loitsch2013]

[Lu2001]

[Lu2007]

[Lu2014]

[Lunceford2012]

[Luo2008]

[Lymperakis2009]

[Lyons2010]

[Mansurov2015]

[Manuel2012]

[Martin1999]

[Maruska\&Tietjen1969]
Li, J., Lin, J.Y. \& Jiang, H.X. Direct hydrogen gas generation by using InGaN epilayers as working electrodes. Appl. Phys. Lett. 93, 2008-2010 (2008).

Li, H., Geelhaar, L., Riechert, H. \& Draxl, C. Computing Equilibrium Shapes of Wurtzite Crystals: The Example of GaN. Phys. Rev. Lett. 115, 085503 (2015).

Lin, F., Bachman, B.F. \& Boettcher, S.W. Impact of Electrocatalyst Activity and Ion Permeability on Water-Splitting Photoanodes. J. Phys. Chem. Lett. 6, 2427-2433 (2015).

Liu, R., Bell, A., Ponce, F.A., Chen, C.Q., Yang, J. W. \& Khan, M.A. Luminescence from stacking faults in gallium nitride. Appl. Phys. Lett. 86, 21908 (2005).

Liu, S. T., Wang, X. Q., Chen, G., Zhang, Y.W., Feng, L., Huang, C.C., Xu, F. J., Tang, N., Sang, L. W., Sumiya, M. \& Shen, B. Temperature-controlled epitaxy of InxGa1-xN alloys and their band gap bowing. J. Appl. Phys. 110, 113514 (2011).

Liu, M., Nam, C. Y., Black, C.T., Kamcev, J. \& Zhang, L. Enhancing Water splitting activity and chemical stability of zinc oxide nanowire photoanodes with ultrathin titania shells. J. Phys. Chem. C 117, 13396-13402 (2013).

Loitsch, B., Schuster, F., Stutzmann, M. \& Koblmüller, G. Reduced threading dislocation densities in high-T/N-rich grown InN films by plasma-assisted molecular beam epitaxy. Appl. Phys. Lett. 102, (2013).

Lu, H., Schaff, W. J., Hwang, J., Wu, H., Koley, G. \& Eastman, L. F. Effect of an AIN buffer layer on the epitaxial growth of InN by molecular-beam epitaxy. Appl. Phys. Lett. 79, 1489-1491 (2001).

Lu, Y. S., Huang, C. C., Yeh, J. A., Chen, C. F. \& Gwo, S. InN-based anion selective sensors in aqueous solutions. Appl. Phys. Lett. 91, (2007).

Lu, X., Xie, S., Yang, H., Tong, Y. \& Ji, H. Photoelectrochemical hydrogen production from biomass derivatives and water. Chem. Soc. Rev. 43, 7581-7593 (2014).

Lunceford, C. \& Drucker, J. Characterization of epitaxially grown In islands on Si(111). J. Vac. Sci. Technol. A Vacuum, Surfaces, Film. 30, 061509 (2012).

Luo, W., Liu, B., Li, Z., Xie, Z., Chen, D., Zou, Z. \& Zhang, R. Stable response to visible light of InGaN photoelectrodes. Appl. Phys. Lett. 92, 111-114 (2008).

Lymperakis, L. \& Neugebauer, J. Large anisotropic adatom kinetics on nonpolar GaN surfaces: Consequences for surface morphologies and nanowire growth. Phys. Rev. B 79, 241308 (2009).

Lyons J.L., Janotti, A. \& Van de Walle, C.G. Carbon impurities and the yellow luminescence in GaN, Appl. Phys. Lett. 97, 152108 (2010).

Mansurov, V., Malin, T., Galitsyn, Y. \& Zhuravlev, K. Graphene-like AIN layer formation on Si(111) surface by ammonia molecular beam epitaxy. J. Cryst. Growth 428, 93-97 (2015).

Mánuel, J.M., Morales, F.M., García, R., Aidam, R., Kirste, L. \& Ambacher, O. Threading dislocation propagation in AIGaN/GaN based HEMT structures grown on Si (111) by plasma assisted molecular beam epitaxy. J. Cryst. Growth 357, 35-41 (2012).

Martin, R.W., Middleton, P.G., O'Donnell, K.P. \& Van der Stricht, W. Exciton localization and the Stokes' shift in InGaN epilayers. Appl. Phys. Lett. 74, 263 (1999).

Maruska, H.P. \& Tietjen, J.J. The preparation and properties of vapor-deposited singlecrystal-line GaN. Appl. Phys. Lett. 15, 327-329 (1969). 
[Maruska1969]

[Maruyama2007]

[Matsubara2008]

[Matsuoka1990]

[Matthews1979]

[Mattila1999]

[Mayrhofer2008]

[Mei2014]

[Mei2014b]

[Memming2015]

[Minsky1996]

[Mittemeijer2014]

[Miyamura2002]

[Mohapatra2008]

[Monemar1997]

[Morales2009]

[Morales2013]
Maruska, H.P. \& Tietjen, J.J. The preparation and properties of vapor-deposited singlecrystal-line GaN. Appl. Phys. Lett. 15, 327-329 (1969).

Maruyama, T., Otsubo, H., Kondo, T., Yamamoto, Y. \& Naritsuka, S. Fabrication of GaN dot structure by droplet epitaxy using $\mathbf{N H}_{3}$. J. Cryst. Growth 301-302, 486-489 (2007).

Matsubara, H., Yoshimoto, S., Saito, H., Jianglin, Y., Tanaka, Y. \& Noda, S. GaN PhotonicCrystal Surface-Emitting Laser at Blue-Violet Wavelengths. Science (80-. ). 319, 445447 (2008).

Matsuoka, T., Sasaki T., \& Katsui, A. Growth and properties of a wide-gap semiconductor InGaN. Optoelectr.-Dev. and Tech. 5.1, 53-64 (1990).

Matthews, J.W., "Dislocations in Solids", edited by F. R. N. Nabarro (North-Holland, Amsterdam), Vol. 2, Chap. 7, p. 461 (1979).

Mattila, T. \& Zunger, A. Predicted bond length variation in wurtzite and zinc-blende InGaN and AIGaN alloys. J. Appl. Phys. 85, 160 (1999).

Mayrhofer, K. J. J., Crampton, A. S., Wiberg, G. K. H. \& Arenz, M. Analysis of the Impact of Individual Glass Constituents on Electrocatalysis on Pt Electrodes in Alkaline Solution. J. Electrochem. Soc. 155, P78 (2008).

Mei, B., Permyakova, A.A., Frydendal, R., Bae, D., Pedersen, T., Malacrida, P., Hansen, O., Stephens, I. E. L., Vesborg, P. C. K., Seger, B. \& Chorkendorff, I. Iron-Treated NiO as a Highly Transparent $\mathrm{p}$-Type Protection Layer for Efficient Si-Based Photoanodes. J. Phys. Chem. Lett. 5, 3456-3461 (2014).

Mei, B., Seger, B., Pedersen, T., Malizia, M., Hansen, O., Chorkendorff, I. \& Vesborg, P. C. K. Protection of $\mathbf{p}^{+}-\mathbf{n}-\mathrm{Si}$ Photoanodes by Sputter-Deposited Ir/IrO $\mathbf{I}_{\mathbf{x}}$ Thin Films. J. Phys. Chem. Lett. 5, 1948-1952 (2014).

Memming, R., "Semiconductor Electrochemistry", 2nd ed., Wiley-VCH, Weinheim, Germany (2015).

Minsky, M. S., White, M. \& Hu, E. L. Room-temperature photoenhanced wet etching of GaN. Appl. Phys. Lett. 68, 1531-1533 (1996).

Mittemeijer, E. J. \& Somers, M. A. J. "Thermochemical Surface Engineering of Steels: Improving Materials Performance." P.190 (Elsevier Science, 2014).

Miyamura, M., Tachibana, K., Someya, T. \& Arakawa, Y. Stranski-Krastanow growth of GaN quantum dots by metalorganic chemical vapor deposition. J. Cryst. Growth 237239, 1316-1319 (2002).

Mohapatra, S. K., Raja, K. S., Mahajan, V. K. \& Misra, M. Efficient photoelectrolysis of water using TiO2 nanotube arrays by minimizing recombination losses with organic additives. J. Phys. Chem. C 112, 11007-11012 (2008).

Monemar, B., Bergman, J.P. \& Buyanova I.A." Optical Properties of GaN and Related Materials. In S. J. Pearton (Ed.), GaN and Related Materials." New York: Gordon and Breach Publishers (1997).

Morales, F.M., González, D., Lozano, J.G., García, R., Hauguth-Frank, S., Lebedev, V., Cimalla, V. \& Ambacher, O. Determination of the composition of $\ln _{x} G_{a_{1-x}} N$ from strain measurements. Acta Mater. 57, 5681-5692 (2009).

Morales, F.M., Mánuel, J.M., García, R., Reuters, B., Kalisch, H. \& Vescan, A. Evaluation of interpolations of InN, AIN and GaN lattice and elastic constants for their ternary and quaternary alloys. J. Phys. D. Appl. Phys. 46, 245502 (2013). 
[Moret2009]

[Morita1981]

[Moseley2013]

[Moses2010]

[Moustakas2006]

[Moustakas2008]

[Murray1984]

[Muth1997]

[Nakamura1992a]

[Nakamura1992b]

[Nakamura1993]

[Nakamura1994]

[Nakamura1995a]

[Nakamura1995b]

[Nakato1976]

[Nakato1987a]
Moret, M., Gil, B., Ruffenach, S., Briot, O., Giesen, C., Heuken, M., Rushworth, S., Leese, T. \& Succi, M. Optical, structural investigations and band-gap bowing parameter of GalnN alloys. J. Cryst. Growth 311, 2795-2797 (2009).

Morita, M., Isogai, S., Shimizu, N., Tsubouchi, K. \& Mikoshiba, N. Aluminum Nitride Epitaxially Grown on Silicon: Orientation Relationships. Jpn. J. Appl. Phys. 20, L173L175 (1981).

Moseley, M., Gunning, B., Lowder, J., Alan Doolittle, W. \& Namkoong, G. Structural and electrical characterization of $\operatorname{InN}, \ln G a N$, and $p$-InGaN grown by metal-modulated epitaxy. J. Vac. Sci. Technol. B Microelectron. Nanom. Struct. 31, 03 C104 (2013).

Moses, P.G. \& Van de Walle, C.G. Band bowing and band alignment in InGaN alloys. Appl. Phys. Lett. 96, 21908 (2010).

Zhou, L., Xu, T., Smith, D. J. \& Moustakas, T. D. Microstructure of relaxed InN quantum dots grown on GaN buffer layers by molecular-beam epitaxy. Appl. Phys. Lett. 88, 231906 (2006).

Moustakas, T. D., Xu, T., Thomidis, C., Nikiforov, a. Y., Zhou, L. \& Smith, D. J. Growth of III-nitride quantum dots and their applications to blue-green LEDs. Phys. Status Solidi 205, 2560-2565 (2008).

Murray, J.L. \& McAlister, A.J. The Al-Si (Aluminum-Silicon) system. Bull. Alloy Phase Diagrams 5, 74-84 (1984).

Muth, J. F., Lee, J. H., Shmagin, I. K., Kolbas, R. M., Casey, H. C., Keller, B. P., Mishra, U. K. \& DenBaars, S. P. Absorption coefficient, energy gap, exciton binding energy, and recombination lifetime of GaN obtained from transmission measurements. Appl. Phys. Lett. 71, 2572 (1997).

Nakamura, S. Iwasa, N. Senoh, M. \& Mukai, T. Hole compensation mechanism of p-type GaN films, Jpn. J. Appl. Phys. 31, 1258 (1992).

Nakamura, S. Mukai, T. Senoh, M. \& Iwasa, N. Thermal annealing effects on p-type Mgdoped GaN films, Jpn. J. Appl. Phys. 31, L139 (1992).

Nakamura, S., Senoh, M. \& Mukai, T. P-GaN/N-InGaN/N-GaN Double-Heterostructure Blue-Light-Emitting Diodes. Jpn. J. Appl. Phys. 32, L8-L11 (1993).

Nakamura, S., Mukai, T. \& Senoh, M. Candela-class high-brightness InGaN/AIGaN double heterostructure blue-light-emitting diodes, Appl. Phys. Lett. 64, 1687 (1994).

Nakamura, S., Senoh, M., Iwasa, N., Nagahama, S. Yamada, T. \& Mukai, T. Superbright green InGaN single-quantum-well-structure light-emitting diodes, Jpn. J. Appl. Phys. 34, L1332 (1995).

Nakamura, S., Senoh, M., Iwasa, N. \& Nagahama, S. High-Brightness InGaN Blue, Green and Yellow Light-Emitting Diodes with Quantum Well Structures. Jpn. J. Appl. Phys. 34, L797-L799 (1995).

Nakato, Y., Tonomura, S. \& Tsubomura, H. The Catalytic Effect of Electrodeposited Metals on the Photo-Reduction of Water at p-type Semiconductors. Ber. Bunsenges. Phys. Chemie 80, 1289-1293 (1976).

Nakato, Y., Yano, H., Nishiura, S., Ueda, T. \& Tsubomura, H. Hydrogen photoevolution at p-type silicon electrodes coated with discontinuous metal layers. J. Electroanal. Chem. 228, 97-108 (1987). 
[Nakato1987b]

[Nakato1992]

[Nakato2004]

[Namkoong2008]

[Nanishi2003]

[Nanishi2009]

[Nellist2016]

[Neudeck1989]

[Neugebauer1996]

[Neugebauer2003]

[Ng2002]

[Nguyen2011]

[Norenberg2002]

[Nowok1993]

[Ohashi1977]

[Ohtani1994]
Nakato, Y. \& Tsubomura, H. Remarkably high photovoltages generated at $\mathbf{n}$-type silicon semiconductor electrodes coated with extremely small platinum islands. Berichte der Bunsengesellschaft für Phys. Chemie 91, 405-408 (1987).

Nakato, Y. \& Tsubomura, H. Silicon photoelectrodes modified with ultrafine metal islands. Electrochim. Acta 37, 897-907 (1992).

Nakato, K., Takabayashi, S., Imanishi, A., Murakoshi, K. \& Nakato, Y. Stabilization of n-Si electrodes by surface alkylation and metal nano-dot coating for use in efficient photoelectrochemical solar cells. Sol. Energy Mater. Sol. Cells 83, 323-330 (2004).

Namkoong, G., Trybus, E., Lee, K.K., Moseley, M., Doolittle, W.A. \& Look, D.C. Metal modulation epitaxy growth for extremely high hole concentrations above $10^{19} \mathrm{~cm}^{-3}$ in GaN. Appl. Phys. Lett. 93, 172112 (2008).

Nanishi, Y., Saito, Y. \& Yamaguchi, T. RF-molecular beam epitaxy growth and properties of InN and related alloys. Japanese J. Appl. Physics, Part 1 Regul. Pap. Short Notes Rev. Pap. 42, 2549-2559 (2003).

Nanishi, Y. \& Yamaguchi, T. In Droplet Elimination by Radical Beam Irradiation for Reproducible and High-Quality Growth of InN by RF Molecular Beam Epitaxy. Appl. Phys. express 2, 51001 (2009).

Nellist, M. R., Laskowski, F. A. L., Lin, F., Mills, T. J. \& Boettcher, S. W. SemiconductorElectrocatalyst Interfaces: Theory, Experiment, and Applications in Photoelectrochemical Water Splitting. Acc. Chem. Res. 49, 733-740 (2016).

Neudeck, G.W., The PN Junction Diode; from "Modular Series on Solid State Devices", Vol. 2, Neudeck, G.W., Pierret R.F., Eds, 2nd ed., Addison-Wesley, Reading, Massachusetts (1989).

Neugebauer J. \& Van de Walle, C.G. Gallium vacancies and the yellow luminescence in GaN, Appl. Phys. Lett. 69, 503 (1996).

Neugebauer, J., Zywietz, T., Scheffler, M., Northrup, J., Chen, H. \& Feenstra, R. Adatom Kinetics On and Below the Surface: The Existence of a New Diffusion Channel. Phys. Rev. Lett. 90, 56101 (2003).

Ng, Y.F., Cao, Y.G., Xie, M.H., Wang, X.L. \& Tong, S.Y. Growth mode and strain evolution during InN growth on GaN(0001) by molecular-beam epitaxy. Appl. Phys. Lett. 81, 3960 (2002).

Nguyen, H. P. T., Zhang, S., Cui, K., Han, X., Fathololoumi, S., Couillard, M., Botton, G. A. \& Mi, Z. p-Type Modulation Doped InGaN/GaN Dot-in-a-Wire White-Light-Emitting Diodes Monolithically Grown on Si(111). Nano Lett. 11, 1919-1924 (2011).

Nörenberg, C., Oliver, R. A., Martin, M. G., Allers, L., Castell, M. R. \& Briggs, G. A. D. Stranski-Krastanov growth of InN nanostructures of GaN studied by RHEED, STM and AFM. Phys. Status Solidi Appl. Res. 194, 536-540 (2002).

Nowok, J.W. A universal relation between diffusion, viscosity and surface tension in liquid metals in capillary-like media. Scr. Metall. Mater. 29, 931-935 (1993).

Ohashi, K., McCann, J. \& Bockris, J. O. Hydrogen and electricity from water and light. Int. J. Energy Res. 1, 259-277 (1977).

Ohtani, A., Stevens, K. S. \& Beresford, R. Microstructure and photoluminescence of GaN grown on Si(111) by plasma-assisted molecular beam epitaxy. Appl. Phys. Lett. 65, 61 (1994). 
[Olesinski1985a]

[Olesinski1985b]

[Oliver2008]

[Osterloh2013]

[Parkinson\&Turner2013]

[Pereira2001]

[Perez2009]

[Peter1984]

[Peter2013]

[Petroff1976]

[Pierret1996]

[Piprek2007]

[Ploog1998]

[Pocza1969]

[Ponce2002]

[Ponomarev\&Peter1995]

[Qi2013]

[Ranade2000]
Olesinski, R.W., Kanani, N. \& Abbaschian, G.J. The Ga-Si (Gallium-Silicon) system. Bull. Alloy Phase Diagrams 6, 362-364 (1985).

Olesinski, R.W., Kanani, N. \& Abbaschian, G.J. The In-Si (In-Silicon) system. Bull. Alloy Phase Diagrams 6, 128-130 (1985).

Oliver, R.A., Jarjour, A.F., Taylor, R.A., Tahraoui, A., Zhang, Y., Kappers, M.J. \& Humphreys, C.J. Growth and assessment of InGaN quantum dots in a microcavity: A blue single photon source. Mater. Sci. Eng. B Solid-State Mater. Adv. Technol. 147, 108113 (2008).

Osterloh, F.E. Inorganic nanostructures for photoelectrochemical and photocatalytic water splitting. Chem. Soc. Rev. 42, 2294-2320 (2013).

Parkinson, B. \& Turner, J. "Photoelectrochemical Water Splitting: Materials, Processes and Architectures", Royal Society of Chemistry, Cambridge, UK (2013).

Pereira, S., Correia, M. R., Monteiro, T., Pereira, E., Alves, E., Sequeira, A. D. \& Franco, N. Compositional dependence of the strain-free optical band gap in $\ln _{\mathbf{x}} \mathrm{Ga}_{1-\mathrm{x}} \mathrm{N}$ layers. Appl. Phys. Lett. 78, 2137 (2001).

Perez, R. and Perez, M., "A fundamental look at energy reserves for the planet." The International Energy Agency SHC Programme Solar Update, 50, 2-3, (2009).

Peter, L. M., Li, J. \& Peat, R. Surface recombination at semiconductor electrodes: Part I. Transient and steady-state photocurrents. J. Electroanal. Chem. Interfacial Electrochem. 165, 29-40 (1984).

Peter, L. M., "Photoelectrochemical Water Splitting Materials, Processes and Architectures." RSC Energy and Environment Series No. 9, Cambridge, UK (2013).

Petroff, P. M., Lorimor, O. G. \& Ralston, J. M. Defect structure induced during forwardbias degradation of GaP green-light-emitting diodes. J. Appl. Phys. 47, 1583-1588 (1976).

Pierret, R.F., "Semiconductor Device Fundamentals", McGraw-Hill, New York, pp. 490492 (1996).

Piprek, J. Nitride semiconductor devices, WILEY-VCH, Germany, (2007).

Ploog, K.H. Nucleation and growth of GaN layers on GaAs, Si, and SiC substrates. J. Vac. Sci. Technol. B Microelectron. Nanom. Struct. 16, 2229 (1998).

Pócza, J.F., Barna, A. \& Barna, P.B. Formation Processes of Vacuum-Deposited In Films and Thermodynamical Properties of Submicroscopic Particles Observed by In Situ Electron Microscopy. J. Vac. Sci. Technol. 6, 472 (1969).

Hu, C.W., Bell, A., Ponce, F.A., Smith, D.J. \& Tsong, I.S.T. Growth of self-assembled GaN quantum dots via the vapor-liquid-solid mechanism. Appl. Phys. Lett. 81, 3236 (2002).

Ponomarev, E.A. \& Peter, L.M. A generalized theory of intensity modulated photocurrent spectroscopy (IMPS). J. Electroanal. Chem. 396, 219-226 (1995).

Qi, B., Ólafsson, S., Göthelid, M., Gislason, H.P. \& Agnarsson, B. Photoemission and low energy electron microscopy study on the formation and nitridation of In droplets on $\mathrm{Si}$ (111)7×7 surfaces. Thin Solid Films 531, 61-69 (2013).

Ranade, M.R., Tessier, F., Navrotsky, A., Leppert, V.J., Risbud, S.H., DiSalvo, F.J. \& Balkas, C.M. Enthalpy of Formation of Gallium Nitride. J. Phys. Chem. B 104, 4060-4063 (2000). 
[Rashid2013]

[Ratsch1994]

[Rawdanowicz2004]

[Rawdanowicz2005]

[Rayleigh1879]

[Reichertz2008]

[Reichman1980]

[Reshchikov2005]

[Ristic2008]

[Robins2007]

[Romanyuk2009]

[Rosner1997]

[Ruffenach2005]

[Ruffenach2007]

[Saitoh2008]

[Sakalauskas2010]
Rashid, M. A., Yusuf, A., Humayun, A. Al, Kareem, A., Mahmood, N. \& Tamaki, S. Stability Analysis of Solar Cell Characteristics Above Room Temperature. 10, 1345-1350 (2013).

Ratsch, C., Zangwill, A., Šmilauer, P. \& Vvedensky, D.D. Saturation and scaling of epitaxial island densities. Phys. Rev. Lett. 72, 3194-3197 (1994).

Rawdanowicz, T.A. \& Narayan, J. Epitaxial GaN on Si(111): Process control of SiNx interlayer formation. Appl. Phys. Lett. 85, 133 (2004).

Rawdanowicz, T.A. "Laser Molecular Beam Epitaxial Growth and Properties of IIINitride Thin Film Heterostructures on Silicon." PhD thesis, North Carolina State University, USA (2005).

Rayleigh, L. On the Stability, or Instability, of certain Fluid Motions. Proc. London Math. Soc. s1-11, 57-72 (1879).

Reichertz, L.A., Yu, K.M., Cui, Y., Hawkridge, M.E., Beeman, J.W., Liliental-Weber, Z., Ager III, J.W., Walukiewicz, W., Schaff, W.J., Williamson, T.L. \& Hoffbauer, M.A. InGaN Thin Films Grown by ENABLE and MBE Techniques on Silicon Substrates. MRS Proc. 1068, (2008).

Reichman, J. The current-voltage characteristics of semiconductor-electrolyte junction photovoltaic cells. Appl. Phys. Lett. 36, 574 (1980).

Reshchikov, M.A. \& Morkoć, H. Luminescence properties of defects in GaN. J. Appl. Phys. 97, 061301 (2005).

Ristić, J., Calleja, E., Fernández-Garrido, S., Cerutti, L., Trampert, A., Jahn, U. \& Ploog, K.H. On the mechanisms of spontaneous growth of III-nitride nanocolumns by plasmaassisted molecular beam epitaxy. J. Cryst. Growth 310, 4035-4045 (2008).

Robins, L.H., Bertness, K.A., Barker, J.M., Sanford, N.A. \& Schlager, J.B. Optical and structural study of GaN nanowires grown by catalyst-free molecular beam epitaxy. I. Near-band-edge luminescence and strain effects. J. Appl. Phys. 101, (2007).

Romanyuk, Y.E., Kreier, D., Cui, Y., Yu, K.M., Ager, J.W. \& Leone, S.R. Molecular beam epitaxy of InGaN thin films on Si(111): Effect of substrate nitridation. Thin Solid Films 517, 6512-6515 (2009).

Rosner, S.J., Carr, E.C., Ludowise, M.J., Girolami, G. \& Erikson, H.I. Correlation of cathodoluminescence inhomogeneity with microstructural defects in epitaxial GaN grown by metalorganic chemical-vapor deposition. Appl. Phys. Lett. 70, 420 (1997).

Ruffenach, S., Maleyre, B., Briot, O. \& Gil, B. Growth of InN quantum dots by MOVPE. Phys. status solidi 2, 826-832 (2005).

Ruffenach, S., Briot, O., Moret, M. \& Gil, B. Control of InN quantum dot density using rare gases in metal organic vapor phase epitaxy. Appl. Phys. Lett. 90, 153102 (2007).

Saitoh, H., Utsumi, W. \& Aoki, K. Decomposition of InN at high pressures and temperatures and its thermal instability at ambient conditions. J. Cryst. Growth 310, 473-476 (2008).

Sakalauskas, E., Schley, P., Räthel, J., Klar, T. A., Müller, R., Pezoldt, J., Tonisch, K., Grandal, J., Sánchez-García, M. A., Calleja, E., Vilalta-Clemente, A., Puteran, R. \& Roldhah, G. Optical properties of InN grown on Si(111) substrate. Phys. Status Solidi Appl. Mater. Sci. 207, 1066-1069 (2010). 
[Sam-Giao2013]

[Sanchez-Garcia1998]

[Schenk1999]

[Schuck2001]

[Seger2009]

[Seger2012]

[Seger2013]

[Sekiguchi2008]

[Sekiguchi2010a]

[Sekiguchi2010b]

[Serrano2010]

[Shen2006]

[Shi2015]

[Shockley1961]

[Simmonds2012]
Sam-Giao, D., Mata, R., Tourbot, G., Renard, J., Wysmolek, A., Daudin, B. \& Gayral, B. Fine optical spectroscopy of the $3.45 \mathrm{eV}$ emission line in GaN nanowires. J. Appl. Phys. 113, (2013).

Sánchez-García, M.A., Calleja, E., Monroy, E., Sanchez, F.J., Calle, F., Munoz, E. \& Beresford, $R$. The effect of the III/ $\mathrm{V}$ ratio and substrate temperature on the morphology and properties of $\mathrm{GaN}$ - and AIN-layers grown by molecular beam epitaxy on Si(1 1 1). J. Cryst. Growth 183, 23-30 (1998).

Schenk, H. P. D., Kaiser, U., Kipshidze, G. D., Fissel, A., Kra, J., Hobert, H., Schulze, J. \& Richter, W. Growth of atomically smooth AIN films with a $5: \mathbf{4}$ coincidence interface on Si ( 111 ) by MBE. Mater. Sci. Eng. B 59, 84-87 (1999).

Schuck, P.J., Mason, M.D., Grober, R. D., Ambacher, O., Lima, A. P., Miskys, C., Dimitrov, R. \& Stutzmann, M. Spatially resolved photoluminescence of inversion domain boundaries in GaN-based lateral polarity heterostructures. Appl. Phys. Lett. 79, 952954 (2001).

Seger, B. \& Kamat, P.V. Fuel cell geared in reverse: Photocatalytic hydrogen production using a $\mathrm{TiO}_{2} /$ Nafion/Pt membrane assembly with no applied bias. J. Phys. Chem. C 113, 18946-18952 (2009).

Seger, B., (Max) Lu, G. Q. \& Wang, L. Electrical power and hydrogen production from a photo-fuel cell using formic acid and other single-carbon organics. J. Mater. Chem. 22, 10709 (2012).

Seger, B., Tilley, S.D., Pedersen, T., Vesborg, P.C.K., Hansen, O., Grätzel, M. \& Chorkendorff, I. Silicon protected with atomic layer deposited $\mathrm{TiO}_{2}$ : conducting versus tunnelling through $\mathrm{TiO}_{2}$. J. Mater. Chem. A 1, 15089 (2013).

Sekiguchi, H., Kishino, K. \& Kikuchi, A. Ti-mask Selective-Area Growth of GaN by RFPlasma-Assisted Molecular-Beam Epitaxy for Fabricating Regularly Arranged InGaN/GaN Nanocolumns. Appl. Phys. Express 1, 124002 (2008).

Sekiguchi, H., Kishino, K. \& Kikuchi, A. Emission color control from blue to red with nanocolumn diameter of InGaN/GaN nanocolumn arrays grown on same substrate. Appl. Phys. Lett. 96, 231104 (2010).

Sekiguchi, H., Kishino, K. \& Kikuchi, A. Formation of InGaN quantum dots in regularly arranged GaN nanocolumns grown by rf-plasma-assisted molecular-beam epitaxy. Phys. Status Solidi Curr. Top. Solid State Phys. 7, 2374-2377 (2010).

Serrano, A., Rodríguez de la Fuente, O. \& García, M.A. Extended and localized surface plasmons in annealed Au films on glass substrates. J. Appl. Phys. 108, 074303 (2010).

Shen, C.H., Lin, H.W., Lee, H. M., Wu, C.L., Hsu, J.T. \& Gwo, S. Self-assembled InN quantum dots grown on $\mathrm{AIN} / \mathrm{Si}(111)$ and $\mathrm{GaN} / \mathrm{Al}_{2} \mathrm{O}_{3}(0001)$ by plasma-assisted molecular-beam epitaxy under Stranski-Krastanow mode. Thin Solid Films 494, 79-83 (2006).

Shi, Y., Guo, H., Yang, J., Zhao, M., Liu, J., Xue, C. \& Tang, J. Plasma-Induced Wafer-Scale Self-Assembly of Silver Nanoparticles and Application to Biochemical Sensing. Materials (Basel). 8, 3806-3814 (2015).

Shockley, W. \& Queisser, H.J. Detailed Balance Limit of Efficiency of p-n Junction Solar Cells. J. Appl. Phys. 32, 510 (1961).

Simmonds, P.J. \& Larry Lee, M. Tensile-strained growth on low-index GaAs. J. Appl. Phys. 112, 54313 (2012). 
[Singh1995]

[Sobanska2012]

[Soto2013]

[Srolovitz1986]

[Srolovitz1995]

[Stampfl1998]

[Stevens1994]

[Stranski1937]

[Stringfellow2010]

[Stroscio1994]

[Stutzmann2001]

[Sugahara1998]

[Sugavaneshwar2012]

[Sun2013]

[Sun2015]

[Sun2015b]
Singh, R. \& Moustakas, T.D. Growth of InGaN Films by MBE at the Growth Temperature of GaN. MRS Proc. 395, 163 (1995).

Sobanska, M., Klosek, K., Zytkiewicz, Z.R., Borysiuk, J., Witkowski, B.S., Lusakowska, E., Reszka, A. \& Jakiela, R. Plasma-assisted MBE growth of GaN on Si(111) substrates. Cryst. Res. Technol. 47, 307-312 (2012).

Soto Rodriguez, P.E.D., Kumar, P., Gómez, V. J., Alvi, N. H., Mánuel, J. M., Morales, F. M., Jiménez, J. J., García, R., Calleja, E. \& Nötzel, R. Spontaneous formation of InGaN nanowall network directly on Si. Appl. Phys. Lett. 102, 173105 (2013).

Srolovitz, D.J. \& Safran, S.A. Capillary instabilities in thin films. I. Energetics. J. Appl. Phys. 60, 247 (1986).

Srolovitz, D.J. \& Goldiner, M.G. The Thermodynamics and Kinetics of film agglomeration. JOM 47, 31-36 (1995).

Stampfl, C. \& Van de Walle, C. Energetics and electronic structure of stacking faults in AIN, GaN, and InN. Phys. Rev. B 57, R15052-R15055 (1998).

Stevens, K.S. Growth of group III nitrides on Si(111) by plasma-assisted molecular beam epitaxy. J. Vac. Sci. Technol. B Microelectron. Nanom. Struct. 12, 1186 (1994).

Stranski, I. N. \& Krastanow, L. Zur Theorie der orientierten Ausscheidung von Ionenkristallen aufeinander. Monatshefte für Chemie 71, 351-364 (1937). // Stranski I. N., Krastanow L. 1937 "Zur Theorie der orientierten Ausscheidung von lonenkristallen aufeinander" Sitz. Ber. Akad. Wiss. Wien, Mat. Nat. K1. Ilb 146, 797 (1937).

Stringfellow, G.B. Microstructures produced during the epitaxial growth of InGaN alloys. J. Cryst. Growth 312, 735-749 (2010).

Stroscio, J.A. \& Pierce, D.T. Scaling of diffusion-mediated island growth in iron-on-iron homoepitaxy. Phys. Rev. B 49, 8522-8525 (1994).

Stutzmann, M., Ambacher, O., Eickhoff, M., Karrer, U., Pimenta, A.L., Neuberger, R., Schalwig, J., Dimitrov, R., Schuck, P.J. \& Grober, R.D. Playing with Polarity. Phys. Status Solidi 228, 505-512 (2001).

Sugahara, T., Sato, H., Hao, M., Naoi, Y., Kurai, S., Tottori, S., Yamashita, K., Nishino, K., Romano, L. T. \& Sakai, S. Direct Evidence that Dislocations are Non-Radiative Recombination Centers in GaN. Jpn. J. Appl. Phys. 37, L398-L400 (1998).

Sugavaneshwar, R. P., Nagao, T. \& Nanda, K. K. Fabrication of Highly Dense Nanoholes by Self-Assembled Gallium Droplet on Silicon Surface. Mater. Express 2, 245-250 (2012).

Sun, X. \& Li, H. Gold nanoisland arrays by repeated deposition and post-deposition annealing for surface-enhanced Raman spectroscopy. Nanotechnology 24, 355706 (2013).

Sun, K., Kuang, Y., Verlage, E., Brunschwig, B. S., Tu, C. W. \& Lewis, N. S. Sputtered NiO Films for Stabilization of $\mathbf{p}^{+} \mathbf{n}$-InP Photoanodes for Solar-Driven Water Oxidation. Adv. Energy Mater. 5, 1402276 (2015).

Sun, K., Saadi, F. H., Lichterman, M. F., Hale, W. G., Wang, H.-P., Zhou, X., Plymale, N. T., Omelchenko, S. T., He, J.-H., Papadantonakis, K. M., Brunschwig, B. S. \& Lewis, N. S. Stable solar-driven oxidation of water by semiconducting photoanodes protected by transparent catalytic nickel oxide films. Proc. Natl. Acad. Sci. 112, 3612-3617 (2015). 
[Synge1928]

[Sze2007]

[Takayanagi1987]

[Tamura2001]

[Tan1991]

[Tanaka1996]

[Tang2011]

[Taylor1988]

[Telieps1985]

[Thompson2000]

[Thompson2012]

[Tilley2010]

[Tischer2011]

[Tringides2010]

[Tseng2014]

[Turner1998]
Sun, K., McDowell, M. T., Nielander, A. C., Hu, S., Shaner, M. R., Yang, F., Brunschwig, B. S. \& Lewis, N. S. Stable solar-driven water oxidation to $\mathrm{O}_{2}(\mathrm{G})$ by Ni-Oxide-coated silicon photoanodes. J. Phys. Chem. Lett. 6, 592-598 (2015).

Synge, E.H. A suggested method for extending microscopic resolution into the ultramicroscopic region. Philos. Mag. J. Sci. 6, 356-362 (1928).

Sze, S.M., Ng, K.K., "Physics of Semiconductor Devices." Third edition WileyInterscience, New Jersey, US (2007).

Takayanagi, K., Tanishiro, Y., Kobayashi, K., Akiyama, K. \& Yagi, K. Surface Structures Observed by High-Resolution UHV Electron Microscopy at Atomic Level. Jpn. J. Appl. Phys. 26, L957-L960 (1987).

Tamura, M., López-López, M. \& Yodo, T. GaN growth on (111) Si with very thin amorphous SiN layer by ECR plasma-assisted MBE. Superf. y Vacio 13, 80-88 (2001).

Tan, M. X., Newcomb, C., Kumar, A., Lunt, S. R., Sailor, M. J., Tufts, B. J. \& Lewis, N. S. Chemical modification of $n-G a A s$ photoanodes with group VIIIB Metal ions: stability in contact with $1.0 \mathrm{M} \mathrm{KOH}(\mathrm{aq})-0.10 \mathrm{M} \mathrm{K}_{2} \mathrm{Se}(\mathrm{aq})$ solutions and I-V properties in contact with 1.0 M KOH(aq)-0.3 M K ${ }_{2} \mathrm{Te}(\mathrm{aq})$ electrolytes. J. Phys. Chem. 95, 10133-10142 (1991).

Tanaka, S., Iwai, S. \& Aoyagi, Y. Self-assembling GaN quantum dots on $\mathbf{A l}_{\mathbf{x}} \mathbf{G a}_{1-\mathbf{x}} \mathbf{N}$ surfaces using a surfactant. Appl. Phys. Lett. 69, 4096 (1996).

Tang, J., Photopoulos, P., Tserepi, A. \& Tsoukalas, D. Two-dimensional nanoparticle selfassembly using plasma-induced Ostwald ripening. Nanotechnology 22, 235306 (2011).

Taylor, J. E. \& Cahn, J. W. Theory of orientation textures due to surface energy anisotropies. J. Electron. Mater. 17, 443-445 (1988).

Telieps, W. \& Bauer, E. The $(7 \times 7)-(1 \times 1)$ phase transition on Si(111). Surf. Sci. 162, 163-168 (1985).

Thompson, C. V. Structure Evolution During Processing of Polycrystalline Films. Annu. Rev. Mater. Sci. 30, 159-190 (2000).

Thompson, C. V. Solid-State Dewetting of Thin Films. Annu. Rev. Mater. Res. 42, 399434 (2012).

Tilley, S. D., Cornuz, M., Sivula, K. \& Gratzel, M. Light-induced water splitting with hematite: Improved nanostructure and iridium oxide catalysis. Angew. Chemie - Int. Ed. 49, 6405-6408 (2010).

Tischer, I., Feneberg, M., Schirra, M., Yacoub, H., Sauer, R., Thonke, K., Wunderer, T., Scholz, F., Dieterle, L., Müller, E. \& Gerthsen, D. I $\mathbf{2}$ basal plane stacking fault in GaN: Origin of the $\mathbf{3 . 3 2} \mathrm{eV}$ luminescence band. Phys. Rev. B 83, 35314 (2011).

Tringides, M.C. \& Hupalo, M. Surface diffusion experiments with STM: equilibrium correlations and non-equilibrium low temperature growth. J. Phys. Condens. Matter 22, 264002 (2010).

Tseng, W.J., van Dorp, D.H., Lieten, R.R., Vereecken, P.M. \& Borghs, G. Anodic Etching of n-GaN Epilayer into Porous GaN and Its Photoelectrochemical Properties. J. Phys. Chem. C 118, 29492-29498 (2014).

Khaselev, O. \& Turner, J.A. A Monolithic Photovoltaic-Photoelectrochemical Device for Hydrogen Production via Water Splitting. Science 280, 425-427 (1998). 
[Ueda1997]

[Uosaki\&Kita1985]

[Urban2013]

[Urban2015]

[Vajpeyi2009]

[Valdueza-Felip2014]

[Veal2006]

[Vechten1992]

[Vurgaftman2001]

[Vurgaftman2003]

[Wacaser2009]

[Wagner1964]

[Walther2000]

[Walther2012]

[Walukiewicz2011]

[Wang2001]
Ueda, O., Isozumi, S., Kotani, T. \& Yamaoka, T. Defect structure of $\langle\mathbf{1 0 0}\rangle$ dark lines in the active region of a rapidly degraded $\mathrm{Ga}_{1-\mathrm{x}} \mathbf{A l}_{\mathbf{x}}$ As LED. J. Appl. Phys. 48, 3950 (1977).

Uosaki, K. \& Kita, H., in: “Modern Aspects of Electrochemistry No. 18", editor: Bockris, A.M., Plenum Press, New York, (1985).

Urban, A. "Position-controlled selective area growth of Ga-polar GaN nanocolumns by molecular beam epitaxy: A versatile approach towards semipolar GaN and the characterization of single nanocolumns", PhD Thesis, Georg-August-Universität Göttingen, Germany (2013).

Urban, A., Müller, M., Karbaum, C., Schmidt, G., Veit, P., Malindretos, J., Bertram, F., Christen, J. \& Rizzi, A. Optical Emission of Individual GaN Nanocolumns Analyzed with High Spatial Resolution. Nano Lett. 15, 5105-5109 (2015).

Vajpeyi, A.P., Ajagunna, A.O., Tsagaraki, K., Androulidaki, M. \& Georgakilas, A. InGaN nanopillars grown on silicon substrate using plasma assisted molecular beam epitaxy. Nanotechnology 20, 325605 (2009).

Valdueza-Felip, S., Bellet-Amalric, E., Núñez-Cascajero, A., Wang, Y., Chauvat, M.P., Ruterana, P., Pouget, S., Lorenz, K., Alves, E. \& Monroy, E. High In-content InGaN layers synthesized by plasma-assisted molecular-beam epitaxy: Growth conditions, strain relaxation, and In incorporation kinetics. J. Appl. Phys. 116, 233504 (2014).

Veal, T. D., Jefferson, P. H., Piper, L. F. J., McConville, C. F., Joyce, T. B., Chalker, P. R., Considine, L., Lu, H. \& Schaff, W. J. Transition from electron accumulation to depletion at InGaN surfaces. Appl. Phys. Lett. 89, 202110 (2006).

Van Vechten, J.A., Zook, J.D., Horning, R.D. \& Goldenberg, B. Defeating compensation in wide gap semiconductors by growing in $\mathrm{H}$ that is removed by low temperature deionizing radiation, Jpn. J. Appl. Phys. 31, 3662 (1992).

Vurgaftman, I., Meyer, J. R. \& Ram-Mohan, L. R. Band parameters for III-V compound semiconductors and their alloys. J. Appl. Phys. 89, 5815 (2001).

Vurgaftman, I. \& Meyer, J.R. Band parameters for nitrogen-containing semiconductors, J. Appl. Phys. 94, 3675 (2003).

Wacaser, B.A., Dick, K.A., Johansson, J., Borgström, M.T., Deppert, K. \& Samuelson, L. Preferential Interface Nucleation: An Expansion of the VLS Growth Mechanism for Nanowires. Adv. Mater. 21, 153-165 (2009).

Wagner, R. S. \& Ellis, W. C. Vapor-Liquid-Solid mecahnism of single crystal growth. Appl. Phys. Lett. 4, 89 (1964).

Walther, C., Blum, R.P., Niehus, H., Thamm, A. \& Masselink, W.T. Modified fermi-level pinning of the (100) GaAs surface through InAs quantum dots in different stages of overgrowth. J. Electron. Mater. 29, 504-509 (2000).

Walther, R., Litvinov, D., Fotouhi, M., Schneider, R., Gerthsen, D., Vöhringer, R., Hu, D. Z. \& Schaadt, D. M. Microstructure of PA MBE-grown InN layers on Si(111). J. Cryst. Growth 340, 34-40 (2012).

Walukiewicz, W. \& Gherasoiu, I. "Tandem Photoelectrochemical Cell for Water Dissociation." US 2011/0005590 A1 (2011).

Wang, X.-S., Zhai, G., Yang, J., Wang, L., Hu, Y., Li, Z., Tang, J. C., Wang, X., Fung, K. K. \& Cue, N. Nitridation of Si(111). Surf. Sci. 494, 83-94 (2001). 
[Wang2009]

[Wang2011]

[Wang2013]

[Wang2016]

[Watanabe2000]

[Widmann1998]

[Williams1993]

[Williams1996]

[Wilson1977]

[Winterbottom1967]

[Witten1981]

[Wu2002b]

[Wu2002a]

[Wu2003]

[Wu2004b]

[Wu2004a]

[Wu2006]
Wang, H., Jiang, D. S., Zhu, J. J., Zhao, D. G., Liu, Z. S., Wang, Y. T., Zhang, S. M. \& Yang, H. Kinetically controlled InN nucleation on $\mathrm{GaN}$ templates by metalorganic chemical vapour deposition. J. Phys. D. Appl. Phys. 42, 145410 (2009).

Wang, D., Pierre, A., Kibria, M. G., Cui, K., Han, X., Bevan, K. H., Guo, H., Paradis, S., Hakima, A.-R. \& Mi, Z. Wafer-Level Photocatalytic Water Splitting on GaN Nanowire Arrays Grown by Molecular Beam Epitaxy. Nano Lett. 11, 2353-2357 (2011).

Wang, K., Araki, T., Yu, K. M., Katsuki, T. \& Mayer, M.A. P-type InGaN across the entire alloy composition range $\mathrm{P}$-type InGaN across the entire alloy composition range. Appl. Phys. Lett. 102111, (2013).

Wang, P., Yuan, Y., Zhao, C., Wang, X., Zheng, X., Rong, X., Wang, T., Sheng, B., Wang, Q., Zhang, Y., Bian, L., Yang, X., Xu, F., Qin, Z., Li, X., Zhang, X. \& Shen, B. Lattice-PolarityDriven Epitaxy of Hexagonal Semiconductor Nanowires. Nano Lett. 16, 1328-1334 (2016).

Watanabe, K., Koguchi, N. \& Gotoh, Y. Fabrication of GaAs Quantum Dots by Modified Droplet Epitaxy. Jpn. J. Appl. Phys. 39, L79-L81 (2000).

Widmann, F., Simon, J., Daudin, B., Feuillet, G., Rouvière, J. L., Pelekanos, N. T. \& Fishman, G. Blue-light emission from GaN self-assembled quantum dots due to giant piezoelectric effect. Phys. Rev. B 58, R15989-R15992 (1998).

Williams, R.H., Shen, T.H., \& Hooper, S., "Semiconductor Interfaces at the SubNanometer Scale", ed. H.W.M Salemink and M.D. Pashley; Dordrecht, the Netherlands: Kluwer Academic Publishers (1993).

Williams, D.B., Carter, C.B. "Transmission Electron Microscopy: A Textbook for Materials Science", Plenum Press (1996).

Wilson, R. H. A model for the current-voltage curve of photoexcited semiconductor electrodes. J. Appl. Phys. 48, 4292 (1977).

Winterbottom, W. Equilibrium shape of a small particle in contact with a foreign substrate. Acta Metall. 15, 303-310 (1967).

Witten, T. A. \& Sander, L. M. Diffusion-Limited Aggregation, a Kinetic Critical Phenomenon. Phys. Rev. Lett. 47, 1400-1403 (1981).

Wu, C.L., Hsieh, J.L., Hsueh, H.D. \& Gwo, S. Thermal nitridation of the Si(111)-(7×7) surface studied by scanning tunneling microscopy and spectroscopy. Phys. Rev. B 65, 45309 (2002).

Wu, J., Walukiewicz, W., Yu, K. M., Ager, J. W., Haller, E. E., Lu, H. \& Schaff, W. J. Small band gap bowing in $\ln (1-x) G a(x) N$ alloys. Appl. Phys. Lett. 80, 4741 (2002).

Wu, C. L., Wang, J. C., Chan, M. H., Chen, T. T. \& Gwo, S. Heteroepitaxy of GaN on $\mathrm{Si}(111)$ realized with a coincident-interface $\mathrm{AIN} / \boldsymbol{\beta}-\mathrm{Si}_{3} \mathrm{~N}_{4}(0001)$ double-buffer structure. Appl. Phys. Lett. 83, 4530-4532 (2003).

Wu, C.-L., Chou, L.-J. \& Gwo, S. Size- and shape-controlled GaN nanocrystals grown on Si(111) substrate by reactive epitaxy. Appl. Phys. Lett. 85, 2071 (2004).

Wu, J., Walukiewicz, W., Li, S. X., Armitage, R., Ho, J. C., Weber, E. R., Haller, E. E., Lu, H., Schaff, W. J., Barcz, A. \& Jakiela, R. Effects of electron concentration on the optical absorption edge of InN. Appl. Phys. Lett. 84, 2805-2807 (2004).

Wu, C.-L., Shen, C.-H., Chen, H.-Y., Tsai, S.-J., Lin, H.-W., Lee, H.-M., Gwo, S., Chuang, T.F., Chang, H.-S. \& Hsu, T. M. The effects of AIN buffer on the properties of InN epitaxial 
films grown on $\mathrm{Si}(111)$ by plasma-assisted molecular-beam epitaxy. J. Cryst. Growth 288, 247-253 (2006).

[Wu2009]

[Wulff1901]

[Xia1993]

[Yahyaie\&Lewis2011]

[Yamada2002]

[Yamaguchi2003]

[Yamaguchi2013]

[Yamamoto2013]

[Yamazaki2009]

[Yan2015]

[Yang2014]

[Yeh1992]

[Yip2012]

[Yoshizawa1997]

[Yu2014]
Wu, J. When group-III nitrides go infrared: New properties and perspectives. J. Appl. Phys. 106, 11101 (2009).

Wulff, G. "XXV. Zur frage der geschwindigkeit des wachsthums und der auflösung der krystallflächen." Zeitschrift für Kristallographie-Crystalline Materials 34.1, 449-530 (1901).

Xia, H., Xia, Q. \& Ruoff, A. L. High-pressure structure of gallium nitride: Wurtzite-torocksalt phase transition. Phys. Rev. B 47, 12925-12928 (1993).

Yahyaie, I., McEleney, K., Walter, M., Oliver, D. R., Thomson, D. J., Freund, M. S. \& Lewis, N. S. Electrical Characterization of Si Microwires and of Si Microwire/Conducting Polymer Composite Junctions. J. Phys. Chem. Lett. 2, 675-680 (2011).

Yamada, M., Mitani, T., Narukawa, Y., Shioji, S., Niki, I., Sonobe, S., Deguchi, K., Sano, M. \& Mukai, T. InGaN-based near-ultraviolet and blue-light-emitting diodes with high external quantum efficiency using a patterned sapphire substrate and a mesh electrode, Jpn. J. Appl. Phys. 41, L1431 (2002).

Yamaguchi, T., Saito, Y., Morioka, C., Yorozu, K., Araki, T. \& Suzuki, A. Effect of AIN buffer layer on the growth of InN epitaxial film on Si substrate. 432, 429-432 (2003).

Yamaguchi, T., Uematsu, N., Araki, T., Honda, T., Yoon, E. \& Nanishi, Y. Growth of thick InGaN films with entire alloy composition using droplet elimination by radical-beam irradiation. J. Cryst. Growth 377, 123-126 (2013).

Yamamoto, A., Mihara, A., Hironaga, D., Sugita, K., Bhuiyan, A. G., Hashimoto, A., Shigekawa, N. \& Watanabe, N. MOVPE growth of $\ln _{x} G_{a} a_{1-x} N(x \sim 0.5)$ on Si(111) substrates with a pn junction on the surface. Phys. status solidi 10, 437-440 (2013).

Yamazaki, S., Yatsui, T. \& Ohtsu, M. Room-temperature growth of UV-emitting dendritic GaN fractal nanostructures using photochemical vapor deposition. Appl. Phys. Express 2, (2009).

Yan, L., Jahangir, S., Wight, S. A., Nikoobakht, B., Bhattacharya, P. \& Millunchick, J. M. Structural and Optical Properties of Disc-in-Wire InGaN/GaN LEDs. Nano Lett. 15, 15351539 (2015).

Yang, H. Bin, Miao, J., Hung, S.-F., Huo, F., Chen, H. M. \& Liu, B. Stable Quantum Dot Photoelectrolysis Cell for Unassisted Visible Light Solar Water Splitting. ACS Nano 8, 10403-10413 (2014).

Yeh, C.Y., Lu, Z.W., Froyen, S., \& Zunger, A. Zinc-blende/wurtzite polytypism in semiconductors, Phys. Rev. B 46, 10086-97 (1992).

Yip, H.L. \& Jen, A. K.Y. Recent advances in solution-processed interfacial materials for efficient and stable polymer solar cells. Energy Environ. Sci. 5, 5994 (2012).

Yoshizawa, M., Kikuchi, A., Mori, M., Fujita, N. \& Kishino, K. Growth of Self-Organized GaN Nanostructures on Al2O3(0001) by RF-Radical Source Molecular Beam Epitaxy. Jpn. J. Appl. Phys. 36, L459-L462 (1997).

Yu, I.S., Chang, C.P., Yang, C.P., Lin, C.T., Ma, Y.R. \& Chen, C.C. Characterization and density control of GaN nanodots on $\mathrm{Si}$ (111) by droplet epitaxy using plasma-assisted molecular beam epitaxy. Nanoscale Res. Lett. 9, 682 (2014). 
[Zamir2001]

[Zhao2016]

[Zhu2013]

[Zhuang\&Edgar2005]
Zamir, S., Meyler, B. \& Salzman, J. Thermal microcrack distribution control in GaN layers on Si substrates by lateral confined epitaxy. Appl. Phys. Lett. 78, 288 (2001).

Zhao, S. \& Mi, Z. InN Nanowires: Epitaxial Growth, Characterization, and Device Applications. Semicond. Semimetals 1-38 (2016).

Zhu, D., Wallis, D.J. \& Humphreys, C.J. Prospects of III-nitride optoelectronics grown on Si. Rep. Prog. Phys. 76, 106501 (2013).

Zhuang, D. \& Edgar, J.H. Wet etching of GaN, AIN, and SiC: a review. Mater. Sci. Eng. R Reports 48, 1-46 (2005). 